Béatrice Haenggeli-Jenni

\title{
L'Éducation nouvelle entre science et militance
}

Débats et combats à travers la revue Pour l'Ère Nouvelle (1920-1940) 
Cet ouvrage étudie l'Éducation nouvelle dans ses dimensions scientifiques et militantes. Porté par des praticiens convaincus qu'une réforme de l'école pourra transformer le monde, le mouvement attire aussi des scientifiques persuadés que la science a un rôle à jouer dans la quête d'une harmonie entre les hommes. Tous se rejoignent dans la volonté de construire une éducation basée sur une connaissance scientifique de l'enfant: selon eux, la pédagogie ne doit plus être un art mais une science.

Ce livre s'intéresse à la relation qu'entretiennent ici la science et la militance : quelle est la part de l'une et l'autre dans les revendications de l'Éducation nouvelle? Comment le discours scientifique se mêle-t-il au discours militant ? Qui sont les acteurs engagés dans le mouvement et comment concilient-ils ces deux facettes dans leur activité ?

Dans une démarche d'histoire sociale, cet ouvrage puise ses apports dans la lecture fine d'une revue emblématique du mouvement, organe de la Ligue internationale pour l'Éducation nouvelle. II étudie les questions en débat telles que les méthodes d'enseignement, le rôle du maître, la place des savoirs scolaires ou la liberté en éducation. Ce livre attache une importance particulière aux acteurs engagés pour cette cause et s'intéresse à leurs profils, réseaux et activités aux niveaux local et international.

Béatrice Haenggeli-Jenni est collaboratrice scientifique à l'Université de Genève, rattachée aux Archives Institut J.J. Rousseau et à l'Équipe de Recherche en Histoire Sociale de l'Éducation (ERHISE). Ses travaux portent sur les mouvements de réformes pédagogiques au $20^{\mathrm{e}}$ siècle, sur l'internationalisation de l'éducation et le rôle qu'y jouent les femmes, dans une perspective transnationale. 
L'Éducation nouvelle entre science et militance 
La pluralité des disciplines et des perspectives en sciences de l'éducation définit la vocation de la collection Exploration, celle de carrefour des multiples dimensions de la recherche et de l'action éducative. Sans exclure l'essai, Exploration privilégie les travaux investissant des terrains nouveaux ou développant des méthodologies et des problématiques prometteuses.

Collection de la Société Suisse pour la Recherche en Education, publiée sous la direction de Georges Felouzis, Rita Hofstetter, Nicole Rege Colet, Bernard Schneuwly et Bernard Wentzel. 
Béatrice Haenggeli-Jenni

\section{L'Éducation nouvelle entre science et militance}

Débats et combats à travers la revue Pour l'Ère Nouvelle (1920-1940) 
Information bibliographique publiée par «Die Deutsche Nationalbibliothek» «Die Deutsche Nationalbibliothek» répertorie cette publication dans la «Deutsche Nationalbibliografie»; les données bibliographiques détaillées sont disponibles sur Internet sous 〈http:/ /dnb.d-nb.de〉.

Publié avec le soutien du Fonds National Suisse de la recherche scientifique, des Archives Institut J.J. Rousseau, de la Faculté de psychologie et des sciences de l'éducation et la Maison de l'Histoire de l'Université de Genève.

Réalisation couverture: Didier Studer, Peter Lang AG.

ISBN 978-3-0343-2096-2 br.

ISBN 978-3-0343-2906-4 eBook

ISSN 0721-3700 br.

ISSN 2235-6312 eBook

ISBN 978-3-0343-2907-1 EPUB

ISBN 978-3-0343-2908-8 MOBI

DOI $10.3726 /$ b11334

\section{Peter LANG (c)}

Open Access: Cette œuvre est mise à disposition selon les termes de la Licence Creative Commons Attribution - Pas d'Utilisation Commerciale - CC-BY-NC-ND 4.0. Pour consulter une copie de cette licence, visitez le site internet https://creativecommons.org/licenses/by-nc-nd/4.0/

Cette publication a fait l'objet d'une évaluation par les pairs.

Béatrice Haenggeli-Jenni 2017

Wabernstrasse 40, CH-3007 Berne, Suisse

bern@peterlang.com,www.peterlang.com 


\section{Table des matières}

REMERCIEMENTS 1

INTRODUCTION

Un mouvement social, politique et international 8

Sciences de l'éducation, pédologie et sciences contributives 11

$\begin{array}{ll}\text { Méthode et questions de recherche } & 16\end{array}$

CHAPITRE 1

LA LIGUE INTERNATIONALE POUR L'ÉDUCATION NOUVELLE:

ÉMERGENCE ET STRUCTURATION 19

Moments fondateurs et leaders charismatiques 19

Beatrice Ensor $\quad 22$

Elisabeth Rotten $\quad 23$

Adolphe Ferrière $\quad 25$

Charte et principes de ralliement: des textes pour fédérer $\quad 27$

L'âge d'or: ancrage et (re)structuration (1927-1930) 33

Baromètres et boussoles: les congrès internationaux 36

CHAPITRE 2

PLATEFORMES D'ÉCHANGES, PRISMES CULTURELS:

TROIS REVUES FONDATRICES

Das Werdende Zeitalter: une militance empreinte de philosophie $\quad 42$

Une rédaction structurée et partagée $\quad 42$

Des thématiques dominées par la philosophie $\quad 49$

Un large réseau d'auteurs germanophones 53

The New Era: de la théosophie à la «Home Education» 59

Structure et rédaction: sous le contrôle de Beatrice Ensor 59

Une histoire marquée par un changement d'orientation 62

Auteurs et thématiques: une palette de spécialistes

pour des numéros spéciaux 
Pour l'Ère nouvelle: de l'ère Ferrière (1922-1930) à l'œuvre du GFEN (1930-1940) 75

Infrastructure et comité: une histoire en deux temps $\quad 75$

Les coulisses de la rédaction $\quad 89$

La composition des numéros: des choix stratégiques 97

L'influence des membres de la rédaction 102

Les années «après Ferrière»: un comité imprégné du GFEN 107

Évolution des thématiques 112

Des auteurs éclectiques: professions et origines diverses $\quad 122$

Mise en regard: principes communs, différences assumées $\quad 130$

Des thématiques sous influence 135

$\begin{array}{ll}\text { Trois configurations contrastées } & 138\end{array}$

CHAPITRE 3

DES AGENTS ET DES RÉSEAUX:

PROFILS D'UNE MILITANCE INTERNATIONALISÉE 143

Acteurs hybrides et polyvalents 143

$\begin{array}{ll}\text { Trois groupes de professionnels } & 145\end{array}$

Diverses formes d'hybridité 153

Des femmes productrices de savoirs $\quad 161$

Praticiennes de haut niveau $\quad 162$

Des femmes actives dans l'éducation de la petite enfance $\quad 165$

Femmes de l'ombre: secrétaires, trésorières, «épouses de»... 166

Des «passeuses de savoirs» 170

Réseaux de relations aux contours multiples $\quad 172$

Réseaux formels et informels 172

Adolphe Ferrière et les écoles nouvelles 176

Beatrice Ensor et les théosophes $\quad 179$

Elisabeth Rotten et le réseau Quaker 185

Réseaux protestants et chrétiens sociaux 189

Plateformes d'échanges et diffusion de savoirs 191

CHAPITRE 4

ESPACES DE CONSTRUCTION, TERRAINS PARTAGÉS 195

Écoles, classes, instituts: emblèmes d'un mouvement qui s'institutionnalise 196

Écoles et instituts 196 
Institutions de recherche et formation $\quad 200$

Des voyages suscitant l'écriture $\quad 202$

Destinations privilégiées $\quad 204$

Groupes et associations: des lieux privilégiés pour

donner corps à la militance 208

Les associations: diversité et multiplicité 209

Visites et conférences: attirer de nouveaux membres 214

Les sections nationales: des valeurs sûres 217

Les revues: outils de propagande nécessaires pour

«faire mouvement» $\quad 221$

Les revues de francophonie $\quad 221$

Les revues officielles de la Ligue $\quad 225$

Congrès et colloques: terrains de rencontre entre science et militance 233

Un rythme à deux temps $\quad 234$

Des lieux à l'image de ses membres 236

Constituer un inconscient collectif 238

Convivialité, fraternité vs tensions, dissensions 240

CHAPITRE 5

CONCEPTS ET PRATIQUES EN CONTROVERSES 249

Conceptions de l'enfant: de l'être biologique à l'être social 249

Un être biologique et naturel 250

L'enfant sujet 253

Une conception en évolution (1920-1940) 254

Autonomie et apprentissage: deux facettes en tension 259

Apprendre par l'expérience: gage d'autonomie? 260

Autonomie et liberté: synonymes ou incompatibles? 263

Le self-government: une fausse liberté 267

Rôle du maître: entre recommandations et injonctions 269

Connaître, aimer, guider $\quad 270$

Consignes pour un nouveau rôle 276

Renouveler la formation $\quad 278$

Paradoxes et ambivalences $\quad 282$ 
CHAPITRE 6

SOCLES THÉORIQUES ET SAVOIRS EN DÉBATS

Aux origines: théories de référence de l’Éducation nouvelle 287

Les science naturelles 290

Nature, naturalisme et romantisme $\quad 292$

Philosophie, sciences sociales et spirituelles 295

La psychologie et ses dérivés 298

Savoirs scientifiques et savoirs d'expérience:

une légitimité discutée 301

Savoirs d'expérience formalisés 302

Savoirs scientifiques en observation $\quad 305$

Des savoirs «perméables» $\quad 307$

L'enjeu scolaire: programmes et savoirs en débat 311

Alléger les programmes pour libérer l'enfant 313

Évacuer les symboles de l'école traditionnelle $\quad 318$

Des principes en débat: inquiétudes et désaccords 323

Les limites de la liberté: le rôle de l'adulte 326

CONCLUSION 331

Une revue-carrefour $\quad 331$

Science et militance: les paradoxes d'une science en construction 332

Scientifiques et militants à la recherche d'une synthèse 339

BIBLIOGRAPHIE

Sources $\quad 343$

Sources publiées $\quad 343$

Références bibliographiques $\quad 344$ 


\section{REMERCIEMENTS}

Cet ouvrage est une version remaniée de ma thèse de doctorat en Sciences de l'éducation présentée à l'Université de Genève en septembre 2011.

Je remercie les membres du jury ainsi que les deux personnes ayant effectué l'expertise de ce volume dont les commentaires et suggestions ont permis d'enrichir et affiner mon propos.

Ma profonde gratitude à la Professeure Rita Hofstetter, ma directrice de thèse, pour ses relectures attentives, sa disponibilité et son soutien dans la réalisation de cet ouvrage.

Toute ma reconnaissance aux membres de ma famille qui m'ont soutenue et encouragée dans toutes les étapes de cette recherche. Leur patience et leur présence au quotidien m'ont apporté la confiance et l'énergie nécessaires pour aller jusqu'au bout de cette aventure.

Une pensée particulière pour mon père à qui je dédie cet ouvrage en mémoire de son ouverture d'esprit et de son inlassable soutien dans mes projets les plus ambitieux. 


\section{LISTE DES ABRÉVIATIONS}

EN: Éducation nouvelle PEN: Pour l'Ėre Nouvelle

BIEN: Bureau international des Écoles Nouvelles

LIEN: Ligue internationale pour l'Éducation nouvelle NEF: New Education Fellowship

GFEN: Groupe Français d'Éducation nouvelle

BIE: Bureau international de l'Éducation

IJJR: Institut Jean-Jacques Rousseau

SDN: Société des Nations

WFEA: World Federation of Educational Associations

AIJJR: Archives Institut J.-J. Rousseau 


\section{INTRODUCTION}

À la fin du $19^{\mathrm{e}}$ siècle, la construction des États nations et l'avènement des démocraties dans les pays occidentaux entraînent la mise sur pied de systèmes publics d'enseignement et de formation. Ceux-ci favorisent la généralisation de la scolarisation offrant une instruction gratuite, publique et obligatoire. Il s'agit de permettre à tous les élèves, quelles que soient leurs origines et appartenances sociales, d'accéder à une instruction dont le but ultime est de former des citoyens éclairés. Le défi, immense, est relevé avec brio, puisqu'en moins de 20 ans, des milliers d'établissements scolaires sont construits dans les villes comme dans les plus petits villages. Cette scolarisation de masse entraîne un besoin accru d'enseignants dont la formation se met en place au même moment, à travers la création d'institutions de formation, à l'exemple des écoles normales dans les régions francophones. Elle entraîne aussi des difficultés dans la gestion de l'hétérogénéité de ces grandes cohortes d'élèves dont les niveaux sont très divers. Malgré les efforts des administrations scolaires, des mouvements de contestation se forment peu à peu en marge de ces systèmes critiquant les dimensions encyclopédiques et scolastiques des méthodes de l'école publique. Parmi ces mouvements, l'Éducation nouvelle - Reformpädagogik, New Education ${ }^{1}$ - est l'un des plus influents et des plus étendus sur le plan international. Ses promoteurs proposent une autre conception de l'école et des méthodes d'enseignement liées à une posture nouvelle à l'égard de l'enfant. Une posture influencée par des précurseurs - Rousseau, Pestalozzi, Lamarck - dont ils se revendiquent mais aussi par des théories, notamment évolutionnistes qui, transforment le concept de développement et influencent durablement le regard porté sur l'enfant. Incarnant le principe même d'évolution, l'enfant devient un objet d'étude idéal pour observer les lois du développement. Désormais, il prend une importance nouvelle car il est associé à l'image de progrès, de renouveau et d'espoir.

1 Aux États-Unis, le mouvement prend le nom de Progressive Education, dans les pays hispanophones on parle de Nueva Educación, tandis que le Brésil et le Portugal le désignent par Escola Nova. 
Cette posture séduit les responsables directs de l'éducation - parents, instituteurs, inspecteurs, directeurs - mais aussi des intellectuels sensibles aux questions éducatives tels que médecins, psychologues, psychanalystes, biologistes. Aussi différents soient-ils, ces professionnels partagent une immense foi en la science. Le tournant du siècle coïncide avec l'émergence de nouvelles sciences ${ }^{2}$ étudiant l'homme et le social selon une approche empirique qui renouvelle l'appréhension des phénomènes sociaux. Parmi elles, la pédologie 3 ou child study - appelée plus tard psychologie de l'enfant - incarne la nouvelle science de l'éducation, étudiant les phénomènes éducatifs à travers une approche expérimentale articulée à la vie sociale. Pour les promoteurs de l'Éducation nouvelle, elle est symbole de progrès et est investie du pouvoir de résoudre les problèmes sociaux. En lien étroit avec ces nouvelles appréhensions de l'enfance, ils plaident pour un «renversement copernicien» (Claparède, 1905), c'est-à-dire une éducation qui renverse la logique habituelle d'enseignement et place désormais l'élève, et non plus les savoirs (programmes), au centre de l'action éducative. Ce renversement est à la base des revendications de l'Éducation nouvelle qui utilise cet argument pour se démarquer de la pédagogie dite traditionnelle considérée comme inadaptée à l'évolution de la société et aux progrès de la science. Ces nouvelles conceptions de l'enfant entraînent des changements importants dans le monde éducatif que ce soit au niveau des méthodes d'enseignement, des programmes scolaires ou de la formation du corps enseignant.

Pour pouvoir partager leurs expériences, doutes et réflexions, les réformateurs créent des associations, certaines débordant les frontières nationales. Elles prennent une ampleur considérable dans la période d'entre-deux-guerres attestant de l'extension du mouvement. Bien que portées par des praticiens, elles sont fréquentées par de nombreux scientifiques qui y partagent leurs savoirs, leurs réflexions, leurs recherches, contribuant de ce fait à l'élaboration d'une nouvelle science de l'éducation. Ces associations se caractérisent avant tout par leur activité militante visant à étendre le mouvement à l'ensemble des systèmes d'enseignement. Elles tentent de mettre en synergie les connaissances

2 La psychologie, l'anthropologie et la sociologie en sont des exemples.

3 La pédologie tire ses origines du grec: «paîs» signifiant l'enfant et «logia» l'étude, la science. La pédologie désigne donc «l'étude de l'enfant» ou «science de l'enfant». 
amassées jusque-là, tant sur le plan théorique que pratique, dans le but ultime de «changer l'école». Il s'agit surtout, pour ces acteurs, de s'unir pour mieux faire entendre leurs voix, pour construire un discours crédible dans le but de convaincre le politique et l'opinion publique du bien-fondé de leurs méthodes. Plusieurs travaux d'historiens (Hameline, 2002a; Hofstetter \& Schneuwly, 2006, 2009a; Németh, 2006; Savoye, 2004) montrent que c'est vers la science que ces acteurs se tournent pour élaborer leur discours, cherchant auprès d'elle une forme de légitimation, voire de caution. Notre questionnement porte sur cette relation intrigante qu'entretiennent ici la science et la militance: quelle est la part de l'une et l'autre dans les revendications de réforme de l'Éducation nouvelle? Comment le discours scientifique se mêle-t-il au discours militant? Comment les acteurs concilient-ils ces deux facettes dans leur activité? Il nous intéresse de comprendre comment s'élaborent les discours de propagande, comment les théories scientifiques y sont convoquées, et quelle crédibilité les praticiens leur accordent.

Nous avons cherché à identifier les revendications fondamentales de l'Éducation nouvelle, à repérer les sciences de référence qui les façonnent et à connaître les acteurs et actrices qui les promeuvent. Notre questionnement se décline en quatre axes à travers lesquels nous avons étudié les relations entre science et militance: 1) Quels sont les profils professionnels et les réseaux de sociabilité des acteurs de l'Éducation nouvelle? 2) Dans quels espaces œuvrent-ils pour donner corps à leurs propositions de réforme? Quels sont leurs champs d'action privilégiés? 3) Quelles sont leurs conceptions éducatives et convictions profondes en matière d'éducation? Ces conceptions font-elles l'unanimité de points de vue ou sontelles sujettes à controverses? 4) Quels types de savoirs ces acteurs prônent-ils au niveau théorique vs pratique? À quelles sciences ou théories se réfèrent-ils pour construire leur plaidoyer en faveur de la réforme?

C'est à travers l'étude approfondie d'une revue, Pour l'Ère nouvelle, que notre recherche ${ }^{4}$ ambitionne de répondre à cet ensemble de questions. Organe francophone de la Ligue internationale pour l'Éducation

4 Cette recherche a été réalisée dans le cadre d'un mandat d'assistante à l'Université de Genève et a bénéficié d'un subisde candoc du FNS (100013117915) sous la direction de R. Hofstetter. Elle s'inspire du cadre théorique et de l'instrumentation méthodologique construits par ERHISE (Équipe de Recherche en Histoire Sociale de l'Éducation) dont l'auteure était membre depuis le début de cette recherche. 
nouvelle, c'est l'une des revues phares du mouvement en raison d'une large représentativité des partenaires qui s'y expriment, de la diversité des thématiques abordées et de son envergure internationale. Cette analyse est complétée par l'étude de deux autres périodiques fondateurs de la Ligue, The New Era et Das Werdende Zeitalter, dont l'histoire éditoriale, les thématiques et les auteurs se différencient du journal francophone, élargissant la perspective et permettant une compréhension plus nuancée du fonctionnement de la Ligue et ses protagonistes. En tant que moyens de communications officiels, ces supports éditoriaux sont des plateformes d'échanges où les éducateurs font part de leurs expériences pratiques et les scientifiques font connaître leurs recherches. En cela, ils constituent des observatoires privilégiés pour comprendre comment se nouent les relations entre science et militance, et pour étudier comment s'élabore le discours de propagande sur les plans scientifique, éducatif et politique.

Cette recherche s'inscrit dans le sillage de nombreux travaux d'historiens qui, grâce à leurs approches et objets complémentaires, ont contribué à conceptualiser l'histoire de l'Éducation nouvelle et à en révéler toute sa complexité. ${ }^{5}$ Une première série de travaux se situant dans le domaine de l'histoire des idées s'attache à définir ce qu'est ou n'est pas l'Éducation nouvelle, à identifier ses concepts et principes fondamentaux. Ces historiens convergent pour dire que le vocable Éducation nouvelle désigne un «vaste ensemble flou» (Hameline, 1986, p. 64), un mouvement "complexe, multiforme, hétérogène» (Hofstetter \& Schneuwly, 2006b, p. 18), voire une «nébuleuse où voisinent théories philosophiques, pratiques novatrices, idéaux politiques» (Ohayon, Ottavi \& Savoye, 2004, p. 4). Selon Hofstetter et Schneuwly (2006), l'Éducation nouvelle se caractérise par la «commune dénonciation de la non adéquation des pratiques éducatives du système scolaire étatique aux besoins spécifiques de l'enfance» (p. 18). Elle se définit avant tout comme une réaction à l'école traditionnelle, un concept créé par ces réformateurs pour se forger une identité, à distance de l'image convenue de l'école. Selon Ohayon, Ottavi et Savoye (2004), l'adjectif «nouvelle» désigne à la fois une rupture introduite dans la pensée et dans les pratiques d'éducation, et une espérance, encore valable aujourd'hui, «laquelle s'enracine dans une modification de longue durée de notre

5 Les travaux présentés ci-après constituent une sélection faite sur la base de nos questions de recherche et ne prétendent pas à l'exhaustivité. 
rapport à l'éducation» (p. 4). Un adjectif qui n'est pas perçu de la sorte par Oelkers (1995) pour qui «l'Éducation nouvelle n'a sans doute pas été très nouvelle» (p. 47) compte tenu de tous les précurseurs qui l'ont précédée et ont préparé son avènement. Pour Bloch (1968), l'Éducation nouvelle signifie moins une méthode qu'un état d'esprit d'ouverture, et pour Avanzini (1995), une manière de concevoir l'éducation qui laisse à l'instituteur la liberté d'interpréter les méthodes comme il l'entend. Pour d'autres, elle doit être comprise comme «une position éthique consistant à reconnaître la valeur de l'enfance» (Jacquet-Francillon, 2004, p. 39) à laquelle s'ajoute l'idée que l'éducation peut faire progresser la société, que ce soit par l'éducation des élites (Duval, 2009) ou par celle du peuple (Clastres, 2004). Hameline (1986) rappelle enfin que ce mouvement est avant tout celui des écoles nouvelles, "ces internats privés situés à la campagne» qui expérimentent et théorisent les nouvelles méthodes.

L'Éducation nouvelle est aussi un mouvement traversé de tensions et de débats (Gutierrez, 2010, 2011; Mole, 2010, 2012; Savoye, 2004; Vidal, 1997) qui se manifestent à différents niveaux, notamment à celui du vocabulaire et des concepts fondateurs. Si elle ne prône pas une méthode à proprement parler, l'Éducation nouvelle est indissociable de la méthode active (Hameline 2002a). Convaincus que l'élève devrait «agir» plutôt que «subir», ses promoteurs s'inscrivent en faux contre une école passive ou assise et militent pour une école «où l'on s'instruit en travaillant» (Ferrière, 1920). Mais Hameline, Jornod et Belkaïd (1995) montrent les ambiguités autour de ce terme pourtant couramment utilisé comme "porte-drapeau» par les acteurs de l'époque. Il suscite des tensions notamment entre Ferrière et Claparède qui interprètent ces mots différemment. Pour Ferrière, l'École active est une école qui libère l'enfant, où la notion d'activité désigne l'attitude nouvelle qu'adopte ce dernier pour s'approprier les connaissances, attitude faite d'initiative, d'autonomie et de liberté. Pour Claparède, l'École active ne traduit qu'imparfaitement le concept «d'éducation fonctionnelle» (Claparède, 1931) qui doit répondre à une fonction, à un «besoin biologique de connaissance»; dès lors, la notion d'activité désigne le processus d'apprentissage au sens «neurologique» du terme.

Cette recherche s'appuie en outre sur les multiples monographies publiées depuis plus de 20 ans sur des acteurs, des institutions, des associations et des groupes locaux ayant milité pour la promotion de l'Éducation nouvelle. Elles révèlent des spécificités propres aux 
contextes culturels concernés contribuant ainsi à une micro-histoire du mouvement. Ces travaux concernent aussi bien les pays européens ${ }^{6}$, incluant ceux d'Europe centrale ${ }^{7}$, que les contextes anglo-saxons, du côté britannique comme du côté américain ${ }^{8}$ sans oublier l'Australie (Hughes, 2015) et la Nouvelle Zélande (Middleton, 2013, sous presse). Quant aux régions francophones, moult publications sont parues ces dernières années $^{9}$ auxquelles cet ouvrage se réfère abondamment en raison des liens directs avec la problématique traitée. Enfin, des ouvrages collectifs tels que Hameline, Helmchen et Oelkers (1995) mettent en regard différents contextes culturels, notamment l'Allemagne et la France, pour identifier les enjeux spécifiques à ces contextes; d'autres (Hofstetter, Magnin \& Depaepe, 2006) étudient les concepts de l'Éducation nouvelle s'interrogeant sur leurs origines et leurs métamorphoses selon les lieux et les époques.

\section{UN MOUVEMENT SOCIAL, POLITIQUE ET INTERNATIONAL}

Jusqu'alors considérée comme un mouvement pédagogique réformiste, l'Éducation nouvelle est aussi conceptualisée aujourd'hui comme un mouvement politique (Oelkers, 2005) et comme un mouvement social (Brehony, 2004). Selon Oelkers (2005), si l'Éducation nouvelle a marqué l'histoire de l'éducation, c'est parce que ses revendications étaient liées davantage à des questions politiques que psychologiques. Pour lui, la véritable nouveauté qu'elle apporte, à savoir la notion de liberté de l'en-

$6 \quad$ Pour les régions hispanophones et lusophones voir Del Pozo Andrés (2009; 2014); Laudo \& Vilanou (2015); Madeira (2012); Soler Mata (2011, 2012); Rabazas Romero (2015). Sur les régions germanophones, voir Gründer (2007); Helmchen (1995); Mayer (2014a, 2014b); Miller \& Oelkers (2014); Oelkers (2011).

7 Sur la Bulgarie, voir Stefanov \& Terziyska (2006); sur la Hongrie, voir Helmich (2014) et Németh (2006); sur la Pologne, Lepalczyk \& Marynowicz (2003).

8 Voir Boyd \& Rawson (1965), Brehony (2004, 2006), Cunnigham (2001) et Jenkins (2000), pour le contexte britannique; Cremin (1964), Reese (2001) et Wraga (2014), pour le contexte nord-américain.

9 Contextes francophones: Gutierrez (2010, 2011); Gutierrez, Besse \& Prost (2012); Mole (2011, 2012); Savoye (2004, 2006, 2010); Serina-Karsky (2013, 2014); Wagnon $(2013,2015)$. 
fant sur laquelle repose toute sa démarche pédagogique, constitue «l'élément-clé» susceptible d'atteindre un public en dehors des professions de l'éducation et qui, de ce fait, lui octroie une envergure politique. Pour Brehony (2004), l'Éducation nouvelle est avant tout un mouvement social, quelle que soit la définition que l'on adopte de ce concept. Selon lui, elle remplit la majorité des critères proposés par Della Porta et Diani (1999) qui définissent le mouvement social comme «(1) un réseau informel, (2) basé sur des croyances et une solidarité partagées, qui se mobilise (3) autour d'enjeux conflictuels, à travers (4) l'utilisation fréquente de diverses formes de protestation» (p. 27). La Ligue internationale pour l'Éducation nouvelle (New Education Fellowship), au cœur de cet ouvrage, en est une bonne illustration. Comme nous le verrons, il s'agit bien d'un réseau informel (réseau de relations personnelles), basé sur des croyances (principes de ralliement) et une solidarité partagées (visites, rencontres) qui se mobilise autour d'enjeux conflictuels (réaction à l'école traditionnelle), à travers l'utilisation de diverses formes de protestation (conférences, publications de propagande, manifestes).

En plus des caractéristiques d'un mouvement social, l'Éducation nouvelle porte celles d'un mouvement international et internationaliste. Des auteurs tels que Röhrs et Lenhart (1994), Oelkers et Osterwalder (1999) montrent l'étendue du mouvement sur le plan international et observent une croissance similaire dans plusieurs pays avec une apogée à la fin des années 1920. Selon eux, l'internationalisme est un mouvement politique, social et culturel à part entière dont font partie la science comme l'éducation. L'Éducation nouvelle est considérée comme l'incarnation de cet élan internationaliste qui caractérise les années d'aprèsguerre dans de nombreux pays occidentaux. Ce dernier se manifeste à travers la création de mouvements sociaux, culturels et pacifistes émanant d'éducateurs, d'universitaires et de divers réformateurs sociaux qui cherchent à établir des réseaux internationaux pour promouvoir la coopération scientifique, la paix, la compréhension mutuelle et la collaboration professionnelle. Fuchs (2004) appréhende le phénomène à travers l'étude des congrès internationaux d'éducation qui se multiplient à cette époque-là, analysant leur genèse, leur structure, leurs contenus et leurs effets sur les réformes éducatives. Ce même auteur montre comment les réseaux internationaux d'éducation s'institutionnalisent, dans les années 1920 notamment, prenant la forme d'associations ou d'organisations internationales en lien étroit avec la Société des Nations (Fuchs, 2007b). Ainsi, le Bureau international d'Éducation (Hofstetter \& 
Schneuwly, 2013; Magnin, 2002) fondé en 1925 à l'instigation des membres de l'Institut Rousseau (Hofstetter, 2010) serait à comprendre comme un exemple de cette institutionnalisation bien qu'elle soit perçue par certains, en particulier Ferrière (Gerber, 1989), comme le prolongement d'une institution déjà existante. ${ }^{10}$ Depuis une dizaine d'années, nombre d'historiens étudient l'Éducation nouvelle et ses acteurs selon une approche transnationale attentive à la circulation des idées ou modèles pédagogiques (Haenggeli-Jenni, 2015; Mayer, 2014b), ainsi qu'à leur réception et leur adaptation dans divers pays (Bruno-Jofré \& Schriewer, 2012; Del Pozo Andrés, 2009; Hai, Simon \& Depaepe, 2015). Cette approche met en évidence l'ampleur du mouvement au niveau international et l'importance des acteurs dans la circulation des idées pédagogiques. L'étude approfondie de certaines figures, notamment de femmes éducatrices et militantes (Del Pozo Andrés, 2013; HaenggeliJenni, 2015, sous presse; Helmich, 2014; Middleton, 2013), fait apparaître de vastes réseaux internationaux - éducatifs, politiques, religieux, féministes - au sein desquels circulent les conceptions éducatives progressistes contribuant à la rapide expansion de l'Éducation nouvelle dans les années d'entre-deux-guerres.

Pour Charle, Schriewer et Wagner (2004), le désir de coopération et de compréhension internationale donnant lieu à la fondation d'organisations telles que l'Institut international de Coopération Intellectuelle (IICI), provient à la fois de l'interdépendance économique croissante des pays et de la menace que représentent alors les politiques nationalistes. Selon eux, les réseaux intellectuels s'érigent avant tout contre une dimension nationaliste de la science qui est l'héritage du 19e siècle. Ce processus d'internationalisation, qui n'est pas sans susciter des tensions avec le processus «d'indigénisation» peut prendre des formes différentes. Il se manifeste par exemple à travers le transfert de concepts et de modèles scolaires d'une région à une autre (Fontaine, 2015; Matasci, 2012) ainsi qu'à travers «le déploiement transfrontalier des modes de production scientifiques (revues) et des moyens de financement de la recherche» (Gingras 2002). Ces travaux montrent en outre le rôle crucial joué par les revues dans ce processus d'internationalisation, revues scientifiques ou spécialisées - à l'exemple de Pour l'Ère Nouvelle - qui contribuent à faire circuler des savoirs aussi bien théoriques qu'expé-

10 Il s'agit du Bureau International des Écoles Nouvelles (BIEN) que Ferrière fonde en 1899. 
rientiels à une échelle internationale (Haenggeli-Jenni, Fontaine \& Bühler, 2014).

Selon Fuchs (2007b), ce processus est visible également à travers la constitution de réseaux de communication institutionnalisés, à l'exemple des congrès internationaux qui rassemblent les membres de mêmes professions ou disciplines naissantes (Rasmussen, 1990). Alors qu'ils sont très en vogue dans des disciplines telles que la biologie, la physique ou la médecine, les congrès d'éducation débutent au tournant du $20^{\mathrm{e}}$ siècle et prennent rapidement de l'ampleur, étant considérés comme «la forme la plus importante d'internationalisation scientifique ${ }^{11 »}$ (Fuchs, 2004, p. 758). Les premiers ont lieu à l'occasion des expositions universelles (Lawn, 2009; Rasmussen, 1989) où les sujets abordés couvrent aussi bien l'éducation scolaire que l'éducation des adultes, l'éducation spéciale ou l'hygiène scolaire. En raison de la création d'associations spécialisées dans certains domaines (éducation à la paix, éducation morale, Éducation nouvelle), les congrès d'éducation se caractérisent bientôt par leur «fragmentation» créant par là-même une large diversité de types de congrès (Fuchs, 2004).

\section{SCIENCES DE L'ÉDUCATION, PÉDOLOGIE ET SCIENCES CONTRIBUTIVES}

Depuis quelques années, des historiens tels que Brehony (2004, 2006), Depaepe (1998), Fuchs (2004), Helmchen (1995, 1999), Hofstetter et Schneuwly (2006), Labaree (2004, 2006), Norris (2004), Oelkers (1995, 2005), Savoye $(2004,2006)$ étudient l'Éducation nouvelle sous l'angle de ses connexions avec le champ disciplinaire des sciences de l'éducation. Ils cherchent à comprendre quel type de relations entretiennent ces deux phénomènes, dans quelle mesure ceux-ci sont liés ou dissociés, quelle est la participation de l'un au développement de l'autre. Pour Hofstetter et Schneuwly (2006), ces deux mouvements sont intrinsèquement liés, voire fusionnés, partageant le même paradigme qui est à leur origine et qui émerge au tournant du 19e-20e: "placer l'enfant au centre», c'est-à-dire concevoir l'enseignement comme «conditionné et défini par l'enfant»,

11 Dans le texte original: «the most important form of scientific internationalization». 
dont découle la nécessité de construire dorénavant «une science de l'apprenant» plutôt qu'une «science de l'enseignant» (p. 25). Selon eux, «ce changement de paradigme constitue le fondement idéologique et conceptuel commun du champ disciplinaire naissant et de l'Éducation nouvelle dont le mariage s'enracine dans une base solide» (p. 26). Or cette base solide est la nouvelle approche empirique prônée par de nombreux scientifiques qui propose désormais un modèle fonctionnel, voire pragmatiste de l'éducation (Tröhler \& Oelkers, 2005). Une approche qui se pose en rupture avec la tradition de nombreux pays où la pédagogie est alors une discipline essentiellement philosophique (Oelkers, 2006) marquée par les traditions culturelles et par l'herbartianisme (Criblez, 2006; Goodchild, 2006). De ce fait, la diffusion du nouveau paradigme, soutenue par l'Éducation nouvelle comme par les sciences de l'éducation émergentes, génère des tensions et des débats qui ne seront pas sans conséquences pour l'avenir des deux mouvements.

Plusieurs historiens de l'éducation convergent vers une interprétation commune de l'histoire de l'Éducation nouvelle et des sciences de l'éducation. D'une part, ils s'accordent pour dire que les deux phénomènes sont comme «deux faces d'une même médaille» (Houssaye, 2002), qu'ils sont «contigus, devenant poreux l'un à l'autre» (Savoye, 2004), tandis que d'autres parlent de «symbiose» (Savoye, 2006), voire de «fusion» (Hofstetter \& Schneuwly, 2006). Un phénomène qui est visible par la double appartenance des acteurs aux deux milieux à l'exemple des scientifiques qui s'impliquent sur le terrain - dans les écoles - pour mener leurs recherches et à l'inverse, les enseignants qui collaborent avec les chercheurs en faisant passer des tests à leurs élèves. Beaucoup prouvent leur double appartenance en écrivant des articles indifféremment dans des revues représentatives de l'un et l'autre milieu. Enfin, des travaux récents réunis dans Hofstetter et Schneuwly (2009a) s'interrogent sur le type de savoirs que promeut l'Éducation nouvelle, sur les rapports que celle-ci entretient avec les savoirs constitués et sur les conséquences de ces relations sur les réformes scolaires. Alors que Brehony (2009) étudie la question du côté de l'éducation de la petite enfance, Savoye (2009) s'intéresse aux «nouveaux savoirs» dispensés dans les lycées français (1930-1939). Ce dernier montre en quoi l'Éducation nouvelle a non seulement impulsé mais durablement imprégné les savoirs et les programmes scolaires dans cet ordre d'enseignement grâce à l'introduction des méthodes nouvelles, ceci selon quatre axes: l'enseignement selon les «méthodes actives», la coordination entre 
enseignants et entre disciplines, le droit à l'expérience et la «liberté pédagogique», l'éducation du «sens social» ou «initiation à la vie sociale» (pp. 508-510). Si l'engagement des maîtres du secondaire en faveur de la réforme est massif dans les années 1930, celui-ci tend à diminuer à la fin de la décennie voire disparaître après 1945. Selon lui, les nouvelles structures de recherche investies par les sciences de l'éducation après la Deuxième Guerre mondiale font perdre à l'Éducation nouvelle sa fonction de «tête chercheuse», devenant «exsangue» comme «vampirisée par son partenaire» (Savoye, 2006, p. 376). Il nous intéresse de savoir si des éléments d'une telle évolution sont déjà repérables dans la période d'entre-deux-guerres, en particulier dans Pour l'Ére Nouvelle.

Notre choix méthodologique part du postulat selon lequel l'analyse détaillée d'une revue, prise comme source principale d'investigation, permet une compréhension plus fine des discours et des enjeux sociaux et politiques qui l'entourent. Le choix de ce corpus est intrinsèquement lié à notre problématique qui cherche à comprendre les relations complexes entre science et militance au sein du mouvement d'Éducation nouvelle. Au-delà de l'analyse des discours, c'est par l'étude approfondie de l'infrastructure éditoriale et du contexte social dans lequel s'inscrit ce périodique que nous ambitionnons de saisir ces relations. Nous avons donc procédé à une «étude de cas» pour pouvoir comprendre à petite échelle ce qui se joue à plus grande échelle. La mise en relation de Pour l'Ėre Nouvelle avec ses homologues germanophone et anglophone témoigne de la volonté de prendre en compte un contexte plus large, international, pour repérer similitudes et différences entre ces organes et mesurer leur place parmi d'autres supports éditoriaux dédiés à cette cause. Enfin, l'étude fine de ce périodique vise à mieux comprendre la place et le rôle des membres de la Ligue parmi les acteurs appartenant aux divers groupes qui se réclament du mouvement.

En outre, cette recherche s'appuie sur les travaux d'historiens ayant étudié cette même question à partir d'autres sources, celles-ci enrichissant notre travail à travers la confrontation de leurs analyses. Afin de préciser notre propos, c'est auprès d'eux que nous avons cherché une définition du mot revue, à comprendre comme synonyme du mot périodique. Jennings (2007) définit la revue comme «un livre à plusieurs voix», une «coopérative de pensée et de communication» (p. 2) où chaque auteur non seulement exprime ses pensées et savoirs mais aussi suscite le dialogue et l'échange d'idées. En d'autres termes, un périodique est le «passeur indispensable entre le travail individuel et le public auquel il 
est destiné» (p. 3). Pour Julliard (1987), «l'objet-revue est un lieu de culture et de débat d'idées» qui est la caractéristique même du monde intellectuel. La pensée y est en état de "continuel affrontement» puisqu'un article de revue est nécessairement «la confrontation d'un homme avec d'autres hommes, d'un penseur avec son temps» (p. 3). Selon ces auteurs, la revue est donc un lieu privilégié pour développer sa pensée et l'échanger avec d'autres, la périodicité du support favorisant ce dialogue entre pairs.

D'après Mole (2010), les revues pédagogiques, associatives et syndicales constituent des sources privilégiées pour saisir les débats concernant l'institution scolaire: "parce qu'elle est - plus que toute autre institution - une projection sur l'avenir, l'institution scolaire se donne à connaître dans les discours au moyen desquels les acteurs pensent et expriment l'horizon de sens de leur action» (p. 13). Les discours permettent de saisir les «constructions discursives» avant qu'elles ne soient «traduites, synthétisées ou résorbées dans des reformulations simplificatrices, avant que les désaccords et les contradictions ne se trouvent atténués, suspendus ou gommés» (p. 14). En d'autres termes, ces supports éditoriaux offrent à l'historien un accès direct à des discours bruts, non formalisés ni retraduits. Cependant, les revues associatives, politiques ou syndicales sont souvent orientées par les valeurs que défendent ses rédacteurs et peuvent devenir des instruments de propagande, comme l'atteste Caspard (1981, 2006). D'après cet auteur, elles sont utilisées pour promouvoir ou imposer une pensée et les pratiques qui lui sont liées. C'est la raison pour laquelle «l'arme de la presse pédagogique» a d'abord été aux mains de l'État, de l'Église et de l'intelligentsia philanthropique. Au fur et à mesure qu'a progressé la démocratie, les mouvements militants, associatifs et syndicalistes s'en seraient emparé portant à affirmer que «la naissance puis le développement de la presse pédagogique sont consubstantiels à l'histoire même de l'enseignement contemporain» (Caspard, 2006, p. 569). Selon l'auteur, les périodiques constituent un excellent moyen d'appréhender le fonctionnement du champ éducatif car ils abordent des thèmes très divers et constituent des lieux de débats permettant aux savoirs de circuler et se construire. Les revues, poursuit-il, ont un double visage: elles offrent d'une part, «une documentation sur les réalisations éducatives les plus diverses» et d'autre part, une "véritable anthologie de la réflexion pédagogique [...] qui ne se limite pas à ce que pensent les grands pédagogues mais reflète aussi ce que pensent les plus petits, infiniment plus nombreux» (p. 565). 
Pour Hofstetter et Schneuwly (2004), les revues sont constitutives du développement d'une discipline scientifique, considérées comme un «réseau de communication destiné à produire du savoir» (p. 575). Selon eux, elles contribuent à l'établissement d'une "communauté de chercheurs» œuvrant et dialoguant autour de problématiques communes. Les interactions entre acteurs à propos du savoir contribuent à établir une "communauté de discours» et constituent les arènes dans lesquelles les résultats de recherche sont entendus et débattus pour la première fois» (p. 575). Ces auteurs s'appuient sur divers travaux d'histoire et de sociologie des sciences notamment ceux de Schriewer (1998), Keiner (2002), Schriewer et Keiner (1992). Ces derniers étudient les revues selon une approche comparative pour comprendre comment se développe le champ disciplinaire des sciences de l'éducation en France et en Allemagne et mesurer l'importance des aires culturelles dans ce développement. Selon Schriewer (1998), faisant lui-même référence à d'autres travaux, «les revues spécialisées jouent un rôle extraordinaire dans l'émergence, l'institutionnalisation, les processus de consolidation sociale et intellectuelle, ainsi que dans la visibilité tant universitaire que publique et politique, des réseaux de communication, que communément, on qualifie de 'disciplines'» (p. 59). Ces revues se distinguent des revues de type professionnel, syndical ou associatif, et permettent de déceler les processus de développement d'une discipline. Les travaux récents de Fuchs (2012) ou de Tröhler, Oelkers, Horlacher et Fuchs (2015) sur la discipline 'histoire de l'éducation' montrent l'importance des revues dans la constitution de ce champ disciplinaire désormais reconnu. Les études comparatives de Fuchs, Drewek, Zimmer-Müller (2010) ou de Knaupp, Schaufler, Hofbauer et Keiner (2014) soulignent l'importance des revues comme support à la circulation des concepts et théories au niveau international mais relèvent la présence de spécificités culturelles qui façonnent les disciplines différemment selon le contexte dans lequel elles se développent. À la suite de Hofstetter (2004) et Périsset Bagnoud, Schneuwly et Hofstetter (2007), nous prenons en compte le fait que «la production scientifique est plus large que celle contenue dans les revues spécialisées» (p. 410) et qu'il convient dès lors de faire preuve de vigilance dans l'interprétation de nos résultats. 


\section{MÉTHODE ET QUESTIONS DE RECHERCHE}

À la suite de Caspard (2006), nous pensons que l'analyse de la presse pédagogique "permet de repérer, d'agréger et de mettre dans une perspective historique plus fine une infinité d'idées, de volontés et de décisions émanant d'acteurs très divers, dans et hors le système éducatif» (p. 569). C'est pourquoi nous avons fait le choix de concentrer notre attention sur un périodique en particulier et ses équivalents dans d'autres langues. Alors que l'historiographie contemporaine valorise pour des raisons heuristiques - une méthode privilégiant le croisement des sources (Hartog, 2013; Revel, 2012), nous avons choisi de croiser les approches dans l'analyse d'une même source. La combinaison de plusieurs types d'analyses - qualitative, quantitative, comparée - étudiant l'objet à plusieurs échelles - discours, contexte d'énonciation, infrastructure éditoriale - devrait garantir le surcroît d'intelligibilité visé. À cela s'ajoute la position que nous avons adoptée, à savoir la position analytique du chercheur qui établit un dialogue entre les sources et ses questions de recherche que ce dernier restitue avec une conscience vigilante du contexte dans lequel les sources ont été émises. Selon Prost (1996), «les questions tiennent, dans la construction de l'histoire, une place décisive. [...] C'est la question qui construit l'objet historique en procédant à un découpage original dans l'univers sans limites des faits et documents possibles» (p. 79). L'objet historique prend ensuite vie dans le récit qu'en fait l'historien, qui se situe, selon Jablonka (2014), entre le genre littéraire et scientifique, une littérature du particulière qui concoure à une intelligibilité du monde et vise à comprendre le passé ou le présent. Nos démarches d'analyse s'appuient en outre sur celles des historiens qui analysent l'évolution des revues dans une perspective de compréhension du processus de disciplinarisation des sciences de l'éducation (Schriewer, 1998; Keiner, 2002).

L'analyse des discours de Pour l'Ère Nouvelle a été guidée par quatre dimensions correspondant aux quatre axes de réflexion mentionnés dans la problématique (acteurs, cadre institutionnel, conceptions éducatives, savoirs de référence). Elles constituent autant d'indicateurs qui ont été utilisés dans chacune des démarches de l'analyse qu'elle soit quantitative, qualitative ou transversale. Les données issues de ces lectures ont constitué le matériau sur la base duquel nous avons construit notre interprétation et notre récit historique. Afin de donner un statut scientifique à notre récit, nous avons veillé à référer autant que possible au dis- 
cours des auteurs, les citant à de nombreuses reprises pour illustrer notre propos ou développer notre argumentation. Nous avons donc élaboré un discours «qui comprend son autre», c'est-à-dire qui s'organise comme un «texte feuilleté dont une moitié, continue, s'appuie sur l'autre, disséminée, et qui se donne le pouvoir de dire ce que l'autre signifie» (Certeau, 1975). À la suite de Farge (1989), nous pensons que

la citation a le charme de l'étrangeté, celui de la justesse et de l'exotisme mêlés de la langue d'autrefois, et encore celui de l'aveu. [...] Suspendue, [elle] fonctionne comme une halte, comme une note blanche, permettant aux mots habituellement raisonnables de l'historien de se mouvoir autrement autour d'elle. (pp. 91-93)

D'autre part, nous avons veillé à introduire ces citations en précisant leur contexte d'énonciation ainsi que le contexte social et culturel dans lequel s'inscrivent les auteurs. Notre récit tente ainsi d'apporter une intelligibilité aux sources étudiées en privilégiant un regard transversal, rejoignant ainsi l'approche de l'histoire culturelle (Poirrier, 2004) ou plus largement, celle de l'histoire sociale.

Les bornes de la période sur laquelle portent nos investigations sont déterminées par des moments-clés de l'évolution des périodiques étudiés, en lien étroit avec l'histoire globale du mouvement. La première borne se situe en 1920, car elle correspond à l'année de fondation des revues anglaise et allemande, avant même la création de la Ligue. Elle marque le début d'une institutionnalisation du mouvement à laquelle participent la publication de revues dédiées à cette cause et l'organisation de congrès internationaux. L'année 1940 constitute la deuxième borne de notre périodisation car elle correspond à la première interruption $^{12}$ de Pour l'Ère Nouvelle; cette année marque aussi la fin d'une période de gloire du mouvement qui se traduit par la disparition de la Ligue internationale pour l'Éducation nouvelle, du moins dans les régions francophones. Ces bornes ne signifient pas que le mouvement d'Éducation nouvelle se limite à ces années-là. Comme l'affirme

12 La revue cesse de paraître de 1940 à 1945; elle reprend son activité en 1946 en publiant 5 numéros cette année-là avant de disparaître à nouveau pendant une année. Dès 1948, elle reprend un rythme de cinq numéros par an jusqu'à ce qu'elle change de nom en 1967 Les Sciences de l'Éducation - Pour l'Ėre Nouvelle, sous l'impulsion de Gaston Mialaret. 
Hameline (2003), il prend naissance bien avant 1920, avec la fondation des premières écoles nouvelles en Europe et aux USA notamment celle d'Abbostholme en 1889 en Angleterre. De plus, l'Éducation nouvelle s'étend bien au-delà de 1940, puisque ses principes suscitent des débats au sein des systèmes éducatifs dès les années $1950^{13}$ et donnent lieu à de nombreuses initiatives concrètes jusqu'à nos jours.

L'ouvrage se décline en six chapitres organisés selon une logique allant du général au particulier. Les deux premiers présentent le contexte dans lequel se déroule notre enquête, s'intéressant tout d'abord à l'association internationale qui réunit nombre de représentants du mouvement ${ }^{14}$, puis aux supports éditoriaux qu'elle publie, en proposant une analyse fine de leur infrastructure éditoriale, des thématiques principales, du profil des auteurs et des conditions de production. Les chapitres 3 à 6 proposent une analyse approfondie des discours tenus dans la revue francophone Pour l'Ère nouvelle à travers quatre thématiques acteurs et actrices, formes institutionnelles, conceptions éducatives, savoirs de référence - permettant d'appréhender les relations entre science et militance au sein de l'Éducation nouvelle et de comprendre leur caractère à la fois indissociable et inconciliable. Enfin, la conclusion revient sur le statut particulier de Pour l'Ère nouvelle, revue au carrefour entre science et militance, et propose une réflexion sur les ambitions philosophiques de certains scientifiques engagés dans le mouvement.

13 Le congrès de la Société Pédagogique Romande (association professionnelle des enseignants du primaire) de 1950 a pour thème «École publique et Éducation nouvelle».

14 La Ligue ne rassemble pas tous les partisans du mouvement et n'incarne pas toute l'Éducation nouvelle; d'autres groupes régionaux existent en parallèle, certains souhaitant rester volontairement à l'écart de cette association internationale. 


\section{ChAPITRE 1}

\section{LA LIGUE INTERNATIONALE POUR L'ÉDUCATION NOUVELLE: ÉMERGENCE ET STRUCTURATION}

Afin de comprendre le contexte dans lequel s'inscrit Pour l'Ère nouvelle, nous proposons ici une étude de l'association dont elle est l'organe: la Ligue internationale pour l'Éducation nouvelle. Il s'agit de présenter les origines de cette association, ses objectifs, ses fondements, sa structure ainsi que les acteurs-clés qui la font vivre. D'autre part, nous montrerons l'évolution de son organisation au fil de sa croissance durant la période étudiée. Comme la Ligue se dote, dès sa fondation, de trois périodiques correspondant aux trois régions linguistiques du mouvement, le contexte de Pour l'Ère Nouvelle est aussi celui de ses «revues-sœurs». Dès lors, une analyse des revues allemande et anglaise a été indispensable pour pouvoir repérer les spécificités de chacune d'entre elles et saisir les enjeux liés au contexte culturel dans lequel elles s'inscrivent. Les résultats détaillés de cette analyse feront l'objet du chapitre 2 où une mise en regard des trois périodiques mettra en évidence leur rôles respectifs dans la recherche de cohésion du mouvement et l'influence des contextes culturels sur leurs discours et orientations.

\section{MOMENTS FONDATEURS ET LEADERS CHARISMATIQUES}

La Ligue internationale pour l'Éducation nouvelle (LIEN) est fondée le 6 août 1921, à Calais lors d'un congrès international qui réunit près de 200 personnes de douze nationalités différentes. L'initiative du rassemblement vient de la New Education Fellowship dont Beatrice Ensor (18851974) est membre active, éducatrice et inspectrice d'école. Depuis 1920, elle essaie de mettre en contact des enseignants de divers pays en 
organisant des conférences internationales et en publiant une revue, Education for The New Era. À Calais, elle est entourée de plusieurs membres de la Theosophical Fraternity, notamment Leslie Hadden Guest et Harold Baillie-Weaver qui assurent avec elle l'organisation du congrès et dont le second est président. Iwan Hawliczek, membre de Theosophical Educational Trust est le secrétaire général de la manifestation. Si aucune mention de ces appartenances n'est clairement énoncée dans les invitations au congrès, la présence de la théosophie y est évidente au point de mettre à distance certains pionniers de l'Éducation nouvelle en France, tels que Georges Bertier ou Roger Cousinet qui préfèrent rester à l'écart (Brehony, 2004). Les conférenciers sont majoritairement anglais à l'exception de quelques étrangers, notamment Ovide Decroly (Belgique), Adolphe Ferrière (Suisse) et Juliette Decroix (France). D'après Jinarajadasa (cité par Brehony, non publié, p. 6), membre de la Theosophical Society, la New Education Fellowship aurait été fondée pour ceux «qui croyaient aux principes de l'Éducation nouvelle mais qui ne pouvaient accepter les fondements théosophiques sur lesquels ceux-ci reposent». Selon Ferrière, elle rassemble «les pionniers de l'éducation - pédagogues, instituteurs, éducateurs, directeurs d'écoles, psychologues - ainsi que tous ceux qui, parents, philosophes, médecins, s'intéressent à l'enfance et à l'amélioration de sa condition» (Circulaire, PEN, 1922, 1, p. 4). De plus, elle comble un manque dans le paysage associatif de l'époque et se distingue des autres associations par le fait qu'elle émerge de la «base»:

Il n'existe aucune association internationale qui relie entre eux ceux qui trouvent les mesures administratives nécessaires mais insuffisantes, et qui veulent un changement radical du point de départ et du but de l'éducation. [...] Nous les maîtres, nous connaissons les difficultés, les obstacles, les limitations qui nous attendent: pauvreté du matériel, exiguïté des locaux, absence de sympathie et de coopération entre collègues, etc. Nous voudrions savoir comment renouveler notre enthousiasme, fortifier nos convictions par des relations avec d'autres esprits, comment rafraîchir notre courage par le contact avec ceux qui ont remporté la victoire dans des expériences semblables aux nôtres. (Ensor, PEN, 1922, 1, p. 5)

C'est l'initiative d'un "petit groupe de pédagogues», affirme Ferrière, désireux de mettre en synergie les multiples initiatives allant dans le sens d'une nouvelle conception de l'éducation. L'idée est de regrouper les expériences en cours depuis plusieurs années dans les écoles nou- 
velles, en un mouvement international qui réunisse «tous ceux qui croient aux mêmes vertus de l'éducation dans la création d'un monde meilleur»:

Cette Ligue réunit les pionniers de l'éducation publique et privée, les parents et de façon générale, les esprits idéalistes qui voient dans une transformation sérieuse de l'esprit et des méthodes de l'éducation traditionnelle le moyen d'orienter le monde vers plus de réelle fraternité et plus de bonheur. (Ferrière, circulaire PEN, 1922, 1, p.4)

Les motivations sont donc autant pédagogiques que philosophiques, l'esprit de la Ligue étant incarné, pour Ferrière, dans les deux mots qui composent son nom: «ex ducere» signifiant «conduire l'enfant hors d'un état jugé inférieur vers un état supérieur», et «nouvelle» qui signifie non seulement autre mais aussi meilleure (Ferrière, circulaire PEN, 1922, 1, p. 4).

Le groupe fondateur est composé de personnes désireuses «d'unir tous ceux qui s'intéressent à l'Éducation nouvelle, qui perçoivent l'aube d'une ère nouvelle et veulent venir en aide à un monde démoralisé: tous ceux qui ont la vision de ce que pourrait être l'humanité dans un avenir relativement prochain, si l'on transformait l'éducation dans nos écoles» (Ensor, PEN, 1922, 1, p. 7). Parmi ces personnes, trois d'entre elles - Beatrice Ensor, Elisabeth Rotten et Adolphe Ferrière - deviendront des «piliers» de la Ligue pendant les premières années parce que cumulant de nombreuses fonctions en son sein. Ces derniers sont tout d'abord les représentants de leur communauté linguistique, Ensor pour l'anglophone, Rotten pour la germanophone et Ferrière pour la francophone. Ils sont aussi les rédacteurs en chef de la revue rattachée à leur communauté - The New Era, Das Werdende Zeitalter, Pour l'Ėre Nouvelle - chacune étant considérée comme un organe officiel de la Ligue. Ensemble, ils forment le Comité exécutif, le titre de présidente ou «organizing director» étant attribué à Ensor. Tous trois très engagés pour la cause qu'ils défendent, ils propageront l'esprit et les idées de la Ligue dans le monde entier grâce à leurs nombreux écrits et voyages. En raison de leur statut particulier au sein de cette Ligue, du rôle central qu'ils jouent dans son histoire et surtout, de l'énergie colossale qu'ils y investissent, nous nous proposons de tracer brièvement leurs portraits sachant que tous trois constituent des figures centrales pour notre enquête sur la revue $P E N^{1}$.

1 Cet acronyme est utilisé ici, et dans tout le livre, pour désigner la revue Pour l'Ère Nouvelle. 


\section{BEATRICE ENSOR}

Beatrice Ensor (1885-1974), née de Normann, est l'une des figures emblématiques de la Ligue dont l'esprit et le charisme ont imprégné durablement l'association. Ses origines familiales et son parcours de vie - nombreux voyages et différents lieux de vie de la famille - en font une personne cosmopolite, plurilingue, qui entretient des relations dans le monde entier. Au début de sa vie, Ensor est active dans le monde des praticiens de l'éducation. Formée dans le domaine de l'économie

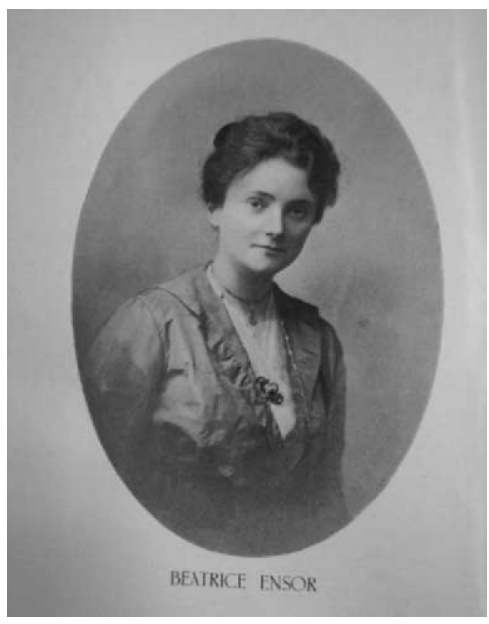
domestique (Domestic Science), elle enseigne d'abord au Training College de Sheffield puis en 1914, devient inspectrice des écoles de filles dans le Glamorgan County Council avant d'assumer ce rôle pour toute la région du sud-ouest de l'Angleterre. Lors d'une visite d'école à Cheltenham, elle découvre la méthode Montessori qui la séduit, ce qui l'incitera à prendre contact avec la doctoresse. Mais au moment de la fondation de la Ligue, sa principale identité est celle d'une théosophe engagée. Membre de la Theosophical Society depuis 1908 et du groupe New Ideals in Education dirigé par Edmond Holmes (Chief Inspector of Elementary Education), elle participe en 1915 à la fondation de la Theosophical Fraternity in Education. Ce groupe grandit rapidement grâce au réseau international de la première Société et établit des sections en France, en Inde, aux États-Unis, en Australie et en Nouvelle Zélande. Beatrice Ensor fait ainsi partie d'un immense réseau de relations international qui, nous le verrons, permettra l'extension rapide de la Ligue et de l'Éducation nouvelle dans ces pays. Elle s'engage peu après dans la Theosophical Educational Trust qui ouvre bientôt une école nouvelle à Letchworth (St-Christopher), dont elle assumera la direction plusieurs années, puis d'autres en Angleterre. Dès 1920, Beatrice Ensor publie une revue - Education for a New Era - qu'elle coédite à ses débuts avec Alexander S. Neill, dans le but de mettre en contact les enseignants théosophes et de faire connaître les pratiques expérimentées dans les Écoles nouvelles. Comme indiqué plus haut, c'est elle qui est à l'origine du Congrès de Calais, entourée pour son 
organisation de plusieurs compatriotes théosophes. Dès la fondation de la Ligue, elle est membre du Comité exécutif aux côtés de Ferrière et Rotten et assume la fonction de présidente générale. Pendant plus de dix ans, elle sillonne le monde entier pour propager les idées de l'Éducation nouvelle grâce à de nombreuses conférences, rencontres, visites, sans compter les innombrables congrès internationaux auxquels elle participe activement. Dès 1933, la mort de son mari l'oblige à s'expatrier en Afrique du Sud où ce dernier avait acquis une grande ferme dont elle doit alors s'occuper. Son éloignement de l'Europe va considérablement réduire son action éducative bien qu'elle reste active, notamment au sein de la section nationale Sud-africaine de la Ligue. En 1937, Ensor donne une conférence très remarquée en Australie à la suite de quoi, elle est nommée Doctor honoris causa de l'Université de Perth. Elle revient en Angleterre plusieurs années après pour se rapprocher de ses enfants et vit à Londres jusqu'en 1974, année où elle décède.

\section{ELISABETH ROTTEN}

Considérée aujourd'hui comme une socialiste, pacifiste et féministe, Elisabeth Rotten (1882-1964) est une personnalité hors du commun qui a joué un rôle important dans la propagande des réformes éducatives - en particulier la Reformpädagogik en Allemagne et à l'étranger. Elle est également connue pour ses actions humanitaires auprès des plus démunis, les enfants et les prisonniers militaires, actions dans lesquelles elle s'engage activement dès 1914 notamment auprès du vice-président de la Croix Rouge Internationale à Genève,

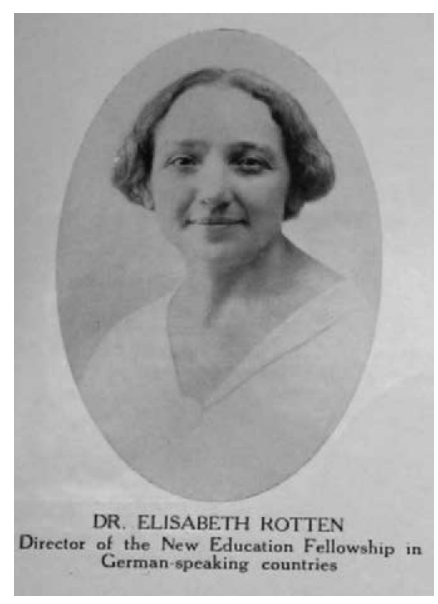
$\mathrm{D}^{\mathrm{r}}$ Frédéric Ferrière, père d'Adolphe. Elle passe sa jeunesse à Berlin où elle étudie la germanistique, la philosophie et les langues modernes et défend une thèse de doctorat en 1912 intitulée Goethes Urphänomen und die platonische Idee (Les archétypes de Goethe et l'idée platonique). Peu après, elle s'engage dans les mouvements de réforme de l'éducation, participe à la fondation de la Bund Entschiedener Schulreformer (Ligue pour une réforme radicale de l'école) puis devient membre de l'association Abteilung für Erziehung der Deutschen Liga für Völkerbund (Ligue 
allemande pour la Société des Nations), associations au sein desquelles elle dénonce l'autoritarisme et le conformisme des méthodes éducatives.

En 1921, elle fonde la Ligue internationale pour l'Éducation nouvelle - Internationalen Arbeitskreis für Erneuerung der Erziehung - aux côtés de Beatrice Ensor et Adolphe Ferrière et prend la direction de la revue allemande Das Werdende Zeitalter. Selon Hameline (2002a), son activité au sein de la Ligue est délicate au lendemain d'un conflit dans lequel l'Allemagne a incarné l'ennemi. Elle assume la «tâche difficile de représenter ce pays au sein de la Ligue «dominée par les Alliés et par l'esprit de la Société des Nations» (p. 270). Jusqu'en 1923, Elisabeth Rotten travaille avec les Quakers ${ }^{2}$ qui assurent un vaste programme d'aide alimentaire (Quäkerspeisung) suite à la Première Guerre mondiale. Elle côtoie les Quakers durant toute sa vie et adhère à la Société des Amis ${ }^{3}$ en 1930.

En 1924, elle participe à la fondation de l'École internationale de Genève et en 1925, à celle du Bureau International d'Éducation (BIE) où elle est nommée directrice-adjointe aux côtés de Ferrière. Elle y est aussi responsable des services d'éducation en faveur des principes de la Société des Nations et des voyages d'études pour instituteurs. Tout comme les deux autres fondateurs de la Ligue, Rotten voyage beaucoup et donne des conférences dans le monde entier. Elle participe notamment à la Conférence internationale sur l'Éducation à Genève en 1919, où son exposé sur les «tentatives d'une nouvelle éducation en Allemagne» est très remarqué. En 1926, elle est appelée à Paris pour donner des conférences au cours desquelles elle rencontre les représentants de plusieurs grandes institutions françaises, La Sorbonne, l'Institut international de Coopération Intellectuelle et l'Association française pour la Société des Nations. Grâce à sa double nationalité suisse et allemande, elle émigre à Saanen $(\mathrm{CH})$ en 1933 suite à l'arrivée au pouvoir d'Hitler. Depuis là, elle poursuivra ses actions dans l'esprit de la Société des Nations, que ce soit en tant qu'éditrice ou traductrice de travaux politico-pédagogiques. En 1944, elle participe à la fondation du Village d'enfants Pestalozzi de Trogen $(\mathrm{CH})$ et contribue activement à la fondation de la Fédération internationale des Communautés d'enfants (FICE). De plus, elle s'investit beaucoup pour la réintégration et la revalorisation des pédagogues allemands dans le discours international politique et pédagogique.

2 Communauté religieuse dérivant du protestantisme qui se caractérise par sa lutte pour le pacifisme et la tolérance envers les autres.

3 Nom désignant la communauté des Quakers. 


\section{AdOLPHE FERRIÈRE ${ }^{4}$}

Adolphe Ferrière (1879-1960) appartient à une famille de protestants établis à Genève depuis le $18^{\mathrm{e}}$ siècle dont le père et le grand-père ont participé à travers leurs métiers à des projets humanitaires notamment au sein de la Croix-Rouge. À moins de 20 ans, il se découvre une vocation de pédagogue après avoir lu le livre d'Edmond Demolins À quoi tient la supériorité des anglo-saxons? (1899). Sur la suggestion de son auteur, Ferrière fonde cette même année le Bureau international des Écoles nouvelles (BIEN) dans le

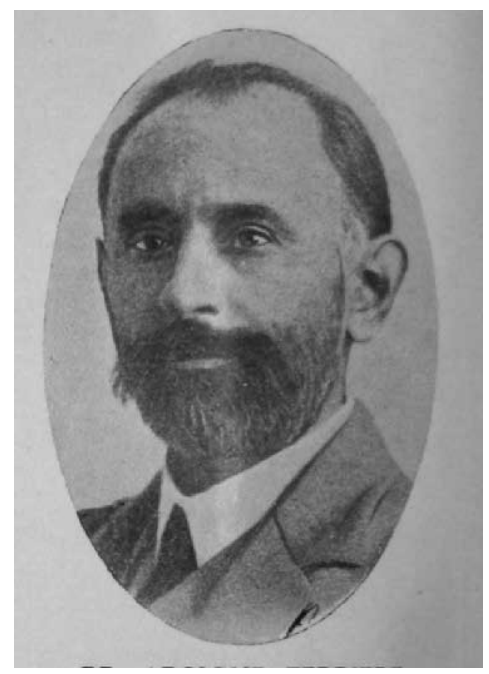
but de consigner les multiples expériences d'Éducation nouvelle et de mettre en réseau tous les éducateurs impliqués dans ces expériences. De 1900 à 1902, il s'engage comme éducateur auprès d'Hermann Lietz qui vient d'ouvrir une école nouvelle à la campagne, à Illsenburg puis à Haubinda (Allemagne). En 1902, avec ses compatriotes Frei et Wilhelm Zuberbühler, il participe à la fondation de Glarisegg, la première école nouvelle de Suisse. De retour à Genève, il étudie la philosophie, la sociologie et la biologie tout en participant à l'activité pédagogique de l'École des Pléiades sur Blonay (CH). À partir de 1912, il donne des cours de psychologie et d'éducation à l'Institut Rousseau tout en terminant l'écriture de sa thèse, La Loi du progrès en biologie et en sociologie publiée en 1915, qui lui vaudra le titre de docteur en sociologie.

En 1921, il participe à la fondation de la Ligue internationale pour l'Éducation nouvelle et prend la direction de son organe francophone, Pour l'Ère Nouvelle. Outre son activité de pédagogue et propagandiste, Ferrière lit et écrit beaucoup; il publie de nombreux ouvrages et articles, en particulier dans les années 1920 au cours desquelles sortent Transformons l'école (1920), L'Autonomie des Écoliers (1921), L'École active (1922, tomes I et II), La pratique de l'École active (1924), Trois pionniers de

4 Ce portrait s'inspire des éléments biographiques contenus dans plusieurs travaux menés sur la vie de Ferrière mais plus particulièrement ceux de Meyhoffer et Gunning (1929) et Hameline (1981). 
l'Éducation nouvelle (1928). À cette époque, il prend conscience de sa surdité croissante, handicap irréversible qui l'éloignera contre son gré d'une activité de praticien et le cantonnera dans ses activités d'écrivain, penseur et publiciste (Hameline, 1981). Pendant la période d'entre-deux-guerres, Ferrière est très actif au sein de la Ligue, il parcourt le monde entier pour donner des conférences, rencontrer des personnalités et visiter des écoles, et propager ainsi les principes de l'Éducation nouvelle. En 1924, il fonde l'École internationale de Genève - qu'il héberge chez lui pour sa première année d'existence - et en 1925, participe à la fondation du Bureau International d'Éducation (BIE). Son Bureau international des Écoles nouvelles (BIEN), devenu le service international de l'Institut Rousseau à partir de 1923 (Hofstetter, 2010), fusionne en 1925 avec ce nouveau BIE dont il est nommé directeur adjoint jusqu'en 1929. Après la Deuxième Guerre mondiale, Ferrière poursuit son activité de publiciste et d'écrivain; il entretient une correspondance volumineuse et se consacre au développement de l'École nouvelle de Bex et à celui du Home «Chez Nous» 5 .

Outre ces trois "piliers», d'autres personnes, présentes à Calais, ont participé à la fondation de la Ligue notamment à travers leur collaboration à la rédaction des «Principes de ralliement»: pour l'Angleterre, Harold Baillie-Weaver, pour la France, Juliette Decroix et M. Bermond6, pour la Belgique, Ovide Decroly et pour la Suisse, Robert Nussbaum (Notre Ligue, PEN, 1922, p. 1). Enfin, de nombreuses autres personnes non présentes à Calais approuvent et saluent la fondation de la Ligue en écrivant des lettres de félicitations et de soutien. Dans Pour l'Ère Nouvelle, les noms des personnes appartenant à la communauté francophone et aux pays latins sont mentionnés dans leur intégralité dès le premier numéro de 1922. Comme en atteste la liste des Français cidessous ${ }^{7}$, la majorité de ces personnes émanent de milieux intellectuels, universitaires et/ou administratifs dont les responsabilités en font des personnalités importantes dans le milieu de l'éducation.

5 Cette école-foyer constitue un modèle d'école nouvelle dont Ferrière loue les pratiques dans de nombreux écrits; il tourne même un film présentant la vie de cette école, qu'il utilisera comme outil de propagande dans ses nombreux voyages. Pour plus de détails sur ce foyer et sur le tournage du film, voir Coquoz (1998).

6 Le prénom complet de ce protagoniste ne figure pas dans PEN ni dans les archives consultées, et nous reste malheureusement inconnu.

7 Pour la Belgique, l'Italie, l'Espagne et les pays d'Amérique latine, voir Notre Ligue, PEN, 1922, 1. 
Georges Bertier: président des Éclaireurs de France, directeur de l'École des Roches et co-directeur de la revue L'Éducation.

Jean Bruhnes: professeur au Collège de France, inspecteur général des études à l'École des Roches.

Ferdinand Buisson: député de la Seine, professeur honoraire à la Faculté des Lettres de l'Université de Paris, directeur honoraire de l'Enseignement primaire au Ministère de l'Instruction Publique.

Roger Cousinet: inspecteur de l'enseignement primaire à Arcis-sur-Aube, co-fondateur de la société pédagogique «La Nouvelle Éducation».

Alice Jouenne: directrice de la première École de Plein air de la Municipalité de Paris et fondatrice de «L'Éducation nouvelle» (Groupe de recherches et d'expériences éducatives).

Pierre Mille: homme de Lettres.

Georges Renard: professeur au Collège de France.

Ludovic Zoretti: professeur à la Faculté des Sciences de l'Université de Caen.

(Notre Ligue, PEN, 1922, 1, p. 1)

\section{CHARTE ET PRINCIPES DE RALLIEMENT: DES TEXTES POUR FÉDÉRER}

À Calais, les fondateurs ${ }^{8}$ de la Ligue adoptent plusieurs «Principes de ralliement», qui constituent les bases idéologiques auxquelles les membres doivent adhérer, du moins à titre d'orientation générale. Ce texte d'orientation vise à rassembler les personnalités très diverses qui soutiennent le mouvement, à fédérer les initiatives d'éducateurs disséminés dans de mutliples régions du monde qui vivent des réalités très différentes dans les écoles. Ce texte figure au début de chaque numéro des revues fondatrices ainsi que dans celles qui deviennent organes officiels de la Ligue dès 1927. Énoncés en sept points, ces principes constituent les bases de l'Éducation nouvelle du point de vue des fondateurs, compte tenu du contexte qui est le leur, en 1921. Mais

8 En fait, l'analyse des archives (AIJJR, Fonds Ferrière, AdF/A) montre qu'Adolphe Ferrière est le principal rédacteur de ces principes de ralliement. Les autres membres fondateurs proposent quelques amendements mineurs, mais l'essentiel provient de la plume du pédagogue suisse. 
cette conception n'est pas partagée par tous les protagonistes du mouvement, raison pour laquelle certains d'entre eux restent volontairement à l'écart de la Ligue. ${ }^{9}$

\section{LIGUE INTERNATIONALE POUR L'ÉDUCATION NOUVELLE}

FONDEE AU CONGRÉS DE CALAIS LE 6 AOUT 1921, ET RATTACHEE AU BUREAU INTERNATIONAL DES ECOLES NOUVELLES, CREE A GENEVVE EN 1899

\section{PRINCIPES DE RALLIEMENT}

1. - Le but essentiel de toute éducation est de préparer l'enfant à vouloir et à réaliser dans sa vie la suprématie de l'esprit; elle doit done, quel que soit par ailleurs le point de vue auquel se place l'éducateur, viser à conserver et à accroltre chez l'enfant l'énergie spirituelle. 2. - Elle doit respecter l'individualité de l'enfant. Cette individualité ne pout se développer que par une discipline conduisant à la libération des puissances spirituelles qui sont en lui.

3. - Les études et, d'une façon générale, l'apprentissage de la vie, doivent donner libre cours aux intérêts innés de l'enfant, c'est-à-dire ceux qui s'éveillent spontanément chez lui et qui trouvent leur expression dans les activités variées d'ordre manuel, intellectuel, esthétique, social et autres.

4. - Chaque age a son caractère propre. Il faut donc que la discipline personnelle et la discipline collective soient organisees par les enfants eux-mêmes avec la collaboration des maitres; elles doivent tendre a renforcer le sentiment des responsabilités individuelles et sociales.

5. - La compétition égoìste doit disparaftre de l'éducation et être remplacée par la coopération qui enseigne à l'enfant à mettre son individualité au service de la collectivité. 6. - La coéducation réclamée par la Ligue, - coéducation qui signifie à la fois instruction et education en commun, - exclut le traitement identique imposé aux deux sexes, mais implique une collaboration qui permette à chaque sexe d'exercer librement sur l'autre une influence salutaire.

7. - L'éducation nouvelle prépare, chez l'enfant, non seulement le futur citoyen capable de remplir ses devoirs envers ses proches, sa nation, et l'humanité dans son ensemble, mais aussi l'être humain conscient de sa diguité d'homme.

\section{BUTS DE LA LIGUE}

1. - D'une façon générale la Ligue s'efforce d'introduire à l'école son idéal et les méthodes conformes à ses principes.

2. - Elle cherche a réaliser une coopération plus étroite : d'une part, entre les éducateurs des différents degrés de l'enseignement, d'autre part entre parents et éducateurs 3. - Elle se propose d'étsblir, par des congrès organisés tous les deux ans, et par les revues qu'elle publie, un lien entre les educateurs de tous les pays qui adhèrent à ses principes et visent des buts identiques aux siens.

4. - Il n'y a pas de cotisation. L'abonnement a la revue "Pour l'Ere Nouvelle" implique l'adhésion à la Ligue. Il suppose done l'adhésion à ses principes de ralliement, tout au moins à titre d'orientation générale.

Ces sept principes contiennent l'idée selon laquelle l'éducation doit libérer l'enfant afin que celui-ci s'épanouisse selon ses besoins et aptitudes personnelles. Quelle que soit la manière dont ils sont formulés, ils insistent fortement sur la notion de liberté. Celle-ci est explicitement évoquée dans les trois premiers où il est dit que l'éducation a pour but de «donner libre cours aux intérêts innés de l'enfant», de «respecter son individua-

9 Nous faisons allusion notamment à Célestin Freinet (1896-1966) qui critique le côté bourgeois des dirigeants de la Ligue (Mole, 2012) ainsi qu'à A. S. Neill qui se retire de la rédaction de The New Era dès 1923 en raison de principes auxquels il n'adhère pas. 
lité», de «libérer les puissances spirituelles qui sont en lui». Une conception de l'éducation fortement influencée par la conviction selon laquelle le développement repose sur des lois, conviction elle-même influencée par la biologie et la théosophie comme nous le verrons plus loin. D'après Hameline (2002b), la «suprématie de l'esprit» et «l'énergie spirituelle» sont des notions directement issues de la théosophie, syncrétisme religieux qui postule la présence d'une «force vitale» en chaque être humain, expression d'une force supérieure divine. De fait, ces principes révèlent une conception de l'enfant centrée sur l'individu où l'interaction avec l'environnement social est peu importante. Cet aspect apparaît cependant dans les points 4 à 6 où les mots comme "collectivité», "collaboration» et «coopération» révèlent la présence d'un monde social dans lequel l'enfant a son rôle à jouer. Un rôle orienté par les valeurs de service, de coopération et de responsabilité qui correspondent aux convictions pacifistes des éducateurs nouveaux qui ambitionnent de transformer la société sur la base de ces valeurs. Le dernier point se distingue des autres dans la mesure où il change d'échelle (la société, la nation, l'humanité) et de perspective (le futur) en proposant un regard sur les effets de l'Éducation nouvelle sur l'être humain et l'humanité.

Selon Hameline (2002b), le contenu et le ton employé dans la rédaction de ces principes de ralliement laissent penser que ces derniers ont été rédigés par Ferrière puis amendés et avalisés par le groupe fondateur présent à Calais. Ils sont imprégnés d'une conception spiritualiste de l'éducation caractéristique de cet auteur mais qui ne fait pas l'unanimité de tous les membres. C'est pourquoi ils sont remplacés, en novembre 1932, par une charte rédigée par le nouveau comité de rédaction de PEN composé principalement des membres du Groupe Français d'Éducation nouvelle (GFEN) ${ }^{10}$ Ce texte révèle la situation de crise que traverse l'Europe en ce début des années 1930 tant sur le plan économique que politique. Il invite tous les parents, éducateurs, administrateurs et travailleurs sociaux à se mobiliser dans un mouvement universel de rénovation de l'école pour «transformer l'ordre social» en instaurant un «esprit de coopération au moyen de l'éducation». Ce texte constitue désormais le socle de ralliement des membres de la Ligue et contient tous les éléments de la nouvelle orientation que prend celle-ci

10 Pour connaître la composition précise de ce comité éditorial, voir le chapitre 2 de ce livre (Pour l'Ėre Nouvelle). Sur le Groupe Français d'Éducation nouvelle, voir Le Roux-Yahiel (1983), Gutierrez (2008, 2011), Savoye (2006). 
à partir des années 1930 (Haenggeli-Jenni \& Hofstetter, 2011), plus particulièrement en 1932 pour la France. Une orientation marquée par un autre regard porté sur l'enfant, désormais perçu comme un être social inséré dans une communauté, qui doit être en mesure «de saisir les complexités de la vie sociale et économique» et de «s'adapter aux exigences de la vie en société».

La crise actuelle appelle la concentration à travers le monde entier de tous les efforts vers une éducation rénovée. En vingt ans, l'éducation pourrait transformer l'ordre social et instaurer un esprit de coopération capable de trouver des solutions aux problèmes de l'heure. A cela, nul effort national ne saurait suffire. G'est pourquoi la Ligue Internationale pour l'Education Nouvelle adresse un pressant appel aux parents, éducateurs, administrateurs et travailleurs sociaux pour qu'ils s'unissent en un vaste mouvement universel.

Seule une éducation réalisant dans toutes ses activités un changement d'attitude vis-à-vis des enfants peut inaugurer une ère libérée des concurrences ruineuses, des préjugés, des inquiétudes et des misères caractéristiques de notre civilisation présente, chaotique et dépourvue de sécurité. Une rénovation de l'éducation s'impose, basée sur les principes suivants :

$1^{\circ}$ L'éducation doit mettre l'enfant en mesure de saisir les complexités de la vie sociale et économique de notre temps.

$2^{\circ}$ Elle doit être conçue de manière à répondre aux exigences intellectuelles et affectives diverses des enfants de tempéraments variés et leur fournir l'occasion de s'exprimer en tout temps selon leurs caractéristiques propres.

3. Elle doit aider l'enfant à s'adapter volontairement aux exigences de la vie en société en remplaçant la discipline basée sur la contrainte et la peur des punitions par le développement de I'initiative personnelle et de la responsabilité.

$4^{\circ}$ Elle doit favoriser la collaboration entre tous les membres de la communauté scolaire en amenant maitres et élèves à comprendre la valeur de la diversité des caractères et de l'indépendance d'esprit.

$5^{\circ}$ Elle doit amener l'enfant à apprécier son propre héritage national et à accueillir avec joie la contribution originale de toute autre nation à la culture humaine universelle. Pour la sécurite de la civilisation moderne, les citoyens du monde ne sont pas moins nécessaires que les bons citoyens de leur propre nation.

\section{Ligue Internationale pour l'Éducation Nouvelle}

Gentre International : 29 Tavistock Square, Londres W. G. I. (Angleterre)

Pour tous renseignements concernant les adhésions, s'adresser au bureau de la Ligue Internationale pour l'Education Nouvelle: 29, Tavistock Square, Londres W. G. I. (Angleterre).

La priorité n'est plus de libérer et épanouir l'enfant mais plutôt de l'éduquer, le guider, «l'amener à apprécier son propre héritage national et à accueillir avec joie la contribution originale de toute autre nation à la culture humaine universelle». ${ }^{11}$ En d'autres termes, le centre d'intérêt s'est déplacé «de l'individu vers le social» comme le remarque Brehony (2004) pour le contexte anglo-saxon. Bien que la dimension humaine et universelle soit toujours présente, le concept de nation apparaît à deux reprises associé à l'idée que l'enfant doit en être fier, que ce soit en "appréciant l'héritage national» ou en faisant figure de «bon citoyen». Un concept révélateur du contexte dans lequel il s'inscrit et se développe. Si la nouvelle charte opère une rupture par rapport à l'ancien texte, elle conserve toutefois certains principes tels que la coopération, la collaboration entre maîtres et élèves ainsi qu'une discipline assumée collectivement, principe

11 Les mots entre guillemets sont issus du texte de la charte. 
auquel s'ajoute la dimension d'initiative personnelle. De fait, le véritable point commun entre les deux textes est celui qui concerne les fondements scientifiques de l'éducation, la conviction selon laquelle l'éducation doit reposer sur une connaissance psychologique de l'enfant. Dans les principes de ralliement, celle-ci apparaît de manière très succincte à travers la phrase «elle doit respecter l'individualité de l'enfant». Dans la charte, cette dimension est renforcée comme en atteste le second point: «Elle doit être conçue de manière à répondre aux exigences intellectuelles et affectives diverses des enfants de tempéraments variés et leur fournir l'occasion de s'exprimer en tout temps selon leurs caractéristiques propres». Dans l'un comme dans l'autre, c'est à la science psychologique qu'il est fait allusion, la seule permettant de connaître véritablement l'enfant et de fonder scientifiquement les revendications de réforme.

Tout comme les principes de ralliement, les buts de la Ligue sont imprimés en $2^{\mathrm{e}}$ page de PEN jusqu'en 1932, avant de disparaître au profit du texte susmentionné. Toutefois, ils se situent à un autre niveau puisqu'ils évoquent les moyens que se donne la Ligue pour opérationnaliser ses principes sur le plan concret. Des moyens révélateurs des ambitions militantes de cette Ligue qui cherche à unifier le mouvement à travers l'organisation de congrès et la publication de revues.

1. D’une façon générale la Ligue s'efforce d'introduire à l'école son idéal et les méthodes conformes à ses principes.

2. Elle cherche à réaliser une coopération plus étroite: d'une part entre les éducateurs des différents degrés de l'enseignement, d'autre part entre parents et éducateurs.

3. Elle se propose d'établir, par des congrès organisés tous les deux ans, et par les revues qu'elle publie, un lien entre les éducateurs de tous les pays qui adhèrent à ses principes et visent des buts identiques aux siens.

4. Il n'y a pas de cotisation. L'abonnement à la revue Pour l'Ėre Nouvelle implique l'adhésion à la Ligue. Il suppose donc l'adhésion à ses principes de ralliement, tout au moins à titre d'orientation générale.

S'il n'y a pas de condition particulière pour devenir membre de la Ligue, le point 4 mentionne toutefois l'exigence d'une adhésion aux principes de ralliement. Mais celle-ci est d'emblée nuancée par la mention «tout au moins à titre d'orientation générale» qui révèle la recherche de consensus parmi les fondateurs. Dans ce groupe, Beatrice Ensor est 
particulièrement sensible à l'esprit d'ouverture et précise à plusieurs reprises que la Ligue «ne se fera l'avocat d'aucune méthode d'éducation, mais recherchera ce qui peut, avec quelque variante, répondre aux besoins des différents pays ou écoles, en leur laissant une pleine liberté dans le cadre présenté» (Ensor, PEN, 1922, 1, p. 7).

Nous ne sommes pas, nous ne voulons pas être les défenseurs d'une doctrine. Nous voulons être les serviteurs de la vérité et de ce progrès réel, historique, infini dans le passé comme dans l'avenir, qui a son fondement dans la vérité. (Ensor, $P E N, 1922,1$, p. 7)

Dans sa conférence d'ouverture au Congrès d'Elseneur (1929), Ensor rappelle cette caractéristique de la Ligue qui se veut ouverte à toutes les tendances et se défend de tout caractère dogmatique. Pour elle, la Ligue n'est pas révolutionnaire mais plutôt évolutionnaire: «Our Fellowship is not revolutionary, it is evolutionary; it is not bound to any one or technique or method. We do not even make a dogma of the New Education» (cité par Boyd \& Makenzie, 1930, p. 3).

Ce désir de neutralité et d'ouverture est paradoxal avec l'attitude propagandiste qu'adoptent les fondateurs de manière générale. En effet, ces derniers s'investissent personnellement pour promouvoir l'Éducation nouvelle, un effort auquel ils appellent chacun à s'associer «en travaillant dans l'esprit de la Ligue», en convaincant les plus réticents du bien fondé et de la nécessité de ses principes pour construire une ère nouvelle. Car, comme le dit Ensor, "plus la Ligue comptera de membres, plus elle sera forte» (Ensor, PEN, 1922, p. 7). De fait, cet effort de propagande constitue une des activités principales de la Ligue. Selon la présidente, le travail de la Ligue peut se comparer à une «croisade» ${ }^{12}$ composée de deux tâches principales: premièrement, la réunion des pionniers de l'éducation de toutes tendances et de tous pays dans un même mouvement organisé, deuxièmement la propagande des principes de l'Éducation nouvelle au moyen de conférences, exposés et de publications (Boyd \& Makenzie, 1930, p. 2). Or, à travers cette activité de propagande, et par les moyens qu'elle se donne pour la mener à bien - congrès, revues, cours du soir, conférences, formation des maîtres - la

12 C'est le mot employé par Beatrice Ensor lors de son discours au congrès d'Elseneur, mot qui lui permet de rappeler les objectifs ambitieux de la Ligue (Ensor, PEN, 1929, 51, p. 217). 
Ligue contribue à l'instauration progressive de la nouvelle «science de l'éducation» dans de nombreux pays. Au fil des ans, l'augmentation de l'instabilité politique en Europe incite le Comité de rédaction de PEN à rappeler les convictions pacifistes de la Ligue et la neutralité de sa mission. Au nom de ces convictions, cette dernière justifie son «droit» d'examiner l'éducation dans tous les pays:

Notre Ligue s'interdit toute appréciation d'ordre politique, national ou confessionnel. Mais les questions qui touchent la justice humaine et à la paix par l'école, celles aussi qui sont d'ordre religieux (au sens non confessionnel du terme): triomphe en l'homme des valeurs spirituelles, raison et amour universels [...] sont au premier plan de ses soucis. C'est pourquoi nous voulons examiner dans un esprit d'impartialité et de vérité l'éducation des enfants dans tous les pays, même ceux qui, pour des motifs politiques, sont «tabous» aux yeux de certains partisans ou journalistes. (Éditorial, PEN, 1928, 35, p. 25)

Enfin, la Ligue défend une éducation pour la paix et par la paix qui s'inscrit dans l'esprit de la Société des Nations: «Par paix, nous n'entendons pas seulement la négation des luttes armées de nation à nation, mais la cessation des haines de races et des causes d'injustice au sein même des nations dont les fils sont divisés entre eux.» (Éditorial, PEN, 1927, 29, p. 110).

\section{L'ÂGE D'OR: ANCRAGE ET (RE)STRUCTURATION (1927-1930)}

Au début, la Ligue n'est pas une association organisée selon les schémas traditionnels; il n'y a ni comité, ni statuts, ni règlements. Les seuls liens sont des liens spirituels - «nos principes et la volonté de les réaliser dans la mesure du possible» - et des liens organisationnels - «nos congrès, tous les deux ans, et nos revues, paraissant en divers pays et en langues diverses; pour le moment en français, anglais et allemand» (circulaire $P E N, 1922,1$, p. 4). Même en 1927, son statut d'association n'est pas clair, ou du moins n'est pas revendiqué:

La Ligue internationale pour l'Éducation nouvelle n'est point proprement une «association» régulièrement constituée; à peine est-elle une fédération. Elle a plutôt l'ambition, comme le dit son statut de fondation, de jouer le rôle de LIEN ${ }^{13}$ entre les éducateurs qui s'intéressent théoriquement ou pratiquement à l'Éducation nouvelle. (Éditorial, PEN, 1927, 26, p. 49)

13 En majuscules dans le texte d'origine. 
Toutefois, il existe un Comité exécutif composé de quatre membres fondateurs, à savoir Ensor, Rotten, Ferrière et Baillie-Weaver, ce dernier assumant le rôle de président de ce comité. En 1925, Baillie-Weaver doit renoncer à sa fonction pour des raisons de santé et est alors remplacé par George S. Arundale (1878-1945) ${ }^{14}$. Les sections nationales affiliées à la Ligue se constituent progressivement, le plus souvent autour d'une personne reconnue qui assume aussi la direction d'une revue éditée dans la langue du pays. En 1925, la croissance rapide de la Ligue conduit les membres du Comité exécutif à constituer un Comité international. Celui-ci est formé d'un représentant par pays «où l'éducation nouvelle réunit un noyau de théoriciens et de praticiens et où une revue d'éducation affiliée à la Ligue concentre les aspirations des novateurs» (Éditorial, PEN, 1927, 26, p. 49). Les pays et les représentants concernés sont: l'Angleterre (B. Ensor), l'Allemagne (E. Rotten), la Belgique (O. Decroly), la Bulgarie (Dr. Katzaroff), la Hongrie (M. Nemes), l'Écosse (G. Krutwell), le Danemark (S. Naessgard), l'Italie (G. Lombardo-Radice), la France (J. Hauser), l'Espagne (L. Luzuriaga) et la Suisse (A. Ferrière).

En 1927, lors du Congrès de Locarno, le Comité international de la Ligue décide de se donner une organisation plus structurée, en raison de l'augmentation considérable de ses membres et des charges assumées par le Comité exécutif.

L'ancienne organisation, selon laquelle la Ligue se bornait à servir de lien entre les éducateurs et psychologues orientés vers une éducation conforme aux données de la psychologie génétique s'est révélée bonne. Mais les ressources n'ont pas crû en proportion des charges - énormes assumées par le Comité exécutif et le secrétariat (Éditorial, PEN, 1927, 33, p. 279).

Cette organisation vise une nouvelle répartition des tâches et pose des conditions précises concernant l'affiliation des sections nationales et des revues. Parmi ces conditions, une contribution financière est désormais demandée à chaque section, ce qui prouve que les raisons ayant

14 Conseiller d'éducation de l'état d'Indore (Inde), professeur d'histoire et principal du Collège Central Hindou de Varanasi dès 1909. En 1917, Arundale fonde avec Annie Besant l'Université nationale Indienne à Chennai dont le premier directeur (chancellor) sera Rabindranath Tagore. Théosophe très engagé, Arundale devient président de la Theosophical Society dès 1934 et fonde à Adyar (près de Madras), cette même année, une école (Besant Memorial School) où Maria Montessori enseignera pendant trois ans (1939-1942). 
présidé à cette nouvelle organisation ne sont pas uniquement structurelles mais aussi financières. En 1930, la Ligue atteint des sommets de participation suite au Congrès d'Elseneur (Danemark, 1929) ce qui la conduit à nommer un Comité consultatif dont la fonction est de soulager la tâche du Comité exécutif. En outre, des «commissions permanentes» sont formées dans le but d'étudier certaines thématiques en dehors des périodes de congrès.

Tableau 1.

Structure de la Ligue internationale pour l’Éducation nouvelle ${ }^{15}$

\begin{tabular}{|c|c|}
\hline Comité exécutif & $\begin{array}{l}\text { Beatrice Ensor, présidente } \\
\text { Adolphe Ferrière, vice-président } \\
\text { Elisabeth Rotten, membre fondatrice }\end{array}$ \\
\hline Comité consultatif & $\begin{array}{l}\text { William Boyd, professeur d'éducation à l'Université de } \\
\text { Glasgow } \\
\text { Ovide Decroly, professeur de psychologie enfantine à } \\
\text { l'Université de Bruxelles } \\
\text { H. C. Bent (B.A.), directeur de l'École Gateway à } \\
\text { Leicester } \\
\text { D. Katzaroff, professeur à l'Université de Sofia } \\
\text { G. Mattson, Fil. Mag. à Upsal } \\
\text { C. Ryan, professeur d'éducation au Collège de } \\
\text { Swarthmore + un représentant des sections nationales } \\
\text { allemande et française + un autre membre pour l'Angleterre }\end{array}$ \\
\hline
\end{tabular}

Comité international 28 pays affiliés: chacun représenté par une personne ${ }^{16}$ et correspondants

\begin{tabular}{ll}
\hline Commissions & $1 . \begin{array}{l}\text { Psychologie et Éducation (4 sous-sections): } \\
\text { permanentes }\end{array}$ \\
& a) Psychologie génétique (chef: Jean Piaget, \\
& Université de Genève) \\
& b) Tests mentaux (chef: Virgil Dickson, Bureau of \\
& Research and Guidance, Berkeley, USA)
\end{tabular}

15 Ce tableau a été fait sur la base des indications de l'éditorial de janvier 1930 (PEN, 54, pp. 1-4). Les noms, prénoms et titres des personnes sont indiqués tels qu'ils figurent dans cet éditorial, c'est pourquoi il n'y a pas d'harmonisation dans la manière de les citer.

16 Pour les noms et coordonnées de ces personnes, se référer à l'éditorial de janvier 1930 (PEN, 54, p. 1) 
c) Psychologie individuelle et Types psychologiques (chef: K.E. Krafft, Zurich)

d) Psychologie de l'inconscient ${ }^{17}$

2. L'enfant dans la famille (chef: A. H. Reeve, présidente de la Fédération du Foyer et de l'École, USA)

3. L'Enfant à l'école maternelle (chef: A. Lynch, un des auteurs de The case of Nursery schools, UK)

4. Refonte des programmes scolaires (chef: H. Rugg, professeur d'éducation à l'Université de Columbia, New York, USA)

5. Préparation des maîtres pour l'Éducation nouvelle

6. L'École dans ses rapports avec la rénovation sociale

\section{BAROMÈTRES ET BOUSSOLES: LES CONGRÈS INTERNATIONAUX}

La croissance de la Ligue est très rapide dès ses premières années d'existence. Au début, elle est visible dans la rubrique «Notre Ligue» de Pour l'Ère Nouvelle qui informe les lecteurs des nouvelles adhésions et des messages de soutien reçus par le comité: «Nombreux sont les messages de chaude sympathie qui nous parviennent de toutes parts. Il en émane de tous les coins du monde latin, de tous les milieux sociaux, de toutes les confessions» (Notre Ligue, PEN, 1922, 2, p. 30). En juillet 1922, la Ligue compte plus de 200 membres en pays latins «sans compter les nouvelles adhésions qui parviennent chaque jour», selon les dires de Ferrière. Six mois plus tard, la rédaction fait état de plus de 260 abonnés à Pour l'Ère Nouvelle sans compter les abonnés aux revues anglaise et allemande. Le rédacteur en chef interprète cette croissance par le fait que contrairement à d'autres sociétés, il ne s'agit pas de «recruter par persuasion des membres nouveaux, plus ou moins convaincus» (Notre Ligue, PEN, 1923, p. 1).

Nos membres [...] étaient en quelque sorte parties intégrantes de notre Ligue sans le savoir, avant de la connaître par le fait seul qu'ils travaillaient à la rénovation de l'éducation dans un esprit de science et de conscience [...]. Nous n'avons eu qu'à organiser les énergies d'ores et déjà actives; moins que cela: à servir de lien entre elles, à les mettre en contact, à les faire connaître les unes aux autres. (Notre Ligue, PEN, 1923, p. 1)

17 L'absence de responsable pour cette sous-section n'est pas explicitée dans cet éditorial, ni ailleurs dans la revue. 
Mais cette visibilité de la croissance s'estompe rapidement. Pendant les trois années suivantes, la rédaction de $P E N$ donne très peu d'informations à ce sujet. Ce n'est qu'en janvier 1926 que l'éditorial signale une brusque croissance des membres en pays latins $(+60 \%)$ suite au Congrès de Heidelberg. Désormais, les congrès deviennent de véritables boussoles et baromètres permettant de mesurer l'expansion de la Ligue et l'évolution de ses orientations: le nombre de participants révèle la croissance des adhérents et les thèmes de congrès indiquent les sujets en débats au cœur du mouvement. Comme le montre le tableau suivant, le nombre de participants double entre le Congrès de Calais (1921) et celui de Montreux (1923), puis augmente considérablement jusqu'au Congrès de Heidelberg. Dès ce moment-là, la croissance devient exponentielle et atteint un sommet au Congrès d'Elseneur (1929) qui marque l'apogée du mouvement et de la Ligue, que ce soit au niveau du nombre de ses membres ou de son étendue géographique.

Tableau 2.

Participants aux congrès internationaux de la Ligue internationale pour l'Éducation nouvelle (1921-1936)

\begin{tabular}{cccc}
\hline Année & Lieu & Thème & Participants \\
\hline 1921 & Calais (F) & L'Expression créatrice de l'enfant & 150 \\
\hline $\mathbf{1 9 2 3}$ & Montreux (CH) & L'École active et l'esprit de service & 300 \\
\hline $\mathbf{1 9 2 5}$ & Heidelberg (D) & $\begin{array}{c}\text { Comment éveiller l'activité spontanée } \\
\text { chez l'enfant? }\end{array}$ & 450 \\
\hline $\mathbf{1 9 2 7}$ & Locarno (CH) & La signification de la liberté en éducation & 1200 \\
\hline $\mathbf{1 9 2 9}$ & Elseneur (DK) & $\begin{array}{c}\text { Vers une éducation nouvelle } \\
\text { Psychologie nouvelle et curriculum }\end{array}$ & 2000 \\
\hline $\mathbf{1 9 3 2}$ & Nice (F) & La transformation sociale et l'éducation & 1600 \\
\hline $\mathbf{1 9 3 6}$ & Cheltenham (UK) & $\begin{array}{c}\text { Les fondations éducatives de la liberté } \\
\text { et de la communauté libre }\end{array}$ & 1400 \\
\hline & &
\end{tabular}

À la lecture de ces chiffres, nous voyons que le développement de la Ligue est spectaculaire en particulier durant la première décennie. Toutefois, cette croissance est fragile car elle est soumise à l'évolution des contextes sociaux et politiques qui influencent l'engagement militant des acteurs. En revendiquant une identité «universelle», la neutralité et 
la tolérance à l'égard de tous, la Ligue défend des valeurs qui correspondent aux années d'après guerre où tous les esprits sont occupés à la construction d'un monde meilleur. Mais dans les années 1930, ce discours est parfois taxé «d'utopiste», de «désuet» voire de «dépassé» dans un contexte où la réalisation des projets énoncés dans les années 1920 devient urgente. Dès lors, la Ligue modifie sa rhétorique en utilisant un discours moins «corporatiste» faisant l'éloge des individualités et du dynamisme des diverses personnalités qui la composent:

Nous sommes une organisation d'âmes vivantes, âmes très fluides d'individualistes très jaloux de leur autonomie spirituelle. Trop de règlements, trop de comités, trop de soucis d'argent seraient sa perte. Elle s'ankyloserait. Elle se cristalliserait en un bloc, solide sans doute, mais lourd, et qui coulerait au fond de l'eau. (Rédaction, PEN, 1931, 64, p. 1)

À partir de 1937, le déclin du mouvement s'accentuant, la Ligue ne peut plus tenir le discours de la neutralité. Elle doit sortir de cette identité «confortable» et prendre position si elle veut rester crédible auprès de ses membres. Elle le fait à travers des manifestes dans lesquels elle exprime ses positions aussi bien à l'égard de l'éducation que de la société, de la démocratie et du monde en général. C'est ainsi qu'elle affirme par exemple, en octobre 1937, son refus de collaborer avec des pays qui n'offrent pas les conditions humaines minimales de vie en société. Ce texte - La Ligue internationale pour l'Éducation nouvelle et le monde actuel - signé par un collectif de membres, marque ainsi pour la première fois, une prise de position politique à l'égard des pays qui bafouent les droits de l'homme et, par là-même, les droits de l'enfant. Un texte qui sera suivi par d'autres, inaugurant ainsi «l'époque des manifestes».

Nous ne pouvons pas donner une éducation digne de ce nom à des êtres qui souffrent de faim, du froid, d'un sentiment d'insécurité et d'injustice; nous ne pouvons pas prendre notre parti de systèmes économiques ou sociaux qui aboutissent à ces maux ou qui les tolèrent. Nous devons garder comme contraire à notre idéal d'éducation toute organisation sociale qui permet l'oppression ou l'exploitation de l'un par l'autre que ce soit à l'intérieur ou à l'extérieur de nos frontières nationales. (Collectif LIEN, PEN, 1937, 131, p. 196) 
Malgré ces prises de position en faveur de la démocratie, le contexte tendu de cette fin des années 1930 se traduit par divers problèmes: ralentissement global des engagements, difficultés au niveau de la mise en application des méthodes nouvelles et problèmes de circulation de l'information en raison de l'interruption de plusieurs revues. Le taux de participation aux congrès diminue, les groupes de partisans se diversifient laissant augurer la fin d'un mouvement international autrefois massif et luttant pour une cause commune. Les manifestations en faveur de l'Éducation nouvelle sont désormais plus discrètes, plus isolées s'accompagnant ou non d'expériences pratiques, conservant toutefois leur caractère international.

Cette analyse de la Ligue internationale pour l'Éducation nouvelle à partir des indications disponibles dans ses revues, nous permet d'observer la rapidité de sa croissance au cours des années 1920 suivie d'un ralentissement dans les années 1930 bien que la fréquentation des congrès soit toujours importante. Pour faire face à cette croissance, la Ligue se structure progressivement dans le but de répartir le travail mais aussi de mieux contrôler ce qui se dit et se fait en son nom. Comme nous l'avons vu, le charisme de ses trois dirigeants joue un rôle important dans les efforts de propagande auxquels ils participent activement afin d'assurer la cohésion du mouvement. 



\section{PlateFormes D’ÉCHANGES, PRISMES CULTURELS: TROIS REVUES FONDATRICES}
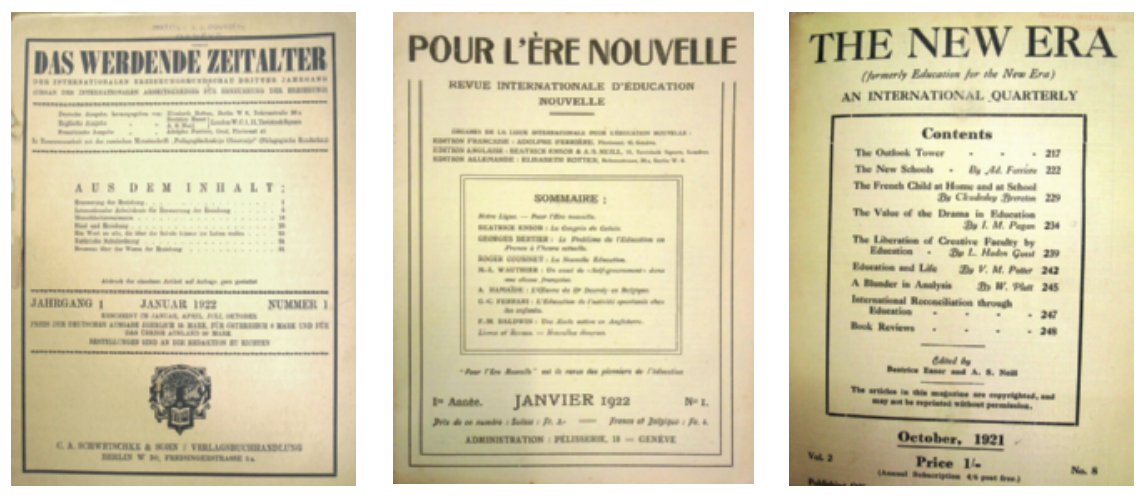

Lors du Congrès de Calais en 1921, les fondateurs de la Ligue décident de publier trois revues comme organes d'échanges entre ses membres: Pour l'Ère Nouvelle, The New Era, Das Werdende Zeitalter. Elles sont dirigées par les trois présidents chacun étant responsable d'une région linguistique. Bien qu'elles portent le même nom ${ }^{1}$, elles sont indépendantes tant sur le plan éditorial que financier: ses rédacteurs ont une grande autonomie dans le choix des articles et des auteurs, mais ils collaborent étroitement pendant les premières années. Il s'agit donc de trois revues parallèles dont les noms figurent respectivement sur les trois supports

1 Les noms et leur signification sont quasi identiques dans les trois cas mais le titre allemand contient une dimension évolutive («werdende»: en devenir, naissant) qui est moins présente dans les titres anglais et français. 
(1922-1926) mais dont le contenu est adapté en fonction du contexte local dans lequel il est diffusé. Ce chapitre propose une analyse de chacun de ces périodiques, présentant son infrastructure éditoriale, ses auteurs et ses thématiques principales. Toutefois, le niveau d'analyse de l'édition francophone est plus approfondi puisqu'elle constitue la source principale de nos investigations. Nous portons une attention particulière à l'histoire de Pour l'Ére Nouvelle, aux profils des auteurs et aux domaines abordés dans les articles de fonds. Cette analyse vise à déceler les synergies entre les trois revues, à identifier leurs points communs mais aussi à voir quelles sont leurs différences et spécificités. Cette approche comparative nous permettra de voir dans quelle mesure le mouvement est uni, si les objectifs sont semblables d'une région à l'autre et surtout, si les aires culturelles influencent le mouvement et les discours tenus à son propos.

\section{DAS WERDENDE ZEITALTER:}

\section{UNE MILITANCE EMPREINTE DE PHILOSOPHIE}

\section{UNE RÉDACTION STRUCTURÉE ET PARTAGÉE}

L'édition germanophone Das Werdende Zeitalter (DWZ) est le prolongement d'une revue qui paraît depuis 1920 sous le nom Internationale Erziehungs-Rundschau (IER) comme supplément mensuel un autre périodique, Die Neue Erziehung. Dirigée par Elisabeth Rotten (1882-1964) à la demande du département d'éducation de la Deutschen Liga für Völkerbund (Ligue allemande pour la Société des Nations), elle est aussi l'organe de la Bund Entschiedener Schulreformer (Ligue pour une réforme radicale de l'école) (Haubfleisch \& Link, 1994). Suite à la fondation de la Ligue, la revue change de nom et s'appelle désormais Das Werdende Zeitalter. ${ }^{2}$ Mais elle poursuit des buts similaires et conserve ses thématiques ainsi que son lectorat. Elle paraît pour la première fois en janvier 1922 sous ses nouveaux noms et statuts. Toutefois, jusqu'en 1925,

2 Ce nom aurait été inspiré d'un ouvrage de Gustav Landauer édité par Martin Buber - deux amis proches d'Elisabeth Rotten - intitulé Der Werdende Mensch (Landauer, 1921). Il s'agit d'un recueil de textes philosophiques, politiques et littéraires écrits par Landauer, membre du mouvement anarchiste allemand. 
elle conserve son ancien nom placé comme sous-titre en page de couverture de chaque numéro bien que son statut d'organe de la Ligue soit désormais mentionné: «Organ des Internationalen Arbeitskreises für Erneuerung der Erziehung» (Organe de la Ligue internationale pour l’Éducation nouvelle).

En 1926, cette indication disparaît au profit d'un nouveau sous-titre «Eine Zeitschrift für Erneuerung der Erziehung» qui change à nouveau en 1928 puisque le journal devient mensuel: «Eine Monatschrift für Erneuerung der Erziehung». Sur cette première page figurent les noms des trois revues fondatrices et de leurs rédacteurs en chef attestant de leur statut commun. L'appartenance à la Ligue est visible, dès 1926, à travers les noms des membres du comité international, ceux des revues affiliées ainsi que les buts et principes de ralliement. Dans DWZ, ceux-ci ornent successivement la 4e (1922-1923), la 3e de couverture (1924-1929) puis disparaissent à partir de 1930, du moins dans l'édition consultée. ${ }^{3}$ Grâce à notre connaissance des deux revues sœurs, nous faisons l'hypothèse que cette disparition n'est pas le signe d'une rupture avec la Ligue mais plutôt la conséquence des changements d'orientation et d'organisation décidés lors du Congrès d'Elseneur de 1929.4 À partir de ce moment-là, il n'est plus du tout évident que DWZ est l'un des organes officiels de la Ligue. En effet, bien que son sous-titre indique qu'il s'agit d'une revue d'Éducation nouvelle, le journal semble prendre davantage $\mathrm{d}^{\prime}$ autonomie publiant essentiellement des auteurs allemands dont les témoignages et thématiques abordés concernent avant tout l'Allemagne. Ainsi, la dimension internationale de la revue diminue au profit de réalités et préoccupations nationales, réalités qui seront bientôt menaçantes pour la survie de la revue et la conduiront à cesser de paraître au cours de l'année 1932.

La fréquence de parution de Das Werdende Zeitlalter est très similaire à celle de Pour l'Ėre Nouvelle, opérant des changements aux mêmes moments que cette dernière. Ainsi, elle paraît trimestriellement - janvier, avril, juillet, octobre - de 1922 à 1925 puis passe au rythme de six numéros

3 L'édition consultée aux archives du Bureau International d'Éducation - UNESCO (Genève) - fait partie d'un volume relié contenant tous les numéros de 1922 à 1930 dont les pages de couverture ont parfois été coupées.

4 Les principes de ralliement disparaissent également de la revue anglophone la même année durant laquelle on remarque d'importants changements d'orientation. 
par an dès 1926 avant de devenir mensuelle (10 numéros par an) à partir de 1927. Chaque numéro contient 7 à 10 articles ce qui représente environ 40 pages de contenu. DWZ se distingue de ses «consœurs» par sa plus courte durée de publication. En effet, son dernier numéro paraît en été 1932 (juillet-août) alors que The New Era et Pour l'Ère Nouvelle poursuivent une publication régulière jusqu'en 1940. Dans la rubrique Mitteilungen der Schriftleitung (Nouvelles de la Rédaction) de ce dernier numéro, les rédacteurs (Wilker et Rotten) annoncent leurs préoccupations concernant la poursuite de la publication de DWZ:

Mit diesem ersten der für 1932 in Aussicht genommen losen Hefte wird der Versucht gemacht, die Verbindung mit unserem Leserkreis wiederherzustellen. Über die Rückkehr zur regelmässig Erscheinungsform kann heute nichts gesagt werden, doch werden alle nur möglichen Anstrengungen gemacht, um sie zu bewerkstelligen. Eine gute Aufnahme dieses Heftes und freundliche Nachsicht für den Ubergangscharakter, den es leider tragen muss, werden uns dafür hilfreich und ermutigend sein. (Rédaction, DWZ, 1932, p. 7)

Cette interruption s'explique d'une part par la crise économique mondiale qui accentue les difficultés financières de la revue et, d'autre part, par la détérioration de la situation politique en Allemagne qui oblige Rotten, tout comme Wilker, à quitter le pays et émigrer en Suisse. Ce dernier s'y installe dès 1933 où il travaille d'abord pour la revue Schweizerische pädagogische Zeitschrift ${ }^{6}$ puis comme co-directeur de l'École nouvelle de Hof Oberkirch de fin 1934 à 1937 avant de quitter le pays pour d'autres contrées. Elisabeth Rotten s'installe elle aussi en Suisse à partir de 1934 d'où elle poursuit son travail de conférences, cours, traductions et publications jusqu'à la fin de sa vie.

De 1922 à 1925, Das Werdende Zeitalter (DWZ) est éditée par Elisabeth Rotten, une personne familière avec le travail d'édition puisqu'elle édite la

5 En français: «Avec ces cahiers isolés de 1932, des tentatives ont été faites de renouer avec le lectorat. À propos du retour à la publication régulière de la revue, nous ne pouvons rien dire aujourd'hui mais tous les efforts seront faits pour l'accomplir. Un bon accueil de ces cahiers et une indulgence amicale de leur caractère malheureusement transitoire nous seront utile et encourageant.»

6 Cette revue qui paraît depuis 1891, en allemand, est l'organe de l'Association des enseignants suisses et poursuit une ambition très explicitement théorique et scientifique (Hofstetter, 2008) 
revue Die Internationale Erziehungs-Rundschau (IER) depuis 1920. Son travail de rédactrice est étroitement lié à son engagement dans diverses associations œuvrant pour une réforme de l'école ainsi que dans les mouvements pacifistes au sein desquels elle milite dès le début de la Première Guerre mondiale. Elle est co-fondatrice en 1914 de la plus importante organisation pacifiste allemande - Bund Neues Vaterland qui deviendra plus tard la Deutsche Liga für Menschenrechte (Ligue allemande pour les Droits de l'Homme) et participe en 1915 à la fondation de Women's International League for Peace and Freedom. ${ }^{7}$ Comme nous le verrons par la suite, l'engagement de Rotten dans ces divers mouvements n'est pas sans influence sur les thématiques abordées dans Das Werdende Zeitalter, en particulier à travers les auteurs qu'elle sollicite pour y écrire.

Dès 1926, un deuxième nom apparaît aux côtés de celui de la rédactrice: Karl Wilker (1885-1980). ${ }^{8}$ Tout comme Rotten, ce dernier a déjà une certaine expérience de l'édition puisqu'il coédite avec Johannes Trüpers la revue Zeitschrift für Kinderforschung de 1910 à 1920 à Berlin. Wilker a une formation de pédagogue et $\mathrm{d}^{\prime}$ enseignant ${ }^{9}$ mais il fait aussi des études de sciences naturelles (botanique, zoologie, minéralogie, géologie) qu'il complète par des études de médecine et de psychologie entre 1910 et 1914. Si le nom de Wilker n'apparaît officiellement qu'à partir de 1926 en page de couverture, il signe déjà de nombreux articles - souvent par ses initiales K.W. - bien avant cette date. De fait, son rôle est crucial dans la publication de $D W Z$ car il assume à lui seul la rédaction de plusieurs rubriques voire de quelques numéros entiers. À partir de 1928, les noms des deux responsables d'édition sont suivis de ceux de plusieurs collaborateurs permanents (ständiger Mitarbeiter). Ces derniers ont le statut particulier de collaborer à la revue de manière régulière à travers la rédaction $\mathrm{d}^{\prime}$ articles ${ }^{10}$ mais ils ne participent pas à la gestion du périodique en tant que tel. Cette liste composée en majorité d'auteurs allemands comporte

7 Ferrière entretient des contacts réguliers avec les membres de cette Ligue, dont le siège est à Genève, auprès de laquelle il donne plusieurs conférences, notamment en 1919 (Gerber, 1989).

8 Cette notice biographique a été rédigée sur la base des informations indiquées dans Haubfleisch (2008), Haubfleisch et Link (1994) et dans https:/ / de.wikipedia.org/wiki/Karl_Wilker (consulté le 8 octobre 2015).

9 Il étudie la pédagogie à Iéna.

10 Il est probable que ces derniers signent un contrat les engageant à livrer un nombre défini d'articles comme c'est le cas pour Ferrière qui, en avril 1915, 
également deux Suisses - Arthur Fischer, Friedrich Kilchenmann - et deux Autrichiens - Irma Sander, Ludwig E. Tesar:

Herausgegeben von E. Rotten und Karl Wilker unter ständiger Mitwirkung von Gertrud Baer (München), Carl Heinrich Becker (Leipzig), Dr. Martin Buber (Heppenheim), Peter Engel (Worms), Otto Erdmann (Burg Nordeck), Dr. Arthur Fischer (Zürich), Dr. Wilhelm Friedrich (Kassel), Paul Geheeb (Odenwaldschule), Dr. med. Clara Happel (Berlin), Dr. Fr. Kilchenmann (Wabern, Bern), William Lottig (Hamburg), Dr. J.W. Mack (Selent), Dr. med. J. Mainzer (Nürnberg), Prof. Karl Metzner (Leitmeritz), Fritz Natorp (Schmalkalden), Gertrud Pincus (Berlin), Dr. Friedrich Schlünz (Düsseldorf), Irma Sander (Innsbruck), Dr. Hans Simons (Berlin), Dr. med. Else Sumpf (VorderHindelang), Ludwig Erik Tesar (Wiener Neustadt, Östereich), Dr. Carl Theil (Jena), Dr. Leo Weismantel (Marktbreit) ${ }^{11}$.

Parmi ces collaborateurs figurent des personnes travaillant dans le milieu de l'éducation, dans celui de la psychologie, de la médecine ou de la politique. La plupart, hommes et femmes confondus, sont porteurs d'un doctorat comme l'indique le préfixe «Dr.» apposé devant leur nom. Les appartenances professionnelles sont variées parmi lesquelles figurent des médecins, des philologues et des philosophes. Mais la majorité d'entre eux sont des «professionnels de l'éducation» que ce soit en tant qu'instituteur, professeur d'école secondaire (Fritz Natorp, professeur de dessin), directeur d'école (Albrecht Merz, Paul Geheeb), professeur dans les écoles normales, dans des instituts de formation d'enseignants (Carl Theil est professeur à l'Institut de Iéna) ou à l'université (Karl Metzner). Les hommes sont majoritaires (18 sur 23) mais les femmes ont le même profil que leurs collègues puisque deux d'entre elles sont médecins (Dr. med. Clara Happel, Dr. med. Else Sumpf). Parmi ces collaborateurs, certains cumulent plusieurs fonctions à l'exemple de Carl Heinrich Becker (1876-1933), éminent philologue et professeur d'histoire et de culture orientale. En parallèle de sa charge de professeur, ce dernier est actif sur le plan politique puisqu'il occupe une fonction au Ministère de la culture pendant la Première Guerre mondiale avant d'être nommé secrétaire d'État puis Ministre de l'Éducation, de la

signe un engagement pour 15 articles avec la revue Éducation, dirigée par Georges Bertier (Gerber, 1989, p. 12).

11 Liste des collaborateurs de la revue telle qu'elle est publiée en première page des numéros de DWZ dès 1928. 
Science et des Arts pour la Prusse dès les années 1920. Parmi ces auteurs, plusieurs participent en 1931 à la fondation de la section allemande et en deviennent les représentants:

C. Heinrich Becker (ministre de l'Éducation, Berlin), J. Gebhard (conseiller scientifique, Hamburg), R. Ulich (professeur de philosophie, Dresden), L. Weismantel (écrivain et pédagogue, Markbreit/M.), H. Doemer (professeur et conseiller d'école supérieure), M. Muchow (conseillère scientifique, Hamburg)

Les profils professionnels de ces auteurs montrent que les questions d'éducation et en particulier la réforme de l'éducation en Allemagne Reformpädagogik - intéressent les plus hauts représentants de la communauté intellectuelle. C'est également là que s'engagent les représentants du champ disciplinaire naissant des sciences de l'éducation comme c'est le cas dans les revues francophone et anglophone.

Pendant toute sa durée de publication, Das Werdende Zeitalter ne comporte pas d'éditorial, les deux pages de couverture étant immédiatement suivies des articles indiqués dans le sommaire. Par contre, dès 1926, une rubrique appelée «Mitteilungen» fait office d'éditorial; on devine alors la présence d'une équipe de rédaction comme l'indique cet ajout dès 1930 «Mitteilungen der Redaktion». Cette rubrique donne des nouvelles diverses concernant la Ligue et la section allemande ainsi que diverses informations concernant les cours, conférences, séminaires, colloques et stages qui ont lieu dans le monde entier. Selon Haubfleisch et Link (1994), cette rubrique constitue une source inestimable d'informations sur l'histoire sociale de la Reformpädagogik et permet de mesurer l'envergure internationale du mouvement à travers les projets, les initiatives et les liens qu'entretiennent les divers acteurs de cette histoire.

Rubrik Mitteilungen ist eine in ihrer Wichtigkeit für eine sozialgeschichtliche Forschung zur Reformpädagogik nicht zu unterschätzende Quelle, denn sie enthält im internationalen Rahmen eine Fülle von Hinweisen auf und Berichten von Tagungen, Kongressen, u.ä. sowie auf Personen, Projekte und Verbindungen einzelner Pädagogen und Initiativen. (Haubfleisch \& Link, 1994 , p. 12$)^{12}$

12 En français [la traduction est nôtre]: «L'importance de la rubrique 'Mitteilungen' ne doit pas être sous-estimée dans le cadre d'une recherche 
Karl Wilker assure également la rédaction de deux autres rubriques (signées occasionnellement par Elisabeth Rotten). Dans la première, intitulée «Bücher» ou «Bücherschau» et qui paraît dès 1923, il fait la recension d'une large palette de livres parus dans les domaines de l'éducation, de la philosophie, de la psychologie voire même de la politique. Selon Haubfleisch et Link (1994), ce ne sont pas moins de 805 recensions qui sont écrites par Wilker en dix ans de publication, parmi lesquelles figurent les titres d'ouvrages de nombreuses figures de l'Éducation nouvelle telles que Claparède, Neill, Price ou Wyneken. D'après ces auteurs, l'étendue de la culture de Wilker en matière de littérature sur l'éducation est précisément l'une des raisons qui, quelques années après la disparition de $D W Z$, incite Friedrich Schneider à engager Wilker comme coéditeur d'une nouvelle revue. Celle-ci porte un nom révélateur de la nouvelle orientation que prend l'Éducation nouvelle après les années 1930: Internationale Zeitschrift für Erziehungswissenschaft (Revue internationale des Sciences de l'Éducation).

Dans la deuxième rubrique intitulée «Zeitschriftenschau» qui paraît dès 1926, Wilker fait le point sur des articles publiés dans une grande diversité de revues. Si la majorité des recensions concerne des revues allemandes, l'importance donnée à des revues étrangères publiées dans des langues aussi diverses que le danois, le hollandais ou le roumain montre non seulement l'étendue géographique du mouvement mais laisse supposer que l'auteur maîtrise de nombreuses langues. Le volume croissant de ces recensions au fil des années atteste de l'augmentation considérable des revues, en particulier à partir de 1928, dans une variété de domaines connexes à l'éducation. Au début (1927-1928), Wilker classe les recensions par thème, ce qui lui permet de regrouper des informations thématiques issues de revues diverses. Cette pratique nous permet d'avoir un aperçu global des thématiques les plus en vogue durant cette période: Kunsterziehung (éducation à l'art), Jugendschriften (écriture des jeunes), Alkoholfrage (alcoolisme), Sexuelle Frage (sexualité), Individual psychologie (psychologie individuelle), Gemeinschaftserziehung (éducation communautaire), Deutsche Auslandschule (école allemande à l'étranger), Jugendbewegung (mouvements de jeunesse), Tierschutz

socio-historique sur l'Éducation nouvelle; c'est une source inestimable d'information de compte-rendus, de rapports de conférences et congrès, ainsi que d'informations sur les personnes, les projets, les initiatives individuelles et les connexions entre éducateurs.» (Haubfleisch \& Link, 1994, p. 12) 
(protection des animaux), Neue Zeitschriften (nouvelles revues), Geschichtunterricht (enseignement de l'histoire), Musikerziehung (éducation à la musique), Arbeitschule (École active), Schulreform (réforme scolaire). Dans cette rubrique, Wilker fait également le résumé de certains articles parus dans les revues The New Era et Pour l'Ère Nouvelle indiquant parfois le sommaire complet du numéro dont il est question.

De manière générale, la revue s'adresse à un public de praticiens ainsi que Rotten l'exprime dans les premières pages du premier numéro de 1920:

Wir möchten einen Spiegel zum Auffangen und Vergleichen recht vieler Lösungsversuche bieten, überzeugt, dass in solchem Suchen die Erzieher aus allen Ländern und Sprachen sich bei aller Mannigfaltigkeit der Wege und Meinungen finden müssen in einer gemeinsamen Zielgebung. Dies immanente Ziel aller echten Pädagogik kann nur sein die Erweckung und Pflege der schöpferischen Seelenkräfte an Stelle der zerstörenden lebensfeindlichen Triebe. (Rotten, IER, 1920, 1, p. 2) ${ }^{13}$

Comme le relèvent Haubfleisch et Link (1994), l'objectif de ce périodique n'est pas de mettre l'accent sur les théories éducatives mais plutôt de privilégier les articles sur les pratiques pédagogiques et les expériences d'application des méthodes nouvelles. Selon ces auteurs, Das Werdende Zeitalter se distingue d'autres revues similaires par l'étendue et la diversité des thématiques qui y sont abordées, autrement dit par l'étendue de son champ d'intérêt: éducation scolaire, éducation spéciale, formation des maîtres, formation d'adultes, enseignement de l'hygiène, méthodes de lecture, éducation artistique, pédagogie générale.

\section{DES THÉMATIQUES DOMINÉES PAR LA PHILOSOPHIE}

Dans les années 1922 à 1926, les articles abordant des questions philosophiques et spirituelles en lien avec l'éducation dominent largement

13 En français [la traduction est nôtre]: «Nous voulons être un miroir qui recueille et compare toutes les tentatives de solution, convaincus que, dans ces recherches les éducateurs de tous les pays et toutes les langues doivent trouver la multiplicité des voies et des opinions dans un but commun. Cet objectif inhérent à toute éducation authentique ne peut être que le renouveau et la libération de la puissance créatrice de l'âme au lieu de la destruction des impulsions hostiles». (Rotten, IER, 1920, 1, p. 2) 
l'ensemble des numéros. On y trouve des titres tels que «Rousseau über das Wesen der Erziehung (aus Emile)» [Rousseau sur la nature de l'éducation] (Rotten, DWZ, 1922), «Goethe: Gemeinschaft durch Freiheit» [Communauté par la liberté] (Rotten, DWZ, 1922), «Zwang und Freiheit» [Coercition et liberté] (Rotten, DWZ, 1926), «Gewalt und Liebe» [Violence et amour] (Buber, DWZ, 1926), "Gewalt der Nichtgewalt» [Violence et non-violence] (Gandhi, DWZ, 1926). D'autres articles, également très nombreux, abordent les dimensions spirituelles de l'éducation à travers la question de la religion, et plus particulièrement de l'enseignement religieux à l'école. ${ }^{14}$

En plus des articles de fond, la revue est truffée, dans ces années-là, de proverbes, ou pensées écrites par divers philosophes à propos de thèmes tels que les missions de l'éducation, les valeurs de la vie, l'éthique de l'éducateur. Ces pensées sont publiées sous forme de petites citations placées au début ou en fin d'article voire même intercalées entre ceux-ci, comme si elles étaient destinées à ponctuer la lecture par des réflexions philosophiques. Les auteurs de ces pensées sont notamment Comenius, Rousseau, Goethe, Paulsen, Pestalozzi, Emerson, Dostoievski, Nietzche, Vischer, James, Gandhi, Tagore.

Nous faisons l'hypothèse que cette dominante thématique est liée à la personnalité et aux intérêts d'Elisabeth Rotten qui pendant ces années-là contrôle l'essentiel de l'édition. En effet, cette dernière a personnellement étudié la philosophie ainsi que la langue et la littérature allemande (Germanistik, Philologie) et voue une véritable fascination pour Goethe. ${ }^{15}$ Ce qui expliquerait pourquoi on trouve tant d'extraits de pensées de Goethe dans DWZ. La récurrence d'articles traitant de questions religieuses ou d'enseignement de la religion (Religionsunterricht) témoigne de la proximité de Rotten avec la spiritualité en particulier avec les Quakers. Mais au-delà de la personnalité d'Elisabeth Rotten, c'est la culture allemande qui se reflète dans cette revue à travers la pré-

14 Notamment: «Über das Religiöse» [Sur le religieux] (Wilker, DWZ, 1922), «Von Religion und Schule» [Sur la religion et l'école] (Ragaz, DWZ, 1923), «Jugendbewegung, Religion und Dogmatismus» [Les mouvements de jeunesse, la religion et le dogmatisme] (Wilker, DWZ, 1923), «Die Erziehungsideale der Quäker» [L'idéal éducatif des Quakers] (Thierney, DWZ, 1923), «Von religiöser Erziehung» [À propos de l'enseignement de la religion] (Ehlen, DWZ, 1928).

15 Elle rédige sa thèse de doctorat sur cet écrivain poète. 
sence forte de la philosophie. Comme le montrent les travaux de Hofstetter et Schneuwly (2007), les régions alémaniques se caractérisent par leur attachement à la philosophie, une discipline phare dont elles peinent à se détacher en raison de traditions culturelles bien ancrées.

Une autre dominante qui se dégage des thématiques est celle des expériences d'Éducation nouvelle, en particulier celles qui sont menées à l'étranger que ce soit aux États-Unis, en Angleterre, en France, en Russie ou en Suisse. ${ }^{16}$ Elisabeth Rotten est elle-même l'auteure de plusieurs articles de ce type, décrivant des écoles, méthodes ou systèmes d'enseignement étrangers avec une affection particulière pour ceux des États-Unis. Ces articles - «Aus Holland», «Eindrücke aus Amerika», «Innen und Aussen», «Eindrücke aus der Neuen Welt» - pour n'en citer que quelques-uns, reflètent à la fois l'intérêt et la mobilité de cette réformatrice qui voyage dans le monde entier à la quête d'idées et méthodes nouvelles. Elle visite plus souvent les pays anglo-saxons car sa maîtrise de l'anglais $^{17}$ lui permet de dialoguer très librement avec ses interlocuteurs. Les expériences menées en URSS (modèle russe) occupent une part importante des articles, même si ceux-ci sont rarement écrits par des Russes mais plutôt par des Allemands qui visitent les écoles de ce pays. ${ }^{18}$

De manière générale, les articles de Das Werdende Zeitalter traitent peu, voire très peu, de psychologie et de psychanalyse. Les quelques auteurs qui abordent ces questions sont principalement des étrangers

16 Les titres révèlent les pays visités: «Das Bildungswesen in Sowiet-Russland» (Wilker, DWZ, 1922), «Selbstregierung in der russischen Mittelschule» (Jucker, DWZ, 1927), «Russland und wir»(Wilker, DWZ, 1923, 3), «Die öffentliche Versuchschulen in Winnetka, Illinois» (Washburne, DWZ, 1923, 3), «Die Gartenschule bei Great Missenden, Bucks» (Hecht, DWZ, 1922, 2), «Die Genfer Lehrer und die Abrüstung» (Descoeudres, DWZ, 1928, 3).

17 Elisabeth Rotten enseigne pendant une année (1913) la littérature allemande à l'Université de Cambridge, cette activité lui conférant un statut de personne bilingue (voir Haubfleisch, 1997).

18 Voir notamment: «Tolstois pädagogische Ideen» (Hubben, DWZ, 1931), «Sowjetrussische Fürsorgeerziehung» (Jacobi, DWZ, 1927, 6), «Selbstregierung in der russischen Mittelschule» (Jucker, DWZ, 1927, 6), «Erziehung in der Sowjetschule» (Jucker, DWZ, 1930, 9), (Krupskaja, IER, 1920), «Bildungswesen in Sowjetsrussland»(Lunatscharski, IER, 1920, 1), «Das system der Volksbildung in der USSR» (Raskin, DWZ, 1930, 9). 
tels que C. G. Jung $(\mathrm{CH})$, A. Ferrière $(\mathrm{CH})$, E. Marcault $(\mathrm{F})$ ou des auteurs allemands qui présentent l'œuvre de psychologues étrangers. En 1922, Carl Heinrich Becker (D) fait la recension d'un ouvrage de Charles Baudouin, Études de psychanalyse, ainsi que de celui de Pierre Bovet, Psychanalyse et éducation, alors que des psychologues alémaniques tels que Wundt ou Meumann ne figurent jamais dans les tables des matières de DWZ. À la suite de Helmchen (1993), Schriewer (1998) et Schriewer et Keiner (1992), nous faisons le constat d'une forte prégnance de la philosophie dans les discours éducatifs durant ces premières décennies du $20^{\mathrm{e}}$ siècle.

Par contre, les auteurs de DWZ portent un intérêt particulier pour les méthodes d'enseignement dans diverses disciplines telles que la langue, la gymnastique, les travaux manuels. Les disciplines artistiques (arts visuels, musique, rythmique) sont particulièrement au goût du jour et font, elles aussi, l'objet de nombreux articles: «Zur Rythmischen Erziehung» (Wilker, DWZ, 1923), «Künstlerische Erziehung» (Natter, $D W Z, 1924)$, «Körperliche Erziehung und Lebensgestaltung» (Steiner, DWZ, 1924), «Erziehung und Kunstwerk» (Wilker, DWZ, 1927) «Werkhauswoche für Erziehungskunst und schöpferischen Arbeit» (Redaktion, DWZ, 1925). Parmi ces méthodes, celles qui viennent de l'étranger occupent une place de choix, en particulier celles développées en Amérique. Ainsi, le «Dalton Plan», les méthodes de la Lincoln School (New York) ou celles des Écoles de Winnetka (Illinois) tiennent le haut du pavé. En revanche, les méthodes développées par Decroly, Cousinet, Freinet ou d'autres pédagogues francophones (méthodes très présentes dans Pour l'Ère Nouvelle) font rarement l'objet d'un article particulier, sauf lorsqu'il s'agit d'un numéro spécial dédié à un congrès.

La revue propose quelques numéros thématiques par exemple à propos de la punition (Das problem der Strafe) ou de la religion (Religionsunterricht) mais dans l'ensemble les numéros sont plutôt éclectiques publiant indifféremment les contributions de chaque auteur. Mais les thématiques d'articles sont très souvent liées à des événements vécus par les auteurs et en particulier par l'un des deux rédacteurs en chef. Par exemple, un voyage de Rotten aux États-Unis donne lieu à plusieurs articles de sa part sur les USA mais aussi de la part d'autres contributeurs. De manière semblable, PEN publie plusieurs articles liés aux voyages de Ferrière tout au long de la période étudiée. Les seuls véritables numéros spéciaux de DWZ sont les comptes-rendus des congrès de la Ligue. Pour exemple, le dernier numéro de 1925 (4. Heft) regroupe 
de nombreux articles sur le thème de la Libération de l'énergie créatrice de l'enfant qui, de fait, sont des traductions des conférences données au Congrès de Heidelberg. Ils sont publiés quasi à l'identique en français dans PEN et en anglais dans The New Era.

\section{UN LARGE RÉSEAU D’AUTEURS GERMANOPHONES}

Dans les premières années (1922-1926), Elisabeth Rotten et Karl Wilker constituent les auteurs les plus prolixes, assumant parfois les trois quarts de l'espace rédactionnel à eux seuls. En effet, Rotten écrit plus de 90 articles sur l'ensemble des années de publication du périodique, auxquels s'ajoutent sept articles écrits conjointement avec Wilker. Ce dernier écrit à lui seul 55 articles auxquels s'ajoute la rédaction de nombreuses rubriques telles que «Mitteilungen», «Bücherschau» et «Zeitschriftenschau» dont nous avons évoqué l'importance et le volume précédemment. D'autres auteurs se distinguent de l'ensemble en raison du nombre d'articles qu'ils signent. Il s'agit de Carl Heinrich Becker (10 articles), Fritz Natorp (6 articles), J. W. Mack (6 articles), Martin Buber (6 articles), Wilhelm Kircher (6 articles), Ernst Jucker (5 articles), Albert Krebs (4 articles) dont nous allons parler plus en détail dans la suite de ce volet. Les autres auteurs apparaissant dans $D W Z$ écrivent en moyenne deux ou trois articles, laissant penser que leurs contributions sont ponctuelles, vraisemblablement sur demande des responsables de la revue. Dans leur recherche archivistique, Haubfleisch et Link (1994) remarquent qu'en 20 ans de publication, 340 auteurs n'ont écrit qu'un seul article! Ce qui signifie que l'essentiel du journal est rédigé par ses éditeurs (Rotten et Wilker) ainsi que par une poignée d'auteurs dans l'entourage proche de ces derniers. Ceci contribue beaucoup à la stabilité de la ligne éditoriale ainsi qu'à l'uniformité de l'idéologie défendue dans Das Werdende Zeitalter.

Au début, les auteurs de DWZ sont avant tout des personnes avec qui Elisabeth Rotten entretient des relations proches, que ce soit en Allemagne ou à l'étranger. Il s'agit d'une part de personnes impliquées dans la vie de la Ligue (Bovet, Ferrière, Ensor, Wyneken) et d'autre part de personnes connues de Rotten par ses relations personnelles ou familiales. Ainsi, plusieurs auteurs suisses écrivent dans DWZ, certains vivant à Zürich (ville d'origine de ses parents) - par exemple Werner Schmidt, Leonhard Ragaz, Dr. Arthur Fischer - d'autres à Berne, comme Friedrich Kilchenmann qui est collaborateur permanent de DWZ et 
membre de la section suisse de la Ligue internationale. ${ }^{19}$ La Suisse est non seulement le pays d'origine de Rotten où se trouvent des attaches familiales, mais aussi celui où elle trouvera refuge en 1933 jusqu'à la fin de sa vie. C'est un pays dans lequel elle a beaucoup d'amis qu'elle rencontre notamment grâce à un important réseau de Quakers qui s'étend dans tout le pays. Ainsi Leonhard Ragaz, ami proche et correspondant de $D W Z$ à Zurich, est un des membres de la section zurichoise Quaker. Marie Butts ${ }^{20}$ en est une autre, avec qui elle collabore au sein du Bureau International d'Éducation dès sa nomination en tant que secrétaire en 1925, alors que Rotten y assume la fonction de directrice adjointe. À Genève, Rotten entretient des relations de travail et d'amitié avec Ferrière, mais aussi avec Bovet alors directeur de l'Institut Rousseau.

En Allemagne, Rotten rencontre beaucoup de personnes dans les milieux éducatifs et militants qu'elle fréquente et qu'elle sollicite pour écrire dans DWZ. C'est le cas de Martin Buber (1878-1965) que Rotten rencontre en 1920 et avec qui elle entretient des relations très proches jusqu'à la fin des années 1950 (Haubfleisch, 1997). Ce dernier, philosophe, écrivain et pédagogue israélite autrichien, est alors rédacteur d'un mensuel juif, Der Jude, et rédige d'importants textes religieux. ${ }^{21}$ En 1924, il devient professeur de philosophie juive à l'Université J.W. Goethe à Francfort mais doit quitter l'Allemagne en 1938 et émigre à Jérusalem. ${ }^{22}$ Un autre auteur que Rotten connaît et apprécie beaucoup est Gustav Landauer (1870-1919), l'une des personnalités les plus marquantes du mouvement anarchiste allemand qu'elle rencontre pendant la Première Guerre mondiale. Ce dernier défend un socialisme utopique, non dogmatique, non marxiste et libertaire auquel se mêlent les idées d'un christianisme "primitif» qui cherche à faire naître une nouvelle réalité «à l'aide d'un idéal orienté par la volonté de l'Homme». ${ }^{23}$ Selon

19 Voir l'éditorial de février 1931 dans PEN, 65.

20 Marie Butts est membre de la Société des Amis (Quakers) et a été formée à l'École Vinet, à Lausanne. Elle y rencontre Ferrière qui visite cette école en 1919 à plusieurs reprises et y donne des conférences. Sur cette école, voir Issenmann (2007).

21 En 1925, il entame à l'aide de Franz Rosenzweig, la traduction de l'Ancien Testament de l'hébreu en allemand.

22 Il est nommé professeur à l'Université hébraïque de Jérusalem.

23 Sur Landauer, voir le site http:/ / fr.wikipedia.org/.wiki/Gustav_Landauer très complet. 
Haubfleisch et Link (1994) les convictions et les écrits de Landauer ont une influence déterminante sur Elisabeth Rotten et façonnent la configuration et les thématiques de $D W Z$ durant les premières années de publication: «Seine Schriften dürften massgeblichen Einfluss auf sie, wie auch ab 1922 auf die inhaltliche Gestaltung des Werdenden Zeitalters ausgeübt haben» (p. 7).

Er trat für einen undogmatischen, nichtmarxistischen und libertären, für einen «utopischen Sozialismus» ein, bei dem sich frühsozialistische, anarchistische und bürgerlich Traditionen mit einer Urchristentum des Ketzerbewegung orientierent Religiösität vermischten. (Haubfleisch \& Link, 1994$, p. 7$)^{24}$

D'autres auteurs, notamment des femmes, font partie des réseaux de sociabilité que la rédactrice construit à travers son engagement dans les mouvements féministes tels que la Ligue Internationale de Femmes pour la Paix et la Liberté (Women's International League for Peace and Freedom) aux congrès de laquelle elle participe régulièrement, notamment en 1915 à La Haye, 1919 à Zurich et 1921 à Vienne. Cette Ligue, dont le siège est à Genève (Rue du Vieux Collège), s'inscrit avant tout dans le mouvement pacifiste d'entre-deux-guerres travaillant pour le désarmement universel et pour la résolution pacifique des conflits. Ce groupement s'intéresse aussi à l'éducation et en particulier aux méthodes nouvelles dans la perspective de réaliser un «état social, politique, et économique basé sur la justice, sans distinction de sexe, de race, de classe ou de foi». ${ }^{25}$ Dans cette optique, il fait appel à Ferrière pour donner des conférences sur l'école active et les méthodes nouvelles dans le cadre de ses cours de vacances ou conférences internationales. ${ }^{26}$ Parmi les contributrices de $D W Z$, figurent plusieurs directrices d'écoles nouvelles à l'exemple de Dora E. Hecht, directrice de Gardenschool ou Mary

24 En français [la traduction est nôtre]: «Il était pour un 'socialisme utopique', non-dogmatique, non marxiste et libertaire, dans lequel les convictions socialistes et anarchistes sont mélangées aux traditions bourgeoises et à un christianisme primitif des hérétiques sur la religiosité.» (Haubfleisch \& Link, 1994, p. 7)

25 Ligue internationale des Femmes pour la Paix et la Liberté dans le Programme officiel du Troisième congrès bisannuel - Third biennal Conference de la World Federation of Education Associations (WFEA).

26 Ferrière est en contact avec cette Ligue internationale de femmes déjà bien avant la fondation de la LIEN. 
M. Hogg, directrice de Fairby Grange, toutes deux en Angleterre. D'autres auteures sont institutrices telles que Gertrud Pincus à Berlin ou Irma Sander à Innsbruck, mais avec un statut de «collaboratrices permanentes» de la revue dès 1928. D'autres encore sont engagées dans les instituts de recherche comme c'est le cas d'Alice Descœudres $(\mathrm{CH})$ qui, au moment où elle écrit des articles pour DWZ (1922, 1923, 1928), enseigne à l'Institut Rousseau.

Dès les premières années de publication, $D W Z$ est aussi marquée par l'influence du réseau Quakers dont plusieurs communautés existent en Allemagne ainsi que dans d'autres pays européens. ${ }^{27}$ Elisabeth Rotten participe aux conférences et assemblées qui se tiennent en Allemagne comme en Angleterre. Si elle adhère officiellement au groupe en 1930 (Haubfleisch, 1997), son engagement dans cette communauté se perçoit bien avant (dès les années 1920) notamment à travers ses écrits et ceux qu'elle sollicite pour publication dans DWZ. Plusieurs d'entre eux abordent la question de la spiritualité, de la religion et de l'enseignement de ces questions à l'école. En avril 1923, un numéro spécial de DWZ est consacré à l'éducation religieuses (Religiöse Erziehung) où figure par exemple un article écrit par Agnes Thierney (Philadelphie) «Die Erziehungsideale der Quäker», preuve des contacts qu'entretient Rotten avec les communautés Quaker américaines. En juillet 1923, un article non signé mais vraisemblablement écrit par Rotten - «Erziehung, Freiheit und Dogma» - est en fait la traduction d'un extrait de l'assemblée générale de la Société anglaise Quaker tenue en juin 1923 à laquelle Rotten a participé. Les valeurs que défendent les Quakers - paix, tolérance, démocratie, égalité des droits - correspondent en tous points aux idéaux que défend Elisabeth Rotten notamment à travers son militantisme dans des associations telles que la Bundes Neues Vaterland (plus tard Deutsche Liga fur Menschen Rechte) ou la Deutsche Liga für Völkerbund - dont elle dirige la section pédagogique - toutes deux promouvant une éducation pour la paix.

Dès 1927, mais surtout à partir de 1928, les auteurs de DWZ se diversifient car la revue passe à un rythme de publication mensuel, raison pour laquelle les deux rédacteurs s'entourent de collaborateurs de choix dont les noms figurent sur la page titre. ${ }^{28}$ Dans les numéros spéciaux,

27 Sur les convictions des Quakers et leur présence en Europe, voir le chapitre 3.

28 La liste de ces collaborateurs est indiquée plus haut dans la partie traitant du comité éditorial. 
Rotten et Wilker font appel à des spécialistes du thème traité. Par exemple, pour un numéro spécial sur l'éducation en Palestine (1929, 2. Heft), H. Bergmann, directeur de la University Library (Jérusalem), Ilek Goldschein, Post Eju Charod et quatre autres auteurs palestiniens sont sollicités. Dans les numéros spéciaux dédiés aux congrès de la Ligue (Heidelberg, 1925; Locarno, 1927; Elseneur, 1929) les auteurs sont les mêmes que ceux qui écrivent dans les deux revues sœurs, puisqu'il s'agit essentiellement de résumés des conférences données lors de ces congrès. On retrouve alors les noms de pédagogues, médecins, psychologues et scientifiques qui, en temps normal, écrivent peu voire pas du tout dans DWZ. À nouveau, on constate une forte influence des ÉtatsUnis car la majorité des auteurs non-germanophones qui y écrivent (excepté les numéros spéciaux) sont des Américains, à l'exemple de C. Washburne, W. Kilpatrick, H. Parkhurst. Cette dominance peut s'expliquer notamment par le fait qu'Elisabeth Rotten maîtrise très bien l'anglais (elle enseigne l'anglais dans plusieurs écoles au cours de sa vie) et peut donc assurer la traduction de ces textes. En revanche, elle maîtrise moins bien le français, raison pour laquelle les écrits des auteurs francophones sont moins présents dans sa revue.

Dès 1929, des indications sur les auteurs sont données dans une page d'introduction à la rubrique «Mitteilungen» où figurent les noms, adresses et fonctions de tous les auteurs qui ont pris part à la rédaction du numéro. On découvre ainsi une grande diversité d'auteurs, où les professionnels de l'éducation paraissent plus nombreux que dans les années précédentes. Parmi eux, certains sont des enseignants des degrés primaire ou secondaire (Albert Leon, maître à Breithorn-Lützel; Bruno Zwiener, maître de dessin) mais la majorité occupe des postes à responsabilité dans des écoles (inspecteur, directeur), dans des instituts de formation ou à l'université: Eberhard Gerlatzek, directeur d'école secondaire (Görlitz); Albert Maeyer, conseiller pédagogique; Wilhelm Kircher, directeur de l'école «Das Haus in der Sonne»; Leo Weismantel, directeur de l'école «Volkschaft für Volkskunde und Erziehungswesen»; Heinz Sieveking, directeur adjoint de la Lichtwark Schule (Altona-Othmarschen). Parmi les étrangers écrivant dans les numéros spéciaux dédiés aux congrès, la majorité occupe des fonctions dirigeantes dans des institutions scolaires, que ce soit en tant que directeur d'école, inspecteur ou directeur d'instituts de formation. De fait, il s'agit des acteurs faisant des interventions dans les congrès qui pour la plupart bénéficient d'une formation de haut niveau. 
Notre analyse de Das Werdende Zeitalter fait apparaître l'importance des traditions culturelles sur les discours en faveur de l'Éducation nouvelle qui se manifestent aussi bien au niveau des thématiques d'articles que des auteurs. À l'exception d'une concordance thématique avec ses revues sœurs dans les numéros spéciaux dédiés aux congrès, le périodique allemand révèle une manière bien spécifique de militer pour l'Éducation nouvelle à une période où le mouvement est en pleine croissance. Alors que PEN et The New Era font état des avancées de la psychologie et de la pédagogie expérimentale, les domaines abordés par $D W Z$ sont fortement imprégnés par la philosophie qui joue ici le rôle de science de référence. Les pensées des philosophes qui ponctuent ses colonnes attestent de l'importance accordée à cette discipline par les rédacteurs. Plusieurs auteurs sont eux-mêmes philosophes, mystiques ou membres de communautés religieuses défendant une conception spiritualiste de l'éducation; par leurs écrits, ils contribuent à ancrer le discours militant dans la philosophie et la spiritualité. Tout se passe comme si l'émergente science de l'éducation - étroitement liée à l'Éducation nouvelle - ne pouvait se construire qu'en référence à la discipline-mère au sein de laquelle celle-ci s'est toujours inscrite, par tradition, dans les régions germanophones.

En cela, nos analyses rejoignent les observations faites par Hofstetter et Schneuwly (2007) à propos des régions alémaniques de Suisse où le développement institutionnel des sciences de l'éducation, concluent-ils, est fortement dominé par la philosophie et par une tradition académique où les disciplines restent à l'écart du monde social. Un mécanisme de développement qui se distingue de celui qui prévaut dans les régions francophones où la science de l'éducation se développe de manière beaucoup plus autonome (institutions dédiées à son étude et à son enseignement) en interaction avec la profession, voire avec la psychologie - pour Genève -, "régions où l'université est dans une relation de plus grande proximité avec la cité» (p. 221). Une impression d'unité de pensée se dégage de $D W Z$ qui contribue à propager l'image d'un mouvement uni, voire uniforme, que nous expliquons principalement par la proximité des auteurs qui y écrivent. Comme nous l'avons vu, ces derniers font partie d'un même réseau de relations au sein duquel les conceptions de l'éducation, en particulier celles de l'Éducation nouvelle, sont proches. De ce fait, ils participent à la construction d'un discours lisse où les débats sont peu présents et où leur militance vise davantage à renforcer l'importance de la philosophie pour l'éducation qu'à pro- 
mouvoir une pédagogie scientifique basée sur une approche empirique des phénomènes éducatifs.

\section{THE NEW ERA: DE LA THÉOSOPHIE À LA «HOME EDUCATION»}

\section{STRUCTURE ET RÉDACTION: SOUS LE CONTRÔLE DE BEATRICE ENSOR}

Tout comme sa consœur allemande, la revue anglophone The New Era ${ }^{29}$ pré-existe à la fondation de la Ligue. Éditée alors sous le nom Education for The New Era, elle paraît dès 1920 en tant qu'organe de la New Education Fellowship anglaise fondée par Beatrice Ensor en 1915. ${ }^{30}$ Son sous-titre «An international quarterly magazine for the promotion of reconstruction in Education» révèle d'emblée les ambitions réformatrices du journal. À ses débuts, il est dirigé par deux personnes: Beatrice Ensor (1885-1974) et Alexander S. Neill (1883-1973). Elle est inspectrice d'écoles de filles en Angleterre puis directrice de la St-Christopher School à Letchworth. C'est dans cette école qu'elle organise le premier congrès du groupe Theosophical Educational Trust en 1920. La revue Education for The New Era qu'elle fonde cette même année vise à "communiquer les idées et les expériences des écoles nouvelles» et à "permettre l'établissement d'une communauté d'enseignants qui se rencontreraient lors de congrès annuels» (Education for The New Era, 1920, 1, p. 1).

De fait, les objectifs de cette première revue sont plus ambitieux que ne le dit cet éditorial de 1920. Dans une lettre envoyée à son ami Wyatt Rawson en 1971 où elle évoque les années 1920, Ensor explique que son objectif était avant tout «de mettre en contact les enseignants théosophes et de propager, à travers eux, les conceptions théosophistes de l'éducation»:

29 Sur cette revue, voir l'étude approfondie de Koslowski (2013).

30 En fusionnant avec la section éducation de la Deutsche Liga für Volkerbund et le Bureau International des Écoles nouvelles lors de la fondation de la Ligue (1921), cette association perd son exclusivité anglaise; mais son nom, New Education Fellowship, est conservé pour désigner désormais la Ligue internationale pour l'Éducation nouvelle en anglais. Ce changement contribue à créer la confusion notamment pour les Anglais. C'est le cas de Percy Nunn pour qui 1915 constitue la date de fondation de la Ligue comme en atteste le titre de sa conférence d'ouverture au congrès de Cheltenham (1936): «Le vingt et unième anniversaire de la Ligue». 
I realised how much Theosophy could change one's approach to the education of a child, especially reincarnation, in which consciousness can manifest on the physical plane. I therefore thought of trying to get teachers who were theosophists into contact through the magazine The New Era and the international conferences. (Ensor, 1971, cité par Brehony, 2004, p. 737)

Si la présence des théosophes est clairement perceptible dans les années 1920, notamment à travers le vocabulaire qu'utilisent certains auteurs, l'appartenance du journal à ce mouvement n'est jamais déclarée ouvertement et cette influence disparaît dès les années 1930.

Le co-rédacteur de la revue, Alexander S. Neill (1883-1973), n'est autre que le fondateur de la célèbre École de Summerhill qui ouvre ses portes en 1924 près de Leiston (Angleterre). Considéré aujourd'hui comme un militant antireligieux prônant une éducation libertaire, Neill est avant tout un praticien qui a passé l'essentiel de sa vie dans les classes, «que ce soit comme élève, élève-maître près de son père, instituteur, ou directeur d'école» (Saffange, 1994, p. 1). Sa position de co-rédacteur de The New Era lui permet d'observer attentivement les expériences d'Éducation nouvelle dont il se fait le propagandiste mais parfois aussi, le critique. En effet, dans l'éditorial du numéro de juillet 1920 - dont il assume la préparation - il attaque ouvertement les crank schools, écoles anglaises publiques, où il prétend que les maîtres imposent leurs propres valeurs aux enfants. Il critique aussi les écoles qui tentent d'appliquer le selfgovernment sous le contrôle des maîtres alors que selon lui «le selfgovernment ne peut réussir que si l'enseignant est convaincu que toute forme d'autorité est dangereuse pour l'enfant» (Neill, Education for the New Era, 1920, 1(3), p. 67). D'autre part, il se positionne farouchement contre Maria Montessori à laquelle il reproche sa scientificité et son moralisme. Il critique plus particulièrement le «Montessorianisme» ou les méthodes Montessori alors même que celles-ci sont parmi les plus valorisées par les théosophes de l'époque (Brehony, non publié). ${ }^{31}$

Selon Brehony, ce qui pousse les théosophes à soutenir les méthodes Montessori n'est pas seulement leur valeur et leur qualité pédagogique

31 De nombreuses écoles anglaises théosophes pratiquent ces méthodes, écoles dont les directeurs écrivent des articles dans The New Era. Voir par exemple Claude A. Claremont, professeur à l'École St. Christopher (fondée par Ensor) et fervent défenseur de ces méthodes qui écrit plusieurs articles à partir de 1920. 
mais c'est surtout parce que Maria Montessori est elle-même une adhérente à la théosophie. Cette dernière rejoint la Theosophical Society en 1899 dont elle rencontre la présidente, Annie Besant (1847-1933), la même année au Congrès international des Femmes à Londres. Besant étant persuadée que les méthodes Montessori sont le résultat de l'introduction des idées théosophistes en éducation, Beatrice Ensor en devient une fervente propagandiste et encourage leur application dans les écoles. ${ }^{32}$ Neill participe à la fondation de la Ligue en 1921, mais il s'en distancie rapidement en raison de certains principes auxquels il n'adhère pas et de «l'ambiance de moralisme» qui y règne (Saffange, 1994, p. 228). Selon Saffange (1994), les positions radicales de Neill provoquent beaucoup d'indignation au sein de la Ligue et créent des tensions entre lui et Beatrice Ensor. Dès 1923, son nom n'apparaît plus sur la première page de The New Era même s'il co-signe encore un éditorial avec Ensor. En 1924, les tensions entre les deux éditeurs se soldent par le départ de Neill qui fonde la même année, l’École de Summerhill.

Dès ce moment-là, c'est Beatrice Ensor qui assume seule la responsabilité éditoriale du journal. En 1928, elle engage une "assistant editor», Miss Clare Soper, qui devient aussi la secrétaire du siège central de la Ligue à Londres. Elle restera fidèle à son poste pendant près de 30 ans, assumant ses tâches alors même que Beatrice Ensor n'est plus en Angleterre, et vit en Afrique du Sud (1932-35). À partir de juillet 1929, les noms de deux compatriotes apparaissent sur la page de couverture: Dorothy V. Halbach et Muriel M. Mackenzie ${ }^{33}$, qui sont toutes deux des théosophes. La première assume cette tâche jusqu'en 1936 alors que la seconde cède sa place dès fin 1931 à une quatrième assistante, Peggy Volkov, qui restera jusqu'en 1940 aux côtés de Beatrice Ensor. Quelles que soient les assistantes qui l'aident dans ses tâches d'édition, Ensor reste la rédactrice en chef et donne le ton général à la revue. Sa présence est particulièrement visible dans les éditoriaux Outlook Tower qu'elle rédige dans chaque numéro. Ces derniers, d'un volume conséquent - 4 à 5 pages - apportent beaucoup d'informations sur la vie de la Ligue et de

32 C'est le cas par exemple d'une section du Collège pour filles de Cheltenham dont elle est l'inspectrice et de l'École St-Christopher. (Brehony, non publié, p. 5).

33 Muriel Mackenzie est l'auteure de plusieurs articles de la revue dès 1920 mais plus particulièrement à partir de 1926 où elle enseigne à la St-Christopher School. 
la section nationale anglaise (dès sa fondation en 1927) ainsi que sur les événements internationaux tels que congrès, séminaires, cours et conférences. Mais surtout, c'est là que la rédactrice s'adresse aux lecteurs, exprime ses opinions, fait part de ses préoccupations. Dans ces éditoriaux apparaît aussi l'idéologie théosophiste dont Ensor est imprégnée perceptible à travers le vocabulaire qu'elle emploie notamment les concepts de fraternité universelle, tolérance entre les peuples, force intérieure, etc.

Parmi les préoccupations d'Ensor, il en est une qui revient de manière récurrente tout au long de la période étudiée: affirmer la neutralité et l'ouverture d'esprit de sa revue et de la Ligue. Ce souci se traduit par des messages fréquents dans les éditoriaux rappelant que la revue ne soutient pas une doctrine, ni une méthode plutôt qu'une autre. Ensor insiste sur sa volonté de faire connaître toutes les expériences du renouveau éducatif, aussi diverses soient-elles, raison pour laquelle elle invite tous les lecteurs à faire part de leur avis et suggestions. La récurrence de ces messages donne à penser qu'il existe des tensions au sein de la Ligue, du moins au sein du lectorat anglophone, même si ceux-ci n'apparaissent pas explicitement dans les colonnes de The New Era (à l'exception des articles de A.S. Neill!). L'un de ces messages apparaît déjà dans l'éditorial d'avril 1921, à peine une année après sa première parution:

Let it not be forgotten criticism is welcomed. We value the frank expression of readers' views because of the assistance this is in discovering how The New Era can be most helpful and effective. When renewing annual subscriptions many friends have taken the opportunity to write kind and flattering opinions of the magazine. Much as we appreciate this, we hope that those who disagree with the views to which expression is given in these pages will also give us the advantage of hearing their side of the case. (The Outlook Tower, The New Era, 1921, 2, p. 5)

\section{UNE HISTOIRE MARQUÉE PAR UN CHANGEMENT D'ORIENTATION}

Dans l'éditorial de janvier 1922, Beatrice Ensor évoque la fondation des deux revues-sœurs (française et allemande) en les présentant comme si elles étaient une émanation de la revue anglaise, laissant accroire que The New Era est la «revue-mère». On sait pourtant que la création des trois périodiques a été décidée lors de la fondation de la Ligue en 1921 et que tous trois ont un statut autonome, certes s'inspirant du modèle des deux journaux préexistants (l'allemande et l'anglaise). 
I am glad to be able to announce that our progress will see the launching of a French and German edition of our ${ }^{34}$ Magazine. The German edition of The New Era will be edited by Elisabeth Rotten who is well known as Secretary to the Educational Department of the German League of Nations Society and co-editor of The International Review of Education. [...] Dr. Rotten will not only undertake the editorship of The New Era in Germany, but she will continue her own vigorous educational work, through which she acts as a link in Germany between different branches of the New Education movement. We shall hope to have many articles from her and through them be kept in touch with educational developments in Germany. (Ensor, The New Era, 1922, 3(9), p. 1)

Ce n'est qu'à la fin du paragraphe - après avoir présenté Ferrière en tant que rédacteur de l'organe francophone - qu'elle indique que chaque revue est indépendante l'une de l'autre, ayant sa propre sélection d'articles même si certains peuvent figurer dans les trois puisqu'elles sont les organes officiels de la Ligue.

The french version will be edited by Adolphe Ferrière, Docteur en sociologie, Director of the Bureau international des Écoles Nouvelles and author of Transformons l'École and Les Écoles nouvelles. The French and the German editions will not be translations of the English edition but will be edited independently in order to meet the special needs of the respective countries. Certain articles, however, will appear in all three editions and each will be the official organ of the New Education Fellowship. (Ensor, The New Era, 1922, 3(9), p. 1)

The New Era connaît un tournant important en 1930 et ce à plusieurs niveaux. Tout d'abord, elle prend un nouveau nom - The New Era in Home and School - changement symbolique de la nouvelle orientation qu'elle prend à ce moment-là. Deuxièmement, elle change de statut puisqu'elle augmente sa fréquence de parution à 10 numéros par an ce qui la hisse au rang de revue mensuelle (monthly magazine), statut davantage reconnu dans le monde des périodiques. Enfin, et c'est là le changement le plus important, The New Era change d'orientation; l'impact se perçoit au niveau de ses contenus, de la composition des numéros, des auteurs et du public visé. Désormais, elle s'adresse aussi bien aux parents qu'aux enseignants; on le voit à son nouveau sous-titre

34 Mis en évidence par nous. 
«A monthly magazine for parents and teachers» et à une nouvelle rubrique "Questions from parents and teachers» qui figure dans chaque numéro. Dès ce moment-là, les thématiques abordées concernent surtout l'éducation au sens global du terme, où l'éducation scolaire et les multiples questions $\mathrm{s}^{\prime} \mathrm{y}$ rapportant cèdent la place à l'éducation familiale et affective de l'enfant.

Dès 1932, chaque numéro contient même une partie séparée s'apparentant à un «supplément» intitulé «Parents and Children», qui contient des articles dédiés aux parents

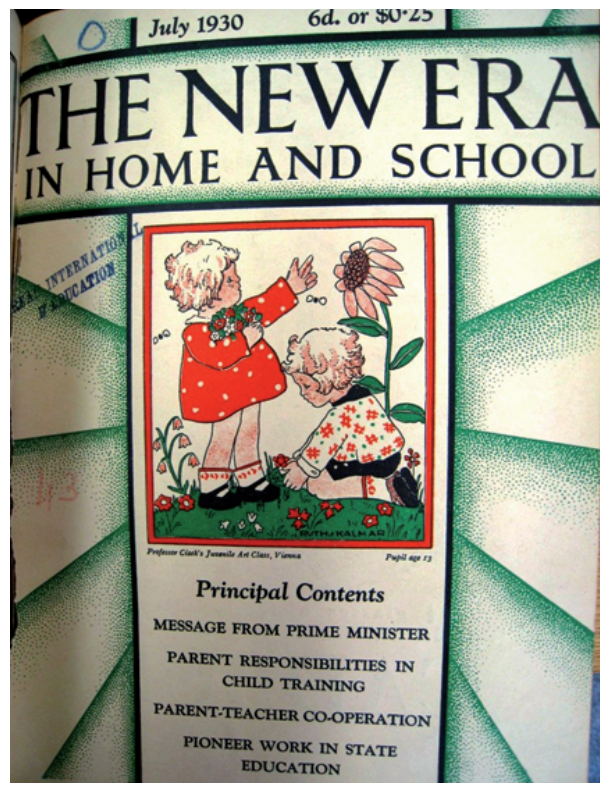
concernant l'éducation dans la famille. La revue est ainsi subdivisée en deux parties: l'une pour les professionnels, l'autre pour les parents. Mais l'espace réservé auparavant aux articles pédagogiques, voire scientifiques, informant de l'avancée des expériences et des recherches pédagogiques diminue considérablement. Dès lors, les contributeurs du périodique changent également, les noms des grandes figures de l'Éducation nouvelle - pédagogues, psychologues, médecins - tendent à disparaître au profit de noms moins connus et dont le profil est différent. Ce sont désormais des représentants d'associations de parents, ou des spécialistes du parenting qui conseillent les parents sur l'habillement des enfants («Clothes for the young child») ou sur l'aménagement intérieur de leur maison. Par exemple, Prudence Maufe, qui a dessiné plusieurs nurseries (jardins d'enfants) et se dit conseillère en aménagement d'intérieur, écrit un article intitulé "Make the furniture fit the Child» (Comment adapter le mobilier à l'enfant) en octobre 1932. Ce supplément dédié à l'éducation familiale disparaît toutefois à partir de 1934.

Les raisons de ce changement d'orientation ne sont pas clairement explicitées mais notre analyse du contexte de l'époque et notre connaissance de l'histoire des revues-sœurs nous permet de faire quelques hypothèses. Tout d'abord, ce tournant correspond manifestement à une 
évolution de la société à laquelle la New Education Fellowship se doit de répondre. Preuve en est l'intervention du premier ministre anglais du moment, J. Ramsay Mc Donald, qui, en saluant l'extension du champ d'intérêt de la revue à la «Home Education», formule une nouvelle demande sociale en sollicitant les spécialistes de l'éducation scolaire pour qu'ils mettent leurs connaissances au service de l'éducation familiale.

In the world of today it is the men and women of character who count, those who can cooperate, bear responsibility, think peace, and extend their sympathies beyond boundaries of their own nation and country. Believing this, it interests me to know that The New Era is widening its field to include home as well as school education. For the children, home is the first school, and the influence of the home can be traced throughout life. The best school in the world can be little of use if the home is unworthy. It is essential that parents should know how to educate their children in the early years before they go to school, and that then home and school should know how to work together to fit boys and girls for the work the world will demand of them as men and women. I will follow with interest the development of «The New Era». (Mc Donald, The New Era in Home and School, 1930, 11(43), p. 1)

De son côté, Beatrice Ensor présente ce changement comme une occasion de progrès, appelant les parents à s'associer aux efforts des éducateurs à travers une coopération active, tant sur le plan pédagogique que financier (sans que cela soit dit explicitement). Elle formule trois objectifs principaux qui révèlent un intérêt désormais prioritaire pour l'éducation de l'enfant à la maison, reléguant les professionnels de l'éducation au second plan. Si le deuxième de ces objectifs concerne les enseignants puisque la revue vise à les informer des progrès de l'Éducation nouvelle, le troisième évoque à peine les scientifiques médecins, psychologues, pédagogues - qui pourtant ont contribué jusque-là à construire cette «science de l'enfant» ou «science de l'éducation» dont l'expertise est réclamée par la société. Ce troisième objectif fait plutôt penser à un plaidoyer pour l'éducation à la paix, incitant au développement d'une pensée éducative commune et harmonieuse dont la revue serait désormais le support.

In its new form the magazine has three main objects. It will focus attention on the child at home, suggest home to prevent and treat undesirable behaviour and deal with topics that interest parents. But it will also remain the teacher's outlook tower on the world of progressive education. Readers who 
are parents may thus gain an insight into school problems, while those who are teachers may obtain an insight into home problems. The third main object will be to serve as a channel for educational thought. [...] By pooling experiences, by reading with the same end in view, by thinking in harmony, we shall create an attitude, a feeling, a mental unity that will foster the cause of peace. (Outlook Tower, The New Era in Home and School, 1930, 11(43), p. 3)

Ce changement d'orientation révèle les nouvelles priorités que se fixent plus globalement les leaders de la Ligue à partir des années 1930. Comme le montre Brehony, à travers l'analyse des éditoriaux de The New Era, ces derniers expriment l'urgence de prendre des mesures concrètes pour tenter de lutter contre les menaces qui planent sur l'Europe. Dans un éditorial de 1931, Beatrice Ensor porte un regard rétrospectif sur les dix années écoulées. Si elle reconnaît que les «expériences radicales» existent toujours et ont encore leur place, elle affirme que la Ligue est désormais préoccupée par «des changements fondamentaux dans les systèmes éducatifs» et par la «nécessité d'ajuster ces systèmes aux nouvelles conditions de la vie moderne» (Outlook Tower, The New Era in Home and School, 1930, 12(49), pp. 1-3). Ce changement d'orientation constitue un véritable tournant ${ }^{35}$ idéologique dans l'histoire de la Ligue qui se manifeste de façon similaire dans Pour l'Ère Nouvelle. Il se traduit par un intérêt nouveau pour l'environnement social de l'enfant, en particulier, et des situations éducatives, en général. Cette nouvelle orientation se confirme lors du Congrès de Nice en 1932 où le thème - La transformation sociale et l'éducation - et les réflexions qu'il suscite, révèlent la préoccupation des éducateurs à prendre en compte davantage les dimensions sociales de l'éducation. Que ce soit dans The New Era ou dans Pour l'Ère Nouvelle, ce congrès est présenté comme un moment charnière dans l'histoire de la Ligue au cours duquel de nombreux auteurs s'expriment en faveur de ce changement. Parmi eux, Harold Rugg, professeur au Teachers College de Columbia University (USA), affirme que les méthodes développées dans les années 1920 sont intéressantes mais insuffisantes compte tenu de la nouvelle civilisation industrielle devenue chaotique. ${ }^{36}$

35 Ce tournant a été analysé et documenté dans Haenggeli-Jenni et Hofstetter, 2011.

36 Voir Rugg, PEN, 1933, 86, pp. 59-64 et Rugg, The New Era, 1932, 13(8), pp. 123-125. 
Un autre changement important s'opère à partir de juillet 1930 dans The New Era, conséquence probable de sa nouvelle orientation: le peu de visibilité de son appartenance à la New Education Fellowship (NEF). À partir de cette date, il est difficile de savoir que The New Era en est l'un de ses organes officiels. En page de titre, l'ancien sous-titre «Organ of the New Education Fellowship» qui indiquait clairement ce rattachement depuis 1922 disparaît à ce moment-là. Les principes de ralliement et les buts de la Ligue, symboles de cette appartenance, disparaissent complètement des pages de couverture. Désormais, elle devient une revue essentiellement anglaise, comme on peut le constater sur la $3^{\mathrm{e}}$ de couverture. Celle-ci comporte un titre - The New Education Fellowship - ainsi que la mention «Chairman of the international Council: Mrs Beatrice Ensor». Puis figurent de manière visible les noms et statuts des membres de la section anglaise, section qui, bien qu'elle ait été fondée en 1927, n'était pas clairement mentionnée dans les numéros antérieurs. Les titres et fonctions de ces personnes révèlent la composition illustre de cette section et confirme le constat, similaire à celui que l'on peut faire dans les revues-soeurs, que le renouveau de l'éducation est entre les mains d'intellectuels de haut rang.

Tableau 3.

Comité de la section anglaise ${ }^{37}$

\begin{tabular}{ll}
\hline President & Prof. Sir Percy Nunn M. A. Dsc. L.L.D. \\
\hline Ex-president & $\begin{array}{l}\text { Sir Michael Sadler C.B., K.C.S.I. (University College, } \\
\text { Oxford) }\end{array}$ \\
\hline Treasurer & B. A. Howard, M.A. (Addey and Stanhope School) \\
\hline Secretary & A. J. Lynch (West Green School) \\
\hline $\begin{array}{l}\text { Assistant } \\
\text { Secretary }\end{array}$ & Miss R. M. Lyle (11 Tavistock Square, W.C.1.) \\
\hline
\end{tabular}

Suite à cette liste de noms figure un texte qui remplace les principes de ralliement et présente, en des termes nouveaux, les objectifs de la Ligue

37 Cette section est fondée en 1927 au congrès de Locarno mais elle n'est publiée officiellement dans The New Era qu'à partir de juillet 1930. Les noms, prénoms et titres des personnes ont été reproduits par nous en respectant la manière dont ils sont cités à l'origine. 
et de la revue. Dans ce texte, la nouvelle orientation est beaucoup moins perceptible. Certes, il mentionne la volonté de «créer un lien entre les administrateurs, les enseignants, les parents et l'enfant» mais l'objectif dominant est de nature scientifique comme l'évoque la citation ci-dessous. Celle-ci affirme qu'il s'agit de rassembler tous ceux qui croient à une éducation plus adaptée aux besoins et intérêts de l'enfant, de faire connaître leurs expériences en publiant leurs articles dans la revue et de participer ainsi à la l'évolution de la recherche en éducation grâce à la publication d'articles évoquant les progrès de l'Éducation nouvelle dans le monde entier.

The Fellowship seeks to draw together those in search of an education more suited to the needs and aspirations of modern life. It brings together educators of different countries and publishes news of their experimental findings. It seeks also a bond between administrators, teachers, parents and child. It presents an open platform from which the leading exponent of progressive education may deliver their message. The New Era, published monthly, endeavors to express the ideals for which the Fellowship stands, and contains articles relating to progressive educational movements in all parts of the world. (Rédaction, The New Era in Home and School, 1930, 11(43)

\section{THE NEW EDUCATION FELLOWSHIP INTERNATIONAL HEADQUARTERS :}

11 TAVISTOCK SQUARE, LONDON, W.C.1, ENGLAND

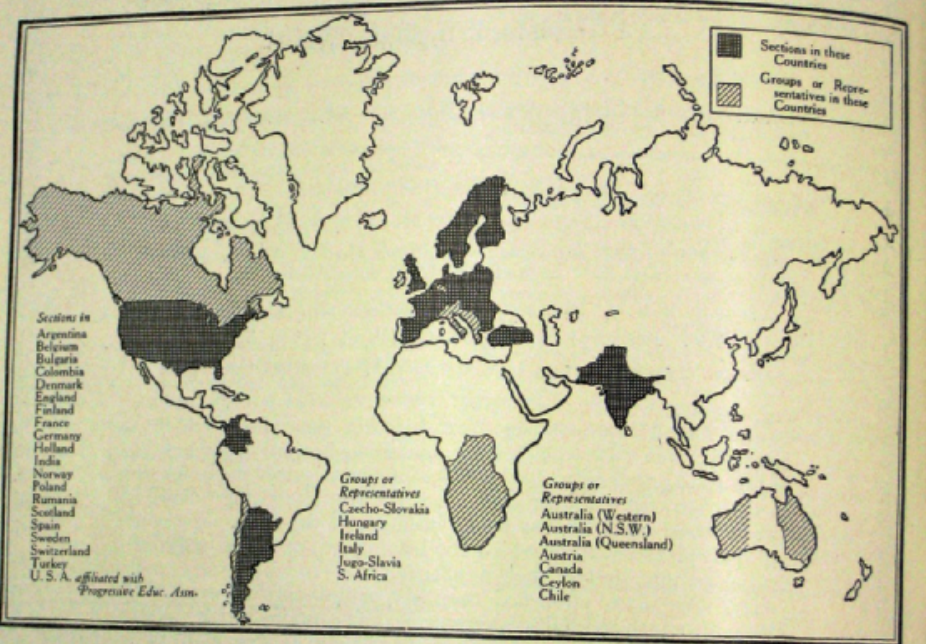


La $4^{\text {e }}$ de couverture constitue un élément supplémentaire indiquant que The New Era est l'organe de la New Education Fellowship. Sur cette page figurent désormais des indications concernant les tarifs et conditions $\mathrm{d}^{\prime}$ affiliation à la Ligue ainsi qu'une carte géographique ${ }^{38} \mathrm{du}$ monde montrant l'étendue de cette dernière et permettant de visualiser où se situent les divers groupes, sections et associations qui lui sont affiliés.

Malgré ces éléments, The New Era devient, à partir de juillet 1930, une revue surtout anglaise publiant des articles de la communauté anglophone $^{39}$ où l'on perçoit une nette augmentation de la participation des dominions (colonies britanniques). On note un intérêt grandissant pour ce qui se passe en Inde et en Afrique du Sud, en particulier de 1932 à 1934, années durant lesquelles Beatrice Ensor séjourne en Afrique du Sud. C'est d'ailleurs dans ce pays qu'elle organise en 1934 un congrès de la New Education Fellowship anglophone dont l'affluence - 6000 participants - signe son succès.

Les changements que connaît The New Era en 1930 sont en partie liés, comme nous l'avons vu, à une évolution de la demande sociale. Mais ils sont également la conséquence de décisions prises au niveau du Comité central lors du Congrès d'Elseneur (1929) car des changements semblables s'opèrent au même moment dans les deux revues-sœurs. La Ligue se structure et désigne des commissions pour étudier des problématiques particulières. En outre, l'esprit général s'oriente vers de nouvelles priorités car les années 1930 sont synonymes de problèmes financiers pour The New Era comme pour ses consœurs francophone et germanophone. En effet, la crise économique de 1929 a un impact direct sur les financements de ces supports éditoriaux qui survivent essentiellement grâce à des dons et des subventions. Beatrice Ensor interpelle régulièrement les lecteurs pour les encourager à faire connaître sa revue, à inciter leur entourage (amis, écoles, institutions, bibliothèques) à souscrire à un abonnement pour The New Era. À chaque fois, c'est l'occasion pour la rédactrice de rappeler les objectifs de cette revue qui, comme elle le répète inlassablement, ne défend aucune doctrine ni méthode, mais

38 Pour plus de lisibilité, la légende de cette carte indique, en noir, les pays possédant des sections nationales et en mode hachuré, les pays comportant des groupes ou des représentants de la Ligue.

39 Cette communauté inclut les États-Unis quoique la majorité des articles écrits par des Américains soient publiés dans leur propre revue Progressive Education. 
vise à faire connaître les expériences pédagogiques nouvelles à tous ceux qui s'y intéressent et qui veulent participer au renouveau de l'éducation.

En 1934, la situation devient vraiment critique en raison des retombées de la crise économique et de la perte d'un financement américain à la fin 1933. De retour d'Afrique du Sud où elle vient de passer deux ans (1932-1934), Ensor écrit une lettre adressée à chaque abonné, glissée dans l'édition de novembre 1934, faisant appel au soutien financier de tous les membres. C'est l'occasion pour elle de repréciser la nouvelle orientation de la revue - amorcée en 1930 - qui s'adresse désormais aussi bien aux enseignants qu'aux parents. Dans cette lettre, on perçoit son intention de toucher voire de «séduire» les parents car ceux-ci sont susceptibles de soutenir The New Era sur le plan financier. Le manque de moyens de cette année-là se remarque par un nombre réduit de numéros et les éditions sont regroupées par deux (mars-avril/mai-juin/septembre-octobre, etc.)

The New Era is not a commercial venture; it is run without profit because we believe that a magazine of this type can be a real service to the cause of peace, internationalism and the new education. It is the only magazine of this kind; it goes to nearly every country in the world. It is concerned with the whole child, not only the child at school or the child at home, or at the nursery school. And therefore it meets the needs of parents, teachers, and social workers. Each issues is designed to give practical help in the problems which continually arise for parents and teachers in dealing with children of various ages, and at the same time to make known the ideals and the philosophy and the attitude to life for which the new education stand and on which it is based. (Ensor, The New Era, 1934, 15, p. 6)

\section{AUTEURS ET THÉMATIQUES: UNE PALETTE DE SPÉCIALISTES POUR DES NUMÉROS SPÉCIAUX}

Au cours de la première décennie, The New Era se caractérise par un grand nombre de numéros thématiques, moins fréquents dans les revues-sœurs. Cette manière de gérer l'édition comporte plusieurs avantages tant pour le comité de rédaction que pour les lecteurs. Pour le comité, les numéros spéciaux peuvent être confiés à des personnes extérieures, anglaises ou étrangères, à l'exemple de personnalités telles que H.C. Dent (membre de la section anglaise) ou P. Bovet (membre de la section suisse) qui sont chargés de la préparation d'un numéro spécial. ${ }^{40}$

40 Bovet assure un numéro spécial sur le thème «Religion et éducation». 
Dans ces numéros, l'éditorial est rédigé par le responsable du numéro qui présente la thématique, ses objectifs ainsi que les auteurs sollicités. Une pratique qui ressemble beaucoup à celle des revues actuelles lorsqu'elles proposent des numéros spéciaux. Pour les lecteurs, ces numéros spéciaux ont l'avantage de réunir des articles de qualité, écrits par des spécialistes autour de problématiques d'actualité. Mais en contrepartie, The New Era comporte moins d'articles «spontanés» émanant de membres de la Ligue désirant faire part de leurs expériences. Comme on peut le voir dans ce tableau ${ }^{41}$, les numéros spéciaux occupent une large place dans l'édition de cette revue.

\section{Tableau 4.}

Numéros spéciaux de la revue The Nerw Era

\begin{tabular}{ll}
\hline January 1922 & Special free time-table numbers \\
\hline April 1922 & A New Method of Primary Teaching \\
\hline October 1922 & An International Record of Experimental Education \\
\hline January 1923 & The Drama in Education \\
\hline April 1923 & The teaching of English under the Dalton Plan \\
\hline January 1924 & Sex Education in School and Home \\
\hline July 1926 & Education in the United States of America \\
\hline July 1927 & Education in Africa and South Africa \\
\hline October 1927 & Liberty in Education \\
\hline January 1928 & Education in Russia \\
\hline January 1929 & Education in Denmark \\
\hline April 1929 & Special edition on Curriculums \\
\hline January 1930 & Pioneer Education in Poland \\
\hline April 1930 & The teaching of History (special edition dedicated to the \\
\hline
\end{tabular}

41 Ce tableau a été confectionné sur la base du dépouillement de The New Era de 1922 à 1940 (Archives BIE). Malheureusement, nous ne pouvons prétendre à son exhaustivité en raison de l'absence de plusieurs numéros dans la collection consultée. 
January 1932 Special edition on Sciences (biology, natural sciences, etc.)

March-April 1934 Education in South Africa

Sept-oct. 1934

New Education Fellowship Conference: South Africa

Outre les numéros spéciaux, les articles des années 1920 se caractérisent par un intérêt dominant pour l'enfant que ce soit pour son développement, son individualité, ses aptitudes ou sa liberté. The New Era accorde aussi une large place aux récits d'expériences effectuées dans les écoles nouvelles, notamment celles qui figurent dans la liste publiée par Ferrière en 1922 dans The New Era, puis en 1925 (dans PEN et The New Era). La plupart de ces articles sont écrits par les directeurs et directrices de ces écoles sous forme de témoignage destinés à «documenter» le mouvement, à montrer à la fois la diversité des «possibles» en Éducation nouvelle et l'étendue du mouvement dans le monde.

Au cours de la deuxième décennie, les numéros spéciaux se font plus rares. Ceux qui paraissent sont en lien direct avec les congrès à l'exemple du numéro spécial sur «L'Éducation en France» (The New Era, 1932, 13, 7) publié avant le Congrès de Nice et des deux numéros spéciaux dédiés à ce congrès (The New Era, 1932, 13, 8). De façon similaire, The New Era propose un numéro spécial sur l'Afrique du Sud en marsavril 1934 (The New Era, 15(4)) en préparation au congrès qui se déroule dans ce pays cette année-là. Le compte-rendu des conférences est publié dans les numéros spéciaux d'octobre et novembre sur le thème du congrès «Education in a Changing world» (très proche de celui du Congrès de Nice). De façon générale, les articles de cette décennie sont plus éclectiques, en lien fort avec la nouvelle orientation de la revue amorcée en 1930. On peut toutefois identifier quelques thèmes dominants. Tout d'abord, de nombreux articles abordent les questions d'éducation familiale évoquant le rôle et la responsabilité des parents dans l'éducation, de même que les questions «d'hygiène domestique», notamment à propos de l'alimentation, de l'habillement, l'entretien et l'aménagement de la maison. Un autre thème fédérateur semble être celui de «l'international»; nombre d'articles abordent cette question en cherchant à définir ce qu'est l'international, l'internationalisme, mais aussi la paix et la tolérance dans le sens d'une compréhension internationale, d'une compréhension mutuelle. Ces articles se multiplient à partir de 1933 reflétant les tensions politiques en Europe et la montée des régimes nationalistes. En lien avec ces tensions, de nombreux articles 
traitent des questions de citoyenneté et de démocratie, en particulier concernant le rôle de l'école dans cette éducation.

L'analyse des sommaires de ces années-là fait apparaître une recrudescence d'articles traitant des dimensions sociales de l'éducation et proposant des méthodes adaptées aux nouvelles conditions de la société en particulier aux exigences du monde du travail et aux problèmes de chômage. Ainsi, on décèle une préoccupation croissante pour la recherche d'applications concrètes des méthodes nouvelles dans le but de transformer en profondeur les systèmes éducatifs. Plusieurs titres d'articles indiquent un intérêt particulier pour les méthodes d'enseignement des disciplines artistiques (musique, art, expression libre) de même que l'enseignement de l'histoire qui occupe une place centrale dans cette réflexion. Enfin, les années 1930 se caractérisent par une prise de conscience progressive des problèmes d'enfants qui dysfonctionnent dans le système éducatif officiel. Ces problèmes sont perçus et traités différemment que dans les années 1920 compte tenu des nouvelles connaissances à disposition. Ainsi, on ne parle plus d'enfants anormaux mais plutôt d'illetrisme, de problèmes psychologiques, de difficultés d'apprentissage (en écriture, lecture, etc.), voire de délinquance. Dès lors, beaucoup de recherches visent à trouver des solutions éducatives alternatives pour ces enfants. Même si ces nouvelles thématiques deviennent dominantes dans la revue, The New Era accorde toujours une place importante aux récits d'expérience. Ainsi, même dans ces années-là, chaque numéro comporte un à deux articles présentant une école, une classe, une expérience pratique, attestant de l'importance accordée à ce type de communication et du rôle documentaire du journal.

La grande majorité des auteurs de The New Era sont des auteurs anglais ou écossais, qu'ils soient théoriciens ou praticiens. Ceux qui écrivent le plus sont actifs dans les instances de la Ligue à l'exemple de William Boyd, professeur à l'Université de Glasgow (Écosse), qui est membre du Comité consultatif de la Ligue pendant plusieurs années. La revue publie également les articles d'auteurs anglo-saxons des dominions parmi lesquels plusieurs font partie du réseau théosophiste construit autour de Beatrice Ensor (Inde, Australie, Nouvelle Zélande, Afrique du Sud). Enfin, quelques contributeurs américains signent des articles dans The New Era, la priorité étant donnée aux auteurs impliqués dans les instances de la Ligue. Les noms les plus fréquemment rencontrés sont ceux de Carleton Washburne, Harold Rugg ou Carson Ryan, tous trois impliqués au sein du Comité international de la Ligue et participant aux congrès internationaux. 
Dès juillet 1930, le changement d'orientation du journal coïncide avec l'arrivée de nouveaux auteurs n'ayant jamais écrit dans The New Era jusquelà: ceux-ci sont spécialisés dans «l'éducation à la maison» (Home Education) ${ }^{42}$ ou l'éducation parentale (Parental education). Une plus grande place est accordée aux parents qui peuvent aussi proposer des articles pour la revue. Cette nouvelle orientation a pour effet de diminuer considérablement pendant quelques années la place accordée aux «spécialistes» de l'éducation (scientifiques, universitaires, directeurs-trices) privilégiant une manière moins scientifique de présenter les choses, le but de la revue étant désormais plus pragmatique visant à être, avant tout, utile aux parents et aux enseignants. Dès ce moment-là, The New Era ne donne que de très brèves indications sur l'appartenance institutionnelle ou professionnelle des auteurs, indications qui figuraient pourtant dès 1922. Les thématiques deviennent plus spécifiques ce qui conduit la rédaction à faire appel à des spécialistes de la question. C'est le cas par exemple pour un numéro spécial sur le thème «Diffusion de l'éducation (broadcasting), film et éducation» où les auteurs choisis sont des spécialistes du cinéma et des médias; il en va de même pour un numéro dédié à la géographie (juillet 1931) où divers spécialistes du domaine écrivent des articles proposant différentes approches de cette discipline selon les ordres d'enseignement concernés et les recherches en cours.

L'analyse de The New Era fait apparaître une manière bien particulière de gérer l'édition ce qui, sur ce plan-là, la distingue de ses revues-sœurs. Les nombreux numéros spéciaux qui jalonnent son histoire éditoriale donnent un aperçu rapide des domaines privilégiés et les étapes de son évolution au fil des ans. Combinée à l'analyse de l'infrastructure éditoriale, cette analyse permet de conclure à la présence de deux phases bien distinctes dans l'histoire de cette revue. La première, allant de 1921 à 1930, se caractérise par une étroite relation de la revue avec la Ligue qui se reflète à la fois dans les thématiques d'articles et dans les noms d'auteurs écrivant durant ces années-là. Les auteurs sont principalement des personnnes engagées au sein de la Ligue, certains y occupant des fonctions dirigeantes. Nombre d'entre eux sont parallèlement engagés dans des groupes théosophistes au sein desquels ils oeuvrent pour le renouveau éducatif. Comme nous l'avons vu, les thématiques qu'ils abordent

42 La revue sollicite notamment Ada Hart Arlitt, professeur et directeur du «Department of Child Care and Training» à l'Université de Cincinatti (Ohio), USA. 
relèvent à la fois de la pédagogie (récits d'expériences pédagogiques, présentation de méthodes d'enseignement) et de la psychologie (tests psychologiques, expériences en laboratoire, recherches sur le terrain) s'intéressant avant tout à l'enfant, à son développement, à ses spécificités psychologiques. Qu'ils soient praticiens ou théoriciens, les articles qu'ils écrivent révèlent leur proximité de vues et d'efforts, comme si leur solidarité était nécessaire pour construire la nouvelle science.

La deuxième phase allant de 1930 à 1940 se caractérise par un changement d'orientation qui se traduit tout d'abord par un intérêt croissant pour l'environnement social de l'enfant considéré désormais comme un facteur important de son développement. Cet environnement étant avant tout celui de la famille, la revue accorde alors une place centrale aux parents et aux spécialistes de l'éducation familiale. Comme nous l'avons $\mathrm{vu}$, les thématiques et les auteurs changent, la science et la recherche sur l'enfant paraissant désormais secondaires. De fait, les scientifiques ne disparaissent pas complètement, mais leur expertise est sollicitée pour faire avancer les connaissances en éducation familiale. Parallèlement, The New Era devient plus anglaise et se distance de la Ligue dont les représentants internationaux écrivent moins dans ses colonnes. Ce tournant rejoint celui que connaît Pour l'Ère Nouvelle au même moment, significatif d'une semblable évolution des discours et des préoccupations. Un tournant manifestement lié au contexte social, économique et politique dans lequel s'inscrit la Ligue dont nous aurons l'occasion de reparler dans la mise en regard des trois revues.

\section{POUR L'ÈRE NOUVELLE: DE L'ÈRE FERRIÈRE (1922-1930) À L'CEUVRE DU GFEN (1930-1940)}

INFRASTRUCTURE ET COMITÉ: UNE HISTOIRE EN DEUX TEMPS

Pour l'Ère Nouvelle paraît pour la première fois en janvier 1922. Alors que ses revues-sœurs existent sous un autre nom auparavant, Pour l'Ère Nouvelle est créée «ex-nihilo» pour répondre à l'un des buts formulés par la Ligue: «établir, par des congrès organisés tous les deux ans, et par les revues qu'elle publie, un lien entre les éducateurs de tous les pays qui adhèrent à ses principes et visent des buts identiques aux siens.» (Buts de la Ligue, PEN, 1922, 1, p. 1). Dans un texte introductif au premier numéro, Ferrière justifie le nom et l'existence de cette revue: 
Jusqu'ici nous avons élevé nos enfants du «dehors au dedans». C'est nous, adultes, qui décidions de cette éducation en premier et en dernier ressort. Demain, c'est l'enfant qui, avec notre concours éclairé, s'élèvera du «dedans au dehors». [...] Est-il exagéré dès lors de parler d'une «Ère nouvelle» de l'éducation? Cette Ėre nouvelle n'est-elle pas le terme des aspirations de beaucoup d'entre nous? N'est-ce pas la plus belle, la plus merveilleuses, la plus enthousiasmante des tâches que de travailler «pour l'Ère nouvelle»? Voilà justifié le titre de cette revue. Voilà justifiées du même coup sa création et son existence. (Ferrière, PEN, 1922, 1, p. 3)

Selon Hameline (2002b), le nom des trois revues révèle aussi l'influence de la pensée théosophiste sur la fondation de la Ligue. Tant Beatrice Ensor, membre d'une Fraternité théosophique en Angleterre, que Ferrière utilisent un vocabulaire imprégné de cette idéologie où le regard sur le monde et les hommes est certes religieux mais aussi «syncrétique, cosmopolite et surtout moderniste» (p. 28).

La métaphore de «l'ère nouvelle» appartient au répertoire d'images de la théosophie «dont la connotation "prophétique» des temps nouveaux est séculière. [...] Dans la mesure où la théosophie est composée aussi de cosmosophie voire de biosophie, cela explique sa tendance à vouloir «changer les manières de voir, de sentir, de penser, de se situer par rapport au vivant et à l'univers» et à prouver aux hommes qu'ils peuvent changer le cours de l'histoire. (Hameline, 2002b, p. 29)

Cette filiation avec le mouvement théosophiste permet de comprendre le vocabulaire utilisé dans la revue à ses débuts, notamment dans la rubrique «Notre Ligue»: «élan de vie spirituelle», «sacrifice pour l'humanité», «portée spirituelle de l'éducation», «libération de l'esprit», «puissance de l'esprit», «libérer l'âme de son prochain». Outre leur connotation spiritualiste, ces termes révèlent la dimension militante du discours de Ferrière qui imprègne fortement la revue durant les premières années.

À ses débuts (1922-1925), Pour l'Ère Nouvelle paraît quatre fois par an et assume une double fonction: «Organe trimestriel de la Ligue internationale pour l'Éducation nouvelle et du Bureau international des Écoles nouvelles». Si la première paraît évidente, la deuxième l'est un peu moins. Comme énoncé plus haut, la fondation de la Ligue est à la fois le fruit d'une initiative anglaise conduite par Beatrice Ensor et celui d'un désir genevois émanant de Ferrière. Ce dernier avait fondé en 1899 à Genève, le Bureau international des Écoles nouvelles (BIEN) «dans le 
but d'établir des rapports d'entraide scientifique entre les différentes écoles nouvelles, de centraliser les documents qui les concernent et de mettre en valeur les expériences psychologiques faites dans ces laboratoires de la pédagogie de l'avenir» (Ferrière, 1919, p. 2). Ferrière cherche à mettre en réseau les expériences des écoles nouvelles à la campagne (Landerziehungsheime) qui se multiplient dès le tournant du siècle. La revue constitue alors un support idéal pour visibiliser ce réseau, pour faire connaître ces expériences et surtout, pour stimuler les échanges directs entre pionniers de l'Éducation nouvelle dont il connaît une grande partie personnellement.

De 1922 à 1924, Ferrière est le seul rédacteur de Pour l'Ère Nouvelle dont il assume aussi bien les tâches éditoriales, qu'administratives et financières. C'est lui qui sélectionne les articles à publier - dont il fait souvent des traductions - et qui rédige la plupart des rubriques, notamment «Notre Ligue» (éditorial), «Nouvelles diverses», «Livres et revues» ainsi que les rapports de congrès. À partir de janvier 1925, il est rejoint par deux personnalités importantes du monde de l'éducation: Ovide Decroly (professeur à l'Université de Bruxelles) et Paul Fauconnet (professeur de psychologie et de pédagogie à la Sorbonne). À eux trois, ils constituent le comité de rédaction qui se maintiendra jusqu'en 1932. Ce changement se traduit par le déplacement de l'administration de la revue de Genève à Paris où celle-ci est désormais prise en charge par Jeanne Hauser, secrétaire du Groupe d'études, de recherches et d'expériences éducatives (futur GFEN). Femme du banquier Lionel Hauser, celle-ci est aussi, selon Ferrière, une «mère de famille extrêmement dévouée à l'œuvre de la Ligue», ainsi qu'une théosophe qui «fait partie de ce petit groupe de personnes qui mettent le bien de l'humanité au-dessus du bien de leur groupe théosophique, comme c'est le cas pour Beatrice Ensor». ${ }^{43}$

En janvier 1926, la revue abrite le «Bulletin international de l'Institut Rousseau» affichant ainsi un lien officiel avec cet institut et ses protagonistes, notamment Édouard Claparède (fondateur) et Pierre Bovet (directeur). Cette année-là, Pour l'Ere Nouvelle sert donc d'organe à trois organismes à la fois: la Ligue internationale pour l'Éducation nouvelle (LIEN), le Bureau international des Écoles nouvelles (BIEN) et l'Institut Rousseau. Comme le souligne Hofstetter (2010), ces deux derniers sont étroitement liés depuis 1923, puisqu'à la demande de Ferrière, le conseil

43 Lettre d'Adolphe Ferrière à Elisabeth Rotten, 16 décembre 1925. AIJJR, Fonds Ferrière, Correspondance alphabétique. Cote: AdF.C.I/ 63 
directeur de l'Institut avait accepté de rattacher le BIEN à son institution: «Dès le $1^{\text {er }}$ janvier 1923, le BIEN sera rattaché à l'Institut Rousseau dont il assumera pour l'étranger le service de renseignements relatif aux diverses questions qui intéressent l'Éducation nouvelle». ${ }^{44}$

Ce rattachement permet d'une part au BIEN de survivre financièrement et de poursuivre son travail. D'autre part, le directeur de l'Institut est intéressé à renforcer une collaboration avec ce bureau en raison de la portée internationale de Pour l'Ère Nouvelle. Dans une lettre du 18 décembre 1922, ce dernier propose d'adopter la revue comme «Organe international de l'Institut»:

Vous voudrez bien nous dire s'il vous paraît indiqué de renforcer d'une manière ou d'une autre les liens entre l'Institut et l'Association internationale pour l'Éducation Nouvelle [en fait, la LIEN] avec laquelle votre Bureau est en rapports si étroits, et en particulier avec le journal Pour l'Ère Nouvelle, si en prenant un certain nombre d'abonnements à placer parmi ses collaborateurs et amis, l'Institut pourrait appuyer votre revue et l'adopter peut-être comme organe international, elle ou ses sœurs allemande et anglaise. ${ }^{45}$

C'est chose faite à partir de janvier 1926, où la revue consacre désormais une à deux pages intitulées «Bulletin international de l'Institut Rousseau» dans les numéros de janvier, mars et avril. Dans cette rubrique figurent des informations sur l'organisation de l'Institut, ses protagonistes ainsi que ses événements et activités telles que cours, conférences, assemblées. Le premier numéro présente l'établissement comme une «École des Sciences de l'Éducation» et insiste sur son triple rôle de «centre d'information, centre de recherches, centre de propagande» (Bulletin IJJR, PEN, 1926, 18, p. 19). La philosophie de l'Institut ${ }^{46}$ y est brièvement expliquée et les principaux événements y sont relatés. Dès le $2^{\mathrm{e}}$ bulletin de mars, $\mathrm{l}^{\prime}$ Institut annonce la récente fondation de son Bureau International d'Éducation (BIE) ${ }^{47}$ qui a eu lieu le 19 décembre 1925. Les noms des membres de la direction sont annoncés: Pierre Bovet

44 Lettre de Pierre Bovet à Adolphe Ferrière, 18 décembre 1922. AIJJR, Fonds général, Dossiers de l'Association de l'IJJR, Dossier BIEN.

45 Lettre de Pierre Bovet à Adolphe Ferrière, 18 décembre 1922. AIJJR, Fonds général, Dossiers de l'Association de l'IJJR, Dossier BIEN.

46 Sur l'histoire de l'Institut Rousseau, voir Hofstetter, 2010.

47 Pour une histoire du BIE, voir Hofstetter \& Schneuwly (2013) et Hofstetter (2015). 
est directeur secondé par Adolphe Ferrière et Elisabeth Rotten, tous deux nommés directeurs adjoints. Selon les indications fournies dans ce numéro, le BIE s'organise comme une «institution libre», et comme un «centre qui n'est pas lié par des attaches gouvernementales et bureaucratiques»:

Ainsi, il peut avoir une plus grande liberté d'allure et une plus grande souplesse pour s'adapter à des besoins variables, et saura mieux collaborer dans un esprit de collégialité avec les associations de maîtres et les chercheurs libres. De cette manière, il sera aussi plus à l'aise pour donner certaines impulsions en ce qui concerne l'éducation pacifique. (Bulletin IJJR, PEN, 1926, 19, p. 44)

Le Bureau International d'Éducation fonctionne donc comme organe de ralliement des innombrables institutions œuvrant pour les mêmes causes, en particulier, la coopération intellectuelle, la solidarité internationale et le renouveau éducatif. Son secrétariat, en relation étroite avec l'Institut, déploie dès 1926 une intense activité de documentation sur les réformes scolaires, l'éducation à la paix, l'enseignement de la lecture, la correspondance interscolaire ou le bilinguisme (Hofstetter, 2015). Cette nouvelle activité explique la rapide mutation du bulletin en «Chronique du Bureau International d'Éducation» où sont relatées les activités du nouveau BIE, notamment ses entreprises de propagande dans le monde entier. En tant que directeur du BIEN, directeur-adjoint du BIE, membre du service des renseignements internationaux de l'Institut Rousseau et rédacteur en chef de la revue, Ferrière se trouve au cœur des informations sur l'Éducation nouvelle et assure ainsi l'essentiel du travail de documentation internationale dont il alimente les diverses rubriques de PEN («Notre Ligue», «Nouvelles diverses», "Chronique du BIE») ainsi que ses écrits personnels. En avril 1926, il informe les lecteurs du nouveau statut de son BIEN désormais fusionné avec le Bureau International d'Éducation:

Dès le printemps 1926, la section «Éducation nouvelle» du Bureau International d'Éducation assume les services d'information du bureau international des Écoles nouvelles. Le rôle de ce dernier se borne dorénavant à assurer comme lors de sa création en 1899, un lien d'ordre scientifique entre les Écoles nouvelles. Le public est donc prié d'adresser toute demande de renseignements au BIE en y joignant la taxe prévue. (Directeur du BIEN, PEN, 1926, 20, p. 70) 
Dès janvier 1927, la Chronique du BIE figure à la fois comme sous-titre de Pour l'Ère Nouvelle et comme rubrique dans le sommaire des numéros et le volume de ses pages ne cesse d'augmenter: des reflets de l'importance croissante que prend ce Bureau. En 1928, la Ligue prospère rapidement grâce à l'adhésion d'un grand nombre de nouvelles sections nationales et de leurs organes respectifs. C'est une année intense marquée par de nombreux voyages des trois membres fondateurs (Ferrière, Ensor, Rotten) qui parcourent le monde entier pour donner des conférences. Si les deux Amériques constituent leurs principales destinations, l'Asie et l'Afrique du Sud reçoivent également leur visite ou celle de membres importants de la Ligue. Ces voyages se traduisent par une avancée considérable du mouvement, notamment aux États-Unis où grâce aux tournées d'Ensor et Rotten, des liens durables sont tissés avec les Américains. Des liens qui se concrétisent par la fondation d'une section officielle de la Ligue composée de membres prestigieux de la Progressive Education Association.

Cette extension se traduit par la multiplication d'associations dans le monde qui, faisant concurrence à la Ligue, font perdre à cette dernière son statut d'exception. Cette situation est perceptible à l'intérieur d'un même pays, notamment en France, où les groupes et associations prolifèrent au point de ne plus savoir quelles sont les spécificités de chacune. À ce propos, Ferrière tente de clarifier la situation en classant les groupes dans des catégories selon leur degré «d'innovation» et d'influence:

Une naturelle division du travail [s'établit] entre les pionniers groupés autour de la «Nouvelle Éducation», les esprits plus prudents que réunit le «Groupe Français» de la Ligue internationale pour l'Éducation nouvelle et la synthèse des œuvres françaises s'occupant de l'enfance, synthèse groupée autour du «Bureau Français d'Éducation» (B.F.E), organe national du B.I.E. de Genève. (Éditorial, PEN, 1929, 44, p. 3)

L'année 1929 est une année clé dans l'histoire de plusieurs institutions impliquées dans la promotion de l'Éducation nouvelle. À la fin de l'année, le Bureau International d'Éducation change de statut: il se détache de l'Institut Rousseau et devient une organisation intergouvernementale qui s'adresse désormais aux ministères de l'instruction publique et oriente ses activités vers l'administration et la gestion des systèmes scolaires nationaux (Magnin, 2002). Ce changement se traduit par la disparition du «Bulletin du Bureau international d'Éducation» publié 
jusque-là dans Pour l'Ère Nouvelle. De son côté, l'Institut Rousseau change aussi de statut en se rattachant à la Faculté des lettres de l'Université de Genève. Il reçoit le mandat d'assumer la formation théorique des enseignants du primaire et change de nom cette année-là pour s'appeler désormais «Institut des Sciences de l'Éducation» (ISE). Selon Hofstetter (2008), ce rattachement constitue «un événement institutionnel majeur et en symbolise l'orientation principale: une clarification structurelle et un ajustement sur le modèle académique pour en garantir le développement». De plus, il atteste de la «reconnaissance dont l'Institut et le champ disciplinaire font l'objet et à laquelle contribue puissamment leur audience internationale» (p. 191)

En Belgique, 1929 est une année importante dans l'histoire de l'Éducation nouvelle puisque c'est à ce moment-là que s'opère la fusion entre le groupe de «Decroly/Hamaïde» et le cercle de travail de Fernand Dubois (Waterloo), fusion qui donne naissance à la section nationale. Celle-ci est dirigée par un comité composé d'Ovide Decroly (président), Amélie Hamaïde, Fernand Dubois, Jozef Emiel Verheyen, Nicolas Smelten et Léon Poriniot. ${ }^{48}$ Ce dernier est le directeur de la revue Vers l'École active qui voit le jour cette même année et constitue désormais l'organe officiel de la Ligue pour la Belgique. Selon Wagnon (2015), cette section nationale est

un outil au service de l'institutionnalisation de l'Éducation nouvelle en symbiose avec les écoles decrolyennes. [...] Elle fut aussi un moyen de légitimer les expériences decrolyennes et placer l'essor de la pédagogie Decroly comme une alternative crédible et reconnue à l'extérieur du pays par les spécialistes des autres pays. (p. 124)

Suite au Congrès d'Elseneur en été 1929, le Groupe Français d'Éducation Nouvelle décide d'intensifier sa collaboration avec la Ligue notamment en assumant la propagande dans tous les pays de langue latine et en prenant une part plus active dans l'organisation des congrès internationaux (analyse des thèmes, choix des personnes à inviter, etc.). Dès cette année-là, le groupe officialise ce lien en devenant une section nationale de la Ligue. Ce changement se traduit par des modifications statutaires au sein du groupe et par l'élection d'un nouveau comité composé de

48 Sur les membres de cette section nationale et Nicolas Smelten en particulier, voir les travaux de Wagnon (2015). 
Paul Langevin (président), Paul Fauconnet (vice-président), Henri Wallon, Henri Piéron et Georges Bertier (membres du comité), ainsi qu'Émilie Flayol (secrétaire) et Jeanne Hauser (trésorière).

Enfin, 1929 constitue un tournant dans l'histoire de Pour l'Ère Nouvelle puisque c'est cette année-là qu'a lieu le transfert officiel de sa gestion administrative au Groupe Français d'Éducation Nouvelle. Les conséquences ne sont pas visibles dans l'immédiat car le comité de rédaction reste le même jusqu'à la fin de l'année 1931, Ferrière conservant son titre de rédacteur en chef. Toutefois, ce dernier passe officiellement le relais cette année-là dans le but de se décharger des tâches administratives de la revue. Dans un numéro spécial consacré à l'histoire du groupe (févriermars 1938), le GFEN relate cet événement important:

En 1929, Mr. Ferrière manifesta le désir d'en remettre la direction en d'autres mains. L'Institut Rousseau et le Groupe Français d'Éducation nouvelle furent envisagés comme successeurs possibles. À la suite d'une consultation qui eut lieu à Genève entre $\mathrm{M}$. Ferrière, $\mathrm{M}$. Bovet, $\mathrm{M}$. Piaget et $\mathrm{M}^{\mathrm{lle}}$ Flayol, il fut décidé que Pour l'Ėre Nouvelle passerait aux mains du Groupe Français, à charge pour lui de conserver à cet organe son caractère international latin et sa liaison avec la Ligue internationale pour l'Éducation nouvelle. (GFEN, PEN, 1938, 135, p. 46)

Du côté de Ferrière, ce n'est qu'en janvier 1931 qu'il évoque l'événement officiellement, dans un éditorial signé de sa main; le transfert de responsabilité apparaît davantage comme une contrainte que comme un choix:

Le rédacteur en chef de cette revue, obligé de restreindre ses activités afin de se concentrer sur la rédaction d'ouvrages qu'il a en chantier depuis longtemps, se voit contraint d'accepter l'offre dévouée du Groupe Français d'Éducation Nouvelle de reprendre à Paris le travail de rédaction. Rien n'est changé pour le moment à nos dispositions sauf que le choix des articles à publier, les comptes-rendus de livres et articles de revues, les nouvelles diverses et la mise en page se feront désormais à Paris. Le rédacteur en chef, seul à la brèche de 1922 à 1930, continuera toutefois d'envoyer des articles originaux ou des comptes-rendus à la revue et prépare pour décembre 1931 une table des matières générale des dix premières années. (Éditorial, PEN, 1931, 64, p. 2)

En février 1931, la rédaction annonce l'installation définitive de la revue au Musée pédagogique à Paris où désormais, toute correspondance concernant l'administration ou la rédaction de la revue (abonnements, 
envois de livres pour comptes-rendus, échange de revues) doit être adressée. Ce siège devient rapidement un lieu de référence du mouvement en francophonie puisqu'il abrite à la fois le bureau de Pour l'Ère Nouvelle, le centre de travail du GFEN ainsi que le siège de la Ligue pour les pays de langue latine. Comme le montre le tableau suivant ${ }^{49}$, le passage officiel de la responsabilité éditoriale au GFEN ne se concrétise qu'en 1932 dans les pages de PEN.

Tableau 5.

Infrastructure éditoriale de Pour l'ère Nouvelle

\begin{tabular}{llll}
\hline Année & Rédaction & Administration & Remarques \\
\hline 1922 & A. Ferrière $(\mathrm{CH})$ & A. Ferrière, Genève & Organe de la Ligue et du \\
& & $\begin{array}{l}\text { (Rue de la Pélisserie) } \\
\text { des Écoles nouvelles } \\
\text { et 2e de couverture }\end{array}$ & Bureau international (BIEN). \\
& & Principes de ralliement et buts \\
& & \\
\hline
\end{tabular}

1923

1924

1925
A. Ferrière:
J. Hauser, Paris
rédacteur en chef $(\mathrm{CH})$
(Av. de l'Observatoire)
P. Fauconnet (France)
O. Decroly (Belgique)

1926

Abrite la Chronique de

l'Institut Rousseau remplacée dès juillet par celle du Bureau

International d'Éducation (BIE)

\begin{tabular}{lll}
\hline 1927 & J. Crémieu, Paris & Chronique du BIE \\
& (Centre de Librairie \\
& française et étrangère)
\end{tabular}

1928

1929 GFEN (dès juillet)(Musée

Pédagogique, Paris)

1930

Disparition de la Chronique du BIE

49 Ce tableau a été fait à partir de notre dépouillement de PEN dans le but de clarifier l'évolution des comités de rédaction et celle des différentes personnes qui en sont membres. 


\begin{tabular}{|c|c|c|c|}
\hline Année & Rédaction & Administration & Remarques \\
\hline \multicolumn{4}{|l|}{1931} \\
\hline 1932 & $\begin{array}{l}\text { O. Decroly (B) } \\
\text { J. Piaget }(\mathrm{CH}) \\
\text { H. Piéron (F) } \\
\text { H. Wallon (F) } \\
\text { E. Flayol (F) (secrétaire) }\end{array}$ & $\begin{array}{l}\text { GFEN:Administration } \\
\text { et rédaction }\end{array}$ & $\begin{array}{l}\text { Dès novembre: suppression } \\
\text { des principes de ralliement } \\
\text { et des buts de la Ligue: } \\
\text { nouvelle «charte» }\end{array}$ \\
\hline 1933 & $\begin{array}{l}\text { A. Hamaïde (B) } \\
\text { J. Piaget }(\mathrm{CH}) \\
\text { H. Piéron }(\mathrm{F}) \\
\text { H. Wallon (F) } \\
\text { E. Flayol (F) (secrétaire) }\end{array}$ & & \\
\hline
\end{tabular}

1934

1935

1936

1937

1938

GFEN

Nouvelle mise en page:

Membres du comité GFEN en

$2^{\mathrm{e}}$ de couverture

\begin{tabular}{|c|c|}
\hline 1939 & $\begin{array}{l}\text { Rédaction: GFEN } \\
\text { Abonnements: Éditions } \\
\text { Bourrelier et Cie }\end{array}$ \\
\hline
\end{tabular}

1940

Seul un numéro (janv.fév.mars) paraît cette année puis la revue s'interrompt jusqu'en 1946

En 1932, le Groupe Français d'Éducation nouvelle (GFEN) est officiellement reconnu comme administrateur et rédacteur de la revue. Un nouveau comité de rédaction est nommé, composé de quatre personnes: Dr. O. Decroly (professeur à l'Université de Bruxelles), Dr. J. Piaget (directeur du Bureau international d'Éducation à Genève), Dr. H. Piéron (professeur au Collège de France), Dr. H. Wallon (professeur à la Sorbonne), les deux derniers étant membres du GFEN. Le nom de Ferrière figure toujours en première page mais en qualité de fondateur et non plus en tant que rédacteur. Dès cette année-là, la revue change de ton. La rédaction n'écrit plus d'éditoriaux - déjà très peu nombreux en 1931 - et les articles de fonds sont rédigés en majorité par des auteurs français. Alors que le groupe avait à charge de conserver «son caractère international latin et sa 
liaison avec la Ligue internationale pour l'Éducation nouvelle» (GFEN, $P E N, 1938,135$, p. 46), ce lien est désormais peu perceptible. Le fameux «esprit de la Ligue» semble avoir disparu et les informations à son sujet sont très succinctes. Durant les années suivantes, la majorité des auteurs sont français et les thématiques portent essentiellement sur des expériences faites en France. La collaboration étrangère est très fluctuante, marquée par les difficultés économiques qui empêchent certains correspondants d'envoyer leurs articles et chroniques.

En novembre 1932, les principes de ralliement sont remplacés par un nouveau texte destiné à rassembler les membres de la Ligue et lecteurs de la revue autour des idées du nouveau comité de rédaction et prenant en compte les difficultés économiques et politiques des années 1930. Cette charte (Gutierrez, 2008; Savoye, 2004) évoque la crise dans laquelle se trouve l'Europe et invite tous les parents, éducateurs, administrateurs et travailleurs sociaux à se mobiliser dans un «mouvement universel» de rénovation de l'école car selon eux, «seule une éducation réalisant dans toutes ses activités un changement d'attitude vis-à-vis des enfants peut inaugurer une ère libérée des concurrences ruineuses des préjugés, des inquiétudes, et des misères caractéristiques de notre civilisation présente, chaotique et dépourvue de sécurité» (Charte, 1932, PEN, 82). Élaborée par le GFEN et avalisée au Congrès de Nice, cette charte marque un tournant vers une orientation plus «pragmatique» de l'Éducation nouvelle, reléguant la dimension spiritualiste, qui caractérisait les principes de ralliement, au second plan. Un texte qui prend en compte la nouvelle réalité de la société et révèle le contexte difficile des années 1930.

L'année 1932 est aussi marquée par le décès d'Ovide Decroly - survenu en septembre - en l'hommage duquel nombre de manifestations sont organisées, dont Pour l'Ère Nouvelle donne un large écho dans le numéro spécial d'octobre 1932 (PEN, 81). Dès 1933, Amélie Hamaïde succède à Decroly au comité de rédaction, afin que celui-ci conserve un représentant de chaque pays, condition pour que la revue garde son statut d'organe latin de la Ligue (Flayol, PEN, 1939, 145, p. 73). Ce comité reste stable jusqu'en 1940 où seul un numéro de Pour l'Ère Nouvelle sera publié, marquant la fin d'une époque interrompue par la guerre. Ce n'est qu'en 1946, après six ans d'interruption, que PEN sera relancée avec de nouveaux objectifs.

Considérée par certains historiens comme une «revue de militantisme pédagogique» (Peyronie, 2002, p. 7), Pour l'Ère Nouvelle est présentée comme une «revue d'orientation»: 
Elle est, diraient les philosophes modernes, d'ordre dynamique. À chacun d'en tirer les éléments statiques conformes à la réalité telle qu'elle se présente à lui. [...] Elle se tient à l'écart de toute attitude confessionnelle ou politique. La vérité humaine est le seul but qu'elle poursuit; la méthode scientifique objective est le seul moyen qu'elle mette en œuvre. (Ferrière, circulaire PEN, 1922, p. 4)

Pour montrer l'originalité de PEN et de ses fondements philosophiques, Ferrière la compare à d'autres revues similaires mais auxquelles elle ne peut être assimilée:

Notre revue ne fait double emploi avec aucune autre. Elle ne fait concurrence à aucune autre. Elle apporte modestement sa collaboration à toutes les autres. Les revues pédagogiques existantes visent des fins professionnelles; rôle utile, nécessaire. Elles visent des fins pédagogiques nationales: perfectionner les programmes et les méthodes de l'école publique; rôle nécessaire, urgent même. Pour l'Ère Nouvelle travaille... pour l'ère nouvelle. Ce qui pour d'autres, constitue un but, un point d'arrivée lointain peut-être, est pour notre revue, un point de départ. (Ferrière, circulaire PEN, 1922, p. 3)

Enfin, Pour l'Ère Nouvelle est présentée comme une revue de spécialistes, tant scientifiques que praticiens, dont les méthodes sont considérées comme particulièrement valables parce qu'elles ont été éprouvées, tel que le préconise la pédagogie expérimentale, science de référence des théories de Ferrière.

Elle étudie ce qui se fait parmi les pionniers de la science de l'enfant et parmi les pionniers de la pédagogie pratique. Elle apporte des matériaux pour reconstruire l'école sur une base meilleure. Matériaux éprouvés, au moyen desquels les pédagogues pourront faire plus et mieux jusqu'ici. Le plus et le mieux possible - ou comme ils sont modestes ils diront: le moins mal possible! (Ferrière, circulaire PEN, 1922, p. 4)

Pourtant, son caractère militant transparaît dès la première page: «La revue Pour l'Ère Nouvelle est la revue des pionniers de l'éducation». Le mot «pionniers» peut être compris comme ceux qui montrent le chemin du progrès, de l'avenir, pour ne pas dire le «droit chemin». Parmi les quatre buts de la Ligue, le quatrième indique: «Il n'y a pas de cotisation. L'abonnement à la revue Pour l'Ère Nouvelle implique l'adhésion à la Ligue. Il suppose donc l'adhésion à ses principes de ralliement, tout au moins à titre d'orientation générale» (Buts de la Ligue, PEN, 
1922, p. 1). Cette mention révèle le côté militant du journal puisque des conditions sont posées d'emblée à ceux qui désirent s'y abonner; elles supposent non seulement un engagement financier (paiement de l'abonnement) mais une adhésion idéologique aux principes de la Ligue. Ce détail est nuancé dès 1923 par une phrase ajoutée au bas du quatrième point: "Ceux de nos abonnés qui désirent n'être pas comptés parmi les membres de la Ligue sont priés simplement d'en aviser la rédaction.» Toutefois, la rédaction se défend de cette image propagandiste qui, à l'époque déjà, semble susciter des critiques de la part du public:

Beaucoup de gens qui ne voient que la couverture de notre revue nous taxent de propagandistes d'un internationalisme de mauvais aloi. [...] S'ils nous avaient lus, et encore mieux, vus à l'œuvre, ils se seraient convaincus depuis longtemps que notre activité est celle de biologistes, psychologues et de sociologues pour qui l'éducation de l'enfant - de tous les enfants du monde - est le souci dominant et pour qui n'existe aucun exclusivisme partiel et partial. (Notre Ligue, PEN, 1923, 7, p. 45)

Au fil des numéros, les nouveaux membres sont remerciés et considérés comme s'ils faisaient partie d'une même famille, poursuivant le même idéal incarné par la construction d'une ère nouvelle.

Que tous ces amis proches ou lointains, que tous ces travailleurs de l'esprit, à la poursuite du même idéal que nous, idéal esquissé dans les principes de notre Ligue internationale pour l'Éducation nouvelle veuillent trouver ici l'expression de notre reconnaissance. Reconnaissance en particulier du rédacteur de cette revue; car celle-ci ne peut accomplir une œuvre féconde que si elle sert de lien entre les hommes et toutes les femmes qui, chacun à son poste, travaillent Pour l'Ère Nouvelle. (Ferrière, PEN, 1922, 1, p. 2)

Pour Ferrière, cette ère nouvelle est la raison profonde qui doit mobiliser, ou «mettre en route», tous ceux qui sont concernés par l'éducation, tant professionnels que parents. Il s'agit donc de propager l'esprit et les méthodes nouvelles, en comptant sur le principe de "contamination» opérationnelle à partir de ceux qui sont déjà acquis à la cause, par exemple, les abonnés de la revue. C'est pourquoi, la rédaction lance régulièrement des appels qui révèlent la tâche à la fois «unificatrice» et «recrutante» de la revue: 
L'Ėre nouvelle ne se fera pas sans nous, sans nos recherches, notre patience et notre effort. [...] Que nos amis ne discontinuent pas de soutenir notre travail et de répandre autour d'eux la conviction qu'une éducation nouvelle et meilleure est possible, réalisée en petit, réalisable en grand, désirable, indispensable, urgente. (Ferrière, PEN, 1922, 1, p. 3)

Mais ces appels se chevauchent parfois avec ceux lancés par d'autres associations créant ainsi des confusions. Ces chevauchements sont révélateurs de l'effervescence militante de l'époque qui se traduit d'ailleurs par la naissance d'une multitude d'associations et de revues d'éducation dont les objectifs et les principes sont souvent similaires:

Pour la France, notre appel, venant de la Ligue Internationale pour l'Éducation Nouvelle s'est trouvé coïncider avec celui de «L'Éducation nouvelle», d'où, comme nous l'avons su, confusion bien naturelle de la part des personnalités auxquelles se trouvait ainsi lancé un double appel. Étant donné l'identité du but poursuivi leur adhésion donnée à «L'Éducation nouvelle» constitue une adhésion implicite à l'idéal de notre Ligue, puisque celle-ci n'est pas un organisme fermé, mais une orientation vers des aspirations. (Avis, PEN, 1923, 6, p. 25)

Les appels sont souvent liés aux problèmes financiers que rencontre la revue. Car si les manifestations de soutien sont bienvenues, elles ne permettent pas à la revue de survivre financièrement. Aussi, la recherche se fait tant du côté des abonnés que du côté de la publicité (annonces) car son ambition est bien de «vivre d'abord» puis de «s'accroître».

Trop peu nombreuses sont les adhésions morales à notre Ligue qui s'accompagnent d'une adhésion matérielle! [...] Il faut que les membres de notre Ligue nous aident. L'existence de ces pages, lien matériel qui nous unit, est l'une des conditions les plus importantes du lien spirituel qui doit nous permettre de nous instruire les uns les autres et d'agir d'un commun accord. (Notre Ligue, PEN, 1922, 2, p. 31)

Ainsi, l'évolution de la revue se caractérise par deux phases ou deux périodes d'environ dix ans chacune, 1922/1931 et 1932/1940; elles se distinguent l'une de l'autre, d'une part par leur contexte social, politique et économique très différent, et d'autre part, par le comité éditorial qui «colore» différemment mais tout aussi profondément l'une et l'autre période. Dans la première, Pour l'Ère Nouvelle se caractérise par son caractère international, jouant pleinement son rôle d'organe de la Ligue, 
évoluant dans un contexte d'expansion du mouvement auquel elle contribue activement. Dans les années 1922 à 1925, son envergure est très large se faisant l'écho des pays du monde entier où progresse le mouvement, alors que les années 1926 à 1930 sont marquées par un resserrement du «champ d'action» sur les pays latins. Ferrière contribue beaucoup à l'envergure internationale de la revue en raison de ses réseaux de relations très étendus et de son âme de propagandiste, militant pour une cause à laquelle il dédie toute sa foi et son énergie.

Dans la deuxième période, Pour l'Ère Nouvelle donne l'impression de se «replier sur elle-même» car elle devient beaucoup plus nationale (française), donnant beaucoup d'informations sur ce qui se passe en France, les auteurs étant majoritairement français. Elle s'apparente dès lors à une «revue de section», une section française où la Suisse et la Belgique n'ont plus tellement leur place et lui faisant perdre son caractère international. ${ }^{50}$

Dès 1936, année du Congrès de Cheltenham, la revue connaît une sorte de «réouverture» sur l'international, attestant d'un nouvel intérêt pour ce qui se passe à l'étranger (même si les «valeurs sûres» restent en France). Une ouverture liée à la situation de crise qui suscite un élan de solidarité international (surtout européen) et une prise de conscience de la nécessité de s'associer à tous les efforts pour tenter de sauver la situation.

\section{LES COULISSES DE LA RÉDACTION}

À ses débuts, la croissance de Pour l'Ère Nouvelle est directement liée à celle de la Ligue puisque les membres sont abonnés automatiquement à la revue. En 1923, la rédaction annonce que la Ligue grandit plus vite que prévu «étendant ses ramifications sur toute l'Europe et sur les autres continents». Ceci se traduit par une recrudescence d'abonnés mais aussi par la naissance de nouvelles revues nationales qui se concurrencent:

$\mathrm{M}^{\mathrm{me}}$ Montessori parle de créer une revue nouvelle. Pourquoi tant de revues? $\mathrm{Ne}$ vaudrait-il pas mieux réserver à $\mathrm{M}^{\mathrm{me}}$ Montessori et à ses collaborateurs quelques pages dans chaque numéro? Toutes choses à examiner en temps et

50 Elle est davantage la revue du Groupe Français d'Éducation nouvelle que de la Ligue internationale pour l'Éducation nouvelle. 
lieu. À ce titre seul, sa présence à Territet serait bien désirable! (Notre Ligue, PEN, 1923, 7, p. 44)

Une année plus tard, les objectifs qui avaient été fixés pour 1924 ne sont pas atteints. Ferrière entreprend plusieurs changements tant dans la structure que dans la gestion de la revue. Il la fait imprimer à Paris à raison de 10 fascicules de 16 pages au lieu de 4 fois 20 pages (par année), sollicite ses contacts pour augmenter le volume des annonces et recherche la collaboration d'autres rédacteurs:

Je suis en présence d'une décision difficile à prendre concernant Pour l'Ère Nouvelle. Jusque-là le déficit annuel prévu était couvert par la Ligue internationale pour l'Éducation nouvelle. Ce subside cesse dès janvier 1926, les «trustees» ayant des intérêts ailleurs et n'ayant promis d'ailleurs leur appui que jusqu'à fin 1925 dans l'espoir que dès lors la revue volerait de ses propres ailes. [...] Faut-il fusionner avec le Bulletin de la Nouvelle Éducation? [...] Ce matin ma femme me suggère l'idée de vous proposer la fusion avec l'Intermédiaire des Éducateurs (6 numéros par an). Cela me serait plus sympathique puisque le BIEN fait partie de l'IJJR. Mais, est-ce faisable? [...] Ce serait une belle augmentation du champ d'activité de l'Institut J.J. Roussseau. ${ }^{51}$

Finalement, aucune fusion n'aura lieu car pour Claparède, «l'Intermédiaire des Éducateurs n'a pas d'existence indépendante ${ }^{52}$ et les propositions de Madeleine Guéritte ne sont pas convaincantes en raison du peu d'espace proposé et du prix prohibitif que coûteraient ces parutions. La brusque croissance des membres de la Ligue après le Congrès de Heidelberg (1925) entraîne une nouvelle embellie pour PEN ce qui permet à la rédaction d'augmenter le nombre de pages et de publier désormais 6 numéros par an. L'augmentation des annonces (4 pages par numéro) et l'élévation du prix des abonnements, ainsi que la diffusion à de grandes associations d'enseignants assureront la santé financière pour l'année 1926:

51 Correspondance de direction, 9 octobre 1925, AIJJR, Fonds Ferrière, FG.F.I 22.

52 C'est Claparède qui répond car Bovet lui a transmis la demande, tous deux étant concernés par la question. Lettre de Claparède à Ferrière, 15 octobre 1925, AIJJR, Fonds Ferrière, FG.F.I/ 22. 
Nous tenons à remercier le Ministre de l'Instruction publique, le Syndicat national des Instituteurs et des Institutrices qui compte près de 80000 membres, le Bureau International des Fédérations nationales du Personnel de l'Enseignement Secondaire Public, la plupart des grandes revues pédagogiques de la France et de l'étranger, la Société Pédagogique Genevoise et son dévoué président Emmanuel Duvillard, ainsi que l'Institut J. J. Rousseau qui a bien voulu choisir notre revue en qualité de Bulletin international et lui assurer son appui. (Éditorial, PEN, 1926, 18, p. 1)

Dès 1927, Pour l'Ère Nouvelle est élevée au rang d'une revue mensuelle», rythme qu'elle conservera jusqu'en 1937. Ce statut lui confère plus de prestige et devrait lui assurer une certaine aisance financière. L'administration est alors reprise par Julien Crémieu, directeur du «Centre de Librairie française et étrangère à Paris. Mais la cause n'est pas gagnée. Dans son éditorial de janvier 1927, la rédaction appelle les lecteurs à faire connaître $P E N$ le plus largement possible, quels que soient les moyens financiers des personnes intéressées. Dans une conjoncture florissante où se multiplient les revues de toutes sortes ${ }^{53}$, la concurrence est dure:

Nous voudrions que tous les amis de l'Éducation nouvelle la connaissent, ceux de France, ceux de Belgique, ceux de Suisse romande. Beaucoup d'entre eux reçoivent d'autres revues. Pourquoi pas la nôtre? Est-ce une question de dépense? Dans ce cas, qu'ils veuillent bien nous l'écrire et nous sommes prêts à en faciliter l'acquisition à qui nous en adressera la demande. (Éditorial, PEN, 1927, 24, p. 1)

Malgré tout, la revue continue de grandir - l'accroissement des abonnés au cours de 1927 est estimé à 72\% - ce qui augure un redressement financier. De plus, le Comité exécutif décide lors du Congrès de Locarno (1927) de demander une contribution financière à toutes les revues qui désirent devenir organes officiels de la Ligue. Mais ces cotisations ne suffisent pas à couvrir les frais d'édition de PEN ni le travail colossal qu'investit son rédacteur. En mai 1928, Ferrière écrit une lettre à Bovet signifiant son souhait de transmettre la responsabilité à quelqu'un d'autre:

53 Pour la conjoncture en Suisse Romande, voir Clavien, Le Dinh \& Vallotton (1993). 
Je compte voir M. Bertier et M. Baucomont pour me débarrasser en 1929 de la «cuisine» de la revue Pour l'Ère Nouvelle. J'en ai assez de me tuer au travail non rémunéré et propter vitam vitae perdere causas. J'ai autre chose à faire que je ne fais pas, faute de temps! Donc veuillez me dire si vous croyez que l'Institut Rousseau, vous, Ed. Claparède, comptez avoir en 1929 une revue de l'Institut Rousseau. Je vous passerais ma revue et ses quelques 900 abonnés, gardant seulement quelques pages pour la Ligue. Si l'Institut Rousseau ne dispose ni des personnes ni de l'argent pour faire faire ce travail et s'il renonce à son projet, alors je verrais s'il y a quelque chose à faire du côté de M. Bertier ou de M. Faucher, ou des deux ensembles. ${ }^{54}$

Finalement, c'est auprès du GFEN qu'il trouvera des forces pour le suppléer. Le transfert officiel de la gestion administrative de la PEN au GFEN a lieu en été 1929, suite à une décision prise au Congrès d'Elseneur. Mais ce transfert n'a pas d'impact décisif sur la santé financière de PEN. En janvier 1930, des déficits budgétaires sont annoncés changement d'imprimeur qui engendre des frais considérables et augmentation des prix d'impression et du papier -, ce qui implique des efforts d'économies plus grands ainsi qu'une recherche accrue de dons et d'annonces.

Tous les amis de notre œuvre sont priés de nous aider, soit en comblant le déficit, soit en nous procurant de nouveaux abonnés ou, si possible, des annonces. Nous fournissons sur demande le tarif des annonces. (Éditorial, PEN, 1930, 54, p. 3)

Dès 1930, les membres du GFEN qui s'abonnent à Pour l'Ère Nouvelle font l'objet d'une adhésion distincte et bénéficient d'une cotisation propre. Cette année-là, les avis pressant les lecteurs à payer leur abonnement se succèdent. Les éditoriaux se faisant très rares, peu d'éléments sont communiqués à ce sujet. Seul l'éditorial de décembre 1930, signale que la fédération mondiale - World Federation of Education Associations - semble résoudre temporairement le problème des finances. ${ }^{55}$

54 Correspondance de direction, 8 mai 1928, AIJJR, Fonds Général, FG.C.I / 2.

55 La World Federation of Education Associations est fondée en 1923 lors de la conférence mondiale sur l'éducation à San Francisco. Ses objectifs visent à découvrir une «méthode à travers laquelle l'idéal de la paix mondiale puisse être diffusé à travers l'éducation» (Fuchs, 2004, p. 776). 
Notre Fédération mondiale, dont la création a été ratifiée à Elseneur, paraît désormais comme l'un des moyens les plus sûrs d'assurer nos finances. Cette fédération ne revêt pas les caractères d'une affiliation et ne modifie en rien l'activité générale de la Ligue. (Éditorial, PEN, 1930, 63, p. 253)

Dès 1931, la revue et le GFEN sont désormais liés et la gestion des abonnements se fait par Jeanne Hauser à Paris (Rue de l'Observatoire). Mais les problèmes financiers persistent si l'on en croit les avis qui jalonnent les pages du périodique et le ton sur lequel ceux-ci sont formulés.

Nous attirons cette année, d'une façon toute spéciale, l'attention de nos abonnés sur l'intérêt qu'il y a pour eux à nous faire parvenir le montant de leur abonnement sans tarder en utilisant la formule de mandat chèque cijointe. En effet, la récente augmentation des taxes postales a rendu les recouvrements par poste si onéreux que nous nous trouverons dans la nécessité de majorer d'environ cinq francs le prix de l'abonnement pour nos lecteurs qui attendront la présentation de leur quittance. Nous mettrons en recouvrement tous les abonnements qui ne nous auraient pas été pas été payés à la date du 28 février. (Rédaction, PEN, 1932, 83, p. 303)

En 1934, le rapport d'activité du GFEN rédigé par Émilie Flayol fait état des difficultés que traverse la revue en raison de la crise générale de 1933. Celle-ci leur a fait perdre beaucoup d'abonnés étrangers en raison des difficultés qu'imposent les gouvernements pour la transmission d'argent. "Cette crise nous a aussi privés petit à petit de presque toutes les ressources que nous procuraient les annonces» (Flayol, PEN, 1934, 96, p. 69).

À ces raisons s'ajoutent la défection des écoles normales dont les crédits «bibliothèque» ont été supprimés, l'augmentation des frais d'impression - qui oblige l'administration à changer trois fois d'imprimeur et les coûts de la publicité qui empêchent l'envoi habituel d'appels aux directeurs d'écoles et de lycées en automne. Si la revue boucle néanmoins son budget cette année-là, le rapport rappelle la «nécessité vitale» que représente l'augmentation du nombre d'abonnements et encourage les lecteurs à tout faire pour aider la revue financièrement. En 1935, le rapport annonce l'adhésion de nouveaux abonnés mais ils ne suffisent pas à compenser les pertes de ceux qui, parfois les plus anciens, ne se réabonnent pas, pour des raisons économiques. La quantité totale d'abonnés est donc inférieure à celle de 1932. Selon Flayol, la collaboration étrangère qui avait beaucoup diminué en 1932 et 1933 reprend son 
activité et montre plus de dynamisme. ${ }^{56}$ À ce propos, le rapport insiste sur le fait que «Pour l'Ère Nouvelle doit rester la revue des peuples de langue latine» et espère pouvoir augmenter sa clientèle surtout en Amérique Latine (Flayol, PEN, 1935, 106, p. 69). Or, ne serait-ce qu'en France, la diffusion n'est pas satisfaisante car, PEN n'est pas consultable dans les bibliothèques d'universités ni dans celles des circonscriptions de l'enseignement primaire. Pour parer à cette lacune, le comité de rédaction envoie une circulaire adressée aux doyens des facultés des lettres pour encourager leurs bibliothèques à s'abonner. Mais comme le montre le tableau suivant ${ }^{57}$, cette circulaire a peu d'effet.

Tableau 6.

Nombre d'abonnés à Pour l'Ère Nouvelle

\begin{tabular}{lcccccc}
\hline & $\mathbf{1 9 3 1}$ & $\mathbf{1 9 3 2}$ & $\mathbf{1 9 3 3}$ & $\mathbf{1 9 3 4}$ & $\mathbf{1 9 3 5}$ & $\mathbf{1 9 3 6}$ \\
\hline France & $/$ & 551 & 571 & 438 & 456 & 409 \\
\hline Etrangers & $/$ & 248 & 233 & 189 & 169 & 153 \\
\hline Total & 715 & 799 & 804 & 677 & 625 & 562 \\
\hline
\end{tabular}

Ces chiffres permettent de constater la diminution progressive des abonnés au fil des années 1930, reflet du déclin que connaît le mouvement dans ces années-là même si les discours des rédacteurs tendent à camoufler cette réalité. Nous pourrions ajouter à ce tableau le chiffre de 900 abonnés évoqué par Ferrière dans sa lettre à Bovet en mai 1928. Un chiffre plausible compte tenu de ceux dont nous disposons ici.

En mars 1936, le rapport d'activité annonce que la composition et les conditions matérielles de la revue sont de jour en jour plus difficiles. Les abonnés français sont en baisse, surtout parmi le personnel enseignant. En revanche, les abonnements étrangers sont plutôt dans une phase de reprise. Le rapport insiste sur le rôle international que doit continuer de jouer Pour l'Ére Nouvelle, en tant qu'organe des pays de langue latine. À ce titre, il précise que l'année 1935 a été l'occasion de publier un rapport

56 Une information nuancée par les chiffres du tableau ci-dessous, ce qui prouve que les rapports de Flayol ne sont pas tout à fait précis et visent avant tout à dynamiser le mouvement alors en train de s'essouffler.

57 Les chiffres indiqués dans ce tableau sont issus du Fonds Paul Langevin (ESPECI, L 58/15) et publiés par Gutierrez en 2011 (p. 34). 
de congrès à Bruxelles ainsi que de soutenir la création de deux groupes rattachés à la Ligue en Grèce et à Cuba. En 1936 et 1937, les difficultés financières perdurent voire s'aggravent pour des raisons similaires aux années précédentes: diminution importante des abonnements - français et étrangers, individuels et institutionnels - augmentation des prix du papier et de l'impression (45\%), etc. Au niveau des contenus, ces deux années sont marquées par le Congrès international de Cheltenham dont la revue fait largement écho en y consacrant pas moins de six numéros spéciaux (trois en 1936, trois en 1937) dans lesquels elle publie les communications sont traduites de l'anglais en français. Si l'année 1937 est déficitaire, le rapport de 1938 relève que de nombreuses revues, privées ou officielles, se sont créées (en France et ailleurs) ce qui expliquerait la baisse générale des abonnés. Si leur inspiration est similaire, PEN garde toutefois ses spécificités:

Sans doute notre revue conserve encore une originalité propre, qui est de ne restreindre ni son intérêt, ni sa documentation à une technique particulière, à une personnalité pédagogique, à une catégorie d'expériences, à une période de l'éducation de la jeunesse; et tout en mettant l'accent - et fortement - sur la pédagogie française, de la situer sans cesse dans l'ensemble du mouvement mondial d'éducation. (Flayol, PEN, 1938, 136, p. 115)

Dans cette situation difficile, le GFEN décide en 1937 de donner au journal une apparence plus moderne en changeant l'aspect esthétique de sa couverture et la disposition des articles. À partir de ce moment-là toute indication concernant les revues affiliées à la Ligue disparaît et la Charte est déplacée à la fin du numéro, signe que le rôle d'organe international de la Ligue est désormais secondaire. Les difficultés financières perdurent et le comité de rédaction lance de nouveaux appels à ses lecteurs. Mais cette fois, le soutien recherché n'est plus seulement financier:

Nous prions instamment nos amis de nous venir en aide en nous donnant des articles, en en recherchant pour nous, en nous signalant les personnes dont la voix se ferait utilement entendre. La Revue est une des manifestations de l'activité du Groupe Français d'Éducation nouvelle qui ont le plus d'étendue et servent le mieux la cause de l'Éducation nouvelle en France et à l'étranger, tous nos membres sont intéressés à la soutenir et à l'améliorer. (Flayol, PEN, 1939, 145, pp. 73-74) 
En 1938, la revue retrouve un nouveau dynamisme grâce au soutien moral de deux personnalités importantes. L'une est le recteur d'Académie, ancien président d'un groupe d'Éducation nouvelle, qui manifeste son soutien en signalant à ses collaborateurs l'intérêt que comporte Pour l'Ėre Nouvelle pour les bibliothèques des établissements du second degré. L'autre personne est Albert Châtelet ${ }^{58}$, alors directeur de l'Enseignement Secondaire qui avait suivi de près les activités du Groupe du Nord des Amis de l'Éducation nouvelle:

Une publication de ce genre est nécessaire, car l'opinion publique et les éducateurs doivent être éclairés sur la nécessité et le sens des transformations qui doivent être introduites dans les méthodes, pour que ces tentatives trouvent déjà des maîtres résolus et capables de les réaliser et d'y entraîner leurs collègues. (Châtelet cité par Flayol, PEN, 1939, 145, p. 73)

L'année 1938 marque aussi un nouveau tournant dans la vie administrative de la revue: le GFEN décide de remettre aux mains d'un technicien, M. Bourrelier, son administration commerciale. C'est lui qui est désormais responsable de la vente de PEN. À partir de ce jour, M. Bourrelier est admis au comité de rédaction en tant que conseiller technique et «se charge des relations avec les imprimeurs, de la comptabilité, de la publicité, des abonnements et rend compte tous les ans de sa gestion au Groupe Français d'Éducation Nouvelle». Toutefois, le groupe «conserve la propriété de la revue et de son titre, reste chargé de la direction intellectuelle et de la rédaction: recherche, choix des articles, composition des numéros, etc.». Par cette division du travail, le groupe espère réaliser «une diffusion plus grande et rendre possible son adaptation plus complète aux besoins de l'heure présente» (Flayol, PEN, 1939, 145, p. 74).

Malgré les événements, l'année 1939 se déroule à peu près normalement avec la publication de huit numéros, dont un est consacré à la présentation des travaux préparés pour le prochain congrès international prévu en France. De fait, ce congrès n'aura pas lieu malgré l'état avancé de son organisation; il est reporté et se tiendra finalement à Ann Arbor (USA) en 1941. Le dernier numéro de 1939 (novembre-décembre) fait état de la situation de guerre générale et consacre ses pages à des articles évoquant les perturbations scolaires engendrées par la guerre aux-

58 Sur Albert Châtelet, voir Condette et Savoye (2011). 
quelles la rédaction propose des solutions et des aménagements. Le numéro s'ouvre sur un texte de la Ligue dans lequel celle-ci annonce son intention de poursuivre son travail, l'importance de son existence et du maintien des relations internationales qu'elle a établies jusque-là. Suit un texte écrit par le bureau du GFEN «Appel aux amis de l'Éducation nouvelle» dans lequel ce dernier signale aux lecteurs que son activité ne sera pas suspendue, mais, au contraire, intensifiée:

En face d'une crise, deux attitudes sont possibles: ou se résigner à ses conséquences soi-disant fatales, ou s'en faire une occasion, un moyen de progrès, en redoublant d'ingéniosité et de hardiesse pour trouver aux difficultés des solutions, dont beaucoup pourront devenir, par delà les routines reconnues impuissantes, des solutions d'avenir. (Bureau GFEN, PEN, 1939, 150, p. 224)

De plus, ce texte rappelle les valeurs défendues par l'Éducation nouvelle pour lesquelles est fait un véritable plaidoyer; il s'agit envers et contre tout, de développer chez l'enfant les vertus de la démocratie et le préparer à son triomphe une fois que la guerre sera finie. Le Bureau annonce qu'il met sa revue Pour l'Ère Nouvelle à la disposition de tous ceux qui s'intéressent aux progrès de l'éducation pour se faire l'écho des grandes nouveautés que les circonstances s'apprêtent à susciter. Enfin, un texte intitulé «Le GFEN et les tâches qu'il envisage» clôt la partie introductive de ce numéro. Le groupe GFEN consacre un chapitre à l'avenir de la revue dans lequel plusieurs demandes sont faites: les abonnés sont priés de faire savoir s'ils ont l'intention de continuer leur abonnement en 1940, de devenir membre du GFEN pour soutenir le groupe et la revue, s'ils désirent - pour ceux qui le peuvent - continuer à être membre et payer leur cotisation pour couvrir les frais de secrétariat. Le groupe demande aux sections départementales de ne pas se dissoudre, de nommer des remplaçants au besoin et de donner tous les renseignements nécessaires pour assurer la bonne diffusion de la revue. Enfin, un appel est fait à tous pour que les lecteurs communiquent au comité de rédaction toutes les expériences et réalisations d'Éducation nouvelle dont ils sont témoins afin d'alimenter et faire vivre la revue.

\section{LA COMPOSITION DES NUMÉROS: DES CHOIX STRATÉGIQUES}

Notre source principale d'investigation étant une revue, il importe de se demander comment celle-ci se «fabrique», autrement dit comment se fait 
le choix des articles, comment se gère la composition des numéros, quel est le rôle des membres du comité de rédaction et, de manière plus générale, quels sont les enjeux qui président à la constitution de cette revue.

Malgré une analyse approfondie de Pour l'Ère Nouvelle, très peu de choses sont dites sur la manière de composer les numéros. Quelques informations figurent dans les éditoriaux mais celles-ci concernent surtout les aspects financiers et statutaires de la Ligue. Par contre, les encarts intitulés «Avis de la rédaction» apportent des éléments. En janvier 1926, un avis annonce que la rédaction compte «limiter de plus en plus la place réservée aux articles théoriques» quoiqu'elle achèvera «la publication de ceux qui sont déjà composés» (Avis, PEN, 1926, 18, p. 21). Une indication importante qui explique la dominance d'articles pratiques ou "praxéologiques» dans $P E N$ à cette période. Si les raisons de cette option ne sont pas explicitées, nous supposons qu'il s'agit de raisons financières car le même avis annonce également la hausse des prix d'abonnements ainsi que la suppression de certains échanges avec d'autres périodiques. Cet avis nous apprend encore que Pour l'Ère Nouvelle ne cite - ou ne publie - un article d'une autre revue qu'à condition que celle-ci, en échange, mentionne les articles de PEN et soutienne l'action de la Ligue: «Les périodiques qui n'ont pu, en 1925, contribuer au progrès de notre action en citant des articles de notre revue ou en la recommandant voudront bien cesser leurs envois» (Avis, PEN, 1926, 18, p. 21). Cette décision est révélatrice du contexte de concurrence dans lequel $P E N$ doit survivre. Concernant les rapports de congrès, la rédaction informe que $P E N$ publie en priorité les conférences des orateurs francophones et que celles des orateurs germanophones et anglophones sont à consulter dans les deux revues sœurs. Sur la composition des numéros, l'éditorial d'octobre 1924 indique que la répartition des articles vise à conserver le caractère "supra-national» de la revue, celle-ci devant jouer le rôle de «lien entre toutes les parties du monde»:

À l'avenir, nous comptons consacrer en moyenne un tiers du total des pages à faire connaître à l'étranger les expériences pédagogiques novatrices de la France; un tiers à parler de la Belgique et de la Suisse romande; et un tiers à faire connaître aux pays de langue française ce qui se fait d'intéressant à l'étranger. (Éditorial, PEN, 1924, 12, p. 61) 
Plusieurs années plus tard, un avis de la rédaction évoque le caractère «avant-gardiste» de la revue et affirme sa volonté de privilégier les articles évoquant des réalisations concrètes d'Éducation nouvelle. Un choix éditorial qui sera de courte durée puisque la revue cesse de paraître à partir d'avril 1940.

Modification à Pour l'Ère Nouvelle: elle doit rester une revue d'avant-garde pédagogique, jamais satisfaite des progrès réalisés toujours animée du désir du mieux. [...] La revue Pour l'Ėre Nouvelle doit maintenant porter le principal de son effort, non plus sur les principes mais sur les réalisations. Aussi nous proposons-nous de réserver désormais plusieurs pages à des descriptions détaillées de techniques et d'organisations nouvelles. (Rédaction, PEN, 1939,147, p. 133)

Pour en savoir plus sur la «fabrication» de la revue, nous avons consulté la correspondance de Ferrière, en particulier celle que celui-ci échange avec Elisabeth Rotten, rédactrice de la revue allemande Das Werdende Zeitalter. Une lettre datée du 19 mars 1924 de Ferrière adressée à Rotten nous apporte des éléments de compréhension sur ces questions:

Chère amie,

Voici l'article que je vous propose. Il est destiné à Ère 10 (j'appelle, entre nous, ma revue: «Ėre», tout court). Vous en tirerez ce que vous voudrez; si vous ne le modifiez que peu, inutile de m'en soumettre le texte allemand. Si vous y faites des adjonctions ou modifications profondes, dans mon esprit, destinés à toucher telle ou telle corde plus particulièrement sensible de l'âme allemande, je serai heureux de revoir le texte pour y modifier, s'il y a lieu, telle ou telle nuance. Je crois en effet: 1) qu'il faut profiter de cet échange de vues entres nos trois revues pour dire à nos («nos»!!, un bien grand mot) peuples respectifs certaines choses qui doivent être dites; et 2) que chacun de nous trois est mal placé pour tout à fait bien comprendre ce qui doit être dit et comment cela doit être dit. Je ne veux pas dire par là que ce soit à vous de faire en entier les articles de Beatrice Ensor et les miens, que nous n'aurions qu'à signer ensuite si nous sommes d'accord. Mais il est peut être bon que vous repreniez, modifiiez et adaptiez nos articles. Vous avez d'ailleurs pressenti ce rôle de collaboration en me suggérant ce que je pourrais dire et ce qu'il convient de ne pas dire. ${ }^{59}$

59 Les passages soulignés le sont par l'auteur. Lettre d'Adolphe Ferrière à Elisabeth Rotten, 19 mars 1924. AIJJR, Fonds Ferrière, Correspondance alphabétique. Cote: AdF.C.I./ 63. 
Cet extrait de lettre fait apparaître les relations étroites qu'entretiennent les rédacteurs des trois revues, relations qui passent notamment par l'échange et la traduction d'articles destinés à être publiés dans leurs revues respectives. Manifestement, chaque rédacteur maîtrise suffisamment les trois langues pour assurer lui-même les traductions. Les propos de Ferrière dévoilent aussi le pouvoir de censure ou d'adaptation que détiennent ces rédacteurs; en effet, ceux-ci se donnent le droit d'adapter les textes qu'ils traduisent afin qu'ils soient conformes à "ce qu'il est convenable de dire» selon les traditions sociales, culturelles et politiques du pays. Un pouvoir qui sous-entend que chaque rédacteur se donne le droit, aussi, de taire ce qui est moins «convenable». La nécessité d'adapter certains articles révèle le poids des différences nationales et la sensibilité de certains pays - ici, l'Allemagne - aux discours et aux images que peuvent véhiculer certains écrits. Sur ces différences, Ferrière va plus loin dans la suite de sa lettre en mentionnant le décalage qui existe entre la France et l'Allemagne concernant les expériences novatrices.

J'ai cru comprendre que l'éducation de l'individu (à laquelle on ne commence pas encore à viser en France, ou à peine dans 10 ou 15 écoles enfantines et primaires) est chose largement dépassée en Allemagne. Vous auriez voulu que je parle de la Gemeinschaftschule en pays latins. Mais, à part les très petits essais de Roger Cousinet, cela n'existe pas, simplement! C'est pourquoi je me contente de vous envoyer ceci qui montre qu'il y a un réveil. Mais ni la psychologie expérimentale, ni (moins encore) l'intuition orientée selon les lois de la biologie, n'ont droit de cité, en pays latins, sauf chez de rares originaux. Donc prenez mon article comme base, comme canevas et brodez là-dessus ce qui doit être dit par une âme latine, mais supranationale comme la mienne, au monde allemand. Si vous le voulez bien, je tenterai de procéder de même avec votre article pour l'Ère, que je vous prie de m'envoyer pour fin mai.

Dans cette partie, Ferrière répond manifestement à une demande adressée par Rotten pour un article sur l'Éducation nouvelle en France. Elle nous permet de constater les différences manifestes qui existent entre les deux pays, non seulement au niveau idéologique - révélant la puissance des traditions culturelles des deux pays - mais surtout au niveau pratique où manifestement, l'avancée du mouvement est très lente en France. Une «lenteur» remarquée aussi par Gutierrez (2008) qui constate une sorte de retrait de la section française du paysage de la Ligue dès 1926 et «l'absence quasi-totale (en 1926-1927) d'articles de fond sur les 
expériences pédagogiques menées en France» (p. 82). Ce deuxième extrait de lettre nous permet en outre de comprendre comment se «fabriquent» certains articles de la revue. Ainsi, dans certains cas, le rédacteur se donne le droit de rédiger un article sur la base d'indications fournies par son collaborateur (ici Ferrière sur la France) et à partir desquelles ce dernier est libre de «broder», de «dire ce qui doit être dit» au peuple à qui il s'adresse. Une manière de faire étonnante qui sous-entend une grande proximité de vues entre les protagonistes ainsi qu'une confiance mutuelle. Si Ferrière donne ici «carte blanche» à Rotten pour rédiger l'article qu'elle juge adéquat pour l'Allemagne, le Genevois semble tenir toutefois à ce que son «âme latine mais supranationale» soit respectée et mise en valeur.

Grâce à cet extrait, nous pouvons donc affirmer, par extrapolation, que certains articles de PEN sont le résultat de commandes, adressées par le rédacteur de la revue à un auteur en particulier. Or, dans la suite de sa lettre, Ferrière donne de précieux indices sur la manière dont se déroulent ces commandes. Dans l'extrait ci-dessous, il fait une commande à Elisabeth Rotten - «l'article pour l'Ère que je vous prie de m’envoyer pour fin mai» - en précisant dans le détail ce qu'il souhaite y trouver.

Ce qui me paraît devoir être dit, par vous à nous, c'est à peu près ceci:

1) dans chacun de nos pays il y a, au point de vue pédagogique, une droite réactionnaire, antinovatrice et nationaliste. Le meilleur moyen de lutter contre elle n'est pas de fusionner par opportunisme les «gauches» novatrices de diverses sauces, mais a) de s'appuyer sur la science et l'expérience qui est vraie partout et toujours, bien que nous la découvrions lentement et laborieusement; b) de s'associer à ceux qui, en tous pays, prennent cette base pour point d'appui.

2) Passant du général au particulier, on peut dire que l'Allemagne ne sera sauvée du péril immense des droites que si - non pas les «gauches» prises en bloc, mais l'élite des esprits novateurs se sent soutenue, appuyée, encouragée par ceux qui sentent et pensent de même dans le monde entier. Un Völkerbund der gleichgesinnten Menschen. ${ }^{60}$

Cette manière de commander un article, que l'on pourrait qualifier de «téléguidée» tant les détails du contenu souhaité sont précis, donne une

60 Lettre d'Adolphe Ferrière à Elisabeth Rotten, 19 mars 1924. AIJJR, Fonds Ferrière, Correspondance alphabétique. Cote: AdF.C.I./ 63. 
indication importante sur le contexte qui règne en France et dans les pays latins à ce moment-là; un contexte peu favorable dans lequel l'ampleur des mouvements «anti-novateurs» qui œuvrent contre l'Éducation nouvelle, ou du moins qui résistent à en appliquer les principes, semble être importante. Ferrière dévoile ici ses tendances politiques à travers une prise de position particulièrement claire en faveur de «l'élite des esprits novateurs» dont il fait partie (appartenance qui lui sera d'ailleurs reprochée souvent) de même que de nombreux membres de la Ligue. Enfin, cette manière de procéder pour commander un article révèle une véritable «stratégie de propagande» sur laquelle les trois rédacteurs - du moins Ferrière et Rotten - semblent être d'accord. Une stratégie qui frise la manipulation dans la mesure où, comme le montre l'extrait suivant, Ferrière «utilise» le discours d'Elisabeth Rotten, pour montrer aux lecteurs de PEN que le mouvement novateur est plus fort que celui des résistants:

Dites aussi, dans l'Ère, à la France et à la Belgique qu'ils sont plus nom-

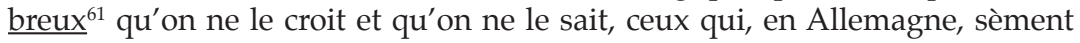
dans l'âme de la jeunesse l'esprit de libre critique et l'esprit d'amour interhumain et qui sauront un jour tenir tête au nationalisme étroit. (p. 5)

\section{L'INFLUENCE DES MEMBRES DE LA RÉDACTION}

L'analyse des éditoriaux et de l'infrastructure éditoriale nous permet de faire des hypothèses sur le rôle joué par les divers membres du comité de rédaction de $P E N$ tout au long de la période étudiée. Comme nous l'avons montré dans le volet précédent, Ferrière est le seul rédacteur de PEN de 1922 à 1924, raison pour laquelle il considère que Pour l'Ère Nouvelle est «sa» revue. Pendant ces années-là, c'est lui qui sélectionne les articles à publier, qui rédige les chroniques de congrès de même que plusieurs rubriques telles que «Notre Ligue», "Nouvelles diverses», «Livres et revues» même si celles-ci ne sont pas explicitement signées de son nom. ${ }^{62}$ Grâce à sa maîtrise des langues c'est aussi lui qui fait les traductions d'articles issus des deux revues sœurs. Mais dès 1925, il est rejoint par deux éminents collègues, Paul Fauconnet pour la France et Ovide Decroly pour la Belgique.

61 Souligné par l'auteur dans la lettre d'origine.

62 Contrairement à la revue allemande, par exemple, dans laquelle Karl Wilker signe de son nom ou de ses initiales (K.W.) toutes les rubriques qu'il rédige. 
Pourtant, l'arrivée de ces deux personnes n'a pas d'influence particulière la ligne éditoriale de la revue car ces deux personnes semblent absentes de la vie de PEN. À aucun moment, l'un ou l'autre ne signe un éditorial pour signifier sa présence ni n'intervient sur la composition des numéros. Même si les éditoriaux restent anonymes, il y a fort à penser que ce soit Ferrière qui les rédige lui-même avec ou sans l'aval de ses deux collègues. En effet, dans le seul éditorial signé explicitement de son nom - dans lequel il remercie ses amis pour la célébration de son anniversaire Ferrière s'adresse à ses lecteurs en disant: «Pour une fois, c'est le Rédacteur en chef, en son nom personnel et non en celui de la Rédaction de la revue qui prend la plume» (Éditorial, PEN, 1929, 51, p. 211). C'est à se demander si ces trois personnes fonctionnent réellement comme un comité de rédaction. Leur correspondance est peu abondante et, vivant dans trois pays différents, on peine à imaginer que leurs rencontres soient fréquentes. Des deux nouveaux membres du comité de rédaction, seul Decroly paraît impliqué dans la vie de la revue, et cela même avant son arrivée officielle au comité. Ce dernier publie cinq articles dans PEN dans ces années-là (1925 à 1928), et dix au total depuis 1922. Parmi les articles qu'il écrit certains sont documentaires présentant des écoles nouvelles qu'il visite à l'étranger, d'autres sont scientifiques apportant des connaissances sur la psychologie de l'enfant en particulier sur les troubles mentaux. Si le rôle de Decroly dans PEN est assez discret, sa présence dans les manifestations de la Ligue est évidente. Il participe aux congrès que celle-ci organise dès $1921^{63}$, congrès dans lesquels il donne des conférences plénières et participe à des groupes de travail, notamment en psychologie. Comme le montre Wagnon (2013), c'est dans cette discipline naissante que Decroly est le plus actif et impliqué. Il fait partie d'un réseau international de psychologues intéressés par l'enfance qui dialoguent et diffusent leurs recherches à travers une abondante correspondance, des publications et des congrès constituant ainsi une sorte $\mathrm{d}^{\prime}$ «internationale de la psychologie». Son importance dans le domaine est perceptible dans la revue à travers les multiples références dont il fait l'objet dans les articles d'autres auteurs, attestant que Decroly constitue une figure centrale pour les membres de la Ligue. Peu après son décès, c'est Amélie Hamaïde qui prend le relais au comité de rédaction et devient la représentante de la Belgique au sein de la Ligue. Sa vie durant, Hamaïde sera une fervente

63 À l'exception de celui de Nice en 1932 auquel il ne peut assister pour des raisons de santé. Il décède peu de temps après ce congrès, en septembre 1932. 
propagandiste de la pédagogie Decroly que ce soit en tant que directrice de l'École de l'Ermitage (1924-1934), fondatrice de sa propre école (dès 1934) ou rédactrice d'ouvrages et articles sur la méthode des centres d'intérêts. Ayant secondé le médecin belge pendant de nombreuses années, «elle pérennise sa pédagogie sans chercher à l'adapter mais au contraire en cultivant ce rôle de gardienne du temple» (Wagnon, 2015, p. 132).

Le rôle de Paul Fauconnet dans le comité de rédaction est plus mystérieux; ce dernier paraît totalement absent de la revue dans laquelle il ne signe aucun article durant la période étudiée. Il est également absent des grands rendez-vous de la Ligue (les congrès) auxquels il ne participe sous aucune forme. Grâce aux travaux de Gutierrez (2008; 2010) et Guey (2012), nous savons que Fauconnet, disciple de Durkheim, est tout d'abord maître de conférences, chargé du cours intitulé «Science de l'éducation et sociologie» à l'Université de Paris de 1921 à 1928. Or, la page de couverture de $P E N$ où il apparaît pour la première fois en octobre $1924^{64}$, le présente comme un «Professeur de Psychologie et de Pédagogie à la Sorbonne»; d'après Gutierrez (2008), cette dénomination pourrait s'expliquer par le fait que Fauconnet rejoint cette année-là la section de Pédagogie de l'Institut de Psychologie fondé à Paris en 1921. En 1926, il obtient le titre de «Professeur sans chaire» à la Faculté des Lettres et se voit attribuer une charge de cours en sociologie. Il faut attendre 1932 pour qu'il soit nommé «Professeur de sociologie» à la Sorbonne tandis que la chaire de Science de l'éducation change de nom et s'intitule désormais chaire de «Psychologie et Pédagogie». Parallèlement, Fauconnet est nommé président du GFEN en 1929, puis vice-président dès 1931, fonction qu'il occupera jusqu'en 1937; dès lors, il paraît étonnant que ce dernier ne concède aucun article à Pour l'Ère Nouvelle dont il est pourtant l'un des membres du comité de rédaction. Certes, il semble avoir une santé fragile (Guey, 2012) qui le limite dans ses activités, mais il est surtout très accaparé par ses tâches à Paris.

L'analyse de la correspondance de Ferrière dans ces années-là nous permet de mieux comprendre les raisons de ce silence. Dans une lettre adressée à Elisabeth Rotten, Ferrière décrit brièvement les personnes que celle-ci va rencontrer à Paris lors de ses prochaines conférences, parmi lesquelles Paul Fauconnet:

64 Numéro 13, «Hors série», dédié à la Semaine de Villebon, Rencontre francophone des membres de la Ligue internationale pour l’Éducation nouvelle. 
M. Fauconnet est professeur de psychologie et de pédagogie à la Sorbonne. C'est un disciple de Durkheim, chef comme vous le savez d'une école sociologique qui attribue à la société dans son ensemble une influence prépondérante et à mon sens exagérée sur l'éducation de l'individu. [...] M. Fauconnet est toujours surchargé de travail; il n'a accepté de figurer sur la couverture de ma revue que si on ne lui demandait aucun article. En fait, je ne l'ai pas vu depuis plus d'une année et nous avons à peine échangé deux ou trois lettres depuis lors. Il a perdu une fille; il a été malade mais à part cela, ses sentiments à notre égard sont très chaleureux. ${ }^{65}$

Cet extrait de lettre nous permet de mieux comprendre pourquoi Pour l'Ėre Nouvelle ne contient aucun article de Fauconnet. Il s'agit donc d'une demande de sa part et d'un accord entre lui et Ferrière sur le fait que son nom figure sur la page de titre de PEN mais qu'aucune contrepartie ne soit exigée en échange. Fauconnet apparaît dès lors comme une personne «alibi» choisie pour représenter la France et dont la présence au comité de rédaction vise avant tout à garantir le caractère international de la revue. Toutefois, on peut se demander pourquoi Ferrière choisit Fauconnet pour jouer ce rôle-là. Nous faisons l'hypothèse que le poste qu'occupe Fauconnet à l'Université de Paris au moment de la fondation de la Ligue constitue la principale raison de ce choix; sa position de chargé de cours en «Science de l'éducation», interprété par Ferrière comme celle de «Professeur de Psychologie et de Pédagogie à la Sorbonne ${ }^{66^{\prime}}$, constitue un atout majeur pour cautionner le mouvement d'Éducation nouvelle et la revue. D'autre part, on peut penser que Ferrière a quelque sympathie pour Fauconnet puisque tous deux ont étudié la sociologie et soutenu une thèse dans ce domaine, Ferrière en 1915 à Genève - La Loi du progrès en biologie et en sociologie - et le Français en 1920 à la Sorbonne - La Responsabilité: une étude sociologique.

Ainsi, malgré la présence de Decroly et Fauconnet au comité de rédaction, Ferrière a une réelle mainmise sur la revue tout au long des années 1920 (voire au-delà). Comme le montre Hameline (2002b), le ton et le vocabulaire utilisés dans les éditoriaux de PEN trahissent la présence de Ferrière dont la philosophie spiritualiste est facilement identifiable à travers certaines phrases:

65 Lettre d'Adolphe Ferrière à Elisabeth Rotten, 16 décembre 1925. AIJJR, Fonds Ferrière, Correspondance alphabétique. Cote: AdF.C.I/ 63.

66 C'est ainsi qu'il est présenté sur la page de couverture de Pour l'Ère Nouvelle. 
Le plaidoyer pour la «suprématie de l'esprit», «l'accroissement de l'énergie spirituelle» de l'enfant, pour la «libération des puissances spirituelles qui sont en lui», c'est du Ferrière. Car, sa philosophie pédagogique est un énergétisme basé sur la conviction que chaque individu est animé du «dedans vers le dehors» d'une «force formatrice que l'éducation doit à la fois libérer et discipliner, qu'elle doit transformer en capacité d'autonomie et de coopération. (Hameline, 2002b, p. 33)

Or ces éditoriaux se font de plus en plus rares à partir de 1930: seuls deux sont publiés cette année-là (janvier et décembre), de même qu'en 1931 qui publient les deux derniers de la période étudiée, tous deux étant manifestement rédigés par Ferrière. La disparition de ces éditoriaux coïncide avec le passage progressif de la revue au GFEN, que Ferrière annonce officiellement qu'en $1931^{67}$ :

Le rédacteur en chef de cette revue, obligé de restreindre ses activités afin de se concentrer sur la rédaction d'ouvrages qu'il a en chantier depuis plusieurs années, se voit contraint d'accepter l'offre dévouée du Groupe Français d'Éducation Nouvelle de reprendre à Paris le travail de rédaction. (Éditorial, PEN, 1931, 64, p. 2)

À partir de cette date-là (février 1931), la revue ne publie plus d'éditorial, un signe qui marque ainsi la fin d'une époque - dix ans exactement (1922-1931) - dominée par la présence de Ferrière, non seulement sur le plan administratif mais aussi sur le plan «idéologique». Selon Hameline (2002b), cette "prise de possession» de la revue ne revêt pas seulement un caractère «d'opportunité pragmatique et politique» mais elle est aussi d'ordre «doctrinal». Contrôler la rédaction d'une revue permet de promouvoir les personnes et les courants de pensée que l'on soutient, mais aussi de diffuser ses propres idées, ses propres convictions. Selon l'auteur, bien qu'elle ne soit jamais énoncée comme telle, sa doctrine «anthropologique et pédagogique», imprégnée de la pensée théosophiste, influence considérablement l'idéologie de la Ligue ainsi que le discours tenu dans la revue. D'après l'auteur, la présence doctrinale de Ferrière dans la constitution de la Ligue est déterminante; les principes de ralliement rédigés par lui puis adoptés au Congrès de Calais en sont tout imprégnés.

67 Voir le volet «infrastructure éditoriale» dans ce même chapitre. 
Ainsi, derrière l'anonymat qui caractérise les rubriques de $P E N$, la présence de Ferrière est évidente. Sa présence à tous les niveaux de la Ligue et sa mainmise sur la revue contribuent d'ailleurs à donner une impression d'homogénéité des discours tenus dans PEN tout au long des années 1920. Cette homogénéité se rapproche même de «l'indiscernabilité» au point de créer la confusion comme le relèvent Hofstetter, Schneuwly, Lussi et Haenggeli-Jenni (2006) à propos du statut, du travail, des écrits et du rattachement de Ferrière. En effet, durant ces années-là, Ferrière joue un rôle majeur dans plusieurs institutions à la fois, assumant le travail de documentation internationale simultanément de l'Institut Rousseau, du BIEN, du BIE et de Pour l'Ère Nouvelle. Dès lors, ses écrits se ressemblent à s'y méprendre et «semblent d'ailleurs s'interchanger sans problème» (Hofstetter, Schneuwly, Lussi \& Haenggeli-Jenni, 2006, p. 130). ${ }^{68}$ Dans Pour l'Ėre Nouvelle, Ferrière met à profit sa position de rédacteur en chef pour faire un peu de "publicité pour ses propres œuvres». D'une part, il tente de faire connaître son Bureau international des Écoles nouvelles en diffusant les informations qu'il récolte dans ce cadre - il publie par exemple la liste des écoles nouvelles établie sur la base des «Trente points» qu'il établit lui-même pour «labelliser» ces écoles ${ }^{69}$. D'autre part, il imprime sur les pages de couverture la liste de ses publications qu'il met à jour au fil des ans. Dès 1932, la revue change donc de cap et de ligne éditoriale, reflétant désormais les positions du GFEN et celle de scientifiques (Wallon, Piéron, Piaget) fermement décidés à utiliser Pour l'Ėre Nouvelle comme tribune de leurs idées.

\section{LES ANNÉES «APRÈS FERRIÈRE»: UN COMITÉ IMPRÉGNÉ DU GFEN}

Dès 1932, le ton de la revue change, portant désormais la marque de son nouveau comité éditorial. De fait, Ovide Decroly - Amélie Hamaïde dès 1933 - et Jean Piaget jouent un rôle mineur dans cette deuxième phase de vie de Pour l'Ére Nouvelle: leur fonction se borne à représenter leur

68 Comme le mentionnent ces auteurs, le «bulletin de l'Institut Rousseau» publié dans $P E N$ de janvier à mars 1926 change simplement de nom à partir du mois de juillet pour s'appeler «Chronique du Bureau International d'Éducation».

69 Ce texte établit en «Trente Points»les critères que doit remplir un établissement pour obtenir le «label» d'école nouvelle (Ferrière, 1919). 
pays au sein du comité. À l'inverse, Emilie Flayol, secrétaire dès 1932, joue un rôle important au sein du comité; nous aurons l'occasion de la présenter plus longuement dans un autre volet de ce chapitre. Les véritables figures phares de cette deuxième période sont Henri Wallon et Henri Piéron, tous deux psychologues reconnus en France comme à l'étranger. Ces derniers vont imprégner profondément la revue de leurs idées et de leurs recherches qui tentent, de manière différente, de mettre en pratique les principes de l'Éducation nouvelle.

Henri Wallon ${ }^{70}$ est probablement celui qui est le plus soucieux de cette articulation «théorie-pratique» en raison de son parcours professionnel. Formé initialement à l'école normale supérieure, Wallon est reçu à l'agrégation de philosophie en 1902 ce qui lui ouvre une carrière d'enseignant du second degré. Mais après deux ans d'enseignement au lycée, celui-ci entame des études de médecine puis s'oriente vers la psychologie. Engagé dans différents services hospitaliers, il fait beaucoup de psychiatrie et s'intéresse plus particulièrement aux anomalies motrices et mentales de l'enfant. ${ }^{71}$ Dès 1919, Wallon porte un intérêt marqué pour la psychologie de l'enfant raison pour laquelle il est sollicité pour donner des conférences sur le sujet à la Sorbonne. En 1922, il fonde un petit laboratoire de psychobiologie dans une école près de Paris (Boulogne-Billancourt) pour observer l'enfant dans un de ses milieux de vie. Ce laboratoire devient bientôt un lieu de recherche et d'enseignement pour les étudiants et les inspecteurs s'intéressant au développement de l'enfant. Au cours des années 1930, plusieurs collaborateurs tels qu'Hélène Gratiot Alphandéry ou Maurice Prudhommeau viennent l'y rejoindre, tous deux écrivant notamment dans PEN. Tout au long de sa carrière, Wallon a le souci constant de concilier ses recherches sur l'enfant avec une application concrète à l'éducation. Même en tant que directeur du «Laboratoire de psychobiologie de l'enfant» à Paris où il est nommé en 1925, il continue de mener de front à la fois recherche et enseignement. Son réseau de relations se compose principalement de scientifiques engagés pour la cause de l'éducation tels que Langevin, Piéron, Laugier, Lahy, Claparède, Piaget, Decroly, Ferrière, Freinet ou Faucher. De fait, ce qui caractérise avant tout la personnalité de Wallon,

70 Les indications biographiques mentionnées ci-après proviennent des travaux de Huber (2013) et Gratiot-Alphandéry (1994/2000).

71 Il écrit une thèse intitulée «L'enfant turbulent: étude sur les retards et anomalies du développement moteur et mental» en 1925. 
$c^{\prime}$ est son militantisme ${ }^{72}$ que ce soit au sein d'associations telles que la «Société française de pédagogie», le "Comité de vigilance des intellectuels antifascistes», la "Commission sur les rapports de la Science et de la Société» ou le «Cercle de la Russie Neuve» ou au sein du Parti communiste et de groupements marxistes. Ainsi, lorsqu'il arrive au comité du GFEN ${ }^{73}$ en novembre 1928, c'est avant tout son caractère militant qu'il va mettre au service du groupe. Wallon cherche à rallier un maximum de gens autour de sa cause, raison pour laquelle il participe activement à l'organisation du Congrès de Nice en 1932 qui réunira de nombreux militants des divers groupes d'Éducation nouvelle en France. De fait, il participe aussi à la rédaction de la nouvelle «charte $»^{74}$ de la Ligue présentée au congrès - dans laquelle il supprime toute référence à la spiritualité dans les buts assignés à l'éducation - et qui témoigne de cette volonté de poser «l'acte éducatif comme un acte politique» (Gutierrez \& Ohayon, 2010, p. 77).

Dans Pour l'Ėre Nouvelle, Wallon écrit plusieurs articles «scientifiques» qui font écho à ses travaux en psychologie de l'enfant. ${ }^{75}$ Mais il écrit aussi des articles à caractère plus militant ${ }^{76}$ dans lesquels il défend une position très ferme sur la nécessité de prendre en compte le milieu social et environnemental pour étudier et éduquer l'enfant. C'est une des raisons qui explique son important soutien à Freinet, notamment au Congrès de Cheltenham en 1936, dont il défend la conception d'une école proche de

72 Un engagement qui fut à l'origine d'une certaine réserve de la part de son milieu scientifique et lui coûta certaines amitiés. (Gratiot-Alphandéry, 1994/2000).

73 Il est nommé vice-président du GFEN tout comme Henri Piéron, alors que Paul Langevin prend la tête du groupe à ce moment-là.

74 Cette nouvelle charte remplace les «principes de ralliement» qui depuis la fondation de la Ligue en 1921, fédéraient ses membres.

75 «Les composantes neurologiques du caractère» (PEN, 1929, 52), «Les origines du caractère chez l'enfant» (PEN, 1934, 98), «La méthode des tests et l'étude du caractère» (PEN, 1937, 124), «Le conflit des automatismes instinctifs et de l'émotion chez l'enfant de 0 à 3 ans» (PEN, 1934, 98).

76 «Culture générale et orientation professionnelle» (PEN, 1932, 81), «Pédagogie concrète et psychologie de l'enfant» (PEN, 1933, 91), «L'expression des émotions et leurs fins sociales» (PEN, 1934, 99), «L'imprimerie à l'école» (PEN, 1936, 121), «Les rapports de la science avec la formation des personnalités libres» (PEN, 1936, 123). 
la vie, en contact étroit avec la communauté dans laquelle elle s'insère. En tant que membre de la rédaction de PEN et président du GFEN de 1933 à 1935, Wallon va donner une couleur très «française» et militante à la revue, ce qui fera fuir certains «anciens» de la Ligue - Ferrière notamment - dont les conceptions spiritualistes de l'éducation n'ont plus leur place. Les auteurs qui écrivent dans PEN à partir de 1932 sont essentiellement des Français, engagés dans divers projets de mise en application des méthodes nouvelles, tous désireux de passer «à l'action». À la fin des années 1930, plusieurs projets de réforme voient le jour en France «classes d'orientation», «loisirs/activités dirigés» - qui contribuent à leur manière à la constitution progressive d'un autre projet de plus grande envergure, le plan Langevin-Wallon, déposé en 1944 au ministère de l'éducation nationale, au sein duquel Wallon jouera un rôle central.

Tout comme Wallon, Henri Piéron (1881-1964) fait d'abord des études en lettres et obtient son agrégation de philosophie à la Sorbonne. ${ }^{77}$ Mais très rapidement, Piéron s'emploie à lutter contre l'idée qui prévaut à l'époque selon laquelle «la psychologie est subordonnée à la philosophie» (Fessard, 1949, p. 7). Il prend contact avec les fondateurs de la psychologie scientifique française que sont Ribot, Janet ${ }^{78}$ ou Lévy-Brühl, mais aussi avec les représentants des sciences naturelles tels que Giard (biologie animale) et Dastre (physiologie). C'est en sciences naturelles qu'il présente sa thèse sur un sujet physiologique «Le mécanisme du Sommeil», un domaine qui influencera considérablement sa conception de la psychologie. Il se forme au contact des laboratoires ${ }^{79}$, mais aussi au contact de la nature (mer) et surtout du milieu hospitalier ${ }^{80}$ où il découvre la psychiatrie dans le domaine de laquelle il écrit une deuxième thèse en 1912. Cette année-là, il reprend le Laboratoire de Psychologie physiologique de Binet en raison de la mort prématurée de ce dernier. Il s'ensuit une carrière brillante marquée par sa nomination au Collège de France en 1923 (chaire de «physiologie des sensations» créée pour lui), par la fondation de l'Institut National d'Orientation

77 L'essentiel des indications biographiques sur Henri Piéron sont tirées de l'article de Fessard (1949).

78 Janet prend Piéron comme secrétaire à la clinique neurologique de la Salpêtrière.

79 Il fréquente notamment le Laboratoire de Psychologie physiologique dirigé alors par Alfred Binet.

80 Il collabore avec le psychiatre Édouard Toulouse à l'asile de Villejuif. 
Professionnelle ${ }^{81}$ en 1928, et par l'introduction en 1944 de la psychophysiologie à la Faculté des Sciences dont il rédige le programme d'un nouveau certificat de licence (Fessard, 1949).

Dès lors, l'essentiel de ses recherches et de ses écrits concernent la psychophysiologie sensorielle. Pourtant, les articles qu'il publie dans PEN concernent plutôt les liens entre psychologie et pédagogie, les recherches psychotechniques à l'école, la méthode des tests, voire même les examens et la «docimologie». ${ }^{82}$ De fait, quand Piéron arrive au GFEN à la fin $1928^{83}$, il vient de fonder son Institut d'Orientation Professionnelle. Dès lors, la décennie qui va suivre sera essentiellement consacrée à des travaux dans le cadre de cet institut où il bénéficie de l'aide de sa femme qui contribue activement à la gestion comme aux recherches de l'institution. C'est d'ailleurs elle qui signe l'article sur «Les recherches psychotechniques à l'école et la collaboration des éducateurs» publié dans PEN en 1931 (PEN, 73) et en co-signe un autre sur la «valeur sélective du certificat d'études en comparaison avec une épreuve par tests» (PEN, 1935, 105). Ainsi, la revue $P E N$ va permettre à Piéron de publier quelques-uns de ses résultats de recherches en particulier ceux concernant l'application dans le domaine éducatif de la psychotechnique ainsi que ceux sur l'orientation professionnelle. Si ce domaine prend de l'ampleur en France au cours des années 1930, c'est en grande partie grâce à lui qui s'associe à d'autres chercheurs tels qu'Henri Laugier pour mener ses recherches. Toutefois, l'éclectisme des sujets qu'il aborde dans ses articles porte à croire que Pour l'Ère Nouvelle ne constitue pas son premier lieu de publication, ni une véritable tribune pour ses idées, écrivant davantage dans des revues scientifiques de psychologie ou de physiologie. Dès lors, le rôle de Piéron est assez discret au sein de PEN tout comme il semble l'être au GFEN, deux lieux où il fait figure de «second» aux côtés de Wallon et Langevin qui sont, eux, davantage médiatiques et «politiques», $\mathrm{du}$ fait de leur militantisme plus engagé.

81 INOP qui deviendra par la suite l'INETOP.

82 «La notion d'aptitude» (PEN, 1929, 49); «Les recherches psychotechniques à l'école et la collaboration des éducateurs» (PEN, 1931, 73); «Le problème des examens. Éducation nouvelle et décimologie» (PEN, 1931, 73); «Comment créer une culture adaptée à l'époque moderne» (PEN, 1932, 82); «Sur les Examens» (PEN, 1934, 101); «Étude critique sur la valeur sélective du certificat d'études et comparaison de cet examen avec une épreuve par tests» (PEN, 1935, 105); «Les méthodes de mesure» (PEN, 1938, 142).

83 Il est nommé vice-président aux côtés de Wallon. 


\section{ÉVOLUTION DES THÉMATIQUeS}

Dans ce volet, nous proposons une analyse des domaines abordés dans les articles de Pour l'Êre Nouvelle et l'évolution de ces thématiques durant les deux décennies étudiées. Les résultats sont issus d'une base de données dans laquelle chaque article a été catégorisé selon le genre et le domaine abordé. Nous avons également pris en compte les titres d'articles qui dans certains cas permettent de faciliter cette catégorisation. Le tableau récapitulatif suivant fait apparaître les tendances générales et permet de mieux comprendre à quel type de revue appartient PEN et quelles sont les questions au cœur des débats.

Tableau 7.

\section{Domaines abordés par les articles de Pour l'Ère Nouvelle ${ }^{84}$}

\begin{tabular}{|c|c|c|c|}
\hline Domaine & Sous-domaine & $\begin{array}{l}\text { Nombre } \\
\text { d'articles }\end{array}$ & Total \\
\hline Pédagogie & $\begin{array}{l}\text { Documentation pédagogique } \\
\text { Pédagogie générale } \\
\text { Méthode d'éducation/d'enseignement } \\
\text { Éducation nouvelle (propagande) } \\
\text { Formation des maîtres } \\
\text { Programmes / plans d'étude } \\
\text { Examens / évaluation } \\
\text { Pédagogie expérimentale } \\
\text { Éducation familiale/relations famille - école } \\
\text { Théorie générale des Sciences de l'éducation }\end{array}$ & $\begin{array}{c}281 \\
96 \\
81 \\
34 \\
20 \\
18 \\
16 \\
14 \\
10 \\
3\end{array}$ & 573 \\
\hline Psychopédagogie & $\begin{array}{l}\text { Orientation scolaire et professionnelle } \\
\text { Psychologie appliquée à l'éducation } \\
\text { Tests psychologiques dans pratique éducative }\end{array}$ & $\begin{array}{c}17 \\
15 \\
6\end{array}$ & 38 \\
\hline $\begin{array}{l}\text { Ce tableau } \\
\text { dans laquel } \\
\text { gorisés. Les } \\
\text { en Histoire } \\
\text { sur l'histoir } \\
\text { 1998; 2002, }\end{array}$ & $\begin{array}{l}\text { a été réalisé à l'aide d'une base de donn } \\
\text { e tous les articles figurant aux sommaires } \\
\text { catégories s'inspirent de celles qu'ERHISE } \\
\text { Sociale de l'Éducation) a élaborées dans le } \\
\text { des sciences de l'éducation en Suisse (Ho } \\
007 \text { ). }\end{array}$ & $\begin{array}{l}\text { ées (File } 1 \\
\text { de PEN o } \\
\text { Équipe de } \\
\text { cadre de } \\
\text { stetter \& }\end{array}$ & $\begin{array}{l}\text { er Pro) } \\
\text { té caté- } \\
\text { cherche } \\
\text { travaux } \\
\text { neuwly, }\end{array}$ \\
\hline $\begin{array}{l}85 \text { Dans cette } \\
\text { Ligue (obje } \\
\text { que des ma }\end{array}$ & $\begin{array}{l}\text { atégorie ont été classés les textes qui parle } \\
\text { ctifs, ambitions, convictions) ou de l'Édu } \\
\text { hifestes ou des textes de propagande. }\end{array}$ & $\begin{array}{l}\text { at explicit } \\
\text { ation no }\end{array}$ & $\begin{array}{l}\text { nt de la } \\
\text { le ainsi }\end{array}$ \\
\hline
\end{tabular}




\begin{tabular}{llll}
\hline \multirow{2}{*}{ Psychologie } & Psychologie de l'enfant/développement & 45 \\
& Théorie psychologie générale & 25 \\
& Tests psychologiques, mesures des aptitudes & 10 & $\mathbf{8 0}$ \\
\hline \multirow{2}{*}{ Philosophie } & Philosophie, religion & 54 & \\
& Éducation morale & 12 & $\mathbf{6 6}$ \\
\hline Sciences naturelles & Biologie, médecine, psychiatrice (psychanalyse) & 14 & \\
& & & $\mathbf{1 4}$ \\
\hline Autres & Biographies & 12 & \\
& Commémoration, histoire & 17 & $\mathbf{2 9}$ \\
\hline Non classables ${ }^{86}$ & Chroniques, annonces, comptes-rendus, etc. & 99 & $\mathbf{1 4 6}$ \\
\hline Total articles classés & & $\mathbf{9 4 6}$ \\
\hline
\end{tabular}

Une large dominance de la pédagogie

Tout d'abord, nous observons que les articles traitant de questions de pédagogie sont largement dominants dans cette revue. Un résultat qui confirme la nature professionnelle de ce périodique, s'adressant avant tout à un public de praticiens aux prises avec les réalités du «terrain». Dans ce domaine, deux grandes catégories dominent les autres: «documentation pédagogique» qui révèle l'importance des récits d'expérience, sortes de

86 Sont considérés comme «non classables» les articles qui ne relèvent pas d'un domaine spécifique mais qui transmettent des informations générales sur une diversité de domaines; ces articles ne comportent pas de développement argumentatif et ne présentent pas de résultats, leur fonction est plutôt «documentaire» ou informative. Nous ne les avons pas classés dans la catégorie «documentation pédagogique» car l'éclectisme des domaines abordés en fait des articles d'un autre ordre, considérés comme «non classables». Dans cette catégorie se trouvent notamment les «chroniques» qui donnent des informations sur un pays (ex: Chronique Amérique latine, Chronique roumaine), sur une institution (Chronique de l'Institut Rousseau) ou qui se font l'écho de diverses revues d'un pays (ex: Chronique française). D'autres articles de type informatif tels que les chroniques de congrès, listings d'institutions ou de notices bibliographiques, annonces de colloques et conférences, extraits de la correspondance entretenue par la rédaction font également partie de cette catégorie «non classable». 
témoignages qui visent à faire connaître des expériences pédagogiques menées dans divers contextes scolaires pour permettre à d'autres de les reproduire. C'est dans cette catégorie que s'expriment la plupart des praticiens qui partagent avec d'autres leurs questions, leurs doutes mais aussi leur enthousiasme et leurs réussites. De fait, ces récits contribuent à la propagande du mouvement en prouvant la faisabilité des méthodes nouvelles tout en attestant de leur étendue à diverses régions, personnes et institutions. Le nombre imposant d'articles dans cette catégorie confirme le caractère documentaire de $P E N$ tout comme c'est le cas également des revues sœurs. La deuxième catégorie dominante est celle de la "pédagogie générale» qui rassemble un très grand nombre d'articles portant sur les questions générales d'éducation: finalités, problèmes, liens avec la société, mandats, etc. De fait, les sujets abordés dans ces articles sont si vastes que dans certains cas, la catégorisation a été délicate en particulier pour les textes touchant des questions philosophiques. Cette difficulté à catégoriser révèle la proximité entre ces deux domaines dans les débats sur l'éducation et plus encore, dans le contexte de réforme qu' est celui de PEN.

Cette catégorie est suivie de près par celle des «méthodes d'éducation et d'enseignement» attestant une fois de plus que cette revue s'adresse à des praticiens. Ces articles présentent une méthode testée par l'auteur décrite de manière à ce que le lecteur puisse la reproduire. Ils indiquent ainsi que la science qu'ils contribuent à construire est expérimentale et que les élèves comme les maîtres doivent s'y initier, la revue jouant ainsi le rôle de transmetteur de savoirs pédagogiques. Si la grande majorité des articles contiennent des phrases ou portions de textes qui adulent les méthodes nouvelles et montrent leur valeur, nous avons placé dans une catégorie à part ceux qui se dédient entièrement à la présentation des activités et des convictions de la Ligue tant ceux-ci se distinguaient des autres par leur caractère propagandiste. Nous les avons placés dans le domaine «pédagogie» car tous abordent des questions pédagogiques bien que celles-ci soient fortement teintées de philosophie ou de réflexion sur la science et son rôle dans l'éducation. Parmi ces articles figurent plusieurs manifestes que publie $P E N$ - rédigés par divers groupes ou sections affiliés à la Ligue - vers la fin des années 1930, véritables plaidoyers en faveur du renouveau de l'éducation dont le ton est profondément militant.

Toujours dans le domaine de la pédagogie, notre analyse fait apparaître un nombre important d'articles traitant de la formation des maîtres (20), des programmes ou plans d'études (18) et des questions 
concernant les examens ou plus globalement de l'évaluation (16). Ces chiffres permettent de mettre en lumière les sujets au cœur des débats et à propos desquels des discussions et des articles sont nécessaires pour clarifier les orientations de chacun. Comme nous le verrons plus loin, ces questions suscitent des controverses parmi les membres de la Ligue en raison des bouleversements que génèrent les nouvelles conceptions de l'enseignement en particulier pour les enseignants. De fait, la formation des maîtres est l'une des questions les plus brûlantes car c'est là que des changements cruciaux doivent être effectués. Une formation qui devrait comporter désormais une dimension théorique sur la psychologie de l'enfant et une dimension pratique à travers l'initiation des étudiants à la pédagogie et psychologie expérimentale.

\section{La psychologie: science de référence de l'Éducation nouvelle}

Les résultats de cette analyse font apparaître, aux côtés de la pédagogie, l'importance de la psychologie de l'enfant. Ce domaine est celui qui fait l'objet du plus grand nombre d'articles (44) en psychologie, attestant par là-même de la référence cruciale que joue ce domaine dans l'Éducation nouvelle. Les principaux auteurs de ces articles sont des éminents spécialistes de la question - Wallon, Piéron, Piaget, Decroly qui constituent des figures de référence auxquelles le mouvement fait appel régulièrement pour cautionner les propositions de réforme. Car c'est bien cette psychologie de l'enfant, ou science de l'enfant, qui légitime le «renversement copernicien ${ }^{87}{ }$ et les nouvelles méthodes d'enseignement que revendiquent les acteurs de l'Éducation nouvelle. Comme on peut le voir, la psychopédagogie, incluant trois sous domaines (voir tableau), suit de près la psychologie de l'enfant avec 39 articles au total. Ce résultat confirme l'importance, pour les acteurs de l'EN, de la psychologie comme science de référence en particulier dans sa version «appliquée», c'est-à-dire jouant pleinement le rôle de «guide» de la pédagogie. Une science qui a pour rôle de diagnostiquer les aptitudes et inaptitudes des élèves (tests) afin d'orienter au mieux les élèves dans leur parcours scolaire, et dans leur futur parcours professionnel (orientation professionnelle).

87 Décrit par Claparède (1905), ce renversement consiste à inverser la logique en plaçant l'enfant et non plus les savoirs scolaires au centre de l'action éducative. 
Dans les autres domaines et disciplines, la philosophie et la religion sont les plus fréquemment abordés. Comme nous l'avons mentionné plus haut, la catégorisation dans ce domaine est particulièrement délicate tant les sujets de pédagogie générale sont proches des questions philosophiques voire spirituelles. De fait, les propositions de réforme sont étroitement liées à la philosophie puisqu'elles visent à former un nouvel homme ou être humain - tolérant, pacifique, autonome, critique, etc. - pour transformer la société et le monde en général. En ce sens, les questions philosophiques sont sans cesse présentes en particulier dans des articles "pédagogiques» où les auteurs évoquent les changements que les nouvelles méthodes vont opérer - ou ont opéré (dans le cas d'un récit d'expérience, par exemple) - sur la personnalité de l'enfant. Des questions suscitées aussi par certains thèmes de congrès tels que «La liberté en éducation» (Locarno), «La transformation sociale et l'éducation» (Nice) ou «Éducation et liberté» (Cheltenham) puisqu'ils interrogent la relation entre l'éducation, l'individu et la société. Comme l'indiquent les chiffres, un autre domaine très présent dans les articles de PEN est celui de la militance. De fait, chaque article pourrait être classé dans la catégorie militance puisque Pour l'Ère Nouvelle est une revue militante et ne s'en cache pas. Cependant, nous n'avons retenu dans cette catégorie que les articles dont le titre indique clairement une volonté de «convaincre», de faire passer un message «persuasif».

Fin des années 1920: un tournant épistémologique

L'analyse de PEN sous l'angle des thématiques permet de déceler une évolution au cours des deux décennies étudiées. Tout d'abord, les années 1920 se caractérisent pas un intérêt pour l'individu, en particulier l'enfant, que l'on étudie pour mieux le connaître, le comprendre, adapter l'enseignement à ses stratégies d'apprentissage (jeu, activité, expérience). En pédagogie, cet intérêt se perçoit à travers une multitude d'articles sur «l'Art et l'Enfant» liée à la conviction selon laquelle l'enfant a des potentiels et des énergies créatrices que l'éducation a pour mission de libérer. Dès lors, on observe une recrudescence d'articles traitant des méthodes et des disciplines permettant de libérer ces énergies telles que la musique, le théâtre, la rythmique, le dessin, les travaux manuels. Cet intérêt se perçoit également à travers les thèmes de congrès des années 1920 dont deux sont consacrés à ces questions: Calais (1921) «L'expression créatrice de l'enfant» et Heidelberg (1925) «Comment éveiller l'activité spontanée chez l'enfant?». Sur un autre 
plan, cet intérêt pour l'enfant se traduit par une préoccupation concernant son éducation morale. Par exemple, le thème de la co-éducation fait l'objet de plusieurs articles dans Pour l'Ère Nouvelle dont la majorité sont écrits dans les années 1920, à l'exemple de «La coéducation des sexes» (Deman, PEN, 1922, 2 et 4), «La coéducation à Bedales» (Powell, PEN, $1925,17)$, «La conservation de l'enfance et la coéducation» (Johnson, PEN, 1927, 31). Comme le montre Raymond (2002), l'éducation morale est une question récurrente dans l'histoire de la Ligue car «l'Éducation nouvelle s'oblige à penser la moralisation de l'enfant d'une nouvelle manière». Ces réformateurs ont la conviction «qu'éduquer selon la nature les enfants dans la liberté, c'est donner à l'enfant toutes ses chances de réaliser plus tard un monde moral» (p. 7). Dès lors, les articles ayant trait à la nature de l'enfant (ainsi qu'à la nature en tant que lieu de vie) et à la liberté (de l'enfant, de l'éducation) occupent une place centrale dans $P E N$ quelle que soit la période où elle se trouve. Selon cet auteur, ces deux thèmes sont des concepts fondateurs de l'Éducation nouvelle qui traversent la période étudiée comme en attestent les congrès qui les choisissent comme thème de la manifestation (Heidelberg, Locarno, Cheltenham).

En psychologie, cet intérêt pour l'individu se traduit par une quantité d'articles sur la psychologie de l'enfant et sur des tests psychologiques. Cette décennie se caractérise par une psychologie centrée sur l'individu (dite psychologie individuelle) et par une psychologie génétique dont les liens avec la biologie et la physiologie sont très étroits. C'est une sorte de continuité de l'approche des années 1900-1920 où l'on cherche à connaître l'enfant dans ses moindres détails, à étudier ses réactions dans diverses situations, à mesurer ses aptitudes notamment par l'usage de tests et d'échelles métriques (échelle métrique de l'intelligence). Durant cette décennie, la psychologie s'intéresse à l'enfant parce qu'elle cherche à montrer les lois, biogénétiques, de son développement et de son épanouissement. Une psychologie qui tente aussi de catégoriser les individus selon des «types psychologiques» bien que tous ne partagent pas cette approche. De fait, Pour l'Ère Nouvelle fait apparaître une diversité de courants en psychologie dans les numéros spéciaux dédiés aux congrès. Lors de ces manifestations, des groupes de psychologues sont constitués parmi lesquels des représentants de divers courants sont mis en présence, suscitant des prises de position parfois très contrastées.

Ces groupes permettent de déceler une diversification des tendances en psychologie qui atteint son apogée au Congrès d'Elseneur en 1929. À 
l'occasion de ce congrès, cinq courants sont présentés faisant l'objet de conférences et ateliers distincts: psychologie individuelle, psychologie de l'inconscient, psychologie de l'enfant, psychologie génétique et «synthèse psychologique» (Psychological synthesis). Les noms des psychologues permettent de repérer les courants en présence à ce moment-là: Lorenzo Luzuriaga (E), William Boyd (UK), William Boven (CH), Adolphe Ferrière $(\mathrm{CH})$, Alfred Adler (AUT), Nicolas Pende (médecin endocrinologue, I), Ovide Decroly (B), Elsa Koehler (D), Robet Nussbaum (CH), Charles E. Krafft (astrologue, CH), Kurt Lewin (D), Henri Wallon (F). Des psychologues tels que Nussbaum, Adler, Krafft, Boven - tous amis proches de Ferrière - focalisent leurs recherches sur les types psychologiques, convaincus que l'on peut classer les individus dans des catégories et affirmant que «le caractère est inséparable de l'intelligence, de l'esprit, qui n'en est que la manifestation la plus mouvante et la plus sublime». Alors que d'autres, tels que Wallon et Lewin se distancent de ce groupe en affirmant que «ce mode de classer les individus est insuffisant» et qu'il faudra «remplacer peu à peu la classification par la conception de types basés sur des concepts conditionnels et génétiques et faisant appel à l'idée de construction conceptuelle» (Lewin, PEN, 1929, 52, pp. 251-252). De son côté, Wallon remet en cause la conception mystique, voire métaphysique, de la force de l'homme, ce que certains appellent la lumière intérieure, ou la force divine. Selon lui, «il n'y a pas d'acte psychique qui ne dépende de l'individu tout entier et la psychologie n'a de sens que si elle rattache au comportement d'êtres particuliers les faits observés, quelles qu'en soient la fréquence et la généralité» (Wallon, PEN, 1929, 52, p. 254). En d'autres termes, Wallon s'élève contre une étude psychologique trop focalisée sur l'individu qui néglige l'influence de l'environnement, proclamant que «l'individu est un tout» et «fait partie d'un tout» qui inclut le monde social et environnemental (Wallon, PEN, 1929,52, p. 255). Des affirmations révélatrices du tournant que constitue la fin des années 1920 concernant les conceptions psychologiques de l'éducation.

Que ce soit en pédagogie ou en psychologie, les années 1920 se caractérisent aussi par un intérêt plus particulier pour le «petit enfant» ou «l'enfant jeune». En effet, la majorité des expériences durant cette décennie concerne des enfants scolarisés dans des écoles maternelles ou primaires, que ce soit dans le secteur public (classes nouvelles) ou privé (écoles nouvelles). C'est dans ces établissements que s'expérimentent les premières mises en pratique des méthodes nouvelles puisque la plupart 
d'entre elles sont destinées à des enfants jeunes ${ }^{88}$ comme en attestent ces quelques titres d'articles: «L'éducation des tout petits par le travail manuel» (Prior, PEN, 1923, 6), «À la Maison des Petits» (Rédaction, PEN, 1923, 7), «La gymnastique analytique du Dr. Tissié chez les tout-petits» (Ferrière, PEN, 1926,19), "Rythmes et gestes chez les tout petits» (Jousse/Ferrière, PEN, 1928, 39), «Psychologie et pédagogie de la personnalité chez les tout petits (Koehler, PEN, 1929, 52). En psychologie, les études de chercheurs tels que Claparède, Decroly ${ }^{89}$ ou Piaget portent aussi sur les petits enfants, à commencer par les nourrissons que ces derniers observent dans leurs moindres faits et gestes, visant à comprendre comment se construisent la pensée, le langage, l'intelligence (Piaget, 1923, 1936).

Dans les années 1930, l'intérêt se déplace progressivement «de l'individu vers le social» (Brehony, 2004; Haenggeli-Jenni \& Hofstetter, 2011) que ce soit dans les recherches en pédagogie ou en psychologie. En psychologie, les écrits de Piaget et Wallon se rejoignent sur l'idée que l'enfant se construit en interaction avec son environnement social, stimulé par ce dernier et qu'il sollicite à son tour. Aux préoccupations focalisées sur le développement et l'épanouissement de l'individu font place des interrogations sur les manières d'adapter l'éducation aux changements de la société. Le Congrès de Nice (1932) spécifiquement dédié à ce thème - «L'Éducation dans ses rapports avec l'évolution sociale» - en est une illustration, marquant ainsi un changement de perspective dans l'appréhension des phénomènes éducatifs. Un tournant qui se reflète dans Pour l'Ère Nouvelle que ce soit au niveau des titres d'articles ou dans leurs contenus. Par exemple, durant la première décennie (19221931), seuls deux articles comportent le mot «social» (ou un dérivé) dans leur titre, alors qu'on en compte 19 dans la deuxième décennie (19321940). De plus, le domaine de «l'orientation scolaire et professionnelle» - quoique déjà existant dans les années 1920 - prend une importance nouvelle dans les années 1930; époque où l'on cherche à optimiser l'adéquation entre les aptitudes individuelles et les exigences du monde

88 C'est le cas notamment des méthodes Montessori conçues avant tout pour des enfants d'âge préscolaire.

89 Ce dernier tourne plusieurs films montrant les réactions d'enfants âgés de 0 à 4 ans dans diverses situations créées par le chercheur (Wagnon, 2013). De façon similaire, c'est une fillette de 4 ans qui est le personnage central du film le Home «Chez Nous» dirigé par Ferrière en 1927-1929. (Coquoz, 1998). 
économique. Dans Pour l'Ère Nouvelle, deux articles contenant le mot «orientation» figurent aux sommaires de la première décennie alors que l'on en dénombre 17 dans la deuxième. De nombreux chercheurs et auteurs français étudient ces questions comme en attestent les articles de Laugier, Piéron, Weinberg, Gal, en lien notamment avec la fondation par Henri Piéron de l'Institut National d'Orientation Professionnelle (INOP) en 1928.

D'autre part, l'intérêt pour le «petit enfant» tend à faire place à un intérêt pour le «grand enfant», notamment l'adolescent et le jeune adulte. Un âge qui, selon les psychologues, se caractérise par l'importance de l'environnement social à partir duquel - et en interaction avec lequel - l'enfant construit son identité (liens d'amitié, «couples enfantins», «bandes d'enfants»). Par définition, l'adolescent et le jeune adulte (davantage que le petit enfant) sont des êtres «sociaux» qui sont appelés à jouer un rôle actif dans la société, raison pour laquelle on se préoccupe de leur orientation scolaire et professionnelle. Ce changement - qui correspond au tournant «social» des années 1930 - se traduit dans PEN par une recrudescence d'articles concernant les expériences pratiques menées dans l'enseignement secondaire ${ }^{90}$ : «L'École active dans l'enseignement secondaire en France» (Cazamian, PEN, 1932,80), «La mission éducative du Professeur d'Enseignement secondaire (Grand-Duché du Luxembourg)» (Frieden, PEN, 1934, 101), «Les idées de la Doctoresse Montessori sur l'enseignement secondaire» (Niot, PEN, 1935, 112), «La formation professionnelle du personnel enseignant secondaire» (Publication BIE, PEN, 1935, 112). Cet intérêt est particulièrement présent en France où de nombreux enseignants du secondaire, aussi bien des collèges que des lycées, se mobilisent pour parvenir à concrétiser des projets d'Éducation nouvelle, à l'exemple des classes d'orientation..$^{91}$ La réforme des lycées qui se met en place à partir de 1936 est inspirée des principes de l'Éducation nouvelle et voit le jour sous le ministère de Jean Zay (1936-1939). Selon Savoye (2009), «il s'agit moins d'une refonte des savoirs enseignés qu'un changement de leurs modes de transmis-

90 Sur 10 articles contenant le mot «secondaire» dans leur titre, seuls deux ont été écrits au cours de la première décennie de $P E N$, les huit autres ayant été écrits entre 1932 et 1940.

91 Les «classes d'orientation» constituent l'un des projets phares de ces années-là qui sera mis en pratique dans plusieurs établissements de 1936 à 1939 en France (Savoye, 2004; Seguy, 2010). 
sion» (p. 507). ${ }^{92}$ Le Congrès du Havre (1936) $)^{93}$ qui réunit pour la première fois tous les enseignants du secondaire constitue un exemple marquant de cette mobilisation dont les efforts seront malheureusement interrompus par la guerre trois ans plus tard.

Outre un intérêt pour l'enfant "plus âgé», l'analyse de Pour l'Ère Nouvelle fait apparaître un intérêt croissant pour l'éducation des adultes. Celui-ci se manifeste à travers l'organisation, croissante dans les années 1930, de divers cours du soir et cycles de conférences. Organisées par des institutions ou des associations ${ }^{94}$, ces manifestations sont destinées à un public d'adultes intéressés par les questions d'éducation et, dans certains pays (Angleterre), à des parents ouvriers désireux de compléter leur éducation de base. Comme le montre Cazamian (Chronique des revues anglaises, PEN, 1935, 113) certaines associations comme la Workers' Educational Association ou Adult Education Movements ouvrent des «clubs pour ouvriers sans travail» ainsi que des «tutorial classes» fonctionnant selon le principe d'une conférence d'une heure chaque quinzaine, suivie d'une discussion de même durée. Des cours qui remportent un immense succès, mis en valeur par l'Université d'Oxford attribuant le honorous Degree à ceux qui ont suivi régulièrement les tutorial classes pendant quatre ans.

De manière générale, les articles des années 1930 font beaucoup allusion aux difficultés que traverse le monde et auxquelles il apparaît urgent de trouver des solutions. Ils révèlent une prise de conscience de la nécessité de rendre l'Éducation nouvelle efficace dans l'espoir qu'elle puisse inverser le cours des choses en édifiant un «rempart solide à toute injustice et violence». Le contexte politique tendu dans lequel s'élaborent ces discours incite le comité éditorial à prendre une position plus engagée fondamentalement distincte de celle de ses premiers membres. Au Congrès de Nice, certains conférenciers - notamment Wallon et Langevin

92 Les transformations proposées sont appliquées selon quatre principes: 1. méthodes actives - «travail actif»; 2 . coordination entre disciplines et entre enseignants; 3. expérience et liberté pédagogique (liberté d'action du maître, «droit à l'expérience»); 4 . éducation du «sens social» et initiation à la vie sociale. (Savoye, 2009, pp. 508-511).

93 Sur le congrès du Havre, voir Condette \& Savoye (2011), Savoye (2006), Gutierrez (2008).

94 Le Groupe Français d'Éducation nouvelle organise des cycles de conférences pendant les hivers 1932 et 1933. L'Institut Rousseau fait de même à Genève mais sur une période plus longue. 
- font un état de situation mitigé après dix ans d'efforts entrepris par la Ligue; s'ils reconnaissent la valeur des changements effectués, ils en montrent les limites et appellent les «amis de l'enfance» à changer d'orientation, prenant davantage en compte les dimensions sociales, ainsi que politiques et économiques, pour rendre l'éducation plus opérationnelle dans sa mission de transformer la société.

\section{DES AUTEURS ÉCLÉCTIQUES: PROFESSIONS ET ORIGINES DIVERSES}

En comptabilisant le nombre d'articles écrits par chaque auteur, des noms apparaissent de façon dominante, dont les idées et tendances marquent la ligne éditoriale. À l'inverse, cette analyse permet de repérer ceux qui écrivent peu ou peu souvent. À ce propos, nous pouvons relever que parmi les 127 auteurs français, plus de la moitié n'écrit qu'un à deux articles au total. De ce fait, l'impact de ces auteurs sur la ligne éditoriale est assez minime au regard d'auteurs étrangers, certes moins nombreux, mais qui écrivent plus régulièrement et imprègnent la revue de façon plus significative. Nous présentons ici les auteurs principaux qui émergent de cette analyse mais une analyse plus approfondie des profils de groupes est proposée dans le chapitre 3 de ce livre.

De façon évidente, cette analyse confirme la dominance considérable de Ferrière sur Pour l'Ère Nouvelle. Malgré son retrait officiel du comité de rédaction en 1929, ce dernier poursuit une activité éditoriale intense pour PEN à travers la rédaction d'articles, de rubriques et de comptes rendus jusqu'à la fin de 1934. Toutefois, comme le montre le tableau suivant, une brusque diminution de cette activité intervient dès 1935, date à partir de laquelle Ferrière ne signe plus que 15 articles au total jusqu'en 1940, alors que ce chiffre correspond auparavant au nombre d'articles qu'il signe chaque année (en moyenne). Une diminution qui s'explique à la fois par son éloignement de la section française de la Ligue et par des problèmes de santé auxquels il doit faire face (Hameline, 1981). 
Tableau 8.

Auteurs principaux de $P E N^{95}$

\begin{tabular}{llccc}
\hline Auteur & Nationalité & 1922-1930 & 1931-1940 & Total \\
\hline A. Ferrière & Suisse & 73 & 57 & 130 \\
\hline E. Delaunay & France & 14 & 16 & 30 \\
\hline H. Wallon & France & 4 & 16 & 20 \\
\hline M.-A Carroi & France & 2 & 16 & 18 \\
\hline H. Piéron & France & 2 & 13 & 15 \\
\hline O. Decroly & Belgique & 11 & 2 & 13 \\
\hline M.-L. Cazamian & France & - & 13 & 13 \\
\hline E. Flayol & France & 3 & 9 & 12 \\
\hline B. Ensor & Angleterre & 7 & 4 & 11 \\
\hline G. Bertier & France & 4 & 6 & 10 \\
\hline P. Bovet & Suisse & 2 & 5 & 7 \\
\hline L. Brun-Laloire & France & - & 6 & 6 \\
\hline A. Hamaïde & Belgique & 2 & 4 & 6 \\
\hline A. Jadoulle & Belgique & - & 6 & 6 \\
\hline C. Washburne & USA & 3 & 3 & 6 \\
\hline R. Cousinet & France & 5 & - & 5 \\
\hline H. Laugier & France & - & 5 & 5 \\
\hline G. Lapierre & France & - & 5 & 5 \\
\hline M. Montessori & Italie & 2 & 3 & 5 \\
\hline E. Rotten & Allemagne & 3 & 5 \\
\hline P. Geheeb & Allemagne & 1 & 5 \\
\hline & & & 5 & 5 \\
\hline
\end{tabular}

95 Dans cette récolte de données, nous avons fait le choix d'inclure l'année 1930 dans la première décennie afin d'obtenir un équilibre du nombre d'années concernées: 1922-1930 (9 ans); 1931-1940 (9 ans + 1 numéro en 1940). Parmi les articles de ce tableau, certains ont été écrits par deux auteurs et d'autres sont le fruit d'un collectif, mais le nom de l'auteur concerné figure toujours parmi les signataires. 
Tableau 9.

Nombre d'articles écrits par Ferrière dans PEN

\begin{tabular}{cccccccccc}
\hline 1922 & 1923 & 1924 & 1925 & 1926 & 1927 & 1928 & 1929 & 1930 & 1931 \\
\hline 1 & 7 & 5 & 7 & 14 & 12 & 8 & 9 & 10 & 7 \\
\hline & & & & & & & & & \\
\hline 1932 & 1933 & 1934 & 1935 & 1936 & 1937 & 1938 & 1939 & 1940 & Total \\
\hline 9 & 12 & 13 & 5 & 1 & 3 & 5 & 2 & - & 130 \\
\hline
\end{tabular}

Cette analyse des auteurs ayant le plus écrit dans PEN fait apparaître la prégnance des membres des comités de rédaction bien que soulignant, à l'inverse, l'absence de certains autres. Ainsi, le nombre d'articles écrits par Wallon et Piéron confirme l'usage que ceux-ci font de PEN qu'ils investissent comme un lieu de diffusion de leurs idées et de leurs découvertes scientifiques. ${ }^{96} \mathrm{Il}$ en va de même pour Decroly qui, en tant que membre du comité de rédaction, utilise la revue pour transmettre ses conceptions à la fois scientifiques et pédagogiques. À l'inverse, il est intéressant de noter l'absence totale de Fauconnet, pourtant membre du comité de rédaction de 1925 à 1929, et la présence très discrète de Piaget, qui ne publie que trois articles durant les années où il siège au comité de rédaction (1932 à 1940). À dessein, nous ne nous attardons pas plus longuement sur ces auteurs puisqu'ils ont fait l'objet de notre attention dans le volet concernant le comité de rédaction. Du côté des autres auteurs, la présence d'Eugène Delaunay ${ }^{97}$ en seconde position de la liste s'explique par sa contribution à la revue en tant que responsable de la «Chronique française» qu'il assure de 1926 à 1939 et dans laquelle il donne son point de vue, souvent aiguisé, sur la vie politique et syndicale en France dans ses rapports avec l'éducation» (Gutierrez, 2008, p. 82). Pour Ferrière, Delaunay est «un des pionniers les plus sérieux de l'École active dans le cadre de l'école publique» (Éditorial, PEN, 1926,18, p. 3) et sa présence au sein de PEN est importante puisqu'en se chargeant de cette chronique et du service des annonces, il décharge Ferrière d'une part importante de son travail.

\footnotetext{
96 Voir le volet concernant le comité de rédaction de $P E N$ dans ce même chapitre.

97 Instituteur à Coulombs par Creully (Calvados).
} 
Dès lors, mis à part les membres du comité de rédaction et le «chroniqueur» qu'incarne Delaunay, ce sont trois femmes qui apparaissent clairement dans le "groupe de tête» des auteurs écrivant le plus dans PEN; trois femmes auxquelles nous proposons de nous intéresser ici. Tout d'abord, il s'agit de Marie-Anne Carroi (1898-1976)98, auteure de 18 articles publiés entre 1928 et 1938 sur des sujets ayant trait à l'éducation des filles et à l'enseignement secondaire. Elle signe ses articles en tant que professeur de lycée, engagée tout d'abord au lycée pour jeunes filles à Tunis (1925-1931), puis au Lycée Lamartine à Paris où elle enseigne la philosophie de 1935 à 1947. Dans ses textes, Carroi se positionne en faveur du droit de tous à l'éducation ${ }^{99}$, militant en particulier pour l'éducation des filles en lien avec le contexte dans lequel elle écrit. ${ }^{100}$ En 1932, elle quitte la Tunisie et voyage aux États-Unis ${ }^{101}$ puis en Allemagne où elle visite des gymnases à Berlin qui pratiquent les méthodes nouvelles et dont elle loue les bienfaits dans un article en 1933. ${ }^{102}$ Carroi est particulièrement intéressée par la méthode de «travail par équipes» à propos de laquelle elle fait une intervention ${ }^{103}$ au Congrès du Havre en 1936, premier congrès entièrement dédié aux questions relatives à l'enseignement du second degré (Savoye, 2006). Cette même année, elle participe à deux autres manifestations, le Congrès international de pédagogie à Utrecht (Pays-Bas) et le Congrès international de la Ligue à Cheltenham. Parmi ses objets d'intérêts figure aussi l'éducation artistique comme en témoigne son intervention sur «L'art et la personnalité libre» (PEN, 1936, 123) au congrès de la Ligue. Dès 1936, Marie-Anne Carroi collabore de manière régulière à l'Information Pédagogique, première revue dédiée à la réforme de l'enseignement secondaire, dirigée par Marcel Ginat et Alfred Weiler. ${ }^{104}$ Une année plus tard, elle figure

98 Sur la trajectoire de cette femme, voir les travaux de Renier (2015), Riondet \& Renier (2013).

99 Elle écrit un article sur $1^{\prime}$ «Éducation des indigènes en Tunisie» (PEN, 1931, 68).

100 Elle est alors professeur en Tunisie et écrit: «Départ de la première Tunisienne pour une Université française» (PEN, 1931, 72).

101 Elle écrit: «Dans les collèges féminins d'Amérique» (PEN, 1932, 74).

102 «Méthode classique - méthode nouvelle dans les gymnases de Berlin» (PEN, 1933, 87).

103 «Développement individuel et travail par équipes» (PEN, 1936, 119).

104 Sur ces deux acteurs du Havre, voir Savoye (2006). 


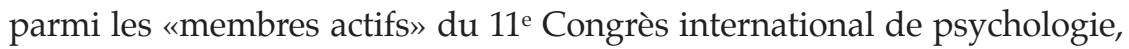
se déroulant du 25 au 31 juillet 1937 à Paris (Riondet \& Renier, 2013). Par ailleurs, Marie-Anne Carroi se distingue par sa maîtrise des langues et ses compétences de traduction. Elle traduit les textes de plusieurs pédagogues, notamment Giuseppe Lombardo-Radice (1929)105 et John Dewey (1947) ${ }^{106}$ à propos duquel elle écrit un mémoire suite à son séjour en Pennsylvanie en 1920-21. Enfin, elle sera membre du comité directeur de L'École Nouvelle Française fondée par Roger Cousinet dès 1945. Ces divers éléments attestent de l'intense activité de Marie-Anne Carroi, de sa mobilité et son engagement pour les causes qu'elle défend. Son activité d'écriture constitue une part centrale de son emploi du temps puisqu'elle collabore non seulement à Pour l'Ėre Nouvelle mais aussi à plusieurs autres revues françaises. Elle contribue ainsi activement à l'élaboration de nouveaux savoirs sur l'éducation dont elle est une spécialiste reconnue pour les degrés secondaires. Pour Savoye (2006), «elle est une des premières 'têtes de pont' de l'Éducation nouvelle en direction de l'enseignement secondaire» (p. 363).

La deuxième femme figurant dans le «groupe de tête» des auteurs de PEN est Marie-Louise Cazamian. Contrairement à sa compatriote, Cazamian n'écrit dans PEN qu'à partir de 1932, en lien avec sa participation au Congrès de Nice. Elle est alors professeur au niveau secondaire comme l'indique son article «L'École active à l'école secondaire en France» (PEN, 1932, 80). Dans PEN, elle rédige en 1934 une «Chronique américaine» ainsi que plusieurs «Chroniques des revues anglaises et américaines» (1935, 1937 et 1938) nous laissant supposer que Cazamian maîtrise bien l'anglais sachant que son mari, Louis Cazamian (18771965), est professeur de littérature anglaise à la Sorbonne de 1925 à 1945. De fait, le couple Cazamian est surtout actif au sein des Compagnons de

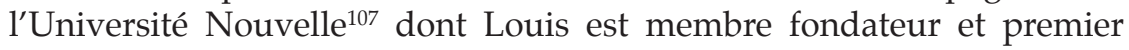

105 Sur le conseil d'Adolphe Ferrière, elle traduit Les Petits Fabre de Porto Maggiore de G. Lombardo Radice ayant elle-même observé et étudié les pratiques pédagogiques de ce pédagogue italien.

106 Elle traduit le célèbre ouvrage Démocratie et Éducation publié en 1947 aux éditions Bourrelier, réédité en 1967 et 2011 aux éditions Armand Colin. Sur la réception de la pensée de John Dewey en France, et les liens avec MarieAnne Carroi, voir Go (2013) et Riondet (2013a).

107 Groupe fondé en 1918, composé à l'origine d'intellectuels désireux de réorganiser l'enseignement public français afin de permettre une sélection plus 
président dès 1919 (Garnier, 2008). Alors que ce groupe reste à distance de la Ligue pendant plusieurs années, un rapprochement s'opère à partir de 1929 lors de la réorganisation du GFEN et de l'arrivée à sa tête de Paul Langevin. ${ }^{108}$ Comme le montre Gutierrez (2008), le Congrès de Nice en 1932 célèbre ce rapprochement grâce à une thématique qui permet aux uns et aux autres de s'exprimer selon leur point de vue. À partir de ce moment-là, la présence simultanée de certains acteurs comme Cazamian, Bertier, Hauser et Brun-Laloire dans les deux groupements incarne les liens désormais plus proches entre ces deux groupements (p. 98). Cazamian participe à plusieurs congrès de la Ligue: à Nice en 1932, où elle fait une intervention sur «La coopération des maîtres et des parents» et sur «l'École active dans l'enseignement secondaire en France» (PEN, 1932, 80) et à Cheltenham en 1936 où, grâce à ses connaissances d'anglais, elle rédige le rapport de la «Commission internationale pour la préparation des professeurs» (PEN, 1937, 126, pp. 79-81). Tout comme Carroi, Cazamian participe cette même année au Congrès du Havre qui réunit les enseignants du degré secondaire, suite auquel elle rédige un article dans PEN intitulé «Échos du Congrès du Havre» (PEN, 1937, 126). Elle en écrit aussi sur les relations famille-école, sur la coopération maîtres-parents et sur l'orientation scolaire (1938).

Enfin, la troisième femme à investir la revue PEN de façon significative est Émilie Flayol (1873-1976). Son nom apparaît pour la première fois dans la revue en 1929 à l'occasion d'un numéro spécial consacré à l'Éducation nouvelle en France. Elle y écrit un article sur «L'esprit des écoles nouvelles et des écoles normales en France» (PEN, 1929, 49) étant alors encore toute imprégnée de sa fonction de directrice d'école normale. L'hypothèse se confirme à travers l'intervention qu'elle fait au Congrès d'Elseneur cette même année, «Comment améliorer la préparation intellectuelle et morale des éducateurs» (PEN, 1929, 53). À partir de

démocratique des élites (principe de l'école unique). Leurs premiers articles et manifestes sont publiés dans la revue L'Opinion puis dans la revue du groupe, L’Université Nouvelle (Garnier, 2008).

108 Ce dernier étant convaincu que la démocratisation de l'école publique doit se combiner avec une rénovation des méthodes pédagogiques, essaie de rallier un maximum de personnes à sa cause, que ce soit parmi les réformateurs «pédagogiques» de la section française de la Ligue (GFEN) ou parmi les défenseurs d'une réforme structurelle de l'enseignement public que sont les Compagnons. (Gutierrez, 2008, p. 97) 
1932, Émilie Flayol assume la fonction de secrétaire au sein du nouveau comité de rédaction de la revue. Très active au sein du GFEN, c'est elle qui en rédige les rapports annuels du comité de $P E N$, de même que le numéro spécial de 1938 qui retrace l'historique du groupe et de la revue. ${ }^{109}$ Elle participe aux diverses activités de propagande organisées par le GFEN telles que visites d'écoles, interventions dans les écoles normales, articles de presse, conférences à Paris et en province. Lors du cycle de conférences organisé par le groupe durant l'hiver 1932-1933 au Collège libre des Sciences sociales ${ }^{110}$, Émilie Flayol donne deux conférences ${ }^{111}$, l'une sur la «Critique des méthodes actuelles d'éducation» et l'autre sur «L'Éducation et la Paix». ${ }^{112}$ D'autre part, Émilie Flayol participe aux congrès de la Ligue des années 1930 (Nice en 1932, Cheltenham en 1936) ainsi qu'à d'autres manifestations comme le Congrès du Havre en juin 1936 où elle représente le GFEN aux côtés de Paul Langevin. Dès cette année-là, Flayol figure dans la liste du comité de rédaction de l'Information Pédagogique.

Les profils de ces trois femmes se rejoignent à plusieurs niveaux: leur activité professionnelle d'enseignement au niveau secondaire signifie que toutes trois ont bénéficié d'une formation de haut niveau dans une ou plusieurs disciplines; leur sensibilité particulière aux questions pédagogiques laisse penser qu'elles ont fréquenté l'école normale supérieure les préparant à enseigner au second degré; leur engagement militant au sein de groupes d'Éducation nouvelle, qu'il s'agisse du GFEN, des Compagnons de l'Université Nouvelle ou du groupe dédié à l'Organisation de l'enseignement du Second degré, en fait des figuresclés au sein du mouvement en France. De fait, leur action ne se situe pas seulement au sein de ce mouvement mais aussi à un niveau politique plus global, à travers leur militance au sein de mouvements féministes nationaux et internationaux. Ainsi, c'est grâce à l'intervention de ces trois femmes auprès du Conseil national de Femmes que le GFEN obtient, en

109 Selon Gutierrez (2008), Émilie Flayol rédige ces rapports en omettant à dessein les conflits et tensions qui existent entre les différents groupes d'Éducation nouvelle, notamment entre le GFEN et La Nouvelle Éducation.

110 Hôtel des Sociétés Savantes, Rue Serpente, Paris.

111 Le GFEN est alors en difficultés financières et cherche par tous les moyens à mieux se faire connaître auprès du public. (Gutierrez, 2008, p. 350)

112 Ces deux conférences ont lieu le 19 janvier et le 16 février 1933 (Communications, PEN, 1932, 74). 
1936, une mention dans les vœux de ce Conseil concernant «l'efficacité des méthodes nouvelles dans le système d'éducation pour les enfants dévoyés ainsi que de l'importance d'y former les maîtres». ${ }^{113}$ Comme nous le verrons plus loin, la militance des femmes au sein de l'Éducation nouvelle se combine souvent d'un engagement militant dans des mouvements féministes qui constituent des réseaux puissants au sein desquels celles-ci entretiennent des relations et font valoir leurs compétences d'intellectuelles (écriture, conférences, etc.) (Pavillon, 2007).

À cette étape de notre recherche, nous pouvons faire plusieurs remarques à propos de Pour l'Ère Nouvelle. Tout d'abord, nous concluons à la présence de deux phases distinctes dont les caractéristiques sont perceptibles aussi bien au niveau des thématiques d'articles, des auteurs principaux que de l'infrastructure éditoriale. Comme nous l'avons vu, la première décennie se caractérise par la dominance d'articles s'intéressant à l'enfant en tant qu'individu dont il s'agit de comprendre les aptitudes, les caractéristiques psychologiques, les phases de son développement dans le but ultime de mieux adapter l'enseignement. Parallèlement, la revue se fait l'écho d'une multitude d'expériences pédagogiques attestant que les méthodes nouvelles sont applicables, et plus globalement, que le mouvement est en marche dans divers contextes. Cette décennie est marquée par la personnalité de Ferrière qui imprègne la revue de sa conception spiritualiste de l'éducation mais aussi de son souci de donner à cette revue une dimension internationale. Pour cela, il agit comme porte-parole de la Ligue, se fait le relais des multiples initiatives et utilise la revue comme outil de propagande pour fédérer le mouvement selon les orientations de la Ligue. Les auteurs qui écrivent durant cette première phase reflètent le caractère cosmopolite du mouvement à cette époque-là, ces derniers appartenant aussi bien aux régions francophones européennes (France, Suisse, Belgique), aux régions latines (Europe du Sud, Amérique latine) qu'aux autres pays dont des personnalités militent au sein de la Ligue.

La deuxième phase de l'histoire de PEN se caractérise par une dimension plus nationale, en raison de sa prise en main par le Groupe Français d'Éducation nouvelle. Ce dernier utilise la revue surtout comme moyen de faire connaître l'Éducation nouvelle française même si plusieurs correspondants étrangers continuent d'y écrire. De fait, ce

113 Émilie Flayol dans le rapport de l'AG du GFEN de mars 1936 cité par Gutierrez (2008, p. 351). 
«repli national» s'explique aussi par le contexte politique et économique du moment dont la crise force les partisans de l'EN à réduire leurs ambitions et à orienter leurs efforts sur des initiatives plus locales et plus concrètes. Ce tournant se traduit par un changement significatif au niveau des auteurs, ceux-ci étant principalement français, membres du GFEN ou d'autres groupes français d'Éducation nouvelle. Au niveau des thématiques, cette deuxième phase se caractérise par un déplacement de l'intérêt de l'individu vers le social, où la prise en compte de l'environnement (social, familial, culturel) de l'enfant est désormais centrale, que ce soit par les praticiens ou par les théoriciens. Elle se traduit par une recrudescence d'articles s'intéressant à l'enfant sous l'angle de ses relations avec la famille, avec ses pairs, avec la communauté, ainsi qu'à son orientation scolaire et professionnelle.

De manière plus globale, l'analyse de $P E N$ fait apparaître le caractère hybride de cette revue dans laquelle s'expriment à la fois des psychologues, des philosophes, des médecins, des intellectuels, et tous les représentants du monde scolaire (instituteurs, éducateurs, inspecteurs, directeurs, etc.). Cette caractéristique favorise la rencontre et la mise en réseau de ces divers acteurs qui participent à la construction de savoirs sur l'enfant et l'éducation. L'analyse révèle en outre que les rédacteurs de ce journal tentent de fédérer le mouvement autour de valeurs chères à l'Éducation nouvelle à travers des discours de type militant et propagandiste. Comme le dit Hofstetter (2008), «Pour l'Ėre Nouvelle se présente donc comme une revue au carrefour entre approche scientifique, travail documentaire et comptes rendus d'expériences qui œuvre à l'échelle nationale et internationale pour la promotion d'un mouvement et de son idéologie» (p. 248).

\section{MISE EN REGARD: PRINCIPES COMMUNS, DIFFÉRENCES ASSUMÉES}

À l'issue de nos investigations, nous proposons une mise en regard des trois revues étudiées afin de mieux comprendre comment se développe l'Éducation nouvelle à une échelle internationale, en particulier dans les régions linguistiques concernées. Si ces périodiques partagent un certain nombre de points communs qui contribuent à la cohésion du mouvement, ils se distinguent par des spécificités liées au contexte dans lequel ils sont publiés. Nous proposons d'analyser l'influence des traditions 
culturelles sur l'infrastrcuture et les diverses composantes de ces périodiques afin de saisir dans quelle mesure le mouvement est fédéré et quel rôle joue ici la militance.

Au niveau du contenu, ces trois organes se composent d'articles de fond ${ }^{114}$ - signés par divers auteurs - ainsi que de plusieurs rubriques rédigées principalement par les membres du comité éditorial. Parmi celles-ci, mentionnons la rubrique «Nouvelles diverses» dont l'équivalent s'intitule «Mitteilungen» dans la revue allemande et «International Notes» dans la revue anglaise, bien que dans cette dernière la rubrique n'apparaisse qu'en 1930, au moment du changement d'orientation. On trouve aussi la rubrique «Book reviews» qui trouve son équivalent sous le nom de «Livres et Revues» dans l'édition française et «Bücher» dans la revue allemande. Dès 1928, cette rubrique se scinde en deux dans ces deux éditions, distinguant désormais les «Livres» («Bücher») d'une part et les «Revues» («Zeitschrift») d’autre part. Si elles sont fréquemment signées de la main de Karl Wilker dans la revue allemande, le nom du signataire ne figure pas de façon claire dans les revues-sœurs. Toutefois, ce genre de tâche exigeant une large connaissance publications dans le domaine, on peut penser que seuls les responsables d'édition pouvaient en assumer la rédaction. Ainsi, dans Pour l'Ère Nouvelle, les événements et publications évoqués dans ces rubriques sont manifestement sélectionnés par Ferrière qui en assume la rédaction jusqu'en 1929 voire même au-delà. Seules quelques revues de la presse pédagogique française sont signées de la main de Louis Brun Laloire mais pas de façon systématique. Concernant The New Era, Beatrice Ensor étant longtemps seule à assumer l'édition de la revue, c'est elle qui se charge de ce travail.

Concernant la fréquence de parution, les trois revues paraissent d'abord au rythme de quatre numéros par an; les revues allemande et française augmentent leur rythme dès 1926 (six numéros par an) puis deviennent mensuelles l'année d'après (dix numéros par an). The New Era conserve plus longtemps le rythme de quatre numéros annuels ce qui explique le volume plus important de ses numéros - entre 40 et 50 pages contre 15 à 20 pages dans les revues sœurs - en particulier dans les années 1926 à 1928. Alors que ses consœurs sont mensuelles à partir

114 Le nombre des articles par numéro oscille entre 4 et 10, ce nombre étant toutefois plus stable dans PEN (4-6 articles par numéro) tout au long de la période étudiée. 
de 1927, la revue anglaise ne les imite qu'en juillet 1930, au moment où elle connaît d'autres changements importants. ${ }^{115} \mathrm{Ce}$ changement de rythme est significatif puisqu'il fait l'objet d'un article dans la rubrique «Revues d'Éducation nouvelle» de PEN au mois de mai 1930:

Pendant ces six dernières années, la revue [The Neww Era] a été tout à la fois un lien entre les pionniers de l'Éducation nouvelle et un centre de discussion des idées éducatives en général, sans distinction de doctrines philosophiques, psychologiques, de méthodes ou de couleurs nationales. Trimestrielle, elle manquait toutefois de l'espace nécessaire, non seulement pour rendre compte de façon adéquate des problèmes éducatifs tels qu'ils se posent dans le monde entier, mais encore pour donner une attention suffisante aux intérêts particuliers des parents. The New Era pourra désormais rendre des services d'importance vitale et pratique aux parents comme aux maîtres. Sous sa forme nouvelle, elle exposera les problèmes d'éducation au point de vue des premiers comme à celui des seconds, et cherchera à créer une coopération active entre l'école et la famille. (Livres et Revues, PEN, 1930, 58, p. 133)

Comme nous l'avons évoqué dans les pages précédentes, le tournant des années 1930 suscite des changements importants pour chaque revue que ce soit au niveau de leur structure administrative - la responsabilité éditoriale de Pour l'Ère Nouvelle est transférée au GFEN - ou au niveau de leur orientation thématique/idéologique - The New Era étend son champ d'intérêt à l'éducation familiale tandis que PEN s'ajuste aux préoccupations sociales et pratiques du GFEN. Pour la revue allemande, le tournant se situe plutôt en 1928, année durant laquelle elle diversifie significativement les thématiques de ses articles grâce à la collaboration officielle de plusieurs compatriotes. Finalement, Das Werdende Zeitalter cesse de paraître en 1932, au moment où les changements amorcés en 1929-30 dans les revues-sœurs prennent toute leur ampleur. En effet, c'est en 1932 que The New Era concrétise véritablement sa nouvelle orientation vers l'éducation familiale en subdivisant chacun de ses numéros en deux parties - l'une pour les maîtres, l'autre pour les parents - et en faisant appel à de nouveaux contributeurs-trices, spécialistes du «parental education». C'est en 1932 également que le transfert de Pour l'Ère Nouvelle au GFEN prend vraiment forme: outre sa responsabilité administrative (acquise en 1929), le GFEN a désormais l'entière responsabilité sur le plan rédactionnel pour lequel il nomme un nouveau comité éditorial.

115 Sur ces changements, voir le chapitre concernant The New Era. 
Le tournant des années 1930 est aussi une période de changement au sein de Ligue qui, en raison de son étendue à la fois géographique et démographique, décide d'une restructuration importante aux niveaux des rôles et des responsabilités des pays membres. Désormais, dit Ferrière, il est nécessaire de «décentraliser les lieux» et de «répartir les tâches entre diverses personnes» (Ferrière, PEN, 1930, 54, p. 3). C'est ainsi que cinq bureaux - France, Suisse, Allemagne, Pologne, Angleterre - sont désignés comme sièges officiels, chacun d'entre eux étant placé sous la responsabilité d'un directeur et d'un secrétaire. Au niveau des personnes, le Comité central désigne un nouveau "Comité consultatif» dans le but de décharger le travail des trois présidents-fondateurs (Ensor, Rotten, Ferrière), un «Comité international» composé des correspondants de chaque pays ainsi que des «commissions permanentes» chargées d'étudier des thématiques particulières. Cette restructuration a des répercussions rapides dans les trois revues à commencer par la publication dans leurs pages de couverture des noms et rôles des nouveaux comités et commissions. D'autre part, c'est à partir de cette date que disparaissent, dans The New Era et DWZ, les principes de ralliement adoptés à Calais en 1921. Si cette disparition est plus tardive dans Pour l'Ėre Nouvelle (novembre 1932), elle est tout aussi significative des nouvelles orientations que prend la Ligue à ce moment-là.

Dans les trois revues, les principes de ralliement sont remplacés par un nouveau texte qui, pour la première fois, est différent d'une revue à l'autre. Tout se passe comme si désormais, chaque revue devenait autonome, prenant des décisions pour elle-même, en fonction des orientations qui lui sont propres. Dans The New Era, le texte est relativement court et reprend les grandes idées d'origine notamment à propos des «méthodes d'éducation adaptées à l'enfant» qu'elle continue de promouvoir. Il insiste toutefois sur la neutralité des positions de la Ligue qui, selon le texte, cherche à rassembler les éducateurs de divers pays et dont la revue permet de publier les expériences. La nouvelle orientation de la revue prise en juillet 1930 apparaît à travers «le souhait de créer des liens plus serrés entre les représentants de l'administration, les maîtres, les parents et les enfants».

The Fellowship seeks to draw together those in search of an education more suited to the needs and aspirations of modern life. It brings together educators of different countries and publishes news of their experimental findings. It seeks also a bond between administrators, teachers, parents and child. It 
presents an open platform from which the leading exponent of progressive education may deliver their message. The New Era published monthly, endeavors to express the ideals for which the Fellowship stands, and contains articles relating to progressive educational movements in all parts of the world. (The New Era, 1930, 11, p. 45, 3e de couverture)

Dans Pour l'Ère Nouvelle un texte plus conséquent et plus engagé remplace les principes de ralliement à partir de novembre $1932\left(2^{\mathrm{e}}\right.$ numéro spécial sur le Congrès de Nice) qui révèle le contexte de crise dans lequel se trouve le monde. De manière similaire aux anglophones, le texte de PEN lance un pressant appel «aux parents, éducateurs, administrateurs et travailleurs sociaux pour qu'ils s'unissent dans un vaste mouvement universel». Toutefois, les auteurs du GFEN le font de manière plus incisive en utilisant un vocabulaire révélateur du militantisme dans lequel ils sont engagés et du contexte socio-économique très difficile ${ }^{116}$ qui règne à ce moment-là justifiant l'urgence des mesures à prendre:

Seule une éducation réalisant dans toutes ses activités un changement d'attitude vis-à-vis des enfants peut inaugurer une ère libérée des concurrences ruineuses, des préjugés, des inquiétudes et des misères caractéristiques de notre civilisation présente, chaotique, et dépourvue de sécurité. Une rénovation de l'éducation s'impose, basée sur les principes suivants. («Charte», PEN, 1932, 82, p. 262)

Comme nous l'avons vu, les «nouveaux» principes, au nombre de cinq, se rapprochent de ceux d'origine sur certains points, notamment dans la volonté que les méthodes éducatives respectent l'individualité de l'enfant et ses caractéristiques personnelles. «Elle [1'éducation] doit être conçue de manière à répondre aux exigences intellectuelles et affectives diverses des enfants de tempéraments variés et leur fournir l'occasion de s'exprimer en tout temps selon leurs caractéristiques propres» (PEN, 1932, 82, p. 262). Par contre, ils s'en distinguent par l'importance qu'ils accordent désormais à l'éducation sociale, aux efforts que l'éducateur doit consentir pour «mettre l'enfant en mesure de saisir les complexités de la vie sociale et économique de notre temps» pour l'aider à «s'adapter volontairement aux exigences de la vie en société». Dans Das Werdende Zeitalter, les principes de ralliement disparaissent de la revue

116 La crise économique de 1929 se traduit par un fort taux de chômage, une dévaluation des monnaies et d'importants problèmes sociaux. 
en janvier 1930. À notre connaissance, aucun texte ne les remplace à partir de ce moment-là ${ }^{117}$ contrairement aux revues sœurs. Les travaux de Haubfleisch et Link (1994) sur DWZ ne font mention d'aucun texte de ce genre même si leur étude porte avant tout sur les acteurs et les thématiques du périodique.

\section{DES THÉMATIQUES SOUS INFLUENCE}

Les changements des années 1930 ne sont pas seulement structurels et administratifs. Nous constatons comme d'autres (Brehony, 2004; Gutierrez, 2008; Peyronie, 2002; Raymond, 2002), un virage important sur le plan idéologique et conceptuel qui transparaît dans les articles. Un tournant qui s'opère dans les trois revues ${ }^{118}$ en écho à des changements sociaux liés au contexte politique et économique mais qui reflète aussi, nous semble-t-il, une évolution de la conception de l'homme et de l'enfant dans son contexte. Un virage que Brehony (2004) définit comme un «déplacement de l'intérêt allant de l'enfant vers la société, pour les besoins individuels vers les besoins sociaux» (p. 750) ou encore comme «une rupture épistémique» (Brehony, 2009, p. 585). Comme nous l'avons montré ailleurs (Haenggeli-Jenni \& Hofstetter, 2011), les auteurs abordent désormais la question du renouveau éducatif sous l'angle de ses implications sociales et concrètes dans une optique de transformation des systèmes éducatifs à large échelle. Les psychologues qui siègent au comité éditorial de $P E N$, comme d'autres, s'orientent désormais vers une psychologie plus sociale - moins centrée sur l'individu - et vers une psychologie appliquée qui permet de mieux comprendre les mécanismes d'apprentissage.

Dans les années 1920, nous constatons une certaine concordance thématique entre les trois revues. D'une part, elles publient des articles identiques (traduits) au même moment ou à quelques mois d'intervalle. Il en va de même pour les comptes-rendus de congrès publiés quasiment au même moment dans les trois revues. D'autre part, il n'est pas rare de trouver des articles publiés d'abord dans The New Era en anglais,

117 Cependant, cette absence est peut-être liée aux volumes que nous avons dépouillés dont les numéros ont été reliés et dépourvus de leurs pages de couverture.

118 Das Werdende Zeitalter est à traiter quelque peu différemment vu sa disparition en 1932. 
puis traduits et publiés en français dans PEN ou en allemand dans DWZ quelques mois plus tard. ${ }^{119} \mathrm{Au}$ niveau des thématiques, nous remarquons, dans les trois revues, une forte prégnance de sujets en lien avec la philosophie, la spiritualité, le vitalisme, le spiritualisme, les lois universelles, la fraternité, l'énergie créatrice. Une concordance qui peut s'expliquer par l'importance de la théosophie au sein de la Ligue dans ces années-là de même que par la sensibilité spirituelle des trois rédacteurs qui transparaît à la fois dans leurs propres discours et dans celui des auteurs qu'ils publient.

Dans DWZ, c'est à travers Elisabeth Rotten que les conceptions de l'enfant comme potentiel de vie circulent. Docteur et professeur de philosophie, elle est sensible aux questions spirituelles d'autant plus qu'elle collabore étroitement avec les communautés Quaker ${ }^{120}$ auxquelles elle adhère officiellement en 1930. Elle partage ainsi l'idée que l'Homme est un être doté d'une énergie créatrice et d'une vitalité universelle et que l'éducation ne doit aucunement restreindre ni réprimer cet élan. Les nombreux articles sur la question publiés entre 1922 et 1925 prouvent cet intérêt. Elle semble attirée par les philosophies ésotériques, voire mystiques, au sein desquelles est laissée une grande liberté d'action à l'être humain. C'est ainsi qu'elle se lie d'amitié avec Martin Buber ${ }^{121}$, un philosophe aux idées mystiques, et qu'elle sollicite la contribution de penseurs et écrivains indiens tels que Rabindranath Tagore ou Mahatma Gandhi pour enrichir la réflexion sur les dimensions spirituelles de l'éducation. ${ }^{122}$ Elle partage aussi les convictions de certains membres du mouvement anarchiste allemand tel que son ami Gustav Landauer ${ }^{123}$, pour qui la liberté de l'Homme ne peut être garantie que par l'absence de règles, de normes, de codes. Dans PEN, c'est Ferrière qui relaie les

119 Ce phénomène est similaire pour les deux autres revues dont certains articles sont traduits et publiés à l'identique dans une des revues sœurs.

120 Pour des détails sur l'obédience Quaker, voir le chapitre 3.

121 Sur cet acteur le chapitre sur la revue allemande Das Werdende Zeitalter.

122 L'idéologie et la spiritualité hindoues inspirent également les théosophes comme le prouve l'étroite collaboration de Beatrice Ensor avec diverses personnalités indiennes depuis la fondation dans ce pays de la Theosophical Educational Trust en 1913. En 1929, une délégation indienne participe au Congrès d'Elseneur comme l'atteste une photo de groupe dans The New Era (1929, 40, p. 203).

123 Voir le chapitre sur la revue allemande Das Werdende Zeitalter. 
idées théosophistes et spiritualistes, convaincu de l'existence de lois universelles qui régissent à la fois l'individu et l'humanité toute entière. Ces idées influencent sa conception du développement de l'enfant qu'il considère comme l'incarnation d'un processus qu'il ne faut ni interrompre, ni orienter, ni formater. Ses idées sont perceptibles à l'intérieur de ses textes mais apparaissent surtout dans les éditoriaux de PEN des années 1922 à 1926. Dans DWZ, c'est plutôt dans les titres d'articles que l'on perçoit cette influence - la revue ne publie pas d'éditoriaux - que ce soit dans les articles écrits par Rotten ou par d'autres auteurs qui partagent ses convictions.

Cette concordance thématique prouve l'existence d'une certaine unité théorique et conceptuelle au sein de la Ligue au cours des premières années, ses objectifs étant alors de transformer l'humanité selon des «principes universels et spirituels». Cette concordance se perçoit également au niveau de la place accordée aux expériences menées dans les écoles, classes, établissements de différents pays. Comme le rappelle Beatrice Ensor à de multiples reprises, il s'agit d'inciter les lecteurs-praticiens à «faire de même», à «se lancer» en s'inspirant des expériences relatées dans les revues. C'est pourquoi les trois revues contiennent beaucoup d'articles de genre «documentation pédagogique» comme nous l'avons montré pour PEN. D'autre part, les articles écrits par les trois rédacteurs en chef sont fortement liés à des événements vécus par ces derniers, à l'exemple des conférences qu'ils donnent ou auxquelles ils assistent, des congrès et surtout, des voyages durant lesquels ces derniers visitent des écoles et rencontrent des personnalités du mouvement. Ainsi, le voyage d'Elisabeth Rotten aux États-Unis en 1928 donne lieu à trois articles de sa part - «Eindrücke aus Amerika» (DWZ, 1928, 7) «Eindrücke aus der Neuen Welt» (DWZ, 1928, 7), «Das andere Amerika» (DWZ, 1929) - ainsi qu'à un numéro spécial en fin d'année composé de cinq articles écrits par des Américain-es sur les expériences pédagogiques aux USA (DWZ, 1928, 7, pp. 473-524). Dans Pour l'Ère Nouvelle, on constate le même genre de pratique de la part de Ferrière. Suite au long voyage qu'il fait en Amérique latine ${ }^{124}$ en 1930, au cours duquel il donne 78 conférences, ce dernier écrit plus de cinq articles occupant quasi la totalité des premiers numéros de 1931 (PEN, 1931, 64, 65, 67) dans lesquels il décrit en détail les écoles visitées, les expériences en cours et les

124 Voir l'éditorial de janvier 1931 (PEN, 64) signé de Ferrière dans lequel il donne des détails de son voyage et de ses observations. 
personnes rencontrées, tout en faisant un état des lieux de l'avancée du mouvement sur ce continent. Dans The New Era, les événements vécus par Beatrice Ensor sont également très présents mais apparaissent peu dans ses articles et c'est plutôt dans les éditoriaux qu'il faut en chercher la trace. En effet, cette dernière investit beaucoup les «Outlook Tower» pour relater, commenter ou donner son opinion que ce soit sur des thématiques générales ou suite à des congrès, conférences, voyages.

\section{TROIS CONFIGURATIONS CONTRASTÉES ${ }^{125}$}

Mais cette apparente homogénéité thématique s'estompe au fil des ans, en particulier à partir de 1929 où les trois revues deviennent plus nationales privilégiant des sujets plus spécifiques aux pays qu'elles représentent. De fait, dès le début, chacune revêt certaines caractéristiques propres à l'environnement culturel dans lequel elle se développe. Ainsi, la place qu'occupe la psychologie dans Pour l'Ère Nouvelle est propre à ce journal, ne trouvant aucun équivalent dans les deux autres. Dès ses débuts, $P E N$ est très imprégnée par la psychologie, considérée comme $L A$ science de référence sur laquelle s'élaborent les nouvelles méthodes pédagogiques. Si notre analyse par domaines fait apparaître une dominance d'articles pédagogiques, celui de la psychologie - et tous les sous-domaines qui en découlent - occupe une place très importante. Au début, ce poids de la psychologie peut s'expliquer par la présence de Ferrière de 1922 à 1929, même s'il est rejoint par Decroly et Fauconnet en 1925. Ferrière est membre de l'Institut Rousseau et très influencé par les activités de recherche menées par d'illustres représentants de cette discipline (André Rey, Édouard Claparède, Charles Baudouin, Jean Piaget). Il est aussi un citoyen de Genève où la psychologie se développe de manière précoce dès le tournant du siècle avec la mise sur pied d'un laboratoire de psychologie par Théodore Flournoy au sein duquel Claparède se forme et procède à ses recherches (Hofstetter, 2010; Ratcliff \& Ruchat, 2006). Il apporte dans sa revue les valeurs et les concepts d'une science alors en pleine expansion dont il se fait le relais dans ses propres textes de même que dans le choix des articles et des contributeurs. La présence de Decroly au comité éditorial et aux

125 Les termes configurations contrastées sont empruntés à Hofstetter et Schneuwly (2007) qui les utilisent pour désigner les différentes formes que prend la disciplinarisation des sciences de l'éducation en Suisse. 
congrès de la Ligue contribue également à donner de l'importance à la psychologie, puisque ce dernier est lui-même un psychologue renommé. À partir de 1929, Ferrière se fait plus discret mais la dominance de la psychologie se poursuit à travers la présence de Wallon, Piéron et Piaget au comité éditorial. De plus, la revue porte la marque de l'évolution considérable que connaît la psychologie en France depuis la fondation en 1920 de l'Institut de Psychologie de l'Université de Paris par Henri Piéron et dont plusieurs représentants écrivent dans PEN (Ohayon, 2006).

Dans la revue allemande, les thématiques relèvent en grande majorité de la philosophie. Cette tendance est visible aussi bien au niveau des titres d'articles que des auteurs qui y écrivent. Ainsi, les textes de Buber, Gandhi, Tagore ou les pensées de philosophes tels que Goethe, Rousseau, Hegel imprimées en encarts dans les colonnes de Das Werdende Zeitalter montrent l'importance de cette discipline dans le champ éducatif germanophone. Le fait qu'Elisabeth Rotten soit une philosophe, fascinée par l'œuvre de Goethe, est aussi une des raisons de cette dominance. Cependant, grâce aux travaux de Helmchen (1993, 1995), Schriewer (1998), Schriewer et Keiner (1992), nous pouvons aussi la comprendre comme la manifestation d'une tradition culturelle allemande où la philosophie opère une influence considérable sur le développement de la pédagogie en général et sur celui du champ disciplinaire des sciences de l'éducation en particulier (Schriewer \& Keiner, 1992). Comme le montre Helmchen (1995), la Reformpädagogik porte l'héritage de l'idéalisme allemand «que domine l'individualisme et la théorie du sujet, c'est-à-dire de la personnalité» (p. 15) où l'on pense que le caractère de l'enfant se révèle de lui-même. Elle est aussi marquée par la conception allemande de l'enfant selon laquelle le développement de la personnalité récapitule celui d'une communauté plus archaïque qui existerait avant toute société (Helmchen, 1995); une conception qui rappelle celle de Ferrière et d'autres membres de l'Institut Rousseau qui pensent que l'ontogenèse refait la phylogenèse (Hameline, 2002a). Dès lors, des concepts tels que la Nature, la pureté, le respect des intérêts et aptitudes de l'enfant ne sont que quelques-unes des valeurs défendues par les promoteurs de la Reformpädagogik dont nous trouvons les marques dans DWZ. Pour leur part, Hofstetter et Schneuwly (2007) pensent que l'emprise de la philosophie qui caractérise les régions alémaniques découle d'un ensemble de facteurs imbriqués: «le mécanisme de différenciation interne des disciplines par élargissement des domaines; une défense explicite et militante 
des disciplines bien établies; la nécessité d'une reconnaissance académique de haut niveau [...]; la difficulté de concevoir la pédagogie comme science théorique autonome» (p. 220). Ainsi, les facteurs se combinent, qu'ils soient culturels, sociaux ou institutionnels, et contribuent à définir une conception de l'éducation et de la science qui l'étudie d'une manière propre à chaque région. Ces observations nous permettent de mieux comprendre pourquoi $D W Z$ revêt un caractère quelque peu isolé, différente des deux autres, où les thèmes en débat au sein de la Ligue révélant le processus de construction d'une nouvelle «science de l'éducation» ne semblent pas au cœur de ses préoccupations.

De son côté, la revue anglaise connaît une évolution marquée par deux phases dont l'année 1930 constitue le moment charnière. La première se caractérise par des thématiques à dominante pédagogique fortement inspirées de la théosophie dont l'influence se perçoit aussi bien dans les objectifs philosophiques attribués à l'éducation que dans la conception de l'enfant et de son développement. Ainsi, l'idée selon laquelle il y aurait une «force intérieure» en chaque enfant que l'éducation a pour tâche de stimuler et libérer constitue une des convictions fortes des auteurs de The New Era. L'analyse des sommaires permet également de repérer la présence de nombreux articles évoquant les recherches menées en psychologie notamment sur le développement de l'enfant, sur les types psychologiques et sur les divers tests grâce auxquels les éducateurs devraient pouvoir mesurer les aptitudes de leurs élèves. Progressivement, la vision spiritualiste du début des années 1920 fait place à une conception beaucoup plus pragmatique de l'éducation s'inspirant de progressistes américains tels que Dewey ${ }^{126}$, Kilpatrick ou Washburne, ces deux derniers écrivant dans la revue. Une évolution qui débute dans les années 1926-1927 et qui trouve son apogée en 1930, date à laquelle cette évolution se concrétise par un changement radical d'orientation. Dès ce moment-là, la revue adopte un discours résolument tourné vers les dimensions pratiques et appliquées de l'Éducation nouvelle, accordant une place beaucoup plus importante encore aux diverses expériences pratiques à l'œuvre dans différents contextes. Désormais, les théoriciens n'ont presque plus leur place, la revue privilégiant clairement les contributions des praticiens, des parents et des spécialistes de la Home Education. Cette nouvelle orientation évolue toutefois au cours des

126 Sur John Dewey et les fondements philosophiques de sa pensée, voir Oelkers (2009), Renier (2013) et Bruno-Jofré, Johnston, Jover \& Tröhler (2010). 
années 1930 vers une version plus nuancée des discours se traduisant par une répartition plus équilibrée des thématiques scientifiques issues de la recherche et de celles issues des expériences du terrain.

Grâce à l'analyse comparative de ces trois revues, nous constatons comme d'autres (Charle, Schriewer \& Wagner, 2004; Hofstetter \& Schneuwly, 2007) l'importance des schémas nationaux et des traditions culturelles dans les supports éditoriaux qui se veulent et se disent «internationaux». Une contradiction qui rejoint celle que relèvent Charle, Schriewer et Wagner (2004) à propos des institutions académiques qui, depuis le $19^{\text {e }}$ siècle, se caractérisent par une "persistante contradiction entre les revendications de cultures académiques définies nationalement d'une part, et par l'universalisme de la science, d'autre part» (p. 11). Ces derniers relèvent aussi la tension qui émerge inévitablement entre la forme nationale et l'orientation transnationale - voire universelle - dans laquelle la pratique des intellectuels est simultanément engagée. Notre analyse comparative des revues de la Ligue met en évidence l'influence des traditions culturelles sur les discours pédagogiques qui se développent dans des contextes locaux et nationaux dont ils portent la marque durablement. Les travaux récents de Hofstetter et Schneuwly et leurs collaborateurs (2007) confirment une tendance similaire à propos de l'émergence du champ disciplinaire des sciences de l'éducation en Suisse. Au terme de leur enquête, ils concluent à la présence de "configurations contrastées» marquées par les traditions culturelles des régions dans lesquelles le champ se développe, la région germanophone se caractérisant par une configuration "philosophicopédagogique» dominée par le modèle allemand (sous l'emprise de la philosophie). Quant à elle, la région francophone (Suisse romande) se caractérise par une configuration "pédagogique» (Lausanne, Fribourg, Neuchâtel) où le champ disciplinaire se développe en articulation étroite avec la profession tandis que Genève se distingue par une configuration «psycho-pédagogique» (Genève) où la psychologie orientée vers l'international détermine fortement l'évolution du champ. Ces remarques rejoignent celle de Célestin Bouglé (1870-1940)157 qui affirme que «les sciences sociales, plus que toutes autres disciplines académiques, conservent la marque de leur origine sociale» (Bouglé, 1938, cité par Schriewer et Keiner, 1992, p. 24). Selon Schriewer et Keiner (1992), cette

127 Philosophe et sociologue français, professeur de sociologie à la Sorbonne en 1901 et directeur de l'École normale Supérieure de Paris dès 1935. 
citation confirme le constat que les sciences sociales sont profondément «ancrées dans» et «affectées par» les traditions intellectuelles et par le cadre institutionnel qui prévalent dans différents contextes nationaux et socioculturels» (p. 25).

Enfin, l'analyse des trois périodiques révèle une grande diversité d'acteurs qui se différencient par leurs appartenances professionnelles, culturelles, confessionnelles ainsi que par les multiples influences qu'ils subissent en lien avec la région dans laquelle ils vivent et les personnes qu'ils côtoient. Dès lors, on peut se demander quelle est la raison qui pousse tous ces acteurs à se rassembler et partager leurs savoirs, leur expérience, leur intelligence pour améliorer l'éducation et le sort de l'enfant. Selon Raymond (2002), ce qui mobilise ces pédagogues, bien plus que les découvertes psychologiques, c'est un idéal d'ordre pédagogique et même politique: «celui de la régénération de l'homme par une éducation renouvelée de l'enfant» (p. 201). Cette interprétation rejoint celle de Mari (2011) pour qui éduquer signifie «agir de manière prospective», c'est-à-dire «agir à partir du moment présent en quête d'un résultat futur». Selon lui, c'est la raison pour laquelle l'éducateur a besoin d'un «modèle anthropologique» - qu'il ne s'agit pas d'appliquer comme un protocole abstrait - mais qui doit «être fondé pour être justifié» (p. 43). Comme le montrent nos analyses, la science joue ici un rôle crucial, servant de justification à des ambitions qui sont tout sauf scientifiques, appartenant plutôt à des ambitions d'ordre philosophique. Selon Soëtard et Le Bouëdec (2011), ces ambitions participent de la «foi du pédagogue» et permettent de dépasser la «rationalité éducative» qui caractérise l'école, de nos jours comme dans le passé (p. 8). 


\section{ChAPITRE 3}

\section{DES AGENTS ET DES RÉSEAUX: PROFILS D'UNE MILITANCE INTERNATIONALISÉE}

Ce chapitre propose une étude approfondie des acteurs et actrices du mouvement d'Éducation nouvelle, ceux et celles qui l'ont façonné à travers leurs expériences, discours et réflexions, qu'ils soient praticiens, théoriciens, scientifiques ou penseurs. Ces derniers sont à la fois les artisans et les porteurs du discours militant: ils le formulent, l'adaptent et le diffusent à travers leurs écrits et leurs actions. Leur discours n'est pas neutre, car il exprime toujours une part de leur identité, de leur culture et leur ancrage professionnel. Selon les personnes, un discours scientifique, politique, religieux se mêle à leurs pensées éducatives, le mélange étant plus ou moins prononcé selon les contextes et les enjeux. Dans ce chapitre, nous procédons d'abord à une analyse des diverses sphères d'activité des acteurs en s'intéressant à leurs appartenances professionnelles et extra-professionnelles afin de mieux comprendre comment se construit leur discours et d'où il est produit. Dans un second temps, nous proposons une focale sur les femmes engagées pour la cause de l'Éducation nouvelle afin de cerner le rôle qu'elles jouent dans la diffusion du mouvement et dans la production de savoirs sur l'enfant et l'éducation. Enfin, nous étudions les réseaux sociaux dans lesquels sont insérés les acteurs, que ce soit sur le plan privé ou professionnel. Plus globalement, il nous intéresse de repérer l'existence de réseaux internationaux dont la Ligue aurait bénéficié pour étendre son influence et de repérer ainsi dans quel type d'internationalisation elle s'inscrit.

\section{ACTEURS HYBRIDES ET POLYVALENTS}

L'analyse quantitative de Pour l'Ère Nouvelle permet d'identifier les activités professionnelles des auteurs. Toutefois, les chiffres du tableau 
suivant sont indicatifs car dans de nombreux cas, les données sont incomplètes: activité professionnelle non précisée, activités multiples ${ }^{1}$, changement de profession au cours de la période étudiée.

Tableau 10. Activités professionnelles des auteurs de PEN

\begin{tabular}{lccc}
\hline Profession & Hommes & Femmes & Total \\
\hline Instituteur & 8 & 12 & $\mathbf{2 0}$ \\
\hline Inspecteur & 15 & 7 & $\mathbf{2 2}$ \\
\hline Directeur & 46 & 30 & $\mathbf{7 6}$ \\
\hline Professeur & 49 & 15 & $\mathbf{6 4}$ \\
\hline université & 19 & 1 & $\mathbf{2 0}$ \\
\hline lycée & 5 & 3 & $\mathbf{8}$ \\
\hline non précisé & 25 & 11 & $\mathbf{3 6}$ \\
\hline Secrétaire & 7 & 7 & $\mathbf{1 4}$ \\
\hline Médecin & 4 & 2 & $\mathbf{6}$ \\
\hline Psychologue & 7 & 1 & $\mathbf{8}$ \\
\hline Philosophe/profs de philo & 9 & 2 & $\mathbf{1 1}$ \\
\hline Autres & & & $\mathbf{3}$ \\
\hline Total & 3 & $\mathbf{9 1}$ & $\mathbf{2 8 8}$ \\
\hline
\end{tabular}

Les profils des auteurs sont donc difficiles à définir tant ceux-ci sont flous et multiformes. La plupart cumulent des fonctions dans les sphères à la fois professionnelles, associatives, politiques et religieuses et changent d'appartenance au cours de la période étudiée. Ce volet vise à étudier de plus près l'activité de ces acteurs à l'aide d'une analyse croisée des données issues de la revue et d'indications biographiques provenant de recherches complémentaires sur les acteurs principaux de la Ligue. Il s'inspire en outre de travaux d'historiens ayant étudié cette question à

1 Par exemple, Ovide Decroly est à la fois médecin, psychologue, professeur (Université de Bruxelles) et directeur d'école (École de l'Ermitage).

2 Autres: 1 préfet; 1 maître de conférence; 1 agrégé. 
l'exemple d'Hameline (2002a), Hofstetter (2010), Hofstetter et Schneuwly (2006), Middleton (2013), Savoye (2006).

Tout d'abord, l'analyse de la revue permet d'identifier une grande hétérogénéité d'acteurs au niveau de leur nationalité, profession, culture, langue, activités professionnelle et extra-professionnelle. Certes, tous ces acteurs militent pour l'Éducation nouvelle, mais à des niveaux différents (statut, position hiérarchique, niveau de responsabilité) avec des références différentes (culturelles, sociales, politiques, économiques) et à des échelles différentes: échelle locale (classe, école, établissement), échelle régionale (ex. inspecteur de région, inspecteur d'académie, etc.), échelle nationale (ministres de l'éducation, politiciens impliqués dans l'administration scolaire), échelle internationale (penseurs, philosophes, fondateurs de la Ligue). Dans Pour l'Ère Nouvelle, cette hétérogénéité apparaît d'abord comme une richesse, voire une force qui donne une puissance particulière à la «mission» dont ces acteurs se sentent investis: réformer l'école sur la base d'une connaissance scientifique de l'enfant pour construire un homme nouveau capable de transformer la société. Mais dans certains cas, cette diversité donne lieu à des tensions qui se traduisent dès les années 1930, par des scissions voire la création de groupes parallèles à la Ligue. De fait, cette hétérogénéité constitue la caractéristique même de l'Éducation nouvelle et plus encore de la nouvelle science en train de se construire. L'analyse de la revue montre que cette science se construit précisément grâce à cette diversité de références théoriques, d'horizons disciplinaires, d'approches, de méthodes, de concepts, qui l'enrichissent et en font sa principale originalité.

\section{TROIS GROUPES DE PROFESSIONNELS}

Les acteurs dont il est question dans Pour l'Ère Nouvelle proviennent de milieux professionnels divers parmi lesquels on trouve les professions de l'éducation (enseignants de niveau préscolaire, primaire, secondaire), de l'administration scolaire (inspecteurs, directeurs, administrateurs), de la santé (médecins, neurologues, psychiatres, psychologues), de la politique (ministres de l'instruction publique, directeurs de départements d'instruction publique, de régions, etc.) ainsi que diverses professions issues des sciences sociales (philosophe, anthropologue, sociologue) et des sciences naturelles (physiciens, biologistes, zoologues). Toutefois, selon Jenkins (2000), cette diversité professionnelle est relative car il s'agit en fait de «professions reliées» (related professions) (p. 139), 
certaines catégories socio-professionnelles étant totalement absentes de ce panorama: ouvriers, commerçants, artisans, industriels. En effet, malgré des références conceptuelles différentes, les professionnels que l'on rencontre dans la revue partagent un objet d'étude commun - l'enfant et son éducation - et surtout une curiosité générale pour l'être humain, que ce soit sous l'angle de son fonctionnement physiologique, biologique, psychologique ou de son rôle dans l'ordre du monde et de la vie. Globalement, nous pouvons distinguer trois grands groupes de professionnels constituant les principaux protagonistes du mouvement d'Éducation nouvelle: les praticiens, les représentants de l'administration et les théoriciens. ${ }^{3}$

\section{Les praticiens}

Nous appelons praticiens les professionnels de l'éducation qui pratiquent un métier éducatif quel que soit le nom qu'on leur donne (éducateur, instituteur, maître, enseignant) et l'ordre d'enseignement dans lequel ils travaillent. Malgré la diversité de leur terrain d'application, ces professionnels se ressemblent par le fait qu'ils sont en contact quotidien avec des apprenants (enfants, adolescents, jeunes adultes) et sont responsables de leur faire acquérir un certain nombre de connaissances et de compétences, dans une durée limitée dans le temps. Nous excluons de ce groupe les professeurs de niveau universitaire qui, même s'ils sont enseignants, pratiquent en parallèle une activité de recherche; de ce fait, ils appartiennent avant tout au monde académique, ce qui les place plutôt dans le troisième groupe appelé «théoriciens».

L'analyse des discours montre des positions diverses concernant les éducateurs «nouveaux», qui selon certains auteurs, ne seraient pas des «enseignants comme les autres». Plusieurs auteurs, à l'exemple de John Eades $^{4}$ affirment que les membres de la Ligue sont différents de la «masse», qu'ils n'appartiennent pas à ce «corps enseignant conserva-

3 Ces trois groupes auraient pu être appelés différemment - par exemple les praticiens pourraient être appelés «éducateurs» mais nous reprenons ici les termes utilisés dans des travaux antérieurs utilisant ces mêmes catégories (Hofstetter \& Schneuwly, 2002, 2007).

4 Directeur de l'école d'application de l'École normale de Kirkstall à Londres (GB). 
teur», «opposé aux changements», à qui «toute nouvelle idée est suspecte», et qui ne «voit pas la raison d'être de la nouvelle psychologie ou de la nouvelle pédagogie» (Eades, 1923, PEN, 8, p. 89). Ce parti pris nous semble très «sectaire» et marque une différence nette entre un corps enseignant considéré comme conservateur et les partisans de l'Éducation nouvelle qui seraient des novateurs avertis. Georges Bertier, directeur de l'École des Roches, est plus nuancé sur la question. Selon lui, ce qui distingue les éducateurs «nouveaux» des enseignants d'écoles publiques, ce qui fait leur «raison d'être», c'est leur «audace, la hâte de réaliser, la soif de liberté, la passion du progrès, la haine du préjugé et de la routine, l'amour enthousiaste de l'enfant» (Bertier, 1923, PEN, 8, p. 97). Une analyse approfondie de cette revue montre que ces praticiens se caractérisent par leur esprit d'ouverture, leur goût pour l'innovation, plus globalement, une attitude distanciée par rapport à leur métier et à leur pratique. Nous pourrions les rapprocher de ce que Perrenoud (2001) appelle des "praticiens-réflexifs», c'est-à-dire des enseignants qui s'interrogent sur leur profession, leur mandat vis à vis des élèves, leur rôle par rapport à la mission de l'école, et qui s'investissent pour se former et progresser dans leur métier. Cette attitude se traduit par une activité d'écriture qui implique non seulement une prise de distance par rapport à sa pratique professionnelle mais aussi une prise de conscience de l'existence de "collègues» faisant partie d'une «corporation», d'une «profession» (professionnalisation). Comme le montrent les travaux de Lussi Borer (2008) et Hofstetter (2008), le corps enseignant se professionnalise à travers la mise en place progressive de formations de haut niveau au cours du premier $20^{\mathrm{e}}$ siècle exigées notamment par la nouvelle appréhension de l'enfance et de son éducation.

On assiste ainsi à Genève, comme dans de multiples contrées, à l'apparition d'un nouveau type d'enseignants, des praticiens qui s'initient progressivement à la recherche expérimentale, notamment psychologique dont on attend des réponses pour résoudre divers problèmes pédagogiques et qui n'hésitent pas à mener eux-mêmes des investigations empiriques (Depaepe, 1993; Hofstetter, 2010).

Nombre de ces praticiens se réunissent en associations pour mettre en commun leurs pratiques, leurs expériences mais surtout pour mieux faire entendre leur voix et ainsi avoir un impact sur l'ensemble du corps enseignant. De fait, ceux qui se regroupent ainsi le font d'abord en faveur d'une cause, révélant par là-même des prises de position et un engagement idéologiques que l'on peut appeler militance. Ainsi, les 
promoteurs de l'Éducation nouvelle se distingueraient des enseignants avant tout et surtout, par leur engagement militant, notamment en devenant membre de la Ligue.

Pour réussir la réforme totale de l'enseignement, il faut d'abord obtenir l'entente entre tous les éducateurs qui, réunis en une vaste corporation enseignante (tels les maçons qui au Moyen-Age construisaient les cathédrales) se mettront ensemble à l'œuvre pour «rebâtir la maison de fond en comble. [...] Ces maîtres-constructeurs ne seront plus ceux que l'on appelait avec dédain «des intellectuels» $[. .$.$] «des rationalistes» [. .$.$] «des individualistes» qui ne$ savent pas que l'union fait la force, mais des réalisateurs, vivant et agissant dans le présent et pour l'avenir. (Decroix, 1922, PEN, 2, p. 35)

En publiant leurs témoignages et récits d'expériences dans Pour l'Ère Nouvelle, ces enseignants contribuent à la mise en commun de connaissances sur l'éducation, autrement dit à la construction de la nouvelle science. Ils confient ainsi leurs questions, leurs doutes, leurs difficultés mais aussi leur enthousiasme et leurs crédos à tous ceux qui œuvrent pour la même cause.

Si nous voulons réussir notre tâche d'aider chaque enfant à développer son âme, nous devons considérer de nouvelles idées et de nouvelles méthodes. Nous devons être prêts à les essayer et adopter celles que nous trouvons être bonnes dans leur application. Il est aussi important pour l'instituteur de se tenir au courant des derniers développements de la science et de la pratique pédagogique, qu'il est important pour le médecin de se tenir au courant des derniers développements de la science médicale et chirurgicale: c'est ainsi seulement que nous rendons notre travail digne de porter le nom de «profession». (Eades, 1923, PEN, 8, p. 89)

Tout comme dans le précédent extrait de Decroix qui utilise les métaphores de «bâtisseurs» et «maîtres-constructeurs», John Eades défend ici une conception du métier d'enseignant qui s'impose progressivement, selon laquelle ce métier est à considérer comme une réelle "profession», comparée ici à celle de médecin. L'Éducation nouvelle participe activement à ce processus de professionnalisation (Lussi Borer, 2009) en reven-

5 L'auteur de cet article intitulé «Les Compagnons et l'École unique» est membre des Compagnons de l'Université Nouvelle, groupe qui revendique une école unique et un corps enseignant uni. 
diquant un nouveau type de formation pour les enseignants. Selon le fondateur de l'Institut Rousseau, cette formation doit être de haut niveau et comporter une dimension à la fois théorique et pratique, offrant la possibilité aux étudiants de s'initier aux méthodes scientifiques expérimentales.

\section{Les représentants de l'administration}

Le deuxième groupe est constitué des professionnels de l'éducation représentant l'administration scolaire: directeur-trices (d'écoles et/ou d'instituts de formation) et inspecteur-trices qui se distinguent des praticiens par le fait qu'ils assument un rôle de direction ou de contrôle. Contrairement aux acteurs du premier groupe, ces derniers ont pour tâche première de diriger non plus des enfants mais des adultes. En ce sens, même si l'essentiel de leur activité se déroule sur le «terrain» (écoles ou autres établissements éducatifs), ils ne sont pas en contact direct avec les élèves et n'ont pas de responsabilité de leurs apprentissages. Ils assument un rôle d'intermédiaire entre les enseignants, les parents et les représentants de l'autorité scolaire, ce qui implique des tâches administratives. Dans les établissements privés, les directeurs doivent gérer à la fois les aspects pédagogiques (programmes, projets, cursus) et les aspects «pratiques» tels que les finances, l'engagement du personnel, l'entretien des bâtiments, l'achat des fournitures. Cette double fonction rend leur tâche complexe mais celle-ci a l'avantage de pouvoir être assumée hors de tout contrôle hiérarchique et exigence institutionnelle. C'est précisément ce qui différencie les directeurs des inspecteurs: alors que les premiers sont libres d'assumer leur rôle comme «bon leur semble» - pour autant que l'établissement fonctionne - les seconds sont soumis à des exigences institutionnelles liées à leur fonction (cahier des charges, attitude, éthique). Les méthodes d'enseignement revendiquées par les partisans de l'Éducation nouvelle nécessitent non seulement une nouvelle formation des enseignants, mais aussi un ajustement du rôle des inspecteurs. Selon les auteurs de la revue, ceux-ci devraient désormais connaître les nouvelles manières d'enseigner et respecter la «liberté intellectuelle» des enseignants de même que la liberté «dans le choix et dans l'application des méthodes». La fonction d'inspecteur fait donc l'objet de nombreuses recommandations, à l'exemple de celles qui sont adoptées lors de la VIe Conférence internationale de l'Instruction Publique réunie à Genève du 19 au 23 juillet 1937, sur la convocation du Bureau International d'Éducation: 
Une fois formés, ils [les maîtres] doivent être soutenus, encouragés et guidés et ce rôle incombe essentiellement et tout naturellement aux inspecteurs. Cela implique que les inspecteurs soient choisis après enquête sérieuse sur leurs aptitudes morales et intellectuelles à l'exercice d'une fonction délicate entre toutes; que nul ne puisse être appelé à cette fonction s'il n'a préalablement affirmé sa curiosité et sa compréhension des questions relatives à l'éducation, ceci par une préparation spéciale comportant l'étude de la pédagogie comparée et celle des systèmes d'organisation scolaire d'autres pays. [...]. Les inspecteurs doivent en outre se maintenir au courant du mouvement pédagogique, en avoir non seulement le devoir mais le loisir, pouvoir voyager à l'étranger, participer à des stages et cours spéciaux, aux travaux de commissions d'études. (Ferrière, 1939, PEN, 144, pp. 41-42)

Comme l'évoque cet extrait, les inspecteurs ont le «devoir» de se tenir au courant des avancées pédagogiques et pour cela, de participer à des manifestations, stages et cours présentant les dernières découvertes sur le sujet. Pour cela, les administrations scolaires devraient leur permettre d'y participer en leur accordant du temps et de la liberté dans la pratique de leur fonction. Cette recommandation permet de mieux comprendre pourquoi les inspecteurs constituent l'un des groupes d'acteurs les plus nombreux ${ }^{6}$ aux congrès de la Ligue ainsi que parmi les auteurs de la revue, car ils ont aussi pour mission d'écrire et faire part de leurs expériences.

\section{Les théoriciens}

Le troisième groupe est formé d'intellectuels, universitaires, provenant aussi bien des sciences naturelles (médecins, psychologues, psychiatres, biologistes) que des sciences sociales (philosophes, pédagogues, anthropologues); tous, avec les outils conceptuels de leur domaine, étudient l'enfant et œuvrent pour améliorer son éducation. Nous utilisons le terme théoriciens pour qualifier ces personnes qui ont une activité de recherche visant à mieux connaître l'enfant, qui élaborent des théories - psychologiques ou pédagogiques - en lien avec leurs découvertes dans le but de transformer les pratiques éducatives. Ce groupe d'acteurs partage plusieurs points communs, à commencer par leur formation initiale. Celle-ci est à la fois spécialisée et générale: il s'agit d'une formation multiple qui s'effectue soit simultanément, soit successivement, dans plusieurs champs disciplinaires (se traduisant par plusieurs titres ou

6 Le groupe le plus important est celui des directeurs-trices. 
diplômes). Ainsi, il n'est pas rare de voir des acteurs étudier parallèlement (ou successivement) la médecine, la philosophie, la théologie, la psychologie, la biologie, la sociologie. Des formations qui s'effectuent, pour la plupart, selon le cursus classique universitaire mais aussi, dans certains cas, sous forme de stages prolongés (plusieurs mois ou années) auprès de spécialistes confirmés. C'est le cas notamment de ceux qui désirent se former en psychologie puisqu'au début du $20^{\mathrm{e}}$ siècle cette science ne possède pas encore de cursus universitaire spécifique. Pour exemple, voici un bref aperçu des formations initiales de quelques figures de l'Éducation nouvelle:

Pierre Bovet: études de théologie, licence en lettres, doctorat en philosophie, puis professeur de philosophie et pédagogie expérimentale.

Ovide Decroly: études de médecine, psychiatrie, neurologie, psychologie. Édouard Claparède: études en sciences naturelles et en médecine; psychologie auprès de Wundt (Leipzig) et Flournoy (Genève).

Henri Piéron: études en lettres, agrégation de philosophie, psychologie auprès des psychologues français (Ribot, Janet, Lévy-Bühl), doctorat en sciences naturelles (physiologie).

Henri Wallon: études à l'école normale supérieure, agrégation de philosophie, études de médecine, études de psychologie et psychiatrie, thèse en psychologie.

Elisabeth Rotten: études de philosophie, littérature allemande, puis littérature anglaise, une année à Cambridge.

Adolphe Ferrière: licence en sciences sociales, doctorat en sociologie (thèse sociologie et biologie), puis pédagogie auprès de $\mathrm{H}$. Lietz en Allemagne.

Maria Montessori: études de mathématiques, sciences physiques et naturelles, études de médecine, puis psychologie et philosophie; enseigne l'histoire de l'anthropologie, puis s'initie à la logopédie dans le cadre de l'école pour enfants anormaux qu'elle dirige.

Comme nous pouvons le voir, nombreux sont ceux qui cumulent différentes formations et donc différentes approches. Ceci explique d'une part la richesse de leur perception des questions éducatives, et d'autre part, leur ouverture aux idées des autres spécialistes dont ils peuvent comprendre les conceptions et systèmes de pensée. C'est précisément cette diversité d'approches qui permet à ces intellectuels d'envisager 
l'éducation à plusieurs niveaux, que ce soit sur un plan psychologique, médical, philosophique, scientifique, religieux, politique ou pédagogique et qui prouve que pour appréhender l'enfant dans toute sa complexité, cette diversité est nécessaire. Ce constat explique pourquoi le champ disciplinaire des sciences de l'éducation, alors en construction, est d'emblée un champ «pluriel» - pluridisciplinaire - fait d'une multitude d'approches qui loin de s'exclure se combinent, se complètent, se nuancent (Hofstetter \& Schneuwly, 2007).

Ces théoriciens se caractérisent en outre par leur engagement multiple dans diverses associations, commissions ou fondations au sein desquelles ils assument des fonctions dirigeantes. On peut les qualifier de «cosmopolites» car ils effectuent beaucoup de voyages au cours desquels ils donnent des conférences, font des visites, participent à des réunions et des congrès. Ils se caractérisent par une activité d'écriture conséquente que ce soit sous forme d'ouvrages (livres), d'articles dans des revues (spécialisées, professionnelles, de vulgarisation) ou dans la presse, de compte-rendu, de rapports, ou plus simplement, de correspondance personnelle. Ces acteurs se caractérisent enfin par leur rôle «médiatique», que ce soit sur les scènes éducative, associative ou politique. Ils y interviennent en donnant des conférences, en rédigeant des manifestes mais aussi en soutenant des projets concrets destinés à faire évoluer l'école (projets de lois, chartes, règlements, etc.) et sur lesquels leur renommée peut avoir une influence. ${ }^{7}$ Sur le plan politique, ils se situent plutôt à gauche de l'échiquier politique et peuvent être qualifiés de «solidaristes, socialistes ou radicaux» (Ohayon, 2006, p. 18) quoique les termes ne signifient pas la même chose dans tous les pays. Selon Vidal (1989), les membres de l'Institut Rousseau et du Bureau International d'Éducation pratiquent un «socialisme modéré», certains d'entre eux contribuant activement au mouvement du «christianisme social». En France, Bertier est considéré comme un «catholique social» participant à certains mouvements syndicaux (Poucet, 2007); les psychologues comme Wallon ou Piéron fréquentent les loges de la francmaçonnerie avant de s'orienter vraiment à gauche dès les années 1930 en devenant compagnons de route du Parti communiste (Ohayon, 2006).

7 Ainsi, la composition illustre de la commission Langevin-Wallon n'est-elle pas sans influence sur la réception du plan de réforme au niveau du ministère. Cette influence s'avèrera toutefois insuffisante à la mise en application complète du projet (Gutierrez \& Kounelis, 2010). 


\section{DIVERSES FORMES D'HYBRIDITÉ}

Grâce à une analyse fine des activités de plusieurs acteurs de la Ligue, nous constatons que ceux-ci cumulent plusieurs rôles et fonctions conduisant à un statut hybride reliant des sphères a priori distantes l'une de l'autre. Nous pouvons dégager plusieurs «types d'hybridité» définis par les sphères qui sont ainsi mises en relation, sachant toutefois que pour certains acteurs, ces types se combinent, se croisent voire se superposent. Cette hybridité explique en partie la difficulté de les catégoriser dans un groupe ou un autre. Par exemple, les théoriciens qui écrivent dans Pour l'Ėre Nouvelle sont souvent très impliqués sur le terrain car ils y effectuent leurs recherches au moyen des méthodes expérimentales (psychologie et/ou pédagogie expérimentale); en ce sens, les écoles constituent leur terrain de recherche et jouent le rôle de laboratoire. Par exemple, Decroly, en tant que médecin psychiatre appartient au troisième groupe défini plus haut. Mais parallèlement, il assume la direction de l'École de l'Ermitage, et de ce fait, appartient aussi au deuxième groupe des directeurs d'école. Il en va de même pour de nombreux scientifiques (Piaget, Claparède, Wallon, etc.) qui œuvrent à la fois dans leurs laboratoires et sur le terrain, en particulier dans les écoles dites «écoles d'application», rattachées aux instituts de recherche. Que ce soit Claparède à la Maison des Petits, Dewey à la Laboratory School de Chicago ou Wallon dans l'école qu'il crée en banlieue de Paris pour mener ses recherches, tous sont actifs simultanément dans les deux sphères. Cette hybridité se manifeste également dans leur double activité d'enseignement et de recherche qui, de fait, caractérise la plupart des universitaires. Ainsi, Decroly est à la fois médecin (neurologie, psychiatrie), directeur d'école (École de l'Ermitage), professeur de psychologie de l'enfant à la Section de Pédagogie de l’Université de Bruxelles (dès 1920) et chargé de cours d’hygiène éducative et médico-pédagogique (1921) dans cette même université. Claparède est à la fois médecin, psychologue, directeur du Laboratoire de psychologie expérimentale de Genève, fondateur et rédacteur de la revue Archives de Psychologie, professeur de psychologie expérimentale à l'Université de Genève (1908-1940) tout en étant membre puis président de la Société Pédagogique Genevoise (19141916). Cette hybridité a pour conséquence d'agrandir les réseaux dans lesquels ces acteurs évoluent puisqu'ils sont impliqués à la fois dans des réseaux scientifiques et des réseaux éducatifs militants. 
Du côté des praticiens, nombreux sont ceux qui collaborent avec les théoriciens, par exemple en pratiquant des tests ou des enquêtes dans leur classe ou simplement en mettant à disposition leurs élèves pour des entretiens ou des observations. Dès lors, ces praticiens sont davantage que de «simples» instituteurs-trices puisqu'ils sont sollicités pour participer à la recherche, faire part de leurs observations, évaluer des méthodes, donner leur avis sur des élèves. Ils sont donc amenés à écrire, à rédiger des rapports, à publier des articles dans lesquels ils formalisent leurs observations et donc leurs savoirs. De ce fait, nous pouvons dire qu'ils se situent au carrefour des premier et troisième groupes tant l'interdépendance des deux mondes est cruciale. Les praticiens ont besoin des théoriciens pour légitimer leurs idées de réforme tandis que les théoriciens ont besoin des praticiens pour éprouver leurs théories en les testant sur les élèves.

\section{Hybridité science-politique}

Parmi les acteurs les plus actifs au sein de la Ligue, nombreux sont ceux qui, en parallèle de leur activité professionnelle, sont engagés dans des milieux associatifs ou politiques. Si ces deux sphères paraissent distinctes de prime abord, nous avons choisi de les réunir ici car le type d'activité que les acteurs y engagent se ressemble à plus d'un titre. En effet, l'activité y est de type militant dans le sens où ces acteurs défendent une cause à laquelle ils dédient leur temps, leur savoir - parfois même leur argent dans le but ultime de transformer la réalité des choses, que ce soit au niveau des mentalités ou de la société. Toutefois, elles se situent à deux niveaux différents: la sphère associative correspondant à un premier niveau de militance, où les idées prennent forme, évoluent, s'affinent à travers les débats et les échanges d'idées, la sphère politique correspondant à un deuxième niveau plus opérationnel, où les idées émises dans la première sphère se concrétisent à travers des projets d'application ou de lois (pour autant que la volonté politique soit là).

Les acteurs dont il est question dans Pour l'Ère Nouvelle sont tous engagés à des degrés variables dans des mouvements associatifs, à commencer par la Ligue. Certains y assument des fonctions telles que président, secrétaire, trésorier et appartiennent à plusieurs groupes en parallèle. À titre d'exemple, voici quelques noms de personnes cumulant ces diverses fonctions: Georges Bertier est directeur de l'École des Roches et président des éclaireurs de France; Charles Baudouin est professeur à l'Institut Rousseau et président de la Société internationale de 
psychagogie et psychothérapie; Eleanor Crosby Kemp est psychologue clinique et vice-présidente de la Ligue pour l'hygiène mentale infantile à New York; C. Philippi van Reesema est directrice des cours pour instituteurs et institutrices de La Haye et présidente de l'Association «Pour Promouvoir l'Esprit de $\mathrm{M}^{\text {me }}$ Montessori». Si l'association dont ils sont membres édite une revue, certains sont membres du comité de rédaction à l'exemple de Gertrud Hartman (USA), secrétaire de Progressive Education Association et éditrice de la revue du même nom.

Les acteurs de la Ligue se caractérisent par leur proximité à la sphère politique, que ce soit à une échelle locale, nationale ou internationale. De fait, tous aspirent, à une échéance plus ou moins proche, à la généralisation des méthodes nouvelles à l'ensemble des écoles d'un pays, voire à celles de tous les pays si l'on considère les discours universalistes des dirigeants de la Ligue. Ce lien «science-politique» devient crucial dans les années 1930 où le contexte politique et économique rend urgente une application des méthodes nouvelles à large échelle, c'est-à-dire dans l'enseignement public. Comme le relève Jenkins (2000), l'une des forces de la Ligue est d'avoir des acteurs engagés dans les instances politiques à tous les niveaux. Ces implications à diverses échelles leur permettent d'agir stratégiquement pour se faire reconnaître comme les spécialistes du renouveau éducatif. Or, ces acteurs «doubles» sont le plus souvent des savants, des philosophes ou des personnalités reconnues dans leur domaine qui jouent un rôle de «caution» auprès des instances politiques.

Cet engagement politique est particulièrement visible dans les discours des années 1930. Le Congrès de Nice constitue un moment charnière à ce propos puisque c'est suite à ce dernier que plusieurs projets de grande envergure dans l'enseignement public sont envisagés notamment en France et en Belgique. L'arrivée de Langevin à la tête du GFEN en 1929, et de Wallon et Piéron au comité, accélère ce mouvement. Langevin, savant physicien, considère que la science ne peut avoir d'impact que si elle est le «bien de tous» (Bensaude-Vincent, 1987) et qu'elle est enseignée largement à tous les élèves. C'est pourquoi ce dernier s'engage au sein du groupe des Compagnons de l’Université Nouvelle dont il est le président à partir de 1930; convaincu que la réforme de l'enseignement doit s'assortir d'une réforme des méthodes pédagogiques, il se tourne ensuite vers les représentants de la Ligue pour en faire des alliés. Petit à petit, fort de sa célébrité et de son envergure internationale, Langevin va se placer dans les instances politiques 
françaises pour élaborer un plan de réforme - le célèbre plan LangevinWallon - qui ne sera déposé qu'en 1944, mais qui est le fruit d'une longue lutte menée durant plus d'une décennie. De fait, Langevin n'est pas seul dans son entreprise, et c'est à l'aide de plusieurs personnes telles que Wallon, Piéron, Laugier usant tout comme lui de leur notoriété pour militer dans des sphères politiques - notamment le Parti communiste - qu'il pourra mettre sur pied ce projet (Gutierrez \& Kounelis, 2010). On sait par ailleurs que le GFEN tente de trouver des alliances auprès du Groupe du Nord car Albert Châtelet (recteur de l'Académie de Lille), Émile Marcel Ginat (professeur au Lycée du Havre) et Alfred Weiler (professeur au lycée de Rouen et collaborateur de Laugier) sont des personnes influentes (Gutierrez, 2008; Savoye, 2006).

Dans les pays où les innovations sont encore marginales, les relations politiques au niveau national jouent un rôle crucial. Ainsi, en Amérique latine, plusieurs initiatives voient le jour grâce à ce type de relations, en particulier en Bolivie, en Argentine ou au Guatemala. C'est ainsi que le gouvernement accorde en 1932 à Hermogenes Gonzales, l'ouverture d'une école nouvelle à grâce à ses relations avec le Ministre de l'instruction publique au Guatemala. ${ }^{8}$ À l'inverse, les efforts entrepris dans ces pays sont souvent avortés en raison de l'arrivée de nouveaux régimes politiques qui suppriment certains projets novateurs encouragés par le gouvernement antérieur. ${ }^{9}$ De façon générale, l'engagement politique à diverses échelles paraît indispensable pour que des projets d'Éducation nouvelle soient mis en œuvre dans une école, une ville, ou une région. À Vienne, c'est grâce à l'implication d'Otto Glöckel, ministre de l'instruction publique et président du conseil scolaire de la ville, qu'une réforme peut se mettre en place dans l'enseignement public primaire au cours des années 1920. En Belgique, c'est grâce à l'action à la fois politique et pédagogique au niveau régional - grâce à la participation d'Hubert Frere, préfet de l'institut provincial de Morlanwelz - qu'un nouveau plan d'études est introduit en 1937. Enfin, en Angleterre, c'est au niveau local que des membres de la Ligue tentent d'œuvrer pour une application des méthodes nouvelles dans les écoles publiques:

8 Voir Éditorial, PEN, 1934, 95, p. 38

9 À ce sujet, voir notamment la «Chronique d'Amérique Latine» (PEN, 1934, 95) rédigée par Ferrière. 
Ce pays si fortement traditionaliste y apparaît cependant comme une terre d'élection pour l'Éducation nouvelle, grâce à une heureuse entente de l'autorité centrale et des autorités locales, des directeurs, des inspecteurs, et des maîtres et grâce aussi à l'intérêt largement répandu qu'on y rencontre pour l'école, l'instruction à tous les degrés, et les moyens de la culture. (Cazamian, 1935, PEN, 113, p. 306)

Au niveau international, c'est à Genève que la Ligue trouve son champ d'action politique le plus favorable puisque c'est là que siègent de multiples organisations internationales. De fait, c'est essentiellement à ce niveau que les membres de l'Institut Rousseau sont engagés; leur implication au niveau local vise essentiellement à trouver des appuis financiers pour faire vivre l'Institut quand ceux-ci viennent à manquer (Hofstetter, 2010). Car c'est bien à une échelle internationale que Ferrière, Bovet, Claparède, Piaget cherchent à faire reconnaître leurs ambitions réformatrices comme l'atteste la fondation, en 1925, du Bureau international d'Éducation. Selon Hofstetter (2015), la fondation de ce bureau est non seulement un acte de promotion des réformes éducatives, mais aussi un acte politique situant d'emblée l'échelle à laquelle ceux-ci ambitionnent de transformer l'éducation.

Quelle que soit l'échelle d'action de ces réformateurs, il est important de relever que les plus engagés sont des intellectuels, acteurs se caractérisant par leur haut niveau de formation et bénéficiant d'une certaine notoriété dans leur domaine où ils font figure de spécialiste, voire «d'expert». Une position qui leur permet de jouer un jeu subtil où il s'agit de rester dans la marge de l'État tout en étant suffisamment performant et innovateur pour «se faire désirer» par celui-ci. Un jeu stratégique où l'expert sollicité de la sorte parvient à une position de force dans laquelle ses idées acquièrent un poids déterminant. Un jeu que les dirigeants de la Ligue semblent avoir bien compris en préservant à sa tête un groupe d'avant-garde:

Dans le but d'élargir son cadre, de répondre à la croissance rapide de cet organisme, une réorganisation est nécessaire, réorganisation qui, tout en permettant d'agir sur un champ plus vaste, conservera pourtant l'esprit qui était à l'origine du mouvement mondial dans le domaine de l'éducation ayant à sa tête une avant-garde qui, tout en restant libre du contrôle de l'État, soit suffisamment importante pour que son concours soit recherché par les autorités. (Ensor, 1929, PEN, 51, p. 218) 


\section{Hybridité science-spiritualité}

Grâce à l'analyse des réseaux des membres de la Ligue, un troisième type d'hybridité apparaît de façon évidente: la double appartenance de nombreux acteurs aux milieux scientifiques et religieux. Comme nous l'avons montré, la plupart d'entre eux sont des intellectuels, c'est-à-dire des personnes qui pensent, réfléchissent, écrivent et pour lesquels «l'esprit» est important. Ils se distinguent par leur refus du matérialisme et du monde industriel et manifestent une certaine attirance pour l'Orient. L'Inde, en particulier, constitue une destination de choix que ce soit pour les Anglais (Ensor, Baillie Weaver, Claremont, etc.) ou pour les Suisses (Ferrière, Bovet) ansi que pour certains qui y séjournent plusieurs années, à l'exemple de Maria Montessori qui y passe les six années de guerre (1939-1945). Suite à nos analyses, nous constatons que plusieurs acteurs sont à la recherche d'une spiritualité nouvelle, loin des dogmes et des Églises établies. Ils cherchent une manière de concilier «esprit et science», ou plus précisément «spiritualité et science», cette double appartenance se déclinant de plusieurs manières.

Tout d'abord, il y a ceux qui adhèrent à la théosophie et aux crédos que celle-ci véhicule à propos de l'existence de lois universelles et d'une force intérieure présente en chaque individu: Ensor, Baillie-Weaver, Montessori, Sadler, Ferrière, Sola de Sellares, Mackinder, Nunn, pour n'en citer que quelques-uns. Ensuite, il y a ceux qui adhèrent au mouvement Quaker (Rotten, Marie Butts, Ragaz, Bovet, Ferrière) et à l'idée que l'individu possède une lumière intérieure en lui, qui lui donne l'énergie et l'envie d'apprendre et de découvrir. Certains acteurs se tournent vers l'Église libre ou l'Église indépendante (Monastier, Bovet, Privat) tandis que d'autres s'initient de façon sérieuse aux religions orientales (Becker, Ulich). D'autres encore cherchent une spiritualité authentique au sein du judaïsme à l'exemple de Buber, Mirski ou Wyneken. À ces réseaux religieux se combinent souvent des réseaux pacifistes par l'intermédiaire d'amitiés entre acteurs impliqués simultanément dans les deux. Ainsi, on peut constater que plusieurs figures emblématiques font partie de mouvements pacifistes et non-violents.

Cette double appartenance aux milieux scientifiques et religieux est particulièrement forte chez les psychologues et psychanalystes qui étudient l'esprit humain et notamment l'inconscient. Que ce soit Freud, Jung, Krafft, Lewin, Baudouin, tous cherchent à comprendre comment fonctionne l'esprit, quel est son pouvoir sur le corps, quels sont les mécanismes d'apprentissage, etc. Dans la revue, de nombreux articles 
abordent la notion de l'inconscient, questionnant quelle est sa force, sa place, son rôle, ses bienfaits, ses méfaits dans la vie de l'homme. De fait, la plupart de ces scientifiques expérimentent personnellement diverses méthodes permettant d'étudier l'inconscient que ce soit l'hypnose, la méditation, la suggestion ou l'auto-suggestion. Ceci permet de comprendre leur intérêt pour les religions orientales, notamment hindoues et bouddhistes. De fait, la double appartenance de ces acteurs aux milieux religieux et scientifiques révèle une grande proximité entre ces deux mondes. Une proximité confirmée par les propos de Langevin selon lequel «la science est un effort séculaire de pénétration et de domination du monde par l'esprit». ${ }^{10}$ Comme le dit Ensor à Elseneur, la frontière entre religion (spiritualisme) et psychanalyse est un «sentier étroit» puisque tous deux s'interrogent sur l'esprit humain, son fonctionnement, ses liens avec l'au-delà (Ensor, 1929, PEN, 51). De fait, nous pouvons dire que les deux mondes - scientifique et religieux - se rejoignent car ils se questionnent sur la place de l'Homme dans l'univers (ses origines, son devenir) et manifestent un intérêt accru pour les phénomènes de la Nature, qu'ils soient physiques, biologiques ou chimiques.

\section{Hybridité science militance}

Au terme de cette analyse, plusieurs caractéristiques communes se dégagent. Tout d'abord, ces acteurs bénéficient d'un haut niveau de formation puisque la plupart d'entre eux ont fait des études secondaires voire universitaires. Ensuite, ils manifestent un intérêt à se former aux nouvelles méthodes en participant à divers cours, congrès, stages. Leurs profils sont «hybrides» dans le sens où ils appartiennent simultanément à plusieurs milieux dans lesquels ils déploient une activité importante. Par exemple, la double appartenance de certains à la sphère scientifique et politique leur permet d'agir à plusieurs niveaux pour faire reconnaître la science qu'ils sont en train de construire. Plusieurs acteurs se caractérisent par leur double appartenance aux milieux scientifique et religieux dont le point commun serait leur foi en la science (scientisme) qui incarne tous les espoirs de renouveler l'éducation; cette conviction se combine à une spiritualité plus ou moins déclarée. Enfin, ce qui réunit les acteurs de l'Éducation nouvelle est leur double appartenance à la science

10 Phrase prononcée par Langevin dans son discours au Congrès de l'Association Française pour l'Avancement des Sciences (AFAS), citée par Ferrière dans un éditorial de 1928 (PEN, 1928, 34). 
et à la militance comme en atteste leur engagement au sein d'associations ou divers groupes de pression. Leur militance s'exprime essentiellement à travers l'activité d'écriture dont la diversité des supports contribue à la vaste diffusion de leurs idées: livres, articles, manifestes, comptes rendus, rapports, correspondance. Mais les supports privilégiés par ces derniers sont les périodiques, comme en atteste le nombre important d'acteurs qui dirigent des revues au sein desquelles ils assument une grande part de la rédaction (éditoriaux, recensions, informations). Plusieurs femmes assument également cette fonction quoique moins nombreuses. Ces rédacteurs-trices sont des personnes influentes, souvent polyglottes, qui assurent une correspondance régulière avec le comité de la Ligue et participent régulièrement aux manifestations. Ce faisant, ils contribuent à la diffusion des savoirs éducatifs d'avant-garde non sans influencer l'orientation et le ton de leur périodique. Cette position leur permet aussi de propager leurs propres convictions personnelles utilisant la revue comme tribune de leurs idées. C'est le cas notamment de Wallon et Piéron lorsqu'ils prennent la direction de Pour l'Ère Nouvelle. Comme le souligne Ohayon (2006) «leur rapport au savoir est militant» en raison de la cause pour laquelle ils se battent qui dépasse largement leur activité professionnelle. Une cause pour laquelle ils s'engagent dans divers milieux tels que la Ligue de l'enseignement, les Universités populaires, l'Éducation nouvelle» (pp. 18-19). Selon Oelkers, les scientifiques qui militent pour l'Éducation nouvelle acquièrent un statut particulier du fait de leur double appartenance. Un statut qui peut avoir des conséquences sur leur reconnaissance et leur crédibilité au sein de leur communauté.

Les acteurs rendent publiques leurs exigences de rénovation et leurs opinions sur l'Éducation nouvelle en échappant largement au contrôle d'une recherche observatrice qui vérifierait les hypothèses de façon indépendante. [...] L'on accepte un paradoxe: le pédagogue innovateur est un acteur armé d'une théorie qui se veut être scientifique sans toutefois pouvoir être corrigée par l'action. (Oelkers, 1995, p. 33)

Selon les contextes sociaux et politiques, les scientifiques qui consacrent leur temps et leurs savoirs à la défense d'une cause récoltent la méfiance de leurs collègues à l'égard de leurs théories. ${ }^{11}$

11 En France, l'engagement de Wallon au sein du Parti communiste se traduit par une certaine méfiance de la part de ses confrères et remet en cause sa crédibilité scientifique (Gratiot-Alphandéry, 1994/2000). 
À l'issue de cette analyse, l'hybridité apparaît comme une caractéristique centrale des acteurs de l'Éducation nouvelle comme si celle-ci était consubstantielle de leur militance. Leur activité professionnelle se double d'un engagement pour une cause sociale au service de laquelle ils mettent leurs savoirs, leur intérêt et leur intelligence. Ce constat rejoint celui fait par d'autres (Drewek \& Lüth, 1998; Hofstetter \& Schneuwly, 2002) qui montrent que les sciences sociales émergent précisément au moment où l'on cherche à résoudre la question sociale: elles sont alors toutes imprégnées de l'idée que la science peut améliorer l'humanité. Selon Hofstetter (2008), l'engagement social de ces acteurs constitue une cohérence qui dépasse la diversité de leurs profils: «pour tous, l'approche scientifique des problèmes sociaux, et avant tout de l'éducation, constitue un outil indispensable pour un engagement social plus efficace; le travail scientifique se prolonge dès lors presque naturellement et souvent sans transition perceptible dans l'engagement social» (p. 201). Pour cet auteur, le caractère hybride de ces acteurs ne caractérise pas seulement ceux qui s'emploient à construire la nouvelle science mais elle conditionne aussi la structure cognitive du champ disciplinaire alors en cours de construction qui prend une «forme hybride alliant discipline et militance dans un mélange inextricable» (p. 294).

\section{DES FEMMES PRODUCTRICES DE SAVOIRS ${ }^{12}$}

Pour l'Ère Nouvelle donne à voir une grande diversité de personnes œuvrant pour l'éducation et la cause de l'enfant dans de nombreuses régions du monde. Parmi ces personnes, notre analyse révèle une part importante de femmes engagées au sein de la Ligue et dans le mouvement en général. Elles représentent plus de 30\% des auteurs de PEN mais elles constituent près de $50 \%$ des participants aux congrès et aux cours de formation dans les instituts spécialisés. La plupart sont praticiennes et se caractérisent par leur engagement militant pour la cause de l'enfant, certaines assumant des fonctions dirigeantes au sein d'institutions scolaires (directrice d'école, inspectrice) ou d'associations (présidente, adjointe, responsable). Grâce à leurs écrits, leurs expériences se

12 Ce chapitre a inspiré la rédaction d'un article publié dans un ouvrage collectif dirigé par Droux et Hofstetter sur la globalisation des mondes de l'éducation (Haenggeli-Jenni, 2015) 
font connaître au-delà de leur contexte local et participent à l'extension du mouvement. La revue contribue ainsi à la reconnaissance de ces protagonistes travaillant dans l'ombre, mais sans qui le mouvement n'aurait pu s'étendre à une si large échelle.

\section{PRATICIENNES DE HAUT NIVEAU}

Parmi les profils de femmes qui écrivent dans PEN, celui qui domine est celui de praticiennes, c'est-à-dire d'institutrices qui travaillent dans des écoles nouvelles ou dans les classes expérimentales d'écoles publiques. Nombre d'entre elles sont à la fois institutrices et directrices, voire fondatrices de l'établissement qu'elles évoquent dans leurs récits. Formées dans des écoles normales, la plupart ont pratiqué leur métier quelques années puis fondent leur propre école en marge du système public. C'est le cas de Cécile Riedel (1906-1989)13, formée à l'École normale de Melun-Sud et qui fonde quelques années plus tard l'école «L'Enfance Heureuse» ${ }^{14}$ à Pau (Basses Pyrénées), ${ }^{15}$ où elle pratique les méthodes Montessori. C'est aussi le cas d'Alice Jouenne (1873-1954) ${ }^{16}$ qui, formée à l'École normale de Paris, ouvre en 1921 la première École de Plein Air de la Municipalité de Paris (Boulevard Bessières, Paris 17e), ou encore de Suzanne Roubakine, institutrice puis directrice de l'école de Clamart (transférée par la suite à Bellevue, France). De fait, toutes ces institu-

13 De langue maternelle allemande, Cécile Riedel grandit dans une famille protestante de l'Église Réformée. En 1918 (12 ans), elle fréquente un orphelinat dirigé par deux demoiselles où elle découvre sa vocation. Elle obtient son brevet élémentaire d'institutrice en 1922. Après un séjour d'une année à Vienne, elle suit une formation de jardinière d'enfants au Collège Sévigné (1926-1928), puis fonde sa propre école à Pau (Quin-de Stoppani, 2007).

14 Cet internat mixte considéré comme un modèle acquiert une notoriété rapide surtout après la tenue de l'assemblée annuelle du groupe La Nouvelle Education (Guéritte \& Cousinet) à Pau, presque entièrement dédiée à l'Enfance Heureuse et à la méthode Montessori. (Quin de Stoppani, 2007).

15 Elle est transférée à Vaucresson (Paris) en 1930, deux ans après sa fondation.

16 Institutrice, militante, socialiste, syndicale et coopératiste, Alice Jouenne publie les principales orientations de son école dans son livre «Une expérience d'Éducation nouvelle: l'École de Plein Air» (1927, Paris, Radot). Pour de plus amples informations sur Alice Jouenne, se reporter à Gutierrez (2008). 
trices complètent leur formation initiale par des stages, des cours d'été ou des semestres de formation dans divers instituts renommés. Ainsi, c'est lors de la formation de jardinière d'enfants qu'elle suit au Collège Sévigné ${ }^{17}$ (1926-1928) après l'obtention de son brevet d'enseignement primaire (1922) que Cécile Riedel découvre les classes expérimentales Montessori. Il en est de même pour Andrée Jadoulle (1896-1975), institutrice formée à l'École normale de Liège, qui après la guerre 1914-18, complète sa formation en suivant des cours à l'Université libre de Bruxelles tout en enseignant dans une école pour déficients mentaux à Seraing (Belgique). ${ }^{18}$ Hofstetter (2010) fait le même constat concernant Mina Audemars (1882-1971) et Louise Lafendel (1872-1971) qui, suite à leur formation initiale ${ }^{19}$, s'initient aux méthodes froebeliennes, la première au Froebel Institute de Londres, la seconde lors d'un cours de formation Froebel à Genève (1908). Quelques années plus tard, elles se forment aux méthodes Montessori lors d'un cours donné par $M^{\text {mes }}$ Bontempi et Barrère à Genève (1913). En 1913, ces deux institutrices prennent la tête de la «Maison des Petits», école d'application de l'Institut Rousseau tout en assumant des charges de cours dans cet institut. Une autre femme, Alice Descoeudres (1877-1963), y est responsable des cours de psychologie et pédagogie des enfants anormaux ${ }^{20}$, sa formation de base étant celle d'une institutrice:

17 Le Collège Sévigné est fondé en 1880 par la Société pour la propagation de l'instruction parmi les femmes. En 1928, il est à la pointe de l'enseignement féminin, œuvrant en faveur de l'accès des femmes à l'enseignement supérieur et à la vie active (Quin-de Stoppani, 2007).

18 À la demande de René Jadot, gendre d'Ovide Decroly et professeur de pédagogie et psychologie à l'École normale de Liège, Andrée Jadoulle prend la direction du Laboratoire expérimental de pédagogie et psychologie fondé à Angleur (Belgique) en 1928. Patronné par une équipe de savants (Decroly, Wallon, Piaget), ce laboratoire devient une école spécialisée pratiquant les méthodes nouvelles qui œuvre encore aujourd'hui. Après la Libération, Jadoulle met sur pied les premiers CEMEA en Belgique et reste active dans ce milieu jusqu'à la fin de sa vie.

19 Mina Audemars obtient le diplôme de la section pédagogique de l'École Secondaire et Supérieure de Jeunes Filles (ESSJF) de Genève et Louise Lafendel se forme à l'École normale de Malagnou (Genève).

20 Ce terme figurant dans les programmes de cours de l'Institut Rousseau (AIJJR, Fonds général, 1912-1913, FG.1) était utilisé pour désigner les cours d'éducation spécialisée. 
Fidèles collaboratrices des premières heures, trois femmes, toutes trois enseignantes, épaulent les deux fondateurs: Alice Descoeudres (1877-1963), Mina Audemars (1882-1971) et Louise Lafendel (1872-1971). Elles ont toutes un haut niveau de formation et d'expertise qu'elles déploient dans les domaines en vogue de l'Institut que sont l'éducation des enfants arriérés et celle des petits, considérés comme lieux par excellence du renouvellement des approches éducatives. Elles associent toutes trois étroitement leurs activités d'éducatrices et de formatrices à leurs activités de recherche et publication. (Hofstetter, 2008, p. 195)

D'autres promotrices de l'Éducation nouvelle - qui n'écrivent pas dans Pour l'Ère Nouvelle - choisissent de se former dans des instituts spécialisés tels que l'Institut Rousseau: Hélène Antipoff (1892-1974)21 (Campos, 2001, 2012) et Marguerite Soubeyran (1929-1940)22 (Ruchat, 2007). Ces dernières se forment durant une année dans cet institut (été 1927 à hiver 1928) avant de fonder chacune de leur côté une école pratiquant les méthodes d'Éducation nouvelle: à Belo Horizonte (Brésil) pour la première, à Dieulefit (France) pour la seconde.

Comme nous l'avons vu précédemment, trois femmes - Marie-Louise Cazamian, Marie-Anne Carroi, Émilie Flayol - se distinguent des autres par leur contribution conséquente à Pour l'Ère Nouvelle en raison du nombre important d'articles qu'elles y publient. Ces dernières bénéficient également d'un haut niveau de formation puisque les premières sont des professeurs de lycée tandis que la troisième, institutrice de formation, se perfectionne à travers son activité d'inspectrice puis de directrice d'école normale ainsi que par sa participation à de nombreux congrès de la Ligue.

21 Elle est une élève de la première volée de l'Institut Rousseau (1912-1913), institutrice stagiaire à la Maison des Petits puis assistante du Laboratoire de psychologie expérimentale dirigé par Claparède. En août 1929, Hélène Antipoff part au Brésil pour enseigner la psychologie et diriger le Laboratoire de Psychologie dans l'École de Perfectionement des Enseignants du Minas Gerais. Elle y remplace Léon Walther en 1932 puis elle ouvre sa propre école en 1940, école rurale de la Fazenda do Rosàrio, destinée aux enfants présentant des difficultés d'apprentissage. Pour une biographie intellectuelle d'Hélène Antipoff, voir Campos (2001, 2012).

22 Infirmière de formation, Marguerite Soubeyran est élève à l'Institut Rousseau en 1927-28 où elle fait la connaissance d'Hélène Antipoff; elle fonde en 1929, avec Catherine Krafft, l'École de Beauvallon à Dieulefit (Delpal, 2014). Leurs premiers élèves sont le fils d'Hélène Antipoff et le fils adoptif de Catherine, un ancien élève du Home «Chez Nous». 


\section{DES FEMMES ACTIVES DANS L'ÉDUCATION DE LA PETITE ENFANCE}

De l'analyse des profils des auteures de PEN, nous pouvons faire un autre constat: ces femmes sont essentiellement actives dans l'éducation de la petite enfance. En effet, qu'elles écrivent en tant qu'institutrice, inspectrice, ou directrice d'école, la majorité d'entre elles œuvrent dans des établissements de niveau préscolaire tels que jardins d'enfants, écoles maternelles, foyers ou écoles nouvelles. Ainsi, que ce soit dans les écoles de Clamart (S. Roubakine), de Vaucresson (C. Riedel) ou du Home «Chez Nous» ${ }^{23}$ - pour n'en citer que quelques-unes - ce sont avant tout de jeunes enfants que ces femmes éduquent selon les méthodes nouvelles. De fait, les méthodes Montessori contribuent largement à cette dominante puisque celles-ci se destinent avant tout aux enfants d'âge préscolaire. Ainsi, que ce soit dans les écoles nouvelles (privées) ou dans les classes maternelles Montessori comme celles évoquées par Alice Jotte (Paris) $^{24}$ ou par Louise Briod (Lausanne), il semble que seules les femmes pratiquent ces méthodes ${ }^{25}$. Les articles consacrés à ces méthodes sont tous écrits par des femmes, à l'exemple de Maria Valli $(\mathrm{CH})$, Maria Boschetti-Alberti (CH), Alice Jotte (F), M $\mathrm{M}^{\text {mes }}$ De Ligt van Rossem (NL), Philippi van Reesema (NL) ou Joosten. ${ }^{26}$ Ces éducatrices ont été formées dans des «Écoles normales Montessori» délivrant une formation spécifique Montessori ou ont suivi l'un des cours internationaux donnés par la doctoresse chaque année à l'étranger.

Du côté des instituts spécialisés de formation, nous constatons également que les femmes sont avant tout spécialistes de la petite enfance. Comme nous l'avons vu à l'Institut Rousseau, Mina Audemars et Louise Lafendel sont spécialisées dans ce domaine - comme en attestent leurs enseignements ainsi que leurs recherches et leurs écrits. De manière

23 Foyer d'Éducation nouvelle à la campagne (La Clochatte s/Lausanne, Suisse) dirigé par Marthe Fillion, Lili Lochner et Suzanne Lobstein et qui accueille des enfants âgés de 6 mois à 12 ans.

24 Voir les articles d'Alice Jotte dans PEN: «Une classe Montessori à Paris» (1928, PEN, 34, pp. 3-5 et 32-34).

25 Pour plus de détails sur ces femmes, voir le compte-rendu du premier Congrès international Montessori à Elseneur, en août 1929.

26 Les prénoms de ces trois personnes nous sont malheureusement inconnus, raison pour laquelle nous utilisons le préfixe $« \mathrm{M}^{\mathrm{me}}$ » pour signifier qu'il s'agit de femmes. 
générale, on constate ainsi l'existence d'un important réseau de femmes dans ces niveaux d'enseignement. Cette tendance s'inverse quelque peu dans les années 1930, du fait que les femmes qui écrivent alors dans la revue sont davantage impliquées dans les niveaux secondaires de l'enseignement, à l'exemple de Marie-Anne Carroi ou Marie-Louise Cazamian. Dans le secteur public, les actrices de l'Éducation nouvelle sont principalement actives dans les réseaux d'éducation féminins que ce soit en tant qu'institutrice ou inspectrice dans les écoles primaires et secondaires de filles ou en tant que formatrice dans les écoles normales pour filles. Cette tendance est particulièrement marquée dans les régions anglo-saxonnes où les écoles sont très rarement mixtes et où la responsabilité de l'éducation des filles revient avant tout aux femmes. Comme le montrent Rogers et Thébaud (2014), l'enseignement est l'une des rares carrières qui s'offre aux femmes instruites à la fin du $19^{\mathrm{e}}$ siècle. Certaines femmes enseignent au niveau primaire ou post-primaire dans des écoles réservées aux filles, pour former de «bonnes mères et épouses dévouées». D'autres, bénéficiant d'un plus haut niveau de formation, s'investissent dans l'enseignement secondaire des jeunes filles, dont les programmes se distinguent de ceux des garçons par l'absence du grec et du latin, et privilégient les humanités modernes.

FEMMES DE L'OMBRE: SECRÉTAIRES, TRÉSORIÈRES, «ÉPOUSES DE»...

Certaines femmes ne sont pas des professionnelles de l'éducation et pourtant, jouent un rôle crucial dans le fonctionnement d'une revue ou d'un groupement oeuvrant pour l'Éducation nouvelle. ${ }^{27}$ Il s'agit en particulier des secrétaires qui, en tant que rédactrices des rapports et synthèses, sont les mieux à même de saisir les enjeux des controverses éducatives. Pourtant, leurs noms et leurs rôles sont souvent peu connus et peu valorisés. Ainsi, des noms tels qu'Émilie Flayol, Jeanne Hauser, Muriel Mc Kenzie, ou Clare Soper apparaissent de manière récurrente sur les pages de couverture des périodiques concernées sans que l'on sache vraiment de qui il s'agit, ni quel est leur rôle dans la vie de la revue et du groupement. Parmi celles-ci, Émilie Flayol est sans doute celle qui signe le plus grand nombre d'articles dans Pour l'Ėre Nouvelle même s'il s'agit principalement des rapports d'activité du GFEN (dès 1934), groupe

27 Toutes les sections nationales de la Ligue possèdent un secrétaire, comme en atteste la liste publiée dans PEN à partir de 1930. 
dont elle est aussi la secrétaire. Le rôle de Muriel Mc Kenzie est assez peu connu à l'exception de sa participation à la rédaction du rapport complet du Congrès d'Elseneur (1929) qu'elle co-signe avec William Boyd (Boyd \& Mackenzie, 1930). Pourtant sa fonction «d'assistante éditrice» aux côtés de Beatrice Ensor au début de sa carrière laisse supposer qu'elle avait une activité d'écriture importante et assumait les tâches administratives qu'exige une telle fonction. Grâce aux récentes recherches de Middleton (2013), le rôle de Clare Soper est maintenant un peu mieux connu, en tant que secrétaire du siège de la Ligue à Londres pendant plus de 30 ans. Ces travaux montrent l'importance cruciale de cette personne, en particulier au niveau de la correspondance qu'elle entretient avec les différents pays membres de la Ligue pour lesquels elle constitue une sorte de «centrale d'échange et d'information». C'est à travers elle que s'opéraient tous les échanges de livres, articles, brochures, à la fois vers et en provenance de l'étranger. Selon Middleton (2013), l'intense activité d'écriture de Clare Soper a permis de conserver les liens avec les multiples sections de la Ligue, même pendant le temps de guerre. C'est aussi grâce à son hospitalité dans les bureaux de Bloomsbury que les visiteurs de la Ligue pouvaient se rencontrer et échanger à propose des expériences menées dans leur pays ou région. Quant à Jeanne Hauser, administratrice puis trésorière de la revue et du GFEN, elle n'est ni institutrice ni directrice, mais selon Ferrière, «elle est une mère entièrement dévouée à la cause de $l^{\prime}$ Éducation nouvelle» ${ }^{28}$. Son rôle précis et ses écrits sont encore peu connus aujourd'hui mais elle a sans doute œuvré à la circulation des idées et savoirs sur l'éducation à travers son activité. De fait, le rôle de ces secrétaires est crucial dans la vie d'un groupe ou d'une revue car ce sont elles qui écrivent divers comptes rendus tels que les rapports de réunions, rapports d'activité, rapports de congrès, etc. Ce faisant, elles jouent un rôle de mémoire et de synthèse des savoirs sur l'Éducation nouvelle qui contribue à la diffusion et à l'extension du mouvement. Malgré l'objectivité apparente de ces écrits, certains textes sont souvent teintés d'une marque personnelle, d'un certain «ton», dans lequel transparaît des prises de position ou des censures..$^{29}$

28 Voir lettre de Ferrière à Rotten, 16 décembre 1925. AIJJR, Fonds Ferrière, Correspondance alphabétique, AdF. C.1/63

29 Certains rapports annuels d'Émilie Flayol sont écrits de manière à camoufler les tensions et controverses qui existent au sein du GFEN (Gutierrez, 2008). 
Une autre catégorie de femmes apparaît de façon encore plus discrète, que l'on découvre à travers quelques remarques d'auteurs, sur des photos de congrès ou dans les listes de comités d'organisation de manifestations diverses. Il s'agit des «épouses de» pédagogues, directeurs, psychologues, figures-clé de l'Éducation nouvelle. C'est en s'intéressant à la biographie de ces derniers que l'on prend conscience de l'importance de certaines d'entre elles. C'est le cas de Madame Ferrière évoquée par Meyhoffer et Gunning (1929) dans leur ouvrage édité à l'occasion du $50^{\mathrm{e}}$ anniversaire de la naissance $\mathrm{d}^{\prime}$ Adolphe Ferrière. De fait, seule une phrase parmi les 30 pages de cet ouvrage mentionne cette femme alors que celle-ci semble jouer un rôle crucial dans la vie de son époux. Elle apparaît comme son «bras droit», comme une femme au service d'un homme qui n'aurait sans doute pas été si prolixe et célèbre sans sa présence au quotidien:

Lui et Madame Ferrière - laquelle infatigablement l'accompagne partout avec une abnégation et un esprit de consécration admirables, prenant sans cesse des notes pour lui, et l'aidant dans ses travaux de secrétariat et dans ses travaux d'écrivain - ont peine à faire face à tant d'activités multiples. (Meyhoffer \& Gunning, 1929, p. 15)

La présence d'Isabelle Ferrière aux côtés de son époux n'est jamais mentionnée dans Pour l'Ėre Nouvelle alors que l'analyse du Petit Journal ${ }^{30}$ (Gerber, 1989) révèle l'importance de cette dernière dans le quotidien de son mari. En effet, celui-ci étant sourd, c'est elle qui fait le lien avec les personnes qu'il rencontre, que ce soit en réunion, en congrès ou lors d'entrevues personnalisées. Dans certains cas, c'est à travers les réseaux de sociabilité de sa femme qu'Adolphe entretient des contacts dans le milieu éducatif. Ainsi, c'est grâce à l'amitié qu'Isabelle entretient avec Ellen Key (1849-1926) qu'il sera mis en contact avec cette figure précurseur de l'Éducation nouvelle avec qui il entretient des relations professionnelles. De plus, Isabelle Ferrière participe aux grands rendez-vous de la Ligue comme en témoigne cette photo $^{31}$ prise en 1929 au Congrès d'Elseneur.

30 Journal tenu par Ferrière tout au long de sa vie dans lequel il consigne ses activités quotidiennes et les personnes qu'il rencontre (AIJJR, Fonds Ferrière, AdF/D/2/3/6-8).

31 Photo prise au Congrès d'Elseneur (DK) en 1929 publiée dans The New Era, 1929, 40, p. 203 (n spécial dédié à ce congrès) Nous reproduisons ici la légende de la photo: 


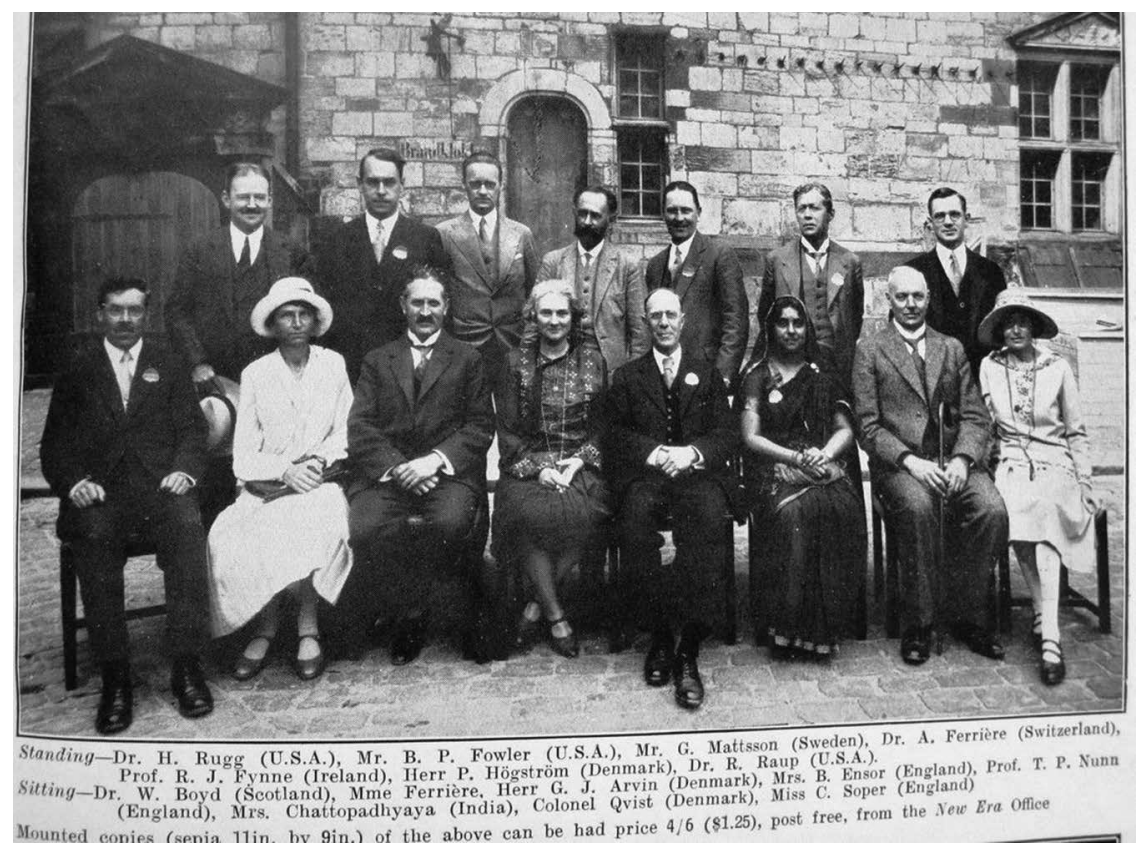

Compte tenu de la surdité quasi totale qui handicape Ferrière dès les années 1900 (Hameline, 1994), nous supposons que Madame Ferrière était présente aux autres congrès de la Ligue ainsi qu'à d'autres manifestations semblables pour soutenir son mari. Comme nous l'avons évoqué plus haut, certaines épouses de grandes figures apparaissent dans les listes des comités d'organisation des manifestations. C'est le cas par exemple du Congrès de la World Federation of Education Associations (WFEA) organisé à Genève en 1929. Dans la liste du comité d'organisation figurent notamment les noms de $\mathrm{M}^{\text {me }}$ Claparède et $\mathrm{M}^{\mathrm{me}}$ Hochstaetter en tant que membres du «Comité des dames», sans que leurs prénoms soient mentionnés. Cette indication laisse penser que ces femmes jouent

Standing: Dr. H. Rugg (USA), Mr. B. P. Fowler (USA), Mr. G. Mattson (Sweden), Dr. A. Ferrière (Switzerland), Prof. R. J. Fynne (Ireland), Herr P. Högström (Denmark), Dr. R. Raup (USA).

Sitting: Dr. W. Boyd (Scotland), $\mathrm{M}^{\mathrm{me}}$ Ferrière, Herr G. J. Arvin (Denmark), Mrs. B. Ensor (England), Prof. T. P. Nunn (England), Mrs. Chattopadhyaya (India), Colonel Qvist (Denmark), Miss C. Soper (England). 
un rôle important dans l'organisation de manifestations de ce type alors qu'il n'en n'est jamais fait mention explicitement dans les rapports de congrès, du moins dans PEN. Ces femmes œuvraient donc pour les mêmes causes que leurs époux mais dans l'ombre de ces derniers. Seule une «épouse de savant», Madame Piéron, est rendue visible dans la revue en raison des articles qu'elle co-signe avec son mari. De fait, celle-ci est une des chevilles ouvrières de la mise sur pied de l'Institut d'Orientation Professionnelle que fonde son mari à Paris en 1928. C'est elle qui en assure l'administration et la direction, de même que le secrétariat, tout en participant à certaines recherches menées par son conjoint sur l'orientation professionnelle. ${ }^{32}$

\section{DES «PASSEUSES DE SAVOIRS»}

Quelle que soit leur fonction, leur niveau de formation et leur insertion (ou non) dans le milieu professionnel, ces femmes partagent un certain nombre de traits communs même si en apparence, elles semblent avoir des rôles bien spécifiques. D'après les textes qui les décrivent, ces femmes se ressemblent au niveau de leur attitude; elles auraient toutes des qualités intrinsèques qui seraient propres au genre féminin telles que la faculté d'abnégation, l'intuition, la douceur, l'écoute, le dévouement, la clairvoyance, «l'amour pour les petits, les fiables les opprimés» (Ferrière, 1926, PEN, 19). Cette faculté d'abnégation caractérise notamment les directrices d'école nouvelle qui sont le plus souvent célibataires, dédiant leur vie à éduquer les enfants dont elles ont la charge pour la cause de l'Éducation nouvelle. ${ }^{33}$ «L'esprit de service» ainsi que la notion de vocation sont alors des valeurs qui caractérisent la plupart des

32 Son nom figure sur quelques publications mais toujours en qualité de « $\mathrm{M}^{\mathrm{me}}$ Piéron», son prénom n'étant pas indiqué. Elle rédige un article dans $P E N$ intitulé «Les recherches psychotechniques à l'école et la collaboration des éducateurs» (PEN, 1931, 73, pp. 279-281) et un autre aux côtés d'Henri Wallon et Henri Laugier «Étude critique sur la valeur sélective du certificat d'études et comparaison de cet examen avec une épreuve par tests. Contribution à une docimastique rationnelle» (PEN, 1935, 105, pp. 47-51).

33 Marthe Fillion, Lili Lochner et Suzanne Lobstein qui dirigent le Home «Chez Nous» sont toutes trois célibataires (Coquoz, 1998). Il en va de même pour Cécile Riedel, Marguerite Soubeyran et d'autres directrices d'école qui dédient toute leur vie aux enfants de leur établissement. 
métiers féminins. Que ce soit en dirigeant des écoles nouvelles, en gérant la vie d'une association ou d'une revue (secrétaires) ou en contribuant bénévolement à l'organisation de manifestations, ces femmes contribuent à la diffusion des méthodes nouvelles et à la construction d'une nouvelle science.

Cependant, ces femmes se distinguent aussi par leur ouverture d'esprit, par leur désir de se perfectionner (professionnellement), par leur intérêt pour la recherche et la science dans un mouvement visant à améliorer la condition de l'enfant par son éducation. C'est dans cet esprit qu'elles participent aux congrès de la Ligue, aux conférences et autres manifestations organisées par les promoteurs de l'Éducation nouvelle. En outre, la plupart d'entre elles s'engagent également pour la cause des femmes en militant au sein d'associations telles que l'Union des Femmes $(\mathrm{CH})$, la Ligue internationale des Femmes pour la Paix et la Liberté (Woman's International League for Peace and Freedom) ou d'autres mouvements féministes et pacifistes internationaux. Certaines s'engagent aussi pour les populations «en danger», que ce soit les enfants «moralement abandonnés», les enfants et les prisonniers militaires pendant la Grande guerre (Rotten en Allemagne) ou pour les victimes de guerre (Ensor en Autriche). Selon Hofstetter (2010), cet intérêt pour les «populations en danger» se traduit plus tard par un intérêt marqué pour l'éducation des arriérés et pour l'éducation de la petite enfance, à l'exemple de Descoeudres, Audemars et Lafendel qui mènent des recherches dans ces domaines au sein de l'Institut Rousseau.

De manière générale, ces femmes se caractérisent par leur caractère militant à tous niveaux, ainsi que par leur personnalité volontaire et charismatique. «Femmes de caractère», elles expriment leurs idées et leur perception du monde dans leur métier ainsi qu'à travers les écrits qu'elles publient, que ce soit des articles dans les revues pédagogiques ou dans des journaux féminins. Comme on peut le voir dans Pour l'Ère Nouvelle, ces articles sont avant tout des témoignages, récits d'expérience ou descriptions de méthodes expérimentées dans les écoles où elles enseignent; la majorité d'entre eux sont de type «intermédiaire» faisant à la fois référence à une pratique d'enseignement et apportant des éléments de connaissance contribuant à la construction d'une «science de l'éducation». À travers ces articles apparaissent aussi des interrogations de type philosophique sur les missions de l'école, sur le rôle du maître et des parents, sur la place de l'éducation dans la société. Si ces articles ne comportent pas de «savoirs savants» en tant que tels, ils 
contribuent à l'accroissement des connaissances sur l'enfant et l'éducation à travers la diffusion de «savoirs expérientiels» très valorisés par la profession. En ce sens, ces femmes peuvent être considérées comme des «passeuses de savoirs» qui diffusent leurs connaissances dans des réseaux éducatifs mais aussi dans des réseaux spécifiquement féminins tels que les groupes féministes. En publiant leurs articles, Ferrière contribue à leur reconnaissance dans le domaine éducatif mais aussi à leur émancipation personnelle ${ }^{34}$ car les textes pédagogiques sont les premiers écrits «autorisés» pour publication sans faire recours au pseudonyme (Castanotto, 2007).

\section{RÉSEAUX DE RELATIONS AUX CONTOURS MULTIPLES}

Pour saisir les liens entre science et militance au sein du mouvement d'Éducation nouvelle, une étude des réseaux s'avère indispensable. En effet, repérer les relations professionnelles et amicales entre acteurs leurs lieux de rencontre, leurs interlocuteurs privilégiés, leurs systèmes d'échanges - va nous aider à comprendre comment circulent les savoirs au sein de la Ligue et à l'extérieur. En analysant ces réseaux, nous pouvons mettre en évidence les personnes les plus impliquées, repérer la place qu'y occupent les scientifiques vs les praticiens, identifier des profils et des appartenances et faire des hypothèses sur l'impact de ces appartenances sur les orientations de la Ligue. Cette partie propose donc une analyse des réseaux de relations des acteurs à travers diverses approches et catégorisations.

\section{RÉSEAUX FORMELS ET INFORMELS}

De notre analyse de Pour l'Ère nouvelle émergent d'emblée deux types de réseaux dans lesquels circulent les acteurs de la Ligue. En référence

34 Ferrière valorise beaucoup le travail des femmes engagées pour la cause de l'Éducation nouvelle et collabore étroitement avec elles que ce soit au niveau pratique sur le terrain, au niveau éditorial ou au niveau de ses enseignements. En effet, il enseigne dans plusieurs écoles de filles, notamment l'École Secondaire et Supérieure de Jeunes Filles (ESSJF), l'École Sociale pour Femmes à Genève, l'École Vinet à Lausanne et donne des conférences à la Ligue internationale des Femmes pour la Paix et la Liberté. 
aux travaux de Fuchs (2007b), nous les distinguerons en parlant de réseaux formels et informels. ${ }^{35}$ Par «réseaux formels», cet auteur entend les diverses institutions (écoles, instituts, centres de recherche) et formes de rencontres institutionnalisées (associations, groupes, congrès) ${ }^{36}$ mises en place par les éducateurs. Par «réseaux informels» ou «sociaux», il entend les «relations entre acteurs sociaux» (p. 200) que ceux-ci entretiennent en lien étroit avec leur appartenance familiale, confessionnelle, culturelle ainsi que leur activité professionnelle, politique, sociale, associative. Dans PEN, les réseaux formels sont les plus visibles et les plus documentés, puisque cette revue est l'organe d'une association internationale. Comme nous l'avons montré, les institutions constituent un lieu important d'échanges et de rencontres. Leur diversité de taille, de population, d'objectifs, entraîne une diversité d'acteurs qui y séjournent et qui construisent dans ces lieux des réseaux sociaux et professionnels.

Si les réseaux formels nous donnent des indications sur les lieux visités et les possibles relations qui ont pu s'y tisser, l'analyse des réseaux informels permet d'appréhender avec plus de finesse comment s'opèrent ces relations, comment certains protagonistes vivent leur double identité de scientifique et militant, et plus largement, comment se construit et s'étend le réseau international que constitue la Ligue. Car l'analyse de $P E N$ sous cet angle-là nous amène d'emblée à une échelle internationale. En effet, la Ligue se caractérise par une grande diversité culturelle; pourtant ses membres partagent de nombreux intérêts communs et forment ainsi une «communauté internationale de pratique» (White, 2001) ou une «communauté d'esprits» (Hameline, 2002a). Comme nous allons le voir, cette internationalité n'est pas seulement due à la participation de plusieurs pays aux activités et manifestations de la Ligue, mais bien davantage aux «réseaux d'acteurs transnationaux» (Saunier, 2004) qui existent en parallèle et dont celle-ci bénéficie pour se propager à une échelle internationale. Si certains d'entre eux s'estompent au fil du temps, d'autres prennent le dessus par la suite, attestant de l'évolution

35 D'autres auteurs parlent de réseaux «horizontaux» et «verticaux» (Cunningham, 2001) pour distinguer ces deux catégories.

36 On devrait ajouter ici les divers supports éditoriaux (livres, revues, brochures, etc.) qui sont les lieux privilégiés d'échanges de savoirs sur l'Éducation nouvelle. Mais nous avons traité la question dans le chapitre précédent. 
que connaît la Ligue durant la période étudiée et de la diversité des acteurs qui la composent.

L'étude biographique des acteurs principaux fait apparaître l'ampleur de «réseaux cachés», du moins peu visibles à une première lecture, dont l'impact sur les orientations de la Ligue, et plus globalement sur l'idéologie de l'Éducation nouvelle, est indéniable. Il s'agit de réseaux religieux - surtout dans les années 1920 - et de réseaux politiques - plus visibles dans les années 1930 - sans parler des réseaux d'amitié présents tout au long de la période étudiée. Considérant que l'analyse des réseaux informels est la «meilleure approche pour faire apparaître la structure de ces réseaux» (Fuchs, 2007b, p. 200), c'est à cette analyse que nous proposons de procéder maintenant; celle-ci ne prétend pas être exhaustive et vise plutôt à repérer les réseaux dominants.

À ses débuts, la Ligue se construit principalement grâce aux réseaux de ses trois fondateurs, Adolphe Ferrière, Elisabeth Rotten et Beatrice Ensor. Ces trois derniers entretiennent des relations professionnelles et d'amitié qui se traduisent par une imposante correspondance. Car à leurs relations «à trois» $s^{\prime}$ ajoutent toutes celles que chacun entretien avec son réseau personnel illustrant le célèbre dicton, «les amis de tes amis sont mes amis». L'analyse biographique d'autres acteurs de la Ligue confirme cette observation: à quelques exceptions près, tous font partie des réseaux de relations de ces trois acteurs-clés.

Ainsi, nous pouvons observer que les collaborateurs permanents de Das Werdende Zeitalter sont tous des amis d'Elisabeth Rotten avec lesquels celle-ci partage ses idées, sa philosophie ainsi que sa conception du monde et de l'éducation. Parmi eux figure un nombre important d'adhérents au mouvement Quaker ${ }^{37}$ dont elle fait partie. Ces derniers militent pour l'éducation mais aussi pour une transformation de la société selon des principes démocratiques visant la paix et la liberté. De façon similaire, Beatrice Ensor fait appel à des personnes de son réseau pour écrire dans The New Era ou pour fonder des écoles nouvelles. Ce réseau est constitué principalement de personnes appartenant au mouvement théosophiste dont des branches existent dans plusieurs pays du monde. Quant à Ferrière, son réseau social est composé des relations qu'il a nouées à travers son Bureau international des Écoles nouvelles, mais aussi de celles qu'il a construites à travers son activité à l'Institut

37 Des précisions concernant ce mouvement figurent dans la suite de ce chapitre. 
Rousseau. De fait, nous le verrons ci-après, Ferrière s'inscrit simultanément dans plusieurs réseaux, que ce soit ceux de Rotten, d'Ensor, de l'Institut Rousseau ou de la Société des Nations. Ces milieux constituent d'une part des lieux d'inspiration dont il se nourrit pour élaborer ses théories éducatives et d'autre part, des endroits où il sollicite des personnes pour écrire dans $P E N$ ou pour l'aider à réaliser ses projets.

Si ces trois réseaux constituent sans conteste le «noyau dur» des premières années de la Ligue (1922-1932), d'autres existent quelque peu en marge, à la fois parallèles et imbriqués, mais dans lesquels de nombreux membres sont impliqués. Il s'agit tout d'abord des réseaux disciplinaires construits par les «théoriciens» de l'Éducation nouvelle auxquels appartiennent en particulier les psychologues, les médecins et les philosophes. Nous verrons ici l'importance du «réseau de pédologie» qui se constitue bien avant la fondation de la Ligue, dont le Congrès de Bruxelles en 1911 (Depaepe, 1987) marque à la fois le lancement et l'apogée du mouvement, congrès auquel participent de nombreux acteurs de l'Éducation nouvelle. Si ces théoriciens s'engagent au sein de la Ligue dès les années 1920, la plupart continuent d'entretenir des relations professionnelles dans leur réseau disciplinaire que ce soit par la fréquentation ou l'organisation de congrès ou par leur activité éditoriale dans les revues spécialisées de cette discipline. Un autre réseau à la fois parallèle et imbriqué à la Ligue est le «réseau Montessori» qui se constitue lui aussi avant la fondation de cette dernière, et d'emblée à une échelle internationale. ${ }^{38}$ Composé principalement $d$ 'institutrices formées aux méthodes de la «Doctoresse» ${ }^{39}$, ce réseau est visible dans PEN à travers les nombreux articles que ces dernières écrivent en tant qu'institutrices ou directrices d'école. L'analyse de PEN permet d'observer l'évolution de ce réseau qui, d'abord cantonné aux régions d'Italie et du Tessin $(\mathrm{CH})$, s'étend rapidement à la France, aux Pays-Bas et aux États-Unis où de nombreuses écoles maternelles publiques et privées s'en réclament. ${ }^{40}$

38 Les premières Casa dei Bambini ouvrent leur portes en 1907.

39 Nom couramment donné à Maria Montessori par les partisans de ses méthodes.

40 L'Association Internationale Montessori est fondée en 1936 aux Pays-Bas et le premier Congrès international Montessori a lieu en 1929 à Elseneur lors du congrès de la Ligue, s'apparentant ainsi à un congrès dans le congrès. 
À partir des années 1930, les réseaux des trois fondateurs s'estompent, laissant la place à d'autres qui se distinguent des premiers par leur échelle désormais nationale ou régionale plutôt qu'internationale. En France, le nouveau réseau est avant tout celui du Groupe Français d'Éducation nouvelle qui reprend en main la revue à partir de 1929. De tendance plus politique, ce réseau est composé d'une grande quantité de praticiens qui désormais écrivent dans PEN mais aussi de scientifiques tels que Wallon, Langevin ou Piéron qui dirigent le groupe et utilisent la revue comme tribune de leurs idées. En Angleterre, les changements sont moins évidents puisque Beatrice Ensor reste à la tête de la revue jusqu'à la fin de la période étudiée. Mais le réseau théosophiste qui lui avait permis de lancer son entreprise à une échelle internationale s'estompe et fait place à un réseau plus restreint basé sur des relations administratives et politiques locales et nationales.

\section{ADOLPHE FERRIÈRE ET LES ÉCOLES NOUVELLES}

Comme le dit Ensor dans son discours au Congrès d'Elseneur, la première «croisade» de l'Éducation nouvelle date du début du siècle ${ }^{41}$ où «des pionniers, tels que Parker, Reddie, Lietz, Badley, Demolins ont fondé les écoles nouvelles parce qu'ils étaient mécontents de l'éducation ordinaire» (Ensor, 1929, PEN, 51, p. 218). C'est aussi à ce momentlà, en 1899, que Ferrière fonde son Bureau International des Écoles nouvelles sur la suggestion d'Edmond Demolins, alors directeur de l'École des Roches. Ferrière s'emploie alors à recenser toutes les écoles nouvelles en constituant des fiches documentaires dont les informations visent d'une part à renseigner les parents intéressés à inscrire leur enfant dans l'un de ces établissements et d'autre part, à établir une grille d'évaluation destinée à garantir le «label» d'école nouvelle (Hameline, 2002a). Pour récolter ces informations, Ferrière visite luimême ces établissements dans lesquels ils séjourne parfois plusieurs semaines, nouant ainsi des relations avec directeurs et directrices dans toute l'Europe. Ainsi se tisse progressivement autour de lui, un immense réseau d'acteurs oeuvrant à l'élaboration de nouveaux savoirs sur l'éducation dans les écoles nouvelles. Outre les pionniers anglais et

41 Hameline fait même débuter le mouvement avant le début du $20^{\mathrm{e}}$ siècle, plus précisément en 1889, date de la fondation de l'École nouvelle d'Abbostholme par Cecil Reddie, en Angleterre (Hameline, 2003). 
allemands ${ }^{42}$ avec lesquels il est en contact depuis le tournant du siècle, Ferrière entretient des relations d'amitié avec Georges Bertier (École des Roches), Paul Geheeb (Odenwaldschule), Wilhelm Frei et Wilhelm Zuberbühler (École de Glarisegg) ainsi qu'avec Elisabeth Huguenin (École de Bex) et les directrices du Home «Chez Nous», Marthe Fillion, Lili Lochner et Suzanne Lobstein, pour n'en citer que quelques-uns. Si ces derniers figurent parmi ses amis les plus proches, Ferrière a des relations plus «formelles» mais néanmoins soutenues avec les directeurs des principales écoles nouvelles à l'étranger. La volumineuse correspondance qui l'occupe quotidiennement témoigne de ses multiples relations dans le monde que ce soit en Europe ou sur le continent américain. ${ }^{43}$

Ces pionniers sont décrits comme des personnes marginales et charismatiques qui transmettent non seulement leurs savoirs et savoir-faire mais aussi, leur conception de la vie, du monde et de l'éducation. De fait, l'étude biographique de leur parcours montre qu'il ne s'agit pas de praticiens comme les autres. Il s'agit d'intellectuels, possédant une formation de haut niveau ${ }^{44}$, dotés d'une volonté ferme de réformer la société dont ils sont insatisfaits. Si certains se connaissent et se rendent visite mutuellement, la plupart travaillent seuls dans leur école, développant parfois un domaine spécifique de l'Éducation nouvelle. ${ }^{45} \mathrm{C}^{\prime}$ est là que Ferrière et son Bureau international des Écoles contribuent de façon décisive à la mise en synergie de ces pionniers, créant par la même un réseau de personnes militant pour l'Éducation nouvelle et favorisant la circulation des savoirs sur l'éducation. Au moment de la fondation de la Ligue, le réseau est alors bien implanté, totalisant plus de 50 écoles

42 Cecil Reddie, James Badley, Hermann Lietz.

43 Dans les années 1920, Ferrière alors vice-président de la Ligue, rédige plus de 4000 lettres par an à des correspondants dans le monde entier (Meyehoffer \& Gunning, 1929).

44 Cecil Reddie (École d'Abbostholme) est titulaire d'un doctorat en chimie (obtenu en Allemagne); Hermann Lietz (Écoles de Haubinda et Illsenburg) fait des études de théologie, de philosophie, d'histoire et de pédagogie. Il est l'assistant de W. Rein au séminaire pédagogique de l'Université de léna (Hameline, 2002a). Georges Bertier (École des Roches) fait des études de philosophie à Paris et est professeur de lettres à l'École des Roches. (Raillon, 1998).

45 C'est le cas par exemple d'Émile J. Dalcroze qui met au point sa méthode de «gymnastique rythmique» dans une école à Hellerau (1910-1914) puis à Genève dès 1915. 
dans de nombreux pays européens. ${ }^{46}$ Ce sont d'ailleurs ces pionniers qui constituent l'essentiel des participants au Congrès fondateur de Calais en 1921. Ainsi, la Ligue bénéficie d'emblée d'un réseau international d'acteurs acquis à sa cause, ce qui lui permettra de se propager rapidement à une échelle internationale.

Ce réseau d'écoles nouvelles va jouer un rôle crucial dans la circulation des savoirs pédagogiques mais aussi dans la circulation des personnes. D'autant plus qu'à cette époque, le mode du voyage est très prisé parmi les éducateurs. En effet, ceux-ci voya-

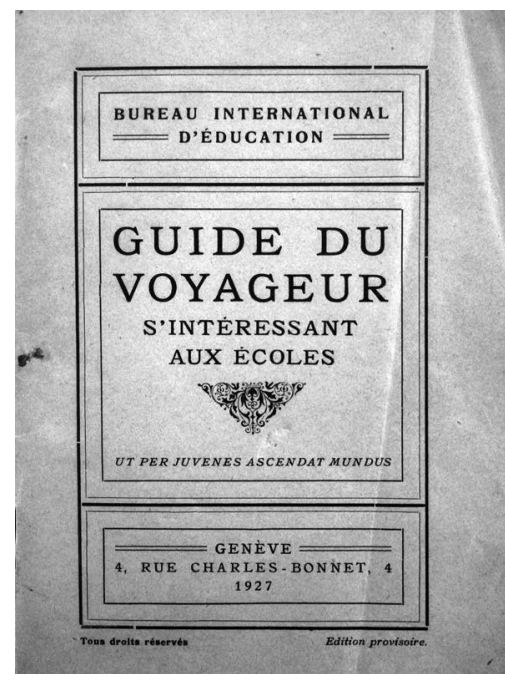
gent d'école en école pour s'imprégner de la vie propre à chaque établissement et pour y voir pratiquer les nouvelles méthodes pédagogiques. Pour nombre d'entre eux, le but est aussi de rapporter chez eux leurs observations et leur savoir-faire afin d'ouvrir, à leur tour, une école expérimentale. Ce mode d'imprégnation par le voyage est d'ailleurs si populaire qu'un Guide du voyageur est édité en 1927 par le Bureau International d'Éducation ${ }^{47}$ pour orienter les voyageurs dans leur périple d'école en école à travers l'Europe.

Les voyages sont vivement recommandés par la Ligue et par d'autres groupes d'Éducation nouvelle ${ }^{48}$ car leurs dirigeants y voient bien plus

46 En 1922, Ferrière ne recense pas moins de 56 écoles réparties principalement en Angleterre (19), Allemagne (18), Suisse (10) et France (6). La Belgique, les Pays-Bas et la Suède arrivent en queue de liste avec seulement une école par pays (Ferrière, PEN, 1922, 3, pp. 64-65).

47 Ce guide fournit pour chaque pays (Allemagne, Autriche, France, Suisse, Belgique, Italie, Danemark, Pologne) des informations sur le système éducatif public et présente les diverses institutions «d'avant-garde» - c'està-dire acquise aux méthodes nouvelles - qui méritent d'être visitées.

48 Les dirigeants de La Nouvelle Éducation encouragent leurs lecteurs à «faire le voyage de Pau» où se trouve «l'école-modèle» L'Enfance Heureuse, "pour éprouver dans cette école, la joie et l'émerveillement que tous les congressistes y ont éprouvés». (Rapport de la 9e assemblée, La Nouvelle Éducation, 1930, cité par Quin-de-Stoppani, 2007). 
qu'une rencontre entre collègues. Selon Ensor, les voyages ont une grande valeur car ils favorisent «la compréhension et l'estime réciproques, [...] une des voies qui hâtera l'avènement de cet esprit international contre lequel le monde se bute aujourd'hui» (Ensor, PEN, 1922, 1, p. 5).

\section{BEATRICE ENSOR ET LES THÉOSOPHES}

Dès le début de siècle, les théosophes sont très impliqués dans les milieux de l'éducation progressiste en raison de l'idéologie qui les anime. Aussi, ils constituent une part importante des adhérents à la Ligue durant sa première décennie de vie. La personne de Beatrice Ensor joue un rôle central dans ces adhésions puisqu'elle est engagée simultanément dans plusieurs groupes théosophistes qu'elle contribue à fonder dans certains cas. Tout comme la société fondatrice, Theosophical Society, ces derniers sont fondés en Angleterre mais se propagent rapidement à une échelle internationale. Ainsi, les théosophes entretiennent d'étroites relations avec l'Inde, où ils séjournent régulièrement ${ }^{49}$, mais aussi avec les États-Unis et divers pays d'Europe. Dans ces pays, il s'agit soit d'individus isolés mais rattachés à des sociétés internationales, soit de groupes d'échelle variable organisés dans leur propre pays. Du fait de leur dispersion dans une grande diversité de pays, les théosophes ont pour habitude d'organiser des congrès internationaux afin de permettre aux membres de la communauté de se rencontrer et créer des liens. Ceci explique pourquoi à Calais en 1921, les congressistes de même que les intervenants sont essentiellement des théosophes, la plupart anglais. ${ }^{50}$ Comme le montre Brehony (2004), que ce soit Haden Guest (représentant du London County Council), Claremont ${ }^{51}$ (fervent défenseur des méthodes Montessori), Brereton, Wilson ou Baillie-Weaver, tous sont des

49 En 1928, une section nationale indienne est fondée dont la revue s'intitule Brahmacharya tandis que deux ressortissants indiens rejoignent le Comité international de la Ligue. (Éditorial, PEN, 1928, 39)

50 Le thème du congrès, «The Creative Self-expression of the Child» est particulièrement en adéquation avec les thèses théosophistes selon lesquelles l'éducation doit permettre de faire émerger la «force», le «potentiel» qui existe au fond de chaque être humain.

51 Claude Claremont enseigne pendant plusieurs années à la St-Christopher School (fondée et dirigée par Beatrice Ensor) avant de prendre la tête du département Montessori de cette école, de même qu'à la St Georges School, toutes deux situées à Letchworth. Il est aussi le directeur des écoles 
théosophes. Du côté des internationaux, au nombre de cinq, Ferrière et Nussbaum fréquentent probablement des groupes théosophistes en Suisse ${ }^{52}$, mais nous ne savons rien des sympathies de Decroly, Hamaïde, Decroix et Loiseau qui interviennent au congrès sur des thèmes avant tout pédagogiques. ${ }^{53}$

Bien que le réseau théosophiste soit moins étendu en France qu'en Angleterre, certains membres de la Ligue et d'autres groupes français d'Éducation nouvelle en font partie. C'est le cas par exemple de Jeanne Hauser, administratrice puis secrétaire-trésorière de $P E N$, également trésorière du Groupe Français d'Éducation Nouvelle à partir de 1929. Dans une lettre que Ferrière écrit à Rotten, celle-ci est présentée comme une «théosophe» faisant partie de ce «petit nombre de personnes qui mettent le bien de l'humanité au-dessus du bien de leur groupe théosophique, comme c'est le cas de Beatrice Ensor». ${ }^{54}$ Selon Ferrière, son mari, Lionel Hauser, banquier parisien impliqué dans le groupe des Compagnons de l'Université Nouvelle est aussi théosophe. ${ }^{55}$ Le fait que Juliette Decroix, présente à Calais, soit aussi membre de ce groupe laisse penser qu'un réseau théosophiste existe au sein des Compagnons de l'Université Nouvelle. ${ }^{56}$ Enfin, nous savons que Paul Langevin (1872-1946) prend la

normales Montessori (Montessori training Colleges) à Londres et Cranleigh. Claremont constitue une figure centrale du réseau Montessori dans lequel il s'investit pendant plusieurs années.

52 La contribution de Nussbaum «L'enfant est-il capable de puissance créatrice» tend à le confirmer de même que certaines indications du Petit Journal tenu par Ferrière. Amis de longue date, ces deux compatriotes font le voyage ensemble à Calais (Gerber, 1989).

53 Decroly présente «Une expérience de programme primaire avec activité personnelle de l'enfant», Hamaïde une conférence intitulée «Application de la méthode du Dr. Decroly» tandis que Decroix intervient sur «Une expérience pédagogique de co-éducation» et Loiseau sur «La valeur éducative du scoutisme». (The New Era, novembre 1921, numéro spécial).

54 Lettre de Ferrière à Rotten, 16 décembre 1925. AIJJR, Fonds Ferrière, Correspondance alphabétique, AdF. C.1/63

55 Comme le montre Gutierrez (2008), le couple Hauser est très engagé au sein du groupe des Compagnons de l'Université Nouvelle où Lionel Hauser assume la fonction de trésorier-adjoint dès 1925, sa femme étant élue membre du bureau de ce groupe cette même année.

56 De plus amples investigations mériteraient d'être menées sur ce groupe, en particulier sur Alice Jouenne car celle-ci est à la fois membre des 
tête des Compagnons ${ }^{57}$ en 1929 et qu'Henri Wallon fréquente les milieux théosophistes puisqu'il donne une allocution en 1932 lors d'une projection de films soviétiques «chez les théosophes», à Paris (Gerber, 1989, p. 70). Si cette appartenance est à notre connaissance peu connue et non documentée, il est en revanche plus admis que Wallon fréquente les milieux francs-maçons, tout comme d'autres psychologues français tels qu'Henri Piéron (Ohayon, 2006). Or, de nombreux travaux (Bandini, 2010; Soler Mata, 2011; Washington, 1999) montrent que les réseaux théosophistes et francs-maçons sont pour ainsi dire confondus, du moins perméables l'un à l'autre car partageant une philosophie commune. Dès lors il n'est pas rare de voir des personnes appartenir conjointement aux deux réseaux. À ce propos, Soler Mata évoque le cas de Manuel Trevino Vila (1865-1939), l'une des figures marquantes du théosophisme en Espagne. Ce dernier, éminent égyptologue, rédacteur de la revue Sophia, et fondateur en 1893 du groupe madrilène de la Société théosophique (Rama Madrid) est aussi membre de la Société maçonnique internationale «Le Droit Humain» incarnant par là-même la proximité de ces idéologies et réseaux. ${ }^{58}$

Si le réseau théosophiste semble peu étendu en France, il l'est davantage en Espagne où des groupes se réunissent dès 1891 à Barcelone, Valence et Madrid (Soler Mata, 2011). C'est à Barcelone que le réseau est le mieux implanté et au sein duquel Maria Sola de Sellares (1899-1998), éminente représentante de la théosophie espagnole, s'engage dès sa jeunesse dans les œuvres éducatives de la ville. C'est en tant que membre du groupe barcelonais de la Theosophical Society qu'elle participe à plusieurs congrès, notamment à celui de la Ligue à Calais en 1921. Seule

Compagnons de l'Université Nouvelle et de la Ligue au sein de laquelle elle participe activement aux diverses activités dès les premières années. Elle écrit quatre articles dans PEN entre 1922 et 1929, et participe aux congrès de Montreux et Elseneur. De même, il serait intéressant d'en savoir plus sur les activités d'Henri Laugier (1888-1973), pacifiste et membre des Compagnons de l'Université Nouvelle, connu pour ses travaux sur la biotypologie qui pourraient être en adéquation avec les thèses du mouvement théosophiste.

57 Il est nommé la même année président du GFEN et président de la Société Française de Pédagogie (Gutierrez, 2008).

58 Durant les années de guerre civile (1936-1939), son appartenance à ces divers réseaux lui causera beaucoup d'ennuis et se soldera par une mort brutale, à Madrid, en 1939. 
ressortissante espagnole de ce congrès, Maria Sola de Sellares va désormais représenter son pays au sein de la Ligue jusqu'en 1930, date à laquelle elle est rejointe par Lorenzo Luzuriaga (Madrid) représentant une délégation plus institutionnelle de l'Éducation nouvelle..$^{59}$ En 1926, elle devient directrice (avec Ricardo Crespo) ${ }^{60}$ de l'École nouvelle Dàmon ${ }^{61}$ inspirée par les méthodes Montessori et le Plan Dalton. Cette école met en pratique les principes de base de l'Éducation nouvelle telle que la co-éducation, le self-government, l'enseignement en plein air, la rythmique, l'éducation physique et artistique. En 1939, Maria Sola doit $\mathrm{s}^{\prime} \mathrm{exiler}^{62}$ pour des raisons politiques mais continue son œuvre en Amérique Latine en contact notamment avec la pédagogie Waldorf (Soler Mata, 2010). Par son exil, cette pédagogue contribue à la diffusion des méthodes nouvelles et de l'idéologie théosophiste sur le continent sud-américain.

En Europe, le réseau théosophiste possède également des adhérents en Italie. C'est le cas de Maria Montessori qui entretient des relations très étroites avec ce mouvement puisqu'elle est elle-même théosophe et qu'une large part des membres de la Société soutient ses méthodes éducatives. La philosophie éducative qu'elle défend convient particulièrement bien aux théosophes à la recherche de méthodes pédago-

59 N'ayant pu bénéficier d'une formation de haut niveau, Maria Sola est avant tout autodidacte s'instruisant par la lecture et les conférences publiques et traduisant en catalan plusieurs œuvres de Ferrière et d'autres pédagogues. Maria Sola entretient des relations amicales avec Ferrière principalement à travers l'échange de correspondance; ce dernier lui rend visite en route pour son voyage en Amérique latine en 1930 (Gerber, 1989).

60 Instituteur catalan, ce dernier fait une partie de ses études en Angleterre pour se former aux méthodes nouvelles; grâce à sa maîtrise de l'anglais, il traduit en espagnol de nombreux textes didactiques de la collection El tesoro del maestro des Éditions Labor pendant les années 1930. (Soler Mata, 2011)

61 Fondée par le groupe barcelonais de la Theosophical Fraternity in Education, elle est affiliée au Bureau international des Écoles nouvelles (BIEN).

62 Après quelques mois en France, c'est en Amérique latine que Maria Sola poursuit sa carrière. Elle dirige des écoles normales pour jeunes filles successivement au Salvador (1940-45) et au Guatemala (1945-48) avant de se réfugier au Mexique en 1954 où elle continue à œuvre pour l'Éducation nouvelle. 
giques favorisant la créativité et la liberté. Montessori, qui considère l'enfant comme une "continuation de l'acte de création", a une foi immense dans la possibilité d'améliorer l'humanité par l'éducation. Une foi qui n'est pas seulement intellectuelle ou idéologique mais aussi spirituelle et pratique. En effet, la réflexion, la contemplation et la méditation sont des notions importantes dans sa vie personnelle (elle pratique régulièrement la méditation) comme dans son programme éducatif (Röhrs, 1994/2000). ${ }^{63}$ Cette pratique spirituelle se perçoit également dans l'organisation du réseau qu'elle construit dès le tournant du siècle en Europe ainsi que dans la figure qu'elle incarne auprès de nombre de ses adeptes. Montessori est volontiers considérée comme la "grande prêtresse» des droits humains, alors que les maisons d'enfants (Casa dei Bambini) qu'elle crée dans plusieurs pays deviennent de véritables «lieux saints» où les éducateurs se rendent presque en «pèlerinage» (Röhrs, 1994/2000). Ses conférences attirent des milliers de personnes en particulier en Angleterre où elle remporte un succès sans précédent. ${ }^{64}$

Comme le montre Cunningham (2001), les réseaux des progressist innovators se combinent et s'entrecroisent en raison de l'appartenance multiple de certains acteurs qui s'engagent simultanément dans plusieurs réseaux. C'est le cas des réseaux théosophistes et «Montessoriens» qui, dans certains pays comme l'Angleterre, se chevauchent en raison des affinités de leurs membres pour la théosophie ${ }^{65}$. Soucieuse que ses méthodes soient appliquées au plus près de sa conception de l'éducation, Montessori établit un réseau important d'institutions dans toute l'Europe, réseau composé à la fois d'instituts de formation - accueillant les étudiants et instituteurs désirant se former aux méthodes Montessori - et d'écoles d'application auprès desquelles ces derniers sont tenus de faire leurs stages. Or, l'Angleterre constitue le pays où se concentre le plus grand nombre de ces institutions. Selon Cunningham (2001), un important «mouvement Montessorien» y prend naissance peu avant la Grande Guerre sous la forme d'une Société Montessori (Montessori Society) dont plusieurs personnes sont membres au moment de la fondation de la

63 Montessori entretient des contacts avec l'Inde où elle fait plusieurs séjours.

64 En 1919, près de 3000 personnes affluent de toute l'Angleterre pour écouter une conférence publique qu'elle donne à Westminster (Londres).

65 Sur la théosophie dans le contexte anglais et néo-zélandais, voir Middleton (2013) et Middleton (sous presse). 
Ligue. Parmi eux, mentionnons Claude Claremont ${ }^{66}$ qui sera l'une de ses figures dirigeantes, ainsi que Michael Sadler qui est membre du comité pendant plusieurs années. Alors que le premier est professeur d'école secondaire à Letchworth (école fondée par Beatrice Ensor), le second est un des plus éminents représentants de la recherche éducative en Angleterre. Après un travail de pionnier à la tête du «Bureau des recherches spéciales» (Office of Special Inquiries), Sadler est alors vicedoyen de l'Université de Leeds, considéré dans son pays et à l'étranger comme la "plus grande autorité sur les questions d'éducation en Angleterre» (Cunningham, 2001). ${ }^{67}$ En 1927, Michael Sadler est nommé président de la Section nationale anglaise de la Ligue aux côtés de Percy Nunn (vice-président) et d'Albert J. Lynch (secrétaire). ${ }^{68}$ À ses débuts, la Société Montessori est dirigée conjointement par Michael Sadler, Lord Lytton et Albert Mansbridge. Or, en 1914, plusieurs membres désirent étendre leurs intérêts à une plus grande diversité d'innovation éducative. Michael Sadler en fait partie et contribue activement à la transformation de cette société en un groupe clairement théosophiste, nommé New Ideals in Education, auquel Beatrice Ensor adhère pendant plusieurs années. Mais pour les «purs Montessoriens»(Cunningham, 2001), ce nouveau groupe n'est pas suffisamment orthodoxe; c'est pourquoi, ils créent ensuite le London Montessori Study Circle qui réunit essentiellement des éducateurs et éducatrices initié-e-s aux méthodes Montessori. Les partisans des méthodes Montessori en Angleterre constituent donc un groupe d'influence important dans le milieu de l'éducation progressive ${ }^{69}$ qui s'entrecroise avec les réseaux théosophistes car revendiquant et pratiquant des méthodes éducatives très similaires.

66 C. Claremont participe au congrès de Calais et de Heidelberg comme en témoignent ses articles dans Pour l'Ère Nouvelle et The New Era.

67 Michael Sadler est aussi l'un des membres les plus impliqués dans le programme "International Examination Inquiry» (IEI) financé par la Carnegie Foundation dans les années 1930 (Lawn, 2008).

68 En 1930, Nunn devient le président de la section, Sadler figurant dans la liste du comité en tant que «ex-president» (The New Era, 1930, July).

69 L'influence de ce groupe devint telle qu'elle suscita bientôt des conflits avec un autre groupe bien établi, les «Froebeliens», dont les buts et les intérêts sont similaires (Brehony, 2009; Cunnigham, 2001). 


\section{ELISABETH ROTTEN ET LE RÉSEAU QUAKER ${ }^{70}$}

Lorsque l'on s'intéresse de plus près à la biographie des acteurs de la Ligue, un réseau informel très vaste apparaît, celui des Quakers. L'étude de l'idéologie de ce mouvement permet de comprendre les convictions pacifistes et libérales qui animent les membres de la Ligue, en particulier dans les années 1920. Le Quakerisme se caractérise par son «pacifisme intégral» (De Dardel, 1961) qui se manifeste à travers le refus du service militaire et par des actions sociales telles que l'aide humanitaire, le secours aux blessés et prisonniers de guerre. En Europe, des groupes existent en Angleterre $^{71}$, en Allemagne, en France ${ }^{72}$ mais Genève constitue un véritable «centre international» à partir de 1920. La présence dans cette ville de la Société des Nations favorise la venue de nombreux Quakers en raison de l'esprit pacifiste et internationaliste qui y règne. Déjà en 1917, un groupe ${ }^{73}$ est fondé, composé principalement d'internationaux anglophones, pour la plupart américains. Mais quelques Genevois en font partie, notamment certains membres de l'Institut Rousseau, à commencer par son directeur, Pierre Bovet. Ce dernier met à disposition les locaux de l'Institut (au 5, rue de la Taconnerie) durant toute l'année 1920 pour les assemblées dominicales du groupe. Avec l'aide de sa femme Amy, elle aussi membre active, Pierre Bovet contribue à la diffusion des idées de ce groupe en traduisant les textes de grandes figures du Quakerisme mondial. ${ }^{74}$ Par la suite, le

70 Plusieurs documents concernant le réseau international Quaker ont été mis à notre disposition par $\mathrm{M}$. Ruchat, notamment une recherche historique sur les groupes Quakers en Suisse (Royston, 2005) ainsi que des indications concernant les membres de l'Institut Rousseau données par Michel Megard, archiviste et bibliothécaire du centre Quaker actuel de Genève.

71 Elisabeth Rotten participe à l'une de leur «General Assembly Meeting» à Londres en 1923 (Haubfleisch, 1997).

72 L'École de Nîmes, en relation avec les Quakers, donne le ton à la fin du $19^{e}$ siècle en associant la paix à l'organisation et au droit international (Biondi, 2000 cité par Ruchat \& Vilbrod, 2011).

73 Un ancien groupe existe depuis le 19e siècle réuni autour d'Ami Bost (17901874).

74 Ils traduisent notamment l'ouvrage d'Inazo Nitobé (1927) Qu'est-ce que le Quakerisme? («A Japanese view on Quakerism») et Journal de George Fox (1935), traduit de l'anglais par $\mathrm{M}^{\mathrm{me}} \mathrm{A}$. Bovet et abrégé par $\mathrm{H}$. van Etten. Inazo Nitobé (1862-1933), premier secrétaire général de la SDN, est une des grandes figures du Quakerisme international. 
groupe devient une branche officielle de la SDN qui lui vaut le nom de "Centre international Quaker» désormais considéré comme une des «ambassades Quakers» de la SDN. ${ }^{75}$ En 1926-27 est fondé le "Quaker International Student Hostel» destiné à accueillir des étudiants fréquentant

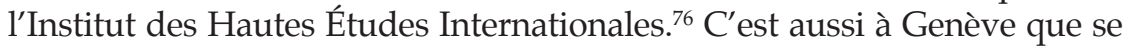
tient, en 1928, l'une des plus grandes conférences sur la paix dans l'organisation de laquelle de nombreux Quakers sont impliqués.

Outre le couple Bovet, d'autres personnes de l'Institut et du Bureau International d'Éducation sont membres ou «amis des Amis» ${ }^{77} \mathrm{du}$ mouvement Quaker. Ainsi, en est-il d'Adolphe Ferrière. Dans la «Chronologie de son existence», Gerber (1989), relève que Ferrière prend contact en 1925, avec Donald Gray, sous-directeur de l'École Quaker de York (USA) où il va donner des conférences sur le BIE, l'Institut Rousseau et le Bureau international des Écoles nouvelles. Chez les Ferrière, Adolphe n'est pas le seul sympathisant puisque sa sœur cadette, Maya Ferrière (1891-1967), enseignante Montessori, fréquente régulièrement les assemblées du groupe genevois dès les années 1920 et en devient membre officielle en 1944. De plus, Ferrière donne plusieurs conférences à l'École Vinet (Lausanne) dans laquelle se forment plusieurs femmes Quakers impliquées dans les réseaux éducatifs à l'exemple de Mary Butts (secrétaire générale du BIE) et Hélène de Monastier (institutrice), femmes qui deviendront des amies de Ferrière.

Plusieurs groupes existent en Allemagne, pays dans lequel se tiendront trois congrès importants ${ }^{78}$ qui seront à l'origine du groupe officiel allemand (fondé en 1925), l'un des plus importants d'Europe. Parmi ses membres dévoués figure Carl Heinrich Becker, proche ami de Rotten et collaborateur régulier de $D W Z$, et rédacteur de plusieurs rapports de congrès. Dans les autres régions germanophones, Vienne et Zurich constituent aussi des lieux de rassemblement. À Zurich, il est intéressant

75 D'autres «ambassades» Quakers sont installées la même année à Paris, Vienne, New York, associées à la SDN (Royston, 2005).

76 Ce foyer héberge des étudiants jusqu'en 1942, date à laquelle il ferme ses portes, faute de pensionnaires.

77 Se dit des personnes qui sont sympathisantes du mouvement sans pour autant en être membres, des personnes familières et habituées au culte Quaker, appelés aussi les «attenders».

78 Ces congrès ont lieu à Eisenach (1923), Wilhelmshöhe (1924) et Eisenach (1925) (Hall, 1938). 
de relever la présence de Leonard Ragaz, collaborateur régulier de DWZ et ami d'Elisabeth Rotten. Pasteur et professeur de théologie à l'Université de Zurich, il est aussi socialiste-chrétien, fondateur et leader des Socialistes religieux (Religiöse Sozialisten). De plus, c'est un ami proche d'Hélène Monastier qui fréquente aussi les libres penseurs du mouvement anarchiste allemand. Comme nous l'avons vu, Gustav Landauer qui écrit dans DWZ en est l'un des plus éminents représentants. Tout comme Hélène Monastier, Leonard Ragaz est d'obédience Quaker et participe à la fondation du groupe de Zurich (dès 1919) dont il accueille chez lui les assemblées dominicales. En 1918, c'est lui qui fait découvrir le mouvement Quaker à Pierre Cérésole (fondateur du Service Civil international) en emmenant ce dernier à Bilthoven (Pays Bas) où se déroule un grand meeting réunissant des Quakers d'Angleterre, d'Allemagne, des États-Unis et de Suisse. Parmi les participants se trouvent Elisabeth Rotten et Ruth Fry qui décident alors de mettre sur pied le vaste programme d'aide alimentaire Quaker (Quakerspeisung) en Allemagne.

En Angleterre, l'étendue du mouvement est moins connue. On sait toutefois qu'un des groupes les plus importants sur le plan international est à Londres (Hall, 1938), ville dans laquelle la SDN ouvrira aussi une Quaker Embassy. D'autre part, on sait que c'est sous l'impulsion de Henry Hodgkin, fondateur du «British Council for Promoting an International Christian meeting» (Royston, 2005) que s'organise le meeting de Bilthoven. Enfin, Isabelle Fry, directrice de la ferme-école de Mayortone-Manor à Wendover fait partie de la «Société des Amis» tout comme le reste de sa famille (dont Ruth Fry). Cette dernière écrit des articles dans The New Era, traduits dans DWZ et Pour l'Ère Nouvelle, et participe aux congrès de la Ligue dans lesquels elle présente les expériences menées dans sa «ferme-école» (Fry, PEN, 1925, 17, p. 40).

Aux États-Unis, la communauté Quaker est bien implantée en Pennsylvanie. C'est dans cet état qu'est fondé en 1864, le Swarthmore College, à l'initiative d'un comité de Quakers. ${ }^{79} \mathrm{Si}$ le collège n'affiche plus d'affiliation religieuse dès le tournant du siècle, il enseigne toutefois des valeurs issues de ses fondateurs telles que le développement

79 Ce comité est essentiellement composé de Quakers des groupes de Philadelphie, Baltimore, et New York, villes dans lesquelles sont fondées les universités américaines parmi les plus prestigieuses. Lucretia Mott fait également partie du comité de fondation. 
intellectuel, l'engagement personnel et la responsabilité sociale, valeurs que l'on retrouve dans les principes de l'Éducation nouvelle. Or, c'est dans ce collège qu'enseigne W. Carson Ryan, professeur de pédagogie, éditeur associé du journal School and Society et président de l'Association Nationale Américaine d'Orientation Professionnelle. ${ }^{80}$ Pendant l'entredeux-guerres, Carson Ryan est un membre actif de la Ligue comme en atteste sa participation aux Congrès de Heidelberg, Locarno et Cheltenham. Il fait des exposés sur «Les méthodes individuelles dans les écoles primaires», sur la «Préparation des maîtres aux écoles nouvelles» (Carson Ryan, PEN, 1927, 31), ainsi que sur la question du rapport entre l'éducation publique et les écoles nouvelles (Carson Ryan, PEN, 1925, 17). Il est aussi membre de la commission permanente de la Ligue chargée d'étudier les examens en lien avec les tests psychologiques. Son nom apparaît aussi dans The New Era et DWZ en raison de ses interventions dans les congrès.

Comme nous pouvons le voir, le réseau Quaker est fait d'un maillage très serré, où les connexions sont favorisées par les acteurs et actrices qui en sont membres; les valeurs qu'ils défendent telles que la liberté, l'altruisme, l'entraide, l'engagement social et l'esprit de service sont aussi celles de l'Éducation nouvelle ce qui explique pourquoi de nombreux acteurs de la Ligue y sont mêlés d'une manière ou d'une autre. Comme le relèvent Ruchat et Vilbrod (2011) «les Quakers forment des réseaux qui se croisent et s'entrecroisent autour du pacifisme, de l'aide sociale et de l'Éducation nouvelle» (p. 157). Toutefois, de plus amples investigations seraient nécessaires pour identifier d'autres membres de la Ligue qui sympathisent avec ce groupe.

\section{RÉESEAUX PROTESTANTS ET CHRÉTIENS SOCIAUX}

En parallèle des trois réseaux liés aux fondateurs de la Ligue, le protestantisme est un réseau important auquel appartiennent de nombreuses figures militant pour l'Éducation nouvelle. C'est le cas, par exemple, d'Édouard Claparède qui reste volontairement à l'écart de la Ligue car il ne partage pas les affinités théosophistes, voire mystiques, de Ferrière et

80 En 1928, il écrit un rapport d'évaluation sur le programme éducatif des Indiens d'Amérique (Meriam Report) puis est nommé responsable de ce programme entre 1930 et 1935. En 1952, il succède à Beatty à la tête de la Progressive Education Association. 
ses acolytes. Originaire d'une lignée de pasteurs fixée à Genève depuis la révocation de l'Édit de Nantes, Claparède établit l'essentiel de ses relations au sein du réseau protestant de Suisse romande au sein duquel il «fait jouer les solidarités culturelles et les solidarités de rang» (Cicchini, 2007, p. 357). Un réseau de «proximité culturelle» qui influence les nominations des professeurs de l'Institut Rousseau comme en témoignent celles de Claparède, Ferrière, Bovet et Piaget - figures clé de l'institution - toutes issues de familles protestantes, les deux derniers venant de Neuchâtel. ${ }^{81}$ Par ailleurs, Claparède évolue dans d'autres réseaux, en particulier les réseaux scientifiques de la psychologie et de la pédologie $^{82}$ (Hofstetter, 2010). Le réseau protestant s'étend également à d'autres pays tels que la France, l'Allemagne, les États-Unis où militent de nombreux partisans de l'Éducation nouvelle. Les protestants sont aussi présents dans d'autres lieux de promotion du savoir pédagogique tels que les Congrès international d'Éducation morale (CIEM) ${ }^{83}$ ou les conférences organisées par des institutions comme l'Institut Rousseau ou l'École Vinet. Selon Issenmann (2007), ces manifestations visent autant à transmettre des connaissances sur l'éducation qu'à permettre la rencontre "de gens de bonne compagnie», autrement dit, de gens de «proximité culturelle». Des conférences qui, en raison du «vent de laïcisation» insufflé par les changements politiques et sociaux des années 1840, tendent à se substituer aux prêches des pasteurs» (Issenmann, 2007, p. 19).

Ainsi, davantage que les credos religieux, c'est plutôt un même esprit, de mêmes valeurs, de mêmes causes qui rassemblent ces acteurs et les constituent en un «réseau de sociabilité». Selon Hameline (1993a), le protestantisme de Claparède «est plus proche des courants libéraux que de ceux de l'orthodoxie ecclésiastique ou du revivalisme mystique. Il est surtout fait d'esprit d'initiative et d'indépendance, incarné dans un individualisme entreprenant, gros de multiples solidarités voulues et cultivées» (p. 162). C'est aussi un même esprit et de mêmes valeurs qui rassemblent les membres de la Ligue et qui contribuent à la formation

81 Neuchâtel est un canton de Suisse romande considéré comme un des creusets du protestantisme suisse.

82 Il se forme notamment auprès de Wundt à Leipzig et collabore avec des scientifiques tels que Flournoy, Binet, Decroly, Piaget, Wallon.

83 Dans l'édition de 1922 du CIEM, qui a lieu à Genève, le comité se compose presque exclusivement de protestants genevois. 
d'un langage commun, voire d'une communauté d'esprits. Hofstetter (2008) fait une observation similaire à propos des membres de l'Institut Rousseau pour qui les questions sociales et morales rejoignent les causes pacifistes et internationales:

Une profonde cohérence se dessine au-delà de la diversité des profils. Pour tous, l'approche scientifique des problèmes sociaux, et avant tout de l'éducation, constitue un outil indispensable pour un engagement social plus efficace; le travail scientifique se prolonge dès lors presque naturellement et souvent sans transition perceptible dans l'engagement social, au prix parfois d'une disparition des frontières. Mais la réciproque semble vraie tout autant: leurs convictions sociales constituent un ressort puissant de leur travail scientifique et contribuent à en définir l'orientation. (Hofstetter, 2008, p. 199)

En ce sens, ils partagent un esprit que l'on pourrait qualifier d'humaniste, voire «d'humanitaire»: le dévouement et l'engagement personnels n'ont pas de limite, et la culture intellectuelle personnelle se met au service de la cause à défendre. Si cet esprit caractérise les grandes figures du mouvement - les fondateurs de la Ligue ou les dirigeants de l'Institut Rousseau - un même esprit habite ceux qui gravitent autour de ces figures, donnant ainsi l'impression que tous font partie d'une même famille.

La plupart d'entre eux, à l'instar des fondateurs et des figures du noyau central, se caractérisent par leur engagement social, au service de ce que l'on appelle alors le «bien public», souvent concrétisé par une affiliation à des institutions sociales et philanthropiques. Leurs convictions religieuses y contribuent, la plupart confessant un protestantisme ou un christianisme social que l'on pourrait appeler «à la genevoise», tant le réseau de relations entre les familles genevoises d'ancienne souche et les institutions sociales et philanthropiques qu'elles patronnent de longue date est ici serré. (Hofstetter, 2008, p. 200)

Comme le relève cette auteur, l'engagement social au service du «bien public» s'inscrit dans un courant plus large appelé «christianisme social» qui émerge au tournant du siècle dans un contexte marqué par l'inquiétude croissante des élites face aux conséquences de la longue dépression économique des années 1874-1890. En Suisse romande, les conservateurs protestants développent un important réseau d'associations dont les objectifs couvrent un large spectre de questions à l'ordre 
du jour: l'économie sociale et domestique, la prostitution, l'alcoolisme, la protection et l'éducation de la jeunesse; en d'autres termes l'hygiène sociale et morale sous toutes ses facettes (Pavillon \& Schoeni, 2007)! Ces associations sont dirigées par une diversité de professionnels, médecins, pasteurs, enseignants, banquiers qui ont comme particularité de ne pas s'adresser aux seules élites mais aux classes dites moyennes «dont la fragilisation économique les alarme particulièrement». De plus, ils tentent d'enrôler dans leur mouvement toutes les femmes bénéficiant d'une instruction, à savoir celles de la bourgeoisie habituées à œuvrer dans les sociétés de bienfaisance mais aussi, fait innovant, les femmes de condition plus modeste «qui cherchent à satisfaire ces nouvelles attentes ou à influer sur leur propre avenir» (Pavillon \& Schoeni, 2007, pp. 281-282). ${ }^{84}$ Ce contexte général de christianisme social et d'intérêt pour l'enfant permet de mieux comprendre la diversité des acteurs qui composent la Ligue internationale pour l'Éducation nouvelle, elle aussi engagée pour la cause de l'enfance.

\section{PLATEFORMES D'ÉCHANGES ET DIFFUSION DE SAVOIRS}

Ces divers groupes sociaux et religieux sont non seulement engagés dans maintes œuvres sociales, mais la plupart d'entre eux produisent des écrits destinés à propager leurs idées au plus grand nombre. Ainsi, les réseaux constituent des véritables plateformes d'échanges et de diffusion des savoirs idéales pour la propagande de nouvelles idées. L'activité de propagande par l'écriture se fait d'une part par la traduction de textes dans des langues du réseau de proximité, à l'exemple de Maria Sola de Sellares qui traduit en catalan les textes des pionniers de l'Éducation nouvelle. C'est ainsi que les livres de certaines grandes figures deviennent de véritables manifestes en raison de leurs traductions dans d'innombrables langues. D'autre part, le support privilégié

84 Ces associations se multiplient au lendemain de la Grande Guerre en particulier à Genève où sont fondées successivement L'Union de Secours aux Enfants, les associations Gouttes de lait, d'Hygiène scolaire, et de Protection de l'Enfance, pour n'en citer que quelques-unes. Investies par de nombreuses femmes, elles sont aussi le lieu de rencontre d'une grande diversité d'acteurs tels que médecins, psychologues, pédologues, pédagogues tous engagés, selon leur appartenance disciplinaire, dans l'étude l'enfant (Fuchs, 2004). 
pour diffuser les idées nouvelles est celui des revues qui se multiplient dans tous les domaines, que ce soit les milieux scientifiques, pédagogiques ou religieux. Par exemple, les théosophes anglais éditent des revues qu'ils diffusent à large échelle d'Angleterre en Australie en passant par l'Afrique du Sud grâce au réseau immense que constituent en eux-mêmes les pays du Commonwealth. Du côté des Quakers, diverses revues sont également publiées que ce soit à une échelle locale visant des groupes régionaux, ou à une échelle internationale fédérant divers groupes nationaux. Ainsi, Hélène Monastier fonde en 1940 la revue Entre Amis au moment même où son équivalent en France, l'Écho des Amis disparaît. ${ }^{85}$ De fait, ces revues locales sont étroitement liées au mouvement de «christianisme social» dans lequel s'inscrivent les Quakers et plus largement, les protestants.

Le journal $L^{\prime} E s s o r$ constitue ici un témoin intéressant de l'engagement «chrétien social» de plusieurs personnalités genevoises de l'Éducation nouvelle et en particulier de l'Institut Rousseau. Fondé en 1905 par quatre pasteurs de l'Église évangélique libre ${ }^{86}$, ce journal qui se présente dans son sous-titre comme un journal «social, moral et religieux» travaille au «rapprochement entre les humains et à leur compréhension réciproque». Parmi les auteurs qui y collaborent, Pierre Bovet constitue l'un des plus fidèles tandis qu'Auguste de Morsier ${ }^{87}$ y assure une chronique (Ruchat \& Vilbrod, 2011). À partir de 1919, Ferrière en devient le rédacteur en chef et ce jusqu'en 1925. Ferrière écarte la dimension religieuse du journal au profit d'une dimension éducative comme en atteste le nouveau sous-titre du journal désormais «social, moral, éducatif». Dès 1922, Ferrière propose un nouveau titre - Le Nouvel Essor - qui fait étrangement écho à Pour l'Ère Nouvelle qui voit le jour cette même année. Un changement de nom qui occasionne quelques remous auprès des collaborateurs ${ }^{88}$ mais est finalement accepté. Pendant ces années, Ferrière uti-

85 Voir Royston (2005, p. 52).

86 Nom donné aux pasteurs qui refusent de signer un contrat avec l'État et préfèrent rester «libres» de la tutelle de l'État. L'appellation «Église évangélique libre» est utilisé pour désigner ce mouvement à Genève, l'appellation «Église libre» désignant le mouvement qui apparaît au même moment dans le canton de Vaud.

87 Un cousin d'Édouard Claparède.

88 En octobre et novembre 1921, Ferrière évoque dans son Petit Journal les difficiles négociations pour changer le titre de la revue au point d'en faire une «affaire Essor» (Gerber, 1989, p. 23). 
lise L'Essor comme tribune de propagande pour l'Éducation nouvelle, L'Essor constituant ainsi l'une des premières revues à parler d'Éducation nouvelle en Suisse romande (Ruchat \& Vilbrod, 2011).

La publication de PEN à partir de 1922 renforce encore l'importance de Ferrière qui peut ainsi diffuser ses idées à une double échelle, à la fois régionale et internationale. De 1933 à 1943, Le Nouvel Essor est repris en mains par Albert Sechehaye, soutenu par l'Église évangélique libre, qui poursuit la diffusion d'un esprit internationaliste, pacifiste, chrétien social et favorable aux pédagogies nouvelles. Au cours de ces dix ans, plusieurs personnalités de l'Institut y collaborent, notamment Édouard Claparède et Alice Descoeudres. Dès 1944, c'est au tour d'Edmond Privat de prendre la tête du journal. Ce dernier, membre de plusieurs associations pacifistes et très engagé pour la diffusion de l'esperanto, est aussi un membre du groupe genevois de la «Société des Amis». Proche ami de Romain Rolland et de Gandhi, Edmond Privat influence durablement Le Nouvel Essor de son charisme et son engagement pour la cause pacifiste. Si L'Essor/Le Nouvel Essor est avant tout un journal Suisse romand, il entretient des contacts étroits avec le journal français Chrétien Libre dont le rédacteur Léon Revoyre rencontre Ferrière à Genève en 1925 (Gerber, 1989, p. 34).

Au terme de cette analyse, nous constatons l'importance cruciale que jouent les réseaux informels dans la diffusion des idées de l'Éducation nouvelle et plus largement, dans l'internationalité du mouvement. Durant les années 1920, ces derniers sont étroitement liés aux appartenances confessionnelles de ses trois dirigeants dont les réseaux s'étendent d'emblée à une échelle internationale. Comme le montre Fuchs (2007a), l'institutionnalisation de ces réseaux au lendemain de la guerre contribue à l'extension et à la reconnaissance de certaines causes déjà présentes dans les discours d'avant-guerre qui se manifestaient alors sous des formes plus isolées. Les réseaux facilitent la circulation des personnes et des savoirs à un niveau international et contribuent ainsi à la construction de la nouvelle science. ${ }^{89}$ Selon nous, ils constituent en outre un tremplin essentiel pour une militance «à grande échelle» dont la Ligue se sert pour s'étendre rapidement au cours des années 1920. Cependant, cette dernière bénéficie également d'un contexte politique et

89 Nos recherches récentes sur les trajectoires et réseaux d'acteurs montrent l'importance des femmes dans la circulation et la diffusion des idées de l'Éducation nouvelle (Haenggeli-Jenni, 2015). 
économique propice aux discours militants. Ce contexte contribue à transformer l'internationalité de la Ligue en un internationalisme synonyme de lutte pour la paix, où l'international devient une cause en soi qu'il convient de défendre à travers les principes éducatifs de l'Éducation nouvelle. Comme l'observe Hofstetter (2008) au sujet de l'Institut Rousseau, «l'internationalisme de l'Institut n'est plus seulement un moyen pour faire circuler les idées et élargir les connaissances, mais devient un but en soi pour construire la paix et la coopération mondiale» (p. 222). Pour l'Institut comme pour la Ligue, «l'ambition se révèle bientôt démesurée» puisque l'un comme l'autre se voient contraints de réduire l'ampleur de leur champ d'action dès les années 1930, l'Institut se concentrant désormais sur ses tâches scientifiques et la Ligue focalisant son énergie sur la mise en application de ses principes à une échelle locale ou régionale. 


\section{CHAPITRE 4}

\section{ESPACES DE CONSTRUCTION, TERRAINS PARTAGÉS}

Pour donner corps à leurs revendications de réforme, les acteurs de l'Éducation nouvelle s'activent dans divers lieux institutionnels, publics ou privés, qui constituent leur «terrain d'expérimentation». Parallèlement, ils s'engagent au sein de divers groupes et associations qui, de par leur caractère institutionnel, apportent une dimension «formalisée» à leurs revendications et contribuent à leur reconnaissance. Nombre d'acteurs participent aux manifestations organisées par ces groupements et rédigent des articles pour les revues ou bulletins qu'ils publient. Comme le montrent les travaux de Hofstetter et Schneuwly (2002, 2007), l'implication des acteurs dans ces diverses structures s'inscrit dans un processus d'institutionnalisation et de disciplinarisation des sciences de l'éducation auquel les mouvements réformistes tels que l'Éducation nouvelle sont étroitement liés. Pourtant, dans les premières décennies du $20^{\mathrm{e}}$ siècle, le mouvement se développe «selon des formes disciplinaires traditionnelles (associations de chercheurs, institutions, revues et congrès) et peine à trouver une assise institutionnelle» (2002, p. 10). Selon ces auteurs, «ni la pédagogie expérimentale, ni la pédologie, ni même la psychologie de l'éducation ne s'installent de plein droit à l'université» (2002, p. 11). Il faudra attendre les années 1960 pour qu'une institutionnalisation durable des sciences de l'éducation se développe dans la plupart des pays occidentaux.

Ce chapitre vise à mieux comprendre comment se manifestent les prémices de cette institutionnalisation. Plus précisément, il s'agit de repérer quels sont les acteurs qui y participent, selon quelles modalités et autour de quels enjeux. Il nous intéresse de mieux connaître l'ampleur de ces premières formes disciplinaires afin de mesurer leur importance dans le processus de construction de la nouvelle science. Pour pouvoir répondre à ce questionnement, nous avons analysé Pour l'Ère Nouvelle à 
travers quatre thématiques correspondant aux quatre «formes institutionnelles» qui se mettent en place à ce moment-là: les institutions, les associations, les supports éditoriaux et les manifestations. Elles constituent les quatre volets de ce chapitre. L'analyse de la structure de la Ligue (associations, sections nationales, revues affiliées, congrès) combinée à une étude des discours nous ont permis de comprendre comment les acteurs évoluent à l'intérieur de ces structures mais aussi comment les savoirs y circulent et se formalisent au contact de ces diverses formes d'institutionnalisation.

\section{ÉCOLES, CLASSES, INSTITUTS: \\ EMBLÈMES D'UN MOUVEMENT QUI S'INSTITUTIONNALISE}

La lecture de Pour l'Ère Nouvelle permet d'identifier une grande variété d'institutions dans lesquelles s'élaborent et s'expérimentent les méthodes d'Éducation nouvelle. Par le terme institutions, nous entendons d'abord tous les types d'écoles - définies au sens de «skolé» comme un «lieu réservé à l'apprentissage» (Hofstetter \& Schneuwly, 2007) qu'elles soient privées, publiques, nouvelles, traditionnelles, maternelles, primaires ou secondaires. Ce terme inclut également les diverses institutions de formation, en particulier celles qui forment aux métiers de l'éducation (écoles normales, instituts des sciences de l'éducation, teaching colleges, etc.). Enfin, nous appelons institutions tous les établissements qui poursuivent un objectif de formation quel que soit le public auquel ils s'adressent: jeunes ou moins jeunes, secteur ordinaire ou spécialisé, etc. Ce volet vise à identifier les divers lieux institutionnels dans lesquels les acteurs de l'Éducation nouvelle s'engagent au quotidien, quels sont les lieux privilégiés, quels en sont les fonctions et les usages, les types de rencontres qui s'y produisent; il s'agit aussi de repérer les échos qu'en donne la revue, selon quelle fréquence, à travers quels auteurs et sous quelle forme.

\section{ÉCOLES ET INSTITUTS}

Grâce à une analyse quantitative, nous constatons la présence massive d'articles comportant le mot école dans leur titre. Dans la plupart des cas, ce mot est ensuite assorti d'une série de qualificatifs qui apportent des précisions à différents niveaux: statut de l'école (école maternelle, enfan- 
tine, primaire, secondaire, école normale, etc.), appartenance au secteur privé ou public (école publique rénovée, école populaire), fonction dans la formation (école ménagère, rurale, internationale) ou spécificité parmi les écoles nouvelles (école expérimentale, école active, école de Plein air). Certaines écoles se définissent par le nom d'une figure du mouvement dont elles se réclament (École Montessori, École Decroly, École Winnetka) ou simplement par le nom que leur a donné leur fondateur souvent lié au lieu où réside l'école - (École des Roches, École de l'Odenwald, École de l'Ermitage, St-Christopher School). Enfin, certains termes utilisés pour qualifier une école révèlent son aspect particulièrement novateur ou prometteur: «une école miraculeuse», «école de l'Humanité», «école normale américaine de l'avenir».

Parmi les 184 articles contenant le mot école dans leur titre, nous constatons une répartition inégale.

Tableau 11.

Articles contenant le mot école dans leur titre

\begin{tabular}{ll}
\hline École nouvelle & 32 \\
École active & 24 \\
École expérimentale & 14 \\
École maternelle & 13 \\
École primaire & 12 \\
École rurale école-ferme & 8 \\
École rénovée & 7 \\
École internationale & 7 \\
École de Plein air & 6 \\
École secondaire & 4 \\
École supérieure & 3 \\
École normale & 3 \\
École de jeunes filles $^{\text {École unique }}$ & 3 \\
Total $^{1}$ & 3 \\
\hline
\end{tabular}

1 Les 35 autres titres d'articles contenant le mot école sont suivis d'un autre adjectif désignant un pays ou une particularité de cette école (10), d'autres éléments de précisions tels qu'un nom (École Decroly, École Winnetka, École des Roches) (8) ou d'un groupe de mots composant l'ensemble du titre («L'école adaptée aux besoins des enfants» «Le dessin et le théâtre à l'école», «L'enfant et son école») (17). 
Comme nous pouvons le voir, les écoles nouvelles arrivent en tête de liste. Cette dominance s'explique par le fait qu'elles constituent le modèle d'école valorisé par la Ligue, modèle dont celle-ci fait la propagande à travers ses revues. ${ }^{2}$ Telles qu'elles sont présentées dans PEN, ces écoles nouvelles sont des institutions privées dont les effectifs sont modestes (20-30 élèves en moyenne) et sont dirigées par un directeur ou une directrice. Les directrices sont nombreuses notamment en France où plusieurs écoles sont fondées par des institutrices de maternelle pratiquant des méthodes pour jeunes enfants, notamment les méthodes Montessori. ${ }^{3}$ Malgré leur diversité, ces institutions sont présentées comme des lieux d'expérimentation des méthodes nouvelles. Pour Bertier, elles constituent les «laboratoires d'expérimentation nécessaires à une généralisation future des méthodes dans les écoles publiques» (Bertier, 1923, PEN, 8). Beatrice Ensor rappelle le statut particulier des écoles nouvelles, établissements dans lesquels le mouvement a pris naissance:

La première croisade de notre mouvement date d'il y a trente ans: des pionniers, tels que Parker, Reddie, Lietz, Badley, Demolins ont fondé les écoles nouvelles parce qu'ils étaient mécontents de l'éducation ordinaire. Ces écoles privées n'étaient pas soumises au contrôle de l'État; elles se caractérisaient par une éducation active et dynamique, non passive et statique. Elles ont créé pour l'enfant une ambiance où celui-ci peut se développer spontanément et joyeusement et ont largement contribué aux changements survenus dans l'instruction publique. On peut les considérer comme les laboratoires de l'Éducation nouvelle. (Ensor, 1929 PEN, 51, p. 21)

Bien que beaucoup moins nombreux (11 articles), certains articles de PEN évoquent des instituts, autres lieux où se rencontrent les acteurs et les savoirs sur l'enfance. Comme le montrent Hofstetter et Schneuwly (2004), le terme institut prend des significations différentes selon les époques, désignant successivement une organisation de type religieux, une communauté de scientifiques $\left(18^{\mathrm{e}} \mathrm{s}\right.$.), une institution pour la

2 Dans cette catégorie sont inclues les «Écoles nouvelles à la campagne» bien que celles-ci ne représentent que deux articles sur les 32, tous deux étant rédigés par Ferrière et consistant en un listing des écoles nouvelles dans le monde.

3 Pour exemple, PEN évoque à plusieurs reprises les écoles Clamart, de Sèvres, de Vaucresson, de Cours Moulin qui sont toutes dirigées par des femmes. 
recherche et l'éducation $\left(19^{\mathrm{e}} \mathrm{s}\right.$.) ou «une organisation pour la promotion et la poursuite d'une cause» (p. 582). Dans la revue, les instituts sont avant tout des lieux de recherche et de formation où sont enseignés à la fois les savoirs scientifiques issus de la recherche (psychologie, pédagogie expérimentale) et les savoirs issus de l'expérimentation pratique effectuée dans des écoles qui leur sont rattachées. ${ }^{4}$ Un aperçu des titres ${ }^{5}$ de ces 11 articles permet d'emblée de constater la diversité d'institutions qu'ils désignent:

Instituts d'orientation professionnelle (Paris et Lisbonne)

Institut J.-J. Rousseau (Sciences de l'éducation, Genève)

Institut International de Psychagogie et Psychothérapie (Genève et Nancy)

Institut expérimental d'Éducation (Bakulé, Tchécoslovaquie)

Institut d'Iéna (Sciences de l'éducation, Allemagne)

Institut de Vienne (Bundes Erziehungs Anstalten)

Institut ouvrier d'Espagne (Institutos Obreros)

Institut médico-pédagogique (Genève)Institut Decroly (Bruxelles)

Qu'il s'agisse des écoles ou des instituts, le lecteur prend connaissance de ces institutions à travers les écrits des protagonistes qui y enseignent - instituteurs et éducateurs - mais surtout, à travers les articles de ceux qui les dirigent. En effet, la majorité des auteurs qui présentent une institution dans la revue sont les responsables de l'établissement, le plus souvent son directeur ou directrice qui en est souvent aussi le fondateur ou la fondatrice. Ils présentent les objectifs, méthodes et activités de leur institution sous forme de récit; un récit souvent très descriptif et détaillé comme s'il fallait permettre au lecteur de s'imprégner du lieu, d'en saisir toutes les caractéristiques. Ces récits ont pour fonction de faire connaître l'existence d'institutions travaillant selon les méthodes nouvelles dans diverses parties du monde. Il s'agit donc bien de

4 Le nombre réduit d'instituts émanant de l'analyse quantitative est à relativiser car nous n'avons recensé ici que les articles contenant le mot institut dans leur titre. Par conséquent, les instituts qui pourraient être évoqués dans d'autres articles ne figurent pas dans cette liste.

5 Les deux premiers de la liste ci-dessous font l'objet de deux articles chacun, les autres ne concernant qu'un article (comportant le nom de l'institut dans son titre). 
documenter, comme l'indique la catégorie appelée «documentation pédagogique» dans laquelle nous avons classé ce type de récit, autrement dit d'accumuler de la connaissance sur les expériences menées dans diverses parties du monde. Un travail auguré par Ferrière en 1899 déjà, dans son Bureau International des Écoles nouvelles (BIEN) qui avait pour but de centraliser toutes les informations disponibles sur les écoles nouvelles.

Bien que cela soit moins fréquent, certaines institutions sont évoquées par des scientifiques (universitaires, chercheurs, théoriciens), soit parce qu'ils y pratiquent leurs expériences (ex. Montessori à la Casa dei Bambini, Decroly à l'École de l'Ermitage), soit parce qu'ils en ont fait la visite. C'est le cas de Decroly qui écrit plusieurs articles (4) entre 1922 et 1926, suite à des visites d'école aux États-Unis, en Amérique latine et aux Pays-Bas dans lesquels il explique le fonctionnement et les méthodes de ces institutions. Comme nous l'avons vu, le mode du voyage est alors monnaie courante dans le domaine de l'éducation (comme dans d'autres) pour pouvoir s'informer sur le fonctionnement des systèmes éducatifs et méthodes d'enseignement à l'étranger.

\section{INSTITUTIONS DE RECHERHCE ET FORMATION}

Les fonctions principales des institutions évoquées dans PEN sont celles de la recherche, puisque c'est là que s'y expérimentent les méthodes nouvelles, et de la formation en raison des nombreux visiteurs qui s'y rendent pour se perfectionner dans leur métier. Selon nos analyses, le mode de formation privilégié est celui du séjour dans les institutions, que ce soit de courte ou de longue durée. Il fait référence au principe du «compagnonnage» qui jusque-là constituait le mode de formation privilégié pour former les instituteurs (Hofstetter, 2010). Basé sur le principe de l'apprentissage par imprégnation, l'étudiant apprend en observant la pratique de celui ou celle qui l'accueille. Ce mode de formation peut paraître surprenant à une époque où l'institutionnalisation des formations à l'enseignement se met en place dans la plupart des pays occidentaux (Lussi Borer, 2008), notamment à travers la fondation des «écoles normales». Toutefois, l'analyse biographique de plusieurs instituteurs montre qu'ils se forment d'abord dans ces institutions, puis complètent leur formation par des stages ou séjours à l'étranger. Selon Fuchs (2007b), l'intensité des échanges d'étudiants et d'enseignants à cette époque contribue à instaurer une coopération et 
une communication mutuelle à l'échelle internationale. Pour exemple, Ferrière séjourne plus d'une année à Ilsenburg et Haubinda (1898 et 1899) - écoles nouvelles à la campagne (Landerziehungsheime) fondées par Hermann Lietz - où il est engagé comme maître volontaire. Il apprend le métier d'instituteur auprès de ce fondateur que Ferrière présente comme son maître au sens quasi spirituel du terme. Deux autres pédagogues suisses, Wilhelm Frei (1872-1904) et Wilhelm Zuberbühler (1872-1942), y séjournent au même moment et tout comme lui, effectuent un stage dans les Landerziehungsheime allemands. ${ }^{6}$ Comme le montre Duval (2009), l'École des Roches constitue également un lieu de formation pour de nombreux instituteurs et visiteurs qui désirent s'imprégner de la vie et des pratiques d'une école nouvelle. Il en va de même au foyer du «Home chez Nous» (Coquoz, 1998) où les directrices diplômées de l'Institut Rousseau fonctionnent comme formatrices pour les étudiants qui désirent s'initier «grandeur nature» aux méthodes prônées par l'Institut Rousseau.

Au-delà des séjours de type «compagnonnage», la revue témoigne d'une grande mobilité des acteurs dans le but de se former dans des institutions spécialisées à l'étranger. Dans la rubrique «Nouvelles diverses», une large diversité de cours est proposée que ce soit sous forme de stages, cours d'été ou semestres de formation dans les universités. Ils s'adressent aux praticiens mais aussi aux membres des administrations scolaires, aux directeurs et inspecteurs qui désirent compléter leurs connaissances dans les domaines de la pédagogie, psychologie, tests, etc. Parmi ces offres de formation, les cours internationaux organisés par Montessori dès 1909 attirent un grand nombre de praticiens du monde entier. Soucieuse de l'application orthodoxe de ses méthodes, cette dernière organise des cours de formation spécifiques et établit un réseau important d'écoles dirigées par des directrices expérimentées auprès desquelles les étudiants doivent faire leurs stages. Parmi ces écoles, signalons les Casa dei Bambini qui «devinrent parfois de véritables lieux saints où les éducateurs se rendaient en pèlerinage» (Röhrs, 1994 / 2000, p. 2). Quant aux acteurs universitaires du champ, une étude prosopographique de leur parcours (Cicchini, 2007) montre que les

6 Au terme de leur séjour, les trois compatriotes fondent en 1902 la première école nouvelle de Suisse, au château de Glarisegg, dans le canton de Thurgovie. Zuberbühler en assurera la direction pendant 35 ans. (Hameline, 2002a). 
séjours de formation à l'étranger font partie intégrante de leur formation initiale, phase importante de leur «sociabilité disciplinaire» (p. 369). $C^{\prime}$ est à ce moment-là que se constitue l'essentiel de leur réseau de relations à l'intérieur duquel ils développeront par la suite des collaborations scientifiques. Ainsi, tout parcours universitaire comprend une partie de la formation à l'étranger qui s'effectue généralement sous forme de séjour prolongé dans un institut de formation ou dans une université renommée.

\section{DES VOYAGES SUSCITANT L'ÉCRITURE}

Ces visites et séjours de formation s'assortissent le plus souvent de productions d'écrits que ce soit sous la forme de «notes de voyage», de «compte rendu de visite» ou de «récit de voyage». Ces articles, publiés dans des revues pédagogiques telles que Pour l'Ėre Nouvelle, remplissent une fonction documentaire dans le sens où ils contribuent à la connaissance des lieux d'expérimentation et permettent ainsi de cartographier l'étendue du mouvement. Le style d'écriture utilisé dans ces récits s'apparente étroitement à celui que l'on trouve dans les récits anthropologiques voire ethnologiques où le visiteur/auteur consigne avec minutie tous les détails du pays visité. Une pratique largement utilisée à cette époque où l'usage de la photo est encore marginal, et les mots remplacent l'image. Ce mode d'apprentissage par visite est largement répandu parmi les acteurs du mouvement comme en atteste la quantité impressionnante d'articles dans PEN relatant de visites ou séjours dans des établissements divers. De fait, il prolonge une pratique déjà courante au $19^{e}$ siècle où le voyage vise à découvrir d'autres modèles scolaires et méthodes d'enseignement. Comme le montre Matasci (2009), certains gouvernements mandatent même des «missions pédagogiques» pour aller observer les systèmes en vigueur dans d'autres contrées avant d'entreprendre des changements dans leur pays.

Cette activité documentaire est celle qui occupe Ferrière depuis 1899, date à laquelle il fonde son Bureau international des Écoles nouvelles pour lequel il confectionne des milliers de fiches recensant toutes les caractéristiques des écoles nouvelles qu'il visite. ${ }^{7} C^{\prime}$ est aussi lui qui fait

7 Ces fiches contiennent une multitude de renseignements sur les aspects pédagogiques, organisationnels et financiers de l'établissement, de même que sur les personnes qui en sont responsables. Ces renseignements servi- 
connaître aux lecteurs de PEN les multiples expériences menées en Europe de l'Est et en Amérique latine en rédigeant des articles comme «La Turquie et ses écoles nouvelles» (1929), «Voyages d'études en Pologne» (1930), «Notes de voyage: Autriche, Hongrie, Roumanie» (1930), «L'Éducation nouvelle au Chili/au Brésil/en Argentine/en Uruguay» (1931), "Chronique d'Amérique latine» (1934). D'après les articles publiés dans la revue, certaines des plus éminentes figures du mouvement utilisent aussi ce mode d'apprentissage par visites. Ainsi, Decroly visite plusieurs écoles sur le continent américain, celle de Park School en compagnie de Raymond Buyse ${ }^{8}$ et celle de Miss Pratt en compagnie de Juliette Delgoffe ${ }^{9}$ (Decroly \& Delgoffe, PEN, 1923, 7, pp. 46-50) aux États-Unis. En Amérique du Sud, il visite le gymnase moderne de Bogota (Colombie) dont il publie un récit encenseur (Decroly, PEN, 1926, 19, pp. 23-28). Bertier fait aussi partie de ces gens qui voyagent et sont curieux des pratiques des autres. Proche ami de Ferrière, c'est en sa compagnie qu'il visite les Landerziehungsheime de Bieberstein et Haubinda (1906), l'École de Bedales en Angleterre (1908) et les écoles nouvelles de Suisse alémanique (Glarisegg et Hof Oberkirch) en 1911 (Gerber, 1989). Ce même type de récits se trouve dans les revues allemande et anglaise où les articles évoquant des visites d'écoles constituent une part importante des numéros. Nombre d'entre eux sont rédigés par Beatrice Ensor et Elisabeth Rotten, attestant de leur mobilité et de leur propension au voyage. En cumulant les

ront d'une part à établir une liste de critères permettant d'attribuer le «label» d'école nouvelle à un établissement; d'autre part, ils permettent à Ferrière d'établir une liste détaillée des écoles nouvelles qu'il publie dans $P E N$ en 1922 et 1925, destinée principalement aux parents désireux d'inscrire leur enfant dans l'un de ces établissements. Lors de l'incendie de son chalet des Pléïades en 1918, des milliers de fiches sont détruites, mais ceci ne décourage pas Ferrière qui se remet au travail une fois le chalet reconstruit (Gerber, 1989; Meyehoffer \& Gunning, 1929).

8 Docteur en pédagogie et inspecteur de l'enseignement primaire à Bruxelles, Raymond Buyse est membre de la Société Belge de Pédotechnie fondée par Decroly. Ce voyage fait l'objet d'un article dans PEN (1922, (4, pp. 70-75) dont le style, faisant une large place aux aspects bucoliques de cette école, laisse penser que Ferrière a en partie réécrit son contenu pour les besoins de la cause (Hameline, 2002b). Le carnet de route original de ce voyage a été publié dans une édition commentée par Depaepe et D'Hulst (2011). Juliette Delgoffe est professeure à l'École de l'Ermitage à Bruxelles. 
articles des trois revues, certaines destinations apparaissent attirer davantage l'intérêt des voyageurs. Ainsi, dans les années 1920, les États-Unis tiennent le haut du pavé, attirant les regards de nombreux visiteurs européens.

\section{DESTINATIONS PRIVILÉGIÉES}

L'analyse qualitative de Pour l'Ère Nouvelle montre que certaines institutions attirent davantage les visiteurs, constituant ainsi des sortes de pôles $d^{\prime}$ attraction. ${ }^{10}$ Pour la plupart, il s'agit d'institutions de renommée internationale, en raison de l'activité des acteurs qui les dirigent et y travaillent. PEN s'en fait l'écho sous différentes formes: dans les éditoriaux, dans la rubrique «Nouvelles diverses» où sont annoncées les principales manifestations organisées par ces institutions, dans les articles d'acteurs qui y ont séjourné et en font le récit. Dans les années 1920, 1'Institut Rousseau constitue sans conteste l'un des pôles d'attraction du milieu éducatif, sorte de «plaque tournante» (Hofstetter, 2010) où se rencontrent praticiens et universitaires du monde entier. Les séjours de formation et les cours d'été y sont très prisés comme en atteste le grand nombre d'étudiants étrangers qui y sont inscrits. ${ }^{11}$ L'Institut pédagogique d'Iéna, en Allemagne, est un autre lieu de formation important, bien qu'il soit essentiellement fréquenté par des universitaires maîtrisant la langue allemande, c'est-à-dire les Suisses alémaniques, les Autrichiens et les ressortissants des pays d'Europe centrale. Une autre institution fréquemment citée dans PEN est le Teachers College de la Columbia University, à New York. Tout comme l'Institut Rousseau, il fonctionne comme un centre de recherche, de documentation et de propagande, propose des cours d'été ou des séjours de formation et organise divers congrès pour les éducateurs. En cela, il constitue un "pôle d'attraction» où la plupart des grandes figures de l'Éducation nouvelle sont passées ${ }^{12}$, que ce soit pour se former, pour y donner des conférences ou pour y rencontrer des représentants de l'association américaine Progressive Education. Comme le montre l'activité d'un éminent profes-

10 Cicchini (2007) parle par exemple du «phénomène d'attraction» joué par l'Institut J.J. Rousseau sur les «praticiens» (p. 358).

11 AIJJR, Fonds général, programmes de cours 1912-1920.

12 Decroly y fait une partie de sa formation. 
seur de cette institution, Harold Rugg (1886-1960) ${ }^{13}$, le Teachers College est en contact avec la Ligue. Ce psychologue et pédagogue spécialiste de la question des curriculums, participe aux congrès de la Ligue (Locarno et Nice), siège au Comité international comme représentant de son pays et assume la coordination de la commission permanente sur la «Refonte des programmes scolaires».

Outre les instituts de formation reconnus, certaines villes ou pays attirent visiteurs et étudiants en raison d'un modèle scolaire particulier ou de la mise sur pied d'expériences novatrices. Ainsi, la revue fait état de nombreuses visites dans des lieux comme Vienne, Hambourg, la Pologne, la Russie, la Turquie qui ont réorganisé leur système d'éducation sur la base des principes de l'Éducation nouvelle. Ainsi, Vienne attire de nombreux instituteurs, inspecteurs et membres de l'administration scolaire en raison de la mise en place d'une réforme généralisée du système scolaire en 1927. Parmi eux, Frank Walser en fait un compte rendu dans PEN (Walser, PEN, 1928, 42) tandis que Robert Dottrens ${ }^{14}$ publie un ouvrage sur la base de son séjour dans cette ville, Le problème de l'inspection et l'Éducation nouvelle (1931). La Belgique, Bruxelles en particulier, attire également de nombreux partisans de l'Éducation nouvelle qu'ils soient praticiens ou théoriciens. Connue pour être le berceau de la pédologie, la Belgique attire les scientifiques qui étudient l'enfant, en particulier des médecins et des psychologues tels que Claparède ou Binet qui viennent rencontrer Schuyten, Ioteyko et Decroly. Le Congrès de pédologie organisé à l'Université de Louvain en 1911 avait déjà rassemblé nombre de ces scientifiques dans ce pays (Depaepe, 1987).

Du côté des praticiens, l'École de l'Ermitage constitue sans conteste l'un des pôles d'attraction les plus importants d'Europe où affluent quantité de visiteurs du monde entier. De fait, les méthodes éducatives élaborées par Decroly étant fondées sur l'étude des anormaux ${ }^{15}$, cette

13 Ce dernier aura une influence notable sur l'école américaine des années 1930-1960 en raison des manuels - textbooks - qu'il publie dans les années 1930 et qui seront diffusés à plusieurs millions d'exemplaires dans tout le pays.

14 Robert Dottrens (1893-1984), instituteur et inspecteur genevois séjourne à Vienne près d'une année pour observer la mise en place de la réforme et analyser les problèmes que cela suscite pour les inspecteurs.

15 Ce mot est utilisé ici selon l'usage qui en est fait à l'époque, c'est-à-dire pour désigner les enfants nécessitant un enseignement spécialisé. 
école attire aussi bien des praticiens curieux de voir en pratique la méthode des «centres d'intérêts» que des scientifiques intéressés par les recherches menées sur le développement de l'enfant. Notons à titre d'exemple, la visite d'Eugène Dévaud (1876-1942) ${ }^{16}$ qui, en 1932, obtient un congé des autorités cantonales pour se rendre en Belgique auprès de Decroly (Cicchini, 2007; Lussi Borer 2008). De manière plus générale, la figure de Decroly et sa méthode des «centres d'intérêts» est l'une des plus abondamment citée dans les articles de PEN. Que ce soit en Espagne, en Allemagne, en Pologne ou en Amérique latine, Decroly semble connu et apprécié par de très nombreux acteurs de l'Éducation nouvelle. À la fin des années 1930, la Belgique attire de nombreux observateurs en raison de la mise sur pied d'un nouveau plan d'études dans tout le pays (1936). Parmi eux, Henri Wallon et Henri Piéron, alors impliqués dans l'expérience des «classes d'orientation» en France, sont intéressés par une réforme de l'enseignement à large échelle et font un compte rendu détaillé de leur visite dans PEN (Wallon et Piéron, PEN, 1937, 127). En France, en l'absence d'un mouvement largement établi ou d'une institution faîtière, ce sont les expériences plus isolées de Roger Cousinet ${ }^{17}$ ou de Célestin Freinet ${ }^{18}$ qui attirent les visiteurs, de même que les écoles nouvelles pionnières telles que l'École des Roches dirigée par Georges Bertier.

Du côté des universitaires, Cicchini (2007) remarque une forte mobilité au cours de leur formation initiale qui inclut plusieurs mois voire quelques années à l'étranger. Mais cette mobilité diminue sensiblement une fois leur formation achevée celle-ci se limitant alors aux congrès et colloques de leur discipline. ${ }^{19}$ De l'analyse de PEN, nous faisons le

16 Eugène Dévaud (1876-1942) est alors professeur de pédagogie à l’Université de Fribourg $(\mathrm{CH})$ et doyen de la Faculté des lettres.

17 À propos de cette figure, voir les travaux de Raillon (1990) et Ottavi et Gutierrez (2007).

18 Sur Freinet et son mouvement, voir les récents travaux de Go et Riondet (2015) et de Peyronie (2013). Sur Élise Freinet et ses pratiques naturistes, voir Riondet (2013b).

19 Claparède, de même que Piaget, participent essentiellement aux congrès de psychologie ou de médecine (Hofstetter, 2010). Decroly, figure d'exception, participe aussi bien aux congrès de médecine et de psychologie qu'à ceux de la Ligue, attestant de son engagement aussi bien scientifique qu'idéologique. Pour une analyse des réseaux de Decroly, voir Wagnon (2013) et Van Gorp, Depaepe et Simon (2004). 
constat que les praticiens sont parmi ceux qui voyagent le plus, que ce soit au moment de leur formation initiale ou pendant leurs années de carrière. De fait, la majorité des voyageurs sont des éducateurs qui travaillent depuis quelques années et qui, pour moderniser leurs pratiques, visitent les «lieux phares» de l'Éducation nouvelle. Cependant, les acteurs les plus mobiles sont les directeurs d'écoles et les représentants de l'administration scolaire. Nous faisons l'hypothèse que leur fonction dirigeante joue un rôle dans cette mobilité; cette fonction leur permet d'être plus libres de quitter l'établissement qu'ils dirigent, contrairement aux instituteurs qui ont la responsabilité des élèves au quotidien. De ce fait, ce sont eux qui, de retour de leurs voyages, sont les plus à même d'impulser des changements de pratiques dans les écoles dont ils ont la responsabilité. Ainsi, directeurs, inspecteurs, responsables de régions voire ministres de l'instruction publique circulent dans les divers «temples» de l'Éducation nouvelle pour s'en inspirer puis tenter de les adapter à la réalité de leur pays ou de leur école. ${ }^{20}$

Pour conclure, notre analyse fait apparaître une nette dominance des institutions de type «école»: c'est là que s'expérimentent les méthodes nouvelles et que s'effectuent les recherches. Elles constituent donc des lieux de formation privilégiés où convergent les praticiens en cours de formation. Dans les années 1920, ces écoles sont principalement des écoles nouvelles à la campagne, institutions privées, à l'image de celles qui voient le jour dès la fin du 19 e siècle. Grâce à l'extension progressive du mouvement, les institutions se multiplient et se diversifient dans tous les pays. Ce phénomène se traduit par une augmentation des séjours de formation et donc une circulation accrue des savoirs sur l'éducation. Cela se traduit par une recrudescence d'articles dans les revues évoquant ces échanges consistant autant à s'inspirer des méthodes observées sur le lieu d'accueil qu'à partager ses propres expériences. Grâce au réseau international que constitue la Ligue, la circulation des personnes est ainsi facilitée et encouragée contribuant à propager les principes d'Éducation nouvelle à large échelle. À partir des années 1930, les expériences s'étendent aux écoles publiques et à

20 Voir pour exemple le périple de Joaquim Tomas nommé inspecteur chef de l'enseignement primaire au Portugal en 1910 puis promu recteur de l'Académie de Santarém. Il est co-fondateur de la Revista Escolar et collaborateur de la revue A Escola Primaria, organe de la Ligue entre 1921 et 1935 (Verissimo, 2007). 
d'autres ordres d'enseignement, en particulier au réseau du secondaire. Cette extension élargit le réseau des écoles à visiter et donc, le réseau de lieux de formation pour les maîtres. Car malgré l'instauration progressive de cursus et de programmes de formation dans les institutions spécialisées, le mode du compagnonnage reste très prisé en raison de l'importance accordée par les praticiens aux savoirs pratiques et au mode d'apprentissage par imprégnation; selon eux, il s'agit non seulement de se former à de nouvelles méthodes d'enseignement mais aussi de s'imprégner de «l'esprit» qui caractérise les écoles nouvelles.

\section{GROUPES ET ASSOCIATIONS: DES LIEUX PRIVILÉGIÉS POUR DONNER CORPS À LA MILITANCE}

Outre des institutions pratiquant les méthodes nouvelles, la revue se fait l'écho de quantité de groupes et associations dédiés à l'Éducation nouvelle composés principalement de professionnels de l'éducation. Selon Lussi Borer (2008), les associations d'enseignants «témoignent de la mise en place d'une organisation par la profession pour mieux représenter ses intérêts vis-à-vis de l'État, de la commune et du public» (p. 295). Cellesci constituent ainsi le premier interlocuteur de la profession auprès du politique lors des débats relatifs à la formation. Si l'émergence de ces groupes professionnels constitue «une étape clé dans le processus de professionnalisation de l'activité enseignante», cet auteur pense qu'elle «ne répond pas uniquement à un besoin social mais contribue à le créer» (Lussi Borer, 2008, p. 296). Il nous intéresse de voir quel rôle jouent ces associations au sein de la profession enseignante, dans quelle mesure celles-ci contribuent à la reconnaissance de nouveaux principes éducatifs et d'une autre conception de la profession. Ce volet vise aussi à comprendre quelle est leur place au sein de la Ligue, quels types d'acteurs en sont membres et plus largement, quelle est l'importance de ces associations dans l'institutionnalisation progressive du mouvement. Pour ce faire, nous avons croisé une analyse quantitative permettant de mesurer l'étendue et l'évolution de ces groupements avec une analyse qualitative permettant de comprendre quel est le rôle des acteurs en leur sein. 


\section{LES ASSOCIATIONS: DIVERSITÉ ET MULTIPLICITÉ}

L'analyse de PEN permet de constater que les associations constituent l'un des premiers lieux de rencontre des protagonistes du mouvement. Elles constituent la première forme de rassemblement des militants, car elle est sans doute la plus facile à mettre sur pied. En effet, il suffit d'une poignée de personnes pour fonder une association - un président, un trésorier, un secrétaire - et cette forme de regroupement ne comporte pas les contraintes administratives d'une institution. De plus, elle n'exige pas une conformité totale de la part de ses membres et permet de réunir des personnes d'horizons divers, ne pratiquant pas forcément la même profession ni ne partageant les mêmes confessions ou idéaux politiques. En d'autres termes, pour fonder une association, il suffit d'une cause commune et de convictions partagées auxquelles les membres sont prêts à dédier leur temps et leurs savoirs. Alors que tout laisse penser que la Ligue internationale pour l'Éducation nouvelle est une association, celle-ci peine à se considérer comme telle:

La Ligue internationale pour l'Éducation nouvelle n'est point proprement une «association» régulièrement constituée; à peine est-elle une fédération. Elle a plutôt l'ambition, comme le dit son statut de fondation, de jouer le rôle de lien entre les éducateurs qui s'intéressent théoriquement ou pratiquement à l'Éducation nouvelle. (Éditorial, PEN, 1927, 26, p. 49)

En tant qu'organe international, Pour l'Ère Nouvelle fonctionne comme relais de nombreuses associations nationales au sujet desquelles cette revue donne des informations. Parmi elles, nous avons repéré différents types de groupement qui se distinguent par l'échelle à laquelle elles interviennent (local, régional, national), l'ordre d'enseignement auquel appartiennent ses membres (maternel, primaire, secondaire), son degré de proximité avec la Ligue (membre, affiliée, section nationale). Pour recenser ces associations, nous nous sommes intéressés à leurs supports éditoriaux car la plupart publient des périodiques et se rendent visibles à travers ceux-ci. Ainsi, c'est en comptabilisant les revues affiliées à la Ligue que nous avons pu observer l'évolution du nombre d'associations, ceci en s'appuyant sur la rubrique «Livres et Revues» qui paraît dans la plupart des numéros de PEN. Dans le tableau suivant, nous pouvons observer une augmentation significative du nombre de revues dédiées à l'Éducation nouvelle, nombre incluant celles qui sont publiées en dehors 
de la francophonie. Cette croissance se manifeste dès la fondation de la Ligue mais plus particulièrement entre 1925 et $1930^{21}$ où l'on assiste à une «explosion» de revues - et donc d'associations - qui atteignent un pic entre 1928 et 1930.

Tableau 12.

Volume de la rubrique «Livres et revues»

\begin{tabular}{lccc}
\hline Année & $\begin{array}{c}\text { Rubrique «Livres et revues» } \\
\text { (pages) }\end{array}$ & Année & $\begin{array}{c}\text { Rubrique «Livres et revues» } \\
\text { (pages) }\end{array}$ \\
\hline 1922 & 6 & 1932 & 27 \\
\hline 1923 & 10 & 1933 & 51 \\
\hline 1924 & 28 & 1934 & 38 \\
\hline 1925 & 24 & 1935 & 31 \\
\hline 1926 & 37 & 1936 & 34 \\
\hline 1927 & 33 & 1937 & 33 \\
\hline 1928 & 61 & 1938 & 39 \\
\hline 1929 & 47 & 1939 & 40 \\
\hline 1930 & 64 & 1940 & 7 \\
\hline
\end{tabular}

Au-delà d'une représentation chiffrée, l'analyse des éditoriaux permet d'avoir un aperçu global de l'évolution des associations grâce aux informations qu'ils donnent sur la croissance du mouvement en général. Dans la mesure des données disponibles dans $P E N$, nous nous proposons maintenant de faire part des données recueillies sur ces groupements en traversant chronologiquement les éditoriaux de 1922 à 1931 (dès 1932, la revue ne publie plus d'éditorial) tout en prenant en compte les articles (10) dont le titre évoque explicitement une association.

Dans les années 1922-1923, les associations spécifiquement dédiées à l'Éducation nouvelle sont peu nombreuses car la plupart ne sont pas encore constituées. Aussi, les messages de soutien et les demandes d'adhésion à la Ligue proviennent surtout de personnes individuelles; ceci

21 À l'exception des années 1927 et 1929 au cours desquelles la rubrique est partiellement supprimée en raison des numéros dédiés aux rapports de congrès (Locarno et Elseneur). 
prouve que les réseaux sociaux sont alors principalement construits sur la base des relations personnelles et non institutionnelles. Ferrière joue ici un rôle crucial; grâce à son Bureau international des Écoles nouvelles (BIEN), il est en contact avec de nombreuses personnes d'emblée acquises à sa cause puisque celles-ci sont pionnières dans la mise en pratique des méthodes nouvelles. Ceci prouve que le mouvement existe avant 1921 et que la fondation de la Ligue répond à un besoin de rassemblement de ces initiatives. Au cours des premières années de PEN, Ferrière informe régulièrement des nouvelles adhésions qui se succèdent à un rythme soutenu. Dès le premier numéro, il fait la liste des personnes qui ont accepté de "prêter un appui moral» à la Ligue, soit une adhésion idéologique aux buts qu'elle poursuit. Parmi cette liste figurent les noms $\mathrm{d}^{\prime}$ illustres personnes dont les appartenances professionnelles et institutionnelles révèlent leur proximité avec les milieux académiques sciences et lettres - et de l'administration scolaire. En guise d'aperçu, nous reproduisons ci-dessous la liste ${ }^{22}$ pour la France et la Belgique. ${ }^{23}$

\section{France}

Georges BERTIER, président des Éclaireurs de France, directeur de l'École des Roches et co-directeur de la revue L'Éducation.

Jean BRUNHES, professeur au Collège de France, inspecteur général des études à l'Écoles des Roches.

Ferdinand BUISSON, député de la Seine, professeur honoraire à la Faculté des lettres de l'Université de Paris, directeur honoraire de l'enseignement primaire au Ministère de l'Instruction Publique.

Roger COUSINET, inspecteur de l'enseignement primaire à Arcis-surAube, co-fondateur de la société pédagogique La Nouvelle Éducation.

Alice JOUENNE, directrice de la première École de Plein air de la Municipalité de Paris et fondatrice de L'Éducation nouvelle (Groupe de recherches et d'expériences éducatives).

Pierre MILLE, homme de lettres.

Georges RENARD, professeur au Collège de France.

Ludovic ZORETTI, professeur à la Faculté des sciences de l'Université de Caen.

22 Cette liste est extraite de PEN et reproduite telle quelle. C'est pourquoi la manière de citer les personnes n'est pas harmonisée.

23 Selon le rédacteur, les messages provenant d'Italie, d'Espagne et des pays d'Amérique latine ne sont pas encore parvenus à la rédaction. 


\section{Belgique}

Gérard BOON, instituteur, directeur de section à la Policlinique de Bruxelles.

M.-F. BOULENGER, directeur de la Ferme-École provinciale de Waterloo. Hélène BURNIAUX, inspectrice de l'enseignement primaire à Bruxelles. Raymond BUYSE, docteur ès sciences pédagogiques, inspecteur de l'enseignement primaire à St-Gilles (Bruxelles).

L.-E. CARTIER, directrice de l'École moyenne à Bruxelles.

J. DEMOOR, professeur à la Faculté de médecine de l'Université de Bruxelles, directeur de l'Institut de physiologie.

Dr. ENSCH, directeur de la clinique scolaire de Schaerbeek.

H. FRERE, préfet de l'Athénée provincial à Morlanwelz.

M. HENDERICK, directeur de l'École normale d'instituteurs de Liège.

A. HERLIN, directeur de l'Institut provincial de Berchem-Ste-Agathe.

T. JONCKHEERE, directeur de l'École normale, professeur à la Faculté de philosophie et Lettres de l'Université de Bruxelles.

(Éditorial, PEN, 1922, 1, p. 3)

Les premières associations dont $P E N$ fait mention se constituent au début des années 1920. La Nouvelle Éducation fait partie des pionnières, fondée en janvier 1921 par Roger Cousinet et Madeleine Guéritte. Dès 1922, celle-ci publie le Bulletin de la Nouvelle Éducation inséré dans la revue L'Éducation dirigée par Georges Bertier. Ce jeune groupe «a pour objet de réunir tous les éducateurs décidés à favoriser en France l'activité personnelle des enfants, soit à l'école, soit dans la famille» et dont le but est «d'aider les éducateurs, de faire connaître et de répéter leurs expériences, afin que les travaux de chacun puissent profiter à tous» (Éditorial, PEN, 1922,3 , p. 55). Il restera pourtant en marge de la Ligue tout au long de la période étudiée, excepté quelques collaborations éditoriales avec PEN dans ses premières années de parution. ${ }^{24}$ Une année plus tard, un autre groupe français voit le jour à l'initiative d'Alice Jouenne: L'Éducation nouvelle. Groupe d'études, de recherches et d'expériences, ancêtre du Groupe Français d'Éducation nouvelle. Les membres de cette association

24 Pour plus de détails sur cette association, voir Gutierrez (2008) et Savoye (2006). 
révèlent la proximité des militants du terrain éducatif et des milieux académiques et administratifs. Ce groupe, ayant pour objet «l'étude des problèmes concernant la réforme de l'éducation et la recherche de solutions qu'ils comportent», se définit de la manière suivante:

Notre groupe veut réunir tous ceux qui pensent que cette orientation (respect de la personnalité de l'enfant dans l'éducation) est nécessaire. Il étudiera de nouvelles méthodes d'enseignement, en cherchera les applications pratiques et essaiera de les réaliser. Ce sera donc à la fois un groupe d'études, de recherches et de réalisations. Ce sera aussi un groupe de propagande qui ne négligera rien pour répandre les idées qu'il aura jugées indispensables pour l'application des méthodes qu'il préconise. Cette propagande se fera par voie de conférences, de brochures, de tracts et d'affiches. (Rédaction, PEN, 1922, 2, p. 31)

En juillet 1923, la rubrique «Notre Ligue» annonce l'existence d'un groupe catalan à Barcelone, «section espagnole de la Fraternité internationale d'Éducation» fondé et dirigé par Maria Sola de Sellares ${ }^{25}$. Parmi ses activités, le groupe signale la publication d'articles (presse et revues), l'organisation de conférences ainsi que la traduction de livres sur l'École active $^{26}$, tout ceci dans le but de faire connaître en Espagne et à l'étranger les expériences réalisées dans ce pays. Quelques mois plus tard, un autre groupe se constitue à Madrid en raison de la présence dans cette ville d'un «laboratoire pédagogique» créé dans un asile de 500 orphelins où ont lieu des «mensurations anthropométriques et des expériences psychologiques» (Notre Ligue, PEN, 1923, 6, p. 22); une nouvelle preuve que le mouvement se constitue en lien étroit avec la psychologie, voire avec l'anthropologie. Dirigé par Tomas y Samper, professeur à l'École normale Supérieure, ce groupe se rattache rapidement à la Ligue de même que sa revue qui deviendra l'un de ses organes officiels. À propos de ces groupes français et espagnols, Ferrière fait une comparaison qui met en évidence certaines tendances parmi les associations. Selon lui, l'Espagne compte deux groupes poursuivant les mêmes buts, mais ils se distinguent par les moyens qu'ils mettent en œuvre:

Comme «L'Éducation nouvelle» d'Alice Jouenne à Paris, le groupe de $\mathrm{M}^{\text {lle }}$ Maria Sola travaille plutôt à la diffusion psycho-pédagogique des méthodes de l'école active, en insistant sur le côté spirituel de l'œuvre de

25 Sur la vie et l'œuvre de cette auteure, voir Soler Mata (2010).

26 En particulier, les ouvrages de Ferrière. 
rénovation à accomplir; en outre il s'adresse de préférence à l'initiative privée. Le groupe de Tomas y Samper, comme celui de $\mathrm{M}^{\mathrm{me}} \mathrm{T}$. J. Guéritte et de M. R. Cousinet, semble être davantage expérimental, scientifique et chercher ses points d'appui dans les écoles officielles. (Éditorial, PEN, 1923, 7, p. 44)

Cette comparaison permet de prendre conscience de la diversité des associations qui, sous une apparente similitude se distinguent notamment par l'approche qu'elles privilégient (expérimentale, scientifique, spirituelle), et par le public auquel elles s'adressent (public ou privé). Ces indications confortent l'hypothèse selon laquelle La Nouvelle Éducation serait restée en retrait de la Ligue pendant de nombreuses années en raison du public plus «populaire» auquel elle s'adressait. Le côté élitaire de la Ligue $^{27}$ est d'ailleurs maintes fois critiqué par ses détracteurs tels que Freinet (Mole, 2010) qui lui reprochent d'élaborer des théories et des méthodes sur la base d'expériences menées dans des écoles privées dont les conditions n'ont rien à voir avec celles des écoles publiques. ${ }^{28}$

\section{VISITES ET CONFÉRENCES: ATTIRER DE NOUVEAUX MEMBRES}

Les années 1922 à 1925 sont marquées par une intense activité de propagande de la part des dirigeants de la Ligue (Ensor, Rotten, Ferrière). Ces derniers voyagent dans le monde entier pour donner des conférences, visiter des écoles, rencontrer des personnalités, dans le but de faire connaître la Ligue et d'augmenter le nombre d'adhérents. Comme le montrent les éditoriaux, la plupart des conférences sont organisées à la demande d'associations ou de groupes intéressés par les méthodes nouvelles, qu'ils appartiennent ou non au milieu éducatif. Paris figure parmi les destinations privilégiées de ces acteurs, en raison de la présence dans cette ville de plusieurs groupements et institutions demandeurs. En 1925, Ferrière y donne deux conférences, la première à la demande du Groupe d'études philosophiques de la Sorbonne, la seconde à la demande de la Section pédagogique de l'Association fran-

27 Plusieurs de ses membres sont des intellectuels et ont bénéficié de hautes d'études dans des écoles ou instituts privés.

28 Nous reviendrons, dans la conclusion, sur les controverses et débats au sein de la Ligue internationale pour l'Éducation nouvelle. 
çaise pour l'Avancement des Sciences (AFAS) dont le directeur est Paul Langevin (professeur au Collège de France). De son côté, Rotten voyage aux États-Unis où elle donne des conférences dans plusieurs villes de l'est du pays. Elle est aussi invitée à Paris en janvier 1926 à la demande de l'Association française pour l'Avancement des Sciences, de la Société Française de Pédagogie et du Syndicat national des instituteurs/trices. Elle s'exprime sur le thème «Pour le désarmement moral par le rapprochement des instituteurs allemands et français» dont l'accueil est très mitigé. Elle donne une autre conférence au Centre européen de la Dotation Carnegie à Paris qui, selon l'auteur de l'éditorial, reçoit un accueil beaucoup plus chaleureux auprès d'un public composé des «représentants de 35 associations françaises, de représentants de l'université, de l'Institut international de Coopération Intellectuelle et de l'Association française pour la Société des Nations» (Éditorial, PEN, 1926, 19, p. 23).

Ces efforts de propagande portent leurs fruits puisque l'accroissement des membres est spectaculaire dans ces années-là. Pour la rédaction, cette croissance est due aux encouragements de personnes influentes et d'associations d'instituteurs de la francophonie; une preuve que les associations jouent un rôle majeur dans la diffusion du mouvement auprès des praticiens. Cette croissance se confirme au Congrès de Heidelberg qui enregistre une augmentation significative du nombre de participants par rapport aux congrès précédents. ${ }^{29}$ En cela, il constitue une étape importante dans la reconnaissance du mouvement sur le plan international. C'est lors de ce congrès qu'une importante délégation américaine se déplace pour rencontrer les représentants de la Ligue. Cette rencontre est interprétée par la rédaction comme la réunion des «deux plus puissantes associations du monde» qui, de fait, est plutôt la réunion des associations et institutions dans lesquelles Ferrière est impliqué:

Ainsi entrent en contact les deux plus puissantes associations d'éducation du monde, la National Education Association et le Bureau international d'Éducation d'Europe, bureau encore officieux mais formé de groupements actifs depuis des années: La Ligue internationale pour l'Éducation nouvelle (1921), l'Association pour les Congrès internationaux d'Éducation morale

29 Ce congrès compte 450 participants alors que celui de Calais (1921) en comptait 100 et celui de Montreux (1923) 300. 
(décidé en 1922 mais dont le comité existe depuis 1908), l'Institut J.-J. Rousseau (siège du BIE) et le Bureau international des Écoles Nouvelles (1899). (Éditorial, PEN, 1925, 15, p. 1)

La croissance entamée au Congrès de Heidelberg se poursuit au cours des années suivantes, en particulier sur le plan des adhésions d'associations. Celles-ci sont parfois de nature très différente et visent des objectifs très distincts. Ainsi, la Child Study Association ${ }^{30} \mathrm{~s}^{\prime}$ affilie à la Ligue au même titre que l'Association pour les Intérêts du Midi (Rusconi, 1926, PEN, 23), que l'Association internationale pour Films d'Éducation nouvelle fondée en 1927 à Locarno (Ferrière, 1929, PEN, 44) ou encore l'Association Montessori dont il est question dans un article en 1939 (PEN, 147). En mars 1927, PEN fait état de la fondation prochaine de plusieurs associations en France, notamment à Alès, à Nîmes ainsi qu'à Toulouse où le groupe fait l'objet des éloges du rédacteur: «L'esprit du groupe est nettement progressif, parfaitement désintéressé et tolérant, accueillant toute collaboration, pourvu qu'elle soit sincère. Ses membres se proposent de faire prochainement appel aux parents, dont la collaboration est indispensable aux éducateurs» (Éditorial, PEN, 1927, 26, p. 50).

Tableau 13.

Associations d'Éducation nouvelle ${ }^{31}$

\begin{tabular}{llll}
\hline $\begin{array}{l}\text { Année } \\
\text { (fondation) }\end{array}$ & Nom de l'association & Nom du responsable & Pays \\
\hline 1900 & Child Study Association & G. Spencer & USA \\
\hline 1921 & La Nouvelle Éducation & R. Cousinet, M. Guéritte & France \\
\hline 1922 & $\begin{array}{l}\text { L'Éducation nouvelle, } \\
\text { Groupe d'études, de } \\
\text { recherches et d'expériences }\end{array}$ & A. Jouenne & France \\
\hline
\end{tabular}

30 Fondée en 1900 aux États-Unis, cette association est présentée par Garlin Spencer au congrès de Heidelberg (1925).

31 Ce tableau a été conçu sur la base des indications mentionnées dans les éditoriaux de PEN de 1922 à 1927. Il ne prétend à aucune exhaustivité du fait que de nombreuses autres associations voient le jour durant cette période mais $P E N$ n'en parle pas donc nous ne les avons pas fait figurer dans ce tableau. À partir de 1928, le nombre de celles-ci augmente tellement que les éditoriaux ne les mentionnent plus nominativement. 


\begin{tabular}{llll}
\hline 1923 & $\begin{array}{l}\text { Section espagnole de } \\
\text { Fraternité internationale } \\
\text { d'Éducation }\end{array}$ & M. Sola de Sellares & Espagne \\
\cline { 2 - 4 } & $?$ (groupe de Madrid) & Tomas y Samper & Espagne \\
\hline 1924 & $?$ & E. Edelstam & Suède \\
\hline 1926 & $\begin{array}{l}\text { Association pour les } \\
\text { intérêts du Midi }\end{array}$ & O. Rusconi & France \\
\hline 1927 & $\begin{array}{l}\text { Association internationale } \\
\text { de Films d'Éducation } \\
\text { nouvelle }\end{array}$ & A. Ferrière & international \\
\end{tabular}

\section{LES SECTIONS NATIONALES: DES VALEURS SÛRES}

La croissance de la Ligue et des associations affiliées est si rapide qu'il devient difficile de distinguer leurs caractéristiques, leurs objectifs et activités. C'est pourquoi, en 1927, le Comité exécutif décide de clarifier le statut de ces diverses associations. Une décision qui, selon nous, est révélatrice de la volonté de maîtrise des trois dirigeants, soucieux que ces groupes s'inscrivent bien dans l'idéologie de la Ligue. Le texte adopté à cette occasion distingue les associations nationales des sections nationales dont les statuts, les droits et les devoirs sont différents. Selon le texte, «les organisations [associations] peuvent prendre l'une des formes suivantes»:

1. Un représentant travaillant pour l'Éducation nouvelle et reconnu par le Comité international de la Ligue.

2. Un groupe travaillant pour l'Éducation nouvelle et affilié à la Ligue.

3. Dans le cas où plusieurs groupes travaillent dans le même pays à l'Éducation nouvelle, il est possible à plus d'un groupe de s'affilier à la Ligue.

(Éditorial, PEN, 1927, 33, p. 279)

Dans le cas des groupes (2 et 3), une cotisation collective est exigée - à envoyer au siège central de la Ligue en janvier de chaque année - dont le montant est fixé par le groupe lui-même. Selon l'éditorial cité plus haut, la déclaration d'affiliation à la Ligue «doit être renouvelée au bout de deux ans». Quant aux sections nationales, le texte adopté à Locarno 
pose des conditions plus contraignantes parmi lesquelles l'aspect financier est désormais pris en compte:

a) Chaque section est entièrement autonome. Elle accepte les principes et buts de la Ligue mais demeure responsable de son organisation intérieure. Chaque section fixe sa propre contribution au centre national auquel différents groupes peuvent être affiliés.

b) Chaque section est dirigé par un comité d'au moins cinq membres, y compris le président et le secrétaire, formé de personnalités bien connues dans les centres pédagogiques et représentatifs de différents aspects du travail éducatif.

c) Elle adresse une fois par an, en janvier, un rapport complet sur ses activités au siège central de la Ligue.

d) Elle envoie une fois par an, en janvier, au siège central de la Ligue, une contribution financière s'élevant à $10 \%$ du montant de ses cotisations individuelles, indépendamment de la part afférente à l'abonnement de la revue.

e) Un représentant, élu par chaque section nationale, fait partie du Comité international de la Ligue. S'il existe dans un pays une revue et une section, le rédacteur de la revue est aussi membre du Comité International, où il siège au même titre que le représentant de la section, ceci pour les cas où rédacteur et représentant ne sont pas réunis en une seule et même personne. Si dans un pays, il existe une revue mais aucune section, c'est son rédacteur qui la représente au Comité international.

(Éditorial, PEN, 1927, 33, p. 279)

Grâce à ce texte, le statut des associations devient plus clair et pose désormais une nette distinction entre les associations locales et les sections nationales, considérées désormais comme les relais officiels de la Ligue dans chaque pays affilié. L'investissement financier (10\% du montant des cotisations individuelles) et humain (présence d'un délégué au Comité international) auquel s'ajoute l'obligation de rendre des comptes, donne à ces sections un statut particulier, plus officiel et mieux reconnu. De fait, la contribution financière exigée de ces sections permet de couvrir une partie des frais du Bureau international de la Ligue (Londres) et «de soutenir le travail entièrement désintéressé de ses chefs [...] dont la brusque suppression, pour cause d'appui insuffisant serait un désastre» (Éditorial, PEN, 1928, 34, p. 2). 
Ces sections commencent à se constituer dès 1927, la plupart étant des associations actives depuis plusieurs années qui se «transforment» en section nationale suite à l'adoption du texte de Locarno. Certaines concrétisent rapidement leur nouveau statut alors que d'autres ont besoin de plusieurs années pour le faire. Dans certains pays, des groupes opposés défendent leur cause et peinent à trouver un terrain $\mathrm{d}^{\prime}$ entente pour fonder une section nationale. ${ }^{32}$ Selon nous, cette nouvelle organisation permet à la Ligue d'avoir un certain contrôle sur les associations qui se multiplient dans tous les pays à ce moment. Elle traduit la volonté des dirigeants de la Ligue de fédérer le mouvement et d'harmoniser les discours en contrôlant ce qui se dit et s'écrit en son nom. Un contrôle qui devrait permettre de prévenir les «dissidences» et d'orienter le mouvement selon une conception de l'Éducation nouvelle défendue par ses directeurs. Mais comme nous l'avons vu, les divergences iront croissant dès le Congrès de Locarno atteignant leur paroxysme à la fin des années 1930.

Tableau 14.

Fondation des sections nationales ${ }^{33}$

1927 Suisse, Angleterre

1928 Yougoslavie, Chili, Argentine, Pologne, Inde, USA

1929 France, Turquie, Roumanie, Hongrie, Norvège, Belgique

1931 Allemagne

Les éditoriaux renseignent sur les fondations des sections nationales et sur la composition de leurs comités. Ainsi, notons que le comité de la section nationale suisse, fondée au Congrès de Locarno, se compose d'un représentant de chaque région linguistique: Hermann Tobler (Suisse alémanique), Pierre Bovet (Suisse romande) et Maria Boschetti

32 C'est le cas notamment aux Pays-Bas et en Belgique où des groupes influents mais divergents peinent à trouver des orientations communes pour fonder une section nationale.

33 Ce tableau résume les informations contenues dans les éditoriaux des trois périodiques. Elles sont probablement lacunaires en raison de la raréfaction des éditoriaux à partir de 1930 alors que c'est dans ces années-là que se constituent la plupart des sections nationales. Le tableau suivant résumant l'affiliation des revues officielles de la Ligue permet de penser que d'autres pays fondent des sections nationales avec une revue affiliée. 
(Tessin). Au cours des années suivantes, de nombreuses sections nationales déposent leur candidature auprès de la Ligue, la plupart éditant une revue dans la langue du pays. Aux USA, il n'existe pas de section nationale à proprement parler. Pourtant, lors de l'assemblée générale de la Progressive Education Association en mars 1928, un comité se constitue pour être représentant au sein de la Ligue. Comme l'indique l'éditorial d'avril 1928, ce groupe d'une trentaine de personnes est composé «d'hommes éminents et universellement connus, tels que MM. Kilpatrick, Monroe, Del Manzo, Russel (doyen de la Columbia University)». En France, la section nationale à proprement parler n'est fondée qu'en 1929 lors du Congrès d'Elseneur dont le comité se compose de Paul Langevin (président), Paul Fauconnet (vice-président), Henri Wallon, Henri Piéron, Georges Bertier (membres du comité). En fait, il s'agit d'une transformation du GFEN en section nationale, bien que deux autres groupes restent actifs à ses côtés: La Nouvelle Éducation qui joue le rôle «d'association active des pionniers de la liberté de l'enfant» (Éditorial, PEN, 1928, p. 74) et le Bureau Français d'Éducation qui ambitionne d'établir un rapprochement entre les différents groupements français (Gutierrez, 2008). Il est important de signaler que certains groupes restent volontairement à l'écart de la Ligue en choisissant de ne pas devenir une section nationale. ${ }^{34}$

Comme nous pouvons le voir, la croissance de la Ligue se fait avant tout à travers l'adhésion d'acteurs collectifs que sont les associations. Sa structuration progressive par la transformation de certains groupes en sections nationales illustre le mouvement d'institutionnalisation qui va de pair avec un processus d'internationalisation de l'éducation dès les années 1920. Comme le montre Fuchs (2007b), ce processus se différencie de celui qui existe avant la Grande Guerre par la forme de plus en plus institutionnalisée que prennent ses structures et ses réseaux. Si la fondation de la Ligue constitue un premier niveau d'institutionnalisation, la création de sections nationales en son sein en illustre un deuxième où les spécificités nationales sont plus prégnantes. En effet, en participant à la vie de la Ligue, ces sections nationales apportent leur identité culturelle (mentalité, traditions, idéologie), ce qui représente à la fois une richesse mais aussi un obstacle à l'homogénéité que recherche la

34 En France, l'association La Nouvelle Éducation reste en marge de la Ligue puisque c'est le GFEN qui devient section nationale. Aux Pays-Bas, il en va de même pour le groupe dirigé par Philippi van Reesema. 
Ligue tout au long de son existence. Selon White (2001), ces sections prennent bientôt une place si importante que la Ligue ressemble davantage à une "constellation de sections individuelles», dont les particularités s'exacerbent au fil des ans, plutôt qu'à une "communauté internationale de pratique» (White, 2001, p. 73).

\section{LES REVUES: OUTILS DE PROPAGANDE NÉCESSAIRES POUR «FAIRE MOUVEMENT»}

Dans ce volet, nous nous intéressons aux nombreux périodiques dédiés à l'Éducation nouvelle qui, rattachés à la Ligue de près ou de loin, contribuent à son expansion et à celle du mouvement. Il s'agit donc d'élargir encore la perspective qui a été amorcée par l'étude des revues allemande et anglaise dans le deuxième chapitre de ce livre. À l'aide de données récoltées dans les éditoriaux de Pour l'Ère Nouvelle ainsi que dans les rubriques «Livres et Revues» et «Chroniques», nous retraçons l'évolution de ces périodiques entre 1922 et 1940 en s'intéressant à leur nombre, leur région d'influence et leurs rédacteurs principaux. Après une brève présentation du contexte général en francophonie (France, Belgique, Suisse), nous nous intéresserons aux revues de pays plus lointains dont Pour l'Ère Nouvelle se fait l'écho, en particulier celles qui sont affiliées à la Ligue en tant qu'organes officiels.

\section{LES REVUES DE FRANCOPHONIE}

La fondation de Pour l'Ère Nouvelle en 1922 apparaît à une période d'effervescence éditoriale sur le plan international liée aux espoirs et aux désirs de reconstruction après la guerre. Les revues d'éducation prennent une importance particulière en raison des enjeux que représente l'éducation dans le cadre de cette reconstruction. Parmi elles, certaines se consacrent spécifiquement à l'Éducation nouvelle ayant pour objectifs de faire connaître les nouvelles méthodes et de favoriser l'échange entre éducateurs. La plupart émanent d'associations qui militent pour cette cause et dont l'organe est à la fois un lieu d'informations, un support d'échanges et un outil de propagande.

En France, plusieurs revues pédagogiques émergent à cette période, parmi lesquelles La Nouvelle Éducation, dirigée par Madeleine Guéritte et Roger Cousinet, qui paraît dès 1922 et sert d'organe à leur association 
qui porte le même nom. Durant les premières années, Ferrière est en contact étroit avec ses rédacteurs collaborant par des échanges réciproques d'articles. Dans le Nord du pays, deux groupes publient des revues dont le but est de faire connaître les expérimentations des méthodes nouvelles dans la région: Le Bulletin de l'U.T.O. publié dès 1927 par un comité de professeurs de l'Institut catholique de Lille et Le Bulletin des Amis du Nord de l'Éducation nouvelle coordonné par René Hubert dès 1930, organe du groupe du même nom (ANEN) (Gutierrez, 2008). C'est en 1937 que naît L'Information pédagogique fondée par Albert Châtelet (recteur de l'Académie de Lille) qui s'intéresse plus spécifiquement à l'organisation de l'enseignement du second degré. ${ }^{35}$ Deux autres revues se distinguent des précédentes car elles sont créées avant la guerre et n'émanent pas d'associations mais de pédagogues ou scientifiques engagés pour la cause de l'enfance. Il s'agit d'une part de L'Éducation publiée de 1909 à 1934 par Georges Bertier, directeur de L'École des Roches ${ }^{36}$ puis par M. Lesur sous le nom Éducation jusqu'en 1941, Bertier conservant une place importante dans le comité directeur. D'autre part, L'Éducateur moderne est une revue publiée de 1906 à 1914 par J. Philippe et G. Paul-Boncour ${ }^{37}$, collaborateurs d'Alfred Binet à la Société libre pour l'Éducation psychologique de l'enfant. Comme son nom l'indique, son ambition est de former «l'éducateur moderne» qui doit désormais «tenir compte des données scientifiques issues de la recherche expérimentale en éducation dans sa pratique quotidienne» (Programme, L'Éducateur moderne, 1906).

En Suisse romande, Pour l'Ère Nouvelle est la seule revue spécifiquement dédiée à l'Éducation nouvelle. Cependant, deux autres périodiques majeurs contribuent à la propagande des méthodes novatrices dans la région: L'Éducateur, fondé en 1865, organe de la Société des Instituteurs Romands ${ }^{38}$ et l'Intermédiaire des Éducateurs, organe de l'Institut Rousseau.

35 Pour davantage d'informations sur ces revues, consulter le site web dédié à l'histoire du mouvement d'Éducation nouvelle en France (http://hmenf. free.fr) élaboré par Laurent Gutierrez.

36 Pour une histoire détaillée de l'École des Roches et du rôle de Georges Bertier, voir Duval, 2009.

37 Les prénoms complets de ces deux acteurs nous sont inconnus.

38 Cette association prend le nom de Société Pédagogique Romande (SPR) dès 1889, puis celui de Syndicat des Enseignants Romands (SER) en 1998. Voir Durand, Hofstetter et Pasquier (2015) qui retrace les 150 d'histoire de cette association et de sa revue. 
Le premier est l'organe des associations professionnelles d'enseignants du primaire dans lequel sont débattues des questions d'ordre pédagogique, syndical et philosophique/ éthique. Cette revue permet aux éducateurs de faire part de leurs expériences d'Éducation nouvelle, mais elle ne milite pas en tant que tel pour le mouvement. La seconde, émane de l'Institut Rousseau où se côtoient chercheurs, médecins, psychologues et pédagogues dont les textes sont à la fois scientifiques et militants. Si son lectorat est avant tout genevois, ce périodique milite explicitement pour une réforme de l'école sur des bases scientifiques et pour la pédagogie nouvelle prônée par le mouvement d'Éducation nouvelle. Ces deux revues fusionnent en 1920 (Jornod, 1989) pour ne garder que le nom de la première, L'Éducateur. Émanant désormais à la fois de praticiens et de théoriciens, ce périodique conserve une position en faveur des méthodes nouvelles comme en atteste les conclusions du congrès de 1924 sur le thème de l'application des méthodes actives à l'école publique. ${ }^{39} \mathrm{D}$ 'autres revues d'éducation existent en Suisse romande, les plus importantes étant celles publiées par les associations d'enseignants qui se constituent dans chaque canton dès la fin du $19^{e}$ siècle. Ainsi, la Société pédagogique de Neuchâtel (SPN), l'Union des Instituteurs Genevois (UIG) qui fusionne rapidement avec la Société pédagogique Genevoise (SPG) et la Société pédagogique Vaudoise (SPV) se dotent aussi de leur organe. À Fribourg, la Société Fribourgeoise d'Éducation (SFE), de confession catholique, publie un Bulletin pédagogique dont l'objectif premier est de contrer les opinions de la Société des Instituteurs Romands (SIR) face aux projets de centralisme et de laïcité en matière d'éducation (Lussi Borer, 2008).

En Belgique, Vers l'école active, fondée peu après la guerre, émane d'un Cercle de travail dirigé par Fernand Dubois à Waterloo. Le responsable de la revue est Laurent Poriniot mais il est secondé par Fernand Dubois et Octavo Picalausa. Pendant plusieurs années, ces derniers sont actifs dans leur cercle mais restent distincts du groupe créé autour d'Ovide Decroly et Amélie Hamaïde à Bruxelles. De fait, dès sa fondation en 1922, l'organe de la Ligue pour la Belgique est Pour l'Ėre Nouvelle dont Decroly est membre du comité éditorial à partir de 1925. Mais en décembre 1927, suite au Congrès de Locarno, un nouveau périodique belge voit le jour. Il s'agit d'une revue flamande Het Schoolblaad de Actieve school (Revue scolaire d'École active) publiée par Étienne Vincent.

39 Sur ce congrès, voir le rapport officiel de la Société Pédagogique romande (1924). 
Après le Congrès d'Elseneur, les deux groupes fusionnent pour fonder une section nationale belge dont la revue Vers l'École active devient l'organe officiel, publiée en français et en flamand dès l'automne 1929.40

La croissance rapide de la Ligue dans les années 1920 se manifeste à travers une augmentation massive des périodiques bien au-delà de la francophonie. La Ligue étant internationale, c'est à elle que s'adressent les associations qui désirent se joindre au mouvement; et pour nombre d'entre elles, cette affiliation passe par la fondation d'une revue, publiée dans leur langue, qui fait office de relais entre la Ligue et leur pays. En tant qu'organe officiel, Pour l'Ère Nouvelle informe régulièrement ses lecteurs de l'évolution de ces périodiques, d'une part dans ses éditoriaux (1922-1931), d'autre part dans ses rubriques «Livres et Revues» (19221940) et «Chroniques» (1926-1940).

À ses débuts, la rubrique «Livres et revues» consacre une part importante aux résumés des articles de The New Era et Das Werdende Zeitalter. Elle donne aussi résumé de leur éditorial - quand celui-ci est substantiel - ainsi que le sommaire du numéro en question. ${ }^{41}$ Dès 1923, les revues mentionnées dans cette rubrique ne sont plus seulement les revues sœurs, mais aussi toutes celles qui voient le jour en langue française, qu'il s'agisse de revues spécialisées ou de type «grand public». Dès 1926, le nombre de revues mentionnées «explose», laissant deviner la multiplication de ces supports dans tous les pays. La rubrique se subdivise alors en trois sections: revues d'Éducation nouvelle, revues de langue française, revues étrangères. Quant aux ouvrages recensés, nombreux eux aussi, ils sont présentés selon deux catégories: ouvrages de langue française, ouvrages de langue étrangère. Le volume de cette rubrique prend alors des proportions très importantes pouvant atteindre 9 à 10 pages selon les numéros (années 1926 à 1930). Pour des raisons notamment financières, la rédaction doit alors trouver des solutions pour désengorger ces rubriques. En 1927, elle signale qu'il n'y a plus lieu de faire une place prééminente aux revues sœurs d'Allemagne et d'Angleterre, chacune «vivant désormais sa propre vie» alors que $\mathrm{d}^{\prime}$ autres sont venues comme «un essaim» s'ajouter aux premières. ${ }^{42}$ Une autre solution est trouvée dès 1926 grâce à la création d'une nouvelle

40 Voir éditorial de janvier 1930 (PEN, 54, p. 2).

41 Toutefois, la place occupée par ces résumés s'amenuise au fil des ans.

42 Dès ce moment-là, toutes les informations concernant la Ligue et ses revues affiliées sont réunies en $2^{\mathrm{e}}$ page de couverture (voir suite du volet). 
rubrique appelée «Chronique française». Assurée par Eugène Delaunay ${ }^{43}$ de 1926 à 1939, cette chronique donne un aperçu des principales revues françaises d'éducation dont l'auteur résume les articles importants, en particulier ceux concernant l'Éducation nouvelle. À partir de 1932, d'autres Français rédigent des rubriques de ce type tels que François Cattier (1932), Louis Brun-Laloire (1933, 1935), Lucien Boes (1937-1939) ou Roger Gal (1940) intitulant leurs articles «À travers les revues de langue française» ou «Revue de la presse pédagogique française». D'autres chroniques s'ajoutent bientôt à celle-ci, notamment celle des revues anglaises et américaines, publiées par Marie-Louise Cazamian en 1935, 1937 et 1938. On peut faire l'hypothèse que les périodiques de langue anglaise jouent alors un rôle particulièrement important dans le champ de l'éducation et méritent qu'on leur consacre une chronique en français dans une revue francophone. Cette multiplication et diversification de chroniques de revues attestent une fois de plus de l'immensité et l'intensité de l'activité éditoriale dans le domaine de l'éducation et de l’Éducation nouvelle en particulier.

\section{LES REVUES OFFICIELLES DE LA LIGUE}

Au cours des trois premières années de vie de la Ligue, les demandes d'affiliation de nouvelles revues se succèdent à un rythme soutenu. D'après ce qu'en disent les éditoriaux, ces demandes sont acceptées sans condition, la Ligue étant alors dans une phase d'expansion à la recherche de nouveaux membres. À partir de 1925, on assiste à une véritable explosion du nombre de périodiques qui se réclament de près ou de loin de l'Éducation nouvelle. Ce mouvement s'inscrit dans une tendance plus large où les progrès de l'édition démocratisent la publication de revues en tous genres. Que ce soit des revues spécialisées d'éducation, des revues familiales ou de vulgarisation, toutes diffusent à des degrés divers, les principes de l'Éducation nouvelle désormais considérés comme la tendance «à la mode». En avril 1925, l'éditorial mentionne plusieurs revues dont il est difficile de savoir quels sont leur

43 Instituteur à Coulombs par Creully (Calvados), la rédaction de PEN le considère comme «un des pionniers les plus sérieux de l'École active dans le cadre de l'école publique» (Éditorial, $P E N, 1926,18$, p. 3). D'abord chargé du service des annonces, il assure la «chronique française» de 1926 à 1939 selon un rythme d'une chronique par semestre (en moyenne). 
statut, à quel niveau elles se situent et de quelle association elles sont l'organe. Une confusion qui est d'autant plus manifeste quand il s'agit de revues publiées en langues étrangères. Ainsi, aux côtés de revues spécialisées telles que L'Intermédiaire des Éducateurs (Genève), L'Éducation libre (Sofia), Pedagogiska Spormal (Suède), ou Progressive Education (USA) figurent des noms telles que Uudistuva Kasvatus-ja Opetustyö (Le travail novateur dans l'Éducation et dans l'enseignement) (Finlande), ou Die Freunde, Zeitschrift internationaler Jugend (publié à Vienne par Richard Weiss) qui traite d'autres thèmes que d'Éducation nouvelle mais qui sert d'organe à la Gesellschaft für Neue Erziehung (Éditorial, PEN, 1925, 15).

Aux États-Unis, le statut de la revue trimestrielle Progressive Education, qui paraît dès 1924 en tant qu'organe de l'association du même nom, est un peu particulier. Alors que les dirigeants de la Ligue reprochent aux Américains de «faire cavaliers seuls», Stanwood Cobb (chairman du comité exécutif de l'association) déclare dans le premier éditorial que «son association et sa revue travaillent en accord étroit avec la Ligue et ses revues d'Europe ainsi qu'avec le Bureau international des Écoles nouvelles» (Éditorial, PEN, 1924, 11, p. 42). Mais la rédaction de $P E N$ précise que les rédacteurs de cette revue «se tiendront à mi-distance entre les périodiques professionnels et les revues de vulgarisation, s'adressant à un public cultivé: parents et éducateurs» (Éditorial, PEN, 1924, 11, p. 42). Elle publie avant tout les expériences menées aux États-Unis mais se fait aussi l'écho de quelques grands noms du mouvement européen, notamment Decroly et Ferrière.

Dans ce foisonnement d'informations un peu éclectique, il devient urgent pour les dirigeants de la Ligue de canaliser cette expansion. Ferrière, en particulier, voit d'un très mauvais œil cette prolifération de revues qui risque d'affaiblir le rôle de Pour l'Ère Nouvelle déjà en prise avec des difficultés financières. Au Congrès de Locarno de 1927, le Comité de la Ligue décide de poser un certain nombre de conditions d'affiliation pour les revues qui souhaitent être reconnues comme l'un de ses organes officiels. Ces conditions sont annoncées officiellement dans l'éditorial de PEN en décembre 1927 (PEN, 33, p. 279). 
1. Chaque fascicule (revue) reproduit en bonne place les principes et les buts de la Ligue.

2. L'affiliation est valable pour une durée de 2 ans pendant laquelle la revue a le droit de se déclarer l'un des organes de la Ligue; au bout de ce temps l'autorisation doit être renouvelée.

3. Lorsque la revue est imprimée dans une langue autre que l'anglais, le français ou l'allemand, elle donne dans l'une des trois langues un résumé d'une page au moins des articles qu'elle publie. Les revues affiliées s'interdisent la publication de tout article de caractère confessionnel ou politique, ou poursuivant un but autre que l'Éducation nouvelle.

Ces conditions adoptées le 8 août 1927, à Locarno, par le Comité international de la Ligue, laissent subsister celles qui ont été communiquées antérieurement aux revues affiliées, mais modifiées comme suit:

a) Indiquer sur chaque numéro de la revue, outre les principes et buts de la Ligue, les noms des autres revues avec leurs adresses.

b) Reproduire les parties de la chronique du Bureau International $d^{\prime} E$ ducation qui ont une importance pour l'ensemble des nations ou pour la nation particulière où paraît la revue affiliée.

c) Donner de temps à autre un aperçu de l'activité des autres revues.

d) Dans la règle, chaque revue de la Ligue peut reproduire les articles parus dans l'une quelconque des autres: ceci peut se faire sans indication de la source, mais seulement après entente entre les rédacteurs.

Pour le Comité de la LIEN: B. Ensor, A. Ferrière, E. Rotten

Si cette décision révèle la volonté de distinguer les revues affiliées à la Ligue de toutes celles qui se publient à d'autres titres, elle montre aussi la volonté du Comité d'avoir un certain contrôle sur ce qui se publie au nom de la Ligue. Selon nous, les conditions adoptées à Locarno confirment cette position de maîtrise recherchée par les dirigeants en garantissant ainsi une forme «d'orthodoxie» des idées véhiculées dans les périodiques. Par exemple, l'affiliation d'une revue est limitée dans le temps, la demande devant être renouvelée tous les deux ans. Cette mesure est une manière, pour le Comité central, de remettre en question, voire d'écarter, l'une ou l'autre revue qui se serait trop éloignée des principes de ralliement. D'autre part, le Comité exige que les revues publiées dans des langues autres que les trois principales donnent des résumés substantiels de leurs articles. Ce besoin de contrôle révèle la 
volonté de la Ligue de fédérer le mouvement selon ses conceptions. Enfin, l'obligation d'indiquer sur chaque numéro les buts et principes de ralliement ainsi que l'interdiction de publier tout article «de caractère confessionnel ou politique, ou poursuivant un but autre que l'Éducation nouvelle», révèle le souci du comité d'assurer une certaine unité de pensée et de pratiques au sein de la Ligue. Un souci qui est en contradiction avec l'identité que revendiquent ses dirigeants, à savoir celle de la diversité et de la tolérance à l'égard des autres cultures et «courants» de pensée: «L'Éducation nouvelle, dans son orientation, est au-dessus des nations. Nous ne connaissons aucun exclusivisme de race ni de nationalité et l'esprit qui nous anime est un esprit de fraternité universelle». ${ }^{44}$ Un paradoxe qui est présent tout au long de la période de croissance des années 1920 et qui sera source de tensions dans les années 1930.

Compte tenu de cette décision, ce n'est qu'à partir de 1927 que la Ligue accorde une place véritablement officielle aux revues affiliées, en faisant figurer leurs noms en $2^{\mathrm{e}}$ page de couverture de ses revues, page désormais dédiée aux informations concernant la Ligue. Cette manière de les énoncer marque une distinction claire entre les organes officiels et les autres périodiques. Certaines, comme les revues hongroise et bulgare, existent bien avant 1927 mais ce n'est qu'à partir de cette date que leur affiliation est officiellement reconnue. Le tableau ci-dessous présente la liste chronologique d'affiliation des revues sur la base des indications des pages de courverture de Pour l'Ère Nouvelle.

44 Discours de clôture du Congrès de Montreux prononcé par le président du congrès, H. Baillie-Weaver (Ensor \& Baillie-Weaver, PEN, 1923, 8, p. 140). 
Tableau 15.

Revues officiellement affiliées à la Ligue internationale pour l'Éducation nouvelle

\begin{tabular}{|c|c|c|c|}
\hline Année & Titre revue officielle & Pays & Rédacteur en chef \\
\hline \multirow[t]{3}{*}{1922} & The New Era & Angleterre \& Écosse & B. Ensor \\
\hline & Das Werdende Zeitalter & $\begin{array}{l}\text { Allemagne, Autriche, } \\
\text { Suisse alémanique }\end{array}$ & E. Rotten \\
\hline & Pour l'Ère Nouvelle & $\begin{array}{l}\text { France, Belgique, } \\
\text { Suisse romande }\end{array}$ & A. Ferrière \\
\hline \multirow[t]{5}{*}{1927 (janv.) } & $\begin{array}{l}\text { Svobodno Vaspitanie } \\
\text { (L'Éducation libre) }\end{array}$ & Bulgarie & D. Katzaroff \\
\hline & La Nueva Era & Chili & A. Hamel \\
\hline & $\begin{array}{l}\text { A Jövö Utjain } \\
\text { (La voie de l'avenir) }\end{array}$ & Hongrie & M. Nemes \\
\hline & L'Educazione Nazionale & Italie & $\begin{array}{l}\text { G. Lombardo- } \\
\text { Radice }\end{array}$ \\
\hline & Nueva Era / La Obra ${ }^{45}$ & République Argentine & J. Rezzano \\
\hline 1927 (fév.) & Revista de Pedagogia & Espagne & L. Luzuriaga \\
\hline \multirow[t]{5}{*}{1927 (nov.) } & $\begin{array}{l}\text { Het Schoolblad (Revue } \\
\text { scolaire d'École active) }\end{array}$ & Belgique flamande & E. Vincent \\
\hline & $\begin{array}{l}\text { Tijdschrift voor Ervaring- } \\
\text { sopvoedkunde (Revue de } \\
\text { pédagogie expérimentale }\end{array}$ & Pays-Bas & H. G. Hamaker \\
\hline & Educaçao social & Portugal & A. V. Lemos \\
\hline & Pedagogiska Spormal & Suède & $\begin{array}{l}\text { E. Edelstam et } \\
\text { M. Montelius }\end{array}$ \\
\hline & Nové Skoly & Tchécoslovaquie & O. Chlup \\
\hline \multirow{4}{*}{1928 (mars) } & $\begin{array}{l}\text { Pentru Inima Copiilor } \\
\text { (Pour le cœur des enfants }\end{array}$ & Roumanie & J. Nisipeanu \\
\hline & $\begin{array}{l}\text { Radna Skola } \\
\text { (L'École active) }\end{array}$ & Yougoslavie & Y. S. Yovanovich \\
\hline & Interruption de la revue $p$ & portugaise (Educaçao soc & \\
\hline & $\begin{array}{l}\text { Interruption de la revue } \\
\text { sopvoedkunde) }\end{array}$ & hollandaise (Tijdschrift r & voor Ervaring- \\
\hline \multirow[t]{2}{*}{1929} & Vernieuwing & Pays-Bas & J. H. Bolt \\
\hline & Interruption de la revue b & belge flamande (Het Sch & hoolblad) \\
\hline
\end{tabular}

45 Cette revue change de nom en 1928 pour s'appeler désormais La Obra. 


\begin{tabular}{|c|c|c|c|}
\hline Année & Titre revue officielle & Pays & Rédacteur en chef \\
\hline \multirow[t]{3}{*}{1930 (janv.) } & $\begin{array}{l}\text { Den Frie Skole } \\
\text { (L'École libre) }\end{array}$ & Danemark & S. Naasgard \\
\hline & Fikirler & Turquie & M. Rahmi Bey \\
\hline & Vers l'École active & Belgique & $\begin{array}{l}\text { L. Poriniot, } \\
\text { O. Piccalausa \& } \\
\text { F. Dubois }\end{array}$ \\
\hline 1931 (avril) & Escuela activa & Uruguay & B. S. Genovese \\
\hline \multirow[t]{6}{*}{1932 (nov.) } & La Nueva Ensenanza & Paraguay & $?$ \\
\hline & Progressive Education & USA & G. Hartman \\
\hline & A Escuela Primaria & Portugal & $?$ \\
\hline & \multirow{3}{*}{\multicolumn{3}{|c|}{$\begin{array}{l}\text { Disparition de la revue allemande (Das Werdende Zeitalter) } \\
\text { Disparition de la revue chilienne (La Nueva Era) } \\
\text { Disparition de la revue hollandaise (Vernieuwing) }\end{array}$}} \\
\hline & & & \\
\hline & & & \\
\hline 1933 (juill.) & \multicolumn{3}{|c|}{ Disparition de la revue italienne (Educazione Nazionale) } \\
\hline Dès 1938 & \multicolumn{3}{|c|}{ Pour l'Ère Nouvelle ne publie plus la liste des revues affiliées } \\
\hline
\end{tabular}

Pour avoir un aperçu plus précis de l'évolution des affiliations au fil des années 1922 et 1940, ce deuxième tableau les récapitule de manière chiffrée.

Tableau 16.

Récapitulatif du nombre de revues affiliées à la Ligue en tant qu'organe officiel

\begin{tabular}{lccc}
\hline Année & Revues fondatrices & Revues officielles & Total \\
\hline 1922 & 3 & & 3 \\
\hline 1927 (fév.) & 3 & 11 & 9 \\
\hline 1927 (déc.) & 3 & 11 & 14 \\
\hline 1928 (mars) & 3 & $\begin{array}{c}+2 \text { nouvelles } \\
-2 \text { interruptions }\end{array}$ & 14 \\
\hline 1929 & 3 & $\begin{array}{c}11 \\
+1 \text { nouvelle } \\
-1 \text { interruption }\end{array}$ & 14 \\
\hline 1930 & 3 & 14 & 17 \\
\hline
\end{tabular}




\begin{tabular}{llcc}
\hline 1931 & 3 & 15 & 18 \\
\hline 1932 (nov.) & 2 & 16 & 18 \\
& & +3 nouvelles & \\
& & -2 disparitions & \\
\hline
\end{tabular}

\begin{tabular}{llcc}
\hline 1933 (juill.) & 2 & 15 & 17 \\
\hline $1934-1937$ & 2 & 15 & 17 \\
\hline $1938-1940$ & & $\begin{array}{c}\text { Absence d'indications concernant } \\
\text { les revues affiliées }\end{array}$ & \\
\hline
\end{tabular}

Ces tableaux ${ }^{46}$ nous permettent de mesurer la croissance de la Ligue à l'aune de ses supports éditoriaux; une croissance étroitement liée à celle des associations et des sections nationales dont le périodique devient un organe officiel de la Ligue. ${ }^{47}$ L'analyse du tableau 16 nous permet de constater une augmentation significative du nombre de revues entre 1922 et 1933 avec deux périodes de "pic», l'une en 1927-1928 et l'autre en 1930. Ces périodes s'expliquent par la procédure d'affiliation des revues officielles qui procède en deux temps: tout d'abord, la revue dépose sa candidature auprès du Comité exécutif de la Ligue, puis celle-ci est avalisée lors d'une séance plénière. Or, ces séances ont lieu une fois par an, et principalement lors des congrès internationaux. Dès lors, on peut aisément supposer que le premier pic (entre novembre 1927 et mars 1928) est la conséquence d'une séance de comité au Congrès de Locarno qui a avalisé les candidatures de sept nouvelles revues (voir tableau 15). Le deuxième pic, en 1929-1930, est à interpréter comme la conséquence du Congrès d'Elseneur au cours duquel le Comité accepte les candidatures de quatre nouvelles revues dont trois - Chili, Yougoslavie, Roumanie - sont déposées au mois d'avril (Éditorial, PEN, 1928, 37, p. 73). En juin 1928, l'éditorial annonce la formation de nouvelles

$46 \quad$ Ils ont été effectués par nos soins sur la base des indications présentes dans Pour l'Ère Nouvelle afin de clarifier l'ordre d'apparition des revues et pour en mesurer l'ampleur au cours des deux décennies étudiées.

47 Pour des raisons de méthode, nous avons fait le choix de ne recenser ici que les revues dites «organes officiels» de la Ligue, puisque ce sont les seules qui sont indiquées dans $P E N$ de manière systématique tout au long de la période étudiée. En ce sens, il s'agit bien d'un aperçu de l'évolution des supports éditoriaux officiels de la Ligue et non pas de l'évolution des revues d'Éducation nouvelle en général. 
sections nationales, notamment en Pologne, en Argentine et en Inde dont la composition des comités est officiellement annoncée. Toutefois, la Ligue connaît aussi des défections cette année-là, signe que la croissance n'est pas linéaire. Deux revues - Educaçao social (Portugal) et Tijdschrift voor Ervaringsopvoedkunde (Pays-Bas) - annoncent leur retrait en raison de tensions et conflits au sein des associations éditrices. Le climat politique dans le premier cas, les clivages idéologiques dans le second, empêchent la publication d'un organe représentatif du pays. L'année 1932 pourrait être considérée comme une troisième période de «pic» en raison de l'affiliation de trois nouvelles revues (Paraguay, Portugal, USA), peu après le Congrès de Nice. Mais le nombre important de défections cette année-là annonce le début d'une période de troubles dont les conséquences se manifestent à plusieurs niveaux. Tout d'abord, la revue allemande cesse de paraître après avoir publié son dernier numéro en mars 1932. En 1933, c'est le tour de l'Educazione Nazionale (Italie) dont le directeur déplore «l'échec des efforts investis pour agrandir le cercle des lecteurs» (Nouvelles diverses, PEN, 1933, 89, p. 173) et qui subit l'effet de censure du régime nationaliste.

La disparition de Das Werdende Zeitalter est particulièrement symbolique puisqu'elle est l'une des revues fondatrices de la Ligue. Pour les historiens de l'Éducation nouvelle (Hameline, 2002a; Oelkers, 2010; Savoye, 2004), c'est le signe d'une période de déclin qui débute dans plusieurs pays. En francophonie, Pour l'Ère Nouvelle résiste aux troubles politiques et continue de paraître mais elle connaît d'importants changements éditoriaux. Elle est dirigée par le GFEN dont elle devient l'organe principal même si elle conserve son statut de revue de la Ligue. De 1933 à 1937, le nombre de revues affiliées se stabilise, mais celui des articles des pays latins diminue. Dès 1938, le statut d'organe de la Ligue est encore visible dans le sous-titre, mais la «charte» est désormais perdue au milieu des annonces du GFEN. Est-ce le signe d'une volonté de se distancer de la Ligue? Nous pouvons l'interpréter comme tel, le groupe publiant avant tout au niveau français avec des auteurs et des problématiques propres à ce pays.

L'analyse des supports éditoriaux tels qu'ils apparaissent dans PEN permet d'observer l'étendue internationale de la Ligue dont l'apogée se situe à la fin des années 1920. Le phénomène «d'explosion» de ces supports durant cette période révèle le succès du mouvement ainsi que l'importance que jouent les revues dans les efforts de propagande. Car elles constituent de véritables tribunes des idées nouvelles sur l'éduca- 
tion bien qu'elles soient progressivement contrôlées par les dirigeants de la Ligue. En effet, la nouvelle organisation décidée en 1927 instaure une hiérarchisation des périodiques puisque certains acquièrent le statut d'organe officiel alors que d'autres restent au second plan. Ce faisant, les dirigeants appliquent une forme de contrôle sur les écrits publiés au nom de la Ligue qui révèle leur désir d'harmoniser voire d'uniformiser le mouvement. Les revues dites officielles deviennent alors de véritables outils de propagande au service de la Ligue, outils nécessaires pour «faire mouvement», et pour donner l'impression qu'il s'agit d'un corps uni et unanime.

\section{CONGRÈS ET COLLOQUES:}

\section{TERRAINS DE RENCONTRE ENTRE SCIENCE ET MILITANCE}

Comme le montrent les travaux de Rasmussen (1990, 2001), Depaepe (1987), Raymond (2002) et les contributions de Fuchs, Cicchini et Brehony réunies dans Hofstetter et Schneuwly (2004), les congrès internationaux constituent des moments de rencontre privilégiés qui jouent un rôle crucial dans la circulation des savoirs en éducation. Ils permettent la rencontre d'acteurs d'horizons culturels, linguistiques et disciplinaires divers et contribuent à construire une communauté d'intellectuels (Jenkins, 2000) et de chercheurs. Cependant, les congrès peuvent aussi être le lieu où éclatent des tensions liées aux divergences de points de vue de personnes qui d'ordinaire agissent dans leur contexte local. Ces divergences apparaissent dans les résumés des interventions publiés dans les rapports de congrès, même s'ils sont souvent atténués par le chroniqueur qui cherche à donner l'impression d'un discours unanime. Grâce à leur périodicité - tous les deux ans dans le cas de la Ligue - les congrès constituent des sortes de «mises à jour» sur les savoirs, que ce soit sur le plan théorique (recherche scientifique) ou pratique (expériences pédagogiques). De ce fait, leurs comptes rendus constituent une richesse immense pour l'historien qui cherche à comprendre l'évolution des conceptions éducatives à cette époque. ${ }^{48}$ Ce volet vise à comprendre l'importance des congrès dans l'extension du mouvement, à identifier

48 Raymond (2002) étudie ces rapports de congrès sous l'angle de l'éducation morale et tire des conclusions très intéressantes sur l'évolution de ces conceptions entre 1920 et 1940. 
les acteurs qui s'y rencontrent et à repérer les thématiques au cœur des débats. À travers une analyse détaillée des comptes rendus de congrès, il nous intéresse de voir quelle place occupent les sciences, plus particulièrement la psychologie de l'enfant et la pédagogie expérimentale, au regard d'autres thématiques.

Pour l'Ère Nouvelle se fait l'écho d'une multitude de congrès, colloques et autres manifestations que ceux-ci soient, ou non, organisés par la Ligue. Dans les articles, les auteurs les présentent comme des moments intenses au cours desquels les barrières classiques de type race, langue, religion, profession s'amenuisent notamment grâce aux événements informels organisés parallèlement aux conférences et ateliers de travail. Ils constituent des moments importants d'échange de savoirs car les intervenants sont appelés à se prononcer sur un thème commun en fonction de leur ancrage disciplinaire et professionnel. PEN informe régulièrement ses lecteurs de la tenue de ces manifestations, que celles-ci soient organisées à une échelle locale, nationale ou internationale. Si ces informations ne sont pas exhaustives, leur étendue est immense attestant que la revue vise à être l'organe faîtier du mouvement en diffusant un maximum d'informations. Cependant, on peut distinguer deux types de manifestations en fonction de la place que leur accorde la revue dans ses colonnes: d'une part, les congrès de la Ligue dont les comptes-rendus occupent une place très conséquente (plusieurs numéros spéciaux par congrès), d'autre part, les colloques et conférences organisés par d'autres associations/institutions évoqués de manière plus succincte (annonces ou brefs comptes-rendus dans les éditoriaux ou dans la rubrique «Nouvelles diverses»). En raison de l'importance que constituent les congrès de la Ligue dans l'évolution du mouvement international d'Éducation nouvelle, nous avons fait le choix de focaliser notre attention sur ces derniers.

\section{UN RYTHME À DEUX TEMPS}

Dès sa fondation, les congrès figurent parmi les quatre buts que se fixe la Ligue pour créer «un lien entre les éducateurs de tous les pays qui adhèrent à ses principes et visent des buts identiques aux siens». ${ }^{49}$ Selon ses fondateurs, il s'agit de rassembler non seulement les éducateurs, mais aussi les parents et toutes les personnes intéressées par les questions éducatives. Comme nous le verrons, les scientifiques sont présents

49 Buts de la Ligue, 2e de couverture, PEN, 1922-1932. 


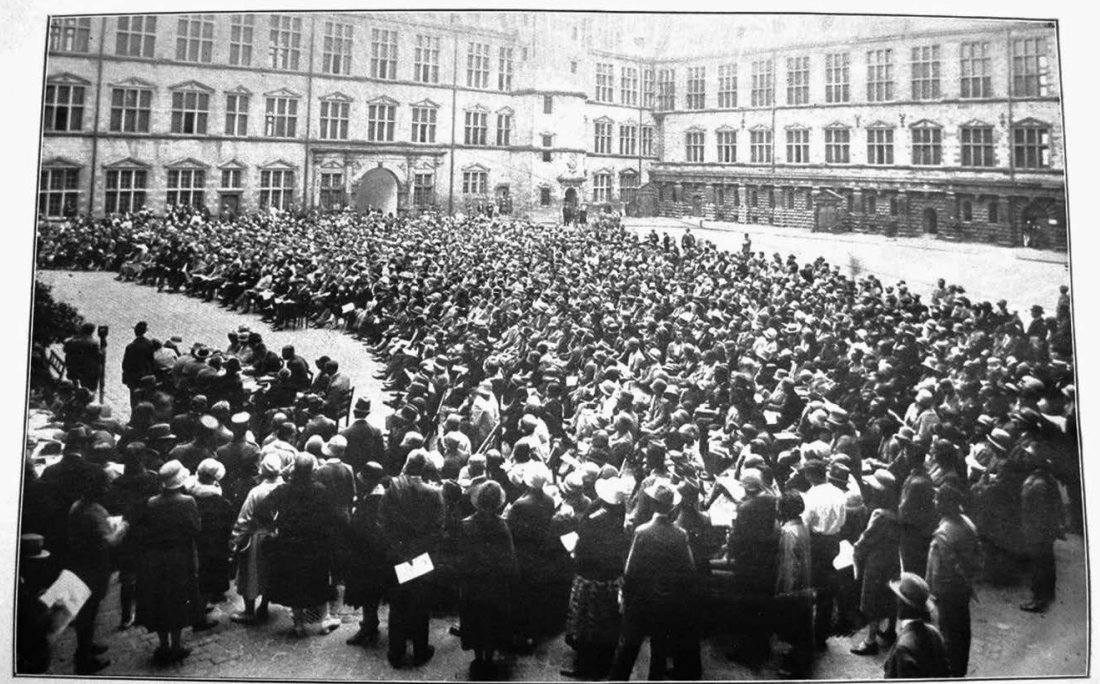

OPENING CEREMONY IN THE COURTYARD OF KRONBORG CASTLE, ELSINORE, FRIDAY, 9th AUCUST, 1929

dans ces rassemblements mais ils y viennent en tant que conférenciers pour transmettre leur expertise sur le thème de la manifestation.

Ces congrès ont lieu pendant 8 à 10 jours, en été, et proposent un thème de réflexion sur la base duquel sont programmés des conférences plénières, des «sections» (présentation d'un orateur suivie d'une discussion), des séances de travail et des ateliers. Ils permettent non seulement de réunir tous les membres de la Ligue, mais aussi la rencontre des sections nationales ou de groupes d'affinités qui ont peu l'occasion de se voir. En dehors des moments de travail, de nombreuses activités récréatives sont organisées - concerts, excursions, expositions - au point que certains disent que ces congrès ressemblent davantage à des «cours de vacances» qu'à des «rencontres scientifiques» (Ferrière, PEN, 1929, 51, p. 223). Cependant, pour les membres du Comité de la Ligue, ils constituent des moments de travail soutenus voire «éprouvants», selon les chroniqueurs. Le Comité s'y réunit quotidiennement, parfois pendant plusieurs heures, pour discuter des projets de la Ligue, de sa structure, de ses finances, ainsi que des aménagements à entreprendre concernant la répartition des tâches. C'est ainsi qu'une importante restructuration 
est décidée lors du Congrès d'Elseneur (1929): diverses commissions de travail sont désignées afin de soulager le travail du Comité central. Cette même année, deux grandes décisions sont prises concernant le Groupe Français d'Éducation Nouvelle: d'une part, c'est à lui que revient désormais la gestion administrative de la revue Pour l'Ère Nouvelle, d'autre part le groupe est désigné responsable pour organiser le prochain congrès international de la Ligue en France. Comme le relève l'éditorial de janvier 1929, les congrès structurent la vie des membres de la Ligue selon un rythme à deux temps: «Il y a les années des congrès: 1921 Calais, 1923 Montreux, 1925 Heidelberg, 1927 Locarno, 1929 Elseneur, où l'on fixe les résultats acquis et où l'on prépare l'activité future. Entre deux, il y a les années où l'on travaille» (Éditorial, PEN, 1929, 44, p. 1).

Cette périodicité se reflète également dans la revue où tous les deux ans, les numéros de septembre à décembre sont dédiés aux rapports de congrès. De fait, l'intégralité des interventions ne peut être publiée en raison du grand volume de pages et donc des frais que cela impliquerait; c'est pourquoi certains rapports font l'objet de tirés à part vendus séparément, à l'exemple de celui du congrès d'Elseneur (1929) publié en anglais par Boyd et Mackenzie (1930). Chaque revue publie donc une sélection de conférences privilégiant, dans le cas de PEN, les interventions en français, faites par les personnalités les plus connues et dont l'objet est le plus en lien avec le thème du congrès. Pour les conférences en allemand et en anglais, la rédaction renvoie le lecteur aux rapports publiés par les revues sœurs qui elles, privilégient les interventions faites dans leur langue de publication. Ces rapports regroupent les versions écrites de conférences données lors du congrès, qui sont le plus souvent résumées mais rédigées et signées par l'orateur. Quand ce n’est pas le cas, il s'agit de résumés effectués par les membres de la rédaction et signalés comme tels au lecteur. À cela s'ajoute le plus souvent le programme du congrès ainsi que les discours d'ouverture et de clôture.

\section{DES LIEUX À L'IMAGE DE SES MEMBRES}

Les congrès de la Ligue sont organisés selon un «tournus» visant à diversifier les lieux où ceux-ci se déroulent et surtout à répartir la charge d'organisation d'un pays à l'autre. 
Tableau 17.

Lieux et thèmes des congrès de la Ligue

\begin{tabular}{llll}
\hline Année & Lieu & Thème & Présidents \\
\hline 1921 & Calais (F) & L'Expression créatrice de l'enfant & $\begin{array}{l}\text { H. Baillie- } \\
\text { Weaver (UK) }\end{array}$ \\
\hline 1923 & Montreux (CH) & L'École active et l'esprit de service & A. Ferrière (CH) \\
\hline 1925 & Heidelberg (D) & $\begin{array}{l}\text { Comment éveiller l'activité } \\
\text { spontanée chez l'enfant? }\end{array}$ & E. Rotten (D) \\
\hline 1927 & Locarno (CH) & $\begin{array}{l}\text { La signification de la liberté } \\
\text { en éducation }\end{array}$ & P. Bovet (CH) \\
\hline 1929 & Elseneur (DK) & $\begin{array}{l}\text { Vers une éducation nouvelle. } \\
\text { Psychologie nouvelle et curriculum. }\end{array}$ & G. J. Arvin (DK) \\
\hline 1932 & Nice (F) & $\begin{array}{l}\text { La transformation sociale et } \\
\text { l'éducation }\end{array}$ & P. Langevin (F) \\
\hline 1936 & Cheltenham (UK) & $\begin{array}{l}\text { Les fondations éducatives de la } \\
\text { liberté et de la communauté libre }\end{array}$ & S. Percy Nunn (UK) \\
\hline
\end{tabular}

Comme nous pouvons le constater, tous ces congrès ont lieu en Europe dont deux pays - la Suisse et la France - les accueillent à deux reprises. Cette «concentration» géographique est révélatrice des dimensions d'une Europe qui, à cette époque, se limite aux grandes villes telles que Paris, Londres, Genève, Bruxelles dans lesquelles la majorité des manifestations de ce type sont organisées (Fuchs, 2004). Si les congrès de la Ligue n'ont pas lieu dans ces grandes villes, ils s'organisent pourtant dans ces mêmes pays (France, Angleterre, Suisse). Nous remarquons aussi que seul un congrès (Elseneur, 1929) est organisé en Europe du Nord (Danemark) et qu'aucun n'a lieu en Europe du Sud. Une indication qui permet de supposer que les pays les plus riches et possédant le plus de membres sont choisis pour l'organisation des congrès. Selon Cicchini $(2004)^{50}$, le choix des lieux d'accueil n'est jamais neutre et fait l'objet de négociations dans lesquelles interviennent autant d'arguments économiques, politiques que relationnels. Malheureusement,

50 Cicchini (2004) se base sur une étude des Congrès internationaux d'Éducation morale dont les protagonistes sont sensiblement les mêmes que ceux des congrès de la Ligue. 
nous n'avons trouvé que très peu d'éléments concernant le choix de ces lieux dans les archives de la Ligue.

Selon Hofstetter et Schneuwly (2004), la «géographie» des congrès est un indicateur de l'extension du mouvement et de sa politique interne. Ainsi, le choix de petites villes telles que Montreux, Locarno, Heidelberg, voire Nice (et non Paris) pour les congrès de la Ligue peut s'interpréter comme la volonté de la part du comité d'être en accord avec les valeurs qu'il défend, notamment la proximité avec la nature, la vie à la campagne, etc. La lecture des rapports de congrès montre que les excursions, les promenades et autres activités de détente sont aussi importantes que les conférences. De plus, le cadre dans lequel se déroulent ces congrès (Château d'Elseneur, Palais Impérial à Nice, hôtels au bord du lac à Montreux et Locarno) est souvent qualifié de «majestueux, somptueux, fleuri, baigné de soleil»; ce qui laisse penser que le choix des lieux de congrès est aussi lié au «standing» auquel sont habitués les membres de la Ligue. La tendance «bourgeoise» qui caractérise certains d'entre eux fait d'ailleurs partie des reproches qui leur sont fréquemment adressés. Enfin, on peut supposer que le choix de ces lieux est influencé par le souci de représenter les principales communautés linguistiques qui composent la Ligue à savoir le français, l'anglais, et l'allemand.

\section{CONSTITUER UN INCONSCIENT COLLECTIF}

Une lecture traversante des rapports de congrès permet de donner un aperçu global des tendances qui s'en dégagent, l'analyse détaillée de chacun d'entre eux ayant été faite dans le chapitre sur l'histoire de la Ligue. Tout d'abord, nous sommes tentés de réunir les trois premiers congrès (Calais, Montreux, Heidelberg) dans un même groupe car ils partagent des caractéristiques semblables: congrès à «petite échelle» (moins de 500 participants), ambiance «familiale» où les idées défendues semblent partagées par la majorité des congressistes, ces derniers visant des objectifs communs. Dès le Congrès de Locarno, le nombre de congressistes augmente à l'image de la croissance rapide de la Ligue à partir de 1925; une croissance qui entraîne une plus grande diversité $\mathrm{d}^{\prime}$ opinions et donc de tensions potentielles parmi les membres.

Le Congrès de 1929 (Elseneur) signe l'apogée du mouvement en raison du record de participation: plus de 2000 participants. Mais le succès est relatif dans la mesure où il est aussi synonyme d'un éclatement du 
mouvement en raison de la multiplicité des méthodes et théories qui sont présentées à ce congrès, révélant la diversité des courants qui se réclament de l'Éducation nouvelle. Le Congrès de Nice (1932) marque un tournant dans l'histoire de la Ligue au regard des causes et des méthodes qu'elle défend depuis sa fondation; il sonne le glas d'une époque durant laquelle tout le monde semblait marcher d'un même pas, et inaugure le début d'un temps marqué par des tensions liées notamment aux différences culturelles - et dès lors conceptuelles - des membres de la Ligue. Les discours concernant les «dimensions sociales de l'éducation» qui ont lieu pendant ce congrès révèlent des positions très différentes selon qu'elles émanent de théosophes, de scientifiques ou de militants venant de France, d'Angleterre, d'Allemagne ou d'Italie. En 1936, à Cheltenham, l'échelle du congrès revient à une dimension plus familiale: nombre de pays sont absents en raison des troubles politiques en Europe mais aussi du déclin que connaît le mouvement dans ces années-là. Les tensions semblent s'être estompées car certains groupes en désaccord se sont alors distancés, suivant leur propre chemin.

Les congrès constituent des moments forts de la vie de la Ligue au cours desquels des liens se créent, des projets de collaboration s'élaborent. Pour les dirigeants, ces moments contribuent à la constitution d'une "culture commune» et d'un «inconscient collectif», siège des principes de l'Éducation nouvelle «qui permet à chaque membre, même éloigné des autres de se sentir lié à tous et $\mathrm{d}^{\prime} \mathrm{y}$ puiser la force nécessaire pour aller de l'avant, même solitaire, même si l'on rencontre des obstacles» (Ensor, PEN, 1923, 8, p. 140). Ces manifestations leur permettent, à intervalles réguliers, de rappeler les convictions, les credo et objectifs qui rassemblent - ou devraient rassembler - les membres de cette association sur la base des «principes de ralliement» élaborés en 1921 à Calais. Mais, les discours tenus dans les congrès des années 1920 diffèrent considérablement de ceux des années 1930, en particulier concernant le but qu'ils visent. Les discours des années 1920 ressemblent à ceux que professent les politiques en période de «campagne électorale» durant laquelle il s'agit de rassembler, convaincre, attirer les nouveaux adhérents.

Dans les années 1930, le ton change: les dissensions augmentent au sein de la "grande famille», les groupes d'Éducation nouvelle se multiplient attirant les membres de la Ligue vers d'autres lieux de rencontre. Dès lors, les discours des dirigeants se modifient, visant à «conserver» 
au sein de la Ligue les membres qui avaient autrefois adhérés aux principes de ralliement. La rhétorique de ces discours s'apparente à celle des politiciens qui, durant leur mandat, cherchent à conserver les membres de leur électorat en mettant en valeur ce qu'ils ont fait. Cette rhétorique est analysée notamment par Le Bart (1998) qui montre que les discours politiques doivent tendre à «légitimer l'action de l'acteur qui le produit» en prouvant que la réalité sociale est transparente, que le monde peut être expliqué par des causes simples et ordonnées, que l'autorité politique est nécessaire, et que cette dernière maîtrise les phénomènes sociaux, la société formant une communauté de citoyens homogènes qui peuvent être régis par les même lois. Cette analyse est particulièrement intéressante car elle est transposable au cas qui nous intéresse: les dirigeants de la Ligue («producteurs de discours») cherchent à montrer que la réalité de la Ligue est «transparente» en ignorant les dissensions qui règnent parmi ses membres; leurs discours visent à montrer que la Ligue est indispensable à l'avenir du mouvement («l'autorité politique est nécessaire») et que celle-ci forme une famille unie («communauté homogène») sur la base des principes de ralliement qu'elle a formulés lors de sa fondation («régie par les mêmes lois»).

\section{CONVIVIALITÉ, FRATERNITÉ vs TENSIONS, DISSENSIONS}

De l'analyse des rapports de congrès publiés dans PEN ressortent plusieurs points forts, sortes de tendances communes qui traversent ces manifestations malgré leur diversité de lieu, de temps, de thème. Tout d'abord, il s'agit de l'esprit qui anime ces manifestations, un esprit décrit comme «fraternel», «familial», «convivial», favorisant les contacts relationnels entre congressistes. De manière récurrente, l'orateur qui prononce le discours d'ouverture évoque «l'esprit de famille» qui réunit les congressistes faisant accroire que ces manifestations sont comme des «réunions de famille» organisées tous les deux ans. L'image de la famille est associée à l'amour, à la chaleur humaine, et surtout au pouvoir que celle-ci aurait de rassembler les personnes les plus diverses. ${ }^{51}$

51 La même image est utilisée dans certains établissements, par exemple à l'Institut J.-J. Rousseau, où les étudiants et professeurs sont présentés comme les membres d'une même famille. 
Un air de famille - le mot «tribu» serait plus juste - pénétrait nos rangs serrés. C'est qu'un même idéal avait servi de centre de ralliement. L'Éducation nouvelle réunissait ici ses pionniers, ses fidèles, ses amis et des curieux: fidèles de demain. (Ferrière, PEN, 1927, 32, p. 262)

Par association, l'image de la famille véhicule celle de la fraternité, plus précisément «l'esprit de fraternité» qui, pour les dirigeants de la Ligue, doit permettre de dépasser les conflits nationaux, internationaux voire personnels afin que puissent s'établir des échanges et des débats dans un climat serein, en particulier lors des congrès. Cette notion de fraternité - dont la proximité avec l'image de la communauté chrétienne est évidente - traverse les décennies en particulier dans les discours d'ouverture des présidents de congrès.

La Ligue internationale pour l'Éducation nouvelle se tient résolument audessus de toutes les différences politiques, religieuses et philosophiques. [...] C'est dans l'esprit de fraternité pour l'Éducation humaine que nous travaillons, pour l'éducation de tout l'homme dans tous les hommes. Et toutes nos différences s'effaceront devant la splendide unité de notre tâche. (Ensor, PEN, 1932, 81, p. 238)

Comme on peut le voir dans cet extrait, Beatrice Ensor exprime la volonté de ne prendre parti pour aucun groupement, de rester neutre par rapport aux appartenances confessionnelles, culturelles, politiques de ses membres. La présidente de la Ligue affirme ainsi son désir d'accepter toutes les tendances, méthodes et courants de pensée avec le souci permanent de ne pas juger ni exclure. Cette préoccupation est particulièrement présente dans les discours des trois fondateurs (Ensor, Rotten, Ferrière) dont elle révèle l'esprit de tolérance et d'ouverture. Cependant, cette volonté «louable» d'accepter toutes les tendances conduit bientôt la Ligue à des problèmes identitaires (Haenggeli-Jenni, 2012) où celle-ci peine alors à définir sa spécificité parmi de nombreuses autres associations d'Éducation nouvelle. Cette diversité «revendiquée» comporte aussi son revers de médaille puisque celle-ci génère des tensions liées aux divers points de vue des protagonistes.

Les congrès de la Ligue sont aussi le lieu de dissensions et tensions, même si celles-ci sont le plus souvent «gommées» dans les articles résumant les interventions dans les rapports de congrès. Aussi, c'est plutôt dans les «chroniques de congrès» que l'on peut constater la présence de ces tensions. Si celles-ci paraissent quasi absentes des deux premiers 
congrès (Montreux, Heidelberg), elles semblent de plus en plus fortes au fil des congrès suivants. À propos de Locarno, Ferrière, en tant que rédacteur de la chronique évoque plusieurs difficultés concernant les aspects organisationnels du congrès (exiguiité des locaux, problèmes de langues, trop d'orateurs...) ainsi que des problèmes liés à la présence de certains participants: "Le clergé a fait obstacle à la participation des Tessinois à notre congrès. Nous avons de suite écrit à l'évêque de Lugano que notre neutralité complète en matière confessionnelle ne justifiait pas cet ostracisme» (Ferrière, $P E N, 1927,32$, p. 263). Mais Ferrière relève surtout la présence indésirable de détracteurs qui ont manifestement troublé «l'esprit de famille» régnant habituellement dans les congrès:

Voici que de l'autre côté du pôle de l'horizon ont surgi des contradicteurs. Des messieurs de Berlin, vêtus à la mode du «prolétariat conscient» ont déclaré trouver certains orateurs de notre congrès trop «bourgeois». À eux aussi nous avons déclaré qu'entre la science pédagogique et la politique il y a un abîme que nous n'avions pas le droit de franchir. Nous, travailleurs intellectuels, déclarons ne pas accepter la subdivision simpliste en classes sociales. En tant que pionniers de l'Éducation nouvelle, nous voyons trop clairement que, sur le chemin de la vérité, chacun doit avancer à son pas, pour oser croire, au succès des appels massifs, à coups de massue adressés à la masse. Les esprits «avancés» devraient comprendre qu'imposer du dehors le progrès à ceux qui ne sont pas mûrs pour l'accepter et pour le vouloir du dedans, c'est se montrer «arriérés». (Ferrière, PEN, 1927, 32, p. 263)

En 1929, le rapport du congrès laisse supposer la présence d'autres contradicteurs de ce genre, sans pourtant les mentionner clairement. Les reproches de ces derniers concernent avant tout les méthodes prônées par l'Éducation nouvelle, en particulier l'absence de programmes et le «chaos toujours nouveau des matières». Rotten y répond en disant que «l'objection a sa valeur relative aussi longtemps que l'on reste à mi-chemin sur les voies nouvelles. Elle s'effondre si l'on va jusqu'au bout» (Rotten, PEN, 1929,51, p. 216). En d'autres termes, Rotten reproche le manque de persévérance de ces détracteurs, affirmant que c'est en allant jusqu'au bout de la logique d'Éducation nouvelle que l'on obtiendra des résultats. C'est en 1932, au Congrès de Nice, que les tensions sont à leur comble, notamment au sein des ressortissants français. Ainsi, Freinet ne partageant pas l'idéologie «bourgeoise» de la Ligue, organise un autre congrès en parallèle, à St-Paul de Vence (village où il enseigne), non loin de Nice (Lamihi, 1997; 
Mole, 2012). La chronique de Ferrière sur ce congrès n'est pas très élogieuse: il le compare à une vaste «foire aux échantillons pédagogiques» dont le programme est un «véritable chaos» (changements tous les jours) et où le «manque de sérieux» de certains congressistes s'ajoute à une diversité culturelle qui ne s'avère pas très fructueuse:

Beaucoup y viennent dans le but unique de fréquenter les coulisses. Et quel kaléidoscope! [...] Le prêtre catholique y coudoie le bolchéviste notoire. Les Sud-américains rivalisent de courtoisie avec les Chinois ou les Japonais. [...] Mais on n'arrive pas toujours à des échanges fructueux. (Ferrière, PEN, 1932, 81, p. 235)

Des remarques similaires sont exprimées par un autre "chroniqueur», Louis Brun-Laloire qui livre ses «impressions de néophyte» dans un article de PEN (Brun-Laloire, PEN, 1932, 82, pp. 263-265). Ce dernier mentionne l'impression d'un "mouvement immense, d'une portée et d'une profondeur variables à l'extrême et difficiles à mesurer» (p. 263) dans lequel se côtoient des divergences nationales liées à la culture, l'esprit, la philosophie. D'autre part, cet auteur déplore le manque de débats et de confrontations, en particulier dans les conférences plénières qui «étaient absorbées par la totalité des congressistes, en quelque sorte passivement, je veux dire sans échanges d'idées, objections, réponses questions, confrontations» (p. 263). Un manque d'autant plus gênant que Brun-Laloire participe au congrès précisément dans le but d'y trouver des débats entre des personnes dont les avis divergent. Au niveau du contenu des interventions, nous constatons un mouvement «contestataire» que l'on peut considérer comme un désaveu à l'égard des théories et méthodes prônées par la Ligue jusque-là. Le contexte politique et économique de crise dans lequel se trouve la société en 1932 conduit une majorité d'intervenants à dire que tout ce que la Ligue a fait jusque-là «n'est pas suffisant». Wallon donne un message clair à ce propos, message symbolique du tournant que connaît la Ligue cette année-là, marqué par un militantisme s'alliant au politique en vue de réalisations concrètes:

Qu'a fait l'Éducation nouvelle jusqu'à présent? Elle a fait une œuvre éminemment utile: elle a proclamé les droits de l'enfant vis-à-vis de l'adulte. [...] Que tirer des droits de l'enfant tels qu'ils ont été défendus par la Ligue? Nous commençons à nous apercevoir que tout cela n'est pas suffisant, que c'est débordé par les événements. (Wallon, PEN, 1932, 81, p. 246) 
Comme nous l'avons montré ailleurs, ce congrès marque une rupture dans l'histoire de la Ligue, augurant une période où les nationalismes s'exacerbent, où chaque pays se préoccupe d'abord de lui-même - comme en témoignent les articles de Pour l'Ère Nouvelle et The New Era-cherchant à mettre en place des projets d'application des méthodes nouvelles dans son contexte national. Le congrès de 1934 organisé par la New Education Fellowship anglaise en Afrique du Sud témoigne de cette nouvelle période durant laquelle les pays prennent des initiatives dont l'échelle est davantage nationale - ou régionale - qu'internationale. Les pays qui y participent sont les pays du Commonwealth, auxquels s'ajoute l'Afrique du Sud, constituant ainsi une forme particulière d'internationalité. Ce congrès, dont l'affluence est immense (près de 6000 participants) est à peine mentionné dans les «Nouvelles diverses» de Pour l'Ėre Nouvelle alors qu'il fait l'objet d'un gros numéro spécial dans The New Era; ce détail confirme le caractère davantage «régional» de cette manifestation. Le même constat peut être fait en 1937 à propos du congrès organisé en Australie par la New Education Fellowship anglaise destinée avant tout aux membres d'Australie et de Nouvelle Zélande: aucune mention n'apparaît dans PEN mais un numéro spécial y est consacré dans The New Era.

Un dernier «grand» congrès international est toutefois organisé avant la guerre, en 1936 à Cheltenham, sur les questions de religion, de spiritualité et de liberté en éducation, thèmes chers aux membres de la Ligue (Raymond, 2002). Pour l'Ère Nouvelle en donne un large aperçu à travers trois numéros spéciaux qui donnent à la fois l'impression d'un retour à une «entente cordiale» entre protagonistes et un accroissement des divergences de point de vue. En fait, ce congrès révèle un véritable «schisme» entre l'aile francophone rationaliste et l'aile anglophone-germanique à tendance idéaliste de la Ligue. Un schisme encore peu perceptible au congrès de Nice, quoique des prémices soient déjà là, mais qui, quatre ans plus tard, se révèle comme une évidence. Pourtant, dès 1938, Pour l'Ère Nouvelle annonce à plusieurs reprises la tenue du prochain congrès européen de la Ligue, prévu pour l'été 1939. Organisé par la section française en collaboration avec le Comité international, il doit avoir lieu à Paris du 3 au 10 août 1939 sur le thème «Les Éducateurs et la réalisation de l'Idéal démocratique». Les conférenciers prévus ${ }^{52}$ sont tous des Européens - à l'exception de deux Américains - de même que le prési-

52 Les conférenciers annoncés sont: G. Bertier (École des Roches, France), Mr. W. B. Curry (Darlington Hall, UK), Dr. J. Davis (Yale University, USA), 
dent qui n'est autre que Paul Langevin. Dans le numéro de juillet 1939 (PEN, 148), le programme est annoncé de même que les derniers détails organisationnels (transport, hôtels, etc.). Un petit encart de la rédaction révèle les intentions pacificatrices de cette manifestation en mentionnant que ce congrès doit être «une réunion familiale plutôt qu'une série de réunions publiques» raison pour laquelle «la présence d'autant de sections et de groupes que possible» est importante (Rédaction, PEN, 1939, 148, p. 172). De fait, ce congrès revêt une importance particulière car, comme l'indique la rédaction, le mouvement est en grandes difficultés:

Nous nous trouvons en présence des difficultés les plus sérieuses qu'ait jamais rencontré notre mouvement et nous devons nous faire une idée nette du rôle que les éducateurs doivent jouer pour mettre l'idéal démocratique effectivement en pratique. (Rédaction, $P E N, 1939,148$, p. 172)

Malheureusement, ce congrès n'aura pas lieu et ses objectifs démocratiques seront lourdement remis en cause par la suite des événements en Europe. Le numéro de septembre 1939 annonce que «le Congrès européen de la Ligue internationale pour l'Éducation nouvelle a dû être reporté à une date ultérieure» suscitant une grande «déception pour les amis de la revue Pour l'Ère Nouvelle» (Rédaction, 1939, PEN, 149, p. 191). ${ }^{53}$ En compensation, la revue annonce qu'elle publiera dans ce

H. Frere (ISC, Belgique), C. Freinet (École Freinet, Vence, France), Dr. H. Green (University College of Wales, UK), Prof. H. R. Hamley (London Institute of Education), Miss F. Hawthrey, formerly principal, Avery Hill training College, Londres), M. Jadot (École normale, Liège, Belgique), P. Langevin (Collège de France), E. Marcault (ancien professeur aux Universités de Pise et Grenoble), L.R. Missen (director of Education, East Suffolk, UK), H. Piéron (Collège de France), F. Redefer (secrétaire, Progressive Education Association, USA), P. Roberts, président, English Association of New Schools), T. A. Rodger, Institut de Psychologie Insdustrielle (Londres), H.G. Stead (chief Education Officer, Chesterfield, UK), Dr. H. Wallon (Collège de France), Dr. L Zilliacus (président NEF, principal Tolo Svenska Samskola, Helsingfors).

53 Ce congrès, organisé par le GFEN, aura lieu en 1946 à Paris, sur le thème «La réforme de l'enseignement et ses rapports avec l'Éducation nouvelle». Malgré le millier de personnes qu'il rassemble dont Ferrière, Dottrens, Cousinet ainsi que «Gal, Piéron, Bloch, Wallon ou Weiler», ce congrès «ne produit pas l'élan international escompté» (Savoye, 2004, p. 255). 
numéro, et dans les suivants, quelques-uns des «travaux» du congrès initialement destinés à être publiés dans les numéros spéciaux. Ceci explique le nombre important d'articles traitant des questions démocratiques dans les derniers numéros de PEN.

L'analyse des congrès de la Ligue apporte des indications précieuses sur les relations et les enjeux qui se jouent au sein du mouvement d'Éducation nouvelle. Ils constituent tout d'abord des moments de rencontre privilégiés au cours desquels les praticiens et les théoriciens peuvent échanger sur des thèmes communs. Ces rencontres sont l'occasion d'établir des réseaux de relations grâce auxquels de futures collaborations (projets de recherche, visites, échanges d'étudiants) peuvent se mettre en place. Des collaborations qui permettent ainsi aux savoirs de circuler, de se construire, de se préciser au-delà des frontières, que ceuxci soient des savoirs pratiques ou théoriques issus de la recherche. Mais comme nous l'avons vu, ces manifestations sont aussi le lieu où éclatent les divergences sur des questions souvent cruciales concernant les fondements mêmes de l'Éducation nouvelle. Véritables «arènes» de transmission des savoirs sur l'éducation (Hofstetter, 2004), ces congrès constituent des moments où les savoirs se confrontent au jugement de la communauté contribuant, ce faisant, à les consolider mais aussi parfois à les fragiliser. De fait, les dissensions qui émergent dans ces moments, en particulier à Nice et à Cheltenham, concernent des principes fondamentaux tels que les conceptions de l'enfant et de la science qui divisent aussi bien les scientifiques que les praticiens. Celles-ci ébranlent considérablement l'unité du mouvement et suscitent le besoin pressant pour les dirigeants de la Ligue, de clarifier leurs conceptions et d'intensifier leur discours de propagande (Haenggeli-Jenni, 2012). Ce discours n'aura qu'un faible impact dans les années 1930 puisque le mouvement s'essouffle au fil des ans bien que de nombreuses initiatives locales résistent aux turbulences.

De façon plus globale, l'analyse de PEN sous l'angle du cadre institutionnel permet de faire un certain nombre de constats. Tout d'abord, nous observons que le mouvement s'institutionnalise progressivement au fil des ans à travers un renforcement général de la structure de la Ligue. Que ce soit au niveau des associations auxquelles elle impose une participation financière ou des revues qui doivent remplir des conditions d'affiliation, la Ligue affiche une volonté de contrôle sur ce qui se dit et se fait en son nom et au nom du mouvement qu'elle prétend fédérer. La structure de la Ligue se renforce comme le montre la constitution de diverses 
commissions destinées à soulager le travail du Comité central dès 1927. Ce renforcement de structure révèle un mouvement d'institutionnalisation qui se fait sur un mode essentiellement «rationnel et bureaucratique» (Hofstetter \& Schneuwly, 2004, p. 579). Chefs de commissions, délégués et autres responsables se multiplient afin de gérer le mouvement, en raison de la dimension incontrôlable que prend ce dernier à la fin des années 1920. Ce processus d'institutionnalisation se traduit en outre par la fondation d'une multitude d'institutions qui favorise la rencontre entre praticiens et théoriciens œuvrant ainsi sur des mêmes terrains d'expérimentation et pour une même cause. Disséminées dans le monde entier, ces dernières participent à l'expansion de l'Éducation nouvelle mais aussi au processus de disciplinarisation des sciences de l'éducation alors en cours (Hofstetter \& Schneuwly, 2002). Un processus auquel participent également les congrès de la Ligue qui, par leur périodicité et leur évolution dynamique au cours de la période étudiée, "permettent à de nouvelles communautés et champs scientifiques ou disciplinaires d'apparaître» (p. 577). 



\section{CHAPITRE 5}

\section{CONCEPTS ET PRATIQUES EN CONTROVERSES}

Ce chapitre restitue les conceptions éducatives des partisans de l'Éducation nouvelle et pointe les enjeux de leurs revendications, en particulier dans la rupture qu'elles prétendent opérer par rapport à celles de l'école dite traditionnelle. Nous tentons d'identifier les convictions à l'origine de la militance de ces acteurs, de repérer les conceptions éducatives qui les mobilisent et les poussent à lutter pour cette cause. Nous proposons d'analyser ces conceptions à travers trois thématiques, intrinsèquement liées, qui transparaissent dans les discours de Pour l'Ère Nouvelle: a) la conception de l'enfant, b) les notions de l'apprentissage et de l'autonomie, c) le rôle du maître. Nous relevons d'une part les tendances dominantes émergeant des discours mais aussi les points de désaccord suscitant des controverses entre acteurs. Ce chapitre est donc divisé en trois volets correspondant aux trois thématiques choisies. Il s'agit en outre de voir si une évolution se dessine au cours des deux décennies étudiées, que ce soit au niveau des acteurs eux-mêmes ou de la Ligue en général.

\section{CONCEPTIONS DE L'ENFANT: \\ DE L'ÊTRE BIOLOGIQUE À L'ÊTRE SOCIAL}

La conception de l'enfant défendue par les auteurs de PEN est avant tout celle d'un être spécifique, qui se distingue de l'adulte par le fait qu'il est un être en devenir, en évolution. Cette conception s'inscrit dans le sillage des précurseurs du $18^{\mathrm{e}}$ siècle tels que Rousseau, Locke, Pestalozzi qui, bien avant eux, reconnaissaient déjà en l'enfant un être différent de l'adulte. Pourtant, l'éducation que les auteurs défendent est considérée comme «nouvelle» parce qu'elle place l'enfant, et non plus les programmes 
(et donc le maître), au centre de l'action éducative. Cette conception de l'enfant est partagée par l'ensemble des partisans du mouvement et c'est précisément parce qu'ils portent un regard nouveau sur l'enfant, affirment-ils, que l'école doit changer.

UN ÊTRE BIOLOGIQUE ET NATUREL

Dans les colonnes de PEN, l'enfant est considéré comme un être naturel au sens biologique du terme. Il est à la fois biologiquement déterminé par son héritage génétique et par les lois de son développement, celui-ci étant appréhendé comme un processus biologique. Toutefois, beaucoup le considèrent aussi comme un être doté d'un "potentiel d'éducabilité» (Hameline, 1986), autrement dit doté d'aptitudes, d'intérêts et de besoins propres à sa condition d'enfant (quoique variables d'un enfant à l'autre) et qui le distinguent de l'adulte. De nombreux articles font part des tentatives de catégoriser ces besoins et aptitudes, de les classifier selon des critères précis pour pouvoir définir des types psychologiques. L'enfant est perçu également, surtout dans les années 1920, comme un être doté d'une énergie vitale inhérente à sa condition humaine, énergie qu'il s'agit de développer et stimuler tout au long de son éducation. Cette conception de l'éducation est d'ailleurs le thème du Congrès de la Ligue de 1925 qui a lieu à Heidelberg: Comment éveiller l'activité spontanée de l'enfant? Comment libérer chez l'enfant les énergies créatrices? Elle est particulièrement présente dans les écrits de Ferrière qui utilise de manière récurrente les termes d'énergie vitale ou de vitalisme pour décrire l'aspect naturel de l'enfant. Ce vocabulaire et cette philosophie imprègnent la revue dans ses premières années (Hameline, 2002b) comme en témoignent les textes d'autres auteurs de la revue, à l'exemple de cette directrice d'école au Tessin"1: "L'Éducation nouvelle est une «éducation qui tient compte des formes et des lois de la nature de chaque individualité, lois déduites directement des lois naturelles de son développement» (Valli, PEN, 1922, 2, p. 37). Cette conception biologique se développe en réaction à une conception mécanique de l'enfant qui, selon eux, est caractéristique de l'école dite traditionnelle.

1 Maria Valli est directrice de L'Asilo d'Infanzia à Bellinzone (Tessin, CH) qui pratique les méthodes Montessori. 
D'une part, l'enfant a été considéré par cette pédagogie (classique) comme un instrument intellectuel qu'on doit pouvoir manier à son gré et qui répondra docilement par le jeu de tel ou tel de ces rouages, aux impulsions que l'on aura su donner aux ressorts mécaniques appropriés, comme si l'on n'avait pas affaire à un être de chair, né d'un germe vivant, doué de potentialités multiples, se déployant au cours de cette merveilleuse féérie qu'est le développement des organismes. (Nunn, PEN, 1936, 121, p. 231)

Dans la revue, l'enfant n'est pas perçu comme un être «mauvais» contrairement aux conceptions du Moyen-Âge qui le considéraient comme un fruit du péché originel (Ariès, 1960; Ruchat, 2003). Il ne s'agit plus de redresser l'enfant ni même de le dresser, mais de le respecter, de l'orienter dans sa croissance et son développement. À l'instar de Claparède, les auteurs de PEN comparent l'enfant à une plante dont il faut prendre soin pour qu'elle puisse grandir et s'épanouir ${ }^{2}$; et pour que cet épanouissement soit optimal, il s'agit de lui donner ce dont elle a exactement besoin à chaque étape de son évolution.

Il n'y a qu'un guide: l'enfant lui-même. Observons-le. [...] Il faut qu'ils [les enfants] vivent une vie naturelle à laquelle, prudemment, nous nous bornerons à ajouter l'éducation et le perfectionnement des sens qui sont chez lui en voie de formation; ceci au moyen des stimulants que la pédagogie scientifique met à leur disposition et de telle sorte qu'ils acquièrent ainsi le moyen de tirer directement de la nature, sans vain raisonnement et sans effort inutile, les expériences et les connaissances les plus vraies et les plus profondes. (Valli, PEN, 1922, 2, p. 37)

Dans les numéros de PEN, l'enfant est considéré comme un être naturel, qui a un intérêt spontané pour la nature en raison de sa proximité avec elle, de son admiration pour elle, comme s'il en était le produit. De manière générale, l'enfant est vu comme un être neuf qui découvre le monde et la civilisation, idée qui rejoint celle de Rousseau pour qui l'enfant est un être naturel car il n'a pas encore été corrompu par la société (Battistolo, 2009). Pour certains auteurs, il va de soi que l'enfant aime la nature:

2 Claparède, tout comme d'autres membres de l'Institut Rousseau (Ferrière, Bovet), utilisent fréquemment «la métaphore jardinière» (Hameline, Jornod \& Belkaïd, 1995) pour désigner leur conception de l'enfant. 
Tous les enfants sains et bien portants, mêmes les malades, aiment les fleurs, la verdure, l'espace, l'eau, le soleil, les insectes. Ils admirent l'arc-en-ciel, les herbes agitées par le vent, les beaux matins et les beaux soirs. (Jouenne, PEN, 1923,8, p. 82)

Cette conception traverse les deux décennies étudiées et constitue un des éléments fédérateurs de la Ligue et plus globalement de l'Éducation nouvelle. Paradoxalement, comme le montre Raymond (2011), la notion de nature et la définition de ce qu'est la nature enfantine, suscite moult controverses au sein de la Ligue tant les définitions divergent. Selon cette auteure, le principe de centrer l'éducation sur l'enfant n'est jamais remis en cause mais les deux positions qui s'affrontent - éducation "psychocentrée» et «sociocentrée» - génèrent des tensions voire des dissensions dès les premiers congrès de la Ligue et plus particulièrement dans ceux des années 1930 (p. 58). Au sein de la Ligue, nombreux sont ceux qui plaident pour un enseignement en contact direct avec la nature car ils pensent que celle-ci permet à l'enfant «d'éclore librement» (Decroly \& Buyse, PEN, 1922, 4, p. 71). Elle est considérée comme une source d'énergie, raison pour laquelle certains estiment «qu'on ne peut négliger cet élément dans l'éducation de l'enfant car, plus que tout autre, il est une force de progrès et de vitalité supérieure» (Jouenne, PEN, 1923, 8, p. 85). Ainsi, plusieurs auteurs attribuent à la nature des vertus bienfaitrices tant pour le développement du corps que pour celui de l'esprit. Elle aurait même un pouvoir thérapeutique sur «les petites âmes en déséquilibre» qui trouvent en elle le «calme suprême et infaillible» dont elles ont besoin. Enfin, certains affirment, même à la fin des années 1930, que la nature constitue «une base solide à la morale individuelle et sociale» capable de développer en l'enfant la générosité et la compréhension qui feront de lui un homme tolérant:

Il faut que l'enseignement, loin de s'attacher à la simple communication de connaissances cataloguées, développe la sensibilité et les possibilités de contact immédiat avec la nature. Ainsi les hommes comprendront mieux les hommes; ainsi les hommes comprendront mieux l'univers. (Coen, PEN, 1936, 115, p. 48)

Mais ces vertus thérapeutiques attribuées à la nature ne sont pas sans ambivalences. L'idée de purifier l'esprit de l'enfant par la nature, de le rendre ainsi perméable aux apprentissages de la vie, peut dissimuler des ambitions quasi doctrinaires. D’après Hameline (2002a), l'éducation qui 
se pratique dans les écoles nouvelles à la campagne - des internats assurant une influence permanente sur l'enfant - vise non seulement l'épanouissement de l'enfant mais aussi l'inculcation de normes et de valeurs chères aux fondateurs de ces écoles. Selon lui, Hermann Lietz - fondateur des trois premiers Deutsche Landerziehungsheime ${ }^{3}$ - ambitionne davantage qu'un épanouissement de la personnalité: "Ce qu'il attend, non sans romantisme, de ce contact rude avec la nature, c'est une régénération de l'âme allemande. Son projet est nationalitaire» (p. 149). Cette observation permet de comprendre pourquoi l'éducation en internat est si souvent recommandée dans $P E N$ et qu'elle figure en tête des «Trente Points» listés par Ferrière pour caractériser les écoles nouvelles. À ce propos, Ferrière affirme que l'éloignement d'avec la famille garantit une plus grande efficacité de la diffusion des valeurs de l'Éducation nouvelle. Ainsi, nous sommes amenée à penser que, sous couvert d'une éducation en contact avec la nature, les écoles nouvelles visent aussi - si ce n'est avant tout - à inculquer une morale correspondant aux valeurs de leurs directeurs, ceux-ci étant de fervents promoteurs du mouvement.

\section{L'ENFANT SUJET}

Dans la revue, plusieurs auteurs à l'exemple défendent une conception de l'enfant où celui-ci est non seulement reconnu comme une personne, mais comme un sujet ayant des droits. C'est le cas de Susan Isaacs, psychologue et pédagogue anglaise ${ }^{4}$ :

Je voudrais affirmer que la qualité humaine de l'enfant, la réalité de ses sentiments, de ses désirs, de ses craintes, même pendant la période où il ne peut s'exprimer, doit être reconnue par ses parents et par ceux qui prennent soin de lui. [...] Ce n'est que lorsque nous reconnaîtrons que l'enfant est, dès les premiers jours, une vraie personne, une personne complète, avec des sentiments et des désirs réels, que d'une part, nous consentirons à nous efforcer de comprendre son mode de développement et à acquérir ainsi la possibilité d'y contribuer; et que, de l'autre, nous serons en état, sans exagération et

3 Écoles nouvelles (internats) à la campagne allemandes.

4 Susan Isaacs (1885-1948) mène des recherches sur le développement intellectuel et social de l'enfant; elle promeut une éducation précoce basée sur le jeu à travers lequel, affirme-t-elle, l'enfant apprend et développe son indépendance. En 1933, elle est nommée directrice du Child Development Department du Institute of Education à l'Université de Londres. 
sans extrême anxiété, d'exercer notre autorité naturelle de parents et de lui demander la reconnaissance de nos droits et privilèges, en tant que personnes réelles. (Isaacs, PEN, 1937, 125, p. 58)

Cette conception se rapproche de celle du psychologue français Henri Piéron qui, dans un article de $P E N$, rend hommage à Ovide Decroly et en particulier à sa pédagogie psychologique, à la base des principes de l'Éducation nouvelle. Cette pédagogie, dit-il, respecte le potentiel naturel «d'activité et d'effort» de l'enfant qui est un principe présent dans tout organisme vivant:

Deux principes dominent la nouvelle éducation. Le premier concerne la nécessité d'adapter les méthodes aux exigences du développement naturel de l'intelligence enfantine, de suivre les stades de ce développement et de ne pas en contrecarrer la marche. Le second implique l'utilisation des leviers naturels d'activité et d'effort qui se rencontrent dans les organismes vivants, par une mobilisation affective adroite des tendances profondes. [...] Or, Decroly s'est bien montré l'un des pionniers de la pédagogie psychologique et biologique moderne en instituant pour répondre au premier principe, la méthode de globalisation et, pour répondre au deuxième, celle des centres d'intérêts. (Piéron, PEN, 1933, 91, p. 236)

Cette conception de l'enfant comme sujet rejoint celle que défendent des penseurs du $18^{\mathrm{e}}$ siècle tels que Pestalozzi, Froebel ou Rousseau. Elle apporte une connotation positive de l'enfant bien qu'il soit aussi considéré, à cette époque-là, comme une personne perfectible par définition dans la mesure où il est un être en devenir (Battistolo, 2009). Selon eux, l'éducation vise donc à perfectionner les facultés physiques, intellectuelles et morales de l'enfant.

\section{UNE CONCEPTION EN ÉVOLUTION (1920-1940)}

Dans les années 1920, les discours tenus dans PEN révèlent que l'enfant est étudié pour lui-même, en tant qu'individu biologique, les chercheurs s'intéressant particulièrement aux spécificités qui le différencient de l'adulte. La méthode des tests est beaucoup utilisée dans le but de déceler les aptitudes de l'enfant à l'aide de tâches précises. L'enfant est considéré comme un être doté d'aptitudes (compétences innées) que le maître a pour tâche de faire émerger et de stimuler, aptitudes que les psychologues s'ingénient à mesurer. En effet, les travaux des psychologues ten- 
tent de définir des profils psychologiques, voire une typologie d'individus, sur la base de leurs études du caractère de l'enfant: «Dans l'étude d'un individu déterminé, elle [la typologie d'individus] permet de réunir par une courbe les notes obtenues pour chaque aptitude particulière et d'établir ainsi son profil psychologique» en comparaison avec un «groupe test» (Wallon, PEN, 1937, 124, p. 3). Dans PEN, cette tendance se traduit par un nombre important d'articles de type scientifique, décrivant les résultats des récentes découvertes effectuées par les chercheurs. En cela, cette revue constitue un des premiers lieux de diffusion des nouvelles connaissances sur l'enfant, lieu d'échange et de visibilisation des savoirs scientifiques. Dans ces années-là, l'enfant est aussi considéré comme le symbole d'immenses espoirs: celui de transformer la société ainsi que la mentalité voire l'éthique de l'homme.

Nous pensons que l'esprit d'amour et de vérité cultivé chez l'enfant éveillera en lui un besoin d'harmonie et de beauté, et que partout où ce résultat sera atteint, les toiles d'araignées que constituent les haines et les malentendus se trouvent balayées, au profit d'une conception plus saine et plus naturelle de la coopération entre les hommes de toutes langues et de toutes nations. (éditorial, PEN, 1927, 29, p. 110)

Dans les années 1930, l'enfant est toujours étudié comme un individu mais on s'intéresse davantage à l'influence du milieu sur son développement. Le milieu est un concept vaste qui englobe beaucoup de choses: la langue, la culture, les traditions, le physique, le matériel et les autres individus. Les psychologues découvrent que l'environnement social a un impact sur le développement de l'enfant en particulier sur son caractère et son psychisme; dès lors, ce développement n'est plus seulement biologique ou génétique, mais il est influencé, voire conditionné par le milieu dans lequel évolue l'enfant. Un milieu dans lequel il y a d'abord et surtout les autres êtres humains:

Jusqu'ici on a trop envisagé la notion de milieu uniquement sous l'angle physique et matériel dans ses rapports avec l'enfant. Les découvertes récentes de la psychologie ont révélé que les aspects les plus importants du milieu sont ceux qui ont trait aux relations humaines, en d'autres termes à l'action de l'individu sur l'individu. (Hunnybun, PEN, 1937, 124, p. 21)

Pour l'Ère Nouvelle se fait l'écho de ces changements en publiant un nombre important de recherches étudiant l'enfant en lien avec les 
personnes qui composent son environnement social. Désormais, la famille devient un objet d'étude privilégié puisqu'elle constitue l'entourage le plus proche de l'enfant. Les articles évoquent des recherches sur les relations parents-enfants, et plus particulièrement sur les relations mère-enfant/père-enfant dans lesquelles les références à Freud sont très présentes (névroses dues aux problèmes relationnels mère-enfant / père-enfant). D'autres évoquent les problèmes dus à la «faillite» de la famille, un modèle de relations dont l'effritement est particulièrement ressenti aux États-Unis où «la désintégration de la vie familiale s'effectue pour une grande part sur une base individualiste et expérimentale» (Matsner-Gruenberg, PEN, 1937, 125, p. 59). Cette démission de la famille est considérée comme une des causes des maux de la société ainsi que comme la responsable de la perte du sens de la nature chez l'enfant.

La famille disparaît alors qu'elle était en mesure de conférer sinon une éducation parfaite, du moins quelques rudiments explicatifs de la vie. L'amenuisement de l'éducation familiale risque de faire disparaître, si l'on ne pare à cette défaillance, la sensibilité, le sens de la nature, le lyrisme et l'originalité des hommes. [...] Nous sommes donc en présence du problème éducatif. La famille éduquait pour hier, assez bien dans un sens, et fort mal dans l'autre. Elle éduque peu, très peu, pour aujourd'hui; elle n'éduquera plus du tout pour demain. [...] Au demeurant, il n'est point sûr que cette disparition progressive de l'éducation soit un mal, si elle est remplacée par un enseignement intelligent de la vie. (Coen, PEN, 1936, 115, p. 47)

Cet extrait d'article révèle une forte ambivalence à propos de la famille dans les discours de PEN. À certains moments, cette dernière est perçue comme la ressource principale de l'enfant pour se construire sur le plan affectif et relationnel, à d'autres, elle est perçue comme responsable de dégâts psychologiques sur l'enfant que l'Éducation nouvelle aurait pour tâche de «réparer», du moins d'amenuiser. En d'autres termes, certains auteurs pensent que la famille peut être aisément remplacée par un enseignement intelligent et de qualité, comme c'est le cas dans les écoles-foyer ou les internats. Par exemple, le «Home Chez Nous» (Coquoz, 1998), foyer d'Éducation nouvelle qu'affectionne beaucoup Ferrière, accueille des enfants «moralement abandonnés», placés dans cette école par le service de tutelle de l'État suite au retrait de la garde parentale à certaines familles. D'autres articles de PEN font état de recherches sur l'enfant dans ses relations avec le maître ou avec l'éduca- 
teur considérant désormais cette relation de façon attentive en raison de ses effets sur la motivation de l'enfant au travail (scolaire). Enfin, l'enfant est étudié dans ses relations avec ses pairs, considéré à la fois comme un individu en interaction avec les autres et comme membre d'un groupe dans le contexte scolaire. Ainsi, les articles sont nombreux sur le travail d'équipes ou le travail libre par groupes ${ }^{5}$ mettant en avant les bienfaits de la coopération sur l'apprentissage.

En 1936, Henri Piéron dédie un long article à la question des influences sur la formation intellectuelle de l'enfant - Le problème de l'action de l'hérédité et du milieu dans la formation intellectuelle (Piéron, PEN, 117, pp. 103-110) - dans lequel il reconnaît la valeur des tests psychologiques (ex. tests Binet) mais pense qu'il faut relativiser leur résultats; car les récentes découvertes de la psychologie montrent qu'il y a «influence du milieu», une donnée importante dont il faut désormais tenir compte. Comme Piéron le dit, cette évolution occasionne des controverses théoriques de fond qui touchent la Ligue comme d'autres milieux dès le début des années 1930.

Ce problème a conduit à des controverses théoriques, extrêmement vives souvent, entre ce qu'on appelle l'esprit biologique et l'esprit sociologique. D'après certains, le niveau intellectuel serait en grande partie fonction de l'action sociale proprement dite, tandis que pour d'autres, ce niveau intellectuel est essentiellement conditionné par des facteurs individuels profonds, des facteurs d'origine héréditaires, de caractère congénital. (Piéron, PEN, 1936,117, p. 103)

Les deux tendances évoquées ici par Piéron constituent le cœur des controverses qui animent la Ligue dans ces années-là du fait que les fondateurs et militants de la première décennie (Ferrière, Rotten, Ensor, Nussbaum, Bertier, Geheeb, etc.) croient essentiellement à un «esprit biologique» tandis que ceux de la deuxième décennie (Wallon, Piéron, Laugier, etc.) adhèrent plutôt à la thèse de «l'esprit sociologique» influencé par l'action sociale. Des controverses qui se solderont par des prises de distance voire des désaffections de certains «anciens» au cours des années 1930, à l'exemple de Ferrière qui n'adhère pas à ces nouvelles conceptions. Celles-ci évoluent aussi en raison de la croissance de la psychologie sociale qui étudie les groupes (à l'intérieur des écoles, des

5 En référence aux travaux de Marie-Anne Carroi (travail d'équipe) et ceux de Roger Cousinet (travail libre par groupes). 
classes) ainsi que les relations interindividuelles qui s'y jouent, notamment les relations de pouvoir.

Les problèmes de la pédagogie moderne ont pris de l'ampleur et ont été très approfondis ces derniers temps grâce au développement d'un groupe de sciences connexes, telles que la sociologie et la psychologie sociale. Ces sciences ont donné l'impulsion à l'étude de la classe scolaire en tant que groupement, dont le travail et la vie sont soumis à des lois particulières. Par le fait même que l'école avec sa vie en groupe est précisément le terrain (soulignés par l'auteur) où se forment et se développent les dispositions sociales des enfants, ces questions exigent un examen approfondi. (Lipszyc, PEN, 1936, 120, p. 195)

Enfin, les années 1930 sont marquées par un intérêt profond pour le développement moral de l'enfant; de nombreux articles sont publiés sur le sujet dans $P E N^{6}$ et le célèbre ouvrage de Piaget - Le jugement moral chez l'enfant - sort de presse en 1932. Désormais, on affirme que l'éducation doit permettre à l'enfant de maîtriser ses pulsions naturelles et ses instincts en l'instruisant aux valeurs morales.

Notre analyse permet de constater les divergences progressives concernant la conception de l'enfant alors que ces acteurs oeuvrent tous à l'amélioration de l'éducation. C'est que ces divergences concernent des questions de fonds à la fois pédagogiques et philosophiques qui contiennent en elles une grande complexité et des contradictions profondes. Des contradictions particulièrement bien résumées par Wilhelm Paulsen (1875-1943) ${ }^{7}$ au Congrès de Locarno dans une conférence intitulée $\mathrm{La}$ Liberté de l'éducateur:

Le problème général est de rebâtir l'éducation sur les besoins vitaux de l'individu et de la société, et de redonner à l'éducation un contact direct avec la nature et la réalité.

Le problème individuel est de préserver intacte la nature de l'enfant, conditionnée par l'hérédité et le milieu, ses forces latentes, ses qualités person-

6 Sur 15 articles contenant le mot «moral-e» dans leur titre, 12 sont publiés dans les années 1930.

7 Pédagogue réformateur allemand, Wilhelm Paulsen est membre du conseil administratif des écoles (Oberstadtschulrat) de la ville de Berlin dès 1921. Très engagé dans le mouvement d'Éducation nouvelle, il est rédacteur de la revue hambourgeoise Pädagogische Reform et auteur de La conquête de l’École. 
nelles, de façon qu'il puisse travailler de lui-même à construire son propre monde.

Le problème social est d'ancrer la jeune vie dans la culture et le système économique d'une communauté de telle sorte que son développement acquière un sens et un stimulant de par ses relations avec la société humaine. [...]

Le problème pratique est de donner à la constitution interne et externe de cette communauté scolaire une forme telle que le conflit entre l'individu et la société disparaisse et que l'enfant trouve dans la sécurité de cette communauté la base la plus précieuse de ses expériences et de son savoir.

Le problème pédagogique est d'éliminer toute espèce d'éducation basée sur un dogme ou une théorie, et de rendre aussi objective que possible l'influence éducative du milieu où vit l'enfant. La coopération, le système économique, l'atelier, les livres, la nature sont des stimulants que la personnalité d'un maître ne saurait compenser. (Paulsen, PEN, 1927, 31, p. 205)

Dans cette intervention, Paulsen relève cinq défis fondamentaux que l'éducation (en particulier l'Éducation nouvelle) devrait permettre de relever. Des défis raisonnables si on les prend isolément, mais de taille si on les considère tous ensemble. Cette énumération vise précisément à montrer l'immensité de la tâche qui incombe aux éducateurs au risque même de paraître décourageante. De fait, ce discours est un plaidoyer pour les principes fondamentaux de l'Éducation nouvelle: une éducation en contact étroit avec la nature et la vie, qui respecte la nature de l'enfant tout en favorisant son épanouissement personnel afin que celui-ci trouve sa place dans la communauté. Un plaidoyer fait des paradoxes de l'Éducation nouvelle puisque l'éducateur n'y occupe qu'une place marginale (seul le dernier point le mentionne): celui-ci doit faire le moins possible acte d'éducation car «la coopération, le système économique, l'atelier, les livres, la nature sont des stimulants que la personnalité d'un maître ne saurait compenser» (Paulsen, PEN, 1927, 31, p. 206). En d'autres termes, ce discours plaide pour une éducation où il ne faut pas éduquer!

\section{AutONOMIE ET APPRENTISSAGE: DEUX FACETTES EN TENSION}

À travers une analyse détaillée des discours, ce volet entend repérer de plus près les conceptions éducatives des auteurs de PEN concernant l'apprentissage et l'autonomie. Ces deux notions sont cruciales car elles touchent les questions plus larges de la liberté et de la discipline (autorité) au cœur des revendications du mouvement et à propos desquelles 
les points de vue ne sont pas unanimes. Dans un premier temps, nous repérerons les différentes conceptions exprimées dans la revue pour pouvoir comprendre, dans un deuxième temps, où se situent les tensions et les désaccords dans le contexte plus large du mouvement.

\section{APPRENDRE PAR L'EXPÉRIENCE: GAGE D'AUTONOMIE}

La conception de l'apprentissage défendue dans la revue se caractérise avant tout par la rupture qu'elle marque, selon eux, avec celle de l'école dite traditionnelle: une activité intellectuelle qualifiée de passive par certains, basée sur la mémorisation de savoirs encyclopédiques dont la maîtrise, ou la non maîtrise, a des conséquences décisives sur la vie future de l'enfant. Comme nous l'avons évoqué ailleurs, la critique de cette conception rallie tous les auteurs en une sorte de consensus qui traverse l'ensemble de la période étudiée.

Toutes nos méthodes d'enseignement sont tournées vers le «bourrage» en vue d'examens ou, pis encore, de concours. On cherche à couler ceux qui ne réussiraient qu'à grande peine à devenir des hommes, dans un monde uniforme. (Coen, PEN, 1936, 115, p. 46)

En opposition à cette l'école dite traditionnelle, les auteurs de PEN plaident pour une conception positiviste de l'apprentissage selon laquelle l'individu ne peut apprendre qu'à travers son activité dans la tâche, son expérience d'une situation vécue et donc à travers les sentiments et émotions que suscitent cette expérience. Ainsi, on affirme que «l'enfant ne peut se développer qu'en agissant et en agissant librement» (Cousinet, 1922, PEN, 1, p. 10). À cette conception s'ajoute celle selon laquelle l'apprentissage serait un besoin naturel au sens psychobiologique du terme. Selon Claparède (1931), l'apprentissage serait une réponse à un besoin ou une fonction naturelle 8 , comme si tout être possédait en lui une curiosité naturelle qui le pousse à découvrir son environnement, à construire de la connaissance sur la base de ses expériences. C'est de cette conception que viennent le vocable éducation fonctionnelle; cette théorie rejoint celle de Dewey (1913) selon laquelle l'apprentissage serait une réponse à un

8 Le mot naturel est à comprendre ici dans le sens de spontané, inné (qui n’a pas besoin d'être appris), autrement dit qui existe dans le sujet comme une tendance préalable à l'expérience. 
intérêt naturel (biologique) suscité par l'expérience auquel l'individu répond par un effort d'adaptation qui génère de l'apprentissage. ${ }^{9}$ Dans la revue, on dénombre une quantité d'articles dédiés à l'observation de l'enfant - la plupart écrits par des psychologues - révélant des résultats de recherches sur les stades de développement et sur les stratégies d'apprentissage. En résumé, il est dit que l'enfant apprend par imitation mais non pas par imitation mécanique comme le singe, mais par imitation intelligente qui reproduit ce qu'il entend ou ce qu'il voit tout en ayant déjà intégré et dépassé ce qu'il vient d'imiter. Si cette conception de l'apprentissage prend des noms différents selon les époques, les régions et les personnes qui la théorisent - éducation fonctionnelle, learning by doing -, elle constitue une des pierres angulaires de l'Éducation nouvelle. On y perçoit l'influence de divers courants de pensée qui en marquent la philosophie, tels que le romantisme, l'évolutionnisme et les théories selon lesquelles l'ontogenèse refait la phylogenèse.

Plusieurs auteurs défendent une conception de l'apprentissage inspirée du modèle scientifique. Pour eux, l'apprentissage de l'enfant doit se faire selon les étapes et la logique de la méthode scientifique: observation, hypothèses, analyse, vérification et loi. L'enseignant doit donc faire en sorte que l'enfant passe tout seul par ces étapes, seule manière, diton, pour que celui-ci intègre durablement les apprentissages, plutôt que $d$ 'intervenir en lui enseignant des notions «toutes faites».

L'observation, l'hypothèse, conçue comme première approximation de l'idée ou de la loi; nouvelles observations pour vérifier l'hypothèse et serrer de plus près, si possible, l'idée ou la loi, voilà la méthode préconisée par $\mathrm{M}^{\mathrm{me}}$ Necker de Saussure. C'est aussi, on le sait, la méthode de la psychologie génétique. (Ferrière, PEN, 1931, 69, p. 150)

Toutefois, cette conception ne fait pas l'unanimité; certains auteurs affirment que pour pouvoir véritablement observer, il faut davantage qu'une rigueur scientifique, il faut ce «quelque chose d'irrationnel» que certains appellent $l$ 'intuition et que d'autres nomment l'imagination. Selon Ferrière, l'imagination est la clef de toute hypothèse car c'est elle qui va orienter l'enfant vers ce qu'il y a à observer. Et, se référant à Vinet, il affirme: «Pour observer, il faut imaginer qu'on découvrira; il faut tourner son

9 Voir la théorie de l'intérêt et de l'effort, notamment dans les travaux de Dewey (1913 et 1916). 
regard sur un certain point et, pour cela, il faut supposer quelque chose; toute la marche de la science est d'hypothèse en fait et de fait en hypothèse» (Ferrière, $P E N, 1931,69$, p. 150).

Une telle conception de l'apprentissage suppose, par définition, que l'enfant puisse bénéficier d'une certaine autonomie. Ce concept-clé fait l'objet de nombreux débats dans la revue car en tenir compte dans l'acte d'enseigner modifie plusieurs de ses composantes: les programmes scolaires, le rôle du maître et de l'élève, les méthodes d'enseignement. Comme nous le verrons par la suite, diverses méthodes sont préconisées pour que le processus d'apprentissage soit le plus naturel possible, autrement dit intégré dans un projet global qui a du sens dans la vie de l'élève et de l'école.

Pour que l'étude soit le plus profitable, il faut permettre à l'enfant d'apprendre à réfléchir en lui donnant l'occasion de redécouvrir par lui-même, seul, sans aide, au moment qui lui est propre, et de la manière qui convient le mieux à son développement, à sa sensibilité, [...] des principes et notions qui sont ordinairement enseignés d'une façon uniforme, au même moment, à tous les élèves d'une classe. (Deschamps-Alexander, PEN, 1928, 39, p. 125)

La notion d'autonomie apparaît comme principe majeur au cœur des conceptions éducatives des auteurs de PEN. Elle est déjà présente dans l'idéologie des pionniers qui pratiquent une autre forme d'éducation dès le début du siècle. Comme le montre Hameline (1984/2002), le tournant du siècle est marqué par une réflexion globale sur la puissance économique des diverses nations. Dans ce contexte, Edmond Demolins, fondateur de l'École des Roches, publie en 1897 un livre qui remporte un franc succès: À quoi tient la supériorité des Anglos-saxons? Selon cet auteur, l'éducation largement particulariste qui caractérise l'éducation anglosaxonne, son ouverture d'esprit et la confiance résolue que les éducateurs font à l'initiative des particuliers expliquent la supériorité de cette population. Aussi, l'Éducation nouvelle des deux premières décennies du siècle est-elle teintée d'une idéologie particulariste où l'autonomie et l'esprit d'initiative sont grandement valorisés. Cette idéologie se reflète dans les articles de PEN des années 1920 où de telles valeurs sont mises en avant dans tous les récits d'expérience relatant la mise en pratique des méthodes nouvelles, que ce soit à l'échelle d'un établissement ou à celle d'une classe. Dans les années 1930, le contexte est différent, l'idéologie a changé, l'éducation particulariste n’a plus sa place. Les préoccu- 
pations sont désormais tournées vers la dimension sociale des nouvelles méthodes: les auteurs encouragent leurs collègues à développer des pratiques de coopération, de partage des tâches, de responsabilisation des élèves à l'égard de la communauté scolaire. Les prises de position au sujet de l'autonomie dans PEN ont lieu essentiellement dans le cadre de débats s'intéressant à deux notions qui lui sont étroitement liées: la liberté et le self-goverment.

\section{AUTONOMIE ET LIBERTÉ: SYNONYMES OU INCOMPATIBLES?}

La question de la liberté en éducation occupe une place très importante aussi bien dans les discours des praticiens que dans ceux des théoriciens. Au sein de la Ligue, deux congrès lui sont dédiés en moins de dix ans - Locarno en 1927 et Cheltenham en 1936 - attestant que la notion de liberté est une des questions qui «obsèdent» les militants de l'Éducation nouvelle (Ohayon, 2006). La liberté fait l'objet de moult débats car les définitions qu'ils en donnent varient en fonction du contexte historique, social, économique du moment ainsi que des théories de référence auxquelles ils se réfèrent. Dans les années 1920, la conception de la liberté est étroitement associée à la notion de créativité, d'invention, d'expression car on pense que c'est en favorisant ces notions que l'on pourra susciter l'activité spontanée de l'enfant, activité dite naturelle. C'est pourquoi, de nombreux articles sont dédiés à l'éducation artistique, et plus particulièrement de 1922 à 1925, en écho au Congrès de 1921 (Calais) qui avait pour thème «L'expression créatrice de l'enfant». Dans ces années-là, certains affirment que supprimer ou empêcher la liberté, c'est comme «tuer le génie», «tuer l'invention, la création de l'enfant». Pour Ferrière, la liberté est synonyme d'élan naturel, de vitalité. Dès lors, laisser l'enfant libre c'est respecter son élan vital, condition indispensable à la manifestation de la spontanéité enfantine. Et, selon lui, si cette liberté est garantie, elle permettra à l'enfant de progresser sans effort:

Pour les révolutionnaires comme pour les enfants et les adolescents, pour tous ceux, en un mot, que soulève l'élan de la vie, le mot de liberté est synonyme de libération. [...] Le progrès pour eux n'est pas un effort mais une joie; il est la satisfaction d'un besoin vivant. Et que pourrait bien signifier le progrès si ce n'est libération de l'esprit, libération à l'égard des forces inférieures de notre être. (Ferrière, PEN, 1923, 7, p. 57) 
Dans la revue, les débats sont âpres sur la question du degré de liberté à accorder aux élèves. Tous les auteurs sont d'accord sur le fait qu'il ne faut pas réprimer et discipliner comme dans les écoles dites traditionnelles; mais les discours révèlent aussi la crainte que la liberté ne se transforme en anarchie, et que ce mot devienne alors négatif. Plusieurs auteurs tentent de rassurer leurs collègues en montrant que le mot liberté peut être - et devrait être - davantage compris dans le sens positif du terme, c'est-à-dire comme un principe organisateur et créateur. Parmi eux, le directeur de la communauté libre de Wickersdorf ${ }^{10}$, Gustav Wyneken ${ }^{11}$ (1875-1964), affirme que d'un «esprit pénétré de liberté peut jaillir une abondance d'idées nouvelles» (PEN, 1927, 31, p. 207), une nouvelle conception du monde. Alors que des avis sceptiques s'expriment à l'égard des écoles ou communautés libres dont il est le directeur, il tente de rassurer ses compatriotes en montrant les avantages d'une liberté «communautairement» assumée:

La communauté scolaire dont je veux vous entretenir a surgi de cette liberté [comprise dans le sens positif] et elle est basée sur elle. Certes elle ne repose pas sur les libertés multiples des individus, libertés toujours subjectives et désorganisatrices, mais sur cette liberté morale, telle que nous la connaissons comme principe du protestantisme (pris dans un sens plus large que le sens ecclésiastique) et qui à son tour peut engendrer des idées fondamentales capables de changer la face du monde. (Wyneken, PEN, 1927, 31, p. 207)

Notre analyse révèle que beaucoup de préjugés circulent à propos de la liberté, véhiculant parfois des conceptions idéalistes de ce que peut être un établissement basé entièrement sur ce principe. En réponse à cette idéalisation, Wyneken tente de démystifier la notion de liberté en montrant que celle-ci a des limites, ne serait-ce que du fait que toute liberté implique des restrictions et des choix.

10 Les Communautés libres en Allemagne (Hambourg, Wickersdorf) ainsi que certaines Free schools aux États-Unis sont très controversées même parmi les partisans de l'Éducation nouvelle.

11 Wyneken étudie la théologie et la philologie à Berlin puis travaille comme enseignant dans des écoles où il fait la connaissance d'Hermann Lietz dont il sera le collègue pendant plusieurs années. Influencé par la pensée de ce dernier ainsi que celle de Martin Buber, il fonde en 1908 la Libre Communauté de Wickersdorf, un internat pratiquant les méthodes d'Éducation nouvelle. 
Il est important de mettre en lumière qu'en optant pour la liberté nous avons par là même établi une restriction à cette liberté. Opter pour la liberté cela veut dire opter pour une certaine conception du monde, choisir un certain type de conception, mais non pas la seule et unique conception possible. (Wyneken, PEN, 1927, 31, p. 207)

Comme l'auteur le précise ici, la liberté est perçue comme un bien communautaire plutôt que comme un bien individuel. Nous savons que ces communautés n'ont pas pu perdurer longtemps en raison de l'oppression croissante qui vise ce genre de groupements en Allemagne dès le début des années 1930.

Si le problème de la liberté en éducation n'est pas neuf, il acquiert aujourd'hui un regain d'intérêt et une puissance nouvelle d'actualité; de plus, il est un des plus complexes et des plus troublants. [...] Prêtons une oreille attentive aux voix de notre temps: jamais il n'a été autant question de liberté. Jamais celle-ci n'a été autant menacée. (Jadoulle, PEN, 1935, 104, p. 9)

Elisabeth Rotten, qui était un des relais des Communautés libres de Hambourg au sein de la Ligue, se voit contrainte d'interrompre en 1932 la publication de sa revue Das Werdende Zeitalter. Comme le montre cette citation, la liberté dans les années 1930 est toujours une question en débat mais dans un climat où celle que l'on revendiquait dix ans auparavant est plus que jamais menacée.

L'Éducation nouvelle porte en elle un paradoxe visible dans de nombreux articles de $P E N$, à savoir la difficile conciliation entre liberté et autorité. Plusieurs auteurs tentent d'élucider la question en fonction de leurs connaissances et de leur appartenance professionnelle. Ainsi, selon un médecin aliéniste hollandais, Groeneveld, ce paradoxe serait intrinsèque à la personne humaine et plus particulièrement à l'individu enfant. Dans un article intitulé Le rôle de l'autorité dans la vie de l'enfant, ce médecin affirme que la nature fondamentale de l'enfant est sans cesse attirée vers deux extrêmes: d'une part un dangereux désir de liberté et d'émancipation, d'autre part, un besoin d'autorité qui pousse parfois l'enfant à une attitude d'obéissance extrême que l'auteur appelle «soumission d'esclave». Selon lui, ce tiraillement entre ces deux pôles engendre des perturbations dans le développement de la personnalité et explique certains comportement d'enfants qui se soumettent de façon très/trop docile à l'autorité, voire qui en ont besoin pour exister. Pour Groeneveld, la vie n'est pas imaginable sans le principe d'autorité car 
«vie» signifie entre autre «ordre» et qui dit «ordre» dit en même temps «hiérarchie» (Groeneveld, PEN, 1936, 120, p. 205).

La difficile conciliation entre liberté et autorité est présente également chez les éducateurs pour lesquels ce paradoxe est réel mais différent de celui de l'enfant. Certains expriment leur tiraillement, non pas entre des «pulsions» extrêmes mais entre des discours contradictoires qui créent la confusion dans leur rôle d'éducateur. «D'une part, les pédagogues modernes demandent qu'on accorde aux élèves la liberté et qu'on respecte leur spontanéité [...]; d'autre part, l'opinion courante désigne comme responsable de tous les abus modernes, le manque d'autorité qui se fait sentir en éducation» (Jadoulle, PEN, 1935, 104, p. 9). À un autre niveau encore, la contradiction se manifeste à propos de certaines méthodes pourtant reconnues par tous comme étant basées sur la liberté, favorisant la spontanéité et la libre expression de l'enfant. Des auteurs dénoncent ces contradictions dans la revue, de façon plus ou moins explicite. Ainsi, il n'est pas rare de trouver des commentaires critiques à propos de la méthode Montessori, au point que certains groupes se forment en marge de la méthode orthodoxe défendue par sa fondatrice (par ex. groupes de Philippi van Reseema, aux Pays-Bas). Dans le commentaire suivant, qui rejoint le sens de ceux faits par d'autres, apparaît un paradoxe entre la volonté de donner de la liberté à l'enfant - de laisser s'exprimer sa personnalité - et le souci de l'orienter vers les objectifs que s'est fixés l'éducateur, objectifs qui, pour certains, ne seraient atteignables que selon un certain ordre et une logique prédéterminés par la méthode.

$\mathrm{M}^{\mathrm{me}}$ Montessori a le mérite d'avoir été une des premières à attirer l'attention des éducateurs sur l'impérieuse nécessité de la liberté en éducation et effectivement sa méthode prévoit à côté d'un matériel mis à la disposition de l'élève, matériel dont il va user dans une certaine latitude, une ambiance scolaire très favorable aux manifestations spontanées de l'enfant. Toutefois, la méthode montessorienne comprend des restrictions, surtout quant à l'utilisation du matériel exclusivement dans le sens prévu par son auteur, et aussi quant aux contraintes d'une adaptation prématurée de l'enfant aux conventions spéciales. (Jadoulle, PEN, 1935, 104, p. 13)

Pour certains auteurs qui tentent de montrer l'aspect réaliste des choses, l'autorité n'est pas à bannir car elle est constructive et utile: elle sert notamment à «redresser les erreurs du milieu familial». Selon eux, l'école a aussi pour mission d'uniformiser, de lisser les individus, de cor- 
riger leurs imperfections, voire leurs tares: «L'autorité est nécessaire pour empêcher les nuisances, pour susciter les intérêts, pour redresser les erreurs du milieu familial, pour empêcher les meneurs d'agir d'une façon néfaste» (Jadoulle, PEN, 1935, 104, p. 12). Des remarques qui heurtent l'esprit sachant qu'on prétend alors vouloir respecter l'individu, faire émerger ses potentiels et développer sa personnalité!

\section{LE SELF-GOVERNMENT: UNE FAUSSE LIBERTÉ}

Dans les articles de PEN, l'autonomie est vue aussi comme une liberté au niveau de la vie en groupe dans la communauté scolaire. Populaire sous le terme de self-government chez les anglo-saxons, traduit par discipline auto-gérée ou par auto-discipline chez les francophones, le principe consiste à gérer de façon autonome la vie de l'école et des élèves. La volonté d'appliquer ce principe de manière intégrale suscite l'ouverture de divers établissements fonctionnant sur ce modèle mais dont les appellations - communauté scolaire, communauté libre, école libre - et les réalités qu'ils recouvrent varient énormément. Si ce principe fait l'objet de nombreux articles dans $P E N$, il suscite un grand intérêt déjà depuis le début du siècle notamment à travers son application dans les premières écoles nouvelles en Angleterre (Abbostholme, Bedales). En 1921, Ferrière publie un livre entier dédié à cette question, L'autonomie des Écoliers, ouvrage qui constitue une référence incontournable en la matière. Pour Ferrière, le devoir de la rénovation scolaire est urgent: la Grande Guerre a montré l'échec d'une école où prévalaient la toute puissance du maître, l'obéissance passive de l'élève, la discipline avec son système de punitions et récompenses engendrant la compétition plutôt que la coopération. L'auteur propose à la place un régime démocratique inspiré du modèle politique où élèves et maître gèrent ensemble la vie de l'école.

Depuis que l'idéal démocratique a pénétré les masses, au point même que les dernières monarchies qui subsistent sont, en fait, des démocraties parlementaires - dernière étape avant la démocratie directe - la tâche apparaît comme urgente d'orienter l'école vers la culture systématique et raisonnée de l'initiative collective, de l'esprit critique, et de la solidarité effective. (Ferrière, $P E N$, 1921, p. 9)

Si pour Ferrière, les objectifs de ce projet sont avant tout pédagogiques, c'est-à-dire destinés à gérer la discipline de façon collective, il s'agit 
aussi, selon Ottavi (2004) d'une éducation à la démocratie: «l'esprit de ces tentatives n'est pas strictement pédagogique mais aussi politique, car self-government peut se traduire par auto-discipline mais aussi par démocratie. L'enjeu est bien une éducation démocratique» (p. 135). Mais pour Jacquet-Francillon (2004), les intentions de telles entreprises sont plus ambitieuses. Selon lui, la tendance à recréer dans les établissements scolaires, comme en modèle réduit, les institutions de l'État démocratique n'a pas pour seul objectif une éducation politique: sous couvert d'une éducation civique, c'est une éducation morale qui est visée avant tout: «aux $19^{\mathrm{e}}$ et $20^{\mathrm{e}}$ siècles, tous les systèmes pédagogiques construits sur le modèle d'une communauté démocratique - enseignement mutuel, républiques d'enfants, classes coopératives - ont essentiellement une fonction morale, on pourrait même dire: une fonction de discipline morale» (Jacquet-Francillon, 2004, p. 41).

À la suite de cet auteur, nous pouvons penser que l'autonomie qui prévaut dans une organisation démocratique en communautés libres constitue, en fait, un outil puissant permettant aux éducateurs «d'exercer» ${ }^{12}$ leur influence sur les enfants qui agissent librement. Ce désir de connaître l'enfant dans les plus infimes détails de sa personnalité pourrait même paraître dangereux, laissant la porte ouverte à des abus de toutes sortes:

L'organisation enfantine qui s'est développée dans notre Institut répond aux inclinations profondes de l'enfant vers une vie sociale et lui donne l'occasion d'exprimer activement ses sentiments, de prodiguer ses efforts, sa volonté et ses instincts. Elle donne ainsi à l'éducateur le meilleur moyen de connaître directement et clairement, dans les manifestations libres de l'enfant, sa vie intime, les normes de sa pensée, la qualité de ses sentiments et la force de sa volonté; elle lui fait connaître même de nombreuses manifestations des instincts qu'on n'a pas l'occasion de connaître à l'école. C'est de la connaissance de ces côtés obscurs de la vie intime de l'enfant que dépend la connaissance des éléments les plus profonds de sa personnalité. Et ce n'est que sur une connaissance parfaite de l'enfant que nous pouvons baser notre éducation solide et consciente de son but, si nous ne voulons pas avoir plus tard, la surprise de voir se manifester des éléments instinctifs de l'esprit que nous n'avions pas su reconnaître et qui se sont développés secrètement et librement en dehors de l'influence de l'éducation. (Bartos, PEN, 1931, 69, p. 160)

12 Ce verbe compris dans le sens «exercer une influence» est utilisé de manière récurrente dans de nombreux articles de PEN. 
La deuxième partie de cette citation permet de mettre le doigt sur une contradiction fondamentale de l'Éducation nouvelle. De manière générale, les auteurs de $P E N$ revendiquent une attitude discrète du maître pour que la nature enfantine puisse se manifester sans entrave; un maître qui, nous l'avons vu, doit rester en retrait, observer l'enfant pour mieux le connaître, et qui doit faire le moins possible acte d'éducation. Or, on voit ici que cet acte est précisément indispensable puisque l'auteur dit que sans lui, on risque d'avoir «la surprise de voir se manifester des éléments instinctifs de l'esprit [...] qui se sont développés secrètement et librement en dehors de l'influence de l'éducation». En d'autres termes, contrairement à la liberté qui est régulièrement prônée, on perçoit ici la nécessité profonde d'éduquer, où ce verbe est à comprendre dans le sens «imprimer des valeurs qui permettent de maîtriser voire d'évacuer les éléments instinctifs». Ici, éduquer est quasi synonyme de contrôler, puisqu'il s'agit d'influencer l'âme de l'enfant, de lui inculquer les bonnes valeurs morales.

Selon nous, cette volonté de contrôle n'est finalement pas très différente de celle que pratique l'école traditionnelle - plus communément appelée discipline - pourtant si décriée par les auteurs de PEN. Elle trahit aussi la peur que la réalité échappe à l'adulte responsable de mener une cohorte d'élèves vers certains objectifs éducatifs. Si ce souci de contrôle est présent dans les articles de PEN, il est souvent camouflé par un discours encenseur sur la liberté de l'enfant et sur l'autonomie. Ces contradictions concernant l'acte d'éduquer ne facilitent pas la tâche du maître dont le rôle est dès lors beaucoup plus complexe, comme nous allons le voir dans le volet suivant.

\section{RÔLE DU MAÎTRE: ENTRE RECOMMANDATIONS ET INJONCTIONS}

Les conceptions éducatives prônées par la Ligue entraînent une redéfinition du rôle et de la place du maître dans l'acte d'enseigner. Ce volet vise à identifier la nature de ces changements, à déceler quelles sont désromais les attentes à l'égard des maîtres et quels sont les impacts de ces changements sur la formation des professionnels. À travers les articles de Pour l'Ėre nouvelle, nous allons tenter de déceler quelles sont les tendances générales exprimées par les auteurs à ce propos et pointer les ambivalences de ce nouveau rôle. 


\section{CONNAITTRE, AIMER, GUIDER}

Une tendance massive se dégage à propos du rôle du maître, exigence devenue presque une évidence: la nécessité de connaître l'enfant. Il se doit de connaître l'enfant en tant qu'être biologique, différent de l'adulte (étapes de son développement, caractéristiques psychologiques) mais aussi en tant qu'être singulier, chaque enfant étant une personne différente des autres. Selon eux, ce n'est que comme ça que le maître pourra le comprendre, déceler ses besoins, ses intérêts, ses stratégies d'apprentissage. Les auteurs plaident donc pour une pédagogie plus individualisée qui implique une réduction importante des effectifs. Au-delà du connaître, le maître doit être capable de diagnostiquer; diagnostiquer les forces et les faiblesses, les aptitudes ou non-aptitudes de ses élèves, et ceci non seulement à travers l'observation mais aussi à l'aide des tests psychologiques. L'objectif final de cette connaissance diagnostique est avant tout de pouvoir mieux orienter l'élève, d'abord au niveau scolaire puis au niveau professionnel.

Tout système d'éducation qui met au premier plan de ses préoccupations le développement intégral de la personnalité de l'enfant doit aboutir à une orientation professionnelle rationnelle. [...] La mission de l'éducateur est, plus encore que d'aider l'enfant à conquérir des parchemins, de l'acheminer vers une activité sociale où il réussira, et où il pourra s'épanouir dans un travail heureux et à rendement élevé. [...]

Toute orientation professionnelle efficace doit reposer sur une connaissance aussi complète que possible du sujet à orienter. [...] Dans une organisation rationnelle de l'éducation, une orientation continue doit s'exercer sur l'enfant. [...] Le livret biotypologique comprendra une partie spéciale dans laquelle le maître d'atelier, le chef d'entreprise, indiqueront le rendement du sujet dans l'apprentissage ou l'exercice de sa profession. (Laugier, Toulouse \& Weinberg, PEN, 1933, 92, p. 249)

Plusieurs auteurs de PEN soutiennent l'idée selon laquelle c'est en laissant l'enfant libre de ses actes et pensées que l'éducateur pourra le mieux connaître l'enfant et observer ses manifestations spontanées. Pour cela, nous l'avons vu, l'éducateur doit se mettre en retrait, être discret, intervenir le moins possible. D'après les discours analysés, cette attitude vise non seulement à mieux connaître l'enfant, mais plus précisément à mieux «pénétrer l'âme enfantine». Selon Piéron, «le premier devoir des 
éducateurs est de pénétrer la mentalité enfantine» (Piéron, PEN, 1933, 91, p. 236). En effet, pour beaucoup, il s'agit de connaître dans ses moindres détails les aptitudes, les besoins, les intérêts de l'enfant mais aussi ses traits de caractère, sa sensibilité, ses sentiments. Tout ceci dans le but final, disent-ils, de mieux adapter l'enseignement aux aptitudes de l'enfant. C'est pourquoi ils privilégient les outils permettant l'expression spontanée tels que les cahiers personnels dans lesquels les enfants consignent leurs observations et découvertes. Pour Sola, c'est seulement au moyen de ces expansions spontanées que le maître arrive à «pénétrer dans l'âme de l'enfant, à connaître ses préférences, à apprécier sa valeur» (Sola, PEN, 1923, 6, p. 34).

Mais derrière cette intention louable, fidèle aux principes d'une pédagogie à l'écoute de l'enfant, n'y a-t-il pas des objectifs moins explicites, à teneur morale, qui motivent les éducateurs à procéder à ces observations? Mieux connaître l'enfant, y compris sa vie intime et ses sentiments, n'est-ce pas une manière de mieux le contrôler, l'orienter, voire le «manipuler» pour lui inculquer certaines valeurs? Si de telles intentions ne sont pas toujours explicites, l'éducation morale constitue néanmoins un enjeu de taille pour les éducateurs nouveaux. Preuve en sont les nombreux débats qui ont lieu pour déterminer la période de l'enfance qui serait la plus propice à cette éducation morale. À ce propos, la Ligue organise des sondages auprès de tous ses membres; les résultats montrent que l'âge de 8-10 ans serait l'une des périodes les plus propices à la constitution d'un fondement moral. Par conséquent, il s'agit d'une période «qu'on ne doit pas laisser échapper» car l'enfant vit là «la partie la plus réaliste de son existence» (Nemes, PEN, 1932, 182, p. 276).

Sur la question de l'éducation morale, Varkonyi ${ }^{13}$ affirme que «l'optimisme naturaliste» qui caractérise la pensée des promoteurs de l'Éducation nouvelle «n'est rien d'autre qu'une théorie de la valeur, une théorie qui considère les forces, facultés et tendances manifestes cachées de la nature humaine comme les valeurs suprêmes et uniques» (Varkonyi, PEN, 1929, 47, p. 88). Selon lui, cette théorie se transforme en norme de valeurs à partir du moment où «l'on présente à la nature et à la volonté humaine des buts et des idéaux plus élevés, s'opposant jusqu'à un certain point aux tendances de la nature humaine et exigeant

13 H. Varkonyi, un père catholique hongrois, écrit dans la revue La pédagogie magyare sur les doctrines de l'Éducation nouvelle notamment sur les écrits de Ferrière. 
possession de soi et victoire sur soi-même» (Varkonyi, PEN, 1929, 47, p. 88). En d'autres termes, il pense qu'on ne peut laisser la nature se manifester en l'enfant que jusqu'à un certain point; dès que l'adulte intervient en y plaçant des objectifs et des attentes, il introduit un système de valeur qui devient le référent et oriente les actions de l'enfant. Autrement dit, respecter complètement la nature de l'enfant serait utopique!

Dans la revue, plusieurs auteurs pensent que l'éducateur a non seulement le devoir de connaître l'enfant mais aussi celui de l'aimer. Ce serait même, selon certains, la condition première de l'épanouissement de l'enfant, en particulier de l'enfant jeune:

Je crois avec certitude ce que la plupart des gens semblent avoir oublié, c'està-dire que les enfants sont des être vivants, plus vivants que les adultes [...]. Aussi est-il de toute nécessité que les enfants aient pour s'y développer, non des écoles, pour y apprendre seulement des leçons, mais un monde dont l'esprit dirigeant soit l'Amour. (Trouillon, PEN, 1933, 93, p. 293)

Pour Piéron, aimer l'enfant est indispensable pour pouvoir mieux le comprendre, saisir ce qui fait sa particularité, afin de l'orienter au mieux dans son éducation.

Le premier devoir des éducateurs est de pénétrer la mentalité enfantine, de s'y intéresser assez pour la comprendre dans ses grandes lignes, dans sa structure générale, et aussi de l'aimer dans ses manifestations individuelles si variées, pour comprendre vraiment chaque cas, édition unique et savoir assouplir les méthodes dans leur adaptation à ces réalités vivantes et complexes que sont les enfants. (Piéron, PEN, 1933, 91, p. 236)

Pour Katzaroff, l'action éducative selon la pédagogie nouvelle s'effectue par «transmission naturelle» que celle-ci provienne d'une personne ou $\mathrm{du}$ milieu. Selon lui, quand l'action éducative provient d'une personne, il faut que la voie entre l'âme de cette personne et celle de l'enfant soit libre pour que la transmission puisse s'effectuer. Or, la seule force capable d'ouvrir cette voie est l'amour: "C'est l'amour du maître pour l'enfant, son estime pour sa personne, sa confiance en lui, sa sincérité envers lui, en même temps que sa supériorité intellectuelle et morale» (Katzaroff, PEN, 1930, 55, p. 34). En d'autres termes, construire une relation de confiance est essentielle pour que l'enfant se livre au maître, pour qu'il manifeste sa spontanéité. 
En raison des nouvelles conceptions éducatives, le rôle du maître se transforme. Il n'y a plus de leçon à proprement parler, car comme le dit Lombardo-Radice «l'École active substitue l'activité personnelle de l'élève à la leçon du maître» (PEN, 1926, p. 178). Pour la plupart des auteurs de PEN, le maître n'est plus le transmetteur de savoirs ni le garant de la discipline, mais plutôt un guide, un «incitateur à l'action», qui se situe en arrière-plan et intervient le moins possible: «Il ne doit ni s'imposer ni s'abstenir. Il doit être le jardinier des petites plantes humaines» (Ferrière cité par Cousinet, PEN, 1925, 15, p. 24). Toutefois, il est responsable de préparer un milieu stimulant dans lequel évolueront les élèves et de laisser à ceux-ci «une liberté guidée par l'emploi-même du matériel d'enseignement» (Harang, PEN, 1927, 27, p. 69). Ce matériel doit être adapté à chaque enfant, c'est-à-dire correspondre à son stade de développement et «contribuer à satisfaire ses besoins de croissance corporelle et spirituelle» (Ferrière cité par Cousinet, PEN, 1925, 15, p. 24). Dans les méthodes prônées par la revue, le matériel occupe une place prépondérante au point qu'il semble remplacer le maître. À l'instar de Montessori en Italie ou $\mathrm{M}^{\text {mes }}$ Audemars et Lafendel à Genève, de nombreux pédagogues mettent au point des jeux éducatifs destinés à la petite enfance car ils sont convaincus que c'est à travers l'expérience suscitée par le matériel que l'enfant apprendra. En d'autres termes, à «l'éducation par les personnes» ils cherchent à substituer «l'éducation par les choses» (Cousinet, PEN, 1925, 15, p. 24). Chez Montessori, l'idée est poussée à l'extrême puisque, selon elle, «ce sont les objets du matériel qui sont moyens de réaction et excitent des réactions psychiques particulières». En d'autres termes, «le matériel doit remplacer presque entièrement le professeur, il n'y a donc aucune interaction directe d'ordre intellectuel entre l'institutrice et l'enfant» (Reesema, PEN, 1926, 23, p. 173).

L'objet est autre chose et plus qu'un objet de simple connaissance pour l'enfant; il joue un rôle bien plus important: celui de stimuler ses instincts latents; il en résulte que l'enfant s'y intéresse avec une concentration merveilleuse. (Montessori, PEN, 1927, 29, p. 113)

Mais cette conception du rôle du maître n'est pas perçue de façon aussi positive par tous les éducateurs. Parmi ceux qui pratiquent la méthode Montessori, certains critiquent la rigidité préconisée dans l'utilisation du matériel: celui-ci ne doit être utilisé que dans l'ordre et dans le but strictement définis par la méthode et risque ainsi de favoriser «la routine et 
l'automatisme». Dès lors, l'enfant «n'est plus libre que dans les limites de la méthode» et «ceux qui s'attendent à voir les enfants manifester un intérêt spontané pour le matériel seront déçus». Ici, le rôle du maître est pris dans un paradoxe: observer l'enfant se mue en contrôler l'enfant pour être sûr qu'il utilise bien le matériel dans le sens prescrit par la méthode.

La directrice doit veiller (à titre d'autorité chargée de guider de façon bienveillante) à ce que les enfants s'en servent pour travailler et non pas pour jouer; elle doit combattre cette dernière tendance avec méthode. En général, on obtient ainsi une classe tranquille... (Reesema, PEN, 1926, 23, p. 173)

Comme le montre Cousinet, la tâche du maître est délicate et subtile, mélange de présence et d'absence, d'observation et d'action. Tout au long de la revue, de nombreux textes s'attachent à décrire l'attitude correcte du maître, précisant à la fois ce qu'il doit et ne doit pas être, ce qu'il doit et ne doit pas faire. Plusieurs articles émanent de la plume d'inspecteurs ou directeurs d'écoles qui se plaignent de l'incapacité des instituteurs à assumer leur nouveau rôle. Selon eux, le manque de formation des enseignants freine, voire empêche, la mise en application des méthodes nouvelles à grande échelle. Or, ces textes apparaissent comme autant de prescriptions à l'égard des maîtres contrastant avec la tendance «libératrice» adoptée à l'égard des élèves. Tout se passe comme si en bannissant les savoirs prescrits et les programmes scolaires, la prescription se tournait vers le maître.

Bien que la présence de deux maîtresses soit prévue, ces maîtresses ne feront œuvre d'éducation que quand le besoin s'en fera impérieusement ressentir. Elles devront surtout observer avec le plus grand soin et d'une façon continuelle l'activité quotidienne des enfants, et prendre note de ces observations; elles devront être attentives à toutes les manifestations de cette activité de manière à les saisir aussitôt qu'elles se présenteront et à fournir ainsi aux enfants, au moment où ils en auront besoin, les matériaux qui leur seront nécessaires. (Cousinet, 1925, PEN, 15, p. 24)

À maintes reprises les auteurs insistent sur le nouveau rôle de l'enseignant: il ne s'agit plus d'instruire ni d'enseigner mais plutôt d'éduquer, accompagner, guider, proposer. En bref il s'agit de préparer un environnement stimulant pour susciter la curiosité et les apprentissages de l'enfant. Le maître n'a plus pour mandat de diffuser des savoirs, et encore moins de faire en sorte que ses élèves obtiennent de bons résultats «sco- 
laires». À ce propos, s'adressant aux éducateurs qui voudraient introduire dans leurs écoles le système du travail libre par groupes, Baldwin, directeur de la St-Christopher School à Letchworth, les met en garde sur les résultats escomptés:

En faisant leur programme, qu'ils ne se leurrent pas de l'espoir de faire cueillir à leurs élèves de beaux succès académiques pendant les premières années. Bornez votre ambition à exciter l'intérêt de l'enfant, non seulement pour ce qu'il fait, mais pour tous les instruments dont il se sert. (Baldwin, PEN, 1922, 1, p. 23)

Pourtant, les messages sont des plus contradictoires car dans nombre d'écoles nouvelles les maîtres utilisent les tests psychologiques pour mesurer le rendement des élèves. Par exemple, dans une école expérimentale aux États-Unis, un directeur se sert des tests pour prouver, vis-à-vis de l'extérieur, la validité de son programme concernant les branches dites scolaires telles que la lecture, l'écriture, l'arithmétique: «L'application des tests de rendement - tests psychologiques comme vous dites - a donnés pour toutes les épreuves des chiffres nettement supérieurs à ceux fournis par les élèves du même âge qui fréquentent les écoles publiques» (Decroly \& Buyse, PEN, 1922, 4, p. 74). Delaunay relève également cette ambivalence en jouant sur les mots enseignement et éducation qui confirme les positions contrastées au sein du mouvement:

Nul doute que les efforts des psychologues contribuent à leur manière à la rationalisation scolaire en permettant d'améliorer le rendement de l'éducation et de l'enseignement. Si nous distinguons ainsi l'éducation de l'enseignement c'est que les novateurs, en fait, sont divisés: les uns s'attachent surtout aux problèmes de culture et de formation, ils veulent vitaliser l'éducation: les autres se préoccupent davantage de meublage et la taylorisation de l'instruction est leur but. (Delaunay, PEN, 1932, 77, p. 114)

Les positions évoquées ici font écho aux conflits relevés par des historiens actuels concernant le clan des progressistes aux États-Unis (Labaree, 2006) et les conceptions opposées de Claparède et Vygotsky sur le savoir et l'éducation (Hofstetter \& Schneuwly, 2009c). Comme le révèle cette citation, nous nous trouvons devant une tension fondamentale et intrinsèque à l'Éducation nouvelle problématisée par plusieurs chercheurs (Depaepe, 1998; Hameline, 1986; Hofstetter, 2010): la recherche de rendement et d'efficacité (à l'aide des tests) alors que 
celle-ci prône l'épanouissement personnel, la non-intervention du maître, le développement naturel de l'enfant, l'apprentissage libre et autonome de l'élève. Comme le dit Hameline (1986), le romantisme de l'enfance que «charie» l'Éducation nouvelle à travers son culte à la nature, son plaidoyer pour une «vie simple et frugale», sa pratique d'activités manuelles et artistiques, ne doit pas faire oublier que «ce même courant, et souvent à travers les mêmes éducateurs, est pénétré de l'importance de la rationnalité. On y est préoccupé au premier chef du rendement de l'école et de celui des élèves» (p. 64).

\section{CONSIGNES POUR UN NOUVEAU RÔLE}

Au fil des articles de $P E N$, une quantité de recommandations sont adressées aux maîtres. La plupart de ces textes commencent par l'énumération des clichés ou images fortes dont doit se défaire le maître, images véhiculées jusque-là par l'école traditionnelle. Par exemple, on demande au maître de se défaire de l'illusion de sa toute puissance, en particulier de celle de son action éducative: «Il doit se défaire de la croyance qu'il peut à lui seul former la personnalité de l'élève, lui donner à lui seul tout le savoir dont il a besoin» (Katzaroff, PEN, 1930, 55, p. 35). Basée sur les postulats que le développement de l'enfant est le résultat d'une adaptation active et non d'une réaction passive aux stimulations du milieu, que ce développement se fait sous l'action continuelle d'un grand nombre d'influences provenant de l'environnement de l'enfant, la pédagogie nouvelle attribue au maître des tâches considérées par les auteurs comme infiniment plus larges et compliquées qu'auparavant.

Certains articles évoquent des métaphores destinées à décrire les attitudes requises du maître pour pouvoir pratiquer les méthodes nouvelles. Par exemple, le maître est comparé à un «mandataire de la vie» qui a pour tâche principale, sinon unique, d'organiser, de réguler, de canaliser les forces éducatrices de la vie et de les laisser agir sur l'enfant. Pour certains, il doit se faire à l'idée qu'il n'est que «l'accoucheur» du développement spirituel de l'enfant, ou encore qu'il doit ressembler à «une locomotive» toujours sous pression «prête à conduire le train dans la direction qui lui sera indiquée par les nécessités vitales du moment». Parmi les qualités requises la liste est longue, mais toutes convergent vers l'idée que le maître doit respecter l'individualité de l'enfant, le laisser s'épanouir librement: il doit avoir une «inclination naturelle pour l'enfant», le don d'observer, le «don de l'intuition», ne rien favoriser ni 
réprimer, «respecter profondément le petit être», surveiller son moi, savoir se dominer pour que sa personnalité s'interpose le moins possible entre l'enfant et la vie. Selon Valli (1922), le maître doit "conserver le calme et la sérénité $[. .$.$] pour donner à chaque phénomène de la vie$ enfantine le temps de se révéler, de mûrir et de s'éclaircir» (PEN, 2, p. 38). Les grandes figures du mouvement contribuent aussi à définir le nouveau rôle du maître à travers des discours qui, d'abord descriptifs, ont tendance à devenir prescriptifs:

L'institutrice a le rôle qui convient dans une pédagogie naturelle: elle est la conseillère discrète, l'organisatrice intelligente du milieu qui doit favoriser l'éclosion spontanée de la pensée personnelle des élèves. (Decroly \& Buyse, PEN, 1922, 4, p. 75)

L'éducateur est celui qui prend et comprend les enfants au point où ils en sont aujourd'hui; qui devine par quelle voie ils en sont venus là et quelles voies ils peuvent suivre à l'avenir; puis qui, choisissant avec eux ou pour eux la voie la meilleure, illumine leur esprit pour que cet esprit se libère des entraves inutiles et noue en un faisceau les tendances utiles à la vie. Partir de ce qui est, s'élever à ce qui doit être. Ne jamais perdre le contact. Savoir mettre en œuvre l'intuition, la raison et l'amour. Ne pas se fixer de but arbitraire, à atteindre coûte que coûte. Respecter - avec les réserves faites tout à l'heure - la croissance de la petite plante humaine. Ne se hâter, à part soi, que pour mettre devant ses pas la nourriture spirituelle dont elle a précisément besoin. Ecarter ce qui peut nuire à sa croissance - ce qui signifie nullement on le comprend, écarter l'effort, surtout quand l'effort est voulu compris parce que conçu comme un moyen de tendre à un but voulu. Enfin, de ne se départir jamais, sinon à bon escient, du calme, de la joie, de la sérénité vraie, celle-ci faite non point de résignation subie, mais de force d'âme et de maîtrise de soi. Telles sont les qualités qui, avec quelques autres, doivent caractériser l'éducateur. (Ferrière, PEN, 1929, 44, pp. 6-7)

On peut se demander pourquoi ces consignes à l'égard des enseignants sont aussi insistantes et récurrentes. Selon Hameline (1984/2002), il y a un écart important entre les pratiques pédagogiques effectives et l'image que l'on en donne. Par exemple, les directeurs d'écoles ont non seulement pour tâche de gérer les dimensions pédagogiques de l'établissement - programmes, pédagogie, manuels - mais aussi les dimensions financières parmi lesquelles la gestion des «ressources humaines» peut être difficile. En effet, ces derniers rencontrent souvent des résistances auprès d'instituteurs-trices qui, de fait, ne sont pas prêt-e-s à enseigner 
de la sorte, ce qui génère une rotation importante du personnel. Dans son journal de $\operatorname{Bex}^{14}$, Ferrière avoue qu'en plus de certains parents et enfants peu coopératifs, «il faut aussi faire avec les enseignants, collaborateurs souvent médiocres, mal payés, n'adhérant que du bout des lèvres au projet éducatif de 'l'original' qui les embauche» (Ferrière cité par Hameline, 1984/2002, p. 152). Comme le montre la citation suivante, $P E N$ se fait l'écho de nombreuses plaintes d'inspecteurs ou directeurs d'écoles qui disent la difficulté de travailler avec des enseignants mal formés n'étant pas préparés à ce type de pédagogie ni motivés par les nouvelles méthodes.

Des directeurs et des directrices ont ressenti autant en Europe qu'en Amérique, la difficulté qu'il y a à se procurer des maîtres suffisamment familiarisés avec la conception moderne du problème de l'éducation, surtout en ce qui concerne la discipline libre et les programmes individuels. Tout cela ne peut être appris qu'en étudiant les méthodes et en assimilant l'atmosphère des écoles où ces idées sont d'un usage constant. (Wicksteed, PEN, 1927, 32, p. 238)

Ces plaintes témoignent du besoin qui se fait ressentir dans tous les pays de renforcer la formation des enseignants et surtout de l'adapter aux nouvelles méthodes prônées par l'Éducation nouvelle (Lussi Borer, 2008). Cette formation doit désormais se situer à un double niveau théorique et pratique - où la pratique devrait s'apprendre, selon Wicksteed, par le mode de l'imprégnation. Un avis qui n'est pas partagé par tous les partisans de l'Éducation nouvelle notamment les scientifiques qui défendent une formation dans des institutions spécialisées.

\section{RENOUVELLER LA FORMATION}

Étroitement liée au nouveau rôle du maître qu'exigent les méthodes nouvelles, la question de la formation des enseignants se pose de façon récurrente dans les discours de PEN. Selon Gerhardt Sieveking (PEN, 1926, 22), directeur de l'Institut des Sciences de l'éducation d'Iéna,

14 À l'aide de Lydie Haemmerlin et Elisabeth Huguenin, Ferrière ouvre en 1921 l'École nouvelle de la Pelouse à Bex $(\mathrm{CH})$, où son expérience en tant qu'éducateur sera marquante quoique de courte durée (1921-1922). 
les exigences de la formation académique des maîtres [...] dépendent non pas de je ne sais quels vœux ou exigences extérieurs, mais d'une nécessité interne profonde, étroitement liée à l'évolution de la conception que nous nous faisons du but et de la nature de l'éducation et de l'enseignement. (p. 109)

En effet, les nouvelles conceptions éducatives impliquent une autre attitude de la part du maître de même que l'utilisation de nouveaux outils et techniques d'enseignement. L'influence majeure, quasi prescriptive, de la psychologie sur la profession enseignante exige des professionnels d'acquérir de larges connaissances en psychologie de l'enfant auxquels doivent s'ajouter, selon certains, des connaissances en biologie afin de connaître les différentes étapes et caractéristiques du développement de l'enfant (phases, processus, régressions, etc.).

Au-delà du contexte de $P E N$, on constate une volonté internationale de renforcer la formation des maîtres. Preuve en sont les congrès et colloques qui se dédient à la question mais aussi les diverses institutions qui voient le jour dès les premières décennies du siècle: Institut Rousseau (Genève), Institut des Sciences de l'éducation (Iéna), Teachers College (New York), Institute of Education (Londres) pour ne citer que les plus connus. Dans la plupart des pays, ces nouvelles institutions remplacent les écoles normales et se caractérisent par l'allongement de la durée des études et par leur contact direct avec la recherche, les professeurs exerçant une double activité d'enseignement et de recherche. Les étudiants y sont initiés à la méthode scientifique et sont formés aux méthodes nouvelles tant sur le plan théorique que pratique grâce aux écoles d'application qui sont rattachées à ces institutions. ${ }^{15}$ Plusieurs articles de PEN nous permettent d'affirmer que les idées circulent, que les modèles voyagent, influençant aussi bien les structures que les contenus de formation. Par exemple, les travaux des psychologues américains ainsi que les expériences faites dans les écoles nouvelles aux États-Unis influencent grandement les Européens. Mais l'inverse est aussi vrai dans de nombreux cas:

De plus en plus, nos étudiants lisent ce que vous publiez dans The New Era et ce qui paraît dans The Progressive Education. [...] Un Comité s'est joint récemment à nos plus grandes institutions, [...] afin d'introduire dans les préparations des maîtres le point de vue de la psychologie nouvelle et la philosophie

15 Par exemple, la Maison des Petits (1913) ou l'École du Mail (1928) pour Genève. 
du nouveau type d'école. Les étudiants sont encouragés à visiter et à étudier les écoles nouvelles [...] et je crois que nos institutions sont en train de modifier leur attitude bien plus encore qu'elles ne s'en rendent compte ellesmêmes. (Ryan, $P E N, 1927,31$, p. 194)

En 1929, une «commission pour la formation des maîtres» est fondée lors du Congrès de la Ligue à Elseneur. Celle-ci voit le jour en raison d'un constat partagé par l'ensemble des membres présents et qui atteste de l'importance que ceux-ci accordent à la formation:

1. Toutes les nations sont aujourd'hui d'accord sur le fait que la préparation des professeurs est le problème le plus important que les éducateurs aient à résoudre.

2. Aujourd'hui, tous les états reconnaissent (c'est un fait d'une portée internationale) que la formation qui a été donnée jusqu'ici aux maîtres est inadéquate; un niveau de capacités supérieur, un niveau vraiment «académique» est nécessaire. [...] C'est cette extension internationale du mouvement qui justifie notre optimisme sur l'amélioration de la préparation universitaire des maîtres. (Schneider, PEN, 1933, 85, p. 31)

Le travail de la commission consiste tout d'abord à faire le point sur les diverses réformes entreprises dans les pays concernés telles que la réforme de la préparation pédagogique en Allemagne (particulièrement en Prusse), les progrès accomplis aux USA, ou encore les initiatives menées en France, en Pologne et en Grande-Bretagne. De manière générale, cette commission défend un renforcement de la formation des maîtres ainsi qu'un certain nombre de principes tels que l'élévation de la formation au niveau universitaire, l'unité de l'enseignement et la préparation commune des deux ordres d'enseignement (primaire, secondaire), le contact plus étroit de la vie et de l'école pendant la préparation ainsi que le développement de l'aspect social de l'enseignement.

Quoiqu'elle soit internationale, la volonté d'améliorer la formation rencontre des adversaires, en particulier parmi ceux qui sont préoccupés par l'aspect économique de la formation. Ces derniers - qui ont aussi leur place dans PEN et dont Schneider se fait l'écho - «pensent qu'une telle préparation sera coûteuse et que les maîtres exigeront alors de plus hauts salaires, tandis qu'à l'heure présente il nous faut chercher à économiser» (Schneider, PEN, 1933, 85, p. 31). Dans les années 1930, une impulsion contraire importante se dessine à l'égard du renforcement de la formation des maîtres; mais selon Schneider, il s'agit d'un phénomène 
temporaire qui ne saurait résister à un mouvement dont les «manifestations internationales» attestent de son ampleur et de son avancée inéluctable ((PEN, 1933, 85, p. 32). L'optimisme de cet auteur se voit confirmé car le mouvement perdure notamment à travers diverses manifestations

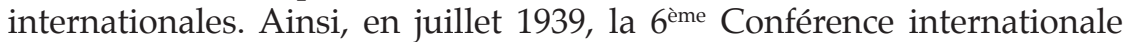
de l'Instruction publique se réunit à Genève sur la convocation du Bureau International de l'Éducation. Au terme de trois jours de discussions, la conférence adopte des recommandations qui, selon Ferrière, revêtent une importance exceptionnelle: «elles ne symbolisent rien de moins que le passage de l'école publique à une ère nouvelle, le passage de la phase littéraire d'hier à la phase scientifique ou technique de demain» (Ferrière, PEN, 1939, 144, p. 41). Argumentées par le fait que «les sciences psychologiques ont fait d'énormes progrès au cours de ces dernières années», les recommandations adoptées par la Conférence internationale insistent sur la dimension scientifique de la formation:

Il importe donc que les futurs éducateurs acquièrent une solide formation psychologique portant spécialement sur la psychologie de l'enfant et de l'adolescent, ceci sans doute au moyen de tests et méthodes métriques, mais aussi et surtout par une étude qualitative du développement mental et de la structure de l'esprit de l'enfant sous les aspects intellectuels et affectifs, individuels et sociaux. [...] Cela suppose: observations, expériences, recherches personnelles; cela suppose préalablement une préparation biologique suffisante pour permettre aux futurs éducateurs de bien comprendre toute la signification des expériences auxquelles ils sont appelés à collaborer. (Ferrière, PEN, 1939, 144, p. 41)

Ces recommandations attestent de l'étroite relation qu'entretient l'Éducation nouvelle avec la formation des enseignants dont elle contribue à accroître la dimension théorique en raison de la nouvelle appréhension de l'enfance qu'elle revendique. De fait, cette relation s'inscrit dans un mouvement plus large, international, qui tend à renforcer la formation des maîtres de l'enseignement primaire comme du secondaire (Criblez \& Hofstetter, 2002; Hofstetter \& Schneuwly, 2007). Comme le montrent les travaux de Lussi Borer (2008), ce processus se traduit par une «systématisation des contenus de formation à travers l'établissement d'une base de savoirs scripturalisés, à la mise en place de cursus aboutissant à des titres ainsi qu'à une évaluation de plus en plus exigeante des savoirs acquis durant la formation» (p. 414). Alors qu'elles prônent une articulation étroite entre la formation théorique et pratique 
au travers d'une approche expérimentale de l'étude de l'enfant, les recommandations de 1939 révèlent une distinction progressive entre les formations pratique et théorique puisqu'elles mentionnent la nécessité de procéder au préalable à des «observations, expériences et recherches personnelles» ainsi qu'à une «préparation biologique suffisante». Lussi Borer (2008) observe une semblable évolution à propos des formations à l'enseignement en Suisse durant cette période: «les formations disciplinaire et professionnelle tendent à devenir de plus en plus distinctes, la formation professionnelle intervenant après la formation disciplinaire pour les enseignants du secondaire, après la formation générale pour ceux du primaire» (p. 414).

\section{PARADOXES ET AMBIVALENCES}

À la lecture de PEN, d'importants paradoxes apparaissent concernant le rôle du maître et les qualités requises pour que celui-ci remplisse au mieux sa fonction. Des paradoxes liés à des messages contradictoires qui souvent placent ce dernier dans le désarroi, ne sachant pas quelle voie il doit suivre. D'un côté, les auteurs revendiquent une formation plus poussée - formation à la psychologie et à la méthode scientifique - et de l'autre, de nombreux textes disent que c'est la personnalité de l'enseignant qui fait tout, personnalité qui devrait être aimante, intéressée, curieuse, stimulante et animée du «feu sacré»!

Pour que les enfants soient libres et «qu'ils veulent ce qu'ils font» [...] il ne suffit pas que l'école qui les accueille offre toutes les ressources de la «Maison des Petits» de Genève et de l'Institut Decroly de Bruxelles. Il y faut surtout la personnalité et l'esprit de l'éducateur. Il faut que celui-ci soit essentiellement un stimulateur d'intérêts, qu'il trouve des occasions d'agir là où personne n'en trouverait [...] comme c'est le cas de tous les vrais éducateurs des masses. [...] Il suffirait que tous les instituteurs et toutes les institutrices fussent animés du feu sacré. (Ferrari, PEN, 1922, 1, p. 20)

Les articles présentant des écoles nouvelles révèlent qu'au-delà de leurs compétences scientifiques et éducatives, les fondateurs d'écoles sont des personnes charismatiques, hors du commun, qui influencent considérablement l'esprit de l'établissement. Cette tendance est particulièrement visible dans les articles des années 1920, où les expériences d'Éducation nouvelle sont encore très localisées dans les écoles nouvelles (alors que dans les années 1930, les expériences se dispersent dans d'autres types 
d'établissements). Selon Hameline (2002a), le charisme de certains pionniers tels que Reddie, Lietz, Badley, Demolins se confond avec leur doctrine ce qui rend aléatoire la poursuite de leur entreprise après leur disparition. Un phénomène également repéré par Savoye (2004) qui pense que cette caractéristique participe de la difficile expansion de l'Éducation nouvelle. L'inauguration des méthodes nouvelles dans plusieurs pays est souvent le fait de personnes charismatiques qui fondent des établissements où leur «esprit» est déterminant: par exemple Carpena en Espagne, Caballero en Colombie, Oropeza au Mexique, Nemes en Hongrie ou Tagore en Inde sont considérés quasi comme des «apôtres» de l'Éducation nouvelle. Sans aller si loin, nous pourrions aussi évoquer les cas de Decroly, Ferrière, Bertier ou Montessori qui tous ont marqué de leur «esprit» les écoles, instituts ou organisations qu'ils ont fondés. Or, ces figures sont charismatiques parce qu'elles possèdent des qualités autres que celles énoncées par les recommandations de la Conférence Internationale. Ils se caractérisent par «un esprit profondément pédagogique», «un instinct d'éducateur», «une âme vibrante», «une intuition éducative», «une autorité naturelle». Ces qualités, difficilement transmissibles dans un programme de formation, apparaissent pourtant comme une condition à la réussite des méthodes nouvelles. Ainsi, certains auteurs expriment leur difficulté à parvenir aux mêmes résultats que ces figures, faute de charisme ou de «foi» dans la méthode:

C'est cette fois-là qu'elle [Montessori] exigeait également de ses collaboratrices; et c'est à cause de cela que la confusion se déclara lorsque les résultats originellement décrits par $\mathrm{M}^{\mathrm{me}}$ Montessori ne se produisirent pas. Les succès obtenus sous sa conduite personnelle et celle de quelques-unes de ses disciples, étaient plutôt, pensait-on, la conséquence de leur intuition pédagogique et de leur autorité naturelle. (Reesema, PEN, 1926, 23, p. 174)

De même, Ferrière reconnaît que le succès des Communautés libres de Wickersdorf ou de l'École de l'Odenwald est lié à un certain nombre de conditions extrinsèques parmi lesquelles la "présence de fortes personnalités adultes qui ont su donner dès le début à ces écoles des traditions d'ordre, de travail et d'idéalisme» (cité par Jadoulle, PEN, 1935, 104, p. 12). En d'autres termes pour qu'une école fonctionne, il faudrait la présence de personnes charismatiques à leur tête, personnalités qui semblent paradoxalement indispensables dans un régime de liberté: «Plus l'instituteur veut limiter son autorité plus il doit la sentir forte, plus il doit posséder une personnalité forte qui lui assure un grand ascendant 
sur ses élèves» (p. 12). Selon l'auteur, ce paradoxe permet de comprendre pourquoi, à l'échelle d'un pays, les gouvernements libertaires ont besoin de dirigeants charismatiques: «Plus un État est libre, plus l'autorité y est limitée. Plus elle est limitée plus elle doit être forte» (p. 12). Les travaux réunis dans l'ouvrage de Miller et Oelkers (2014) qui étudient l'évolution de la Reformpädagogik (Éducation nouvelle) à l'École de l'Odenwald (D) relèvent les mêmes difficultés à poursuivre l'œuvre du charismatique Paul Geheeb (fondateur et directeur de l'école pendant 24 ans) après son exil en Suisse en 1934.

En conclusion, nous pouvons dire que le rôle du maître fait l'objet de multiples recommandations dans $P E N$, que ce soit de la part de personnes acquises aux nouvelles méthodes ou de la part de commissions chargées de réfléchir sur la question. Les enjeux sont grands car la réussite de la réforme dépend de la qualité des maîtres et de leur adhésion aux nouvelles méthodes. De fait, les recommandations concernant l'attitude idéale du maître sont contradictoires mêlant à la fois un haut niveau de connaissances (psychologie de l'enfant) et des aptitudes intuitives, irrationnelles, ayant davantage à voir avec la vocation que la formation.

Nous avons pu voir que les conceptions éducatives présentes dans PEN sont fortement influencées par les théories et les sciences de référence $^{16}$ constitutives de l'Éducation nouvelle qui évoluent au fil des ans. Dans les années 1920, elles sont encore fortement imprégnées de la biologie et des théories de l'évolution dont découle une conception des aptitudes individuelles comme des talents innés, déterminés génétiquement (psychologie génétique). Dans les années 1930, les progrès des diverses sciences appréhendant l'enfance, dont la psychologie, permettent de montrer que l'enfant se développe aussi, et surtout, en interaction avec son environnement et que le milieu a un impact sur son développement. Dans les colonnes de la revue, les auteurs partagent la conception d'un enfant en tant que personne ou sujet actif dans ses apprentissages et dont il s'agit de respecter les spécificités dans l'action éducative. S'il y a consensus sur l'idée que l'enfant doit apprendre par l'expérience personnelle, les avis divergent sur le degré de liberté et d'autonomie à accorder dans cet apprentissage et par conséquent, sur le rôle du maître dans l'action éducative. Là encore, de fortes contradic-

16 Ces théories de référence sont analysées et présentées dans le chapitre suivant. 
tions émergent des discours tenus dans la revue où se mêlent recommandations, injonctions voire prescriptions. Les nouvelles conceptions éducatives impliquent un renforcement de la formation des enseignants en raison des connaissances sur le développement psychologique de l'enfant désormais nécessaires à l'enseignant pour faire son métier correctement. Des exigences contradictoires avec le rôle marginal que l'Éducation nouvelle attribue au maître qui doit rester discret et faire le moins possible acte d'éducation. 



\section{CHAPITRE 6}

\section{SOCLES THÉORIQUES ET SAVOIRS EN DÉBAT}

Ce chapitre met en évidence les savoirs et théories de référence qui constituent le socle théorique de l'Éducation nouvelle. Nous présentons, dans un premier temps, les principales théories auxquelles se réfèrent les auteurs, plus précisément les conceptions philosophiques, scientifiques et éducatives qui façonnent leur pensée. Nous verrons que ces théories ne sont pas partagées par tous et suscitent parfois des divergences entraînant des désaccords sur les orientations de l'éducation. Nous analysons l'évolution de ces théories au cours de la période étudiée en les mettant en lien avec le contexte social, économique et politique du moment. Dans un deuxième temps, ce chapitre s'intéresse aux savoirs professionnels des praticiens qui expérimentent les méthodes nouvelles. Cette analyse vise à identifier les types de savoirs privilégiés dans Pour l'Ère Nouvelle dans une optique de compréhension des relations qui se jouent entre science et militance. Le troisième et dernier volet fait une focale sur les savoirs constitués et les programmes scolaires à travers une analyse fine des discours tenus dans la revue. Cette focale vise à comprendre quelles relations les partisans de l'Éducation nouvelle entretiennent avec ces savoirs supposant que d'autres types de savoirs sont privilégiés en vue de stimuler l'épanouissement de l'enfant.

\section{AUX ORIGINES:}

\section{THÉORIES DE RÉFÉRENCE DE L'ÉDUCATION NOUVELLE}

L'analyse de Pour l'Ère Nouvelle permet de mettre en lumière les principaux constituants d'un socle théorique de l'Éducation nouvelle auquel les auteurs de cette revue se réfèrent. Ce socle constitue une sorte de langage commun à partir duquel se construisent leurs revendications de 
réforme. Beaucoup d'acteurs s'expriment concernant ce socle, que ce soit pour le valider, le nuancer voire l'infirmer au gré de l'évolution de la société qui les entoure.

Les discours tenus dans PEN font apparaître un consensus global autour duquel semblent se réunir tous les auteurs. Il s'agit d'une «foi» immense en la science, foi qui s'inscrit dans le contexte portant l'héritage du $19^{\mathrm{e}}$ siècle. À cette époque, la science est synonyme de progrès et d'espoir; elle est utilisée pour légitimer toutes sortes d'innovations car on estime qu'elle est capable d'affirmer des vérités universelles. Dans la revue, il s'agit d'une science avec un grand «S», comme s'il s'agissait d'un terme générique, mais qui désigne de manière implicite, l'émergente «science de l'enfant» (Hofstetter \& Schneuwly, 2009b; Ottavi, 2001). Selon les auteurs, elle représente la grande nouveauté qui distingue l'Éducation nouvelle de l'école traditionnelle et légitime les revendications réformistes. Les auteurs expriment un changement radical dans la manière de penser le social et l'éducation depuis l'avènement de la science dans les sphères sociales. Tous la défendent et s'en réclament car ils lui attribuent le pouvoir de résoudre les problèmes de la société.

La pédagogie est la science la plus récente, parce que son objet est l'être le plus complexe qui soit sur terre: l'être humain. Il est évident que seule une science mûre peut résoudre des problèmes complexes. Et c'est précisément parce que jusqu'à présent la science n'a pas atteint la maturité, qu'elle a omis de considérer comme une de ses branches la science de l'éducation. Voilà pourquoi, jusqu'ici, la pédagogie a totalement manqué de base scientifique. (Westharp, PEN, 1929, 45, p. 45)

Malgré l'hétérogénéité des profils, nous pouvons dégager trois conceptions de la science sur lesquelles les auteurs se rejoignent. Elles témoignent, chacune à leur façon, que la science représente la nouveauté majeure en éducation, investie de la mission de garantir l'avènement $d^{\prime}$ 'une ère nouvelle.

Premièrement, la science est perçue comme une entité globale, universelle, qui possède son esprit et ses lois, lois vérifiables en tout temps et en tout lieu. Comme le dit Ferrière, «la physiologie et la psychologie recherchent des lois qui doivent être vraies partout et toujours. Elles sont générales et s'appliquent à tous les êtres» (Ferrière, PEN, 1929, 51, p. 213). Parmi ces lois, il en est une qui rassemble plusieurs membres de la Ligue et qui, selon Coquoz (2000), constitue l'un des présupposés que 
l'on rencontre dans les doctrines éducatives de l'École genevoise: il s'agit de la loi selon laquelle l'ontogenèse refait la phylogenèse ou en d'autres termes, «une loi qui présuppose que le développement de l'enfant correspond à celui du progrès de l'humanité» (p. 377). Cette idée est empruntée à la «loi biogénétique fondamentale» du philosophe allemand Ernst Heinrich Haeckel (Coquoz, 2000; Hameline, 2002a) qui suppose l'existence d'une concordance entre la croissance naturelle et spontanée de l'être humain et le perfectionnement de l'espèce. Cette loi, et plus généralement le principe des «correspondances», est aussi défendue par les théosophes, nombreux parmi les membres de la Ligue. Annie Besant, présidente de la Theosophical Society dès 1907, affirme par exemple que «la même série d'événements se répète inlassablement dans des cycles plus vastes ou plus réduits». Selon, Chappuis (2005), toute l'œuvre de cette théosophe est centrée sur la compréhension des lois qui régissent l'infiniment petit et l'infiniment grand. Ainsi, saisir les lois dans le petit cycle permettrait de saisir celles du grand cycle, et à l'inverse, «saisir ces même lois dans le grand cycle et le petit deviendra à son tour intelligible» (p. 8). De manière générale, les théosophes croient à la «loi des correspondances»:

Si vous comprenez un cycle donné, vous les comprenez tous. La constitution d'un système solaire et celle du système humain sont soumises aux mêmes lois. [...] Si vous les comprenez dans l'un, vous pourrez également les comprendre dans l'autre. [...] qu'il s'agisse du développement de la conscience dans l'univers, dans la race humaine ou dans l'individu, les lois à étudier sont les lois générales. (Besant, 1908 cité par Chappuis, 2005, p. 8)

Selon les auteurs de $P E N$, la science ne connaît pas de frontières puisqu'elle s'attache à montrer le fonctionnement de l'enfant, sans distinction de race ou de sexe, et à établir des lois de validité universelle. C'est pourquoi elle apparaît comme le moyen de rassembler les hommes et les nations d'horizons divers autour d'une même cause: améliorer le sort de l'enfance.

La Science est une, elle ne connaît pas de limites territoriales; ce qui est vrai, certainement vrai, l'est partout. Or, la psychologie de l'enfance, branche de la science et l'éducation telle que nous la concevons, n'est autre que l'application pratique à l'enfance de cette branche de la science. (Éditorial, PEN, 1926, 20, p. 47) 
Deuxièmement, la science est considérée comme un instrument de libération et d'émancipation de l'homme, science et liberté apparaissant comme deux notions interdépendantes. Pour Langevin, «la science a une valeur collective, elle représente une vérité sur laquelle tous pensent et doivent être d'accord, un instrument de libération matérielle, intellectuelle et morale» (Langevin cité dans l'éditorial, PEN, 1928, 34, p. 2). La majorité des auteurs est convaincue qu'une éducation qui respecte les phases de développement de l'enfant lui octroie plus de liberté car celuici n'est plus tenu «d'ingérer» des savoirs préétablis. Pour eux, l'enfant doit pouvoir construire ses connaissances en fonction de ses besoins et en harmonie avec ses aptitudes personnelles. Dès lors, une pédagogie par la science est synonyme de liberté, liberté de penser, d'inventer, de créer. Elle concorde avec la méthode empirique (dite inductive) largement privilégiée par les auteurs de la revue: une démarche fondée sur l'observation, l'analyse, puis l'établissement de lois qui correspond à l'approche que plébiscitent les scientifiques. Toutefois, nous verrons plus loin que cette démarche n'est pas sans susciter craintes et débats parmi ceux qui tiennent à une conception «métaphysique» de l'éducation affirmant «l'existence d'une sorte de causalité en soi, ou pour mieux dire, d'un dynamisme sous-jacent aux phénomènes comparés et qui conditionne les relations constatées» (Ferrière, PEN, 1934, 98, p. 134).

Troisièmement, la science est perçue comme l'argument principal qui justifie le changement. Selon les auteurs, les résultats d'investigations scientifiques prouvent en eux-mêmes la nécessité d'une rupture avec le passé - avec l'école dite traditionnelle - et justifient les nouvelles orientations à prendre pour faire advenir une éducation nouvelle. En d'autres termes, les connaissances scientifiques des phénomènes éducatifs constituent les fondements d'une approche renouvelée de l'éducation à même de fonder cette nouvelle ère. Or, de quelle(s) science(s) proviennent ces investigations? À quelles théories les auteurs de PEN font-ils référence pour légitimer leurs revendications de réforme?

\section{LES SCIENCES NATURELLES}

La lecture de la revue fait apparaître l'importance de théories issues de la biologie et des sciences naturelles, constituant des références fondamentales du mouvement. Parmi elles, les théories de l'évolution, énoncées par Darwin au milieu du 19e siècle, influencent non seulement les discours mais façonnent toute la pensée de cette époque. Bien que ces 
théories ne soient pas explicitement énoncées dans les articles, elles transparaissent en filigrane dans les discours tenus sur les conceptions de l'enfant, de l'éducation et de la société. Ainsi, la notion des aptitudes naturelles de l'enfant auxquelles l'enseignement devrait s'adapter au plus près, fait référence à une idéologie imprégnée des théories du développement. Selon ces théories, tout être humain reçoit un patrimoine génétique à sa naissance (dons) qui détermine ses aptitudes et ses potentiels de développement. En d'autres termes, la nature attribuerait à chaque individu des aptitudes différentes qui se manifestent ensuite à travers l'attribution de rôles et de places dans la société. En revendiquant le respect du rythme et des phases de développement de l'enfant, les éducateurs nouveaux cautionnent l'existence d'un ordre social préexistant façonné par les différences interindividuelles, ordre contre lequel il n'y a pas lieu de lutter, selon certains auteurs.

L'important est que nous sachions nettement quels progrès la nature nous permet d'opérer, et que nous ne nous obstinions pas à progresser dans la même mesure que ceux qui ont plus de talent et de facilité que nous. Il ne faut pas oublier que l'inégalité entre les talents constitue aussi un équilibre. S'il n'existait que des personnalités supérieures aptes à accomplir les besognes pédagogiques les plus compliquées, il n'y aurait plus personne pour accomplir les besognes toutes simples. (Dalcroze, PEN, 1922, 3, p. 52)

Pour Ferrière, l'être humain contient un potentiel de vie, il est doté d'un élan vital qui serait le moteur même du développement de la société et un maillon essentiel de son expansion. Cette conception de l'homme et de la vie s'inscrit dans le courant plus vaste du vitalisme qui émane de la philosophie de Bergson, philosophe auquel Ferrière se réfère à plusieurs reprises. Selon Hameline (1993b), ce qui préoccupe Ferrière c'est de «situer le phénomène humain dans le contexte où il prend sa signification, et ce contexte est reconstitué à la fois par l'apport des sciences biologiques et celui des sciences sociales, sur fond d'évolutionnisme» (p. 388).

La psychologie génétique considère la vie comme un mouvement, un déroulement, une évolution ou, pour mieux dire, une croissance, un épanouissement où ce qui est virtuel a d'abord été à l'état potentiel et prépare à son tour une manifestation de l'avenir, un nouveau déploiement des énergies. À la base de toutes ces manifestations, comme moteur central, se trouve ce qu'Henri Bergson a nommé «l'élan vital»; à la base de toute manifestation 
d'ordre spirituel, ce qu'on a proposé d'appeler: l'élan vital spirituel. (Ferrière, PEN, 1931, 69, p. 150)

À cela s'ajoute, chez Ferrière, une conception méritocratique de la société mais qui n'est pas partagée par tous les membres de la Ligue. Dès la fin des années 1920, elle suscite des prises de positions contrastées notamment parmi les ressortissants français et américains qui défendent une conception plus rationaliste et pragmatique de la société.

NATURE, NATURALISME ET ROMANTISME

La nature est très présente dans les discours de $P E N$, écrite souvent avec un grand «N» révélant le véritable culte que certains lui vouent. C'est que la Nature constitue l'un des concepts-clés de l'Éducation nouvelle sur lequel repose toute la logique éducative. D'une part, elle est visible dans la célèbre «métaphore jardinière» qu'utilisent Claparède, Ferrière et d'autres, comparant l'enfant à une plante que l'éducateur a pour tâche de faire pousser en la nourrissant mais sans la dresser. D'autre part, la psychologie génétique postule que «l'être individuel est relié par des liens biogénétiques à l'évolution de la Nature» raison pour laquelle il est indispensable de «faire reposer tout développement individuel, donc toute éducation, sur le respect de ces lois de la Vie» (Helmchen, 1995 p. 91). C'est sur ce postulat que repose la conception du développement de l'enfant, un développement dont Piaget et Wallon, entre autres, définiront les étapes (stades). Ce postulat se traduit en éducation par le respect du rythme et intérêts de l'enfant ainsi que l'adaptation des savoirs scolaires à son développement. De fait, la Nature est intimement liée à la notion d'évolution - issue de la biologie et des théories sur l'évolution des espèces - car, pour les partisans de l'Éducation nouvelle, la Nature représente à la fois l'origine de l'être humain et l'avenir vers lequel celui-ci doit tendre.

C'est pourquoi la Nature est adulée, non seulement en raison de son rôle «biologique» comme nous venons de le voir dans l'évolution des êtres vivants, mais aussi en raison des pouvoirs qu'on lui attribue. Les auteurs en parlent comme une «source de vitalité», un «lieu de régénérescence», comme un «élément clé» pour la santé du corps et de l'esprit. Il faut dire que le contexte de l'époque et les conditions dans lesquelles les enfants sont scolarisés suscite l'inquiétude de nombreuses personnes: 
Dans presque toutes les classes que j'ai visitées à Vienne et dans les environs [...] l'air était mauvais. Fenêtres presque toujours closes, même par le beau temps. [Dans une école] il y avait jusqu'à sept becs de gaz dans une classe. Les élèves étaient aussi endormis et avaient la tête aussi lourde que moimême après deux heures de séjour dans ce mauvais air. (Walser, PEN, 1928, 42, pp. 197-198)

Le «culte» de la nature dont il est question dans de nombreux articles est une forme de réaction à un environnement scolaire jugé malsain voire dangereux pour l'enfant. Rappelons ici que la mise en place de systèmes éducatifs d'État dans le dernier tiers du $19^{\mathrm{e}}$ siècle dans les pays occidentaux entraîne une massification de l'éducation qui se traduit par une urbanisation scolaire importante dont la logique spatiale et organisationnelle s'inspire de celle des industries. Enfin, cet «hymne» à la nature s'inscrit dans un courant de pensée hygiéniste qui se propage dès le tournant du siècle en lien avec les découvertes de la médecine sur le rôle des microbes dans la propagation des épidémies. Dès lors, on attribue à la Nature et au soleil en particulier, le pouvoir d'éloigner les maladies et de fortifier la santé des enfants:

L'héliothérapie a fait des merveilles curatives. L'insolation de l'enfant fait chaque jour des merveilles préventives. La tuberculose et le déséquilibre nerveux dû à une intoxication de l'organisme cèdent devant les mystérieux rayons ultra-violets. [...] Le soleil est le plus grand des remèdes, le plus répandu, et le meilleur marché. Mieux que cela: il rend tout remède inutile si l'on sait faire appel à ses radiations dispensatrices de santé. (Ferrière, 1928, 39, pp. 128-130)

Dans les articles évoquant des écoles nouvelles, les auteurs insistent sur l'emplacement de ces établissements qui, situées à la campagne, favorisent le contact direct des enfants avec la nature, que ce soit pour observer celle-ci (sciences naturelles, botanique) ou pour y travailler (jardinage, travaux des champs). La nature est considérée comme le milieu naturel de l'enfant où il peut à la fois se livrer aux «ébats primitifs» mais aussi y trouver «le calme dont son système nerveux a besoin» (Ferrière, PEN, 1925, 15, p. 4). D'autre part, elle est très présente dans les textes relatant les visites de ces écoles, à travers le vocabulaire utilisé par l'auteur dont on perçoit l'intention évidente de souligner ce qui les distingue des écoles traditionnelles: 
Plus de vilains murs épais qui dérobent à leurs yeux la verdure, les plantes, les oiseaux, les jeux charmants de l'ombre et du soleil. Par le beau temps, l'air doux et léger caresse leurs muscles pleins de vie et ils voisinent avec le moineau, l'abeille et la coccinelle. Quel est le plus grand bienfait que permettre à l'enfant d'éclore librement au sein de la nature? (Decroly \& Buyse, PEN, 1922, 4, p. 71)

Dans Pour l'Ère Nouvelle, plusieurs articles sont dédiés aux écoles de Plein Air ${ }^{1}$ écrits par leurs directeurs-trices. Destinées d'abord aux enfants tuberculeux ou chétifs, celles-ci proposent les méthodes nouvelles dans un esprit particulier où la nature, le soleil, la lumière sont utilisés à des fins thérapeutiques. Dans une perspective à la fois curative et préventive, ces écoles sont construites en collaboration avec des architectes et des médecins de telle sorte que les élèves profitent en toute saison du plein air et du soleil. Comme le montre Luc (2003), ces écoles s'inscrivent dans «l'essor d'une politique de prévention sociale contre la tuberculose» et de manière plus générale, dans une démarche prophylactique «qui inspire notamment des initiatives contre l'insalubrité des logements populaires à partir du milieux des années 1920» (p. 7). Jusque-là, le traitement des enfants tuberculeux consistait en deux phases: le placement des malades en sanatorium et l'envoi temporaire des sujets menacés en colonie de vacances pendant l'été. D'après cet historien, les écoles de Plein Air sont plus ambitieuses: «outre des soins fondés sur la climatothérapie et l'exercice physique, ces écoles offrent un enseignement rénové qui s'avère être le plus fécond dans cet environnement» (Luc, 2003, p. 8). Les articles sur ces écoles occupent une place importante dans la revue, notamment car Ferrière entretient des contacts étroits avec le directeur du Bureau international des Écoles de Plein Air, Jean Dupertuis, qui se fait le porte-parole de ces établissements en Suisse Romande:

Les méthodes de l'école active se sont révélé les seules bonnes à l'école de Plein Air et inversement; le plein air et le soleil rendent l'enfant plus équilibré, plus actif, doué de plus d'initiative, plus vraiment intelligent, toutes qualités qui sont requises pour le succès de l'école active. [...] Les résultats de la pratique solaire sont tellement nets, tellement probants, pour les grands

1 Sur ces écoles et leur étendue à une échelle internationale, voir l'ouvrage dirigé par Châtelet, Lerch et Luc (2003). Voir aussi Savoye (2003) et plus récemment, Châtelet (2011). 
enfants comme pour les petits, que la méthode fait sa propagande elle-même dès qu'elle entre dans un milieu. (Ferrière, $P E N, 1928,39$, p. 128)

\section{PHILOSOPHIE, SCIENCES SOCIALES ET SPIRITUELLES}

Outre les sciences médicales et naturelles, la philosophie et les sciences sociales constituent des références importantes pour les auteurs de PEN. Toutefois, ces derniers s'y réfèrent de manière plus implicite et leurs appartenances culturelles jouent ici un rôle important dans le choix de ces références. Des théories telles que le rationalisme ou le taylorisme sont particulièrement présentes dans les textes écrits par des Américains et par des Français. Les Américains expriment le souci constant de rendement de l'éducation et d'efficacité sociale où le taylorisme apparaît comme une des solutions possibles. Ce souci se traduit par la mise au point de méthodes pédagogiques adaptées, à la fois fidèles aux principes de l'Éducation nouvelle (respect du rythme, besoins et intérêts de l'enfant) mais organisées de manière à garantir une efficacité contrôlable de l'apprentissage. Le Dalton Plan qui propose des paliers de progression composés d'objectifs d'apprentissage facilement repérables et évaluables en est un exemple.

En Europe (surtout en France), les mêmes préoccupations se traduisent à travers la naissance, dès les années 1930, d'une nouvelle science: la biotypologie, appelée aussi par ses inventeurs «science des types humains» (Laugier, PEN, 1935, 109). Elle constitue une sorte de combinaison des recherches menées par la pédagogie expérimentale sur les niveaux d'élèves dans les classes et les recherches menées en psychologie sur les typologies d'individus. Cette science fait l'objet de plusieurs articles dans $P E N$ où elle apparaît comme une solution aux problèmes d'efficacité et de rendement, favorisant une orientation professionnelle rationnelle qui respecte au plus près les caractéristiques individuelles de l'enfant.

Les données biotypologiques recueillies renseigneront sur l'état de maturité psycho-physiologique de l'enfant. Elles permettront autant qu'il est possible, de constituer des classes homogènes de niveau mental et d'âge physique, de préciser la précocité ou le retard des développements, de donner des conseils $\mathrm{d}$ 'hygiène physique convenables et de constituer, pour certains cas particulièrement anormaux, des climats pédagogiques adaptés (écoles spéciales). (Laugier, Toulouse \& Weinberg, PEN, 1933, 92, p. 251) 
En Allemagne et dans d'autres régions germanophones, les théories telles que le romantisme et le naturalisme constituent des références importantes. De fait, celles-ci sont présentes dans la philosophie européenne déjà au $19^{\mathrm{e}}$ siècle et imprègnent la philosophie de l'Éducation nouvelle dès ses débuts. On le perçoit à travers les écrits d'auteurs qui ont séjourné en Allemagne une partie de leur carrière, notamment Ferrière qui a vécu plusieurs mois dans les Écoles nouvelles de Hermann Lietz à Haubinda et Ilsenburg.

Contrairement aux précédentes, la théorie du «positivisme» semble être une référence unanimement partagée par tous les membres de la Ligue quel que soit leur pays d'appartenance. De fait, elle constitue un des piliers du socle théorique de l'Éducation nouvelle puisqu'elle fonde sa démarche, sa posture, son éthique à l'égard de l'apprentissage. En effet, les auteurs se rassemblent autour d'une critique de l'éducation traditionnelle qui affirme que l'apprentissage est le fruit du travail de la raison, de l'intellect. Ils défendent, à l'inverse, une posture positiviste, «expérimentaliste», où l'apprentissage est considéré comme le fruit de l'expérience individuelle vécue à travers les sens et les émotions.

Comme nous l'avons vu, d'autres théories de référence telles que la religion et la spiritualité sont régulièrement évoquées dans les textes de la revue. L'éducation y apparaît comme le moyen suprême de faire triompher l'esprit sur les pulsions matérielles dans l'espoir de former un être naturellement discipliné.

On peut affirmer que ce congrès fut un succès pour les idées fondamentales que notre Ligue travaille à répandre: faire triompher la suprématie de l'esprit sur les mobiles égoïstes, mercantilistes et matérialistes; conserver et accroître chez l'enfant l'énergie spirituelle; l'amener à une discipline individuelle et collective autonome qui le conduise à la libération des puissances spirituelles qui sont en lui. (éditorial, PEN, 1922, 4, p. 69)

Les conceptions religieuses, le plus souvent discrètes apparaissent toutefois dans certains textes de type philosophique où les valeurs du christianisme sont présentées comme un fondement de la pensée occidentale. Ces valeurs sont à prendre en compte dans le contexte des revendications de l'Éducation nouvelle sachant que plusieurs de ses promoteurs sont issus de familles protestantes où l'individu et son éducation constituent des valeurs clés. Mais selon les auteurs, ces valeurs sont mises en péril par l'industrialisation croissante de la société et perdent leur fonction de repère auprès des nouvelles générations: 
Pendant des siècles, la Bible fut le ciment de la civilisation occidentale. Elle ne l'est plus aujourd'hui. [...] La Bible donnait à l'homme une cosmogonie. Elle lui fournissait une explication du monde où il se trouve et de la place qu'il y occupe, puis elle racontait l'histoire générale de l'humanité. [...] Elle le rattachait à toute l'humanité en lui donnant le sens des relations et des devoirs. Elle lui faisait une place dans le monde, elle donnait un sens à sa vie. (M.B., PEN 1926, 22, pp. 107-108)

La philosophie issue de la théosophie qui imprègne la revue durant les premières années est considérée par certains comme une pseudoreligion (Guénon, 1996), pour d'autres comme une «science spirituelle» (Washington, 1999). La notion de science y est bien présente comme en attestent les objectifs de la Société Théosophique, fondée en 1889 par Helena P. Blavatsky et H. Olcott: encourager l'étude des religions, de la philosophie et des sciences; rechercher les lois de la nature et les pouvoirs de l'homme; créer les liens d'une fraternité universelle de l'humanité, sans distinction de race, de sexe, et de religion. Les théosophes sont à la recherche d'une complémentarité entre la raison et l'esprit: une synthèse qui allierait diverses religions, philosophies et sciences à partir d'un dialogue avec la culture orientale. Dès lors, l'éducation théosophiste se caractérise par la synthèse de principes d'orientations diverses. Elle consiste avant tout à «réveiller en nous les facultés de l'Esprit, à nous rendre capable de sentir les grands idéaux, de les vivre dans notre esprit et de les réaliser par nos actes» (Trevino, 1920 cité par Soler Mata, 2011, p. 2). Selon Chappuis (2005), la théosophie se caractérise par trois éléments principaux: «la divinité enfouie en chaque être humain, le fait que l'humanité se trouve sur le chemin de l'évolution, et la nécessité pour les êtres de développer la parcelle divine enfouie en eux par un travail de quelque ordre qu'il soit» (p. 11). Pour découvrir cette "parcelle divine», les théosophes préconisent une méthode par l'expérience personnelle qui, selon eux, est la seule base du vrai savoir. Une expérience qui ne peut toutefois être le seul fait de l'expérience individuelle, mais qui doit être guidée par un maître aidant la personne à trouver la «Voie», à se mettre en route vers la connaissance. Dès lors, les théosophes préconisent une éducation qui respecte l'individualité de l'enfant et qui fasse émerger la force «divine» cachée en chacun d'eux. Ils sont donc favorables aux méthodes d'Éducation nouvelle où le mode d'apprentissage par l'expérience est valorisé et où le maître est un guide plutôt qu'un dispensateur de savoirs.

Ces théories tendent à disparaître dans les années 1930 laissant la place à un discours davantage inspiré de la sociologie. Il s'agit plutôt de 
la «dimension sociale de l'éducation» considérée par beaucoup comme un aspect désormais incontournable des méthodes nouvelles. Cette préoccupation apparaît alors si cruciale qu'elle constituera le thème du Congrès de 1932 organisé par le Groupe Français d'Éducation nouvelle à Nice. Ces questions y seront vivement débattues suscitant des prises de position très diverses aboutissant à des dissensions voire des défections de certains membres de la Ligue (Gutierrez, 2010).

\section{LA PSYCHOLOGIE ET SES DÉRIVÉS}

Bien que les théories énoncées plus haut constituent des références évidentes pour les auteurs de la revue, la psychologie, et toutes les sciences qui en découlent (psychologie de l'enfant, psychologie expérimentale, tests psychologiques), constituent la science de référence dominante, fonctionnant comme une sorte de langage commun à tous les membres de la Ligue. Dans les textes, la psychologie est utilisée pour démontrer la scientificité des méthodes nouvelles, pour démontrer leur validité et la pertinence. Dans les années 1920, la psychologie à laquelle se réfèrent les auteurs est avant tout une psychologie génétique, focalisée sur l'individu, qui vise à montrer les lois biogénétiques de son développement et qui tente de catégoriser les différents types d'individus. La psychologie constituerait d'ailleurs, selon eux, la base scientifique fondamentale qui distingue les écoles nouvelles des écoles traditionnelles, caractéristique que Ferrière place au premier rang des 30 conditions pour obtenir le «label»d'école nouvelle (texte des «Trente Points»²).

L'école nouvelle est un laboratoire de pédagogie pratique. A) Pionnier des écoles d'État, elle prépare le terrain en prouvant l'efficacité des méthodes nouvelles. B) Elle s'appuie sur les données de la psychologie de l'enfant et sur les besoins de son corps et de son esprit. (Ferrière, Pen, 1925, 15, p. 4)

De fait, toute théorie psychologique publiée dans la revue est aussi abordée sous l'angle de ses effets sur l'éducation, des possibles utilisations

2 Texte intitulé «L'École nouvelle et le Bureau international des Écoles nouvelles», publié pour la première fois en 1919, dans lequel Ferrière énumère trente points caractéristiques des Écoles nouvelles. Ce texte fait office de référence pour attribuer le «label» d'École nouvelle aux divers établissements qui voient le jour dès le tournant du $20^{\mathrm{e}}$ siècle. Il est publié dans Pour l'Ère Nouvelle en avril 1925 (Ferrière, Pen, 1925, 15, pp. 2-9). 
de cette connaissance dans le domaine de l'enseignement attestant de l'importance de la psychologie appliquée.

Nous basons notre enseignement sur quelques principes qui tous traduisent notre désir d'appliquer ce que nous connaissons de la psychologie de l'enfant et d'éviter, autant que faire se peut, de faire violence aux jeunes mentalités. (Deschamps-Alexander, Pen, 1928, 39, p. 125)

La dominance de la psychologie se perçoit à plusieurs niveaux. D'une part, elle apparaît à travers la quantité d'articles écrits par des scientifiques (médecins, psychologues, pédologues) apportant de nouvelles connaissances dans le domaine. Ces contributions, parfois conséquentes (publiées sur deux numéros), suscitent souvent des réactions d'autres chercheurs, plaçant le lecteur face à une sorte de dialogue entre «savants» qui, par l'intermédiaire de leurs écrits, discutent leurs données, se questionnent ou se répondent. D'autre part, la dominance de la psychologie se voit dans la place qu'occupent les interventions de psychologues dans les congrès. Dans les rapports, celles-ci sont souvent regroupées dans un chapitre à part, sous divers intitulés tels que Point de vue des psychologues (Montreux, 1923), Le groupe des psychologues (Heidelberg, 1925), ou Travaux de la commission de psychologie (Cheltenham, 1936). Enfin, cette prégnance se perçoit au niveau de l'organisation même de la Ligue qui, suite au Congrès d'Elseneur, décide de nommer sept commissions permanentes pour alléger la tâche des membres du Comité central. Parmi elles, la commission de psychologie est d'emblée subdivisée en quatre sous-sections: «psychologie génétique, tests mentaux, psychologie individuelle et types psychologiques, psychologie de l'inconscient» (Éditorial, PEN, 1930, 54, p. 1).

Mais cette posture épistémologique ne remporte pas l'adhésion de tous les membres voire en dérange certains, en particulier les partisans d'une éducation populaire, adaptée aux exigences de la vie sociale et économique. Ces derniers reprochent à la Ligue de privilégier une approche individualiste, teintée d'élitisme au détriment de l'intérêt collectif et d'une vision plus dynamique de l'équilibre social.

Croire que l'éducation existe pour l'individu est une erreur moderne. L'éducation existe pour le bien de la collectivité et de la race; elle existe pour subjuguer l'individu dans l'intérêt du monde et, en fin de compte, dans celui de son propre bonheur. [...] Tout le monde demande des réformes. Mais les réformateurs de l'éducation semblent considérer celle-ci toujours du point de 
vue de l'entreprise scolaire privée et de l'élève individuel, presque jamais du point de vue de l'entreprise publique s'occupant de la collectivité tout entière. De la littérature pédagogique, on ne peut donc rien tirer de pratiquement utile. (M.B., PEN, 1926, 22, pp. 107-108)

Certains prennent d'ailleurs la liberté d'adapter les principes de l'Éducation nouvelle de manière à les rendre plus cohérents avec leurs conceptions. Ainsi, certains pays d'Europe de l'Est (Russie, Pologne) adoptent une posture plus sociale (celle que la Ligue prônera dès les années 1930), par exemple en utilisant la méthode des centres d'intérêts non pas pour favoriser l'épanouissement individuel de l'enfant, mais pour servir les intérêts de la communauté.

Alors que l'enseignement complexe [méthode des centres d'intérêt] dans les autres pays provenait de considérations proprement pédologiques concernant l'appropriation de l'enseignement aux forces et aux intérêts de l'enfant, en Russie, les considérations sociologiques ont occupé la première place. [...] Ainsi, la tendance de l'école soviétique fondant son travail sur le principe complexe est dirigée non pas vers le développement spécial des activités enfantines, mais vers le travail de l'école socialement utile, [...] et vers la participation effective de l'école à l'édification de la nouvelle vie socialiste. (Ivanoff, PEN, 1928, 40, p. 177)

À l'issue de cette analyse, nous pouvons affirmer que l'Éducation nouvelle repose sur un socle théorique solide composé de diverses théories se combinant et se complétant. Ce socle constitue les racines de convictions profondes sur la valeur de l'homme et de l'enfant qui perdurent à travers toute la période étudiée malgré les courants contraires qui ébranlent l'unité de la Ligue au cours des années 1930. De fait, certaines bases théoriques restent communes à tous (droits de l'enfant, respect de l'individualité, critique de la discipline autoritaire) constituant une sorte de langage commun qui contribue à la construction d'une nouvelle science, la science de l'éducation. Ce constat rejoint celui d'Hofstetter et Schneuwly $(2002,2007)$ qui décrivent la dimension plurielle du champ disciplinaire des sciences de l'éducation et le comparent à une mosaïque où les sciences contributives constituent des composantes essentielles du champ. Comme le montre Depaepe (1998), la science de l'éducation qui se construit au début du $20^{\mathrm{e}}$ siècle porte l'héritage de traditions culturelles et épistémologiques européennes telles que le positivisme, le darwinisme, l'intérêt pour la recherche expérimentale et les réformes 
éducatives qui façonnent une certaine conception de l'enfant et de l'éducation. À celles-ci s'ajoutent des théories plus spécifiques à certaines régions, par exemple le romantisme allemand ou le pragmatisme anglosaxon. Selon cet auteur, la pédologie tente de se distancer de ces influences et cherche à construire une connaissance de l'enfant de manière progressive et systématique à partir d'une «tabula rasa» (p. 38) grâce à une démarche inductive inspirée des sciences naturelles. L'analyse des discours tenus dans PEN montre que ces théories de référence sont bien présentes et que cette science qui se construit est faite de multiples théories, références, approches qui malgré leurs différences, ont le mérite d'être toutes tolérées au sein de la Ligue attestant que celles-ci peuvent cohabiter au sein d'une même communauté, voire d'un même individu.

\section{SAVOIRS SCIENTIFIQUES ET SAVOIRS D'EXPÉRIENCE: UNE LÉGITIMITÉ DISCUTÉE}

Ce volet vise à comprendre comment se mêlent la science et la militance à l'échelle des savoirs produits par les acteurs de l'Éducation nouvelle. Il cherche à déceler quels sont les types de savoirs diffusés dans Pour l'Ère Nouvelle et plus particulièrement quelle place y occupent les savoirs scientifiques (théoriques) par rapport à d'autres types de savoirs, tels que les savoirs d'expérience (pratiques). Il s'agit de voir quelle est la proportion de ces savoirs sur l'ensemble de la période étudiée, dans quels types d'articles ceux-ci apparaissent, quelle est leur forme, par qui ils sont utilisés et à quelles fins. En outre, il s'agit d'identifier les domaines dans lequels ces savoirs sont le plus présents afin de voir si ceux-ci sont propres à un domaine ou s'ils sont souples et perméables. En prolongement des travaux d'ERHISE (Hofstetter \& Schneuwly, 2007; 2009a), cette analyse vise à mieux comprendre comment se construit la nouvelle science de l'éducation en s'intéressant à la manière dont les savoirs se combinent, se confrontent et se discutent au sein d'une revue où s'expriment praticiens et scientifiques.

Dans les articles de Pour l'Ère Nouvelle, deux grands types de savoirs se donnent à voir à travers les discours: le premier que nous appellerons savoirs d'expérience et le second, savoirs scientifiques (ou théoriques). Par savoirs scientifiques nous entendons les discours dont le but principal est d'apporter des connaissances (sur l'enfant, l'éducation, la psychologie); il 
s'agit de savoirs formalisés, émanant des milieux de la recherche et transmis par des scientifiques. Nous appelons savoirs d'expérience tous les discours dont le but principal est de transmettre des savoirs professionnels acquis à travers une pratique éducative quelle qu'en soit la durée, le lieu et l'échelle; il s'agit donc de savoirs transmis par des praticiens. L'analyse quantitative de Pour l'Ère Nouvelle permet de faire un constat général sur l'ensemble de la période: les articles de genre connaissance sont largement minoritaires par rapport aux articles de genre intermédiaire ou praxéologique ${ }^{3}$ dans lesquels les savoirs d'expérience dominent. ${ }^{4}$ Pour pouvoir affiner cette observation, nous avons croisé l'analyse par genre d'article avec une analyse par domaine afin de voir dans quels domaines les savoirs scientifiques les savoirs d'expérience étaient les plus présents. Il ressort que ces savoirs sont fortement influencés par les ancrages disciplinaires de ceux qui les produisent, les sphères professionnelle et disciplinaire se démarquant assez clairement l'une de l'autre.

\section{SAVOIRS D'EXPÉRIENCE FORMALISÉS}

Dans la revue, les savoirs d'expérience sont présents dans les articles de genre intermédiaire, textes qui comportent à la fois une dimension cognitive et une dimension praxéologique. On les trouve également dans les articles des domaines documentation pédagogique et méthode d'enseignement. Dans la grande majorité, il s'agit de textes de type narratif et descriptif présentant une expérience ou une méthode effectuée sur le terrain

3 Ces trois catégories donnent une indication sur le genre d'article: le genre connaissance a été attribué aux articles dont le but principal est de transmettre des connaissances, autrement dit qui se caractérise par ses apports cognitifs (certains articles contiennent aussi une dimension praxéologique mais celle-ci est mineure). Le genre praxéologique, «visant à mobiliser des savoirs d'action» (Lessard \& Bourdoncle, 2002), a été attribué aux articles dont le but est de mobiliser le lecteur, de transformer ses pratiques et/ou ses manières de penser (à l'aide de conseils pratiques). Le genre intermédiaire a été attribué aux articles comportant un mélange d'apports cognitifs et de conseils pratiques dont le contenu apporte des connaissances destinées à transformer les pratiques et les manières de penser l'enseignement.

4 Sur 946 articles, nous recensons 189 articles de genre connaissance, 174 de genre praxéologique, 393 de genre intermédiaire et 129 «autre» (non catégorisable). 
(une classe, une école, un jardin d'enfants). Ils comportent beaucoup $d^{\prime}$ informations sur la manière dont les protagonistes $s^{\prime} y$ sont pris pour mener à bien leur expérience: le contexte de l'expérience (nombre d'enfants, âge, niveau scolaire, environnement), les moyens mis en œuvre (matériel, organisation des activités, durée) et les résultats (conséquences, effets sur les élèves sur le plan cognitif et comportemental). Ces informations sont transmises à l'aide d'un vocabulaire très détaillé comme si le but était de permettre au lecteur de reproduire dans le menu détail l'expérience présentée par l'auteur. Le vocabulaire est celui de professionnels - instituteurs, éducateurs, professeurs - prouvant qu'il $\mathrm{s}^{\prime}$ agit de textes avant tout destinés à des pairs. Le but de ces articles est double: d'une part, ils servent à prouver aux praticiens, en particulier à ceux qui ne sont pas encore convaincus, qu'il n'est pas si difficile d'appliquer les méthodes nouvelles (forte dimension praxéologique); d'autre part, ils servent à montrer aux scientifiques que leurs théories sont applicables et qu'elles portent leurs fruits.

Nous percevons dans la rhétorique de ces articles une volonté de convaincre, un désir de lutter contre les préjugés, de démystifier tout ce qui se dit sur l'Éducation nouvelle. Pour ce faire, les auteurs présentent leur expérience de manière très détaillée, prouvant que l'application des méthodes nouvelles est à la portée de tous. Dans certains textes, l'auteur utilise une manière quasi cinématographique de raconter son expérience, comme si une caméra filmait la scène. Le but est à nouveau de prouver la réalité des faits avec un souci particulier de réalisme, l'idée étant de «donner à voir», comme si l'image virtuelle ajoutait à la véracité des faits. Ces textes donnent des détails très précis sur l'environnement de l'établissement, l'ambiance qui y règne, les sons, les odeurs, la lumière, dans un style quasi poétique. Ces détails participent de la dimension praxéologique du texte qui vise à créer l'adhésion du lecteur.

Suivez-moi à St-Christophe et vous aurez un autre spectacle. C'est le matin; le temps est clair et beau. En approchant de Letchworth, qu'apercevez-vous? Une rangée de moineaux? Eh non, ce sont des enfants qui travaillent làhaut...et seuls! Pour visiter l'établissement je vous confie à une grande fille, car les garçons sont tous occupés ce matin à l'atelier. Voici un élève absorbé par la solution d'un problème de mathématiques... Voyez ce petit garçon: travaille-t-il vraiment tout seul celui-là? [...] Vous le questionnez... Vous vous informez alors de son âge... (Baldwin, PEN, 1922, 1, pp. 21-23) 
Nous avons identifié trois types de récits d'expérience correspondant à un mouvement allant du particulier au général: 1) des récits d'expérience «directs» (l'auteur est la personne qui a vécu et mené l'expérience), 2) des récits d'expérience «indirects» (l'auteur est une personne qui a coordonné ou mis sur pied l'expérience, par exemple un inspecteur ou directeur d'école) et 3) des récits de visite (l'auteur est une personne extérieure qui visite une école/classe). Les savoirs expérientiels qui apparaissent dans ces trois types de récits se distinguent par le degré de prise de distance par rapport à l'expérience relatée. Les récits du premier type (1) font apparaître des savoirs d'expérience très proches de la réalité, peu analytiques, ciblés sur le déroulement d'une activité, donnant des informations sur ses étapes, le matériel nécessaire, etc. Les récits d'expérience «indirects» (2) donnent à voir des savoirs davantage distants de l'expérience menée, avec des informations sur le contexte, l'environnement, le nombre d'élèves et les conditions de réussite de l'expérience. Dans le troisième type de récit (3), il s'agit de compte rendu de visites d'école où l'auteur a une position encore plus distante puisqu'il écrit en tant qu'observateur d'une expérience menée par d'autres personnes, dans un contexte le plus souvent étranger. Ces textes contiennent davantage d'informations de type organisationnel et présentent des savoirs relayés par l'auteur qui sont mis en relation avec des expériences vécues par ce dernier.

Au niveau des auteurs de ces récits, nous observons que ceux-ci sont différents selon le type de texte. Ainsi, les auteurs des récits de type 1 sont majoritairement des instituteurs enseignant à l'école maternelle ou primaire (écoles privées et publiques), une minorité de récits concernant des expériences menées au secondaire. Les auteurs des récits de type 2 sont principalement des directeurs d'école ou des inspecteurs, en d'autres termes des anciens praticiens occupant des fonctions de contrôle dans leur établissement ou leur région. Enfin, les auteurs des récits de type 3 sont plus difficiles à catégoriser puisque nombreux sont ceux qui adoptent le mode du voyage et de la visite pour se former et découvrir d'autres méthodes d'enseigner; toutefois, parmi ceux qui écrivent dans $P E N$, nous remarquons la présence de plusieurs figures-clés de la Ligue et du mouvement, très habitués à ce genre de visites.

Les savoirs d'expérience sont également présents dans des articles de genre praxéologique bien que ceux-ci soient moins nombreux que la catégorie intermédiaire. Dans ces textes, les savoirs d'expérience sont utilisés à des fins de «propagande»: il s'agit de convaincre le lecteur du bien 
fondé de la méthode ou de l'expérience présentée, de démontrer son efficacité, son utilité, sa «faisabilité», en d'autres termes de faire la preuve que la mise en pratique de l'Éducation nouvelle est possible, fructueuse et avantageuse! De ce fait, ces textes se caractérisent par leur type injonctif visant non seulement à mettre en marche le lecteur dans le sens des idées prônées mais aussi à transformer sa pratique voire sa pensée et ses convictions. Ce style injonctif est particulièrement présent dans les articles de militance prônant les bienfaits de l'Éducation nouvelle tels que certains comptes-rendus de congrès et les manifestes.

\section{SAVOIRS SCIENTIFIQUES EN OBSERVATION}

Comme nous l'avons dit, les savoirs scientifiques dans PEN se situent essentiellement dans des articles de genre connaissance et sont écrits par des auteurs de formation universitaire menant des recherches dans divers domaines (psychologie de l'enfant, pédagogie expérimentale, physiologie, médecine, etc.). Ces articles se caractérisent par leur dominante cognitive dont le but est de faire part de résultats de recherche, d'élargir ainsi les connaissances de tous et de susciter la réflexion sur certains thèmes en débat. Mais si le fond est scientifique, la forme l'est beaucoup moins. En effet, le style d'écriture qui est utilisé dans ces articles s'apparente plus au récit d'expérience utilisé dans les articles de type intermédiaire ou praxéologique qu'au genre scientifique (thèse, hypothèses, résultats, discussion) comme ce serait le cas dans des revues de médecine ou de biologie. De fait, Pour l'Ėre Nouvelle contient une grande quantité d'articles faisant état de tests, d'enquêtes, de recherches expérimentales dont les résultats sont présentés parfois dans leur version préliminaire.

Ces articles se font l'écho d'un mouvement général de recherche, d'essais, d'ébauches et reflètent par là-même la dimension expérimentale de la science qui est en train de se construire. Parmi ces récits dits scientifiques, certains font état de recherches menées en laboratoire, sur des échantillons réduits d'enfants observés selon des protocoles bien précis. Les objectifs et les protocoles de ces recherches sont le plus souvent mentionnés dans l'article précisant dans le détail les conditions du déroulement de l'expérience. Par exemple, l'article indique quel type d'examinateur a été sollicité - universitaires formés, ayant contribué ou non à l'élaboration du test - ou s'il s'agit d'une expérience menée avec un élève et un examinateur. Or, la plupart de ces laboratoires sont en fait 
des écoles rattachées à des instituts de recherche, appelées alors école expérimentale ou école d'application, à l'exemple de la Maison des Petits et de l'École expérimentale du Mail rattachées à l'Institut J.-J. Rousseau, ou de la Laboratory School rattachée à l'Université de Chicago (Dewey). Certaines écoles nouvelles privées telles que l'École de l'Ermitage à Bruxelles font aussi office de terrain de recherche, comme en atteste l'intense activité scientifique menée par Decroly - puis par ses étudiants dans cette école.

La revue publie aussi des comptes-rendus de recherches effectuées hors du périmètre scolaire dont les conditions d'expérimentation sont bien explicitées, prouvant la scientificité de la démarche des chercheurs.

Pour ce qui est de nos recherches, en raison des différences des milieux de l'école autonome et de la classe de perfectionnement, nous avons étendu notre étude du milieu scolaire au milieu social et tenu compte des amitiés hors de l'école. [...] L'investigation portera sur cinq années et demie: d'octobre 1928 à avril 1934. [...] Notre enquête n'a porté que sur des garçons, on serait en droit de se demander comment se comportent les filles [...]. Cette question délicate pourrait donner lieu à des enquêtes objectives. Mais n'ayant pas les éléments de l'enquête, nous nous contentons de poser la question. (Prudhommeau, PEN, 1934, 101, pp. 263, 269)

Ces articles font part de résultats d'enquêtes ou recherches menées à large échelle (plusieurs centaines d'élèves) basées sur des expériences réalisées en série. Il s'agit de textes apportant beaucoup de connaissances, écrits dans un style scientifique, tirant leur scientificité de la systématicité avec laquelle ces expériences sont menées. Certaines enquêtes sont effectuées par des scientifiques, en leur nom, dans le but d'obtenir des résultats sur des grandes cohortes d'élèves, d'autres sont menées par des collectifs. Parmi ceux-ci figurent les groupes de travail de la Ligue, par exemple la commission des examens qui publie les résultats de son enquête ${ }^{5}$ dans PEN en 1933. Certaines études sont mandatées par des instances officielles (inspecteur de circonscription, ministère ou département de l'Instruction publique, Bureau International de l'Éducation) dans le but de pouvoir produire des recommandations ou induire des changements à large échelle dans les systèmes éducatifs. Dans ce cas-là, les résultats sont rédigés et publiés par les personnes res- 
ponsables de l'enquête ou qui s'en font le relais. Le plus souvent, il s'agit d'auteurs collectifs qui ne sont pas des scientifiques mais plutôt des membres de l'administration scolaire ou des représentants des instances mandatrices et qui signent au nom du collectif (par exemple: «publications du BIE»).

Les deux types d'enquêtes se distinguent par l'objectif qui les soustend. Dans le premier cas, il s'agit d'un objectif scientifique, visant à contribuer à étendre les connaissances du domaine dans lequel s'inscrit l'étude; dans le deuxième cas, l'objectif est plutôt politique, les conclusions de l'enquête étant utilisées pour modifier des programmes ou pour transmettre des recommandations aux écoles d'une région ou d'un pays. C'est ainsi que la Belgique instaure un nouveau plan d'études en 1937 basé sur les méthodes d'Ovide Decroly. ${ }^{6}$ Dans le cas d'enquêtes mandatées par le Bureau International de l'Éducation, les résultats visent à édicter des recommandations à une échelle internationale. ${ }^{7}$ Dans la revue, certains domaines tels que la formation des enseignants sont particulièrement sensibles et font l'objet de plusieurs enquêtes suivies de recommandations. ${ }^{8}$

\section{DES SAVOIRS «PERMÉABLES»}

L'Éducation nouvelle est avant tout fondée sur une science expérimentale qui formule ses théories sur la base d'expériences réalisées sur le «terrain». Dans PEN il s'agit aussi bien de pédagogie expérimentale que de psychologie expérimentale. Toutes les deux sont convoquées car elles contribuent chacune à leur manière à l'élaboration de la nouvelle science de l'éducation. La lecture de la revue permet d'assister à l'élaboration de cette science puisque ce support permet non seulement une centralisation des expériences mais aussi une «visibilisation» des découvertes. Les savoirs scientifiques présents dans PEN sont produits sur la base d'expériences conduites dans des classes qui font office de laboratoires. Toute recherche dite scientifique implique donc la collaboration d'un ou

6 Voir le numéro spécial sur le Plan d'études Belge dans Pour l'Ère Nouvelle: PEN, 1937, 127.

7 Le Bureau International de l'Éducation est aujourd'hui «l'institut de l'UNESCO spécialisé dans les contenus, les méthodes et les structures de l'éducation» (http:/ / www.ibe.unesco.org).

8 Voir Publications du BIE: PEN, 1935, 12; PEN, 1936, 116; PEN, 1938, 134. 
plusieurs maîtres. Ceux-ci sont souvent sollicités pour faire passer des tests (questionnaires, épreuves) mais aussi pour donner des renseignements complémentaires. En d'autres termes, les instituteurs participent au processus de recherche en récoltant les données.

Il faut qu' on sache aussi que nos observations ont porté sur des milliers d'enfants des deux sexes, depuis deux ans jusqu'à 18 ans, et que ces observations ont été faites dans les meilleures conditions de milieu possibles, par le maître ou la maîtresse habituels, au cours d'un exercice familier. (Bertrand, PEN, 1928, 40, p. 174)

Ainsi, il s'agit bien de savoirs issus de l'expérience (savoirs expérientiels des enseignants) mais qui acquièrent un autre statut du fait qu'ils sont formalisés par des chercheurs dans le cadre d'une recherche scientifique. Ces savoirs transformés, formalisés, contribuent non seulement à agrandir les connaissances dans le domaine pédagogique mais servent aussi à formuler des recommandations voire des conventions. Tous ces savoirs sont à leur manière fondés sur une démarche empirique (observations, données) ainsi que sur des hypothèses, généralisations, inférences et interprétations plus ou moins intuitives.

Une analyse fine du contenu (analyse qualitative) fait apparaître des nuances, des subtilités et montre que les savoirs se côtoient, se mélangent à l'intérieur des catégories énoncées. Ainsi, dans des articles classés connaissance, il n'est pas rare de trouver des références aux savoirs d'expérience des éducateurs ou des recommandations exhortant les praticiens à tenir compte des connaissances qu'on vient de présenter. De même, dans les articles classés praxéologique où les savoirs d'expérience dominent, les auteurs font souvent appel aux savoirs savants pour convaincre le lecteur du bien-fondé de leur technique ou de leur méthode. C'est même une stratégie rhétorique dans le discours de propagande: les textes débutent avec quelques paragraphes énonçant des savoirs scientifiques citant parfois le nom des figures auxquelles l'auteur se réfère - par exemple, les psychologues connus en Europe (Claparède, Wallon, Piaget, Piéron) ou des grandes figures de l'Éducation nouvelle (Decroly, Montessori, Ferrière, Claparède). Puis, l'article se poursuit par le récit d'expérience de l'auteur qui, fort de ces références «savantes», devient alors plus crédible et plus légitime à énoncer ses idées. Ainsi, il n'est pas rare que dans un même article des savoirs scientifiques côtoient des savoirs d'expérience. Selon Hofstetter et Schneuwly (2007), cette proximité des savoirs est précisément ce qui caractérise la période 
d'émergence des sciences de l'éducation où le champ disciplinaire s'imbrique avec les terrains éducatifs et avec d'autres disciplines académiques: «les savoirs issus de la discipline, alors en construction, et ceux de la profession se côtoient, se combinent, la frontière étant alors mouvante, ouverte, perméable» (p. 443).

L'importance accordée aux savoirs expérientiels dans cette revue s'explique aussi par le rôle de l'association dont elle est l'organe, affirmé dès la parution de son premier numéro en 1922:

Nous désirions fonder une association qui fournirait les moyens de se connaître aux instituteurs qu'un même enthousiasme a saisis, qui sont attirés vers ces mêmes idéaux libres et beaux qui commencent à se faire jour dans l'œuvre éducative. Cette Ligue donnerait aux éducateurs le sentiment de l'entr'aide, si bienfaisant à tout cœur humain. [...] Les éducateurs sont les grands facteurs de l'avenir. Il est de notre devoir de faire briller à leurs yeux ce bel idéal, et tout en leur rappelant leur responsabilité de les prémunir contre toute lassitude et tout découragement qui risqueraient de leur faire oublier la grande mission à laquelle ils sont appelés. (Ensor, PEN, 1922, 1, p. 7)

Comme nous pouvons le voir dans cette citation, la Ligue internationale pour l'Éducation nouvelle se considère avant tout comme une association de praticiens, ceux-ci revendiquant leur connaissance des difficultés et des réalités du terrain. En tant qu'organe de cette Ligue, PEN est donc d'abord une revue professionnelle destinée aux professionnels de l'éducation. Cependant, l'importance accordée aux savoirs scientifiques, l'utilisation qu'en font les praticiens et la place croissante que ces savoirs occupent dans la revue montre que PEN peut aussi être considérée comme une revue scientifique. En effet, elle permet aux scientifiques, aux chercheurs spécialistes de «l'étude de l'enfant» (pédologie, pédagogie, psychologie) de faire part de leurs découvertes, d'échanger leurs savoirs, de rendre visible leurs travaux à la communauté scientifique. Tout se passe comme si les acteurs étaient en train de construire un «édifice de la connaissance» auquel chacun apporte sa pierre quelle que soit son appartenance professionnelle, culturelle, linguistique, sociale. Dans ce processus d'édification, la revue constitue un lieu d'échange où chacun peut à la fois prendre et déposer des connaissances, autrement dit, un lieu de transmission de savoirs. En ce sens, elle se situe au carrefour entre théoriciens et praticiens qui dépendent les uns des autres pour faire avancer la science. Comme le montrent Hofstetter et Schneuwly (2006), et comme le révèle notre propre enquête, les premiers ont besoin des praticiens à la 
fois pour tester leurs méthodes et pour connaître les résultats de leur mise en application. De leur côté, les praticiens ont besoin des théoriciens pour synthétiser, formaliser et conceptualiser les savoirs acquis par l'expérience. Car une fois formalisé, le savoir devient plus légitime, plus crédible, à la fois dans le monde scientifique et dans le monde politique. Ainsi, lorsque Ferrière publie la première partie de sa thèse dans les Archives de Psychologie - revue scientifique que dirige Claparède - c'est bien dans le but de «réaliser son œuvre et d'asseoir sa notoriété dans le monde étroit des savants» (Hameline, 1993b, p. 387), en l'occurrence au sein du collège de spécialistes que représente la psychologie. Les praticiens ont donc besoin des savants pour soutenir leurs revendications, pour légitimer et rendre crédibles leurs désirs de réforme.

En ce sens, Pour l'Ère Nouvelle est une revue hybride: à la fois scientifique parce que lieu de publication des représentants des sciences de l'éducation émergentes, et professionnelle parce que lieu d'échange des praticiens qui s'essaient aux méthodes nouvelles. S'il n'est pas explicitement énoncé dans ses colonnes, ce statut hybride est affirmé de façon plus claire par d'autres associations, notamment par La Nouvelle Éducation qui prend naissance au même moment en France. Cette société pédagogique énonce clairement son double statut qui fait partie de ses objectifs principaux:

Nous nous proposions d'abord de travailler à répandre en France et partout où pourrait s'étendre notre activité, les découvertes les plus modernes de la pédagogie moderne. Nous nous proposions en outre de porter remède dans la mesure du possible à un inconvénient particulièrement grave chez nous, où les savants qui travaillent dans le même domaine se connaissent mal et ne profitent guère de leurs découvertes mutuelles, parce que chacun travaille un peu jalousement entre les quatre murs de sa chambre, et où les revues spéciales (surtout les revues pédagogiques) sont trop peu nombreuses pour accueillir tous les travaux qui mériteraient d'être connus. Nous voulions grouper tous ceux qui, acceptant les principes que je viens de dire, les mettaient en œuvre et essayaient d'élever les enfants ou ceux qui leur étaient confiés conformément aux vues de la pédagogie moderne. (Cousinet, PEN, 1922, 1, p. 10)

Le bulletin dont La Nouvelle Éducation se dote dès 1922 sera l'organe de l'association jusqu'en 1939. Elle a pour but, dit son fondateur, «de tenir nos membres au courant de nos travaux et des résultats de nos expériences». Or, les membres de cette société pédagogique ne sont autres que des praticiens puisqu'elle a pour objet 
de réunir tous les éducateurs décidés à favoriser en France l'activité personnelle des enfants, soit à l'école soit dans la famille. Son but est d'aider ces éducateurs à faire connaître et répéter leurs expériences afin que les travaux de chacun puissent profiter à tous et que se fasse plus vite la transformation nécessaire de nos méthodes d'éducation. (Guéritte \& Cousinet, PEN, 1922, 1, p. 1)

Ainsi, tout comme PEN, cette revue est avant tout un lieu d'échange de praticiens mais elle vise aussi à réunir les connaissances de tous, y compris celles des savants qui travaillent pour améliorer le sort de l'enfant.

Pour conclure, nous observons une grande proximité des savoirs scientifiques et des savoirs d'expérience qui au lieu de s'exclure se complètent et se nourrissent les uns les autres. Cette observation rejoint celles que nous avons pu faire à propos des thématiques d'articles ainsi que des profils d'acteurs qui à ces différents niveaux, confirment le caractère hybride et pluridisciplinaire de la nouvelle science de l'éducation, en particulier dans cette période d'entre-deux-guerres. Une pluridisciplinarité qui est toujours valable aujourd'hui comme en atteste le pluriel utilisé dans l'appellation en français «les sciences de l'éducation» (Hofstetter, 2010).

\section{L'ENJEU SCOLAIRE: PROGRAMMES ET SAVOIRS EN DÉBAT}

Cette partie étudie la question des savoirs de l'Éducation nouvelle en s'intéressant plus particulièrement aux savoirs à enseigner et aux programmes scolaires. ${ }^{9}$ Sur la base de recherches effectuées dans différents contextes (Helmchen, 2009; Hofstetter \& Schneuwly, 2009a; Savoye, 2009), nous nous proposons de repérer les types de savoirs privilégiés dans la revue, ceux qui rallient la majorité des auteurs et ceux qui suscitent des controverses. Plus globalement, cette partie vise à identifier les positions adoptées par les réformateurs à l'égard de ces savoirs et à mieux comprendre «quelles transformations des savoirs l'Éducation nouvelle génère» en raison des méthodes d'enseignement qu'elle défend (Hofstetter \& Schneuwly, 2009b, p. 454).

9 Cette partie et les données dont elle est issue a fait l'objet d'un article intitulé: «Savoirs constitués et programmes scolaires: un statut ambivalent dans la pédagogie de l'Éducation nouvelle» (Haenggeli-Jenni, 2011). 
À une première lecture, la question des savoirs et programmes scolaires n'apparaît pas centrale parmi les thématiques abordées dans Pour l'Ère Nouvelle. Si l'on analyse les titres d'articles, très peu contiennent des mots comme programme, plan d'étude, curriculum, ou d'autres mots évoquant explicitement des contenus de savoirs. De fait, la question n'est pas éludée par les auteurs de PEN, mais elle n'est pas visible d'emblée. En effet, il est nécessaire de procéder à une lecture détaillée des articles pour découvrir que cette question est abordée le plus souvent de façon «détournée», par le biais d'autres thématiques plus centrales comme la liberté en éducation, la créativité, l'éducation artistique, l'activité spontanée de l'enfant, le rôle du maître, ou encore les examens et l'évaluation. D'autre part, cette question évolue au fil des ans occupant une place toujours plus importante en particulier dans les années 1930. Comme nous le verrons, le Congrès d'Elseneur (1929) constitue un tournant par rapport à cette problématique puisque la deuxième partie de son thème lui est consacré: «Psychologie nouvelle et curriculums». Les débats qui y ont lieu attestent que la question est toujours plus «brûlante», la Ligue étant appelée à se positionner sur cette question. Les parents demandent une plus grande visibilité des apprentissages - notamment sous forme de programmes clairs - en raison d'un contexte socio-économique en crise dans lequel la préoccupation concernant les débouchés sur le marché du travail devient centrale. Du côté des enseignants, le Congrès de Nice confirme la demande pressante de ces derniers sur le statut des programmes scolaires pour pouvoir "passer à l'action» à plus large échelle. Une demande qui révèle l'extension des méthodes nouvelles aux écoles publiques où la question constitue un des points les plus sensibles pour les représentants de l'administration scolaire. En effet, tant que la réforme reste dans le secteur privé, la question est de moindre importance puisque chaque établissement gère lui-même ses programmes scolaires.

L'analyse de la revue montre enfin que cette question est plus en vogue dans certaines régions, influencée d'une part par les traditions culturelles et d'autre part, par le contexte économique du pays. Ainsi, on peut dire que la question des programmes et curriculums préoccupe beaucoup les Américains comme en atteste leur engagement dans les commissions de la Ligue ${ }^{10}$ sur les programmes et l'évaluation (examens).

10 Harold Rugg est responsable de la commission permanente «Refonte des programmes scolaires» tandis que W. Carson Ryam dirige la commission «Examens». 
Leur culture scolaire est imprégnée d'une logique «pragmatiste» où le rendement et la production constituent des enjeux centraux. De plus, les théories de Taylor influencent profondément la pédagogie américaine qui s'en inspire pour construire des programmes visant à une meilleure efficacité sociale (social efficiency programs).

\section{ALLÉGER LES PROGRAMMES POUR LIBÉRER L'ENFANT}

De la lecture de PEN se dégage un consensus sur la conception du savoir: il doit d'abord «correspondre et répondre à des besoins de l'enfant» où "la valeur éducative des savoirs disparaît comme thématique» (Hofstetter \& Schneuwly, 2006, p. 28). Cette conception considère que désormais l'épanouissement de l'enfant est plus important que les connaissances à acquérir, l'apprentissage englobant une grande diversité de savoirs autres qu'intellectuels. Dès lors, le but de l'enseignement n'est plus de diffuser un savoir donné mais de susciter l'envie d'apprendre et de connaître. Cette conception du savoir est fortement influencée par les théories de référence de l'Éducation nouvelle et en particulier par la conception de l'apprentissage selon laquelle l'enfant doit apprendre par lui-même à travers l'expérience, l'activité, le tâtonnement.

Recevoir n'est pas savoir. Notre but n'est pas d'apporter à l'enfant une science toute faite, $c^{\prime}$ est à lui de la faire. De même qu'un gain acquis difficilement est plus précieux, un gain intellectuel acquis au prix d'un effort est mieux compris et mieux gravé. [...] Il faut que dans tout son travail, l'enfant pense et cherche. Jamais il n'est passif. Avec le savoir bien acquis, cet effort développe l'habitude du travail, l'esprit de recherche. (Trellis, PEN, 1934, 97, pp. 114-115)

Par conséquent, l'apprentissage et la scolarité prennent une toute autre dimension dans la vie de l'enfant. Les auteurs plaident pour le rapprochement de l'école et de la vie affirmant que l'étude n'est pas «un acheminement vers les jouissances de la vie», mais qu'elle en est une en elle-même: «Elle doit donc n'être qu'agréable: c'est pour cela que nous devons permettre à l'enfant de vivre une vie active, libre, dans un milieu riant dont il est maître et responsable» (Deschamps-Alexander, PEN, 1928, 39, p. 125).

Cette volonté profonde de libérer l'enfant, de l'affranchir de tout ce qui pourrait entraver son développement naturel constitue un des consensus principaux des auteurs de la revue. De fait, plus que d'une 
volonté, il s'agit de la condition même de l'Éducation nouvelle qui lui est intrinsèquement liée. En effet, par le fait même de revendiquer une connaissance scientifique de l'enfant, l'Éducation nouvelle suppose la liberté du sujet: pour pouvoir observer l'enfant et déceler ses besoins, il faut que celui-ci soit libre de ses actes et de ses pensées. Certains se réfèrent à Rousseau et à la célèbre phrase de l'Émile «Commencez donc par mieux étudier vos élèves car assurément vous ne les connaissez point», d'autres à la posture scientifique du zoologue:

Si l'enfant se développe en agissant et s'il est indispensable au maître de connaître la façon d'agir et de réagir de l'enfant, cela implique que les réactions de l'enfant doivent pouvoir être observées dans un maximum de liberté. Les classes expérimentales sont à la psychologie aussi nécessaires (et bien davantage) que les «parcs nationaux»ou les «réserves» à l'ornithologiste. (Bovet, PEN, 1927, 31, p. 176)

Cette conviction que l'Éducation nouvelle participe à la libération de l'enfant découle également $\mathrm{du}$ «renversement copernicien» (Claparède) pour lequel plaident ses partisans. En revendiquant une «école sur mesure», ils cherchent à libérer l'enfant des contraintes formelles et institutionnelles qui caractérisent l'école traditionnelle. Pour eux, cette libération passe par la disparition de toute contrainte externe qui pourrait entraver le développement naturel de l'enfant. Afin de faire émerger les savoirs naturels, les potentiels propres à chaque individualité, l'enfant doit être libre de son temps et de ses mouvements. L'analyse de PEN montre que cette libération s'opère à plusieurs niveaux, aussi bien à celui des contenus (programmes scolaires) qu'au niveau organisationnel (horaires, répartition des élèves), institutionnel (hiérarchie, administration) et architectural (locaux, espaces).

Dans une école qui cherche à libérer l'enfant, les programmes scolaires font figures d'obstacles: obstacles au développement de l'enfant car ils orientent voire «formatent» ce dernier selon une conception des connaissances établies a priori. Ces savoirs sont considérés comme inutiles et contraignants, à la fois pour l'élève et pour le maître, puisque l'on plaide pour une pédagogie expérimentale où l'apprenant, par son activité, découvre le savoir par lui-même. Pour Ensor, les programmes et les examens sont «les deux dragons qui ferment la voie du progrès dans les écoles d'État», dragons qu'il s'agit de vaincre! (Ensor, PEN, 1929, 51, p. 220). Aussi, certaines écoles plaident pour une abolition totale des programmes, convaincues que la demande d'instruction viendra de l'en- 
fant le jour où celui-ci en aura besoin, en réponse à son activité et ses découvertes. C'est le cas par exemple des Communautés scolaires de Hambourg ${ }^{11}$ ou des Free schools américaines qui abolissent toute programmation des savoirs à enseigner. Selon certains auteurs, toute planification du travail à partir d'objectifs à atteindre est contraire à la nouvelle manière d'envisager l'enseignement: «La poursuite des résultats fixés à l'avance conduit l'éducateur à négliger ses élèves. Il ne faut pas fixer les résultats à atteindre» (Wauthier, PEN, 1922, 3, p. 59). Dès lors, pour le maître, il s'agit moins de transmettre des savoirs intellectuels que des savoirs-faire permettant à l'enfant «d'apprendre à apprendre» et au final, de se développer en tant que personne. Comme le rappelle Paulsen ${ }^{12}$, cette volonté fait partie des principes fondateurs de l'Éducation nouvelle: «Le but à atteindre par le programme [...] doit être la formation de l'esprit de l'enfant et non pas la matière à enseigner» (Paulsen, PEN, 1927, 31, p. 206). Un principe qui est mis en pratique dans plusieurs écoles nouvelles, en particulier dans les écoles Montessori comme en atteste cette institutrice de la Maison des enfants de Sèvres: «Notre enseignement n'a pas pour but d'arriver à un état de savoir, mais de mettre en marche un esprit et de développer tous ses moyens d'aller de l'avant. Pour cela nous développons l'effort personnel et la pensée... (Trellis, 1934, 97, p. 114) $)^{13}$

Cependant, plusieurs auteurs contestent l'idée d'abolir complètement les programmes; des débats importants ont lieu, notamment au Congrès de Locarno en 1927. Une lecture approfondie des articles de la revue fait apparaître beaucoup de malentendus à propos de la notion d'école sans programme. Selon Rotten (1929), il ne s'agit pas d'abolir les programmes, ni de les «refondre», ni même de les «adapter aux conditions de la vie moderne» («comme beaucoup s'appliquent à le faire de manière zélée», dit-elle); il s'agit plutôt de les adapter aux lois génétiques de l'évolution de l'enfant, ce qui signifie une transformation beaucoup plus profonde et plus essentielle des pratiques d'enseignement. ${ }^{14}$ En

11 Voir les articles de Rotten (PEN, 1923, 6, pp. 30-32), Petersen (PEN, 1923, 8, pp. 107-108) et Lamzus (PEN, 1925, 17, pp. 44-45) sur ces écoles.

12 Wilhelm Paulsen est un ancien membre de l'Oberstadtschulrat à Berlin et auteur de La Conquête de l'École.

13 Les mots en italiques sont soulignés par l'auteur dans le texte d'origine.

14 Le terme «essentiel» est à comprendre ici dans le sens profond du terme relié à la notion d'essence. 
d'autres termes, le programme devrait être organique et dynamique. ${ }^{15}$ Pour la rédactrice allemande, un programme n'est considéré comme dynamique que si le «processus psycho-biologique de l'évolution enfantine dans des circonstances analogues amène spontanément, en vertu des lois génétiques, des résultats analogues.» Par conséquent, «la transformation des programmes doit partir non de telle ou telle exigence des matières scolaires, ni d'un enrichissement de celles-ci, mais de la connaissance psychologique de la croissance spirituelle de l'enfant» (PEN, 51, p. 215). Si cette conception des programmes paraît précise et scientifique dans ce discours, elle ne donne pas beaucoup de pistes aux instituteurs pour en envisager une application pratique. À ce problème, Rotten répond en faisant référence aux Américains qui, pour elle, «sont les spécialistes de la question»:

Il existe mille façons diverses d'exprimer une même loi fondamentale et aucune méthode ne saurait s'ériger en panacée. Mais il y a lieu de montrer les rapports qui peuvent exister entre cette conception nouvelle des programmes et d'autres tendances profondes du temps présent. (Rotten, PEN, 1929,51, p. 216)

De fait, les rapports dont il est question dans cet extrait sont d'ordre métaphysique comme en atteste la suite de l'article de Rotten. Selon elle, le lien «réel», «primordial» entre le programme et la vie est un «lien cosmique qui constitue l'essence de tout ce qui vit» (Rotten, PEN, 1929, 51, p. 217). En d'autres termes, l'organisation du programme doit se calquer sur «l'organisation cosmique» qui existe dans toute matière vivante. Mais, comme elle le précise, «concevoir ce lien du Tout est affaire de divination plutôt que de connaissance rationnelle». Ce genre de discours, qui reste à un niveau cosmique et métaphysique, n'est pas pour rassurer les parents préoccupés par l'avenir de leurs enfants. Ceux-ci sont inquiets de savoir si ces méthodes permettront à leur progéniture d'accéder à un métier, à une place dans la société. Si Rotten semble parfaitement consciente de ces soucis et des reproches qui sont faits à l'Éducation nouvelle, elle les interprète comme une conséquence de l'école ancienne:

15 Un terme qu'utilise également Ferrière pour décrire Pour l'Ère Nouvelle, qui selon lui, a la particularité d'être une revue dynamique. (Ferrière, PEN, 1922, circulaire de présentation, p. 4) 
Les objections que l'on fait au programme dynamique, au chaos toujours nouveau des matières en raison des circonstances de la vie et des étapes de l'évolution psychobiologique de l'enfant, ces objections émanent du monde tel que l'a créé l'école ancienne. Elles tournent presque toujours autour du même point: que le savoir ainsi acquis serait fortuit, fragmentaire, lacunaire, et que si l'enfant n'a pas été obligé d'apprendre telles ou telles choses définies, il aura de la peine à faire sa place dans la vie et que toutes les professions risquent de lui être fermées. (Rotten, PEN, 1929, 51, p. 216)

Au-delà des savoirs et programmes scolaires, la revue fait apparaître une réflexion de fond à propos de l'évaluation et des examens dans le cadre scolaire. De manière générale, c'est toute la logique d'évaluation des résultats, en référence à la pratique en vigueur dans les écoles traditionnelles, qui est remise en cause par les tenants de l'Éducation nouvelle.

Mesurer avec des chiffres le travail qu'a fourni un enfant n'a aucun sens, aucune valeur. On ne doit mesurer que l'effort fourni par l'enfant et cet effort n'est pas mesurable! En résumé il est indispensable d'accepter tous les résultats des travaux fournis par l'enfant, quels qu'ils soient. (Wauthier, PEN, 1922, 3, p. 59)

Dans les écoles nouvelles, les programmes sont allégés et les horaires sont assouplis afin de permettre la pratique d'autres activités telles que la gymnastique, la musique, le dessin, l'expression corporelle. Les savoirs scolaires y sont présents mais ils ne constituent pas la préoccupation centrale des directeurs de ces écoles. Dans certaines d'entre elles, la présence des élèves n'est obligatoire que pour «certaines classes», un à deux jours par semaine, le reste étant réservé aux «études libres». (Baldwin, PEN, 1922, 1, p. 21) À en croire Gerhardt Sieveking, directeur de l'Institut des sciences de l'éducation d'Iéna, la volonté d'allégement des programmes correspond à une évolution globale de la mentalité de l'époque:

La mentalité nouvelle qui s'est fait jour durant ces vingt dernières années dans toute l'Europe exige une attitude nouvelle du maître et, dès lors, un nouveau mode de formation. La transmission des connaissances n'est plus le but ultime; la maîtrise de la matière à enseigner et de la méthode a perdu sa position centrale; à l'école qui se borne à enseigner et à faire apprendre, il faut substituer une école où l'éducation, le travail et l'expérience soient au premier plan. (Sieveking, PEN, 1926, 22, pp. 109-110) 
Si cette observation permet de comprendre le contexte de l'époque, la lecture de Pour l'Ère Nouvelle montre que la réalité est plus complexe et que le sujet occasionne moult discussions parmi les membres de la Ligue. Certes ces derniers se rallient derrière une commune dénonciation de l'enseignement encyclopédique, mais nous verrons plus loin que l'allégement des programmes fait l'objet de nombreux débats notamment par rapport au degré d'application de ce principe.

\section{ÉVACUER LES SYMBOLES DE L'ÉCOLE TRADITIONNELLE}

En lien étroit avec la place minimale accordée aux savoirs scolaires, de nombreux auteurs adoptent une attitude de déni, voire de rejet, à l'égard de tout support contenant des savoirs constitués tels que livres ou manuels scolaires.

Brookwood adopte en matière d'éducation une attitude expérimentale. [...] Les livres sont là pour être consultés, non pas étudiés: filles et garçons coopèrent à la rédaction de leurs manuels, à la classification de leurs découvertes, de leurs expériences. (Rédaction, PEN, 1924, 9, p. 10)

Symboles de l'école traditionnelle, ils sont considérés comme contraignants, parce qu'imposant une certaine vision du savoir qui ne tient pas compte des individualités de l'enfant. À la place, les auteurs préconisent l'utilisation de divers cahiers (cahiers de vie, cahiers d'expériences, cahiers de textes) créés par les enfants dans lesquels ceux-ci écrivent et dessinent librement, au fil de leurs apprentissages et découvertes. Ces cahiers constituent, pour certains auteurs, le seul moyen qui permette vraiment au maître de "pénétrer dans l'âme de l'enfant, de connaître ses préférences, d'apprécier sa valeur» (Sola, PEN, 1923, 6, p. 34).

Ces cahiers, expression d'une énergie vierge qui gaîment et librement s'épanche sans qu'aucun obstacle l'ait déviée, nous donnaient à penser à quel degré d'intellectualité et de vertu arriverait l'homme si tous les maîtres comprenaient la grandeur et cultivaient l'intérêt propre à chaque âge pour initier les enfants et les incliner vers le bien. (Sola, PEN, 1923, 6, p. 34)

Il nous apparaît ici que la liberté d'expression prônée par les éducateurs nouveaux peut être, en fait, un moyen puissant d'orienter voire de formater l'enfant, à son insu. 
Comme nous l'avons évoqué plus haut, l'analyse montre que la «libération de l'enfant» s'opère à plusieurs niveaux. Au-delà d'un premier niveau concernant les savoirs scolaires et leurs supports, on constate une volonté partagée de libérer l'enfant au niveau spatial, voire architectural. Pour les auteurs de PEN, transformer l'école, c'est aussi modifier l'espace dans lequel évolue l'élève à commencer par le local classe, symbole de l'école traditionnelle. En d'autres termes, libérer l'enfant, c'est lui offrir un environnement libre de contraintes qui lui permette de faire éclore tous ses potentiels et ainsi construire une personnalité libre et originale. Si la plupart des écoles nouvelles sont établies à la campagne c'est non seulement pour permettre l'organisation d'activités en plein air, en contact avec la nature, mais aussi pour rompre avec l'image habituelle de «l'école-bâtisse» ${ }^{16}$. On y transforme les classes en ateliers, symboles des écoles et des méthodes nouvelles. De manière générale, les réformateurs veulent faire de l'école un lieu accueillant, sécurisant, où l'enfant puisse s'épanouir sereinement, en lien étroit avec la vie sociale environnante. Dans les écoles publiques, faute de pouvoir «déplacer les murs» les instituteurs s'ingénient à transformer leur classe en un lieu convivial:

J'ai transformé ma petite classe en une espèce d'annexe de l'École de la rue de l'Ermitage. Elle devient un petit atelier où les enfants prennent une part active au travail où l'on rencontre une atmosphère de joie, d'amour, et de sincérité. (Hamaïde, PEN, 1922, 1, p. 17)

Pour évacuer l'image type de l'enseignement frontal, de nombreux instituteurs aménagent leur classe en créant des espaces de travail favorisant à la fois la mobilité et l'interaction des enfants. Plusieurs s'inspirent des principes énoncés par Montessori pour qui l'aménagement de l'espace est une des composantes essentielles de la liberté de l'enfant dans son apprentissage: «Organisation de la classe, interprétation du programme et de l'emploi du temps, établissement de la discipline concourent pour permettre à chaque enfant le plus grand effort dans la plus grande liberté» (Harang, PEN, 1927, 27, p. 74). Elle est une des premières à créer du mobilier et du matériel adaptés à la taille des enfants d'âge préscolaire. Plus qu'un lieu d'apprentissage, les auteurs

16 Dans les années 1970, Jacques Pain et Fernand Oury l'appelleront «écolecaserne» révélant ainsi une même volonté de se distancer de l'école traditionnelle (Oury \& Pain, 1972). 
de PEN pensent que l'école doit être désormais un lieu d'épanouissement où l'enfant apprend selon son rythme et ses intérêts, ceux-ci étant notamment guidés par le jeu. À ce propos, plusieurs psychologues de l'époque, en particulier Claparède (1905, 1931), montrent l'importance $\mathrm{du}$ jeu comme moteur et source d'apprentissage. Certaines écoles y accordent alors une place prépondérante au point d'en faire le cœurmême de leur programme: «L'école se propose de seconder le besoin spontané de jouer qu'éprouvent les enfants afin de le transformer en désir (spontané aussi) d'activité utile» (Ferrari, PEN, 1922, 1, p. 19).

$\mathrm{Au}$ niveau des contenus, on remarque une même volonté de se distinguer de l'école traditionnelle en offrant une autre approche des savoirs scolaires. Dans les écoles nouvelles, les programmes sont réduits pour permettre une gestion plus souple du temps et des activités. Plutôt qu'un enseignement conditionné par des programmes établis a priori, les auteurs de PEN préconisent un enseignement naturel adapté aux besoins de l'enfant et inséré dans un projet qui mobilise ses intérêts. Les méthodes, bien que variables selon les régions, valorisent une organisation des programmes par thématiques plutôt que par discipline. Les savoirs scolaires sont abordés à travers des projets larges qui font appel à des compétences relevant de plusieurs domaines (rédaction, lecture, arithmétique, sciences, etc.). Cette approche est jugée plus naturelle et plus respectueuse des intérêts de l'enfant puisqu'elle ne sépare pas les matières scolaires; selon les auteurs elle permet à l'enseignant une gestion plus souple et plus adaptée des savoirs scolaires en y intégrant tous les objectifs pédagogiques. Cette technique pédagogique donne lieu à un foisonnement de méthodes qui se traduit, dans la revue, par un nombre important d'articles dédiés à leur présentation. Leurs noms varient selon les pédagogues qui les ont élaborées mais toutes relèvent du même principe: Project method (Dalton Plan) ${ }^{17}$, Méthode des centres d'intérêts (Decroly), Méthode de travail libre par groupe (Cousinet), Système complexe d'enseignement (Russie). Malgré leurs spécificités, ces méthodes se ressemblent par le fait qu'elles privilégient une organisation temporelle «groupée» (une discipline par matinée ou par jour) et partagent toutes un principe commun: les savoirs à enseigner ne sont plus découpés par matières mais sont intégrés dans un projet global commun à un groupe d'élèves, projet qui s'insère, selon certains auteurs, dans un pro-

17 Pour une étude sur la réception de cette méthode en Espagne, voir Del Pozo Andrés, 2009. 
jet encore plus vaste, celui du «travail de coordination intellectuelle de l'esprit humain»:

On n'étudie pas les diverses branches à part et chacune pour soi. L'histoire, les sciences économiques et sociales, les arts, la musique, l'économie domestique, la littérature, le journalisme [...] seront présentés comme des aspects divers du grand travail de coordination intellectuelle de l'esprit humain dans son ascension vers la vérité. (Rédaction, PEN, 1924, 9, p. 10)

Désireux de transformer l'école dans son ensemble, les auteurs de PEN sont soucieux de sortir l'école du schéma classique du «lire, écrire, compter». Plusieurs auteurs s'accordent pour dire qu'il ne s'agit plus de faire des «têtes bien pleines» mais «des têtes bien faites» (Decroix, PEN, 1922, 2, p. 35) la priorité allant désormais au développement de techniques $\mathrm{d}^{\prime}$ apprentissage favorisant «l'apprendre à apprendre». Ils revendiquent la prise en compte globale de l'enfant dont l'éducation doit permettre son épanouissement aussi bien intellectuel que physique, artistique et moral: «Il faut que l'éducation soit intégrale pour que la personnalité soit libre, complètement elle-même, sans ces limitations qui l'enserrent si souvent aujourd'hui. Il faut qu'elle s'adresse à tout l'être et lui permette d'épanouir toutes ses virtualités» (Bertier, PEN, 1936, 121, p. 240).

Dans la majorité des textes, le développement des aptitudes intellectuelles sont reléguées au second plan au profit de disciplines visant l'épanouissement de l'enfant ainsi qu'un équilibre entre l'éducation du corps et de l'esprit. Au fil des articles, on constate une tendance allant vers la diversification des matières voire vers un renversement des priorités accordées aux disciplines:

Un premier fait m'apparaît comme des plus significatifs: c'est le renversement des valeurs que nous attribuons aujourd'hui aux diverses disciplines. Celles qui apparaissaient comme fondamentales et de premier plan, vestiges de traditions périmées, ont petit à petit - non sans luttes acharnées - cédé la place à d'autres, considérées autrefois comme des disciplines de luxe, soit au contraire de dernier plan - rabaissées au rôle de simple instrument de jeu et destinées à occuper les heures trop longues pendant lesquelles la maîtresse était la proie d'enfant inquiets et nerveux; je veux parler du dessin, du modelage, de la rythmique de la musique, et d'une manière générale de tous les exercices qui visent à donner une éducation artistique. À notre sens, la préformation esthétique doit précéder sa préformation intellectuelle abstraite. (Coirault, PEN, 1932, 83, p. 319) 
Pour convaincre leur lectorat, les auteurs utilisent une rhétorique puissante qui dévalorise les anciennes méthodes ou disciplines scolaires. Ainsi, ils affirment que c'est pour «rompre avec l'immobilisme des classes habituelles» que les travaux manuels sont désormais préconisés comme une discipline à part entière. Il s'agit aussi bien de travaux des champs, de menuiserie, de bricolage, que de travaux à l'aiguille ou de tâches ménagères pour les filles. De manière générale, les disciplines artistiques prennent une importance considérable dans l'aménagement du temps scolaire. D'après les auteurs, l'éducation par les arts est très naturelle car elle aide les enfants à développer ce qu'ils possèdent déjà naturellement (aptitudes innées). Que ce soit à travers le dessin, les arts plastiques (peinture, modelage, sculpture) ou la musique (chant, rythmique), c'est le développement de l'enfant et son épanouissement personnel qui sont visés avant tout. Les savoirs véhiculés par ces disciplines sont d'un autre ordre que ceux du «lire, écrire, compter» et ont pour but de développer des aptitudes où la performance n'est pas le critère premier.

Il est à mon sens, complètement inutile de soumettre l'esprit des élèves à des opérations difficiles d'ordre mathématique. L'important pour eux n'est-il pas de faire se pénétrer réciproquement l'esprit et le corps? Pour faire participer le corps à l'action cérébrale, il importe que celle-ci n'absorbe pas toutes les forces de l'être. (Dalcroze, PEN, 1922, 3, p. 52)

En lien avec les sciences auxquelles se réfère l'Éducation nouvelle (hygiénisme, vitalisme, naturalisme), l'éducation physique ${ }^{18}$ devient une discipline préconisée dans toutes les écoles; une discipline qui doit se dérouler, si possible, à l'extérieur afin de faire bénéficier les élèves du soleil et du plein air. L'hygiène du corps est considérée comme essentielle pour garantir une hygiène de l'esprit et une éducation naturelle. Ferrière, dans son texte des «Trente Points», en fait un des critères d'attribution du label «d'école nouvelle»:

Point 9: L'École nouvelle assure la culture du corps par la gymnastique naturelle. A. Pratiquée le torse nu ou même en bain d'air complet, elle aguerrit et écarte les maladies. B. Elle rend souple et adroit sans ennuyer l'enfant. C. Elle s'associe aux jeux et aux sports. (Ferrière, PEN, 1925, 15, p. 6)

18 Sur l'histoire de cette discipline et son développement en Suisse, voir Czáka (en préparation). 


\section{DES PRINCIPES EN DÉBAT: INQUIÉTUDES ET DÉSACORDS}

Si la majorité des articles revendiquent l'allégement voire l'abolition des programmes scolaires, les auteurs ne sont pas unanimes sur la question. Le sujet suscite controverses et débats, de nombreuses voix s'élevant pour dénoncer des pratiques extrêmes ou l'interprétation erronée de certains principes. Ces voix émanent à la fois de parents inquiets que leur enfants «n'apprennent rien», de représentants des administrations scolaires qui rappellent le mandat d'instruction de l'école publique, ou de théoriciens de l'Éducation nouvelle qui nuancent leurs théories pour mieux se faire comprendre. Pour l'Ėre Nouvelle est ainsi jalonnée de textes qui tentent de «redresser la barre», de mettre en garde contre les dérives d'une interprétation abusive de l'allégement des programmes.

Parmi les parents, on perçoit une inquiétude quant aux connaissances effectives que leurs enfants auront plus tard. Les débats ne sont pas explicitement ouverts dans la revue, mais apparaissent en filigrane dans les récits d'éducateurs qui relatent des rencontres ou conférences où ils sont interpellés par des parents. Ces discours révèlent les reproches adressés aux méthodes nouvelles et les inquiétudes qu'elles suscitent dans l'opinion publique. Lors d'un congrès de La Nouvelle Éducation en juin 1922, Cousinet et Wauthier répondent à ce genre de questions par des paroles qui se veulent rassurantes tentant de montrer que le savoir n'est pas central et que les programmes peuvent être nuisibles:

Le programme n'est pas nécessaire puisque nous mettons l'ordre nousmêmes dans les connaissances acquises. L'intervention du maître est inutile et serait même nuisible si elle établissait un programme, un ordre, avant que l'enfant ait acquis quoi que ce soit. (Wauthier, PEN, 1922, 3, p. 58)

Cependant, les inquiétudes sont réelles concernant le statut du savoir ainsi acquis. Selon les parents, celui-ci serait fragmentaire, lacunaire dont les conséquences pourraient être considérables si l'enfant ne parvient pas à faire sa place dans la vie en raison des nombreuses professions qui risquent de lui être fermées. Du côté des autorités scolaires, une inquiétude s'exprime en lien avec les engagements qu'elles doivent tenir concernant les programmes scolaires. Les discours émanant des autorités révèlent une méconnaissance de ce qui se fait concrètement sur le terrain: c'est souvent par personnes interposées que des responsables découvrent les nouveautés proposées par l'Éducation nouvelle. Ainsi en 
est-il d'un inspecteur en Belgique qui s'exprime sur la question du rendement de l'école primaire:

De divers côtés on me signale que l'école primaire actuelle, sous prétexte de fonder la formation intellectuelle des élèves sur le seul jeu spontané des tendances enfantines, montre un certain dédain pour l'enseignement systématique et approfondi du savoir lire, écrire, et calculer, etc. (Delaunay citant un inspecteur belge, $P E N, 1934,97$, p. 122)

Parmi les théoriciens de l'Éducation nouvelle qui s'expriment dans la revue, plusieurs s'accordent pour dire que certaines écoles sont allées trop loin dans l'abolition totale des programmes scolaires. Ils déplorent ce genre de pratique qui, au lieu de servir l'enfant, risque de l'handicaper dans sa vie adulte.

Quelques écoles nouvelles sont allées à l'extrême inverse. Elles déclarent que les enfants libres et heureux se développent comme des fleurs; mais à quatorze ans il manque à ceux-ci les fondements solides, nécessaires pour un travail plus avancé. Il nous faut trouver un équilibre: que l'enfant comprenne la nécessité des techniques pour pouvoir s'exprimer lui-même aussi parfaitement que possible. (Ensor, PEN, 1927, 31, p. 179)

Cette attitude de tolérance quasi sans conditions concernant les performances de l'élève rentre en contradiction avec une composante essentielle de la philosophie de l'Éducation nouvelle: la nécessité de rendre l'école plus efficace, mieux adaptée aux besoins de la société afin d'éviter le «gaspillage». Ainsi, on peut affirmer que les tests psychologiques mis en place pour mesurer les aptitudes et le niveau d'intelligence de l'enfant ne répondent pas seulement à une curiosité scientifique des pédagogues et psychologues. Ils sont conçus aussi pour mieux orienter les élèves en fonction de leurs aptitudes et ainsi favoriser une meilleure efficacité de l'école. Si Claparède favorise l'ouverture de classes spéciales, c'est pour permettre une meilleure répartition des élèves dans les cursus et ainsi améliorer l'efficacité de l'école. Dans certains pays, aux ÉtatsUnis notamment, ce souci d'efficacité donne lieu à l'élaboration de programmes scolaires très détaillés définis en fonction de demandes sociales et économiques précises (social efficiency programs, Labaree, 2006). Dans ce cas, les tests psychologiques sont utilisés pour déterminer le programme scolaire qui correspond le mieux à chaque élève et par la suite, pour les orienter dans la voie où ils seront le plus efficaces. 
Il faut avoir vu les enfants à l'œuvre pour se rendre compte de l'esprit d'initiative dont ils font preuve. C'est en plein le régime actif où le travail est orienté dans un sens tout à fait pratique: ces petits américains sont déjà des hommes d'affaires qui aussi bien que leur père, connaissent la valeur des mots «temps et argent» et ils sortiront de l'école préparés à la vie d'action qui les attend. (Delgoffe, PEN, 1923, 7, pp. 46-49)

Intimement liée à l'allégement des programmes, l'individualisation de l'enseignement fait partie des revendications des auteurs de Pour l'Ère Nouvelle car comme le dit Ferrière, elle est la dimension essentielle d'une méthode pédagogique scientifique: «Si nous voulons réaliser l'École active, si nous voulons fonder l'Éducation nouvelle sur une base scientifique, il faut à tout prix individualiser l'enseignement et l'éducation» (Ferrière, PEN, 1929, 51, p. 214). Cette «technique» pédagogique offre une grande souplesse à l'enseignant qui peut ainsi adapter son enseignement aux aptitudes et intérêts de chaque enfant. Si la revue comporte une majorité d'articles louant les bienfaits de cette technique et relatant les expériences de son application, quelques auteurs mettent en garde leurs collègues contre les pièges auxquels un tel enseignement peut conduire. Ainsi, certains affirment qu'une utilisation abusive de cette technique risque de provoquer le contraire du résultat escompté, à savoir d'isoler les aptitudes les unes des autres et d'en supprimer les rapports dynamiques réciproques au lieu de les mettre en synergie. De plus, certains pensent qu'une application trop systématique de l'individualisation peut diminuer voire inhiber la puissance de développement des forces latentes de l'enfant au lieu de les stimuler. Pour Lombardo-Radice, un travail individuel sans programme collectif ne permet pas de nourrir et épanouir suffisamment l'enfant. Selon lui, les programmes sont essentiels à la construction/formation (Bildung) de la personnalité. Ils constituent l'élément «nutritif», le terreau nécessaire pour que la semence puisse germer et grandir. Or, pour lui, un terreau riche est un terreau alimenté par les apports du maître et des programmes.

Le travail individuel qui obéit au choix de l'enfant doit être nourri de l'homme complet qui est en l'enfant. Chaque semence doit être déposée sur un terrain riche d'éléments nutritifs, "complets». Le terrain c'est le programme, qui fait essayer l'enfant dans toutes les directions possibles nécessaires à l'homme tout entier. [...] Tout en entravant le moins possible l'initiative du maître, l'école ne doit-elle pas établir un programme répondant 
à l'idéal éducatif de chaque époque et chaque pays? (Lombardo-Radice, PEN, 1927, 31, p. 183)

\section{LES LIMITES DE LA LIBERTÉ: LE RÔLE DE L'ADULTE}

Dans le même ordre d'idées, plusieurs auteurs rappellent que la liberté de l'enfant n'est pas synonyme d'anarchie. En tant que scientifique et pédagogue, Decroly tente de redéfinir cette liberté pour éviter les abus: «laisser l'enfant libre ne signifie pas qu'on l'abandonne à son libre arbitre capable de déclencher chez lui l'effort et la volonté» (Decroly, $P E N, 1927,31$, p. 185). Si pour beaucoup, «laisser libre» veut dire «tolérer les caprices et les états d'humeurs variables de l'enfant», l'auteur insiste sur l'aspect positif de cette liberté en disant que «laisser l'enfant libre, c'est aussi lui permettre de manifester ses tendances favorables, le laisser libre de bien faire» (p. 185). Selon lui, cela ne signifie pas que l'adulte doit adopter une attitude passive et se mettre en retrait. Au contraire, le régime de liberté exige une attitude dynamique et responsable du maître qui a pour tâche d'organiser cette liberté afin d'en faire quelque chose de constructif et productif.

Ce point de vue est partagé par d'autres auteurs, en particulier par les Américains pour qui le rendement de l'éducation scolaire est une préoccupation constante. On le perçoit dans les articles qu'ils signent dans Pour l'Ère Nouvelle ou dans les textes issus de leurs conférences données dans les congrès. Pour eux, libérer l'enfant ne veut pas dire qu'on le laisse faire «ce qu'il veut quand il veut», ni que le maître se base uniquement sur les intérêts spontanés de l'élève. Certes, il s'agit d'encourager l'enfant à créer, innover, affirmer ses idées mais il faut également l'inciter à prendre en compte ses camarades et à apporter sa contribution au groupe. Ces auteurs rappellent l'importance de la dimension sociale de l'éducation qui, disent-ils, est un aspect primordial dans la formation de l'enfant car il structure son identité en lien avec l'environnement dans lequel il se développe. Selon eux, si la spécialisation à laquelle on tend en individualisant l'enseignement est utile et performante, elle ne doit pas rester isolée et sans intérêts pour les autres. Ils critiquent les écoles qui, en voulant trop individualiser l'enseignement, négligent la responsabilité sociale à laquelle elles doivent former les enfants pour qu'une fois adultes, ceux-ci puissent s'intégrer pleinement dans la communauté. 
Nous devons constamment veiller à développer, en même temps que la faculté créatrice et l'affirmation du moi, un sens profond d'unité sociale, la compréhension du fait que si ce qui est favorable à l'individu contribue au bien-être de la communauté. [...] L'éducation en vue de cette liberté doit donc tenir compte des besoins sociaux et faire naître le sens de la responsabilité sociale. (Washburne, PEN, 1927, 31, pp. 198-199)

C'est pourquoi, d'après eux, l'adulte a un rôle essentiel à jouer, discret mais nécessaire, un rôle d'organisation des savoirs sans lequel l'activité libre ne peut parvenir aux résultats escomptés. Selon Washburne, «cette organisation ne diminuera en rien la liberté» (PEN, 1927, 31, p. 198); au contraire, elle permettra à l'enfant d'acquérir un certain nombre de connaissances dont il pourra faire bénéficier la communauté. Vers la fin des années 1920, aux USA et ailleurs, les écoles sans programmes perdent de leur popularité et des voix s'élèvent pour lutter contre leur tendance trop libertaire.

Dans les écoles libres, les enfants font une quantité de choses mais pas de façon assez longue pour en valoir la peine. C'est pourquoi ces écoles libres ont perdu le respect de la société. [...] Le but de l'éducation est d'obtenir le maximum de croissance avec le minimum de dépense. L'enfant doit apprendre à lire, compter, etc.; il doit apprendre à vivre en société, il doit apprendre à vivre avec lui-même. [...] Aussi le maître doit-il faire des plans et les organiser, avoir un matériel prêt, des projets, des excursions, toutes sortes d'appareils si l'on veut atteindre le maximum de croissance avec le minimum de temps et d'énergie. Il faut faire une sélection; si nous dépendons des intérêts spontanés des enfants, où cela nous conduira-t-il? (Rugg, PEN, 1927, 31, p. 196)

De nombreux auteurs discutent la question de la liberté au cœur des revendications de l'Éducation nouvelle. Ils pointent les limites de cette liberté en mettant en garde contre la dérive possible d'une interprétation abusive de ce principe. Parmi eux, Lombardo-Radice (1927) pense que les écoles dites libres, sans programmes, peuvent emprisonner l'enfant dans des choix forcément limités par ses connaissances, connaissances qui suivent souvent «les manifestations intempestives du choix capricieux [de l'enfant] et prennent un caractère pathologique». Dans ce cas, l'auteur se demande si l'on peut vraiment parler de liberté de l'enfant: «ne serait-il pas plus exact de parler de fausse liberté?»(LombardoRadice, PEN, 31, p. 184). Selon lui, le programme ou curriculum a pour 
but de «provoquer l'intérêt dans le plus grand nombre possible de directions» et doit jouer le rôle de garant contre l'aliénation de l'enfant par manque d'apports extérieurs.

Donnons le plus large développement au travail libre; mais ne permettons pas qu'une activité ou qu'une manifestation spontanée envahisse la place de façon à barrer la route aux autres aptitudes de l'homme en les laissant sommeiller. (Lombardo-Radice, PEN, 1927, 31, p. 183)

Dans le même ordre d'idées, Washburne (1927) s'élève contre ceux qui appliquent la liberté de façon extrême dans les écoles. Il pense que s'il $\mathrm{n}^{\prime} \mathrm{y}$ a pas de programmes ni de savoirs enseignés, l'enfant court le risque de ne pas maîtriser certains savoirs, outils pourtant nécessaires à la vie en société (lecture, écriture, calcul). Selon lui, ces savoirs ne sont pas seulement importants pour la vie d'adulte, ils sont constitutifs de la libertémême de l'individu. Dès lors, un enfant qui ne possède pas ces outils deviendra un adulte moins libre car en raison de limites internes à son être, il sera moins apte à juger, à évaluer, à faire valoir son opinion, à défendre ses intérêts et ceux des siens.

Pour que l'homme soit vraiment libre il faut que son milieu lui soit familier. Un étranger incapable de s'exprimer dans la langue des habitants de la ville où il se trouve est beaucoup moins libre que le natif de cette ville. [...] Les limitations qui viennent de l'être lui-même sont bien plus sévères que celles qui lui sont imposées du dehors. Ceux qui se font les défenseurs de la liberté dans l'éducation perdent parfois ce fait de vue et, en voulant s'écarter du gavage forcé, de l'éducation rigide, ils ne réussissent pas à donner à l'enfant cette maîtrise des connaissances et cette habileté qui lui sont indispensables pour remplir son rôle dans le monde d'aujourd'hui de façon efficace. Cette maîtrise est un droit que tout enfant peut revendiquer. Il est donc nécessaire de choisir intelligemment et avec soin les connaissances et les capacités (compétences) qui seront nécessaires à nos enfants. C'est à nous, adultes, à faire ce choix, plutôt qu'aux enfants, car les instincts et les intérêts naturels nés d'un passé biologique ne peuvent être un guide certain pour l'enfant dans son choix des connaissances et des capacités que demande notre civilisation moderne industrielle, si artificielle et complexe. [...] Se borner à instruire l'enfant et à développer son habileté, ce serait négliger son éducation, le préparer à n'être qu'un automate, un rouage dans le mécanisme de la civilisation industrielle. (Washburne, PEN, 1927, 31, p. 197) 
Enfin, certains auteurs comme Andrée Jadoulle - institutrice puis directrice du Laboratoire d'Angleur (Belgique) - montrent les limites des méthodes de liberté en éducation en raison des conditions d'application parfois difficiles que rencontrent les maîtres, en particulier dans les écoles publiques.

Les écoles publiques sont soumises aux programmes officiels. [...] Même si ces programmes, en Belgique, permettent de nombreuses initiatives et innovations, ils sont muets concernant l'éducation au sens social et sont très lourds et exigeants au niveau des savoirs à acquérir. [...] De plus, les écoles publiques accueillent des enfants de tous les environnements sociaux, éduqués par ces environnements à travers des pratiques et des principes très différents, parfois contradictoires. [...] Les écoles publiques sont soumises aux inspections ce qui signifie obstacles et soucis. C'est pourquoi la méthode de liberté en éducation n'est pas facile à appliquer dans les écoles publiques. (Jadoulle, 1935, PEN, 104, p. 8)

En conclusion, une attitude très ambivalente est présente parmi les auteurs de PEN concernant les savoirs constitués et les programmes scolaires. Ce constat rejoint celui avancé par les auteurs du volume spécial que Paedagogica Historica a dédié à cette problématique en 2009. Considérer les savoirs comme mineurs et artificiels parce que non adaptés aux caractéristiques de l'enfant, les reléguer au second plan parce que désormais l'épanouissement est plus important que l'acquisition de savoirs dits intellectuels, est contradictoire avec l'importance que les auteurs accordent aux savoirs provenant des sciences de référence. En effet, ces savoirs sont régulièrement convoqués pour légitimer leurs revendications puisqu'ils considèrent que désormais, la pédagogie doit être fondée sur la science, en particulier sur la psychologie et la pédagogie expérimentale. Comme nous l'avons vu, cette conception du savoir ne fait pas l'unanimité; elle suscite d'importants débats au sein de la Ligue dès la fin des années 1920. De fait, la question est si cruciale que les positions vont progressivement se durcir au point de générer des scissions au sein du mouvement, scissions qui se manifestent dans divers contextes nationaux et internationaux. Comme le montre Labaree (2004, 2006) la question divise les Américains en deux clans: le premier, composé des progressistes pédagogiques, choisit Dewey comme figure de proue tandis que le deuxième, les progressistes administratifs, se réfèrent avant tout à Thorndike. Ces deux clans se distinguent par leur conception radicalement opposée du savoir et de l'éducation: la première postule que 
l'éducation doit être proche de la vie et permettre à l'enfant de s'épanouir selon ses besoins et intérêts naturels, la seconde affirme que l'école doit transmettre des savoirs pour garantir l'émancipation de l'enfant, mais des savoirs adaptés à l'âge de l'enfant et pensés en fonction des besoins économiques.

Ces deux conceptions antagonistes s'apparentent à celles que relèvent Hofstetter et Schneuwly (2009c) à propos de Claparède et Vygotsky qui défendent des conceptions contrastées du savoir, en particulier des savoirs à et pour enseigner. Claparède, proche de Dewey, revendique une conception fonctionnelle de l'éducation qui s'adapte au développement de l'enfant, où les savoirs enseignés doivent être en lien avec la vie quotidienne et répondre aux besoins naturels de l'enfant. Pour Vygotsy, l'éducation précède et stimule le développement; les savoirs scolaires sont donc centraux, systématiques, différents de la vie quotidienne, et destinés «à transformer la relation à son propre processus psychique» (Hofstetter \& Schneuwly, 2009c, p. 605). Ces deux conceptions rejoignent celles que nous venons d'observer dans la revue où les dirigeants de la Ligue et la majorité des membres se rapprochent des positions de Claparède et Dewey, alors que Washburne et Lombardo-Radice se situent plutôt du côté de Thorndike et Vygotsky. Pourtant, tous ces acteurs partagent l'idée selon laquelle l'éducation doit se baser sur une connaissance scientifique de l'enfant et que celle-ci doit être à la base de la réforme de l'école. En ce sens, on peut dire qu'il y a consensus sur la dimension scientifique de l'éducation, mais les avis divergent quant aux manières de l'appliquer puisqu'elle ambitionne de transformer les pratiques à large échelle. Les débats sur les savoirs scolaires mais aussi sur la formation des enseignants - autrement dit sur les savoirs à et pour enseigner - révèlent de fortes ambivalences sur la nature de ces savoirs, leur place, leur rôle, leur contenu et leur organisation. Des divergences qui sont pour partie liées aux théories et sciences de référence qui constituent le socle théorique de l'Éducation nouvelle. 


\section{CONCLUSION}

\section{UNE REVUE-CARREFOUR}

Au terme de nos investigations dans les revues de la Ligue, et plus spécifiquement dans Pour l'Ère Nouvelle, l'impression dominante qui se dégage est celle d'un vaste chantier. Un chantier où s'affairent des acteurs d'horizons multiples, aux profils et appartenances diverses, mais tous animés d'un même élan, d'une même volonté: améliorer l'éducation pour transformer la société. Ce vaste chantier est celui d'une science en construction à laquelle ces périodiques nous offrent le privilège d'assister. En tant qu'espace de publication et d'information, ils constituent, chacun à leur manière, un lieu de rencontre d'acteurs, d'idées, de savoirs, d'expériences qui participent de cette construction.

L'étude approfondie de Pour l'Ère Nouvelle nous amène à considérer cette revue comme un carrefour et ce à plusieurs niveaux. Tout d'abord parce qu'elle met en contact des personnes à travers lesquelles des pays, des langues, des cultures, des croyances se rencontrent. Ce faisant, elle offre un niveau de regard au-delà des frontières, proposant un aperçu des réalités d'ailleurs, créant par là-même une ouverture d'esprit et un intérêt pour l'autre, pour le différent. Deuxièmement, Pour l'Ére nouvelle suscite la rencontre d'une diversité de savoirs: savoirs pratiques, théoriques, méthodologiques, psychologiques, pédagogiques, ou plus globalement, la mise en contact des savoirs à enseigner et pour enseigner. Elle permet non seulement leur rencontre mais aussi leur confrontation, leur discussion, leur remise en question en raison de la présence d'un lectorat appelé à réagir et à s'exprimer. Troisièmement, cette revue constitue une plateforme où se confrontent plusieurs sciences de référence telles que la pédagogie, la biologie, la médecine, la physiologie, la pédologie, la sociologie, la psychologie, la pédagogie expérimentale. L'analyse des discours nous a permis de mesurer l'importance de ces références, leur place et leur évolution dans ce vaste chantier, donnant à voir d'emblée la pluridisciplinarité des sciences de l'éducation. Quatrièmement, Pour l'Ère Nouvelle est le carrefour de divers courants intellectuels qui imprègnent diversement la pensée des auteurs selon d'où et à quel moment ils 
parlent. Il s'agit de courants tels que le positivisme, le darwinisme, le vitalisme, le romantisme, le naturalisme, l'internationalisme, l'idéalisme, le pragmatisme qui apparaissent souvent «entre les lignes» mais colorent fortement le discours de certains auteurs. Enfin, Pour l'Ère Nouvelle est un carrefour car elle permet la rencontre entre la science et la militance, quelles que soient les formes que prennent l'une et l'autre. La science s'y décline en une quantité de concepts, approches et théories qui toutes participent à l'élaboration d'une science de l'éducation. La militance prend deux types de formes durant la période étudiée: celle d'une conception spiritualiste de l'éducation visant à changer la posture de l'adulte vis à vis de l'enfant, dans les années 1920, et celle d'un engagement politique et social ambitionnant de changer les pratiques d'enseignement et les systèmes éducatifs, dans les années 1930. Si ces deux types de militance se distinguent dans leurs objectifs spécifiques, ils se rejoignent dans la volonté de susciter l'adhésion du plus grand nombre à la cause de l'Éducation nouvelle, à la cause d'une réforme éducative à grande échelle. En cela, Pour l'Ère Nouvelle est une revue militante, puisqu'elle cherche à «faire mouvement», à fédérer ses partisans malgré la diversité de personnes, d'idées et de courants qui la composent.

\section{SCIENCE ET MILITANCE:}

\section{LES PARADOXES D'UNE SCIENCE EN CONSTRUCTION}

À l'issue de notre enquête, nous parvenons à une conclusion paradoxale: dans ce vaste chantier, la science occupe une place à la fois centrale et marginale. Centrale parce qu'elle est constamment présente dans les discours de ses promoteurs, utilisée pour légitimer les revendications de réforme, et marginale, parce que ses caractéristiques emblématiques (savoirs, congrès, institutions, acteurs, thématiques) sont peu visibles. L'analyse quantitative du point de vue des thématiques révèle une présence mineure d'articles à caractère purement scientifique par rapport aux articles abordant des questions pédagogiques (méthodes d'enseignement, pédagogie générale). De même, les articles de genre connaissance mobilisant des savoirs scientifiques sont minoritaires par rapport aux articles dits intermédiaires ou praxéologiques qui privilégient les savoirs d'expérience. Du côté des acteurs, les scientifiques qui écrivent dans ce périodique sont peu nombreux comparativement aux représentants du domaine «pédagogique» (instituteurs, professeurs, inspecteurs, 
directeurs) qui utilisent la revue comme outil de diffusion de leurs idées et expériences. Un même déséquilibre se perçoit dans les congrès de la Ligue dans lesquels la grande majorité des participants est constituée de personnes issues des milieux éducatifs et non des réseaux scientifiques. Enfin, les institutions dont il est question dans PEN sont massivement des institutions pédagogiques telles que classes, écoles, jardins d'enfants, foyers éducatifs alors que les instituts de recherche à caractère plus scientifique sont minoritaires.

En revanche, l'analyse qualitative permet de constater la place centrale qu'occupe la science dans les discours des auteurs. Une science qui est convoquée au sens générique du terme - incluant aussi bien les sciences naturelles que les sciences sociales - dont on glorifie la dimension philosophique: la science est considérée comme universelle, dépassant les frontières, se situant au-dessus des différences de race, de sexe, de culture et de religion. Elle est investie du pouvoir suprême de rassembler les hommes autour de mêmes lois, de mêmes vérités capables de les réunir dans un même mouvement de transformation de la société. Elle porte ici l'héritage du $19^{\mathrm{e}}$ siècle durant lequel on attribue à la science le pouvoir de résoudre les problèmes sociaux, pouvoir auquel les partisans de l'Éducation nouvelle croient fortement. La science est synonyme d'espoir: espoir de solutionner les problèmes scolaires, de faire disparaître le caractère dogmatique, sclérosé et autoritaire d'une éducation contre laquelle ils s'opposent. À ce niveau, le terme science est moins générique qu'auparavant; il désigne ici la psychologie ou la science de l'enfant considérée alors comme le "guide du praticien» car elle seule semble capable d'affirmer des vérités et donc d'orienter les pratiques. Selon les partisans de l'Éducation nouvelle, la science permettra à l'école de mieux réussir car elle donne au praticien les moyens de connaître celui qu'il doit éduquer, à l'instar de Claparède (1905) qui pense que «l'école échoue parce que les éducateurs ignorent l'enfance».

De manière générale, les discours tenus dans la revue font apparaître une double attitude par rapport à la science. D'une part, les acteurs lui accordent une forte crédibilité en raison des pouvoirs et espoirs dont elle est porteuse, d'autre part elle suscite tensions et désaccords parmi ceux qui tentent de mettre ses principes en application. On constate une sorte de consensus général sur la valeur de la science, sur le fait que désormais, la pédagogie doit être fondée sur une connaissance scientifique de l'enfant. C'est précisément ce qui distingue l'Éducation nouvelle de 
l'école dite traditionnelle. Par contre, les manières de traduire les observations scientifiques dans la pratique éducative suscitent débats et controverses. Dans ses colonnes, Pour l'Ère Nouvelle rend visible cette diversité de points de vue: les uns prônent des méthodes par projet, d'autres les centres d'intérêts, d'autres le travail libre par groupes, d'autres encore des plannings de tâches individualisés. Au-delà des méthodes, le débat est âpre sur des questions nodales comme les programmes scolaires, l'évaluation, les examens, questionnant leur but, leur contenu, leur organisation, leur utilisation.

En résumé, tout se passe comme s'il y avait consensus sur le fond mais pas sur la forme. Â ce propos, les congrès constituent des moments particulièrement intéressants pour voir comment se manifestent ces tensions. Alors que les conférences des scientifiques semblent faire l'unanimité, celles des praticiens suscitent des prises de position contrastées. On serait tenté de croire ici qu'il y a accord sur les savoirs apporté par les premiers, mais désaccords sur les applications proposées par les seconds. Pourtant, les discours des scientifiques suscitent aussi des réactions ambivalentes en raison du glissement qui tend à se produire - surtout dans les années 1930 - entre le rôle descriptif et le rôle prescriptif de la science. Alors que les outils issus de la psychologie (tests, mesures, observations, etc.) sont censés aider les éducateurs à mieux connaître l'enfant, ils tendent à devenir prescriptifs et à indiquer la voie prétendue correcte pour garantir l'efficacité des méthodes.

Outre ces différentes fonctions, on peut se demander pourquoi la science est si peu visible dans Pour l'Ère Nouvelle au niveau des thématiques d'articles, des institutions ou des acteurs qui la représentent. À ce stade de nos recherches, nous pouvons l'interpréter de plusieurs manières. Tout d'abord, ces données révèlent le caractère particulier de la science qui est en train de se construire: il s'agit d'une science expérimentale s'intéressant à l'enfant et à son éducation. De ce fait, les expériences se déroulent sur des terrains qui accueillent des enfants dans un but éducatif, à savoir les écoles et autres structures éducatives. C'est pourquoi ce sont ces institutions qui apparaissent massivement dans la revue, les instituts de recherche étant plutôt marginaux. Dans ces écoles, les scientifiques collaborent avec les praticiens qui participent à la conduite des recherches, mènent des entretiens, consignent les résultats d'observation. De ce fait, on peut supposer que ce sont eux qui écrivent l'essentiel des articles relatant des expériences pédagogiques, ceux-ci étant classés dans le domaine «pédagogie générale» ou «documentation 
pédagogique». Enfin, la faible présence des scientifiques dans les congrès s'explique par le fait que ceux-ci y participent avant tout en tant que conférenciers et non comme participants. Ainsi, ils représentent un petit pourcentage de personnes mais dont l'importance est incontestable. Comme le montrent les rapports de congrès, les groupes ou commissions de psychologie saisissent ces occasions pour mener des réflexions communes qui font ensuite l'objet de conférences plénières et de comptes-rendus séparés attestant de l'importance accordée à cette science.

Toutefois, les résultats contradictoires de ces deux analyses apportent des éléments précieux sur la place marginale qu'occupe la science dans l'Éducation nouvelle. De fait, celle-ci apparaît essentiellement comme une science convoquée, sollicitée, voire «utilisée» à des fins moins scientifiques qu'il n'y paraît. À la suite d'autres chercheurs, nos résultats montrent que les militants réformistes convoquent la science pour légitimer leurs revendications de réforme. Ils en ont besoin pour cautionner leurs démarches auprès de leurs pairs, des parents, de l'opinion publique et surtout auprès des pouvoirs politiques et l'administration scolaire. Car, pour pouvoir espérer une généralisation des méthodes nouvelles à l'ensemble du système, il faut que celles-ci soient reconnues par les politiques qui détiennent le pouvoir de voter des lois et mettre en place des réformes. Mais au-delà du plaidoyer pour une pédagogie scientifique s'ajoutent des revendications philosophiques et éthiques sur une certaine conception de l'homme et de la société. L'analyse détaillée des discours montre que la science n'est qu'un des moyens pour atteindre l'idéal de société rêvé par les promoteurs de l'Éducation nouvelle dont la paix, la tolérance, la solidarité et la coopération seraient les fondements. La science est donc utilisée pour cautionner des revendications qui sont marginalement scientifiques, et davantage éthiques et philosophiques.

L'analyse de Pour l'Ère Nouvelle fait apparaître d'autres forces en présence qui occupent une place centrale dans les discours. Il s'agit d'une dimension quasi insaisissable à une première lecture mais qui traverse l'ensemble des articles même si elle tend à s'estomper au cours des années 1930. Il s'agit d'une confiance inébranlable en la science et en l'enfant qui rassemble ces militants dans un même élan, dans un même engagement pour la cause de l'éducation. Une dimension qui prend des noms et des teintes différents selon l'époque et l'auteur qui l'évoque mais que l'on pourrait rassembler derrière le mot confiance, un mot qui 
serait le dénominateur commun entre le spiritualisme de Ferrière, le mysticisme des théosophes et le militantisme des leaders du Groupe Français d'Éducation Nouvelle. Il y a tout d'abord les convictions spiritualistes des dirigeants de la Ligue qui apparaissent clairement dans nombre de leurs écrits. De façon récurrente, ceux-ci utilisent un vocabulaire religieux où des mots tels que «temple», «cathédrale» évoquent les lieux, des verbes tels que «convertir», "prêcher», «libérer l'âme de son prochain» en évoquent les pratiques, tandis que des mots comme «salut de l'enfant», «rénovation spirituelle», «suprématie de l'esprit» évoquent certains des principes-même de l'Éducation nouvelle. Les principes de ralliement en sont fortement imprégnés jusqu'à ce que les membres du GFEN, Wallon en particulier, retirent précisément cette dimension lors de leur reformulation en 1932. Si les références explicites à la notion de «force intérieure» que l'éducation aurait pour tâche de faire émerger disparaissent dans les années 1930, nombre d'articles font référence à un "potentiel d'éducabilité» (Hameline, 1986) se rapprochant des convictions spiritualistes selon lesquelles il existe en chaque enfant un potentiel d'apprentissage. Des convictions qui donnent toute leur raison d'être à l'Éducation nouvelle respectant l'enfant, reconnaissant ses droits et s'attachant à faire émerger ce potentiel. Ainsi, au-delà du spiritualisme des années 1920 et du rationalisme des années 1930, on peut dire que ces acteurs partagent une profonde foi en l'enfant.

Au-delà d'une foi en l'enfant, c'est une véritable foi en la vie 1 qui semble animer ces acteurs. En effet, les ambitions universalistes de l'Éducation nouvelle dépassent les limites de la pédagogie et de la science pour toucher à des considérations philosophiques. Or, cette foi en la vie n'est-elle pas le propre des savants qui étudient le monde du vivant? Nous pourrions ainsi expliquer pourquoi plusieurs figures de ce mouvement s'intéressent au monde animal, à l'exemple de Piaget qui fait ses premières recherches scientifiques sur les mollusques, ou des membres de l'Institut Rousseau qui étudient l'intelligence des singes et des poules. Le monde animal, qui plus est celui d'espèces simples sur le plan génétique, permet d'étudier le concept d'évolution mais il révèle aussi un intérêt passionné pour la vie, sous quelque forme que ce soit. Ainsi, d'un intérêt pour la vie découle naturellement un intérêt pour l'enfant qui conduit à l'idée selon laquelle en protégeant l'enfant, on protège la vie.

1 Une expression tirée du livre d'Ellen Key Le siècle de l'enfant (1908). 
À cette époque l'enfant est considéré comme une incarnation du principe même d'évolution, raison pour laquelle il devient un objet d'étude pour de nombreux savants. Il incarne à la fois l'origine de l'espèce et son devenir puisque c'est en lui que toute évolution se rejoue et se poursuit. Il est investi de tous les espoirs de changer la société car c'est à travers lui, et donc à travers son éducation, que l'on peut espérer former un homme et un monde meilleurs. De plus, l'enfant, et en particulier le petit enfant, constitue une incarnation de la Nature puisqu'il est considéré comme l'être le plus proche de l'état naturel. S'inspirant de Rousseau, les partisans de l'Éducation nouvelle considèrent l'enfant comme un être, bon par nature, qu'il faut protéger des mauvaises influences et éduquer selon ses besoins.

Chez les partisans de l'Éducation nouvelle, ces convictions philosophiques ne restent pas au niveau du discours. Ils concrétisent leurs idées en s'engageant dans des mouvements dédiés à une cause sociale, politique et/ou religieuse. Comme nous l'avons montré, ces acteurs se caractérisent par leur double appartenance aux mondes scientifique et militant étant actifs dans des milieux associatifs ou politiques. Toutefois, il reste difficile à comprendre comment ces acteurs parviennent à cumuler leur activité scientifique et leur activité militante, celle-ci étant même parfois teintée de religiosité (surtout dans les années 1920). Nous pouvons l'expliquer par le fait qu'ils sont tous inscrits dans un courant scientifique qui se questionne sur l'être en devenir, et cherche à comprendre comment fonctionne l'esprit humain. De fait, l'étymologie du mot psychologie veut dire «science de l'âme» et nous savons que les débuts de la psychiatrie et de la psychanalyse partageaient des champs d'études communs investiguant les domaines de l'inconscient au moyen de l'hypnose ou du spiritisme notamment. Les scientifiques qui interviennent dans les premiers congrès de la Ligue (Calais, Heidelberg, Montreux) ne sont autres que C. G. Jung, E. Krafft, E. Marcault, M. Buber, tous très imprégnés de ces courants qui s'apparentent davantage à la psychologie de l'inconscient qu'à la psychologie de l'enfant.

Pour certains, la dimension spirituelle est un équilibre nécessaire pour être forts au milieu d'une société en constante évolution. Un équilibre particulièrement nécessaire à l'éducateur qui doit transmettre des valeurs de sérénité, écoute et tolérance. Selon Ensor, «nous devons dilater notre conscience, intensifier notre vie intérieure» pour pouvoir résister à l'époque de machinisme que traverse la société: 
Dans notre mouvement d'Éducation nouvelle, nous ne pouvons être forts que si notre centre de gravité est ferme, que si nous possédons le calme et l'harmonie, que si nous accordons notre petite conscience personnelle au grand Inconscient. Ainsi seulement nous pouvons aider l'enfant à s'adapter à contrôler ses énergies intimes, et lui enseigner à faire des réserves de force et d'harmonie afin qu'il puisse supporter la tension du monde moderne. (Ensor, PEN, 1929, 51, p. 221)

Si la militance des années 1920 est fortement teintée de spiritualisme, celle des années 1930 prend une toute autre forme qui s'inspire davantage du modèle politique. Nous attribuons ce changement d'une part au contexte environnant (crise économique et montée des nationalismes en Europe) et, d'autre part, aux changements qui ont lieu à la tête de Pour l'Ère Nouvelle dirigée dès 1929 par un groupe engagé politiquement. Le discours philosophique et spiritualiste de la première décennie fait place à un discours politique plus ancré dans la réalité qui traduit la volonté de "passer à l'action» et d'opérer des changements concrets dans le sens proposé par la science. Comme l'ont montré d'autres historiens, la militance du GFEN permettra de susciter le débat, de sensibiliser l'opinion publique, et surtout d'interpeller les instances politiques sur la nécessité d'une réforme profonde de l'école. De fait, c'est surtout la double activité scientifique et militante de personnalités comme Langevin, Wallon ou Piéron qui va permettre de convaincre les milieux politiques français de soutenir des projets de réforme tels que les «classes d'orientation» ou le Plan Langevin-Wallon. Mais cette double activité occasionne aussi des tensions en raison des enjeux de reconnaissance et d'appartenance qui $\mathrm{s}^{\prime} \mathrm{y}$ jouent. En effet, la militance tend parfois à prendre le dessus sur la science, créant ainsi un déséquilibre entre les deux sphères d'activité de ces scientifiques. Que ce soit l'engagement de Wallon au sein du Parti communiste ou celui des dirigeants de l'Institut Rousseau dans d'autres sphères, ils suscitent la méfiance de leur entourage qui leur reproche de négliger leur activité première, soit l'activité scientifique. Ainsi, paradoxalement, les scientifiques les plus militants sont souvent les plus controversés au sein de leur communauté, laissant penser à une incompatibilité entre science et militance.

À l'exception de ces contextes particuliers, on peut dire que la militance qui caractérise l'Éducation nouvelle est avant tout une «force motrice». Qu'elle se manifeste à travers des discours spiritualistes ou des luttes politiques, cette militance paraît consubstantielle à la propagation des principes réformistes et de la science de l'éducation que ses pro- 
moteurs s'attachent à construire. Tout se passe comme si la nouvelle science avait besoin des militants pour se faire reconnaître au sein des communautés scientifique, pédagogique et politique. Les associations telles que la Ligue internationale pour l'Éducation nouvelle et les revues qu'elles publient jouent ainsi un rôle crucial dans ces efforts de reconnaissance permettant aux nouveaux savoirs de circuler et de se confronter aux sphères dans lesquelles ils sont appelés à se déployer.

\section{SCIENTIFIQUES ET MILITANTS À LA RECHERCHE D'UNE SYNTHÈSE}

Un mot revient de manière récurrente dans les discours de Pour l'Ère Nouvelle, que ce soit dans les années 1920 ou les années 1930, dans la bouche des scientifiques ou dans celle des praticiens, dans les régions du Nord ou du Sud: le mot «synthèse». Ce terme s'accompagne très souvent du mot «recherche» pour exprimer ce qui semble être le véritable objectif que poursuivent tous ces acteurs: la «recherche d'une synthèse». Mais de quelle synthèse s'agit-il? Si la réponse à cette question n'apparaît jamais explicitement dans les colonnes de la revue, les investigations menées dans le cadre de cette recherche nous permettent de formuler des hypothèses fortes.

Les scientifiques qui étudient l'enfant expriment de façon récurrente l'objectif qui les anime: la recherche de lois. La loi est comme l'emblème de la méthode scientifique à laquelle ils veulent former les enseignants de même que les enfants dont ceux-ci auront la charge. Une méthode qui se compose de plusieurs étapes où la loi constitue l'étape ultime à laquelle on parvient après être passé par l'observation et par l'analyse des résultats. Une méthode où l'expérimentation constitue l'élément-clé et à laquelle s'initient dès leur plus jeune âge les élèves des écoles nouvelles. La recherche de lois est donc un thème récurrent dans les discours de PEN dont le but est de trouver une forme de synthèse. Ce désir apparaît de manière particulièrement aiguë lors du Congrès d'Elseneur dont le thème, la «Psychologie nouvelle», rassemble une large diversité de psychologues - Lewin, Wallon, Boyd, Boven, Köhler, Krafft - étudiant la question selon différentes approches qui se distinguent au fil des ans (psychologie génétique, psychologie individuelle, psychologie de l'inconscient, etc.). Ainsi, pour les dirigeants de la Ligue, le but du congrès est précisément de parvenir à une synthèse de ces approches, à une synthèse des psychologies pour pouvoir donner 
une orientation claire à la pédagogie nouvelle. Dans ce cas, il s'agit avant tout des lois psychologiques, plus précisément des lois du développement de l'enfant: lois génétiques qui permettraient de déterminer des types psychologiques et les caractères. Mais la revue fait référence à d'autres lois émanant d'autres sciences, à commencer par la biologie et les lois de l'évolution des espèces à partir desquelles il y aurait des lois biogénétiques régissant l'être humain; les lois de la physique et de l'astronomie évoquées en particulier dans les discours de Langevin, qui selon lui, ne doivent pas être établies a priori mais par l'expérience; les lois naturelles qui caractérisent l'évolution de l'enfant; enfin, les lois supérieures à l'homme, celles d'un monde «divin» qui régirait l'espèce humaine, quel que soit le nom que prend cette loi (Dieu, force, esprit.). Par la diversité de ses théories et savoirs de référence, l'Éducation nouvelle incarne une sorte de «synthèse des sciences» où se mêlent à la fois la pédagogie, la philosophie, la physique, la médecine, la psychologie.

Cette recherche de synthèse trouve ses raisons principales dans le désir d'application d'une science plutôt que des sciences afin d'en faciliter la mise en pratique. Alors qu'ils revendiquent une ouverture à toutes les pratiques, méthodes et courants de pensée, les dirigeants de la Ligue cherchent à les rassembler en un seul mouvement fédérateur. Selon eux, c'est ici que réside la particularité de l'Éducation nouvelle car c'est à elle qu'incombe le mandat de réaliser cette synthèse. Pour Ensor, cette synthèse constitue l'une des plus importantes «croisades» de la Ligue à l'échelle universelle:

Depuis que nous sommes assemblés ici, venus de toutes les parties du monde, une question s'est imposée à notre attention: celle de savoir quelle serait l'idée, quel serait le mode d'éducation qui pourrait réunir tous les hommes de tous les pays et tous les hommes de chaque pays. (Ensor, PEN, 1929, 51, p. 218)

Pour Ferrière et Ensor, la synthèse entre théorie et pratique permettra de trouver une voie qui convienne à tous, alliant science et expérience. Il $\mathrm{s}^{\prime}$ agit de trouver un terrain de rencontre entre ce qui représente les emblèmes de ces deux pôles, la psychologie et la pédagogie. Les partisans plaident pour l'application de la psychologie à la pédagogie incarnée par la psychopédagogie - bien que ce passage suscite beaucoup de problèmes en raison des divergences d'interprétation. Comme nous l'avons vu, de nombreux acteurs incarnent une hybridité entre théorie et 
pratique puisqu'ils sont à la fois scientifiques (chercheurs, universitaires) et "praticiens-expérimentateurs» sur le terrain éducatif où ils mènent leurs recherches. ${ }^{2}$ Selon Langevin, développer l'esprit scientifique au niveau de l'éducation permettrait de résoudre les maux sociaux car cet esprit joue un rôle crucial dans la recherche d'une unité de culture entre les hommes. Une unité ou «synthèse» qui dépasse les ambitions de l'éducation:

Le remède au déséquilibre social [dépend] d'une introduction plus large de la science, conçue dans l'esprit plus que dans ses résultats. L'effort d'adaptation de l'esprit à la réalité que représente la science peut et doit servir de base à la synthèse que nous cherchons, fournir le lien nécessaire entre le monde matériel et le monde moral pour préparer à la fois la maîtrise sur les choses et l'harmonie entre les hommes. (Langevin, PEN, 1932, 81, p. 241)

Comme le montre cet extrait, $c^{\prime}$ est bien de la science au sens générique du terme qu'il s'agit. Une science comme «état d'esprit» qui doit contribuer à changer les mentalités. Or, l'esprit scientifique, par essence, est progressiste puisqu'il est en mouvement perpétuel, s'adapte aux changements, se remet en question et construit pour l'avenir. En cela, le terme Progressive Education, utilisé aux États-Unis pour désigner l'Éducation nouvelle, définit bien cet état d'esprit ouvert au changement, tourné vers l'avenir, basé sur une vision progressiste du monde. Pour opérer cette transformation de mentalités à large échelle, l'Éducation nouvelle a besoin des scientifiques car ceux-ci peuvent mettre leur savoir au service de l'éducation, et ainsi garantir le progrès de la société et de l'humanité. Dès lors, les scientifiques ont un rôle crucial à jouer non seulement sur le plan théorique mais aussi sur le plan pratique, en s'engageant pour cette cause, en s'investissant dans des projets concrets qui favorisent ce changement de mentalité. Or, quoi de plus concret que l'éducation et l'enseignement? C'est ainsi que des savants comme Langevin ou Wallon décident de militer au sein des

2 Une synthèse qui n'est pas toujours considérée comme telle par certains praticiens qui persistent à considérer les théoriciens comme détachés de la pratique. C'est ce que montre Jornod (1989) à propos de Claparède qui reçoit des remarques désobligeantes de la part des syndicats d'instituteurs romands au sein desquels les praticiens ne voient pas les théoriciens d'un très bon œil. 
groupes d'Éducation nouvelle car c'est là que se trouvent le dynamisme et la structure nécessaire pour mener à bien leur action.

Pour atteindre une échelle universelle, la synthèse que recherchent les protagonistes de l'Éducation nouvelle doit être aussi une synthèse entre science et morale, voire entre science et spiritualité. Certains courants religieux sont à la recherche de lois et de modes de pensée universels qui ne sont pas loin des valeurs morales défendues par l'Éducation nouvelle. Alors que dans les conceptions occidentales science et morale sont généralement opposées, Langevin pense que ce n'est pas le cas et que l'Éducation nouvelle doit réaliser la synthèse qui prouve qu'elles ne le sont pas:

Il y a une opposition, un conflit aigu entre la thèse morale et l'antithèse scientifique, conflit qui ne peut-être résolu que par une synthèse, et c'est cette synthèse que l'Éducation nouvelle doit contribuer à réaliser par la solution du problème de la culture. (Langevin, PEN, 1932, 81, p. 241)

D'après Langevin, c'est par la «solution du problème de la culture» que l'Éducation nouvelle pourra faire cette synthèse. Sur le plan pratique, cela se traduit par des attitudes et des conceptions pédagogiques. Tout d'abord, explique-t-il, ce sont les savoirs scolaires qu'il faut considérer autant que possible comme un ensemble car «le côte à côte mène toujours au dogmatisme». Comme nous l'avons vu, les éducateurs nouveaux plaident pour ne pas séparer les disciplines scolaires et privilégient des méthodes qui incluent tous les savoirs dans une même activité ou projet: ce faisant, ils opèrent une «synthèse des savoirs». Plusieurs auteurs de $P E N$ revendiquent une synthèse de l'enseignement théorique et pratique en proposant des activités qui favorisent l'observation et l'expérience. Selon eux, l'enseignement doit non seulement diffuser la science - faire connaître les théories scientifiques - mais aussi la pratiquer à travers l'initiation à la méthode scientifique. Pour Ferrière, l'Éducation nouvelle est plus qu'une synthèse scientifique; elle serait en fait, une combinaison entre «la science et le bon sens», une sorte de synthèse entre la connaissance et l'intuition humaine «unies dans une collaboration féconde» (Ferrière, PEN, 1928, p. 3). 


\section{BIBLIOGRAPHIE}

\section{SOURCES}

Archives Institut Jean-Jacques Rousseau (AIJJR)

- Revue Pour l'Ėre Nouvelle (1922-1940)

- Guide du voyageur s'intéressant aux écoles. Genève, Bureau international d'éducation, 1927, 80 pages (édition provisoire). Dossier Guide Baedeker Fonds Général. Archives Institut J.-J. Rousseau, cote: FG.Baedeker

- Fonds général

- Fonds Adolphe Ferrière

Centre de documentation du Bureau International d'Éducation (UNESCO)

- Revue Education for the New Era (1920-1921)

- Revue The New Era (1922-1940)

- Revue Internationale Erziehungs Rundschau (1920-1921)

- Revue Das Werdende Zeitalter (1922-1932)

- Revue Pour l'Ère Nouvelle - Les Sciences de l'Éducation (1946-2000)

\section{SOURCES PUBLIÉES}

Bovet, P. (1932). Vingt ans de vie: L'Institut J.-J. Rousseau de 1912 à 1932. Neuchâtel: Delachaux \& Niestlé.

Claparède, E. (1905/1909/1911). Psychologie de l'enfant et pédagogie expérimentale. Genève: Kündig.

Claparède, E. (1920). L'école sur mesure. Lausanne et Genève: Payot \& Cie.

Claparède, E. (1931). L'éducation fonctionnelle. Neuchâtel: Delachaux \& Niestlé.

Dewey, J. (1913/1922). L'école et l'enfant. Neuchâtel: Delachaux \& Niestlé. Dewey, J. (1916/1944). Démocratie et éducation. Paris: A. Colin.

Dottrens, R. (1931). Le problème de l'inspection et l'éducation nouvelle. Neuchâtel et Paris: Delachaux \& Niestlé. 
Ferrière, A. (1919). L'École nouvelle et le Bureau international des Écoles Nouvelles. Bâle: Azed.

Ferrière, A. (1920). Transformons l'école: appel aux parents et aux autorités. Bâle: BIEN.

Ferrière, A. (1921). L'autonomie des écoliers. Neuchâtel et Paris: Delachaux \& Niestlé.

Ferrière, A. (1922). L'école active. Neuchâtel \& Genève: Forum.

Ferrière, A. (1924). La pratique de l'école active. Neuchâtel \& Genève: Forum.

Ferrière, A. (1928). Trois pionniers de l'Éducation nouvelle: Hermann Lietz, Frantisek Bakulé, Giuseppe Lombardo-Radice. Paris: Flammarion.

Key, E. (1908). Le siècle de l'enfant. Paris: Flammarion.

Landauer, G. (1921). Der werdende Mensch. Aufsätze über Leben und Schriftum. Potsdam: M. Buber.

Meyhoffer, P. \& Gunning, W. (1929). Adolphe Ferrière. Notices biographiques et bibliographiques à l'occasion du cinquantième anniversaire de sa naissance. [s.l.]

Piaget, J. (1923). Le langage et la pensée chez l'enfant. Paris: Delachaux et Niestlé.

Piaget, J. (1936). La naissance de l'intelligence chez l'enfant. Paris: Delachaux et Niestlé.

Programme officiel du Troisième congrès bisannuel - Third biennal Conference of the World Federation of Education Associations (WFEA) (1929). Genève: BIE.

Société Pédagogique Romande (1924). XXI congrès de la Société Pédagogique Romande. Genève: Sonor.

Theosophical Society General, C. \& Jinarajadasa, C. (1925). The golden book of the Theosophical Society: a brief history of the Society's growth from 1875-1925. Adyar, Madras: Theosophical Publishing House for the General Council of the Theosophical Society.

\section{RÉFÉRENCES BIBLIOGRAPHIQUES}

Ariès, P. (1960). L'enfant et la vie familiale sous l'Ancien Régime. Paris: Plon. Avanzini, G. (1995). L'Éducation nouvelle et ses concepts. In D. Hameline, J. Helmchen \& J. Oelkers, L'éducation nouvelle et les enjeux de son histoire (pp. 65-74). Berne: Peter Lang. 
Bandini, G. (2010). La Franc-maçonnerie et le droit à l'instruction: de l'Unité italienne jusqu'au début du XXe siècle. Paedagogica Historica, $46(1 \& 2), 161-177$.

Battistolo, E. (2009). L'Éducation nouvelle et Jean-Jacques Rousseau: entre revendication légitime et filiation fantasmée. Mémoire de licence en sciences de l'éducation, Université de Genève.

Bensaude-Vincent, B. (1987). Langevin (1872-1946). Science et vigilance. Grenoble: Presses universitaires de Grenoble.

Bloch, M.-A. (1968). Philosophie de l'Éducation nouvelle (2e éd.). Paris: PUF.

Boyd, W. \& Mackenzie, M. (1930). Towards a New Education. London: Knopf.

Boyd, W. \& Rawson, W. (1965). The story of the New Education. London: Heinemann.

Brehony, K.J. (2004). A New Education for a New Era: The Contribution of the Conferences of the New Education Fellowship to the Disciplinary Field of Education 1921-1938. Paedagogica historica, 40(5 \& 6), 733-755.

Brehony, K.J. (2006). Representations of Socialists Educational Experiments in the 1920s and 1930s. The Place of the Sciences of Education. In R. Hofstetter \& B. Schneuwly (Eds.), Passion, Fusion, Tension. New Education and Educational Sciences (pp. 271-304). Berne: Peter Lang.

Brehony, K.J. (2009). Transforming theories of Childhood and early childhood Education: child study and the empirical assault on Froebelian rationalism. Paedagogica Historica, 45(4 \& 5), 585-604.

Brehony, K.J. (article non publié). Routinisation wébérienne contre défaite politique: l'innovation pédagogique dans les pages de The New Era (1920-1939).

Bruno-Jofré, R. \& Schriewer, J. (Eds.) (2012). The Global Reception of John Dewey's Thought. London: Routledge.

Bruno-Jofré, R., Johnston, J.S., Jover, G. \& Tröhler, D. (2010). Democracy and the Intersection of Religion and Traditions. The Reading of John Dewey's Understanding of Democracy and Education. Montreal: McGill Queen's University Press.

Campos, R.F. (2012). Helena Antipoff, uma biografia intelectual. Rio de Janeiro: Fundação Miguel de Cervantes.

Campos, R.F. (2001). Helena Antipoff: a synthesis of Swiss and Soviet psychology in the context of Brazilian education. History of Psychology, 4(2), 133-158. 
Caspard, P. (1981). La presse d'éducation et d'enseignement, XVIII siècle-1940 (tome I). Paris: INRP, Éditions du CNRS.

Caspard, P. (2006). La presse d'éducation et d'enseignement. In T. Charmasson (Ed.), L'histoire de l'enseignement, XIX ${ }^{e}-X X^{e}$ siècles. Guide du chercheur (pp. 569-579). Paris / Lyon: CTHS / INRP.

Castanotto, F. (2007). De la mise en scène bourgeoise à l'avant-scène féministe (1879-1912): première socialisation et formation intellectuelle d'Émilie Gourd. Les Annuelles, 10, 47-94.

Certeau, M. de (1975). L'écriture de l'histoire. Paris: Gallimard.

Chappuis, D. (2005). Les états modifiés de conscience dans le théosophisme d'Helena Petrovna Blavatsky et Annie Besant ou les états modifiés de conscience. Mémoire de DEA en Histoire comparée des religions, Université de Lausanne.

Charle, C., Schriewer, J. \& Wagner, P. (Eds.) (2004). Transnational intellectual networks. Frankfurt: Campus.

Châtelet, A.-M. (2011). Le souffle du plein air. Genève: Métis Press.

Châtelet, A.-M., Lerch, D. \& Luc, J.-N. (Eds.) (2003). L'École de Plein Air, Open-Air Schools. Paris: Recherches.

Cicchini, M. (2004). Un bouillon de culture pour les sciences de l'éducation? Le Congrès international d'éducation morale (1908-1934). Paedagogica historica, 40(5\&6), 633-656.

Cicchini, M. (2007). Acteurs et réseaux des sciences de l'éducation: du local à l'international. In R. Hofstetter \& B. Schneuwly (Eds.), Émergence des sciences de l'éducation en Suisse, à la croisée de traditions académiques contrastées (fin du 19e-première moitié du 20e siècle) (pp. 345373). Berne: Peter Lang.

Clastres, P. (2004). Éducation libérale et Éducation nouvelle en France de Victor Lapade à Edmond Demolins. In A. Ohayon, D. Ottavi \& A. Savoye (Eds.), L'Éducation nouvelle, histoire, présence et devenir (pp. 93-121). Berne: Peter Lang.

Clavien, A., Le Dinh, D. \& Valloton, F. (1993). Jalons pour une histoire à faire: les revues romandes (1880-1914). Les Annuelles, 4, 7-28.

Condette, J.-F. \& Savoye, A. (2011). Le congrès du Havre (31 mai-4 juin 1936): Albert Châtelet et la réforme de l'enseignement du second degré. Carrefours de l'éducation, 31, 61-88.

Coquoz, J. (1998). De l'éducation nouvelle à l'éducation spécialisée. Un exemple suisse: le «Home chez Nous». Lausanne: LEP.

Coquoz, J. (2000). Un modèle suisse «d'École active» durant l'entredeux-guerres: images et mirages. Paedagogica Historica, 36(1), 369-388. 
Cremin, L. (1964). The Transformation of the School: Progressivism in American Education, 1876-1957. New York: Knopf.

Criblez, L. (2006). «Experimental pedagogy» in german-speaking Switzerland after 1900. Scientific bases for school reform? In R. Hofstetter \& B. Schneuwly (Eds.), Passion, Fusion, Tension. New Education and Educational sciences (end 19th-middle 20 th century) (pp. 3768). Berne: Peter Lang.

Criblez, L. \& Hofstetter, R. (2002). La professionnalisation des métiers de l'éducation à travers la tertiarisation de la formation. Schweizerische Zeitschrift für Bildungswissenschaften, 24(1), 5-26.

Cunningham, P. (2001). Innovators, networks, and structures: towards a prosopography of progressivism. History of Education, 30(5), 433-451.

Czáka, V. (en préparation). Former les corps et les âmes: histoire sociale et genrée de l'éducation physique en Suisse romande (fin 18e-début $20^{e}$ siècle). Université de Lausanne, Faculté des Lettres.

De Dardel, S. (1961, $1^{\text {er }}$ décembre). Les Quakers de Genève. La Vie Protestante.

Della Porta, D. \& Diani, M. (1999). Social Movements. An Introduction. Malden: Blackwell Publishing.

Delpal, B. (2014). L'album de Beauvallon: fondation et période historique de l'école (1929-1945). Rochechinard: Comptoir d'édition.

Del Pozo Andrés, M. (2009). The transnational and national dimensions of pedagogical ideas: the case of the project method (1918-1939). Paedagogica Historica, 45(4 \& 5), 561-584.

Del Pozo Andrés, M. (2013). Justa Freire o la pasión de educar. Barcelona: Octaedro.

Del Pozo Andrés, M. (2014). Community and the myth of the ideal school: Circulation and appropriation of the Hamburg Gemeinschaftsschulen in Spain (1922-1933). Paedagogica Historica, 50(5), 599-614.

Depaepe, M. (1987). Le premier (et dernier) congrès international de pédologie à Bruxelles en 1911. Bulletin de la Société Alfred Binet et Théodore Simon, 87(612), 28-54.

Depaepe, M. (1993). Zum Wohl des Kindes? Pädologie, pädagogische Psychologie und experimentelle Pädagogik in Europa und den USA, 18901940. Weinheim: Deutscher Studien Verlag.

Depaepe, M. (1998). La recherche expérimentale en éducation de 1890 à 1940: les processus historiques sous-jacents au développement d'une discipline en Europe de l'Ouest et aux États-Unis. In R. Hofstetter \& 
B. Schneuwly (Eds.), Le pari des sciences de l'éducation (pp. 29-56). Bruxelles: De Boeck.

Depaepe, M. \& D'hulst, L. (2011). An Educational Pilgrimage to the United States. Un pèlerinage pédagogique aux États-Unis. Travel Diary of Raymond Buyse, 1922. Carnet de voyage de Raymond Buyse, 1922. Louvain: Leuven University Press.

Drewek, P. \& Lüth, C. (Eds.) (1998). History of Educational Studies. Gent: CSHP.

Durand, G., Hofstetter, R. \& Pasquier, G. (2015). Les bâtisseurs de l'école romande. 150 ans du Syndicat des Enseignants Romands et de l'Éducateur. Genève: Georg.

Duval, N. (2009). L'École des Roches. Paris: Belin.

Farge, A. (1989). Le goût de l'archive. Paris: Seuil.

Fessard, A. (1949). Henri Piéron. L'Année psychologique, 50, 7-13.

Fontaine, A. (2015). Aux heures suisses de l'école républicaine. Un siècle de transferts culturels et de déclinaisons pédagogiques dans l'espace francoromand. Paris: Demopolis.

Fuchs, E. (2004). Educational Sciences, Morality and Politics: International Educational Congresses in the Early Twentieth Century. Paedagogica Historica, 40(5 \& 6), 757-784.

Fuchs, E. (2007a). Networks and the History of Education. Paedagogica Historica, 43(2), 185-197.

Fuchs, E. (2007b). The creation of New International Networks in Education: The League of Nations and Educational organizations in the 1920s. Paedagogica Historica, 43(2), 199-209.

Fuchs, E. (2012). Transnational Perspectives in Historical Educational Research. Comparativ Zeitschrift für Globalgeschichte und Vergleichende Gesellschaftsforschung, 22(1), 7-14.

Fuchs, E., Drewek, P. \& Zimmer-Müller, M. (2010). Internationale Rezeption in pädagogischen Zeitschriften im deutsch-amerikanischen Vergleich 1871-1945/50. Bestandsverzeichnis. Berlin: DIPF.

Garnier, B. (2008). Les combattants de l'école unique. Paris: INRP.

Gerber, R. (1989). Vie et œuvre d'Adolphe Ferrière (1879-1960). Chronologie de son existence. Genève: Université de Genève, FPSE: [s.n.]. Repéré à https: / / www.unige.ch / archives / aijjr / files / 6214/1709/9804/R_Ger ber_petit.pdf

Gingras, Y. (2002). Les formes spécifiques de l'internationalité du champ scientifique. Les Actes de la recherche en sciences sociales, 141-142, 31-45. 
Go, H.-L. (2013). Dewey, penseur de l'éducation. Nancy: Presses universitaires de Nancy.

Go, H.-L. \& Riondet, X. (2015). À côté de Freinet. Nancy: Presses universitaires de Nancy / Éditions universitaires de Lorraine.

Goodchild, L.F. (2006). The Beginnings of Education at American Universities. In R. Hofstetter \& B. Schneuwly (Ed.), Passion, fusion, Tension. Éducation nouvelle et sciences de l'éducation (fin du 19e-milieu du 20 siècle) (pp. 69-105). Berne: Peter Lang.

Gratiot-Alphandéry, H. (1994). Henri Wallon. Perspectives, 24(3/4), 821835.

Gründer, H.U. (2007). Anarchistische Erziehung als libertäre Reformpädagogik. Geschichte-Modelle-Beispiele. Baltmannsweiler: Schneider.

Guénon, R. (1996). Le théosophisme: histoire d'une pseudo-religion. Paris: Éditions Traditionnelles.

Guey, E. (2012). À la croisée de l'Éducation nouvelle et de la délinquande juvénile: la protection de l'enfance en danger moral (19301945). In L. Gutierrez, L. Besse \& A. Prost (Eds.), Réformer l'école. L'apport de l'Éducation nouvelle (pp. 297-304). Grenoble: Presses universitaires de Grenoble.

Gutierrez, L. (2008). L'Éducation nouvelle et l'enseignement catholique en France (1899-1939). Thèse de doctorat en sciences de l'éducation, Université Paris VIII-Saint-Denis.

Gutierrez, L. (2010). La Ligue internationale pour l'Éducation nouvelle. Contribution à l'histoire d'un mouvement international de réforme de l'enseignement (1921-1939). Spirale. Revue de recherches en éducation, 45, 29-42.

Gutierrez, L. (2011). Les premières années du Groupe français d'éducation nouvelle (1921-1940). Recherches et Éducations, 4, 27-39.

Gutierrez, L., Besse, L. \& Prost, A. (Eds.) (2012). Réformer l'école. L'apport de l'Éducation nouvelle. Grenoble: Presses universitaires de Grenoble.

Gutierrez, L. \& Kounelis, C. (Eds.) (2010). Paul Langevin et la réforme de l'enseignement. Grenoble: Presses universitaires de Grenoble.

Gutierrez, L. \& Ohayon, A. (2010). Henri Wallon: le «deuxième homme». In L. Gutierrez \& C. Kounelis, Paul Langevin et la réforme de l'enseignement (pp. 71-86). Grenoble: Presses universitaires de Grenoble.

Haenggeli-Jenni, B. (2011). Savoirs constitués et programmes scolaires: un statut ambivalent dans la pédagogie de l'Éducation nouvelle. Recherches et Éducations, 4, 13-25. 
Haenggeli-Jenni, B. (2012). L'Éducation nouvelle: débats autour de définitions conceptuelles et identitaires (1920-1940). In L. Gutierrez, L. Bresse, \& A. Prost (Eds.), Réformer l'école. L'apport de l'Éducation nouvelle (1930-1970) (pp. 19-29). Grenoble: Presses universitaires de Grenoble.

Haenggeli-Jenni, B. (2015). Le rôle des femmes dans la circulation des savoirs éducatifs au sein de la Ligue internationale pour l'Éducation nouvelle (1920-1940). In J. Droux \& R. Hofstetter (Eds.), Globalisation des mondes de l'éducation. Circulations, connexions, réfractions (19e$20^{e}$ siècles) (pp. 76-96). Rennes: Presses universitaires de Rennes.

Haenggeli-Jenni, B. (sous presse). La Ligue internationale pour l'Éducation nouvelle: réseau international à la croisée de réseaux pacifistes et féministes de l'entre-deux-guerres (1920-1940). Actes du colloque "Femmes et réseaux dans les sociétés modernes et contemporaines. Réalités et représentations». Bordeaux: Presses universitaires de Bordeaux.

Haenggeli-Jenni, B., Fontaine, A. \& Bühler, P. (Eds.) (2014). Une circulation des idées pédagogiques sur papier. Presse d'éducation, transferts et trajectoires transnationales des savoirs (1850-2000). Revue suisse des sciences de l'éducation, 36(1).

Haenggeli-Jenni, B. \& Hofstetter, R. (2011). Pour l'Ėre Nouvelle (19221940). La science convoquée pour fonder une «internationale de l'éducation». Carrefours de l'Éducation, 31, 137-159.

Hai, A.A., Simon, F. \& Depaepe, M. (2015). Translating Ovide Decroly's ideas to Brazilian teachers. Paedagogica Historica, 51(6), 744-767.

Hall, W. (1938). Quaker International Work in Europe since 1914. Thèse en sciences politiques, Université de Genève.

Hameline, D. (1981). Autour d'Adolphe Ferrière et de l'éducation nouvelle (Cahiers de la Section des sciences de l'éducation, $\mathrm{n}^{\circ}$ 25, pp. 9-37). Genève: Université de Genève.

Hameline, D. (1984/2002). Changer l'école, Changer l'école! Il y a cent ans qu'on dit ça... In D. Hameline, L'éducation dans le miroir du temps (pp. 113-120). Lausanne: LEP.

Hameline, D. (1986). Courants et contre-courants dans la pédagogie contemporaine. Sion: ODIS.

Hameline, D. (1993a). Édouard Claparède (1873-1940). Perspectives, 23, 161-173.

Hameline, D. (1993b). Adolphe Ferrière (1879-1960). Perspectives, 23, 379406. 
Hameline, D. (2002a). L'éducation dans le miroir du temps. Lausanne: LEP.

Hameline, D. (2002b). Les premières années de Pour l'Ère nouvelle: militantisme et propagande? Les Sciences de l'éducation. Pour l'Ère nouvelle, $35,27-40$.

Hameline, D., Helmchen, J. \& Oelkers, J. (Eds.) (1995). L'éducation nouvelle et les enjeux de son histoire. Berne: Peter Lang.

Hameline, D., Jornod, A. \& Belkaïd, M. (1995). L'école active. Textes fondateurs. Paris: PUF.

Hartog, F. (2013). Croire en l'histoire. Paris: Flammarion.

Haubfleisch, D. (1997). Elisabeth Rotten (1882-1964) - eine (fast) vergessene Reformpädagogin. Marburg. Repéré à http / / : archiv.ub.uni-marburg.de / sonst/1996/0010.html

Haubfleisch, D. (2008). Elisabeth Rotten (1882-1964) - Netzwerkerin der Reformpädagogik. In M. Meierhofer, Entwicklung, Bildung, Erziehung. Beiträge für eine zeitgemäße Reformpädagogik (pp. 47-61). Zürich: Institut für das Kind.

Haubfleisch, D. \& Link, J.-W. (1994). Archivhilfe 8. Das Werdende Zeitalter (Internationale Erziehungs-Rundschau). Bonn: Archiv der Arbeiterjugendbewegung.

Helmchen, J. (1993) Reformpädagogik als pädagogischer Internationalismus? Eine Untersuchung am Beispiel der Beziehungen zwischen der francophonen Éducation Nouvelle und der deutschen Reformpädagogik im Zeitraum von 1900 bis 1933 (Habilitationsschrift) [Cd-Rom]. Oldenburg: Université d'Oldenburg.

Helmchen, J. (1995). L'éducation nouvelle francophone et la Reformpädagogik allemande - deux histoires? In D. Hameline, J. Helmchen \& J. Oelkers (Eds.), L'éducation nouvelle et les enjeux de son histoire (pp. 129). Berne: Peter Lang.

Helmchen, J. (1999). Wieviele Geschichten der Reformpädagogik gibt es? In J. Oelkers \& F. Osterwalder (Eds.), Die neue Erziehung. Beiträge zur Internationalität der Reformpädagogik. (pp. 69-98). Berne: Peter Lang.

Helmchen, J. (2009). Les savoirs autour de l'Éducation nouvelle. Paedagogica Historica, 45(4 \& 5), 673-684.

Helmich, K. (2014, septembre). Schoolfounder, Scholar, Editor or Cultural Ambassador? The Mediating Activity of Nemesné Müller Márta across Europe (1912-1939). Communication présentée à l'ENIUGH (European Congress on World and Global History), Paris. 
Hofstetter, R. (2004). The Construction of a New Science by Means of an Institute and its Communication Media: the Institute of Educational Sciences in Geneva (1912-1948). Paedagogica Historica, 40 (5 \& 6), 657684.

Hofstetter, R. (2008). L'avènement des sciences de l'éducation: le vivier genevois (fin $d u$ 19e-milieu $d u \quad 20^{e}$ siècle). Habilitation à diriger des recherches, Institut d'Histoire, Paris-Sorbonne IV.

Hofstetter, R. (2010). Genève: creuset des sciences de l'éducation. Genève: Droz.

Hofstetter, R. (2015). Building an «international code for public education»: Behind the scenes at the International Bureau of Education (1925-1946). Prospects, 45(1), 31-48.

Hofstetter, R., Magnin, Ch. \& Depaepe, M. (Eds.) (2006). New Education: Genesis and Metamorphoses. Paedagogica Historica, 42 (1 \& 2).

Hofstetter, R. \& Schneuwly B. (Eds.) (1998). Le pari des sciences de l'éducation. Bruxelles: De Boeck.

Hofstetter, R. \& Schneuwly, B. (Eds.) (2002). Science(s) de l'éducation (19e$20^{e}$ siècles). Entre champs professionnels et champs disciplinaires. Berne: Peter Lang.

Hofstetter, R. \& Schneuwly, B. (Eds.) (2004). The Role of Congresses and Institutes in the Emergence of the Educational Sciences. Paedagogica Historica (Special issue), 40 (5 \& 6).

Hofstetter, R. \& Schneuwly, B. (Eds.) (2006). Passion, Fusion, Tension. Éducation nouvelle et sciences de l'éducation (fin du 19e-milieu du $20^{e}$ siècle). Berne: Peter Lang.

Hofstetter, R., Schneuwly, B., Lussi, V. \& Haenggeli-Jenni, B. (2006). L'engagement scientifique et réformiste en faveur de la «nouvelle pédagogie». Genève dans le contexte international - premières décades du $20^{\mathrm{e}}$ siècle. In R. Hofstetter \& B. Schneuwly (Eds.), Passion, Fusion, Tension. Éducation nouvelle et sciences de l'éducation (fin du 19emilieu du 20e siècle) (pp. 107-142). Berne: Peter Lang.

Hofstetter, R. \& Schneuwly, B. avec la collaboration de Cicchini, M., Lussi, V., Criblez, L. \& Späni, M. (2007). Émergence des sciences de l'éducation en Suisse. À la croisée de traditions académiques contrastées (fin du 19e-première moitié du 20e siècle). Berne: Peter Lang.

Hofstetter, R. \& Schneuwly, B. (Eds.) (2009a). New Education at the Heart of Knowledge Transformations. Paedagogica Historica, 45 (4 \& 5).

Hofstetter, R. \& Schneuwly, B. (Eds.) (2009b). Introduction. Contrasted views of New Education on knowledge and its transformation. 
Anticipation of a new mode or ambivalence? Paedagogica Historica, $45(4 \& 5), 453-467$.

Hofstetter, R. \& Schneuwly, B. (Eds.) (2009c). Knowledge for teaching and knowledge to teach: two contrasting figures of New Education: Claparède and Vygostky. Paedagogica Historica, 45 (4 \& 5), 605-630.

Hofstetter, R. \& Schneuwly, B. (2013). The International Bureau of Education (1925-1968): a platform for designing a «chart of world aspirations for Education». European Education Research Journal, 12(2), 215-230.

Houssaye, J. (2002). Le bon professeur de l'Éducation nouvelle: persistance d'un modèle. Ou: petite histoire des rapports entre les Sciences de l'éducation et l'Éducation nouvelle. Les Sciences de l'éducation. Pour l'Ère nouvelle, 35, 47-62.

Huber, M. (2013). Henri Wallon: de la psychologie génétique aux pratiques émancipatrices de l'Éducation nouvelle. Lyon: Chronique sociale.

Hughes, J.P. (2015). Theory into practice in Australian Progressive Education: the Enmore Activity School. History of Education Review, 44(1).

Issenmann, C. (2007). L'École Vinet et la destination sociale de la femme (1884-1908). Les Annuelles, 10, 13-46.

Jablonka, I. (2014). L'histoire est une littérature contemporaine. Manifeste pour les sciences sociales. Paris: Seuil.

Jacquet-Francillon, F. (2004). L'enfant comme personne: un fondement culturel de l'Éducation nouvelle. In D. Ohayon, D. Ottavi \& A. Savoye, L'Éducation nouvelle: histoire, présence et devenir (pp. 29-45). Berne: Peter Lang.

Jenkins, C. (2000). New Education and its Emancipatory Interests (19201950). History of Education, 29(2), 139-151.

Jennings, J. (2007). Avant propos. Revue Mil neuf cent, 25, 3-5.

Jornod, A. (1989). Théorie/ pratique: fusion ou fission? L'union des journaux des «théoriciens» et des "praticiens» de l'éducation de 1921 à 1932: L'Intermédiaire des Éducateurs et L'Éducateur. Mémoire de licence en sciences de l'éducation, Université de Genève.

Julliard, J. (1987). Le monde des revues au début du siècle. Introduction. Cahiers Georges Sorel, 5, 3-9.

Keiner, E. (2002). Education between Academic Discipline and Profession in Germany after World War II. European Educational Research Journal, 1(1), 83-98. 
Koslowski, S. (2013). Die New Era der New Education Fellowship: ihr Beitrag zur Internationalität der Reformpädagogik im 20. Jahrhundert. Bad Heilbrunn, Allemagne: Julius Klinkhardt.

Knaupp, M. Schaufler, S. Hofbauer, S. \& Keiner, E. (2014). Education Research and Educational Psychology in Germany, Italy, and United Kingdom - an Analysis of Scholarly Journals. Revue suisse des sciences de l'éducation, 1, 83-106.

Labaree, D.F. (2004). The Trouble with Ed Schools. New Haven: Yale University Press.

Labaree, D.F. (2006). Progressisme, écoles, et education schools. Une romance américaine. In R. Hofstetter \& B. Schneuwly (Eds.), Passion, Fusion, Tension. New Education and Educational Sciences (end 19th-middle $\left.20^{\text {th }}\right)$ (pp. 305-324). Berne: Peter Lang.

Lamihi, A. (Ed.) (1997). Célestin Freinet et l'École Moderne. Vauchrétien: Ivan Davy.

Laudo, X. \& Vilanou, C. (2015). Educational discourse in Spain during the early Franco regime (1936-1943): toward a genealogy of doctrine and concepts. Paedagogica Historica, 51(4), 434-454.

Lawn, M. (Ed.) (2008). An Atlantic crossing? The work of the International Examination Inquiry, its Researchers, Methods and Influence. Oxford: Symposium Books.

Lawn, M. (Ed.) (2009). Modelling the Future. Exhibitions and the materiality of Education. Oxford: Symposium Books.

Le Bart, C. (1998). Le discours politique (Que sais-je?). Paris: PUF.

Le Goff, J. (1988). Histoire et mémoire. Paris: Gallimard.

Lepalczyk, I. \& Marynowicz E. (2003). Helena Radlinska: A portrait of the Person, Researcher, Teacher and Social Activist. In S. Hering \& B. Waaldijk (Eds.), History of Social Work in Europe (1900-1960) (pp. 7177). Leske \& Budrich: Opladen.

Le Roux-Yahiel, M. (1983). L'Histoire du Groupe français d'Éducation nouvelle de 1922 à 1962. Mémoire de maîtrise d'histoire contemporaine, Université de Paris, Panthéon-Sorbonne.

Lessard, C. \& Bourdoncle, R. (2002). Qu'est-ce qu'une formation professionnelle universitaire? Conceptions de l'université et formation professionnelle. Revue française de pédagogie, 139, 131-154.

Luc, J.-N. (2003). L'école de plein air: une histoire à découvrir. In A.-M. Châtelet, D. Lerch \& J.-N. Luc, L'école de plein air. Une expérience pédagogique et architecturale dans l'Europe du XXe siècle (pp. 7-14). Dijon: Focales. 
Lussi Borer, V. (2008). Formations à l'enseignement et sciences de l'éducation. Analyse comparée des sites universitaires de Suisse Romande (fin du 19epremière moitié $d u 20^{e}$ siècle). Thèse de doctorat en sciences de l'éducation, Université de Genève.

Lussi Borer, V. (2009). Les savoirs: un enjeu crucial de l'institutionnalisation des formations à l'enseignement. In R. Hofstetter \& B. Schneuwly (Eds.), Savoirs en (trans)formation. Au cour des professions de l'enseignement et de la formation (pp. 41-58). Bruxelles: De Boeck.

Madeira, A.I. (2012). Diffusion-reception Networks of Pedagogical Knowledge. The Circulation of John Dewey's Educational Discourse in the Luso-Afro-Brazilian Space. In R. Bruno-Jofré \& J. Schriewer (Eds.), The global reception of John Dewey's thought (pp. 149-172). New York: Routledge.

Magnin, Ch. (2002). Un survol de l'histoire du BIE, de sa fondation en 1925 jusqu'à aujourd'hui. Exposé présenté en ouverture de la 49e Session du Conseil du BIE, 30 janvier 2002.

Mari, G. (2011). Foi, raison, éducation: de Karl Popper à...Thomas d'Aquin. In M. Soëtard \& G. Le Bouëdec (Eds.), La foi du pédagogue (pp. 43-56). Paris: Don Bosco.

Matasci, D. (2009). Les missions pédagogiques françaises en Allemagne: un exemple de circulation transfrontière des modèles scolaires (18601914). Trajectoires [En ligne], 3. Repéré à https://trajectoires.revues. org $/ 235$

Matasci, D. (2012). L'école républicaine et l'étranger. Acteurs et espaces de l'internationalisation de la "réforme scolaire» en France (1870-première moitié $20^{e}$ siècle). Thèse de doctorat en histoire, Faculté des Lettres, Université de Genève.

Mayer, Ch. (2014a). The Experimental and Community Schools in Hamburg (1919-1933): an introduction. Paedagogica Historica, 50(5), 561-570.

Mayer, Ch. (2014b). Circulation and internationalisation of pedagogical concepts and practices in the discourse of education: The Hamburg school reform experiment (1919-1933). Paedagogica Historica, 50(5), 580-598.

Middleton, S. (2013). Clare Soper's Hat: New Education Fellowship Correspondence between Bloomsburry and New Zealand (19381946). History of Education, 42(1), 92-114.

Middleton, S. (sous presse). New Zealand Theosophists in «New Education» Networks (1880s-1938). History of Education Review. 
Miller, D. \& Oelkers, J. (Eds.) (2014). Reformpädagogik nach der Oldenwaldschule - Wie weiter? Weinheim: Beltz Juventa.

Mole, F. (2010). L'école lä̈que pour une République sociale. Controverses pédagogiques et politiques (1900-1914). Rennes: Presses universitaires de Rennes / INRP.

Mole, F. (2011). L'École rénovée: une revue d'éducation nouvelle entre anarchisme et syndicalisme (1908-1909). Carrefours de l'éducation, 31, 9-22.

Mole, F. (2012). Freinet au Congrès mondial de Nice (1932): une révolution sociale par l'Éducation nouvelle? In L. Gutierrez, L. Besse, \& A. Prost (Eds.), Réformer l'école. L'apport de l'Éducation nouvelle (19301970) (pp. 63-75). Grenoble: Presses universitaires de Grenoble.

Németh, A. (2006). A chapter in the history of an ambivalent relationship Connections between the «new education movement» outside of the universities and academic pedagogy Hungary. In R. Hofstetter \& B. Schneuwly (Ed.), Passion, Fusion, Tension. New education and Educational sciences (end 19th-middle 20th century) (pp. 169-190). Berne: Lang.

Norris, N.D. (2004). The Promise and Failure of Progressive Education. Lanham: Scarecrow Press.

Oelkers, J. (1995). Reformpädagogik: Aktualität und Historie. In W. Böhm \& J. Oelkers (Eds.), Reformpädagogikkontrovers (pp. 23-47). Würzburg: Ergon.

Oelkers, J. (2005). Reformpädagogik: eine kristische Dogmengeschichte. Weinheim: Juventa Verlag.

Oelkers, J. (2006). The Strange Case of German «Geistwissenschaftliche Pädagogik». The Mental Side of the Problem. In R. Hofstetter \& B. Schneuwly (Eds.), Passion, Fusion, Tension. Éducation nouvelle et sciences de l'éducation (fin du 19e-milieu du 20e siècle) (pp. 191-222). Berne: Peter Lang.

Oelkers, J. (2009). John Dewey und die Pädagogik. Weinheim: Beltz Juventa. Oelkers, J. (2010). Reformpädagogik. Entstehungsgeschichten einer internationalen Bewegung. Zug: Klett und Balmer.

Oelkers, J. (2011). Eros und Herrschaft. Die dunklen Seiten der Reformpädagogik. Weinheim: Beltz Verlag.

Oelkers, J. \& Osterwalder, F. (Eds.) (1999). Die Neue Erziehung. Beiträge zur Internationalität der Reformpädagogik. Berne: Peter Lang.

Ohayon, A. (2006). Psychanalyse, éducation nouvelle et éducation morale dans les années 1930 en France. In R. Hofstetter \& 
B. Schneuwly (Eds.) (2006), Passion, Fusion, Tension. Éducation nouvelle et sciences de l'éducation (fin du 19e-milieu du 20e siècle) (pp. 325-340). Berne: Peter Lang.

Ohayon, A., Ottavi, D. \& Savoye, A. (Eds.) (2004). L'Éducation nouvelle, histoire, présence et devenir. Berne: Peter Lang.

Ottavi, D. (2001). De Darwin à Piaget. Pour une histoire de la psychologie de l'enfant. Paris: CNRS.

Ottavi, D. (2004). Observer pour connaître: Niccolò Tomaseo et la découverte de l'enfant. In A. Ohayon, D. Ottavi \& A. Savoye (Eds.), L'Éducation nouvelle, histoire, présence et devenir (pp. 11-28). Berne: Peter Lang.

Ottavi, D. \& Gutierrez, L. (2007). Roger Cousinet, un philosophe à l'épreuve de la pédagogie. Paris: INRP.

Oury, F. \& Pain, J. (1972). Chronique de l'école-caserne. Paris: Maspéro.

Pavillon, M. avec la collaboration de Schoeni, C. (2007). Femmes de lettres, femmes engagées? Écrivaines, journalistes, et réseaux associatifs au tournant du $20^{\mathrm{e}}$ siècle. Les Annuelles, 10, 281-302.

Périsset Bagnoud, D., Schneuwly, B. \& Hofstetter, R. (2007). Infrastructures éditoriales et formes coginitives des Sciences de l'éducation. In R. Hofstetter \& B. Schneuwly, avec la collaboration de M. Cicchini, V. Lussi, L. Criblez \& M. Späni, Émergence des sciences de l'éducation en Suisse. À la croisée de traditions académiques contrastées (fin du 19e-première moitié du 20e siècle) (pp. 409-440). Berne: Peter Lang.

Perrenoud, Ph. (2001). Développer la pratique réflexive dans le métier d'enseignant. Professionnalisation et raison pédagogique. Paris: ESF.

Peyronie, H. (2002). 1922-2002. Pour l'Ère nouvelle et l'Éducation nouvelle 80 ans après. Les Sciences de l'éducation. Pour l'Ère nouvelle, 35, 7-13.

Peyronie, H. (2013). Le mouvement Freinet: du fondateur charismatique à l'intellectuel collectif: regards socio-historiques sur une alternative éducative et pédagogique. Caen: Presses universitaires de Caen.

Poirrier, Ph. (2004). Les enjeux de l'histoire culturelle. Paris: Seuil.

Poucet, B. (2007). Georges Bertier, syndicaliste. Les Études sociales, 145, 19-30.

Prost, A. (1996). Douze leçons sur l'histoire. Paris: Seuil.

Quin-de Stoppani, M.-C. (2007). Cécile Riedel (1906-1989): l'itinéraire d'une pédagogue montessorienne. Les Études sociales, 145, 31-50.

Rabazas Romero, T. (Ed.) (2015). El conocimiento teórico de la educación en España. Evolución y consolidación. Madrid: Síntesis. 
Raillon, L. (1990). Roger Cousinet, une pédagogie de la liberté. Paris: A. Colin.

Raillon, L. (1998). Georges Bertier, pionnier de l'Éducation nouvelle. Les Études sociales, 127-128, 217-228.

Rasmussen, A. (1990). Jalons pour une histoire des congrès internationaux au $\mathrm{XIX}^{\mathrm{e}}$ siècle: Régulation scientifique et propagande intellectuelle. Relations internationales, 62, 115-133.

Rasmussen, A. (2001). 1900. Le tournant internationaliste est-il pensable? Mil neuf cent, 19, 27-41.

Ratcliff, M. \& Ruchat, M. (Eds.) (2006). Les Laboratoires de l'esprit. Genève: Musée d'histoire des sciences.

Raymond, A. (2002). L'éducation morale dans le mouvement de l'Éducation nouvelle. Comment éduquer moralement un enfant? Paris: L'Harmattan.

Raymond, A. (2011). L'éducation naturelle: une idée centrale mais controversée dans les congrès de la Ligue internationale pour l'Éducation nouvelle (1921-1936). Carrefours de l'éducation, 31, 41-60.

Renier, S. (2013). John Dewey et l'enquête de l'enseignant: de l'expérience sociale à la formation du jugement individuel. Éducation $\mathcal{E}$ didactique, 7(1), 165-183.

Renier, S. (2015). Les conditions d'émergence d'une pensée critique: l'itinéraire intellectuel de Marie-Anne Carroi (1920-1940). In A.D. Robert \& B. Garnier (Eds.), La pensée critique des enseignants. Éléments d'histoire et de théorisation (pp. 153-174). Le Havre/Rouen: Presses universitaires du Havre/Rouen.

Reese, W.J. (2001). The Origins of Progressive Education. History of Education Quarterly, 41(1), 1-24.

Revel, J. (2012). Les sciences historiques. In J.-M. Berthelot, Épistémologie des sciences sociales (pp. 19-76). Paris: PUF.

Riondet, X. (2013a). La réception de l'œuvre de Dewey chez les enseignants militants dans les années 50. In H.-L. Go (Ed.), Dewey, penseur de l'éducation (pp. 115-132). Nancy: Presses universitaires de Nancy.

Riondet, X. (2013b). Élise Freinet: des pratiques naturistes aux pratiques de l'École Freinet. Recherches E éducations, 8, 133-146.

Riondet, X. \& Renier, S. (2013). Biographies Le Maîtron: Marie-Anne Carroi. Repéré à http: / / maitron-en-ligne.univ-paris1.fr /

Rogers, R. \& Thébaud, F. (2014). La fabrique des filles. L'éducation des filles de Jules Ferry à la pilule. Vérone: Éditions Textuel.

Röhrs, H. (1994/2000). Maria Montessori. Paris: UNESCO - Bureau international de l'éducation. 
Röhrs, H. \& Lenhart, V. (Eds.) (1994). Die Reformpädagogik auf den Kontinenten. Ein Handbuch. Frankfurt: Peter Lang.

Royston, M. \& E. (2005). Let their lives speak. Switzerland Yearly Meeting. Genève: History \& Biography Project.

Ruchat, M. (2003). Inventer les arriérés pour créer l'intelligence. L'arriéré scolaire et la classe spéciale. Histoire d'un concept et d'une innovation psychopédagogique. Berne: Peter Lang.

Ruchat, M. (2007). Engagement social et esprit de l'Éducation nouvelle: Édouard Claparède, Hélène Antipoff, Marguerite Soubeyran (19291940). Les Études sociales, 145, 7-18.

Ruchat, M. \& Vilbrod, A. (2011). Roland Assathiany: un fonctionnaire militant au service de l'éducation spécialisée. Paris: L'Harmattan.

Saffange, J.-F. (1994). Alexander Sutherland Neill (1883-1973). Perspectives, 24(1-2), 225-236.

Saunier, P.-Y. (2004). Circulations, connexions et espaces transnationaux. Genèses, 4(57), 110-126.

Savoye, A. (2003). École de Plein air et Éducation nouvelle, France 19201954. In A.-M. Châtelet, D. Lerch \& J.-N. Luc (Eds.), L'École de plein air. Une expérience pédagogique et architecturale dans l'Europe du $20^{e}$ siècle (pp. 280-288). Paris: Éditions Recherches.

Savoye, A. (2004). L'Éducation nouvelle en France: de son irrésistible ascension à son impossible pérennisation (1944-1970). In A. Ohayon, D. Ottavi \& A. Savoye (Eds.), L'Éducation nouvelle, histoire, présence et devenir (pp. 145-162). Berne: Peter Lang.

Savoye, A. (2006). Un terrain de rencontre de l'Éducation nouvelle et des Sciences de l'éducation: la réforme de l'enseignement secondaire en France dans l'entre-deux-guerres. In R. Hofstetter \& B. Schneuwly (Eds.), Passion, Fusion, Tension. Éducation nouvelle et sciences de l'éducation (fin du 19e-milieu du $20^{e}$ siècle) (pp. 359-377). Berne: Peter Lang.

Savoye, A. (2009). Nouveaux savoirs et Éducation nouvelle dans les lycées, France 1930-1939. Paedagogica Historica, 45(4 \& 5), 503-514.

Savoye, A. (2010). Langevin et l'expérience des Classes nouvelles, une préfiguration de la réforme de l'enseignement. In L. Gutierrez \& C. Kounelis (Eds.), Paul Langevin et la réforme de l'enseignement (pp. 107-120). Grenoble: Presses universitaires de Grenoble.

Schriewer, J. (1998). Études pluridisciplinaires et réflexions philosophico-herméneutiques: la structuration du discours pédagogique en France et en Allemagne. In P. Drewek \& C. Lüth (Eds.), Histoire des sciences de l'éducation (pp. 57-84). Gent: CSHP. 
Schriewer, J. \& Keiner, E. (1992). Communication Patterns and Intellectual Traditions in Educational Sciences: France and Germany. Comparative Education Review, 36, 25-51.

Seguy, J. Y. (2012). Les classes d'orientation sous le front populaire: une rencontre entre la culture de l'institution scolaire d'état et la culture du courant de l'Éducation nouvelle (1937-1939). In L. Gutierrez, L. Bresse, \& A. Prost (Eds.), Réformer l'école. L'apport de l'Éducation nouvelle (1930-1970) (pp. 19-29). Grenoble: Presses universitaires de Grenoble.

Selleck, R.J.W. (1968). The New Education: The English Background 18701914. London: Pitman and Sons.

Serina-Karsky, F. (2013). Pratiques éducatives et bien-être de l'enfant à l'école: la contribution de l'Éducation nouvelle (1910-2010). Pour un nouveau paradigme éducatif. Thèse de doctorat en sciences de l'éducation, Université de Paris 8.

Serina-Karsky, F. (2014). Un exemple de transfert international de compétences: Louis Raillon, passeur d'Éducation nouvelle entre la France et le Québec. Recherches E éducations, 10, 211-220.

Soëtard, M. \& Le Bouëdec, G. (Eds.) (2011). La foi du pédagogue. Paris: Don Bosco.

Soler Mata, J. (2010). Maria Solà de Sellarés: Teosofia, educació i Escola Nova a Catalunya. In Actes XIX Jornades d'Història de l'Educació (Olot, 18-20 novembre 2010). Annals del Patronat d'Estudis istòrics d'Olot $i$ Comarca (pp. 167-181). Olot: Patronat d'Estudis Històrics d'Olot i Comarca.

Soler Mata, J. (2011). Pedagogía contemporánea y teosofía en Cataluña durante el siglo XX: De los principios a las prácticas educativas. In P. Celada Perandones (Ed.), Arte y oficio de Enseñar. Dos siglos de perspectiva histórica [XVI Coloquio Nacional de Historia de la Educación] (pp. 647-656). El Burgo de Osma (Soria): SEDHE-UVA-CEINCE.

Soler Mata, J. (2012). The Rousseau Institute of Geneva's influence on and presence in Catalan pedagogy in the first third of the $20^{\text {th }}$ century. Catalan Social Sciences Review, 1, 58-87.

Stefanov, S. \& Terziyska, M. (2006). L'influence des idées de l'Éducation nouvelle sur l'émergence et le développement des disciplines pédagogiques en Bulgarie. In R. Hofstetter \& B. Schneuwly (Ed.), Passion, Fusion, Tension. Éducation nouvelle et sciences de l'éducation (fin du 19e-milieu du 20e siècle) (pp. 223-252). Berne: Peter Lang. 
Tröhler, D. \& Oelkers, J. (Eds.) (2005). Pragmatism and Education. Rotterdam: Sense Publications.

Tröhler, D., Oelkers, J., Horlacher, R. \& Fuchs, E. (2015). Challenging national boundaries in the history of education. An outline of the journal «Bildungsgeschichte: International Journal for the Historiography of Education» (IJHE). History of Education $\mathcal{E}$ Children's Literature, 10(1), 23-27.

Van Gorp, A., Depaepe, M. \& Simon, F. (2004). Backing the actor as agent in discipline formation: an example of the «secondary disciplinarization» of the Educational Sciences, based on the networks of Ovide Decroly (1901-1931). Paedagogica Historica, 40(5 \& 6), 591-616.

Verissimo, N. (2007). À travers l'Europe de l'Éducation nouvelle: le périple de Joaquim Tomas (1930). Les Études sociales, 145, 51-58.

Vidal, F. (1989). L'Institut Rousseau au temps des passions. Éducation et recherche, 10, 82-105.

Vidal, F. (1997). L'Éducation nouvelle et l'Esprit de Genève: une utopie politico-pédagogique des années 1920. Équinoxe. Revue de sciences humaines, 17, 81-98.

Wagnon, S. (2013). Ovide Decroly, un pédagogue de l'Éducation nouvelle (1871-1932). Berne: Peter Lang.

Wagnon, S. (2015). Nicolas Smelten (1874-1962), l'infatigable militant de l'enseignement public et des méthodes actives? Cahiers Bruxellois, 47, 112-131.

Washington, P. (1999). La Saga théosophique. De Blavatsky à Krishnamurti (Trad. R. de Claunet). Chambéry: Exergue.

White, M.H. (2001). The New Education Fellowship: an international community of practice. The New Era in Education, 82(3), 71-75.

Wraga, W.G. (2014). Condescension and critical sympathy: Historians of education on progressive education in the United States and England. Paedagogica Historica, 50(1 \& 2), 59-75. 



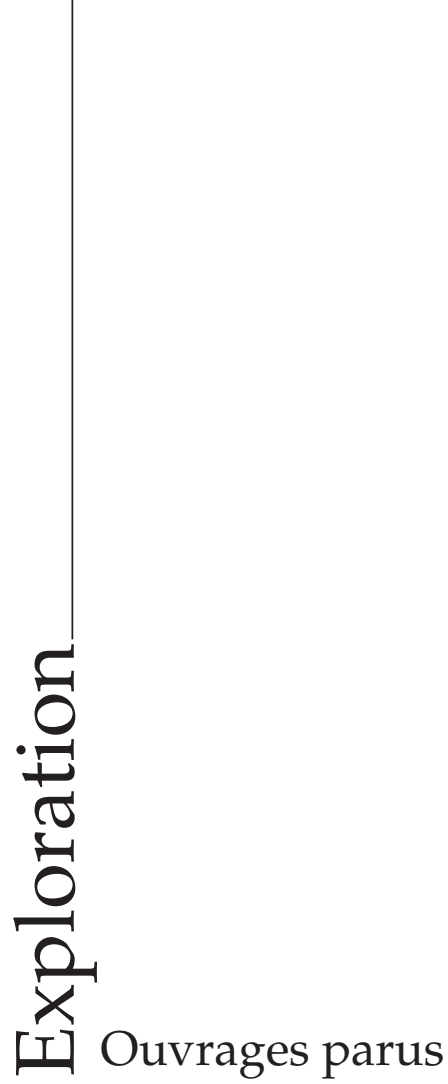

\section{Education: histoire et pensée}

- Catherine Bouve: L'utopie des crèches françaises au XIXe siècle. Un pari sur l'enfant pauvre. Essai socio-historique. 308 p., 2010.

- Loïc Chalmel: La petite école dans l'école - Origine piétiste-morave de l'école maternelle française. Préface de J. Houssaye. 375 p., 1996, 2000, 2005.

- Loïc Chalmel: Jean Georges Stuber (1722-1797) - Pédagogie pastorale. Préface de D. Hameline, XXII, 187 p., 2001.

- Loïc Chalmel: Réseaux philanthropinistes et pédagogie au $18^{e}$ siècle. XXVI, 270 p., 2004.

- Nanine Charbonnel: Pour une critique de la raison éducative. 189 p., 1988.

- Marie-Madeleine Compère: L'histoire de l'éducation en Europe. Essai comparatif sur la façon dont elle s'écrit. (En coédition avec INRP, Paris). 302 p., 1995.

- Jean-François Condette, Jules Payot (1859-1940). Education de la volonté, morale laïque et solidarité. Itinéraire intellectuel et combats pédagogiques au cœur de la III République. 316 p., 2012. 
- Lucien Criblez, Rita Hofstetter (Ed./Hg.), Danièle Périsset Bagnoud (avec la collaboration de/unter Mitarbeit von): La formation des enseignant(e)s primaires. Histoire et réformes actuelles / Die Ausbildung von PrimarlehrerInnen. Geschichte und aktuelle Reformen. VIII, 595 p., 2000.

- Daniel Denis, Pierre Kahn (Ed.): L'Ecole de la Troisième République en questions. Débats et controverses dans le Dictionnaire de pédagogie de Ferdinand Buisson. VII, 283 p., 2006.

- Marcelle Denis: Comenius. Une pédagogie à l'échelle de l'Europe. 288 p., 1992.

- Patrick Dubois: Le Dictionnaire de Ferdinand Buisson. Aux fondations de l'école républicaine (1878-1911). VIII, 243 p., 2002.

- Marguerite Figeac-Monthus: Les enfants de l'Émile? L'effervescence éducative de la France au tournant des XVIII et XIX ${ }^{e}$ siècles. XVII, 326 p., 2015.

- Nadine Fink: Paroles de témoins, paroles d'élèves. La mémoire et l'histoire de la Seconde Guerre mondiale de l'espace public au monde scolaire. XI, 266 p., 2014.

- Philippe Foray: La laïcité scolaire. Autonomie individuelle et apprentissage du monde commun. X, 229 p., 2008.

- Jacqueline Gautherin: Une discipline pour la République. La science de l'éducation en France (1882-1914). Préface de Viviane Isambert-Jamati. XX, 357 p., 2003.

- Daniel Hameline, Jürgen Helmchen, Jürgen Oelkers (Ed.): L'éducation nouvelle et les enjeux de son histoire. Actes du colloque international des archives Institut Jean-Jacques Rousseau. VI, 250 p., 1995.

- Béatrice Haenggeli-Jenni: L'Éducation nouvelle entre science et militance. Débats et combats à travers la revue Pour l'Ère Nouvelle (1920-1940). VIII, 361 p., 2017.

- Rita Hofstetter: Les lumières de la démocratie. Histoire de l'école primaire publique à Genève au XIXe siècle. VII, 378 p., 1998.

- Rita Hofstetter, Charles Magnin, Lucien Criblez, Carlo Jenzer ( $\dagger$ ) (Ed.): Une école pour la démocratie. Naissance et développement de l'école primaire publique en Suisse au 19e siècle. XIV, 376 p., 1999.

- Rita Hofstetter,Bernard Schneuwly (Ed./Hg.): Science(s) de l'éducation (19e-20esiècles) - Erziehungswissenschaft(en) (19.-20. Jahrhundert). Entre champs professionnels et champs disciplinaires - Zwischen Profession und Disziplin. 512 p., 2002.

- Rita Hofstetter, Bernard Schneuwly (Ed.): Passion, Fusion, Tension. New Education and Educational Sciences - Education nouvelle et Sciences de l'éducation. End 19th-middle 20th century - Fin du 19e - milieu du $20^{e}$ siècle. VII, 397 p., 2006.

- Rita Hofstetter, Bernard Schneuwly (Ed.), avec la collaboration de Valérie Lussi, Marco Cicchini, Lucien Criblez et Martina Späni: Emergence des sciences de l'éducation en Suisse à la croisée de traditions académiques contrastées. Fin du $19^{e}$ - première moitié du $20^{e}$ siècle. XIX, 539 p., 2007.

- Jean Houssaye: Théorie et pratiques de l'éducation scolaire (1): Le triangle pédagogique. Préface de D. Hameline. 267 p., 1988, 1992, 2000.

- Jean Houssaye: Théorie et pratiques de l'éducation scolaire (2): Pratique pédagogique. 295 p., 1988. 
- Alain Kerlan: La science n'éduquera pas. Comte, Durkheim, le modèle introuvable. Préface de N. Charbonnel. 326 p., 1998.

- Francesca Matasci: L'inimitable et l'exemplaire: Maria Boschetti Alberti. Histoire et figures de l’Ecole sereine. Préface de Daniel Hameline. 232 p., 1987.

- Pierre Ognier: L'Ecole républicaine française et ses miroirs. Préface de D. Hameline. 297 p.,1988.

- Annick Ohayon, Dominique Ottavi \& Antoine Savoye (Ed.): L'Education nouvelle, histoire, présence et devenir. VI, 336 p., 2004, 2007.

- Johann Heinrich Pestalozzi: Ecrits sur l'expérience du Neuhof. Suivi de quatre études de P.-Ph. Bugnard, D. Tröhler, M. Soëtard et L. Chalmel. Traduit de l'allemand par P.-G. Martin. X, 160 p., 2001.

- Johann Heinrich Pestalozzi: Sur la législation et l'infanticide. Vérités, recherches et visions. Suivi de quatre études de M. Porret, M.-F. Vouilloz Burnier, C. A. Muller et M. Soëtard. Traduit de l'allemand par P.-G. Matin. VI, 264 p.,2003.

- Martine Ruchat: Inventer les arriérés pour créer l'intelligence. L'arriéré scolaire et la classe spéciale. Histoire d'un concept et d'une innovation psychopédagogique 1874-1914. Préface de Daniel Hameline. XX, 239 p., 2003.

- Jean-François Saffange: Libres regards sur Summerhill. L'œuvre pédagogique de A.-S. Neill. Préface de D. Hameline. 216 p., 1985.

- Michel Soëtard, Christian Jamet (Ed.): Le pédagogue et la modernité. A l'occasion du 250 anniversaire de la naissance de Johann Heinrich Pestalozzi (1746-1827). Actes du colloque d'Angers (9-11 juillet 1996). IX, 238 p., 1998.

- Alain Vergnioux: Pédagogie et théorie de la connaissance. Platon contre Piaget? 198 p., 1991.

- Alain Vergnioux (éd.): Grandes controverses en éducation. VI, 290 p., 2012.

- Marie-Thérèse Weber: La pédagogie fribourgeoise, du concile de Trente à Vatican II. Continuité ou discontinuité? Préface de G. Avanzini. 223 p., 1997.

\section{Recherches en sciences de l'éducation}

- Sandrine Aeby Daghé: Candide, La fée carabine et les autres. Vers un modèle didactique de la lecture littéraire. IX, 303 p., 2014.

- Linda Allal, Jean Cardinet, Phillipe Perrenoud (Ed.): L'évaluation formative dans un enseignement différencié. Actes du Colloque à l'Université de Genève, mars 1978. 264 p., 1979, 1981, 1983, 1985, 1989, 1991, 1995.

- Claudine Amstutz, Dorothée Baumgartner, Michel Croisier, Michelle Impériali, Claude Piquilloud: L'investissement intellectuel des adolescents. Recherche clinique. XVII, 510 p., 1994.

- Bernard André: S'investir dans son travail: les enjeux de l'activité enseignante. XII, 289 p., 2013

- Guy Avanzini (Ed.): Sciences de l'éducation: regards multiples. 212 p., 1994. 
- Daniel Bain: Orientation scolaire et fonctionnement de l'école. Préface de J. B. Dupont et F. Gendre. VI, 617 p., 1979.

- Jean-Michel Baudouin: De l'épreuve autobiographique. Contribution des histoires de vie à la problématique des genres de texte et de l'herméneutique de l'action. XII, 532 p., 2010.

- Véronique Bedin \& Laurent Talbot (éd.): Les points aveugles dans l'évaluation des dispositifs d'éducation ou de formation. VIII, 211 p., 2013.

- Ana Benavente, António Firmino da Costa, Fernando Luis Machado, Manuela Castro Neves: De l'autre côté de l'école. 165 p., 1993.

- Jean-Louis Berger: Apprendre : la rencontre entre motivation et métacognition. Autorégulation dans l'apprentissage des mathématiques en formation professionnelle. XI, 221 p., 2015

- Denis Berthiaume \& Nicole Rege Colet (Ed.): La pédagogie de l'enseignement supérieur: repères théoriques et applications pratiques. Tome 1: Enseigner au supérieur. 345 p., 2013.

- Anne-Claude Berthoud, Bernard Py: Des linguistes et des enseignants. Maîtrise et acquisition des langues secondes. 124 p., 1993.

- Pier Carlo Bocchi: Gestes d'enseignement. L'agir didactique dans les premières pratiques d'écrit . 378 p., 2015.

- Dominique Bucheton: Ecritures-réécritures - Récits d'adolescents. 320 p., 1995.

- Sandra Canelas-Trevisi: La grammaire enseignée en classe. Le sens des objets et des manipulations. 261 p., 2009.

- Jean Cardinet, Yvan Tourneur (†): Assurer la mesure. Guide pour les études de généralisabilité. 381 p., 1985.

- Felice Carugati, Francesca Emiliani, Augusto Palmonari: Tenter le possible. Une expérience de socialisation d'adolescents en milieu communautaire. Traduit de l'italien par Claude Béguin. Préface de R. Zazzo. 216 p., 1981.

- Evelyne Cauzinille-Marmèche, Jacques Mathieu, Annick Weil-Barais: Les savants en herbe. Préface de J.-F. Richard. XVI, 210 p., 1983, 1985.

- Vittoria Cesari Lusso: Quand le défi est appelé intégration. Parcours de socialisation et de personnalisation de jeunes issus de la migration. XVIII, 328 p., 2001.

- Nanine Charbonnel (Ed.): Le Don de la Parole. Mélanges offerts à Daniel Hameline pour son soixante-cinquième anniversaire. VIII, 161 p., 1997.

- Gisèle Chatelanat, Christiane Moro, Madelon Saada-Robert (Ed.): Unité et pluralité des sciences de l'éducation. Sondages au cour de la recherche. VI, 267 p., 2004.

- Florent Chenu: L'évaluation des compétences professionnelles. Une mise à l'épreuve expérimentale des notions et présupposés théoriques sous-jacents. 347 p., 2015.

- Christian Daudel: Les fondements de la recherche en didactique de la géographie. 246 p., 1990.

- Bertrand Daunay: La paraphrase dans l'enseignement du français. XIV, 262 p., 2002.

- Jean-Marie De Ketele: Observer pour éduquer. (Epuisé)

- Jean-Louis Derouet, Marie-Claude Derouet-Besson (éds.): Repenser la justice dans le domaine de l'éducation et de la formation. VIII, 385 p., 2009. 
- Ana Dias-Chiaruttini: Le débat interprétatif dans l'enseignement du français. IX, 261 p., 2015.

- Joaquim Dolz, Jean-Claude Meyer (Ed.): Activités métalangagières et enseignement du français. Actes des journées d'étude en didactique du français (Cartigny, 28 février - 1 mars 1997). XIII, 283 p., 1998.

- Pierre Dominicé: La formation, enjeu de l'évaluation. Préface de B. Schwartz. (Epuisé)

- Pierre-André Doudin, Daniel Martin, Ottavia Albanese (Ed.): Métacognition et éducation. XIV, 392 p., 1999, 2001.

- Pierre Dominicé, Michel Rousson: L'éducation des adultes et ses effets. Problématique et étude de cas. (Epuisé)

- Andrée Dumas Carré, Annick Weil-Barais (Ed.): Tutelle et médiation dans l'éducation scientifique. VIII, 360 p., 1998.

- Jean-Blaise Dupont, Claire Jobin, Roland Capel: Choix professionnels adolescents. Etude longitudinale à la fin de la scolarité secondaire. 2 vol., 419 p., 1992.

- Vincent Dupriez, Jean-François Orianne, Marie Verhoeven (Ed.): De l'école au marché du travail, l'égalité des chances en question. X, 411 p., 2008.

- Raymond Duval: Sémiosis et pensée humaine - Registres sémiotiques et apprentissages intellectuels. 412 p., 1995.

- Eric Espéret: Langage et origine sociale des élèves. (Epuisé)

- Jean-Marc Fabre: Jugement et certitude. Recherche sur l'évaluation des connaissances. Préface de G. Noizet. (Epuisé)

- Georges Felouzis et Gaële Goastellec (Éd.): Les inégalités scolaires en Suisse. École, société et politiques éducatives. VI, 273 p., 2015.

- Monique Frumholz: Ecriture et orthophonie. 272 p., 1997.

- Pierre Furter: Les systèmes de formation dans leurs contextes. (Epuisé)

- Monica Gather Thurler, Isabelle Kolly-Ottiger, Philippe Losego et Olivier Maulini, Les directeurs au travail. Une enquête au cœur des établissements scolaires et socio-sanitaires. VI, 318 p., 2017.

- André Gauthier (Ed.): Explorations en linguistique anglaise. Aperçus didactiques. Avec JeanClaude Souesme, Viviane Arigne, Ruth Huart-Friedlander. 243 p., 1989.

- Marcelo Giglio \& Francesco Arcidiacono (Eds): Les interactions sociales en classe: réflexions et perspectives. VI, 250 p., 2017.

- Patricia Gilliéron Giroud \& Ladislas Ntamakiliro (Ed.): Réformer l'évaluation scolaire: mission impossible. 264 p. 2010.

- Michel Gilly, Arlette Brucher, Patricia Broadfoot, Marylin Osborn: Instituteurs anglais instituteurs francais. Pratiques et conceptions du rôle. XIV, 202 p., 1993.

- André Giordan: L'élève et/ou les connaissances scientifiques. Approche didactique de la construction des concepts scientifiques par les élèves. $3^{e}$ édition, revue et corrigée. 180 p., 1994.

- André Giordan, Yves Girault, Pierre Clément (Ed.): Conceptions et connaissances. 319 p., 1994. 
- André Giordan (Ed.): Psychologie génétique et didactique des sciences. Avec Androula Henriques et Vinh Bang. (Epuisé)

- Corinne Gomila: Parler des mots, apprendre à lire. La circulation du métalangage dans les activités de lecture. X, 263 p. 2011.

- Armin Gretler, Ruth Gurny, Anne-Nelly Perret-Clermont, Edo Poglia (Ed.): Etre migrant. Approches des problèmes socio-culturels et linguistiques des enfants migrants en Suisse. 383 p., 1981, 1989.

- Francis Grossmann: Enfances de la lecture. Manières de faire, manières de lire à l'école maternelle. Préface de Michel Dabène. 260 p., 1996, 2000.

- Jean-Pascal Simon, Francis Grossmann (Ed.): Lectureà l'Université. Langue maternelle, seconde et étrangère. VII, 289 p., 2004.

- Michael Huberman, Monica Gather Thurler: De la recherche à la pratique. Eléments de base et mode d'emploi. 2 vol., 335 p., 1991.

- Institut romand de recherches et de documentation pédagogiques (Neuchâtel): Connaissances mathématiques à l'école primaire: J.-F. Perret: Présentation et synthèse d'une évaluation romande; F. Jaquet, J. Cardinet: Bilan des acquisitions en fin de première année; F. Jaquet, E. George, J.-F. Perret: Bilan des acquisitions en fin de deuxième année; J.-F. Perret: Bilan des acquisitions en fin de troisième année; R. Hutin, L.-O. Pochon, J.-F. Perret: Bilan des acquisitions en fin de quatrième année; L.-O. Pochon: Bilan des acquisitions en fin de cinquième et sixième année. 1988-1991.

- Daniel Jacobi: Textes et images de la vulgarisation scientifique. Préface de J. B. Grize. (Epuisé)

- René Jeanneret (Ed.): Universités du troisième âge en Suisse. Préface de P. Vellas. 215 p., 1985.

- Samuel Johsua, Jean-Jacques Dupin: Représentations et modélisations: le «débat scientifique» dans la classe et l'apprentissage de la physique. 220 p., 1989.

- Constance Kamii: Les jeunes enfants réinventent l'arithmétique. Préface de B. Inhelder. 171 p., 1990, 1994.

- Helga Kilcher-Hagedorn, Christine Othenin-Girard, Geneviève de Weck: Le savoir grammatical des élèves. Recherches et réflexions critiques. Préface de J.-P. Bronckart. 241 p., 1986.

- Georges Leresche $(\dagger)$ : Calcul des probabilités. (Epuisé)

- Francia Leutenegger: Le temps d'instruire. Approche clinique et expérimentale du didactique ordinaire en mathématique. XVIII, 431 p., 2009.

- Even Loarer, Daniel Chartier, Michel Huteau, Jacques Lautrey: Peut-onéduquer l'intelligence? L'évaluation d'une méthode d'éducation cognitive. 232 p., 1995.

- Georges Lüdi, Bernard Py: Etre bilingue. 4e édition. XII, 223 p., 2013.

- Brigitte Louichon, Marie-France Bishop, Christophe Ronveaux (Ed.): Les fables à l'école. Un genre patrimonial européen? VII, 279 p., 2017.

- Valérie Lussi Borer: Histoire des formations à l'enseignement en Suisse romande. X, 238 p., 2017.

- Pierre Marc: Autour de la notion pédagogique d'attente. 235 p., 1983, 1991, 1995. 
- Jean-Louis Martinand: Connaître et transformer la matière. Préface de G. Delacôte. (Epuisé)

- Jonas Masdonati: La transition entre école et monde du travail. Préparer les jeunes à l'entrée en formation professionnelle. 300 p., 2007.

- Marinette Matthey: Apprentissage d'une langue et interaction verbale. XII, 247 p., 1996, 2003.

- Paul Mengal: Statistique descriptive appliquée aux sciences humaines. VII, 107 p., 1979, 1984, 1991, 1994, $1999\left(5^{\mathrm{e}}+6^{\mathrm{e}}\right), 2004$.

- Isabelle Mili: L'œuvre musicale, entre orchestre et écoles. Une approche didactique de pratiques d'écoute musicale. X, 228 p., 2014.

- Henri Moniot (Ed.): Enseigner l'histoire. Des manuels à la mémoire. (Epuisé)

- Cléopâtre Montandon, Philippe Perrenoud: Entre parents et enseignants: un dialogue impossible? Nouvelle édition, revue et augmentée. 216 p., 1994.

- Christiane Moro, Bernard Schneuwly, Michel Brossard (Ed.): Outils et signes. Perspectives actuelles de la théorie de Vygotski. 221 p., 1997.

- Christiane Moro \& Cintia Rodríguez: L'objet et la construction de son usage chez le bébé. Une approche sémiotique du développement préverbal. X, 446 p., 2005.

- Lucie Mottier Lopez: Apprentissage situé. La microculture de classe en mathématiques. XXI, 311 p., 2008.

- Lucie Mottier Lopez \& Walther Tessaro (éd.): Le jugement professionnel, au cœur de l'évaluation et de la régulation des apprentissages. VII, 357 p., 2016.

- Gabriel Mugny (Ed.): Psychologie sociale du développement cognitif. Préface de M. Gilly. (Epuisé)

- Romuald Normand: Gouverner la réussite scolaire. Une arithmétique politique des inégalités. XI, 260 p., 2011.

- Sara Pain: Les difficultés d'apprentissage. Diagnostic et traitement. 125 p., 1981, 1985, 1992.

- Sara Pain: La fonction de l'ignorance. (Epuisé)

- ChristianePerregaux:Lesenfantsàdeuxvoix. Deseffetsdubilinguismesuccessifsurl'apprentissage de la lecture. 399 p., 1994.

- Jean-François Perret: Comprendre l'écriture des nombres. 293 p., 1985.

- Anne-Nelly Perret-Clermont: La construction de l'intelligence dans l'interaction sociale. Edition revue et augmentée avec la collaboration de Michèle Grossen, Michel Nicolet et Maria-Luisa Schubauer-Leoni. 305 p., 1979, 1981, 1986, 1996, 2000.

- Edo Poglia, Anne-Nelly Perret-Clermont, Armin Gretler, Pierre Dasen (Ed.): Pluralité culturelle et éducation en Suisse. Etre migrant. 476 p., 1995.

- Jean Portugais: Didactique des mathématiques et formation des enseignants. 340 p., 1995.

- Laetitia Progin: Devenir chef d'établissement. Le désir de leadership à l'épreuve de la réalité. 210 p., 2017. 
- Nicole Rege Colet \& Denis Berthiaume (Ed.): La pédagogie de l'enseignement supérieur: repères théoriques et applications pratiques. Tome 2. Se développer au titre d'enseignant. VI, 261 p., 2015

- Yves Reuter (Ed.): Les interactions lecture-écriture. Actes du colloque organisé par THÉODILE-CREL (Lille III, 1993). XII, 404 p., 1994, 1998.

- Philippe R. Richard: Raisonnement et stratégies de preuve dans l'enseignement des mathématiques. XII, 324 p., 2004.

- Marielle Rispail et Christophe Ronveaux (Ed.): Gros plan sur la classe de français. Motifs et variations. X, 258 p., 2010.

- Yviane Rouiller et Katia Lehraus (Ed.): Vers des apprentissages en coopération: rencontres et perspectives. XII, 237 p., 2008.

- Guy Rumelhard: La génétiqueet ses représentations dans l'enseignement. Préface de A. Jacquard. 169 p., 1986.

- El Hadi Saada: Les langues et l'école. Bilinguisme inégal dans l'école algérienne. Préface de J.-P. Bronckart. 257 p., 1983.

- Muriel Surdez: Diplômes et nation. La constitution d'un espace suisse des professions avocate et artisanales (1880-1930). X, 308 p., 2005.

- Valérie Tartas: La construction du temps social par l'enfant. Préfaces de Jérôme Bruner et Michel Brossard XXI, 252 p., 2008.

- Sabine Vanhulle: Des savoirs en jeu aux savoirs en «je». Cheminements réflexifs et subjectivation des savoirs chez de jeunes enseignants en formation. 288 p., 2009.

- Joëlle Vlassis: Sens et symboles en mathématiques. Etude de l'utilisation du signe «moins» dans les réductions polynomiales et la résolution d'équations du premier degré à inconnue. XII, 437 p., 2010.

- Gérard Vergnaud: L'enfant, la mathématique et la réalité. Problèmes de l'enseignement des mathématiques à l'école élémentaire. V, 218 p., 1981, 1983, 1985, 1991, 1994.

- Nathanaël Wallenhorst: L'école en France et en Allemagne. Regard de lycéens, comparaison d'expériences scolaires. IX, 211 p., 2013.

- Jacques Weiss (Ed.): A la recherche d'une pédagogie de la lecture. (Epuisé)

- Martine Wirthner: Outils d'enseignement : au-delà de la baguette magique. Outils transformateurs, outils transformés dans des séquences d'enseignement en production écrite. XI, 259 p., 2017.

- Richard Wittorski, Olivier Maulini \& Maryvonne Sorel (éds). Les professionnels et leurs formations. Entre développement des sujets et projets des institutions. VI, 237 p., 2015.

- Tania Zittoun: Insertions. A quinze ans, entre échec et apprentissage. XVI, 192 p., 2006. 